\title{
Living and Fossil Brachiopod Genera 1775-1979 LISTS AND BIBLIOGRAPHY
}

\section{REX A. DOESGHER}




\title{
SERIES PUBLICATIONS OF THE SMITHSONIAN INSTITUTION
}

Emphasis upon publication as a means of "diffusing knowledge" was expressed by the first Secretary of the Smithsonian. In his formal plan for the Institution, Joseph Henry outlined a program that included the following statement: "It is proposed to publish a series of reports, giving an account of the new discoveries in science, and of the changes made from year to year in all branches of knowledge." This theme of basic research has been adhered to through the years by thousands of titles issued in series publications under the Smithsonian imprint, commencing with Smithsonian Contributions to Knowledge in 1848 and continuing with the following active series:

\author{
Smithsonian Contributions to Anthropology \\ Smithsonian Contributions to Astrophysics \\ Smithsonian Contributions to Botany \\ Smithsonian Contributions to the Earth Sciences \\ Smithsonian Contributions to the Marine Sciences \\ Smithsonian Contributions to Paleobiology \\ Smithsonian Contributions to Zoology \\ Smithsonian Studies in Air and Space \\ Smithsonian Studies in History and Technology
}

In these series, the Institution publishes small papers and full-scale monographs that report the research and collections of its various museums and bureaux or of professional colleagues in the world cf science and scholarship. The publications are distributed by mailing lists to libraries, universities, and similar institutions throughout the world.

Papers or monographs submitted for series publication are received by the Smithsonian Institution Press, subject to its own review for format and style, only through departments of the various Smithsonian museums or bureaux, where the manuscripts are given substantive review. Press requirements for manuscript and art preparation are outlined on the inside back cover.

\author{
S. Dillon Ripley \\ Secretary \\ Smithsonian Institution
}


SMITHSONIAN CONTRIBUTIONS TO PALEOBIOLOGY $\bullet$ NUMBER 42

\title{
Living and Fossil Brachiopod Genera 1775-1979 LISTS AND BIBLIOGRAPHY
}

\author{
Rex A. Doescher
}

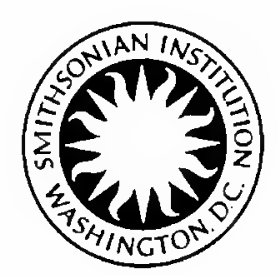

SMITHSONIAN INSTITUTION PRESS

City of Washington 1981 


\section{A B S T R A C T}

Doescher, Rex A. Living and Fossil Brachiopod Genera 1775-1979: Lists and Bibliography. Smithsonian Contributions to Paleobiology, number 42, 238 pages, 1981.-All genera and subgenera of fossil and living brachiopods described from 1775 to 1979 are listed with comments on their current nomenclatural status. A computerized list presents all valid brachiopod genera (and subgenera), including subjective synonyms, with additional information regarding type-species, superfamily classification, and range (by period) of each genus. A bibliography supplements both lists.

OfFicial publication Date is handstamped in a limited number of initial copies and is recorded in the Institution's annual report, Smithsonian Year. Series cover design: The trilobite Phacops rana Green.

Library of Congress Cataloging in Publication Data

Doescher, Rex $\Lambda$

Living and fossil brachiopod genera $1775-1979$.

(Smithsonian contributions to paleobiology; no. 42)

Bibliography: p.

1. Brachiopoda. 2. Brachiopoda, Fossil. 3. Brachiopoda-Bibliography. 4. Brachiopoda, Fossil-Bibliography. I. Title. II. Series: Smithsonian Institution. Smithsonian contributions to paleobiology; no. 42.

QE701.S56 no. 42 [QL395] 560s [594'.8] 80-607773 rev 


\section{Contents}

Introduction

Acknowledgments

1

Annotated List of Genera and Subgenera $\ldots \ldots \ldots \ldots \ldots \ldots \ldots \ldots$

Computerized List of Valid Genera and Subgenera ............. 44

Literature Cited

187 



\title{
Living and Fossil Brachiopod Genera 1775-1979 LISTS AND BIBLIOGRAPHY
}

\author{
Rex A. Doescher
}

\section{Introduction}

Fifteen years have elapsed since the Treatise on Invertebrate Paleontology, Part $H$ : Brachiopoda ${ }^{1}$ became the standard reference for brachiopod workers worldwide. The Treatise recognized approximately 1700 distinct genera and placed an additional 500 in synonomy; subsequently, over 1200 new genera of brachiopods have been proposed. In less than two decades the generic list grew to a size 50 percent greater than that amassed in the preceding 204 year interval. Some of this growth was described in Richard E. Grant's presidential address to the Paleontological Society in November $1979 .^{2}$

It became apparent that an updated brachiopod generic list was necessary to complement both the Zoological Record and Neave's Nomenclator Zoologicus, which have often lagged years behind current research. One aspect of the effort to compile such a list was the creation of a file that

\footnotetext{
${ }^{1}$ Moore, Raymond C., editor, Treatise on Invertebrate Paleontology, Part H: Brachiopoda, 2 volumes (Lawrence, Kansas: The Geological Society of America and University of Kansas Press, 1965).

${ }^{2}$ Grant, R. E., "The Human Face of the Brachiopod," Journal of Paleontology, volume 54, number 3 (1980), pages 499-507, 9 figures.
}

Rex A. Doescher, Department of Paleobiology, National Museum of Natural History, Smithsonian Institution, Washington, D.C. 20560. contains photocopies or reprints of over 3800 references to new brachiopod genera. Also, in recognition of the benefits relating to computer data retrieval, a computer data file was built utilizing a Honeywell (Series 60) Model 66/05 computer under the Time Sharing and Text Editor subsystem. Henceforth, if a researcher has a specific area of interest, e.g., Permian Productacea, the data can be sorted on the desired fields exclusively, thereby limiting the printout to his area of interest and also providing him with an updated list of all brachiopod genera in this category within months, rather than years, of publication. The present study is the outgrowth of the compilation of these manual and computer files.

The "Annotated List of Genera and Subgenera" contains all the names of brachiopod genera and subgenera published between 1775 and 1979 . Literature citations for original publications are given for all valid genera and subgenera, including subjective synonyms. Symbols or annotations designate junior subjective synonyms, junior objective synonyms, junior homonyms, misspellings, nomina nuda, replacement names, and other nomenclatural problems. In literature citations, the page or plate number locates the designation of the type-species. Multiple citations are given if the taxon has been presented as "new" in more than one publication. The "Literature Cited" is 
composed of those works referenced in the "Annotated List."

Notice of the publication of several genera was received too late to include them in the "Computerized List"; however, they have been added to the "Annotated List." These genera are as follows: Achunoproductus Ustritsky, 1971; Agyrekia Koneva, 1979; Edreja Koneva, 1979; Infurca Percival, 1979; Ivshinella Koneva, 1979; Palaeoschmidtites Koneva, 1979; Parvulaltarostrum Sartenaer, 1979; Pseudoprotathyris Modzalevskaja, 1979; Svalbardia Afanasjeva, 1977a; Tethorotes Manankov, 1979; Tythothyris Zezina, 1979; and Uraloproductus Ustritsky, 1971.

The terminology used in the column heads of the "Computerized List of Valid Genera and Subgenera" is explained below. A number in parentheses marks each of the eight sortable data fields.

Genus(1): All nomina nuda, misspellings, junior objective synonyms, junior homonyms, ichnogenera, and other invalid names are omitted from the list. All subjective synonyms that are so indicated by the Treatise in 1965, however, are included in deference to each researcher's opinion.

Author(2): The author responsible for establishing the new genus or subgenus. The name is spelled in the manner in which it appeared on the original publication. For computer compatibility, lengthy multiple names are designated by the primary author plus et al. Some authors have published under several names: Jin Yu-gan has used Jing and Ching Yu-kan; G. A. Afanasjeva was once known as G. A. Besnossova; and A. Rollet became by marriage A. Boullier. Cross references, where name variations are known, are given in the "Literature Cited."

Date(3): The year in which the genus or subgenus was published.

Type-Species: The primary type of a genus or subgenus. In this category, the generic(4) as well as specific(5) term is sortable. The author/date(6) of publication of the type-species is included.

Superfamily(7): Superfamily designations come primarily from the Treatise. All new genera or subgenera after 1965 are placed in the superfam- ily designated or created by the author. The author and date of publication of the superfamily is noted.

Range(8): Range designations come solely from the Treatise. No attempt has been made to expand the ranges as indicated by the Treatise or the ranges of all new genera subsequent to 1965 .

It is hoped that all future publications that include new brachiopod genera will be sent to me so that pertinent data can be entered in the computer file and updated lists can be published periodically. I would also like to be informed of any errors or omissions in these lists.

Acknowledgments.-To Dr. G. A. Cooper, I owe special thanks for creating the generic and specific files, for spending numerous hours assisting me with problems of nomenclature, and for reviewing this manuscript; to Mrs. Josephine W. Cooper, whose dedication to the maintenance of the bibliographic files and whose translation of the Russian literature made this work possible; to the entire staff of the National Museum of Natural History Library, especially Jack Marquardt, Carolyn Hahn, Betty Jean Swartz, Amy Levin, and Susan Mackler for their patient response to my many requests; to Dante Piacesi, who, along with Karen Collins, wrote the programs to enable me to modify and extract the raw data in a meaningful way; to Harrison Sheng and Yin Hongfu for their help in transliterating and translating the Chinese literature references; to Drs. Jess Johnson, Howard Brunton, and Bert Rowell, who kept me abreast of new brachiopod genera; to Barbara Spann, editor of series publications with the Smithsonian Institution Press, for assisting me in the researching and compiling of the bibliographic information; to Dr. Curtis Sabrosky, research entomologist, for his help with the International Code of Zoological Nomenclature; to Dr. Richard E. Grant, for allowing me the time to pursue this goal and for reviewing this manuscript. Finally, I would like to dedicate this work, not only to the many un-named friends who had a part in this project, but also to my wife, Joan, whose encouragement was a constant inspiration to me. 


\title{
Annotated List of Genera and Subgenera
}

\author{
$\left({ }^{*}=\right.$ junior subjective synonym (Treatise); ${ }^{* *}=$ junior objective synonym; \\ $\dagger=$ junior homonym; $\ddagger=$ not included in "Computerized List")
}

$A$, Legrand-Blain, 1968 [un-named]

Abichia Askerov, 1964; 1965; 1967 [nomina nuda]

Abrekia Dagys, 1974:98

Abyssothyris Thomson, 1927:190

Acambona White, 1865:27

Acanthambonia Cooper, 1956c:212

Acanthatia Muir-Wood and Cooper, 1960:158

Acanthocosta Roberts, 1971:104

Acanthocrania Williams, 1943:71

Acanthoplecta Muir-Wood and Cooper, 1960:171

Acanthoproductus Martynova, 1970:59

Acanthorhynchia Buckman, 1914 [nomen nudum]; 1917:69

Acanthorthis Neuman, 1977:22

†Acanthospira Weller, 1914 [replaced by Acanthospirna]

Acanthospinfer Menakova, 1964:34

Acanthospirina Schuchert and LeVene, 1929:119 [new name for Acanthospira]

Acanthothiris Orbigny, 1850:323

Acanthothyris._Bronn, 1862; Paetel, 1875 [misspelling of Acanthothiris]

Acanthothyropsis Kamyschan, 1973, in Kamyschan and Babanova, 1973:64

Acculina Misius, 1977, in Misius and Ushatinskaia, 1977: 113

$\ddagger$ Achunoproductus Ustritsky, 1971:21

Acidotocarena Liu, 1979:507

Acolosia Cooper and Grant, 1976a:2051

Acosarina Cooper and Grant, 1969:2

Acrites.-Davidson and King, 1874 [misspelling of Acritis]

**Acritis Volborth, 1869 [= Aulonotreta]

Acrilosia Cooper and Grant, 1969:5

${ }^{*}$ Acrosaccus Willard, 1928:259 [= Schizotreta]

Acrospirifer Helmbrecht and Wedekind, 1923:952

Acrothele Linnarsson, 1876:21

Acrothyra Matthew, 1901:303

Acrothyres Hou, 1963:419

Acrotrela Kutorga, 1848:275

Acrotretella Ireland, 1961:1140

Actinoconchus McCoy, 1844:149

Actinomena Öpik, 1930:166

Acuminothyris Roberts, 1963:15

Acutatheca Stainbrook, 1945:55

Acutella Ljaschenko, 1973:119

(Acutilineolus) Amsden, 1978:31 [subgenus of Eospirifer]
Adiaphragma Xian, 1978b:299

Adminiculoria Waterhouse and Gupta, 1978b:133

Admixtella Rozman, 1978:84

Adolfia Gürich, 1909:137 [replacement by Guerichella invalid, Adolfia not preoccupied]

Adolfispirifer Krylova, 1962:75

Adrenia Chatterton, 1973:126

Adriana Gregorio, 1930a:29

Adygella Dagys, 1959a:25

Adygelloides Dagys, 1959a:28

Aegiria Öpik, 1933a:55

Aegiromena Havlíček, 1961b:450

Aegironetes Havlícek, 1967a:46

Aemula Steinich, 1968a: 193

Aenigmastricklandia Ziegler, 1966:347

Aenigmastrophia Boucot, 1971:156

Aenigmathyris Cooper, 1971:F2

Aequspiriferina Yang and Yin, 1962, in Yang et al., 1962: 110

Aerothyris Allan, 1939:245

Aesopomum Havliček, 1965a:293

Aetheia Thomson, 1915b:389

Afghanispira Dagys, 1968 [nomen nudum]

Afghanospirifer Plodowski, 1968:255

Afilasma Stehli, 1961b:460

Agastapleura Xu, 1978:211

Agelesia Cooper and Grant, 1969:6

Agernella Pajaud and Patrulius, [1965]:582

Agramatia Sokolskaja, 1948:39

Agulhasia King, 1871:109

$\ddagger$ Agyrekia Koneva, 1979:60

Ahizella Opik, 1932:37

Airtonia Cope, 1934:275

Ajukuzella Ovtsharenko, 1977 [nomen nudum]

Aklassia Popov, 1976, in Nazarov and Popov, 1976:37

Ala Nalivkin, 1979:135

Alaskospira Kirk and Amsden, 1952:61

Alatiformia Struve, 1963:499

Alatoproductus Ching and Zhu, 1978, in Jing and $\mathrm{Hu}, 1978$ : 120

Aldanispirifer Alekseeva, 1967:96

Aldanotreta Pelman, 1977:51

Aldingia Thomson, 1916b:501

Aldynkulirhynchia Ovtsharenko, 1977 [nomen nudum] 
Alexenia Ivanova, 1935, in Ivanov, 1935:89

Alichovia Gorjansky, 1969:97

Aliconchidium St. Joseph, 1942:247

†Alifera Muir-Wood and Cooper, 1960 [replaced by Alitaria]

Alimbella Andreeva, 1960, in Markowskii, 1960:293

Alipunctifera Waterhouse, 1975:17

Alisina Rowell, 1962:141

Alispira Nikiforova, 1961, in Nikiforova and Andreeva, $1961: 243$

Alispirifer Campbell, 1961:434

Alilaria Cooper and Muir-Wood, 1967:808 [new name for Alifera]

**Allanaria Crickmay, 1953 [invalid new name for Allanella, Allanella not preoccupied]

Allanella Crickmay, 1953a:5 [replacement by Allanaria invalid, Allanella not preoccupied]

Allanetes Boucot and Johnson, 1967b: 142

Allorhynchoides Savage, Eberlein, and Churkin, 1978:392

Allorhynchus Weller, 1910:509

Almerarhynchia Calzada Badía, 1974a:94

Almerasithyris Tchorszhewsky, 1973 [nomen nudum]

*Alphachoristiles Gatinaud, 1949:492 [= Choristiles]

*Alphacyrtiopsis Gatinaud, 1949:490 [= Cyrtiopsis]

*Alphaneospirifer Gatinaud, 1949:491 [= Neospirifer]

*Allaestrophia Bublichenko, 1956:96 [= Leptodontella]

Altaethyrella Severgina, 1978:38

Allajella Kulkov, 1962:653

Aliplecus Stehli, 1954:349

*Altorlhis Andreeva, 1960, in Markowskii, 1960:288 [ = Nothorthis $]$

Alwynella Spjeldnaes, 1957:85

Alwynia Stehli, 1961b:464

Ambikella Sahni and Srivastava, 1956:207

Amblotrema Rafinesque, 1831 [incertae sedis]

Amblytrema.-Agassiz, 1846 [misspelling of Amblotrema]

Ambocoelia Hall, 1860:71

Amboglossa Wang and Zhu, 1979:60

Ambonorthella Bassett, 1972:66

Ambothyris George, 1931:42

Amesopleura Carter, 1967a:364

Ameloria Cooper and Grant, 1975:1055

Amissopeclen Havlícek, 1960:243

Amoenospirifer Havlícek, 1957b:439

Amosina Boucot, 1975:360

Amphiclina Laube, 1865:28

Amphiclinodonta Bittner, 1888a:288

Amphigenia Hall, 1867b:163

Amphipella Cooper and Grant, 1969:11

Amphistrophia Hall and Clarke, 1892:292

Amphistrophiella Harper and Boucot, 1978b:157

Amphithyris Thomson, 1918:20

Amphitomella Bittner, 1890:298

Amsanella Boucot, 1975 [nomen nudum]

Amsdenella Tillman, 1967:1251
Amsdenina Boucot, 1975:357

(Amsdenostrophiella) Harper and Boucot, 1978b:158

[subgenus of Amphistrophiella]

Amurothyris Koczyrkevicz, 1976b:77

Amygdalocosla Waterhouse, 1967a: 101

Anabaia Clarke, 1893, in Hall and Clarke, 1893:141

Anabaria Lopushinskaja, 1965:27

Anabolotrela Rowell and Henderson, 1978:8

Anadyrella Dagys, 1974:185

Anarhynchia Ager, 1964:63

Anastrophia Hall, 1867b:163 [new name for Brachymerus]

Anathyrella Khalfin, 1960b:476

Anathyris Peetz, 1901:378

Anatrypa Nalivkin, 1941:172

Anazyga Davidson, 1882:128

Anchigonites Öpik, 1939:136

Ancilloloechia Havlíček, 1959b:78

Ancistrocrania Dall, 1877:13 [new name for Cranopsis]

Ancistrorhyncha Ulrich and Cooper, 1942:624

Ancorhynchia Ching and Ye, 1979:101

Ancylostrophia Harper, Johnson, and Boucot, 1967:423

Andobolus Kozlowski, 1930:316

Aneboconcha Cooper, 1973b:28

Anechophragma Neuman, 1977:37

†Anelasma Ivanov, 1925 [replaced by Brachythyrina]

**Anelasmina Semichatova, 1939 [ = Anelasma]

Anemonaria Cooper and Grant, 1969:8

Aneuthelasma Cooper and Grant, 1976b:2906

Angarella Asatkin, 1932:486

Angulotrela Palmer, 1954:769

Angusticardinia Schuchert and Cooper, 1931:244

Angustothyris Dagys, 1972d:31

Anidanthus Hill, 1950:10

Anidanthus Whitehouse, 1928 [nomen nudum; = Anidanthus

Hill, 1950]

Anisactinella Bittner, 1890:302

Anisopleurella Cooper, 1956c:805

Anomactinella Bittner, 1890:300

Anomalesia Cooper and Grant, 1976b:2819

Anomaloglossa Percival, 1978:125

Anomaloria Cooper and Grant, 1969:16

Anomalorthis Ulrich and Cooper, 1936b:622

Anoplia Hall and Clarke, 1892:309

Anopliopsis Girty, 1938:281

Anoplotheca Sandberger, 1855:8

Anoplambonites Williams, 1962:170

Ansehia Termier and Termier, 1970:456

Anteletes.-Orbigny, 1850 [misspelling of Enteletes]

Antelocoelia Isaacson, 1977b:171

Anteridocus Cooper and Grant, 1976a:2055

Anthracospirifer Lane, 1963:388

Anligalealella Sapelnikov and Rukavischnikova, 1975b:74

Antigonamboniles Öpik, 1934:147

Antrgoniarcula Elliott, 1959:146 
Antinomia Catullo, 1851:75

Antinomya.-Davidson, 1853 [misspelling of Antmomia]

Antiplychina Zittel, 1880:704

Antiquatonia Miloradovich, 1945:499

Antirhynchonella Oehlert, 1887, in Fischer, 1887:1311

Antirhynchonella Quenstedt, 1871 [nomen nudum; = Antirhynchonella Oehlert, 1887]

Antispirifer Williams, 1916, in Williams and Breger, 1916: 116

Antistrix Johnson, 1972:121

Antronaria Cooper and Grant, 1976a:2036

Apatomorpha Cooper, 1956c: 709

Apatorthis Öpik, 1933b:6

Aperispirifer Waterhouse, 1968a:35

Aphanomena Bergström, 1968:13

Aphaurosia Cooper and Grant, 1976a:2048

Aphelesia Cooper, 1959:41

*Apheoorthina Havlíček, 1949a:99 [= Apheoorthis]

Apheoorthis Ulrich and Cooper, 1936b:620

Apicilirella Su, 1976:179

Apleurotis Rafinesque, 1819 [nomen nudum]

Apollonorthis Mitchell, 1974:392

Apomatella Schuchert and Cooper, 1931:245

Apopentamerus Boucot and Johnson, 1979:104

Aporthophyla Ulrich and Cooper, 1936b:625

Aporthophylina Liu, 1976: 144

A pouszella Carter, 1972:732

Aprongia Gregorio, 1886:23

Apsilingula Williams, 1977:403

Apsocalymma McIntosh, 1974:213

Apostreta Palmer, 1954:770

Araratella Sartenaer and Plodowski, 1975:5

Aratanea Schmidt, 1967:92

Araxathyris Grunt, 1965, in Ruzhentsev and Sarytcheva, 1965:240

Araxilevis Sarytcheva, 1965, in Ruzhentsev and Sarytcheva, 1965:221

Araxina Askerov, 1965; 1967 [nomina nuda]

Arbizustrophia Garcia-Alcalde, 1972c:57

Arcelinithyris Almeras, 1971:173

Arceythyris Rollet, 1964:38

Archaeorthis Schuchert and Cooper, 1931:243

Arcosarina Ching, Sun, and Ye, 1979:185

Arctilreta Whitfield, 1908:57

Arctochonetes Ifanova, 1968:29

Arclohedra Cooper, 1936:210

**Arclolaballa Dagys, 1977 [= Pseudolaballa]

Arctomeristina Amsden, 1978:33

Arclospirifer Stainbrook, 1950:382

Arctothyris Dagys, 1965:140

Arcualla Walmsley and Boucot, 1975:79

Arcuatolhyris Popiel-Barczyk, 1972:136

(Arduennensisspirifer) Kutscher and Mitmeyer, 1970 [subgenus of Acrospirifer; nomen nudum]
(Arduspirifer) Mittmeyer, 1972:101 [subgenus of Acrospirifer; elevated to genus by Mittmeyer in 1973]

Arenaciarcula Elliott, 1959:147

Arenorthis Havlícek, 1970b:29

Areostrophia Havlícek, 1965a:293

Argentiproductus Cooper and Muir-Wood, 1951:195 [new name for Thomasella Paul, 1942]

$\dagger$ Argiope Deslongchamps, 1842 [replaced by Megathiris]

Argovithyris Rollet, 1972:95

Argyope.-Davidson 1850 [misspelling of Argiope]

Argyrotheca Dall 1900:44 [new name for Cistella]

Arionthia Cooper and Grant, 1976b:2750

Arktikina Grunt, 1977a:69

Aromasithyris Almeras, 1971:544

Artimyctella Liu, 1979:508

Artiotreta Ireland, 1961:1138

Aseptalium Hou and Xian, 1975:37

Aseplella Martínez and Winkler, 1977:18

Aseplonetes Isaacson, 1977a: 177

**Asioproductus Chan, 1977; 1978; 1979 [= Transennatia]

Asperlinus Waterhouse and Piyasin, 1970:132

Aspidothyris Diener, 1908:59

Astegosia Cooper and Grant, 1969:16

Astraborthis Williams, 1974:69

Astulorhyncha Havlíček, 1961a: 105

Asymphylotoechia Ross, 1970:60

Asyrinx Hudson and Sudbury, 1959:46

Asyrinxia Campbell, 1957:80

Atelelasma Cooper, 1956c:517

Atelestegastus Cooper and Grant, 1975:829

Atelithyris Smirnova, 1975c:77

*Athabaschia Crickmay, 1963:10 [= Ladogioides]

*Athyrella Renz, 1913 [nomen nudum; = Spirigerella]

Athyrhynchus Johnson, 1973c:467

Athyris McCoy, 1844:147

Athyrisina Hayasaka, 1922:52

†Athyrisinoides Chen and Wan, 1978

Athyrisinoides Jiang, 1978:303

Atlantida Havliček, 1971c:37

**Atretia Jeffreys, 1870 [= Cryptopora]

Atribonum Grant, 1965:38

Atrypa Dalman, 1828:127

Atryparia Copper, 1966a:982

Atrypella Kozlowski, 1929:30

Atrypina Hall and Clarke, 1893: 162

Atrypinella Khodalevich, 1939:45

Atrypoidea Mitchell and Dun, 1920:272

*Atrypopsis Poulsen, 1943:44 [= Dubaria]

Atrythyris Struve, 1965a:218

Attenuatella Stehli, 1954:343

Auchmerella Struve, 1964c:521

Aulacella Schuchert and Cooper, 1931:246

Aulacophoria Schuchert and Cooper, 1931:247 
*Aulacorhyna Strand, 1928:37 [new name for Aulacorhynchus; = [sogramma]

$\dagger$ Aulacorhynchus Dittmar, 1872 [replaced by Aulacorhyna] Aulacothyris Douvillé, 1879:277

Aulacothyroides Dagys, 1965:155

Aulacothyropsis Dagys, 1959b:99

Auldosprra Williams, 1962;252

Aulonotreta Kutorga, 1848:282

Auloprotonia Muir-Wood and Cooper, 1960:273

Aulosteges Helmersen, 1847:331

Auriculispina Waterhouse, 1975:13

Austinella Foerste, 1909a:224

Australiarcula Elliott, 1960:26

Australina Clarke, 1912:10

Australirhynchia Savage, 1968b:731

Australocoelia Boucot and Gill, 1956:1174

Australosia McKellar, 1970:12

Australospirifer Caster, 1939:159

Australostrophia Caster, 1939:83

†Austriella Bittner, 1890 [replaced by Austriellula]

**Austriellina Schuchert and LeVene, 1929 [invalid new name for Austriella, already renamed]

Austriellula Strand, 1928:38 [new name for Austriella]

Austrirhynchia Ager, 1959:325

Austrochoristites Roberts, 1971:230

Austronoplia Isaacson, 1977a: 178

Austrospirifer Glenister, 1956:58

Austrothyris Allan, 1939:238

Avisyrinx Martínez, 1975:33

Avonia Thomas, 1914:259

Avonothyris Buckman, 1917:102

Axiodeaneia Clark, 1917:375

Azerithyris Askerov, 1964; 1965; 1967 [nomina nuda]

Babukella Dagys, 1974:208

Backhausina Pajaud, 1966b:125

Bactrynium Emmrich, 1855:449

Bagnorthis Levy and Nullo, 1974a:184

Bagrasia Nalivkin, 1960, in Sarytcheva et al., 1960:231

Bailliena Nelson and Johnson, 1968:723

Bajanhongorella Rozman, 1977:124

Bajanorthis Andreeva, 1968, in Markowskii, 1968:73

Bajkuria Ustritsky, 1963, in Ustritsky and Tschernjak, 1963:116

Balakhonia Sarytcheva, 1963, in Sarytcheva et al., 1963: 231

Balanoconcha Campbell, 1957:86

Balatonspira Dagys, 1974:137

Bancroftina Sinclair, 1946:295 [new name for Raymondella Whittington, 1938]

Bandoproductus Ching and Sun, 1979 [nomen nudum]

Barbaestrophia Havlícek, 1965b:7

Barbarorthis Öpik, 1934:183

**Barrandella Hall and Clarke, 1893 [= Antirhynchonella]
Barrandina Booker, 1926:132

Barroisella Hall and Clarke, 1892:63

Barzellinia Gregorio, 1930b:8

Bashkiria Nalivkin, 1979:144

Basilicorhynchus Crickmay, 1952:1

Basiliola Dall, 1908:442

Basiola.-Thomson, 1915 [misspelling of Basiliola]

†Bathycoelia Foerste, 1909 [replaced by Pionodema]

Bathymyonia Muir-Wood and Cooper, 1960:245

Bathynanus Foster, 1974:80

Bathyrhyncha Fuchs, 1923:854

Baturria Carls, 1974:167

Bazardarella Ovtsharenko, 1977 [nomen nudum]

Beachia Hall and Clarke, 1893:260

Beckmannia Mohanti, 1972:166

Beecheria Hall and Clarke, 1893:300

Beichuanella Chen, 1978:339

Beitaia Rong and Yang, 1974:199

Bejrutella Tchorszhewsky, 1972:40

(Bekkerella) Reed, 1936:39 [subgenus of Rafinesquina]

†Bekkerella Rosenstein, 1943 [replaced by Elsaella]

Bekkeromena Rōōmusoks, 1963:235

Belbekella Moisseiev, 1939:195

Beleutella Litvinovich, 1967:55

Bellaclathrus Winters, 1963:26

Bellimurina Cooper, 1956c:854

Belothyris Smirnova, 1960:117

*Benignites Havlícek, 1952:409 [= Merciella]

Bergalaria Schmidt, 1975:101

Beschevella Poletaev, 1975:51

*Betachoristites Gatinaud, 1949:492 [= Choristites]

*Betacyrtiopsis Gatinaud, 1949:490 [= Cyrtiopsis]

* Betaneospirifer Gatinaud, 1949:491 [= Neospinfer]

Biarea Torbakova, 1959, in Khodalevich et al., 1959:75

Bibatiola Grant, 1976:136

Bicarinatina Batrukova, 1967 [nomen nudum]; 1969:59

Bicia Walcott, 1901:677

Biconostrophia Havlícek, 1956:562

Bicornes Quenstedt, 1852 [invalid name, Opinion 907 ICZN]

Bicuspina Havlícek, 1950:18

Bidentatus Khodalevich and Breivel, 1972, in Malygina et al., 1972:202

Biernatella Balinski, 1977:179

Biernatium Havlícek, 1975:234

Bifida Davidson, 1882:27

Bifolium Elliott, 1948:3

Bihendulirhynchaa Muir-Wood, 1935:104

Bihenithyris Muir-Wood, 1935:111

Bilaminella Babanova, 1964:66

Bullingsella Hall and Clarke, 1892:230

†Billingsia Ford, 1886 [replaced by Elkania]

Bilobia Cooper, 1956c: 760

**Bilobites Linné, 1775 [= Dicoelosia] 
Bilolina Reed, 1944:109

Bimuria Ulrich and Cooper, 1942:623

Biparetis Amsden, 1974:54

Biseptum Khodalevich and Breivel, 1959, in Khodalevich et al., 1959:39

Bisinocoelia Havlíček, 1953:7

*Bispinoproductus Stainbrook, 1947:311 [= Leioproductus]

Bistramia Hoek, 1912, in Steinmann and Hoek, 1912:247

Bisulcata Boucot, 1975 [nomen nudum; = Bisulcata Boucot and Johnson, 1979]

Bisulcata Boucot and Johnson, 1979:117

Bisulcatella Boucot, 1975 [nomen nudum]

Bisulcina Titova, 1977:81

Bittnerella Dagys, 1974:77

Bittnerula Hall and Clarke, 1894:764

*Blairella Miller and Gurley, 1896 [nomen nudum; = Rhipidomella]

*Blasispirifer Kulikov, 1950:6 [= Spiriferella]

Blochmannella Leidhold, 1921:356

Blyskavomena Havliček, 1976:369

Bobinella Andreeva, 1968, in Markowskii, 1968:80

Bodrakella Moisseiev, 1936:47

Bohemeella Schuchert and Cooper, 1931:242

Bohemirhynchia Nekvasilova, 1973:78

(Boicinetes) Havliček and Racheboeuf, 1979:73 [subgenus of Chonetes]

Bojarinovia Aksarina, 1978, in Aksarina and Pelman, 1978: 106

Bojodouvillina Havlíček, 1967a:171

Bojothyris Havlícek, 1957 [nomen nudum]; 1959a:247

Boloria Grunt, 1973, in Grunt and Dmitriev, 1973:119

Booralia Campbell, 1961:449

Boreadorthis Öpik, 1934:184

Borealirhynchia Su, 1976:194

Borealis Boucot, Johnson, and Rubel, 1971:274

Boreiospira Dagys, 1974:126

Boreiothyris Dagys, 1968:110

Bornhardtina Schulz, 1914:363

Borszhaviella Tchorszhewsky, 1973 [nomen nudum]

Bosquetella Smirnova, 1969a:83

Bothrionia Cooper and Grant, 1975:985

Bothrostegium Cooper and Grant, 1974:330

Botsfordia Matthew, 1891:148

Boubeithyris Cox and Middlemiss, 1978:419

Bouchardia Davidson, 1850b:65

Bouchardiella Doello-Jurado, 1922:200

*Bouchardopsis Maillieux, 1933 [nomen nudum; = Tylothyris]

Boucotella Bowen, 1966:186

Boucotia Gill, 1969:1227

Boucotides Amsden, 1968:44

Boucotinskia Brunton and Cocks, 1967:180

Bouskia Havlíček, 1975:233

Brachiopus Rafinesque, 1831 [sine type]

†Brachymerus Shaler, 1865 [replaced by Anastrophia]
Brachymimulus Cockerell, 1929:105 [new name for Mimulus] (Brachyprion) Shaler, 1865:63 [subgenus of Strophodonta] Brachyspirifer Wedekind, 1926:198

Brachythyrina Fredericks, 1929:385 [new name for Anelasma] Brachythyrinella Waterhouse and Gupta, 1978a:425

Brachythyris McCoy, 1844:128

Brachyzyga Kozlowski, 1929:31

Bracteoleptaena Havlíčk, 1963b:224

Brahimorthis Havlíček, 1971c:29

Branconia Gagel, 1890:62

Brandysia Havlícek, 1975:231

Branikia Havlícek, 1957b:437

*Branxtonia Booker, 1930:30 [= Wyndhamia]

†Brasilia Clarke, 1913 [replaced by Brasilina, Chapadella, and Oliveirella]

Brasilica.-Greger, 1920 [misspelling of Brasilia]

* Brasilina Clarke, 1921:139 [new name for Brasilia; = Paranaia]

Brasilioproductus Mendes, 1959:48

Brevicamera Cooper, 1956c:560

Brevilamnulella Amsden, 1974:62

Brevilamula Amsden, 1974 [nomen nudum]

(Breviseptum) Sapelnikov, 1960b:109 [subgenus of Conchidium]

Brevispirifer Cooper, 1942:231

Brochocarina Brunton, 1968:31

Broeggeria Walcott, 1902:605

Bronnothyris Popiel-Barczyk and Smirnova, 1978:134

Brooksina Kirk, 1922:3

Browneella Chatterton, 1973:117

Brunnirhyncha Havlícek, 1979:97

Brynella Bancroft, 1933 [nomen nudum]

Bryorhynchus Cooper and Grant, 1969:11

Buchanathyris Talent, 1956:36

Bufocephalus Linné, 1779 [incertae sedis]

Bulahdelia Roberts, 1976, in Roberts et al., 1976:213

Bullarina Ching and Sun, 1979 [nomen nudum]

(Bullothyris) Sučić-Protić, 1971:21 [subgenus of Loboidothyropsis]

Burmirhynchia Buckman, 1915 [nomen nudum]; 1917:49

Burrirhynchia Owen, 1962:59

Bursula Klein, 1753 [nonbinomial]

Buxtona Thomas, 1914:259

Buxtonioides Mendes, 1959:43

Bystromena Williams, 1974:146

**Cacata Strand, 1928 [new name for Thomsonia; = Coptothyris]

Cacemia Mitchell, 1974:394

Cactosteges Cooper and Grant, 1975:850

Cadomella Munier-Chalmas, 1887, in Fischer, 1887:1285

Caenanoplia Carter, 1968:1143

Caenotreta Cocks, 1979a:94

Caeroplecia Williams, 1974:121 
Calcirhynchia Buckman, 1914 [nomen nudum]; 1917:30

California.-Bassler, 1915 [misspelling of Cliftonia]

(Callaiapsida) Grant, 1971:323 [subgenus of Camerisma]

Callicalyptella Boucot and Johnson, 1972:301

Calliglypha Cloud, 1948:468

Calliomarginatia Ching, 1976, in Zhang and Ching, 1976: 181

Callipentamerus Boucot, 1964a:887

Callipleura Cooper, 1942:228 [new name for Cyclorhina]

Calliprolonia Muir-Wood and Cooper, 1960:246

Callispirina Cooper and Muir-Wood, 1951:195 [new name for Mansuyella]

Callospiriferina Rousselle, 1977:157

Calvinaria Stainbrook, 1945:44

Calyplolepla Neuman, 1977:35

Camarelasma Cooper and Grant, 1976b:2900

Camarella.-Dall, 1877; Oehlert, 1887 [misspelling of $\mathrm{Ca}$ merella]

* Camarium Hall, 1859b:43 [= Merisla]

Camarophorella Hall and Clarke, 1893:215

Camarophoria.-Herrmannsen, 1846 [misspelling of Camerophoria]

Camarophorina Licharew, 1934a:213

Camarophorinella Licharew, 1936b:63

Camarospira Hall and Clarke, 1893:82

Camarothyridina Linnik, 1966:129

Camarotoechia Hall and Clarke, 1893:190

Camarotoechioides Rzonsnitzkaja, 1978:178

Cambrolrophia Ulrich and Cooper, 1937:78 [new name for Eostrophia]

Camdella Ustritsky, 1971 [nomen nudum? misspelling of Gamdaella?]

Camerella Billings, 1859b:301

Camerisma Grant, 1965:64

** Camerophoria King, 1844 [nomen nudum]; 1846 [= Stenoscisma]

Camerophorina Schmidt, 1941:43

Camerothyris Bittner, 1890:318

Campages Hedley, 1905:43

Campylorthis Ulrich and Cooper, 1942:621

Canadospira Dagys, 1972c:40

†Canavaria Merla, 1928 [replacement by Gemmellaria invalid, Gemmellaria preoccupied; replaced by Sicelia]

Cancellospirifer Campbell, 1953:10

Cancellothyris Thomson, 1926:525

Cancrinella Fredericks, [1928]:784

Cancrinelloides Ustritsky, 1963, in Ustritsky and Tschernjak, 1963:85

Candispirifer Havliček, 1971 a:26

Canthylotreta Rowell, 1966:5

† Capellinia Hall and Clarke, 1893 [replaced by Capelliniella] Capelliniella Strand, 1928:38 [new name for Capellinia]

**Capillifera Jing and Hu, 1978:120; Ching and Ye, 1979:86 [invalid new name for Chilianshania, Chilianshania not preoccupied]

Capillirhynchia Buckman, 1914 [nomen nudum]; 1917:58

Capillirostra Cooper and Muir-Wood, 1951:195 [new name for Rhynchonellopsis Böse, 1894]

Capillithyris Katz, 1974:258

Capillonia Waterhouse, 1973:37

Caplinoplia Havlícek and Racheboeuf, 1979:102

Carapezzia Tomlin, 1930:24 [new name for Geyeria]

Carbocyrtina Ivanova, 1975:81

Cardiarina Cooper, 1956a:527

Cardinirhynchia Buckman, 1917:74

Cardinocrania Waagen, 1885:746

Cardiadonla Hall, 1850 [nomen nudum]

Cardialhyris Roberts, 1971:178

Caricula Grant, 1976:128

Carinagypa Johnson and Ludvigsen, 1972: 128

(Carnastrophia) Gratsianova, 1975:64 [subgenus of Areostrophia)

Carinatina Nalivkin, 1930:192

Carinatinella Gratsianova, 1967:97

Carinalrypa Copper, 1973:496

Cariniferella Schuchert and Cooper, 1931:246

Carinokoninckina Jin and Fang, 1977:59

Carlinia Gordon, 1971:259

*Carlopsina Reed, 1954:183 [= Schellwienella]

Carmanella Boucot, 1975 [nomen nudum]

Carneithyris Sahni, 1925:364

Carpatolhyris Smirnova, 1975a:135

Carlorhium Cooper and Grant, 1976a:2191

Caryogyps Johnson, Boucot, and Murphy, 1976:53

Caryorhynchus Crickmay, 1952:1

Casquella Percival, 1978:128

Cassianospira Dagys, 1972a: 103

Cassidirastrum McLaren, 1961:2

Caslellaroina Boucot, 1972, in Amos, 1972:11

Calazyga Hall and Clarke, 1893:157

Cathaysia Ching, 1966, in Wang et al., 1966:372

Caucasella Moisseiev, 1934:83

Caucasilhyris Askerov, 1964; 1965; 1967 [nomen nudum]

Caucasorhynchia Dagys, 1963:66

Caucasothyris Dagys, 1974:192

*Causea Wiman, 1905:53 [= Mickwilzia]

Cavalisinurostrum Sartenaer, 1972:2

Cedulia Racheboeuf, 1979:256

Celebetes Grant, 1976:137

Celsifornix Carter, 1974:677

Cenorhynchia Cooper and Grant, 1976a:1986

Centronella Billings, 1859a:131

Centronelloidea Weller, 1914:247

Centrorhynchus Sartenaer, 1970:11

Centrospirifer Grabau, 1931 [nomen nudum; = Centrospirifer Tien, 1938]

${ }^{*}$ Centrospirifer Tien, 1938:111 [= Cyrlospirifer] 
Ceocypea Grant, 1972:226

Ceratreta Bell, 1941:233

Cererithyris Buckman, 1914 [nomen nudum]; 1917:109

Chaeniorhynchus Cooper and Grant, 1976a: 1999

Chaganella Nikitin, 1974:65

Changshaispirifer Zhao, 1977:428

*Chaoella Licharew, 1931:161 [= Keyserlingina]

Chaoiella Fredericks, 1933:31

Chaoina Ching, 1974:308

*Chapadella Greger, 1920:70 [new name for Brasilia; = Paranaia]

Chapinella Savage, Eberlein, and Churkin, 1978:388

Charionella Billings, 1861b:148

Charionoides Boucot, Johnson, and Staton, 1964:817

Charltonithyris Buckman, 1915 [nomen nudum]; 1917:106

Chascothyris Holzapfel, 1895:234

Chathamithyris Allan, 1932:15

Chattertonia Johnson, 1976:789

Chatwinothyris Sahni, 1925:368

(Chaulistomella) Cooper, 1956c:432 [subgenus of Multicostella]

Cheirolhyris Rollier, 1919:338

Cheirothyropsis Makridin, 1964:267

Chelononia Cooper and Grant, 1974:327

*Chemungia Caster, 1939:107 [= Strophonelloides]

Cheniothyris Buckman, 1915 [nomen nudum]; 1917:128

Chianella Waterhouse, 1975:13

${ }^{*}$ Chilianshania Yang and Ting, 1962, in Yang et al., 1962: 86 [= Monticulifera $]$

${ }^{*}$ Chilidiopsis Boucot, 1959b:25 [= Fardenia]

Chilidorthis Havlíček, 1972, in Havlíček and Josopait, 1972: 342

Chimaerothyris Paulus, Struve, and Wolfart, 1963:463

Chivatschella Zavodowsky, 1968, in Markowskii, 1968:125

Chlidonophora Dall, 1903:1538

Chlupacina Havlícek and Racheboeuf, 1979:86

Chnaurocoelia Johnson, Boucot, and Murphy, 1976:95

Choanodus Cooper and Grant, 1974:402

Choanoproductus Termier and Termier, 1966 [nomen nudum]

Chondronia Cooper and Grant, 1976b:2854

†Chonetella Krotow, 1885 [replaced by Chonetina]

Chonetella Waagen, 1884:657

Chonetes Fischer de Waldheim, 1830, pl. 26

Chonetina Krotow, 1888:500 [new name for Chonetella Krotow, 1885]

Chonetinella Ramsbottom, 1952:13

Chonetinetes Cooper and Grant, 1969:4

Chonetipustula Paeckelmann, 1931 a:31

Chonetoidea Jones, 1928:389

${ }^{*}$ Choniopora Schauroth, 1854:546 [= Acanthocrania]

Chonopectella Sarytcheva, 1966:135 [new name for Chonopectoides Sarytcheva, 1965]

Chonopectoides Crickmay, 1963:23
†Chonopectoides Sarytcheva, 1965 [replaced by Chonopectella]

Chonopectus Hall and Clarke, 1892:312

Chonosteges Muir-Wood and Cooper, 1960:113

Chonostegoides Sarytcheva, 1965, in Ruzhentsev and Sarytcheva, 1965:212

Chonostrophia Hall and Clarke, 1892:311

Chonostrophiella Boucot and Amsden, 1964:881

Choperella Ljaschenko, 1969a:22

Choristitella Ivanov and Ivanova, 1937:163

Choristites Fischer de Waldheim, 1825:8

Choristothyris Cooper, 1942:233

Christiania Hall and Clarke, 1892:299

Chrustenopora Havlícek, 1968:123

Chynistrophia Havlíček, 1977b:301

Cilinella Havliček, 1970b:16

Cimicinella Schmidt, 1943:67

Cimicinoides Anderson, Boucot, and Johnson, 1969:156

Cincta Quenstedt, 1868:25

Cinctifera Muir-Wood and Cooper, 1960:166

Cinerorthis Havliček, 1974:167

Cingulodermis Havlícek, 1971a:15

Cirpa Gregorio, 1930a:40

†Cistella Gray, 1853 [replaced by Argyrotheca]

Cistellarcula Elliott, 1954:726

Clarkeia Kozlowski, 1923:86

Clarkella Walcott, 1908:111

Clathrithyris Smirnova, 1971 [nomen nudum]; 1974:48

Clavigera Hector, 1879:538 [type designated by Marwick, 1953:45]

Clavigerina.-Marwick, 1946 [misspelling of Clavigera]

Clavodalejina Havlícek, 1977a:227

Cleidothyris._Paetel, 1875 [misspelling of Cleiothyris Phillips, $1841 ;=$ Athyris]

Cleiothyridellina Waterhouse, 1978:82

Cleiothyridina Buckman, 1906b:324 [new name for Cleiothyris King, 1850]

†Cleiothyris King, 1850 [replaced by Cleiothyridina]

${ }^{* *}$ Cleiothyris Phillips, 1841 [= Atrypa]

Cliftonia Foerste, 1909b:82

Clinambon Schuchert and Cooper, 1932:115

Clintonella Hall and Clarke, 1893:160

Cliothyridina.-Girty, 1910 [improper emendation of Cleiothyridina]

${ }^{*}$ Cliothyris.-Agassiz, 1846 [misspelling of Cleiothyris Phillips, $1841 ;=$ Athyris $]$

Cliothyris.-Hall and Clarke, 1893 [misspelling of Cleiothyris Phillips, 1841]

Clipsilia.-Dall, 1877 [misspelling of Clipsilis]

Clipsilis Rafinesque, 1820 [nomen nudum]

Clistotrema Rowell, 1963:35 [new name for Orbicella]

Clitambonites Agassiz, 1847:90

Clivospirifer Ljaschenko, 1973:108

Clorinda Barrande, 1879:109

Clorindella Amsden, 1964:236 
Clorindina Khodalevich, 1939:11

Clorindinella Rzonsnitzkaja, 1975:61

Cloudella Boucot and Johnson, 1963, in Boucot, Caster, et al., 1963:123 [new name for Pleurothyris]

Cloudothyris Boucot and Johnson, 1968:B19

Cnismatocentrum Dall, 1920:321

Coelospira Hall, 1863:60

Coelospirella Su, 1976:203

Coelospirina Havlíček, 1956:586

Coeloterorhynchus Sartenaer, 1966b:41

Coenothyris Douvillé, 1879:270

Colaptomena Cooper, 1956c:889

Coledium Grant, 1965:96

Collarothyris Modzalevskaja, 1970:155

Collemataria Cooper and Grant, 1974:434

Collumatus Cooper and Grant, 1969:6

Colongina Breivel and Breivel, 1970b:61

Comalopoma Havlícek, 1950:54

Comelicania Frech, 1901:551

Comioloechia Ljaschenko, 1973:37

Composila Brown, 1849:131

Compressoproductus Sarytcheva, 1960, in Sarytcheva et al., 1960:231

Compsoria Cooper, 1973a:23

Compsothyris Jackson, 1918b: 188

Comuquia Grant, 1976:97

${ }^{*}$ Conchidiella Khodalevich, 1939:32 [= Zdimir]

Conchidium Oehlert, 1887, in Fischer, 1887:1311

Concinnispirifer Boucot, 1975:367

Concinnithyris Sahni, 1929:11

${ }^{*}$ Condrathyris Minato, 1953:69 [= Phricodothyris]

Connecloproductus Donakova, 1974:173

Conodiscus Ulrich and Cooper, 1936b:619

Conomimus Johnson, Boucot, and Gronberg, 1968:406

Conotrela Walcott, 1889:365

** Conradia Hall, 1874 [= Dinobolus]

Contradouvillina Gratsianova, 1975:48

*Coolinia Bancroft, 1949a: 7 [= Fardenia]

Cooperina Termier, Termier, and Pajaud, 1966:332

Coptothyris Jackson, 1918a:479 [new name for Thomsonia]

** Cora Fredericks, 1928 [= Linoproduclus]

Corbicularia Ljaschenko, 1973:29

Cordatomyonia Boucot, Gauri, and Johnson, 1966:164

Corineorthis Stubblefield, 1939:67

Cornwallia Wilson, 1932:388

Coronalosia Waterhouse and Gupta, 1978a:415

**Corrugatella Khalfin, 1948 [= Cymostrophia]

Corrugatimediorostrum Sartenaer, 1970:23

Corlezorthis Johnson and Talent, 1967a: 143

Corvinopugnax Havlícek, 1961a:36

Coscinarina Muir-Wood and Cooper, 1960:138

Coscinophora Cooper and Stehli, 1955:469

Costachonetes Waterhouse, 1975:2

Costacranaena Johnson and Perry, 1976:631
** Costalosia Waterhouse and Shah, 1966 [= Lichareviella $]$

Costanoplia Xu, 1977:65

Coslatrypa Copper, 1973:494

tCostellaria Muir-Wood and Cooper, 1960 [replaced by Costellarina]

Costellarina Muir-Wood and Cooper, 1967, in Cooper and Muir-Wood, 1967:808 [new name for Coslellaria]

Costellirostra Cooper, 1942:231

Costellispirifer Boucot, 1973:51

Costiferina Muir-Wood and Cooper, 1960:277

Costinifera.-Gobbett, 1963 [misspelling of Costiferina]

Costinorella Dagys, 1974:101

Costirhynchia Buckman, 1914 [nomen nudum]; 1917:39

†Costirhynchia Dagys, 1974 [replaced by Costirhynchopsis]

Costirhynchopsis Dagys, $1977 \mathrm{~b}: 139$ [new name for Costirhynchia Dagys, 1974]

Costisorthis Havlícek, 1974:170

Costispinifera Muir-Wood and Cooper, 1960:217

Costispirifer Cooper, 1942:232

Costispiriferina Dagys, 1974:127

Costistricklandia Amsden, 1953: 143

Costistrophonella Harper and Boucot, 1978a:99

Costoconcha Ching, Sun, and Ye, 1979:193

Craigella Reed, 1935:353

Cranaena Hall and Clarke, 1893:297

*Cranaenella Fenton and Fenton, 1924:129 [= Cranaena]

Crania Retzius, 1781:72

Cranicella Rafinesque, 1815 [nomen nudum]

†Craniella Oehlert, 1888 [replaced by Petrocrania]

Craniops Hall, 1859b:84

Craniotrela Termier and Monod, 1978:149

Craniscus Dall, 1871b:27

†Cranopsis Dall, 1871 [replaced by Ancistrocrania]

Craspedalosia Muir-Wood and Cooper, 1960:82

Craspedelia Cooper, 1956c:773

Craspedona Cooper and Grant, 1975:881

Crassalrypa Mizens, 1977b:54

*Crassiorina Havlícek, 1950:36 [= Cryptothyrs]

Crassipunctatrypa Mizens and Rzonsnitzkaja, 1979:63

Crassumbo Carter, 1967a:408

Cremnorthis Williams, 1963:378

Crentspirifer Stehli, 1954:348

Cretirhynchia Pettitt, 1950:1

Cricosia Cooper, 1973c:384

Crinistrophia Havlícek, 1967a:134

*Criopus Poli, 1791:34 /= Crania]

†Crispella Kozlowski, 1929 [replaced by Howellella]

*Cristiferina Cooper, 1956c:961 [= Paucicrura]

Crossacanthia Gordon, 1966:581

Crossalosia Muir-Wood and Cooper, 1960:76

Crozonorthis Melou, 1976:700

Cruralina Smirnova, 1966:32

Cruratula Bittner, 1890:66

Cruricella Grant, 1976:187 
Cruricoelia Sykes, 1964 [nomen nudum]

Crurirhynchia Dagys, 1961a:96

Crurithyris George, 1931:43

Cryopus.-Deshayes, 1836 [misspelling of Criopus]

Cryptacanthia White and St. John, 1867:119

Cryptatrypa Siehl, 1962:196

Cryplonella Hall, 1861:101

Cryplonema._Bigsby, 1868 [misspelling of Cryptonella]

Cryplopora Jeffreys, 1869:136

Cryploporella Bitner and Pisera, 1979:71

Cryplorhynchia Buckman, 1914 [nomen nudum]; 1917:66

Cryplospirifer Grabau, 1931c:405

Cryptothyrella Cooper, 1942:233

Cryptothyris Bancroft, 1945:223

Cryplotreta Pelman, 1977:53

Cienalosia Cooper and Stehli, 1955:470

(Clenochonetes) Racheboeuf, 1976:52 [subgenus of Strophochonetes]

Clenokoninckina Xu, 1978b:283

Cubanirhynchia Kamyschan, 1968:57

Cubanothyris Dagys, 1959a:35

Cudmorella Allan, 1939:242

Cumberlandina Boucot, 1975:371

Cuneırhynchia Buckman, 1914 [nomen nudum]; 1917:35

Cunolites Defrance, 1821 [nomen nudum]

Cuparius Ross, 1971:126

Cupularostrum Sartenaer, 1961c:2

Curranella._Chatterton, 1973 [error for Resserella]

Curticia Walcott, 1905:319

Curtirhynchia Buckman, 1914 [nomen nudum]; 1917:36

Cyclacantharia Cooper and Grant, 1969:7

Cycladigera Havlícek, 1971b:230

Cyclocoelia Foerste, 1909a:227

Cyclomyonia Cooper, 1956c:975

†Cyclorhina Hall and Clarke, 1893 [replaced by Callipleura]

Cyclospira Hall and Clarke, 1893:146

Cyclothyris McCoy, 1844:150

Cyclus Barrande, 1879 [nomen nudum; = Paterula]

Cydimia Chatterton, 1973:131

Cymalorhynchia Buckman, 1914 [nomen nudum]; 1917:53

Cymbidium Kirk, 1926:3

Cymbistropheodonta Harper and Boucot, 1978c:38

Cymbithyris Cooper, 1952b:5

Cymostrophia Caster, 1939:39

Cyphomena Cooper, 1956c:841

(Cyphomenoidea) Cocks, 1968:316 [subgenus of Cyphomena]

Cyphotalosia Carter, 1967a:282

Cypholerorhynchus Sartenaer, 1965b:55

Cyrolexis Grant, 1965:89

Cyrtaena.-Hall, 1867 [misspelling of Cyrtina]

Cyrlalosia Termier and Termier, 1970:455

Cyrtella Fredericks, [1924a]:312

Cyrtia Dalman, 1828:92

Cyrtina Davidson, 1858:66
Cyrlinaella Fredericks, 1916:18

*Cyrinaellina Fredericks, 1926:414 [= Cyrtina $]$

†Cyrtinopsis Fredericks, 1916 [replacement by Davidsonella

Fredericks invalid, Davidsonella preoccupied; replaced by Davidsonina]

Cyrtinopsis Scupin, 1896:247

Cyrtiopsis Grabau, 1923:194

Cyrloniscus Boucot and Harper, 1968:172

Cyrtonotella Schuchert and Cooper, 1931:243

Cyrlospirifer Nalivkin, 1924, in Fredericks, [1924a]:312

†Cyrlotheca Bittner, 1890 [replaced by Thecocyrtella]

Cyrtothyris Middlemiss, 1959:123

Dabashanospira Fu, 1979 [nomen nudum]

Dactylogonia Ulrich and Cooper, 1942:624

Dactylotrela Rowell and Henderson, 1978:3

Daghanirhynchia Muir-Wood, 1935:82

Dagnachoneles Afanasjeva, 1978:66

Dalaia Plodowski, 1968:256

* Dalejina Havlícek, 1953:5 [= Mendacella]

*Dalejodiscus Havlícek, 1961 b:449 [= Plectodonta]

Dalerhynchus Bai, 1978, in Yoh and Bai, 1978:54

Dalinuria Lee and Gu, 1976:245

Dalligas Steinich, 1968b:336

Dallina Beecher, 1893:382

Dallinella Thomson, 1915a:75

Dallithyris Muir-Wood, 1959:303

Dalmanella Hall and Clarke, 1892:205

*Dalmanellopsis Khalfin, 1948:208 [= Levenea]

Danella Pajaud, 1966a: 70

(Danocrania) Rosenkrantz, 1964:515 [subgenus of Crania]

Darvasıa Licharew, 1934a:213

Dasyalosia Muir-Wood and Cooper, 1960:77

Dasysaria Cooper and Grant, 1969:9

Dalangia Yang, 1977:369; 1978a:122; 1978b:268

†Davidsonella Fredericks, 1926 [invalid new name for Cyrtinopsis Fredericks, 1916, Davidsonella preoccupied; replaced by Davidsonina]

Davidsonella Munier-Chalmas, 1880:279

†Davidsonella Waagen, 1885 [replaced by Lakhmina]

Davidsonia Bouchard, 1849:92

Davidsonialrypa Lenz, 1968: 182

Davidsonina Schuchert and LeVene, 1929:120 [new name for Davidsonella Fredericks, 1926]

Daviesiella Waagen, 1884:613

Davoustia Racheboeuf, 1976:68

Dawsonelloides Boucot and Harper, 1968:165

Daya._Koken, 1896 [misspelling of Dayia]

Dayia Davidson, 1881 c:291

* Dearbornia Walcott, 1908:78 [= Acrothele]

Decoropugnax Havlícek, 1960:244

Decurlella Gaetani, 1966:344

Dedzelina Havlíček, 1950:33

Delepinea Muir-Wood, 1962:99 
Dellachania Waterhouse, 1971, in Bamber and Waterhouse, 1971:217

Deltarina Cooper and Grant, 1976a:2012

Deltathyris.-Herrmannsen, 1846 [misspelling of Delthyris]

*Deltatreta Ulrich, 1916 [nomen nudum]; 1926, in Butts, 1926:100 [= Polytoechia]

Delthiridaea._Gray, 1848 [misspelling of Delthyridaea]

Delthyridaea McCoy, 1844 [nomen nudum]

Delthyridea.-Agassiz, 1846 [misspelling of Delthyridaea]

Delthyris Dalman, 1828: 120

*Deltorthis Ulrich, 1927 [nomen nudum; = Polytoechia]

Demonedys Grant, 1976:66

** Denckmannella Schuchert and LeVene, 1929 [new name for Denckmannia]

$\dagger$ Denckmannia Holzapfel, 1908 [replaced by Macroplectane and Denckmannella]

** Dentatrypa Breivel, 1959 [= Vagrania]

Denticuliphoria Licharew, 1956, in Kiparisova et al., 1956: 57

Dentospiriferina Dagys, 1965:109

*Deothossia Gatinaud, 1949:488 [= Cyrtospurifer]

Derbyaeconcha Licharew, 1934, in Zittel, 1934:507

Derbyella Grabau, 1931b:270

Derbyia Waagen, 1884:594

Derbyina Clarke, 1913b:212

† Derbyina Grabau, 1931 [replaced by Grabauellina]

Derbyoides Dunbar and Condra, 1932:115

Dereta Elliott, 1959:147

Desatrypa Copper, 1964:363

Desmoinesia Hoare, 1960:226

Desmorthis Ulrich and Cooper, 1936b:624

Desquamatia Alekseeva, 1960:421

Destombesium Havlícek, 1971c:58

Devonalosia Muir-Wood and Cooper, 1960:84

Devonamphistrophia Harper and Boucot, 1978b: 159

(Devonara) Biernat, 1966:77 [subgenus of Eodevonaria]

Devonochonetes Muir-Wood, 1962:43

Devonogypa Havlícek, 1951:3

Devonoproductus Stainbrook, 1943a:55

Diabolirhynchia Drot, 1963:111

Diambonia Cooper and Kindle, 1936:356

Diandongia Rong, 1974:113

Diaphelasma Ulrich and Cooper, 1936b:629

Diaphragmus Girty, 1910:218

Diazoma Dürkoop, 1970:195

Dicaelosia._King, 1850 [misspelling of Dicoelosia]

Dicamara Hall and Clarke, 1893:73

Dicamaropsis Amsden, 1968:85

Dicellomus Hall, $1871 \mathrm{a}: 3$

Diceromyonia Wang, 1949:35

Dichospirifer Brice, 1970:192

Dichozygopleura Renouf, 1972:121

Diclipsites Rafinesque, 1831 [incertae sedis]

Diclisma Rafinesque, 1831 [incertae sedis]
Dicoelosia King, 1850:106

Dicoelostrophia Wang, 1955:351

*Dicraniscus Meek, 1872:280 [= Triplesia]

Dicrosia Cooper, 1978:12

Dictyoclostoidea Wang and Ching, 1964, in Wang et al., 1964:265

Diclyoclostus Muir-Wood, 1930:103

Dictyonathyris Xu, 1978b:287

Dictyonella Hall, 1868:274

Dictyonina Cooper, 1942:228 [new name for Iphidella Walcott, 1912]

Dictyonites Cooper, 1956c: 187

* Dictyostrophia Caster, 1939:42 [= Megastrophia]

Dictyothyris Douvillé, 1879:267

Dictyothyropsis Barczyk, 1969:66

Dicystoconcha Termier, Termier, Lapparent, and Marin, 1974: 123

Didymelasma Cooper, 1956c:615

Didymoparcium Lenz, 1977:62

Didymospira Salomon, 1895 [a group term, not generic]

Didymothyrts Rübel and Modzalevskaja, 1967:238

Dielasma King, 1859:260

Dielasmella Weller, 1911:447

Dielasmina Waagen, 1882:359

*Dielasmoides Weller, 1911:444 [= Dielasma]

**Dienerella Reed, 1931 [= Waagenites]

**Dienerina Ozaki, 1931 [= Elivina]

Dierisma Ching, Sun, and Ye, 1979:133

Destolhyris Thomson, 1916b:504

*Digitia Gregorio, 1930a:32 [= Leptodus]

Dignomia Hall, 1871 a:2 [nomen nudum]; 1872, plate 13.

Digonella Muir-Wood, 1934:550

Diholkorhynchia Yang and Xu, 1966:24

Dimegelasma Cooper, 1942:232

Dimerella Zittel, 1870:222

*Dinapophysia Maillieux, 1935:5 [= Straelenia]

Dinarella Bittner, 1892:25

Dinarispira Dagys, 1974:131

Dinobolus Hall, 187 la:4

(Dinorthis) Hall and Clarke, 1892:195 [subgenus of Plaesiomys]

Dioristella Bittner, 1890:299

Diorthelasma Cooper, 1956c:997

*Diorthis Kobayashi, 1937 [nomen nudum; = Dinorthis]

Diparelasma Ulrich and Cooper, 1936b:623

Diphyites Schroeter, 1779; Hermannsen, 1846 [nonbinomial]

Diplanus Stehli, 1954:299

Diplonorthis Mitchell, 1977:30

Diplospirella Bittner, 1890:297

Diraphora Bell, 1941:243

Dirinus McCoy, 1844:44

Discina Lamarck, 1819:237

Discinisca Dall, 1871b:37 
*Discinolepis Waagen, 1885:750 [= Schizopholis]

Discinopsis Matthew, 1892, in Hall and Clarke, 1892:106

Discomyorthis Johnson, 1970b:84

Discotreta Ulrich and Cooper, 1936b:619

Discradisca Stenzel, 1964:627

Disculina Deslongchamps, 1884:231

Dispiriferina Siblik, 1965:79

Ditreta Biernat, 1973:65

Divaricosta Cooper and Grant, 1969:11

Dmitria Sidiachenko, 1961:80

Dmitrispirifer Ljaschenko, 1973:126

Dnestrina Nikiforova and Modzalevskaja, 1968:206

Doleroides Cooper, 1930:375

Dolerorthis Schuchert and Cooper, 1931:244

Doloresella Sando, 1957:122

Donella Rotai, 1931:29

Dongbaella Ching and Ye, 1979:127

Dorashamia Sarytcheva, 1965, in Ruzhentsev and Sarytcheva, 1965:217

Dorsisinus Sanders, 1958, in Easton et al., 1958:53

Dorsoplicathyris Almeras, 1971:437

Dorsoscyphus Roberts, 1971:50

Dorytreta Cooper, 1956c:666

(Dotswoodia) McKellar, 1970:19 [subgenus of Whidbornella]

Douvillina Oehlert, 1887, in Fischer, 1887:1282

Douvillinaria Stainbrook, 1945:23

Douvillinella Spriestersbach, 1925:432

Douvillinoides Harper and Boucot, 1978b:152

Dowhatania Waterhouse and Gupta, 1978b:127

Drabodiscina Havlíček, 1972:231

Draborthis Marek and Havlíček, 1967:280

Drabovia Havlícek, 1950:45

Drabovinella Havliček, 1950:50

Dracius Steinich, 1967:1146

Drahanorhynchus Havlíček, 1967a:203

Drahanostrophia Havlíček, 1967a:202

Drepanorhyncha Cooper, 1956c:627

*Drummuckina Bancroft, 1949b:12 [= Sirophomena]

Duartea Mendes, 1959:59

Dubaria Termier, 1936:1266

Dubioleptina Havlíček, 1967a:70

*Dulankarella Rukavischnikova,:1956:135 [= Leptestiina]

Dundrythyris Almeras, 1971:189

Durranella Percival, 1979a:96

Duryeella Boucot, 1975:370

Dushanirhynchia Wang and Zhu, 1979:42

Dyctionella._Oehlert, 1887 [misspelling of Dictyonella]

Dyoros Stehli, 1954:312

Dyschrestia Grant, 1976:101

Dyscolia Fischer and Oehlert, 1890:71

Dyscritothyris Cooper, 1979:15

Dysoristus Bell, 1944, in Lochman and Duncan, 1944:146

Dysozistus._Gorjansky, 1960 [misspelling of Dysoristus]

Dyticospirifer Johnson, 1966b: 1043
Dzieduszyckia Siemiradzki, 1909:85

Dzirulina Nutsubidze, 1945:188

Eatonia Hall, 1857:90

Eatonioides McLearn, 1918:138

Eccentricosta Berdan, 1963:254

Echinalosia Waterhouse, 1967b:167 [new name for Multispinula Waterhouse, 1966]

Echinaria Muir-Wood and Cooper, 1960:248

Echinauris Muir-Wood and Cooper, 1960:222

(Echinirhynchia) Childs, 1969:73 [subgenus of Acanthorhynchia]

Echinocoelia Cooper and Williams, 1935:844

Echinocoeliopsis Hamada, 1968b:17

Echinoconchus Weller, 1914:138

Echinospirifer Ljaschenko, 1973:109

Echinosteges Muir-Wood and Cooper, 1960:102

Ecnomiosa Cooper, 1977:129

Ectenoglossa Sinclair, 1945:63

Ectochoristites Campbell, 1957:71

Ectoposia Cooper and Grant, 1976b:2898

Ectorensselandia Johnson, 1973b: 1105

(Ectorhipidium) Boucot, 1975 [nomen nudum; = Ectorhipidium) Boucot and Johnson, 1979]

(Ectorhipidium) Boucot and Johnson, 1979:102 [subgenus of Rhipidium ]

$\ddagger$ Edreja Koneva, 1979:49

Edriosteges Muir-Wood and Cooper, 1960:103

Ehlersella Boucot and Johnson, 1966a:569

Eichwaldia Billings, 1858:192

Eifelatrypa Copper, 1973:497

(Eilotreta) Amsden, 1968:36 [subgenus of Onychotreta]

Elasmothyris Cooper, 1956c:984

Elassonia Cooper and Grant, 1976a: 1935

Elenchus Aleksandrov, 1973:118

Eleutherocrania Huene, 1899:212

Eleutherokomma Crickmay, 1950:219

†Elina Fredericks, 1924 [replaced by Elinoria]

*Elinoria Cooper and Muir-Wood, 1951:195 [new name for Elina; = Brachythyrina $]$

Elita Fredericks, 1918:87

Eliva Fredericks, [1924a]:319

Elivella Fredericks, [1924a]:316

*Elivina Fredericks, [1924a]:314 [= Spiriferella]

Elkania Ford, 1886:325 [new name for Billingsia]

Ella Fredericks, 1918:87

Elliottella Stehli, 1955b:711 [new name for Psilonotus]

Elliottina Pajaud, 1963:996

*Ellipsothyris Sahni, 1925:371 [= Carneithyris]

Elliptoglossa Cooper, 1956c:242

Elliptostrophia Havlíček, 1963b:225

Elmaria Nalivkin, 1947:133; 1951:28

* Elsaella Alichova, 1960:192 [new name for Bekkerella Rosenstein, 1943; = Heterorthina $]$ 
Elymospirifer Wang, 1974, in Wang et al., 1974:40

Elyla.-Ivanova, 1960 [misspelling of Elita]

Elytha.-Fredericks, 1924 [misspelling of Elita]

Elythyna Rzonsnitzkaja, 1952:61

Emanuella Grabau, 1923:192

Emeithyris Xu, 1978b:301

Enallosia Cooper and Grant, 1976b:2859

Enantzosphen Whidborne, 1893:97

Enantıosphenella Johnson, 1974:67

Enchondrospirifer Brice, 1970:175

** Encuclodema Foerste, 1912 [invalid new name for Cyclocoelza, Cyclocoelia not preoccupied]

Enigmalosia Czarniecki, 1969:272

Enteles._Schellwien, 1892; Clark, 1903 [misspelling of Enteletes]

Enteletella Licharew, 1926:719

Enteletes Fischer de Waldheim, 1825:6

Enteletina Schuchert and Cooper, 1931:247

Enteletoides Stuckenberg, 1905:60

Eoamphistrophia Harper and Boucot, 1978b:154

Eoanastrophia Nikiforova and Sapelnikov, 1973:65

Eobrachythyris Brice, 1970:182

Eochonetes Reed, 1917:915

Eochoristites Chu, 1933:29

Eocoelia Nikiforova, 1961, in Nikiforova and Andreeva, 1961:252

Eoconcha Cooper, 1951:4

Eoconchidium Rozman, 1967:62

Eoconulus Cooper, 1956c:283

Eocramatia Williams, 1974:129

Eodallina Elliott, 1959:146

Eodalmanella Havlí̌cek, 1950:24

Eodevonaria Breger, 1906:534

Eodinobolus Rowell, 1963:37

Eoglossinotoechia Havliček, 1959b:81

Eogryphus Hertlein and Grant, 1944:89

Eohemithiris Hertlein and Grant, 1944:55

Eohemithyris._Cooper, 1959 [rejected emendation of Eohemuthins]

Eohowellella Lopushinskaja, 1976:79

Eokarpinskia Rzonsnitzkaja, 1964:103

Eokirkidium Khodalevich and Sapelnikov, 1970:196

Eolacazella Elliott, 1953:694

Eolissochonetes Hoare, 1960:220

Eolyttonia Fredericks, [1924b]:25

Eomarginifera Muir-Wood, 1930:103

(Eomarginiferina) Brunton, 1966:230 [subgenus of Eomarginifera ]

Eomartiniopsis Sokolskaja, 1941:78

(Eomegastrophia) Cocks, 1967:260 [subgenus of Megastrophia]

Eonalivkinia Vladimirskaya, 1978 [nomen nudum]

*Eoobolus Matthew, 1902:97 [= Obolus]

Eoorthis Walcott, 1908:103
Eoparaphorhynchus Sartenaer, 1961a:2

Eopentamerifera Boucot, 1975 [nomen nudum]

Eoperegrinella Ager, 1964:61

Eopholidostrophia Harper, Johnson, and Boucot, 1967:411

Eoplectodonta Kozlowski, 1929:30

Eoplicanoplia Boucot and Harper, 1968: 168

*Eoreticularia Nalivkin, 1924, in Fredericks, [1924a]:314 |= Reticularnopsis]

Eorhipidomella Hints, 1971:266

Eoschuchertella Gratsianova, 1974:83

Eoseptaliphoria Ching and Sun, 1976, in Ching et al., 1976: 294

Eosophragmophora Wang, 1974, in Wang et al., 1974:36

Eosotrematorthis Wang, 1955:336

Eospinatrypa Copper, 1973:496

Eospirifer Schuchert, 1913, in Zittel, 1913:411

Eospiriferina Grabau, 1931a:494

(Eospingerina) Boucot and Johnson, 1967a:90 [subgenus of Spirigerina]

Eostrophalosia Stainbrook, 1943a:58

(Eostropheodonta) Bancroft, 1949a:9 [subgenus of Strophodonia]

$\dagger$ Eostrophia Ulrich and Cooper, 1936 [replaced by Cambrotrophia ]

Eostrophomena Walcott, 1905:256

(Eostrophonella) Williams, 1950:282 [subgenus of Strophonella]

Eosyringothyris Stainbrook, 1943b:431

Eousella Ovtsharenko, 1976:26

${ }^{* *}$ Epacroplecia Williams, 1962 [= Craigella]

Ephippelasma Cooper, 1956c: 261

Epicelia Grant, 1972:224

Epicyrta Deslongchamps, 1884:275

Epithyris Phillips, 1841:55

Epithyroides Xu, 1978b:306

Epithyrus._Paetel, 1875 [misspelling of Epithyris]

Epitomyonia Wright, 1968:128

(Equirostra) Cooper and Muir-Wood, 1951:195 [new name

for Isorhynchus; subgenus of Porambonites ]

Erectocephalus Xian, 1978a:334

Eremithyris Brügge, 1973:197

Eremotoechia Cooper, 1956c:513

Eremotrema Cooper, 1956c:960

Ericiatia Muir-Wood and Cooper, 1960:172

Eridmatus Branson, 1966:75

Eridorthis Foerste, 1909a:223

Erymnaria Cooper, 1959:64

Erymnia Cooper, 1977:92

Espella Nilova, 1965:102

Estlandia Schuchert and Cooper, 1931:245

Estonirhynchia Schmidt, 1954:236

Etheridgina Oehlert, 1887, in Fischer, 1887:1278

Etymothyris Cloud, 1942:59

Eucalathis Fischer and Oehlert, 1890:72 
Eucharitina Schmidt, 1955:121

Eudesella Munier-Chalmas, 1880:279

Eudesia King, 1850:144

Eudesiles Rioult, 1966: 74

Eudoxina Fredericks, 1929:382

Euidolhyris Buckman, 1915 [nomen nudum]; 1917:101

Eumetabololoechia Sartenaer, 1975:3

Eumetria Hall, 1863:54

*Eunella Hall and Clark, 1893:290 [= Cranaena $]$

†Eunoa Clarke, 1902 [replaced by Oxlosia]

**Euobolus Mickwitz, 1896 [= Obolus]

Euorthisina Havlíček, 1950:16

**Euproduclus Whitehouse, 1928 [= Linoproductus]

Euractinella Bittner, 1890:303

Eurekaspirifer Johnson, 1966b: 1045

**Eurhynchonella Leidhold, 1920 [= Rhynchonella

Eurycolporhynchus Sartenaer, 1968d:566

Euryspirifer Wedekind, 1926:202

*Eurytatospinfer Gatinaud, 1949:487 [= Cyrlospirifer]

Eurythyris Cloud, 1942:63

Eurytrela Rowell, 1966:9

Euterebratula Makridin, 1954 [nomen nudum]

Euthyris Quenstedt, 1869 [rejected name proposed to replace Athyris]

Euxinella Moisseiev, 1936:41

Evanescirostrum Sartenaer, 1965a:8

Evenkina Andreeva, 1961, in Nikiforova and Andreeva, 1961:96

Evenkinorthis Yadrenkina, 1977:27

Evenkorhynchia Rozman, 1969:102

(Exalrypa) Copper, 1967a:123 [subgenus of Spinatrypina]

Excavatorhynchia Jin and Fang, 1977:44

Exceptothyris Sučić-Protić, 1971:37

Faksethyris Asgaard, 1971:385

Falafer Grant, 1972:217

Fallax Atkins, 1960:72

Fallaxispirifer Su, 1976:226

Falsatrypa Havlícek, 1956:584

Famatinorthis Levy and Nullo, 1973:146

Fardenia Lamont, 1935:311

Fascicosla Stehli, 1955c: 71

Fascicostella Schuchert and Cooper, 1931:246

Fasciculina Cooper, 1952b: 7

Fascifera Ulrich and Cooper, 1942:620

Fascistropheodonta Harper and Boucot, 1978c:24

Fascizelina Havlíček, 1975:232

Felinotoechia Havlíček, 1961a:73

Fenestrirostra Cooper, 1955a:57

Fenxiangella Wang, 1978:224

Ferganella Nikiforova, 1937:77

Ferganoproductus Galitzkaja, 1977:33

Ferrax Havliček, 1975:231

Ferrythyris Almeras, 1971:218
Fezzanoglossa Havliček, 1973, in Havliček and Massa, 1973: 273

Ffynnonia Neuman and Bates, 1978:592

Fibulistrophia Garcia-Alcalde, 1972b:43

Fidespirifer Ljaschenko, 1973:129

Filiconcha Dear, 1969:299

†Fimbriaria Muir-Wood and Cooper, 1960 [replaced by Fimbrinia ]

Fimbrinia Cooper, 1972a:450 [new name for Fimbriaria]

Fimbriothyris Deslongchamps, 1884:273

Fimbrispirifer Cooper, 1942:231

Finkelnburgia Walcott, 1905:277

Finospirifer Ying, 1979 [nomen nudum]

Fissirhynchia Pearson, 1977:48

Fissirostra._Orbigny, 1847 [misspelling of Fissurirostra]

* Fissurirostra Orbigny, 1847:269 [= Trigonosemus]

Fistulogonites Neuman, 1971:117

Fitzroyella Veevers, 1959:104

Flabellarhynchia Buckman, 1914 [nomen nudum]; 191 7:65

Flabellocyrtia Chorowicz and Termier, 1975:235

Flabellothyris Deslongchamps, 1884:263

Flabellulirostrum Sartenaer, 1971:5

†Fletcherina Stehli, 1961 [replaced by Fletcherithyris]

Fletcherithyris Campbell, 1965:24 [new name for Fletcherina]

Fletcherithyroides Dagys, 1977a:14

Flexaria Muir-Wood and Cooper, 1960:259

Fluctuaria Muir-Wood and Cooper, 1960:304

Foliomena Havlíček, 1952:413

Fordinia Walcott, 1908:65

Forma Gregorio, 1930a:28

Formosarhynchia Seifert, 1963:177

(Fossatrypa) Mizens and Rzonsnitzkaja, 1979:62 [subgenus of Munieratrypa]

Foveola Gorjansky, 1969:30

Franklinella Lenz, 1973:1405

Fredericksia Paeckelmann, 1931 b:48 [new name for Munia]

*Frenula Dall, 1871 a:55 [= Macandrevia]

Frenulina Dall, 1895:724

Frieleia Dall, 1895:714

Fulcriphoria Carls, 1974:176

Furcirhynchia Buckman, 1914 [nomen nudum]; 1917:59

Furcilella Cooper, 1956c:876

Fusella McCoy, 1844:128

Fusichonetes Liao, 1979 [nomen nudum]

Fusiproduclus Waterhouse, 1975:12

Fusirhynchia Dagys, 1968:68

Fusispirifer Waterhouse, 1966:43

Gacella Williams, 1962:222

Gacina Stehli, 1961b:458

*Gagnella Moisseiev, 1939:193 [= Kolhidaella]

Galateastrophia Harper and Boucot, 1978c:25

Galeatella Muir-Wood and Cooper, 1960:174 
†Galealella Sapelnikov and Rukavischnikova, 1975 [replaced by Galeatellina]

Galeatellina Sapelnikov and Rukavischnikova, 1976:122 [new name for Galeatella Sapelnikov and Rukavischnikova, 1975]

Galliennithyris Rollet, 1966:304

Gamdaella Miloradovich, 1947:92

Gamonetes Isaacson, $1977 \mathrm{a}: 168$

Gamphalosia Stainbrook, 1945:33

Gannania Fu, 1979 [nomen nudum]

Gasconsia Northrop, 1939:161

Gaspesıa Clarke, 1907:277

Gastrodeloechia Sartenaer, 1965a:2

Gefonia Licharew, 1936a:264

Gegenella Lee and $\mathrm{Gu}, 1976: 237$

Geinitzia Hall, 1889 [nomen nudum; = Conotrela]

Gelidorthina Havlíček, 1974:168

Gelidorthis Havlícek, 1968:125

Gemerithyris Siblík, 1977:203

Gemmarcula Elliott, 1947:145

†Gemmellaria Fabiani and Ruiz, 1933 [invalid new name for Canavaria, Gemmellaria preoccupied; replaced by Sicelia]

Gemmellaroia Cossman, 1898:77 [new name for Megarhynchus]

* Gemmellaroiella Mabuti, 1937:16 [= Gemmellaroia]

Gemmulicosia Waterhouse, 1971, in Bamber and Waterhouse, 1971:210

Geniculifera Muir-Wood and Cooper, 1960:187

(Geniculomclearniles) Harper and Boucot, 1978b:132 [subgenus of Mclearnites]

Georgethyris Minato, 1953:68

Geranocephalus Crickmay, 1954:157

Gerassimovia Licharew, 1956, in Kiparisova et al., 1956:59

Gerothyris Struve, 1970:537

Geyerella Schellwien, 1900:12

†Geyeria Carapezzi and Schopen, 1899 [replaced by Carapezzia]

Gibberostrophia Harper and Boucot, 1978a:79

Gibbirhynchia Buckman, 1914 [nomen nudum]; 1917:43

Gibbilhyris Sahni, 1925:372

(Gibbochonetes) Aisenverg, 1971:70 [subgenus of Delepinea]

Gibbospirifer Waterhouse, 1971, in Bamber and Waterhouse, 1971:221

†Gigantella Sarytcheva, 1928 [replaced by Gigantoproductus]

Gigantoproductus Prentice, 1950:437 [new name for Gigantella]

Gigantorhynchus Sapelnikov and Malygina, 1977:62

Gigantothyris Seifert, 1963:180

Gilledia Stehli, 1961a:451

Giraldibella Havlícek, 1977a:87

Giraldiella Bancroft, 1949a:5

Girlasia Gregorio, 1930a:21

Girtyella Weller, 1911:443

Gisilina Steinich, 1963:735
Gjelispinifera Ivanova, 1975:86

Glabrichonetina Waterhouse, 1978:130

Glaciarcula Elliott, 1956:285

Gladiostrophia Havlícek, 1967a:141

Glassia Davidson, 1881b:11

Glassina Hall and Clarke, 1893:97

Glazewskia Pajaud, 1964 [nomen nudum]; 1964:260

Glendonia McClung and Armstrong, 1978:2

Globiella Muir-Wood and Cooper, 1960:305

Globirhynchia Buckman, 1914 [nomen nudum]; 1917:48

Globispirifer Tachibana, 1964:37

Globithyris Cloud, 1942:82

Globosobucina Waterhouse and Piyasin, 1970:123

Globosochonetes Brunton, 1968:48

Glossella Cooper, 1956c:228

Glosseudesia Lobatscheva, 1974:146

Glosshypothyridina Rzonsnitzkaja, 1978:180

†Glossina Phillips, 1848 [replaced by Palaeoglossa]

(Glossinotoechia) Havlícek, 1959b: 79 [subgenus of Glossinulus]

Glossinulina Johnson, 1975:958

Glossinulus Schmidt, 1942:394

Glossoleplaena Havlícek, 1967a: 115

Glossorthis Öpik, 1930:82

**Glossostrophia Williams, 1950 [= Leptodontella ]

Glossothiris.-Gregorio, 1891 [misspelling of Glossothyris]

**Glossothyris Douvillé, 1879 [= Nucleala]

Glossothyropsis Girty, 1934:251

Glotlidia Dall, 1870:157

Glyplacrothele Termier and Termier, 1974:45

Glyplamboniles Cooper, 1956c:713

Glyplerina Boucot, 1970:984

Glypitias Walcott, 1901:675

†Glyploglossa Cooper, 1956 [replaced by Glyploglossella]

Glyploglossella Cooper, 1960:601 [new name for Glyptoglossa]

Glyptomena Cooper, 1956c:881

Glyptoria Cooper, 1976:281

Glyptorthis Foerste, 1914:258

Glyptospirifer Hou and Xian, 1975:72

Glyptosteges Cooper and Grant, 1975:877

Glyptotrophia Ulrich and Cooper, 1936b:627

Gmelinmagas Marinelarena, 1964:268

Gnathorhynchia Buckman, 1914 [nomen nudum]; 1917:29

Goleomixa Grant, 1976:180

Gonambonites Pander, 1830:77

Gondolina Ching and Liao, 1966, in Wang et al., 1966:412

Goniarina Cooper and Grant, 1969:2

Goniclis Rafinesque, 1819; 1831 [nomina nuda]

* Goniocoelia Hall, 1861:101 |= Pentagonia]

Goniophoria Janischewsky, 1910:81

Goniorhynchia Buckman, 1914 [nomen nudum]; 1917:52

Goniothyrella Tchorszhewsky, 1973; 1974 [nomina nuda]

Goniothyris Buckman, 1914 [nomen nudum]; 1917:117 
Goniotrema Ulrich and Cooper, 1936b:626

Gonotrema Rafinesque, 1820 [nomen nudum]

Gorgostrophia Havlíček, 1967a:136

(Gotatrypa) Struve, 1966:129 [subgenus of Alrypa]

Gollandia Dall, 1870 [new name for Trimerella, withdrawn by Dall in 1871]

* Grabauellina Licharew, 1934, in Zittel, 1934:507 [new name for Derbyina Grabau, 1931; = Derbyia ]

*Grabauicyrtiopsis Gatinaud, 1949:490 [= Cyrtiopsis]

* Grabauispirifer Gatinaud, 1949:413 [= Cyrtospirifer]

Gracianella Johnson and Boucot, 1967:868

Gracilotoechia Baranov, 1977:78

Grammetaria Cooper, 1959:58

Grammorhynchus Roberts, 1971:144

Grandaurispina Muir-Wood and Cooper, 1960:306

Grandirhynchia Buckman, 1914 [nomen nudum]; 1917:40

* Grandispirifer Yan, 1959:116 [= Unispirifer]

Grantonia Brown, 1953:60

Granulirhynchia Buckman, 1914 [nomen nudum]; 1917:64

Grasirhynchia Owen, 1968:19

Graticulatia Gupta and Waterhouse, 1979 [nomen nudum]

**Gratiosina Grant, 1976 [= Transennatia ]

Grayina Boucot, 1975:354

Greenfieldia Grabau, 1910, in Grabau and Sherzer, 1910: 148

*Greenockia Brown, 1952:91 [= Hemiplethorhynchus]

Grorudia Spjeldnaes, 1957:61

Gruenewaldita Tschernyschew, 1885:46

Grumantia Ustritsky, 1963, in Ustritsky and Tschernjak, 1963:70

Gryphus Megerle von Mühlfeld, 1811:64

Guangshunia Xian, 1978a:318

Guangxiispirifer Xian, 1978a:316

Gubleria Termier and Termier, 1960:241

**Guerichella Paeckelmann, 1913 [invalid new name for Adolfia, Adolfia not preoccupied]

*Guerichia Wedekind, 1926 [nomen nullum; = Adolfia]

Guicyrtia Wang and Zhu, 1979:59

Guizhouella Yang, 1978a: 124

Gunnarella Spjeldnaes, 1957:149

Gunningblandella Percival, 1979a:111

Gusarella Prosorovskaja, 1962:111

*Guseriplia Dagys, 1963:107 [= Sinucosta]

Guttasella Neuman, 1977:31

Gwynia King, 1859:258

Gypidia Dalman, 1828 [invalid name; = Pentamerus]

Gypidium.-Beyrich, 1837 [misspelling of Gypidia]

Gypidula Hall, 1867b:163

Gypidulella Khodalevich and Breivel, 1959, in Khodalevich et al., 1959:26

Gypidulina Rzonsnitzkaja, 1956, in Kiparisova et al., 1956: 49

Gypospirifer Cooper and Grant, 1976a:2210

Gyrosoria Cooper, 1973c:386
Gyrothyris Thomson, 1918:28

Hadrorhynchia McLaren, 1961:3

Hadrotreta Rowell, 1966:12

Hadyrhyncha Havlícek, 1979:98

Hagabirhynchia Jefferies, 1961, in Hudson and Jefferies, 1961:5

Hallina Winchell and Schuchert, 1892:292

Halorella Bittner, 1884:107

Halorellina Xu, 1978b:276

Halorelloidea Ager, 1960:159

Hamburgia Weller, 1911:446

*Hamletella Hayasaka, 1953:92 [= Orthothetina]

Hamlingella Reed, 1943:78

Hamplonina Rollier, 1919:360

Hanaeproductus Ficner and Havlíček, 1978:65

Hansolrela Krause and Rowell, 1975:46

Hanusitrypa Havlícek, 1967b:443

Haplospirifer Lee and Gu, 1976:280

Harknessella Reed, 1917:862

Harpidium Kirk, 1925:2

Harringtonina Boucot, 1972, in Amos, 1972:10

Hartlella Bell, 1929:149

*Hartlina Hall and Clarke, 1893:292 [= Romingerina]

Havlicekia Boucot, 1963:693

Haydenella Reed, 1944:79

Haydenoides Chan, 1977:352; 1979, in Hou et al., 1979:82

Hebertella Hall and Clarke, 1892:198

Hebeloechia Havlícek, 1959b:79

** Hectoria Trechmann, 1918 [invalid new name for Clavigera, Hectoria preoccupied; replaced by Heclorina; Clavigera not preoccupied]

**Hectorina Finlay, 1927 [invalid new name for Hecloria; Clavigera not preoccupied]

Hederna Boucot, 1957:323

(Hedstroemina) Bancroft, 1929:58 [subgenus of Kjaerina]

Heimia Haas, 1890:87

Helaspis Imbrie, 1959:400

Helenathyris Alekseeva, 1969:1157

Helmersenia Pander, 1861:48

Helvetella Owen, 1977:221

Hemichoneles Racheboeuf, 1979 [nomen nudum]; in Havlíček and Racheboeuf, 1979:83

Hemileurus Cooper and Grant, 1976a:2018

Hemiplethorhynchus Peetz, 1898: 181

(Hemipronites) Pander, 1830:74 [subgenus of Clitamboniles]

Hemiplychina Waagen, 1882:363

Hemithiris Orbigny, 1847:268

Hemithyris.-Bronn, 1848 [misspelling of Hemithris]

Hemithyropsis Katz, 1974:254

Hemiloechia Nikiforova, 1970:103

Hercosestria Cooper and Grant, 1969:7

Hercosia Cooper and Grant, 1969:8

Hercostrophia Williams, 1950:277 
Hercolhyris Cooper, 1979:25

Hesperinia Cooper, 1956c:822

Hesperithyris Dubar, 1942:78

Hesperomena Cooper, 1956c: 744

Hesperonomia Ulrich and Cooper, 1936b:621

Hesperonomiella Ulrich and Cooper, 1936b:622

Hesperorhynchia Warren, 1937:2

Hesperorthis Schuchert and Cooper, 1931:244

Hesperotrophia Ulrich and Cooper, 1936b:630

Heleralosia King, 1938:278

Heleraria Cooper and Grant, 1976a:2276

Helerelasma Girty, [1909]:337

*Helerelasmina Licharew, 1939:120 [= Jisuina]

Helerorthella Harper, Boucot, and Walmsley, 1969:79

Heterorthina Bancroft, 1928b:59

Helerorthis Hall and Clarke, 1892:202

Himalairhynchıa Ching and Sun, 1976, in Ching et al., 1976:292

Hindella Davidson, 1882:130

Hinniphoria.-Bronn, 1862 [misspelling of Hynniphoria]

Hipparionix Vanuxem, 1842:124

Hipparionyx. - Auctorum [improper emendation of Hipparionix by subsequent authors]

Hircinisca Havlícek, 1960:241

Hirnantia Lamont, 1935:313

Hirsulella Cooper and Muir-Wood, 1951:195 [new name for Hirsutina]

†Hirsutina Kirchner, 1933 [replaced by Hirsutella]

Hisingerella Henningsmoen, 1948, in Waern et al., 1948: 388

Hispanirhynchia Thomson, 1927:159

Histosyrinx Massa, Termier, and Termier, 1974:168

Holcorhynchella Dagys, 1974:110

Holcorhynchia Buckman, 1914 [nomen nudum]; 1917:28

Holcospirifer Bassett, Cocks, and Holland, 1976:620

Holcothyris Buckman, 1915 [nomen nudum]; 1917:125

Hollardiella Drot, 1966:877

Holorhynchus Kiaer, 1902:68

Holosia Cooper and Grant, 1976a:1971

Holotricharina Cooper and Grant, 1975:1173

Holledahlina Foerste, 1924:123

Holynatrypa Havlícek, 1973:339

Holynetes Havlícek and Racheboeuf, 1979:97

Homevalia._Campbell, 1959 [error by Campbell, should be Ingelarella]

Homoeorhynchia Buckman, 1914 [nomen nudum]; 1917:36

Homoeospira Hall and Clarke, 1893:112

Homoeospirella Amsden, 1968:90

* Homotreta Bell, 1941:230 [= Prototreta]

Hontorialosia Martínez, 1979:119

Hoplotheca-Bigsby, 1878 [misspelling of Anoplotheca]

Horderleyella Bancroft, 1928a:178

Horridonia Chao, 1927:24

Hoskingia Campbell, 1965:52
Howellella Kozlowski, 1946:295 [new name for Crispella]

Howellites Bancroft, 1945:203

Howitlia Talent, 1956:34

Howseia Logan, 1963:756

Huenella Walcot t, 1908:109

Huenellina Schuchert and Cooper, 1931:247

Hunanella Lee, 1939 [nomen nudum]

Hunanoproductus Hou, 1965:117

*Hunanospirifer Tien, 1938:139 [= Cyrtospirifer]

Hungarispira Dagys, 1972a:102

Hungaritheca Dagys, 1972b:97

Hustedia Hall and Clarke, 1893:120

Hustediella Dagys, 1972a: 100

Hyatella._Cossmann, 1898 [misspelling of Hyattella]

†Hyattella Hall and Clarke, 1894 [replaced by Hyattidina]

Hyattidina Schuchert, 1913, in Zittel, 1913:415 [new name for Hyaltella]

Hyborhynchella Cooper, 1955a:59

Hynniphoria Suess, 1859a:44

(Hyponeatrypa) Struve, 1966:137 [subgenus of Atrypa]

Hypopsia Cooper and Grant, 1974:272

Hypothyridina Buckman, 1906b:324 (new name for Hypothyris King, 1846]

$\dagger$ Hypothyris King, 1846 [replaced by Hypothyridina]

**Hypothyris Phillips, 1841 [= Kallirhynchia]

Hypseloterorhynchus Sartenaer, 1971:2

Hypsiptycha Wang, 1949:17

Hypsomyonia Cooper, 1955a:53

Hysterolites Schlotheim, 1820:247

†Hystricina Stainbrook, 1945 [replaced by Spinalrypa]

Hystriculina Muir-Wood and Cooper, 1960:211

Iberirhynchia Drot and Westbroek, 1966:165

Iberithyris Kvakhadze, 1971 [nomen nudum]; 1972:75

*Icodonta Bell, 1941:213 [= Micromitra]

(Idioglyplus) Northrop, 1939:173 [subgenus of Cymostrophia]

*Idiorthis McLearn, 1924:56 [= Dalmanella]

Idiospira Cooper, 1956c:691

Idiostrophia Ulrich and Cooper, 1936b:631

Iheringithyris Levy, 1961:84

Ikella Tjazheva, 1972:205

Ilmarinia Öpik 1934:125

Ilmenia Nalivkin, 1941:186

Ilmenispina Havlícek, 1959a:257

Ilmospirifer Ljaschenko, 1969a:18

Ilyinella Jassjukevitch, 1973:104

Imatrypa Havlícek, 1977b:300

Imbrexia Nalivkin, 1937:105

Imbricatia Cooper, 1952b:21

* (Imperia) Gregorio, 1930a:31 [subgenus of Lyttonia; = Leptodus]

Implexina Poletaev, 1971, in Aisenverg, 1971:79

Inaequalis Sučić-Protić, 1971:12

Incisius Grant, 1976:103 
(Independatrypa) Copper, 1973:493 [subgenus of Desquamatia]

Indigia Barchatova, 1970 [nomen nudum]; 1973:100

Indorhynchia Ovtsharenko, 1975:124

Indospirifer Grabau, 1931a:359

Inflatia Muir-Wood and Cooper, 1960:227

$\ddagger$ Infurca Percival, 1979b: 185

*Ingelarella Campbell, 1959:341 [= Tomiopsis]

Ingria Öpik, 1930:57

*Iniathyris Besnossova, 1963, in Sarytcheva et al., 1963:312 $[=$ Composita $]$

Innuitella Crickmay, 1968:5

Inopinatarcula Elliott, 1952:2

Institella Cooper, 1942:230

Institifera Muir-Wood and Cooper, 1960:203

Institina Muir-Wood and Cooper, 1960:165

Inversella Öpik, 1933a:21

Inversathyris Dagys, 1968:92

Inverirypa Struve, 1961:334

Iotina Cooper and Grant, 1976a: 1944

Iowatrypa Copper, 1973:495

†Iphidea Billings, 1872 [replaced by Iphidella Walcott, 1905]

*Iphidella Walcott, 1905:304 [new name for Iphidea; = Palerina]

†Iphidella Walcott, 1912 [replaced by Dictyonina]

Irboskites Bekker, 1924:48

Irenothyris Pojariskaja, 1966:27

Irgislella Tjazheva, 1972, in Tjazheva and Zhavoronkova, 1972:90

Irhirea Havlícek, 1971c:44

Iridistrophia Havlíček, 1965a:292

*(Ima) Gregorio, 1930a:32 [subgenus of Lyltonia; = Leplodus]

Iru Öpik, 1934:89

Ishımia Nikitin, 1974:59

*Isjuminella Makridin, 1954; 1955 [nomina nuda; =Isjuminelina]

Isjuminelina Makridin, 1960, in Rzonsnitzkaja et al., 1960: 254

Ismenia King, 1850:245

Isocrania Jaekel, 1902:1063

Isogramma Meek and Worthen, 1870:35

Isophragma Cooper, 1956c:733

Isopoma Torley, 1934:81

†Isorhynchus King, 1850 [replaced by Equirosira]

Isorthis Kozlowski, 1929:29

(Isospinatrypa) Struve, 1966:155 [subgenus of Spinatrypa]

Isovella Breivel and Breivel, 1970a:52

Israelaria Cooper, 1976:281

Isumithyris Hatai, 1948:498

Ithyris.-Bigsby, 1878 [misspelling of Athyris]

*Iubagraspirifer Gatinaud, 1949:487 [= Cyrlospirifer]

*Iugrabaspirifer Gatinaud, 1949:487 [= Cyrlospirifer]

Ivanothyris Havlíček, 1957b:440
Ivanovia Ivanova, 1949 [nomen nudum?]

Ivanoviella Makridin, 1954 [nomen nudum?]; 1955:83

Ivdelinia Andronov, 1961:45

\$Ivshinella Koneva, 1979:54

Iwaispirifer Tachibana, 1964:39

Jaanussoniles Neuman, 1977:26

†Jacobella Patte, 1926 [invalid new name for Austriella,

Jacobella preoccupied; replaced by Austriellula]

Jaffaia Thomson, 1927:254

Jaisalmeria Sahni, 1955:187

(Jakutella) Abramov, 1970:119 [subgenus of Krotovia]

Jakutochoneles Afanasjeva, 1977a:31

Jakutoproductus Kaschirzev, 1959:28

Jamesella Walcott, 1905:252

Janiceps Frech, 1901:551

Janiomya Havlíček, 1967a:174

Janius Havlícek, 1957a:245

Japanithyris Thomson, 1927:251

Jalsengina.-Ivanova, 1960 [transliteration of Yatsengina]

Jisuina Grabau, 1931b:105

Jivinella Havlícek, 1949a:94

Johnsonathyris Savage, Eberlein, and Churkin, 1978:381

Jolonica Dall, 1920:366

Jolvia Sapelnikov, 1960a:56.

Junnanella.-Rzonsnitzkaja, 1958 [transliteration of Yunnanella]

Junnanellina. - Rzonsnitzkaja, 1958 [transliteration of Yunnanellina]

Juralina Kyansep, 1961:29

Juresania Fredericks, [1928]:792

Juvavella Bittner, 1888b:127

Juvavellina Bittner, 1896:132

Jvanoviella. - Makridin, 1955 [transliteration of Ivanoviella]

Kabanoviella Smirnova, 1973:43

Kachathyris Smirnova, 1975b:120

Kadraliproductus Galitzkaja, 1977:36

Kafirnigania Katz, 1962:138

Kallirhynchia Buckman, 1914 [nomen nudum]; 1917:31

Kamoica Hatai, 1936:313

Kaninospirifer Kulikov and Stepanov, 1975, in Stepanov et al., 1975:63

Kansuella Chao, 1928:68

Kaplex Ficner and Havlícek, 1975:362

Karabachia Askerov, 1965; 1967 [nomina nuda]

Karabaghia Askerov, 1964 [nomen nudum]

Karadagella Babanova, 1965:95

Karadagithyris Tchorszhewsky, 1973 [nomen nudum]; 1974:55

Karakulina Andreeva, 1972:55

Karavankina Ramovs, 1966 [nomen nudum]; 1969:253

Kardonikithyris Tchorszhewsky, 1973 [nomen nudum]

Karlicium Havlíček, 1974:169 
Karpalhothyris Tchorszhewsky, 1973 [nomen nudum]

Karpinskia Tschernyschew, 1885:49

Kasakhslania Besnossova, 1968, in Sarytcheva, 1968:179

Kassinella Borissiak, 1956, in Kiparisova et al., 1956:50

Katastrophomena Cocks, 1968:293

Kalunia Kulkov, 1963:54

Kayserella Hall and Clarke, 1892:259

Kayseria Davidson, 1882:21

Kayserlingia.-Beecher, 1891 [misspelling of Keyserlingia]

Kelusia Mamedov, 1978:200

Kendzhilgilhyris Ovtsharenko, 1977 [nomen nudum]

Keratolhyris Tuluweit, 1965:76

Kerpina Struve, 1961:333

*Kestonithyris Sahni, 1925:363 [= Gibbithyris]

Keyserlingia Pander, 1861:46

Keyserlingina Tschernyschew, 1902:475

Khinganospirifer Su, 1976:221

(Khodalevichia) Boucot and Johnson, 1979:116 [subgenus of Kirkidium]

Khodalevilchia Boucot, 1975 [nomen nudum]

Kiaeromena Spjeldnaes, 1957:183

Kiangsiella Grabau and Chao, 1927:103

Kikailhyris Yabe and Hatai, 1941:495

Kilethyris Yasamanov, 1977 [nomen nudum]

Kindleina Savage, Eberlein, and Churkin, 1978:390

Kinelina Ljaschenko, 1969b:35

Kingena Davidson, 1852a:42

Kingenella Popiel-Barczyk, 1968:72

Kingenina Askerov, 1964; 1965; 1967 [nomina nuda]

Kinghiria Litvinovich, 1966:98; 1969, in Litvinovich et al., 1969:252

Kingia.-Schloenbach, 1866 [misspelling of Kingena]

Kinnella Bergström, 1968:11

Kirkidium Amsden, Boucot, and Johnson, 1967:865

Kirkina Salmon, 1942:598

Kisilia Nalivkin, 1979:146

Kalakamilhyris Minato, 1951:374

Kjaerina Bancroft, 1929:43

Kjerulfina Bancroft, 1929:59

Klipsleinella Dagys, 1974:150

** Klitambonites Pander, 1830 [= Clilamboniles $]$

Kochiproductus Dunbar, 1955:107 [new name for Tschernyschewiella]

Koeveskallina Dagys, 1965:172

Kolhidaella Moisseiev, 1939:189

Kolymithyris Dagys, 1965: 148

Komiella Barchatova, 1970 [nomen nudum]

Komispirifer Ljaschenko, 1973:105

Koninckella Munier-Chalmas, 1880:280

Koninckia. —Suess, 1854 [misspelling of Koninckina]

Koninckina Suess, 1853, in Davidson, 1853:92

Koninckodonta Bittner, 1893:137

Konstantia Glazewski and Pajaud, 1970, in Pajaud, 1970: 189
Korlugina.-Davidson, 1871 [misspelling of Kulorgina]

Kosirium Ficner and Havlícek, 1975:362

Kotujella Andreeva, 1962:94

Kozlowskia Fredericks, 1933:29

†Kozlowskiella Boucot, 1957 [replaced by Kozlowskiellina]

Kozlowskiellina Boucot, 1958:1031 [new name for Kozlowskiella]

(Kozlowskites) Havlíček, 1952:406 [subgenus of Eopleclodonta]

Kransia Westbroek, 1967:81

†Kraussia Davidson, 1852 [replaced by Kraussina]

Kraussina Davidson, 1859, in Suess, 1859b:210 [new name for Kraussia]

Kraussinina._Paetel, 1875 [misspelling of Kraussina]

(Krejcigrafella) Struve, 1978:97 [subgenus of Schucherlellopsis]

Krotovia Fredericks, [1928]:780

Kueichowella Yang, 1978b:267

Kullervo Öpik, 1932:70

Kulumbella Nikiforova, 1960:61

Kumbella Khodalevich, 1975:137

Kundalella Aksarina, 1978, in Aksarina and Pelman, 1978: 105

Kurakilhyris Hatai, 1946:98; 1948:495

Kutchirhynchia Buckman, 1914 [nomen nudum]; 1917:54

Kutchithyris Buckman, 1915 [nomen nudum]; 1917:113

Kutorgina Billings, 1861a:8

Kutorginella Ivanova, 1951:329

Kuvelousia Waterhouse, 1968b:1175

Kvesanirhynchia Kvakhadze, 1976:505

Kwangsia Grabau, 1931a:205

Kwangsiella._Grabau, 1932 [misspelling of Kwangsia]

Kwangsirhynchus Hou and Xian, 1975:38

Kymatolhyris Struve, 1970:533

(Kyrlatrypa) Struve, 1966:135 [subgenus of Atrypa]

Labaia Licharew, 1956, in Kiparisova et al., 1956:66

Laballa Moisseiev, 1962, in Dagys, 1962:51

Labriproduclus Cooper and Muir-Wood, 1951:195 [new name for Worthenella]

Lacazella Munier-Chalmas, 1880:279

Lachrymula Graham, 1970:158

Lacunaerhynchia Almeras, 1966:97

Lacunariles Öpik, 1934:175

Lacunites Gorjansky, 1969:103

Lacunosella Wiśniewska, 1932:30

Ladjia Veevers, 1959:125

Ladogia Nalivkin, 1941:165

Ladogiella Öpik, 1934:93

Ladogifornix Schmidt, 1964:505

Ladogilina Ljaschenko, 1973:58

Ladogilinella Ljaschenko, 1973:61

Ladogioides McLaren, 1961:4 
Laevicamera. - Grabau, 1936 [improper emendation of Levicamera]

(Laevicyphomena) Cocks, 1968:317 [subgenus of Cyphomena]

Laevigaterhynchia Wiśniewska-Żelichowska, 1978:112

Laevirhynchia Dagys, 1974:91

Laevispirifer Ushatinskaia, 1977a:137

Laevithyris Dagys, 1974:198

Laioporella Ivanova, 1975:83

* Lakhmina Oehlert, 1887, in Fischer, 1887:1265 [new name for Davidsonella Waagen, 1885; = Neobolus]

Lamanskya Moberg and Segerberg, 1906:71

* Lamarckispirifer Gatinaud, 1949:489 [= Cyrlospirifer]

Lambdarina Brunton and Champion, 1974:819

Lamellaerhynchia Burri, 1953:274

Lamellaconchidium Kulkov, 1968:38

** Lamellispirifer Nalivkin, 1937 [= Mucrospirifer]

Lamellokonunckina Jin and Fang, 1977:57

Lamellosia Cooper and Grant, 1975: 1270

Laminatia Muir-Wood and Cooper, 1960:190

Lamnaespina Waterhouse, 1976:244

Lamnimargus Waterhouse, 1975:10

Lancangjiangıa Jin and Fang, 1977:50

Lanceomyonia Havlícek, 1960:243

Langella Mendes, 1961a:3

Langkawia Hamada, 1969a:261

Laosia Mansuy, 1913:83

Laqueus Dall, 1870:123

Larium Gregorio, 1930a:25

Lateralatirostrum Sartenaer, 1979a:539

Laticrura Cooper, 1956c:980

Latiplecus Lee and Gu, 1976:299

Latiproductus Sarytcheva and Legrand-Blain, 1977:75

Latonotoechia Havlíček, 1960:244

Lazulkinia Rzonsnitzkaja, 1952:152

Leangella Öpik, 1933a:42

Leiochonetes Roberts, 1976:26

Leiolepismatina Yang and Xu, 1966:39

Leioproductus Stainbrook, 1947:308

Leiorhynchoidea Cloud, 1944, in King et al., 1944:57

Leiorhynchoides Dovgal, 1953:140

Leiorhynchus Hall, 1860:75 [replacement by Nudirostra invalid, Leiorhynchus not preoccupied]

Leioria Cooper, 1976:284

Leioseplathyris Wu, 1974, in Wang et al., 1974:42

Lenaloechia Nikiforova, 1970:106

Lenorthis Andreeva, 1955:69

Lenothyris Dagys, 1968:100

Lepidocranıa Cooper and Grant, 1974:246

Lepidocycloides Nikiforova, 1961, in Nikiforova and Andreeva, 1961:212

Lepidocyclus Wang, 1949: 12

Lepidoleplaena Havlícek, 1963b:224

Lepidorhynchia Burri, 1956:689

Lepidorthis Wang, 1955:331
Lepidospirifer Cooper and Grant, 1969:15

* Leprsmatina Wang, 1955:353 [= Psioidea]

Leplaena Dalman, 1828:94

*Leplaenalosia King, 1845 [nomen nudum; = Strophalosia]

* Leplaenella Fredericks, 1918:89 [= Leplaena]

**Leplaenella Sokolskaja, 1952 [= Lepiagonia; junior homonym of Leptaenella Fredericks]

Leplaenisca Beecher, 1890:239

Leplaenoidea Hedström, 1917:5

Leptaenomendax Garcia-Alcalde and Martínez-Chacon, 1978:255

Leplaenopoma Marek and Havlíček, 1967:282

Leplaenopyxis Havlícek, 1963b:224

*Leplaenulopsis Haupt, 1878 [nomen nudum?; = Leplaena]

Leplagona McCoy, 1844:117

Leplalosia Dunbar and Condra, 1932:260

Leptathyris Siehl, 1962:212

Leptella Hall and Clarke, 1892:293

Leptellina Ulrich and Cooper, 1936b:626

Leptelloidea Jones, 1928:385

*Leplembolon Mickwitz, 1896:130 [= Lingulella]

Leplestia Bekker, 1922:363

Leptestiina Havlícek, 1952:412

Leptobolus Hall, 1871b:3

Leplobulus. - Hall, 1871 [misspelling of Leptobolus]

Leptocaryorhynchus Sartenaer, 1970:14

Leptochonetes Havlícek and Racheboeuf, 1979:89

Lepiocoelia Hall, 1857:108

Leplocoelina Johnson, 1970b:170

Leptodonta Khalfin, 1955:237 [new name for Oehlertia]

Leptodontella Khalfin, 1948:254

Lepiodus Kayser, 1883:161

Leplogonia.-Agassiz, 1846 [misspelling of Leplagonia]

*Leptoptilum Öpik, 1930:130 [= Leptestia]

Leploskelidion Amsden, 1974:47

Lepiospira Boucot, Johnson, and Staton, 1964:814

Leptostrophia Hall and Clarke, 1892:288

(Leptostrophiella) Harper and Boucot, 1978a: 74 [subgenus of Lepiosirophia]

Lepiothyrella Muir-Wood, 1965:H855 [new name for Leptothyris]

Leptothyrellopsis Bitner and Pisera, 1979:82

$\dagger$ LeptothyrisMuir-Wood, 1959 [replaced by Lepiothyrella]

Lercarella Mascle and Termier, 1970:188

*Lespius Gregorio, 1930a:24 [= Strepiorhynchus]

Lethamella Liu, 1979:509

Lethamia Waterhouse, 1973:38

Leurosina Cooper and Grant, 1975:1260

Levenea Schuchert and Cooper, 1931:246

Levibiseplum Xian, 1975, in Hou and Xian, 1975:31

* Levicamera Grabau, 1934 [nomen nudum; = Psilocamara]

Leviconchidiella Rzonsnitzkaja, 1960, in Markowskii, 1960: 301

Levigatella Andronov, 1961:38 
Levipustula Maxwell, 1951:10

Levispira Mizens, 1975:47

Levilusia Muir-Wood and Cooper, 1960:295

Lialosia Muir-Wood and Cooper, 1960:87

Libyaeglossa Havlícek, 1973, in Havlícek and Massa, 1973: 275

Libys Massa, Termier, and Termier, 1974:175

Lichareviella Ustritsky, 1959, in Khu et al., 1959:134

Licharewia Einor, 1939:69

Licharewiella Sokolskaja, 1960:219 [replacement by Magniderbyia invalid, Licharewiella not preoccupied]

Lievinella Boucot, 1975:372

Ligula-Cuvier, 1798 [misspelling of Lingula]

Ligularius. _Duméril, 1806 [misspelling of Lingula]

Ligulops.-Hall, 1871 [misspelling of Lingulops]

Liljevallia Hedström, 1917:12

Limbella Stehli, 1954:329

Limbimurina Cooper, 1956c:851

Lindstroemelia._Dunbar and Condra, 1932 [misspelling of Lindstroemella]

Lindstroemella Hall and Clarke, 1890:134

*Lindstromella Hall and Clark, 1889 [nomen nudum; = Lindstroemella]

*Lineirhynchia Buckman, 1914 [nomen nudum]; 1917:59 [= Furcirhynchia $]$

Linguithyris Buckman, 1914 [nomen nudum]; 1917:99

Lingula Bruguière, 1797, p1. 250 [type designated by Lamarck, 1799:89]

Lingulapholis Schuchert, 1913:295

Lingularius.-Schuchert and LeVene, 1929 [misspelling of Ligularius]

Lingulasma Ulrich, 1889:384

Lingulelasma.-Miller, 1889 [improper emendation of Lingulasma]

Lingulella Salter, 1866:333

Lingulepis Hall, 1863:129

*Lingulichnites Szmuc, Osgood, and Meinke, 1976 (ichnogenus; = Lingulichnus]

Lingulichnus Hakes, 1976 [ichnogenus]

Lingulidiscina.-Girty, 1909 [misspelling of Lingulodiscina]

Lingulipora Girty, 1898:387

Lingulobolus Matthew, 1895:261

Lingulodiscina Whitfield, 1890:122

Lingulops Hall, 1871 a:2 [nomen nudum]; 1872, plate 13

Linguopugnoides Havlícek, 1960:242

Linnarssonella Walcott, 1902:601

Linnarssonia Walcott, 1885:115

Linoporella Schuchert and Cooper, 1931:247

Linoproductus Chao, 1927:128

Linoprotonia Ferguson, 1971:551

Linostrophomena Rong, Xu, and Yang, 1974:203

Liocoelia Schuchert and Cooper, 1931:248

Lioleptaena Xian, 1978a:265
Liorhynchus.-Oehlert, 1887 [improper emendation of Leiorhynchus]

Liosotella Cooper, 1953:36

Liospiriferina Rousselle, 1977:164

Liostrophia Cooper and Kindle, 1936:355

Liothyrella Thomson, 1916a:44

** Liothyrina Oehlert, 1887 [new name for Liolhyris; = Gryphus]

Liothyris Douvillé, 1879 [replaced by Liothyrina]

Liralingua Graham, 1970:153

Liraplecta Ching and Sun, 1979 [nomen nudum]

Liraria Cooper and Grant, 1975:1156

${ }^{*}$ Liraspirifer Stainbrook, 1950:381 [= Cyrtospirifer]

Lirellaria Cooper and Grant, 1976a:2031

Liricamera Cooper, 1956c:592

Liriplica Campbell, 1961:440

Lissajousithyris Almeras, 1971:164

Lissalrypa Twenhofel, 1914:33

Lissatrypoidea Boucot and Amsden, 1958: 159

Lissella Campbell, 1961:452

Lissochoneles Dunbar and Condra, 1932:170

Lissocoelina Schuchert and Cooper, 1931:248

*Lissocrania Williams, 1943:71 [= Crania]

*Lissomarginifera Lane, 1962:902 [= Eomarginifera]

Lissopleura Whitfield, 1896:232

Lissorhynchia Yang and Xu, 1966:14

(Lissosia) Cooper and Grant, 1975:1241 [subgenus of Dyoros]

Lissostrophia Amsden, 1949:202

(Lissotreta) Amsden, 1968:37 [subgenus of Onychotreta]

Litocothia Grant, 1976:166

Liothyris Roberts, 1971:237

Ljaschenkovia Batrukova, 1969:71

Ljudmilispirifer Tcherkesova, 1976:90

Llanoella Boucot, 1975:351

Loboidothyris Buckman, 1914 [nomen nudum]; 1917:112

Loboidothyropsis Sučić-Protić, 1971:17

Lobothyrella Tchorszhewsky, 1971 [nomen nudum]

Lobothyrs Buckman, 1914 [nomen nudum]; 1917:107

Lobothyroides Xu, 1978b:307

Lobothyropsis Tchorszhewsky, 1974 [nomen nudum]

(Lobvia) Breivel and Breivel, 1977:97 [subgenus of Theodossia]

*Lochengia Grabau, 1931 [nomen nudum; = Cryptospirifer]

Loczyella Frech, 1901:503

*Loganella Boucot and Amsden, 1958:164 [= Rhipidomella]

Loilemia Reed, 1936:116

Lomatiphora Roberts, 1971:84

Longdongshuia Hou and Xian, 1975:39

Longispina Cooper, 1942:230

Longithyris Katz and Popov, 1974:30

*Longvillia Bancroft, 1933:3 [= Strophomena]

Loperia Walcott, 1905:287

Lophrothyris Buckman, 1914 [nomen nudum]; 1917:114

Lopingia Chan, 1979, in Hou et al., 1979:64 
Lorangerella Crickmay, 1963:11

Lordorthis Ross, 1959:447

Loriolithyris Middlemiss, 1968:176

Losvia Breivel and Breivel, 1976, in Tjazheva et al., 1976: $116 ; 1977: 83$

Lowenstamia Stehli, 1961b:460

Loxophragmus Cooper and Grant, 1974:432

Luanquella Garcia-Alcalde and Racheboeuf, 1978:847

Ludfordina Kelly, 1967:599

Luhaia Rōōmusoks, 1956:1091

Lunaria Ching, Sun, and Ye, 1979:155

Lunpolaia Ching and Ye, 1979, in Ye and Yang, 1979:67

Luofuia Xu, 1977:66

Lutetiarcula Elliott, 1954:727

Lycophoria Lahusen, 1886:221

*Lyra Cumberland, 1818, in Sowerby, 1818:84 [= Terebrirostra]

Lysingothyris. - Hall, 1868 [misspelling of Syringothyris]

* Lytha Fredericks, [1924a]:298 [= Spirifer]

*Lyttonia Waagen, 1883:398 [= Leptodus]

Maakina Andreeva, 1961, in Nikiforova and Andreeva, 1961:170

Macandrevia King, 1859:261 [replacement by Waldheimiathyris invalid, Macandrevia not preoccupied]

Macandrewia. - Bronn, 1862 [improper emendation of $\mathrm{Ma}$ candrevia]

Machaeraria Cooper, 1955a:55

Mackerrovia Cocks, 1968:319

Maclarenella Stehli, 1955a:868

Macrocoelia Cooper, 1956c:891

* Macroplectane Cossmann, 1909:215 [new name for Denckmannia $;=$ Rensselandia $]$

Macropleura Boucot, 1963:690

Macropotamorhynchus Sartenaer, 1970:24

Madarosia Cooper and Grant, 1976a:2002

Magadania Ganelin, 1977:153

Magadina Thomson, 1915d:399

Magadinella Thomson, 1915d:400

Magas Sowerby, 1818:40

Magasella Dall, 1870:134

Magella Thomson, 1915d:396

Magellania Bayle, 1880:240 [new name for Waldheimia]

Megharithyris Farag and Gatinaud, 1960:77

Magnicanalis Rowell, 1962:140

** Magniderbyia Ting, 1965 [invalid new name for Licharewiella, Licharewiella not preoccupied]

*Magnithyris Sahni, 1925:367 [= Carneithyris]

Magniventra Harper and Boucot, 1978a:77

Magnumbonella Carter, 1968:1145

† Maia Fredericks, 1924 [replacement by Mansuyella invalid, Mansuyella preoccupied; replaced by Callispirina]

Majkopella Moisseiev, 1962, in Dagys, 1962b:61

Makridinirhynchia Sučić-Protić, 1969:81
Malayanoplia Hamada, 1969a:255

Malleia Thomson, 1927:283

Malurostrophia Campbell and Talent, 1967:309

Malwirhynchia Chiplonker, 1938:306

Mametothyris Smirnova, 1969, in Smirnova and Pergament, 1969:34

*Mammosum Gregorio, 1930a:25 [= Streplorhynchus]

Manithyris Foster, 1974:55

*Mannia Davidson 1874:156 [= Cryptopora]

Mannia Dewalque, 1868 [nomen nudum]

Manoproductus Boucot, 1975 [nomen nudum]

Mansina Andreeva, 1977:116

† Mansuyella Reed, 1944 [invalid new name for Maia, Mansuyella preoccupied; replaced by Callispirina]

Maorielasma Waterhouse, 1964:175

Maoristrophia Allan, 1947b:440

Marcharella Andreeva, 1968, in Markowskii, 1968:75

Marginalosia Waterhouse, 1978:64

Marginatia Muir-Wood and Cooper, 1960:263

Marginella Gemmellaro, 1897 [nomen nudum]

Marginicinclus Sutton, 1938:561

Marginifera Waagen, 1884:717

Marginirugus Sutton, 1938:561

Mariannaella Sapelnikov and Rukavischnikova, 1975a:7

Marinurnula Waterhouse, 1964:177

** Mariona Nalivkin, 1975 [= Parallelora]

$\dagger$ (Marionella) Bancroft, 1928 [replaced by Marionites; subgenus of Multicostella]

(Marionites) Cooper and Muir-Wood, 1951:195 [new name for Marionella; subgenus of Multicostella]

Markitoechia Havlícek, 1959b:81

Marklandella Harper, Boucot, and Walmsley, 1969:82

*Martellia Wirth, 1936:300 [= Polytoechia]

*Martellispirifer Gatinaud, 1949:487 [= Cyrtospirifer]

Martinella._Lee, 1939 [misspelling of Martiniella]

Martinia McCoy, 1844:139

Martiniella Grabau and Tien, 1931, in Grabau, 1931c:420

?Martinigisis Lebedev, 1926 [incertae sedis]

Martiniopsis Waagen, 1883:525

Martinothyris Minato, 1953:70

Matulella Cooper, 1951:5

Mauispirifer Allan, 1947b:445

Maxillirhynchia Buckman, 1914 [nomen nudum]; 1917:55

Maya._Rakusz, 1932 [misspelling of Maia]

Mcewanella Foerste, 1920:197

† Mclearnia Caster, 1939 [replaced by Mcleamites]

*Mclearnites Caster, 1945:319 [new name for Mcleamia; = Shaleria]

(Mclearnitesella) Harper and Boucot, 1978b:131 [subgenus of Mclearnites]

Medessia Andreeva, 1960, in Markowskii, 1960:295

Mediospirifer Bublichenko, 1956:102

Mediterranirhynchia Sučić-Protić, 1969:53

Meekella White and St. John, 1867:120 
Meekina Walcott, 1905 [nomen nudum]

Megachonetes Sokolskaja, 1950:42

Megaderbyia.-Jing, Ye, Xu, and Sun, 1979 [improper emendation of Magniderbyia]

*(Megakozlow'skiella) Boucot, 1957:322 [subgenus of Kozlowskiella; = Kozlowskiellina]

Megakozlowskzellina Amsden and Ventress, 1963 [name apparently used subgenerically rather than specifically as was intended; = Kozlowskiellina]

Megalanteris._Oehlert, 1887 [misspelling of Meganteris]

Megalopterorhynchus Sartenaer, 1965a:5

Megalorhynchus.-Gregorio, 1930 [misspelling of Megarhynchus]

Megamyonia Wang, 1949:32

Meganterella Boucot, 1959c: 767

Meganteris Suess, 1855:51

Megapleuronia Cooper, 1952a: 117

† Megarhynchus Gemmellaro, 1894 [replaced by Gemmellaroia] Megarites Rafinesque, 1831 [nomen nudum]

Megasalopina Boucot, Gauri, and Johnson, 1966:166

Megasella.-Paetel, 1875 [misspelling of Magasella]

Megasteges Waterhouse, 1975:6

Megastrophia Caster, 1939:37

(Megastrophiella) Harper and Boucot, 1978c: 19 [subgenus of Megastrophia]

Megalhiris Orbigny, 1847:269 [new name for Argiope]

Megathyris.-Bronn, 1848 [misspelling of Megathiris]

Megerlea.-Davidson, 1856 [misspelling of Megerlia]

Megerleia.-Bronn, 1862 [misspelling of Megerlia]

Megerlia King, 1850:145 [replacement by Muehlfeldtia invalid, Megerlia not preoccupied]

Megerlina Deslongchamps, 1884:243

Megorima Rafinesque, 1819 [nomen nudum]

Megorisma._Paetel, 1875 [misspelling of Megorima]

Megousia Muir-Wood and Cooper, 1960:309

Megumatrypa Harper, 1973:72

Meifodia Williams, 1951:106

Mendacella Cooper, 1930:381

Mendathyris Cloud, 1942:125

Mennespirifer Ljaschenko, 1973:101

Mentzelia Quenstedt, 1871:522

Mentzelioides Dagys, 1974:130

Mentzeliopsis Trechmann, 1918:229

Meona Steinich, 1963:732

Merciella Lamont and Gilbert, 1945:655

Merista Suess, 185 1:150

Meristella Hall, 1860:75

Meristina Hall, 1867a:299

Meristorygma Carter, 1974:689

Meristospira, Grabau, 1910, in Grabau and Sherzer, 1910 : 159

Merospirifer Reed, 1949:467

* Mesodalmanella Havliček, 1950:26 [= Howellites]
(Mesodouviella) Harper and Boucot, 1978b:124 [subgenus of Mesodouvillina]

(Mesodouvillina) Williams, 1950:281 [subgenus of Douvillina]

Mesoleptostrophia Harper and Boucot, 1978a:68

(Mesolissostrophia) Williams, 1950:280 [subgenus of Lissostrophia]

Mesolobus Dunbar and Condra, 1932:160

Mesonomia Ulrich and Cooper, 1936b:627

(Mesopholidostrophia) Williams, 1950:280 [subgenus of Pholidostrophia]

Mesoplica Reed, 1943:97

Mesospirifer Wedekind, 1935 [nomen nudum]

Mesolreta Kutorga, 1848:270

Metabolipa Godefroid, 1974:5

Metacamarella Reed, 1917:934

Metaplasia Hall and Clarke, 1893:56

Metorthis Wang, 1955:333

Metriolepis Cooper and Grant, 1976b:2717

Mezounia Havlícek, 1967a:31

Miaohuangrhynchus Yang, 1977:374

Micella Ovtsharenko, 1976:24

Macidus Chatterton, 1973:134

** Mickwitzella Walcott, 1908 [= Thysanotos]

Mickwitzia Schmidt, 1888:5

Micraphelia Cooper and Grant, 1969:4

Microcardinalia Boucot and Ehlers, 1963:51

Micromitra Meek, 1873:479

Microrhynchia Muir-Wood, 1952:124

Microsphaeridiorhynchus Sartenaer, 1970:27

* Microthyridina Schuchert and LeVene, 1929:120 [new name for Microthyrs; = Ornuthella]

$\dagger$ Microthyris Deslongchamps, 1884 [replaced by Microthyridina]

Microtrypa Wilson, 1945:144

*Mikrothyris Quenstedt, 1868 [nomen nudum?; = Alrypa]

Millythyris Almeras, 1971:245

Mimaria Cooper and Grant, 1976b:2907

Mimatrypa Struve, 1964a:436

Mimella Cooper, 1930:376

† Mimulus Barrande, 1879 [replaced by Brachymimulus]

* Minatothyris Vandercammen, 1957:178 [= Warrenella]

(Miniplanus) Waterhouse and Piyasin, 1970:101 [subgenus of Diplanus]

Miniprokopia Havlíček, 1971 b:229

Minororthis Ivanov, 1950, in Ivanov and Miagkova, 1950: 23

(Minutilla) Crickmay, 1967:10 [subgenus of Acutatheca] Minulostropheodonta Harper and Boucot, 1978c:30

Miogryphus Hertlein and Grant, 1944:95

*Miopentamerus Woods, 1937 [nomen nudum?; invalid name; = Pentamerus

Mirantesia Mohanti, 1972:176

Mirifusella Carter, 1971:250 
Mirisquamea Sučić-Protić, 1971:38

* Mirtellaspirifer Gatinaud, 1949:488 [= Cyrtospirifer] Misolia Seidlitz, 1913:179

Miyakothyris Hatai, 1938:100

Mjoesina Spjeldnaes, 1957:137

*Mobergia Redlich, 1899:5 [= Botsfordia]

Modestella Owen, 1961, in Casey, 1961:573

Moeschia Boullier, 1976:333

Mogoktella Andreeva, 1968, in Markowskii, 1968:82

Moisseevia Makridin, 1964:243

Moisseievia Dagys, 1963:46

Molongella Savage, 1974:13

Molongia Mitchell, 1921:547

Monadotoechia Havlícek, 1960:243

Monelasmina Cooper, 1955a:54

Mongolella Alekseeva, 1976:347

Mongoliella Ischnazarov, 1972:69

* Mongolina Grabau, $1931 \mathrm{~b}: 102$ [= Rostranteris]

Mongoliopsis Grunt, 1977c:79

Mongolirhynchia Hou and Zhao, 1976:191

Mongolosia Manankov and Pavlova, 1976:354

Mongolospira Alekseeva, 1977:63

Monobolina Salter, 1866:334

Monoconvexa Pelman, 1977:49

Monomerella Billings, 1871:221

Monorthis Bates, 1968:144

Monsardithyris Almeras, 1971:198

Montanella Ovtsharenko, 1976:21

Montzclarella Wiśniewska, 1932:55

† Monticola Nalivkin, 1930 [replaced by Plectorhynchella]

Monticulifera Muir-Wood and Cooper, 1960:327

Moorefieldella Girty, 1911:63

Moorellina Elliott, 1953:694

Moquellina Ching, Sun, and Ye, 1979:141

Moraviaturia Sahni, 1960:19

Moravilla Havlíček, 1953:4

Moravostrophia Havlíček, 1962:471

Morganella McKellar, 1970:17

Morinorhynchus Havliček, 1965a:291

* Morrisia Davidson, 1852c:371 [= Platidia]

* Morrisina Grabau, $1931 \mathrm{~b}: 98$ [= Hemiptychina]

Morrisithyris Almeras, 1971:131

Moscuella Makridin, 1954 [nomen nudum; = Mosquella]

Mosquella Makridin, 1955:86

Moumina Fredericks, [1924a]:323

Moutonithyris Middlemiss, 1976:63

Mucrospirifer Grabau, 1931c:408

**Muehlfeldtia Bayle, 1880 [invalid new name for Megerlia, Megerlia not preoccupied]

Muirwoodella Tchorszhewsky, 1973 [nomen nudum]; 1974: 52

Muirwoodellopsis Tchorszhewsky, 1973; 1974 [nomina nuda]

Muirwoodia Licharew, 1947:187
(Multicosta) Khodalevich and Breivel, 1977, in Breivel and Breivel, 1977:71 [subgenus of Leviconchidiella]

Multicostella Schuchert and Cooper, 1931:244

Multispinula Rowell, 1962:147

† Multispinula Waterhouse, 1966 [replaced by Echinalosia]

(Multispirifer) Kaplun, 1961:88 [subgenus of Eospirifer]

† Munella Fredericks, 1924 [replaced by Purdonella]

Munhella Neuman, 1971:117

† Munia Fredericks, 1918 [replaced by Fredericksia]

Munieratrypa Mizens and Rzonsnitzkaja, 1979:60

Muriferella Johnson and Talent, 1967b:44

Murinella Cooper, 1956c:844

Murravia Thomson, 1916a:45

Musculina Schuchert and LeVene, 1929:120 [new name for Musculus]

† Musculus Quenstedt, 1869 [replaced by Musculina]

Mutationella Kozlowski, 1929:31

Myotreta Gorjansky, 1969:67

Myriospirifer Havlícek, 1978:105

Mystrophora Kayser, 1871:612

Nabarredia Havlícek and Racheboeuf, 1979:96

Nabiaoia Xu, 1979a:370

Nadiastrophia Talent, 1963:62

Najadospirifer Havliček, 1957a:246

Najdinothyris Makridin and Katz, 1964, in Makridin, 1964: 35

(Nakazatothyris) Minato and Kato, 1977:623 [subgenus of Undispirifer]

Naliukinaria Rzonsnitzkaja, 1968, in Markowskii, 1968:117

Naliukinella Popov, 1974, in Katz and Popov, 1974:23

Nalivkinia Bublichenko, 1928:982

Nanambonites Liu, 1976:145

Nannirhynchia Buckman, 1914 [nomen nudum]; 1917:67

Nanorthis Ulrich and Cooper, 1936b:621

Nanospira Amsden, 1949:203

Nanothyris Cloud, 1942:45

Nantanella Grabau, 1936:72

Nanukidium Jones, 1979:1261 [new name for Rossella]

Naradanithyris Tokuyama, 1958b:2

Nayunnella Sartenaer, 1961b:2 [new name for Yunnanella Grabau 1931]

**Neatretia Fischer and Oehlert, 1891 [= Cryptopora]

(Neatrypa) Struve, 1964b:526 [subgenus of Desquamatia]

Nebenothyris Minato, 1953:72

Nekhoroshevia Bublichenko, 1956:101

Nemalocrania Grant, 1976:31

Nemesa Schmidt, 1941:41

Neobolus Waagen, 1885:758

Neobouchardia Thomson, 1927:270

Neochonetes Muir-Wood, 1962:87

Neocoelia McKellar, 1966:2

Neocyrtina Yang and Xu, 1966:62

Neodelthyris Hou, 1963:413 
Neofascicosta Xu, 1978b:278

Neogypidula Licharew, 1934a:213

* Neohemithyris Yabe and Hatai, 1934:587 [= Bastliola ]

(Neokarpinskia) Mizens, 1977a:95 [sutgenus of Karpinskia]

(Neokjaerina) Levy and Nullo, 1974a:191 [subgenus of Kjaerina]

Neoliothyrina Sahni, 1925:375

Neometabolipa Godefroid, 1974:23

*Neomunella Ozaki, 1931:25 [= Choristites]

Neophricadothyris Licharew, 1934b: 175

Neophricodothyris.-Ivanova, 1960 [misspelling of Neophricadolhyris]

Neoplicatifera Ching, Liao, and Fang, 1974:309

Neoproboscidella Ivanova, 1949 [nomen nudum]

Neoproductella Lee, 1939 [nomen nudum]

Neoretzia Dagys, 1963:130

Neorhynchia Thomson, 1915b:388

Neospirifer Fredericks, [1924a]:311

Neospirifer Nikitin, 1900 [nomen nudum; = Neospirifer Fredericks, 1924]

(Neospirigerina) Rzonsnitzkaja, 1975:72 [subgenus of Spintgerina]

Neostrophia Ulrich and Cooper, 1936b:631

Neothecidella Pajaud, 1970:108

Neothyris Douvillé, 1879:274

Neotrela Sobolev, 1976:132

${ }^{* *}$ Neotrigonella Cossman, 1910 [new name for Trigonella; objective synonym of Tetractinella; subjective synonym of Cheirothyris]

Neowellerella Dagys, 1974:116

Nepasztoechra Havlícek, 1979:91

* Nereidella Wang, 1955:339 [= Paurorthis]

Nervostrophia Caster, 1939:79

Neumanella Harper and Boucot, 1978c:29

Neumayrithyris Tokuyama, 1958a: 120

**Newberna Hall, 1891 [= Rensselandia]

Newberya.-Schuchert, 1897 [improper emendation of Newberria]

Nicolella Reed, 1917:860

Nigeroplica Nalivkin, 1975, in Stepanov, 1975:160

Nikiforovaena Boucot, 1963:67

Ningbingella Roberts, 1971:152

Ninglangothyris Jin and Fang, 1977:60

Nipponithyris Yabe and Hatai, 1934:588

Nisusia Walcott, 1905:248

Niviconia Cooper and Grant, 1974:374

Nix Easton, 1962:45

* Nocturnellia Havlícek, 1950:53 [= Dalmanella]

Nodella Tachibana, 1964:38

(Noellingia) Hall and Clarke, 1893:229 |subgenus of Porambonites]

Nondia Boucot and Chiang, 1974:66

Nordathyris Grunt, 1977b:73

Nordella Ljaschenko, 1973:135
Nordispirifer Ljaschenko, 1973:104

Nordotoechia Tcherkesova, 1965:80

Norella Bittner, 1890:315

**Norgium Breivel and Breivel, 1970 [= Proconchidium]

Notanoplia Gill, 1950:249

Nothopindax Cooper and Grant, 1974:318

Nothorthis Ulrich and Cooper, 1938: 106

Notiochonetes Muir-Wood, 1962:48

* Notoconchidium Gill, 1950:243; 1951:187 [= Karpinskia]

Notoleptaena Gill, 1950 [nomen nudum]; 1951:192

Notoparmella Johnson, 1973a: 1026

Notorygmia Cooper, 1972b:13

Notosaria Cooper, 1959:48

Notospirifer Harrington, 1955:115

Notostrophia Waterhouse, 1973:35

(Notothyrina) Licharew, 1936a:271 [subgenus of Rostranteris]

Notothyris Waagen, 1882:378

Notozyga Cooper, 1977: 105

Novellinetes Havliček and Racheboeuf, 1979:87

Novozemelia Tcherkesova, 1973:28

Nucleata Quenstedt, 1868:25

Nucleatina Katz, 1962:135

Nucleatula Bittner, 1888b: 126

Nucleospira Hall, 1859a:23

Nudauris Stehli, 1954:317

**Nudirostra Cooper and Muir-Wood, 1951 [invalid new name for Leiorhynchus, Leiorhynchus not preoccupied]

Nudirostralina Yang and $\mathrm{Xu}, 1966: 21$

Nudispiriferina Yang and $\mathrm{Xu}, 1966: 47$

Nugnecella Levy and Nullo, 1974b:28

Nuguschella Tjazheva, 1960, in Markowskii, 1960:408

Nurataella Larin, 1973:135

Nurochonetes Ushatinskaia, 1977b:42

Nyalamurhynchia Ching, Rong, and Sun, 1976, in Ching et al., 1976:300

Nyege Veevers, 1959:113

Nymphorhynchia Rzonsnitzkaja, 1956, in Kiparisova et al., 1956:53

Oanduporella Hints, 1975:19

Obesaria Havlícek, 1957b:438

Oblongarcula Elliott, 1959:147

Obnixia Hoover, 1979:11

Obolella Billings, 1861a:7

*Obolellina Billings, 1871:222 [= Rhynobolus]

Obolopsis Saito, 1936:350

Obolus Eichwald, 1829:274

Obovtes Rafinesque, 1831 [nomen nudum]

Obovothyris Buckman, 1927:32

Obturamentella Amsden, 1958:99

Obulus._Quenstedt, 1868 [misspelling of Obolus]

Ochotathyris Dagys, 1974:159

Ochotorhynchia Dagys, 1968:47

Ocnerorthis Bell, 1941:251 
Ocruranus Liu, 1979:506

Odarovithyris Tchorszhewsky, 1971:63

Odontospirifer Dunbar, 1955:155

Oehlertella Hall and Clarke, 1890:132

†Oehlertia Khalfin, 1948 [replaced by Leptodonta]

Oepikina Salmon, 1942:589

* Oepikinella Wilson, 1944:199 [= Strophomena]

Ogbinia Sarytcheva, 1965, in Ruzhentsev and Sarytcheva, 1965:229

Ogilviella Lenz, 1968:180

Okathyris Smirnova, 1975c:71

* Oldhamella Noetling, 1905 [invalid new name for Oldhamina]

Oldharnia.-Zittel, 1910 [misspelling of Oldhamina]

Oldhamina Waagen, 1883:406 [replacement by Oldhamella and Waagenopora invalid]

Oldhaminella Wanner and Sieverts, 1935:232

Oleneothyris Cooper, 1942:233

Oleorthis Havlíček, 1968:124

Olgerdia Grigorjewa, 1977:50

Oligomys Schuchert and Cooper, 1931:243

Oligoptycherhynchus Sartenaer, 1970:20

Oligorhachis Imbrie, 1959:403

Oligorhynchia Cooper, 1935:49

Oligorthis Ulrich and Cooper, 1936b:624

Oligothyrina Cooper, 1956a:526

* Olvveirella Oliveira, 1934:167 [new name for Brasilia; = Paranaia]

Ombonia Caneva, 1906:54

Omnutakhella Lopushinskaja, 1976:66

Omolonella Moisseiev, 1936:39

Omolonia Alekseeva, 1967, in Alekseeva and Nuzhdina, 1967: 138

**Omolonospirifer Simakov, 1970 [= Tenisia $]$

Omolonothyris Dagys, 1968:132

Oncosarina Cooper an Grant, 1969:9

Onniella Bancroft, 1928b:55

Onnizetına Havlíček, 1974:168

Onopordumaria Waterhouse, 1971, in Bamber and Waterhouse, 1971:205

Onychoplecia Cooper, 1956c:529

Onychotreta Ulrich and Cooper, 1936a:339

(Opikella) Amsden, 1968:48 [subgenus of Leangella]

Opisthotreta Palmer, 1954:771

Opsiconidion Ludvigsen, 1974:143

†Orbicella Orbigny, 1847 [replaced by Clistotrema]

Orbicoelia Waterhouse and Piyasin, 1970:144

* Orbicula Cuvier, $1798: 435$ [= Crania]

* Orbicularius Duméril, 1806 [= Crania]

Orbiculoidea Orbigny, 1847:269

Orbiculopora Batrukova, 1969:75

Orbiculothyris Wolfart, 1968, in Wolfart et al., 1968:528

Orbinaria Muir-Wood and Cooper, 1960:150

Orbirhynchia Pettitt, 1954:29
Orbuthele Sdzuy, 1955:9

Orenburgella Pavlova, 1969:76

Orientospira Dagys, 1965:101

Orientospirifer Hou and Xian, 1975:49

Orientothyris Katz and Popov, 1974:28

Orgostrophia Mitchell, 1977:115

Oriskania Hall and Clarke, 1893:270

Orlovirhynchia Dagys, 1968:75

Ornatothyrella Dagys, 1974:210

Ornatothyris Sahni, 1929:45

Ornithella Deslongchamps, 1884:273

*Ornithothyris Sahni, 1925:374 [= Carneithyris]

Ornothyrella Havlícek, 1971c:80

Orthambonites Pander, 1830:81

Orthidiella Ulrich and Cooper, 1936b:621

Orthidium Hall and Clarke, 1892:244

Orthis Dalman, 1828:112

**Orthisina Orbigny, 1847 [= Clitambonites]

Orthisocrania Rowell, 1963:39

Orthoidea Friren, 1876:1

* Orthonomaea Hall, 1859 [nomen nudum]; 1893, in Hall and Clarke, 1893:159 [= Catazyga]

Orthopleura Imbrie, 1959:391

Orthorhynchula Hall and Clarke, 1893:181

Orthorhynchuloides Williams, 1962:241

Orthospirifer Pitrat, 1975:387

Orthostrophella Amsden, 1968:23

Orthostrophia Hall, 1883, pl.36

Orthotetella King, [1931]:51

Orthotetes Fischer de Waldheim, 1829:375

Orthothetina Schellwien, 1900:10

Orthothrix Geinitz, 1847:86

Orthothyris Cooper, 1955b:64

Orthotichia Hall and Clarke, 1892:213

Orthotichina.-Tschernyschew, 1914 [misspelling of Orthotichia]

Orthotoma Quenstedt, 1869:315

Orthotomoides Tchorszhewsky, 1971 [nomen nudum]

Orthotropia Hall and Clarke, 1893, pl.84

Ortotichia.-Tschernyschew, 1914 [misspelling of Orthotichia]

Orulgania Solomina and Tschernjak, 1961:61

Orusia Walcott, 1905:273

Oslogonites Öpik, 1939:133

*Oslomena Spjeldnaes, 1957:161 [= Glyptomena $]$

Osmarella Pearson, 1977:58

(Otariella) Waterhouse, 1978:30 [subgenus of Marginifera]

Otospirifer Hou and Xian, 1975:65

Otusia Walcott, 1905:246

(Ovalella) Walmsley and Boucot, 1975:75 [subgenus of Isorthis]

** Ovalia Nalivkin, 1937 [= Brachythyris]

Ovatia Muir-Wood and Cooper, 1960:312

Overtonia Thomas, 1914:260 
Overtoniina Grunt, 1973, in Grunt and Dmitriev, 1973:94

Oxlosia Ulrich and Cooper, 1936b:619 [new name for Eunoa]

Oxoplecia Wilson, 1913:81

Oxycolpella Dagys, 1962b:68

Oxypleurorhynchia Plodowski, 1973:85

Oxyrhynchus Quenstedt, 1868 [nomen nudum]

Pachiloma Rafinesque, 1831 [nomen nudum]

†Pachyglossa Cooper, 1956 [replaced by Pachyglossella]

Pachyglossella Cooper, 1960:601 [new name for Pachyglossa]

Pachymagas Ihering, 1903:332

**Pachyrhynchus King, 1850 [= Bouchardia]

Pachythyris Boullier, 1976:154

Pacificocoelia Boucot, 1975:361

Pacifithyris Hatai, 1938:216

Paeckelmanella Licharew, 1934a:212

** Paeckelmannia Licharew, 1934 [invalid new name for Tornquistia, Tornquistia not preoccupied]

Pahlenella Schuchert and Cooper, 1931:245

†Pakistania Stehli, 1961 [replaced by Whttspakia]

Palaebolus._Gorjansky, 1960 [misspelling of Palaeobolus]

Palaeobolus Matthew, 1899:202

Palaeochoristites Sokolskaja, 1941:26

**Palaeocrania Eichwald, 1854 [= Pseudocrania]

Palaeoglossa Cockerell, 1911:96 [new name for Glossina]

Palaeoglossina. - Dunbar and Condra, 1932 [misspelling of Palaeoglossa]

$\ddagger$ Palaeoschmidules Koneva, 1979:35

Palaeospirifer Martynova and Sverbilova, 1968:26

Palaeostrophia Ulrich and Cooper, 1936b:627

Palaeostrophomena Holtedahl, 1916:43

*Palaferella Spriestersbach, 1942:187 [= Gruenewaldıa]

Paldiskia Gorjansky, 1969:28

*Palinorthis Ulrich and Cooper, 1936b:625 [= Ingria]

†Pallasiella Renz, 1932 [replaced by Phymatolhyris]

Pamirorhynchia Ovtsharenko, 1977 [nomen nudum]

Pamirotheca Dagys, 1974:79

Pamirothyrts Dagys, 1974:195

†Pamirothyris Ovtsharenko, 1975 [replaced by Pamirothyropsis]

Pamirothyropsis Ovtsharenko, 1979:126 [new name for $\mathrm{Pa}$ mirothyris Ovtsharenko, 1975]

Pammegetherhynchus Sartenaer, 1977:68

Pampoectlorhynchus Sartenaer, 1968c:4

Panderina Schuchert and Cooper, 1931:243

Pantellaria Dall, 1919:251

Papiliolinus Waterhouse and Gupta, 1977:160

Papillostrophia Havlícek, 1967a:132

Paraacanthothyris Kamyschan, 1973, in Kamyschan and Babanova, 1973:67

Parabifolium Pajaud, 1966a:70

(Parabomhardina) Sun and Hou, 1964, in Hou and Xian, 1964:416 [subgenus of Bornharditina]
Paracapillithyris Katz and Popov, 1974:26

Parachonetes Johnson, 1966c:365

Parachoristiles Barchatova, 1968, in Markowskii, 1968:164; 1970:145

Paraconchidium Rong and Yang, 1974:198

Paracostanoplia Xu, 1977:65

Paracraniops Williams, 1963:347

Paracrothyris Wu, 1974, in Wang et al., 1974:42

Paracrurithyris Liao, 1979 [nomen nudum]

*Paracyrtiopsis Gatinaud, 1949:490 [= Cyrtiopsis]

Paradoxolhyris Xu, 1978b:302

Paradygella Liao and Sun, 1974:352

Paraemanuella Yang, 1977:415

* Parakeyserlingina Fredericks, 1916:64 [= Keyserlingina $]$

Paralazulkinia Jiang, 1978:314

Paraldingia Richardson, 1973:122

Paralepismatina Yang and Xu, 1966:38

(Paraleplostrophia) Harper and Boucot, 1978a:70 [subgenus of Mesoleptostrophia]

Parallelelasma Cooper, 1956c:612

Parallelora Carter, 1974:680

Paralytlonia Wanner and Sieverts, 1935:207

Paramarginatia Ýang, 1978a:111

Paramarginifera Fredericks, 1916:64

* Paramarlinia Reed, 1949:471 [= Marlinia]

Paramentzelia Xu, 1978b:293

Paramerista Su, 1976:207

Paramesolobus Afanasjeva, 1975:101

Paramonticulifera Tong, 1978:234

Paranaia Clarke, 1913a, pl. 21

Paranorella Cloud, 1944, in King et al., 1944:59

Paranorellina Dagys, 1974:100

Parapholidostrophia Johnson, 1971:309

Paraphorhynchus Weller, 1905:261

Paraplicanoplia Xu, 1977:67

Parapugnax Schmidt, 1964:505

Parapulchratia Chan, 1979, in Hou et al., 1979:87

Parareticularia Lee and Gu, 1976:298

(Pararhipidium) Boucot, 1975 [nomen nudum; = (Pararhipidium) Boucot and Johnson, 1979]

(Pararhipidium) Boucot and Johnson, 1979:101 [subgenus of Rhipidium]

(Paraschizophoria) Lazarev, 1976:110 [subgenus of Schizophoria]

Paraspinifer Wedekind, 1926:198

Paraspiriferina Reed, 1944:252

(Parastringocephalus) Siruve, 1965b:467 [subgenus of Stringocephalus]

†Parastrophia Hall and Clarke, 1894 [replaced by Parastrophina]

Parastrophina Schuchert and LeVene, 1929:121 [new name for Parastrophia]

Parastrophinella Schuchert and Cooper, 1931:248

Parastrophonella Bublichenko, 1956:93 
Paratetratomia Yang, 1977:394

Parathecidea Backhaus, 1959:55

Paralhyridina Schuchert and LeVene, 1929:121 [new name for Parathyris]

†Paralhyris Douvillé, 1916 [replaced by Parathyridina]

Paralhyrisina Wang, 1974, in Wang et al., 1974:41

Paratreta Biernat, 1973:68

Parazyga Hall and Clarke, 1893:127

**Pareatonia McLearn, 1918 [= Eatonia]

Parenteletes King, [1931]:48

Parmorthina Havlicek, 1975:232

*Parmorthis Schuchert and Cooper, 1931:246 [= Resserella]

Paromalomena Rong, 1979 [nomen nudum]

Paromoeopygma Sartenaer, 1968b:3

Paruncinella Waterhouse and Gupta, 1978b: 132

Parvirhynchia Buckman, 1914 [nomen nudum]; 1917:56

$\ddagger$ Parvulaltarosirum Sartenaer, 1979b:2

Paryphella Liao, 1979 [nomen nudum]

Paryphorhynchopora Simorin, 1956:245

Paryphorhynchus. - Weller, 1914 [misspelling of Paraphorhynchus]

Palagorhynchia Allan, 1938: 199

Paterina Beecher, 1891:345

Paterorthis Havlíček, 1971c:45

Palerula Barrande, 1879:110

Paucicostella Cooper, 1956c:711

Paucicrura Cooper, 1956c:956 [new name for Resserella Schuchert and Cooper, 1932, not Bancroft, 1928]

Paucispinifera Muir-Wood and Cooper, 1960:319

Paucistrophia Wang and Zhu, 1979:23

Paulonia Nalivkin, 1925:282

Paurogastroderhynchus Sartenaer, 1970:25

Paurorhyncha Cooper, 1942:231

*Paurorthina Rübel, 1961:187 [= Paurorthis]

Paurorthis Schuchert and Cooper, 1931:243

Payuella Grabau, 1934:150

Pectorhyncha McLearn, 1918:137

Peculneithyris Smirnova, 1972, in Smirnova and Terekhova, 1972:76

Peetzatrypa Rzonsnitzkaja, 1975:95

Pegmarhynchia Cooper, 1955a:58

Pegmathyris Hatai, 1938:99

* Pegmalrela Bell, 1941:231 [= Linnarssonia]

Pelagodiscus Dall, 1908:440

Peleicostella Havliček, 1971b:230

Pelonomia Cooper, 1956c:699

Pelichia Ching and Sun, 1979 [nomen nudum]

(Pembrostrophia) Bassett, 1971:325 [subgenus of Amphistrophia]

Peniculauris Muir-Wood and Cooper, 1960:279

Pennospiriferina Dagys, 1965:112

Pentactinella Bittner, 1890:301

Pentagonia Cozzens, 1846:158

Pentamerella Hall, 1867b:163
Pentamerifera Khodalevich, 1939:22

Pentameroides Schuchert and Cooper, 1931:248

Pentamerus Sowerby, 1812:73

Penllandella Boucot, 1964b:104

Penllandina Bancroft, 1949b: 13

Penzhinothyris Smirnova, 1969, in Smirnova and Pergament, 1969:36

Perakia Hamada, 1969b: 7

Perdilocardinia Schuchert and Cooper, 1931:246

Peregrinella Oehlert, 1887, in Fischer, 1887:1305

Peregrinelloidea Dagys, 1968:43

Perennithyris Tchorszhewsky, 1973; 1974 [nomina nuda]

**Pereudesia Dall, 1920 [= Coptothyris]

Periallus Hoover, 1979:17

Perichonetes Xu, 1979a:372

Peridalejina Havliček, 1973:337

Peridiolithus Huepsch, 1768 [nonbinomial]

Perigeyerella Wang, 1955:346

Perimecocoelia Cooper, 1956c:593

Perissolhyris Carter, 1967b:588

Peritritoechia Xu, Rong, and Liu, 1974:151

Permianella He and Zhu, 1979:132

Permicola Koczyrkevicz, 1976b:75

Permochonetes Afanasjeva, 1977b: 148

Permophricodothyris Pavlova, 1965:134

* Permorthotetes Thomas, 1958:82 [= Orthotetes]

* Permospirifer Kulikov, 1950:5 [= Licharewia]

Permundaria Nakamura, Kato, and Choi, 1970:295

Perrierihyris Almeras, 1971:423

(Peshiatrypa) Xian, 1978a:298 [subgenus of Desquamatia]

Pesterevatrypa Rzonsnitzkaja, 1975:93

Pelasmaia Cooper and Grant, 1969:10

Petasmatherus Cooper and Grant, 1969:12

Petria Mendes, 1961b:22

Petrocrania Raymond, 1911:229 [new name for Craniella]

Petroria Wilson, 1926:28

Pexidella Bittner, 1890:300

Phapsirhynchia Pajaud, 1976:102

Pharcidodiscus Roberts, 1976:46

* Phareira Bolten, 1798:159 [= Lingula]

Philedra.-Bekker, 1921 [misspelling of Philhedra]

Philhedra Koken, 1889:467

Philhedrella Kozlowski, 1929:28

Phlogoiderhynchus Sartenaer, 1970:18

Phoeniciloechia Havlícek, 1960:242

**Pholidops Hall, 1859 [= Craniops]

Pholidostrophia Hall and Clark, 1892:287

Phragmophora Cooper, 1955a:51

Phragmorthis Cooper, 1956c:509

Phragmostrophia Harper et al., 1967:428

Phragmolhyris Cooper, 1955b:66

Phrenophoria Cooper and Grant, 1969:12

Phricodothyris George, 1932:525 
Phymatothyris Cooper and Muir-Wood, 1951:195 [new name for Palasiella]

Physemella Godefroid, 1974:50

Physetorhyncha Sartenaer and Rozman, 1968:137

Physotreta Rowell, 1966:19

Piarorhynchella Dagys, 1974:110

Piarorhynchia Buckman, 1914 [nomen nudum]; 1917:34

*Piarothyris Sahni, 1925:370 [= Cameithyris]

Piclothyris Thomson, 1927:260

Pinaxiothyris Dagys, 1968:86

Pinghuangella Jiang, 1978:315

(Pinguaella) Boucot, 1975 [nomen nudum; = (Pinguaella)

Boucot and Johnson, 1979]

(Pinguaella) Boucot and Johnson, 1979:114 [subgenus of Kirkidium]

Pinguispirifer Havlícek, 1957a:246

Pioglyplus. _St. Joseph, 1941 [misspelling of Idioglyplus]

Pionodema Foerste, 1912:139 [new name for Bathycoelia]

Pionomena Cooper, 1956c:901

(Pionorthis) Schuchert and Cooper, 1931:244 [subgenus of Plaesiomys]

†Pirgula Gregorio, 1930 [replaced by Pirgulia]

Pirgulia Cooper and Muir-Wood, 1951:195 [new name for Pirgula]

Pirothyris Thomson, 1927:280

Pirotothyrls Sučić-Protić, 1971:31

Pisirhynchia Buckman, 1914 [nomen nudum]; 1917:28

* Pizarroa Hoek, 1912, in Steinmann and Hoek, 1912:246 $[=$ Bistramia $]$

Pizzaroa. - Eastman, 1913 [misspelling of Pizarroa]

Plachiloma Ferussac, 1835 [misspelling of Pachiloma]

Placolhyris Westphal, 1970:38

Placotriplesia Amsden, 1968:40

Plaesiomys Hall and Clarke, 1892:196

Plagiorhyncha McLearn, 1918:138

Planalvus Carter, 1971:248

$\dagger^{*}$ (Planalrypa) Copper, 1967b:231 [subgenus of Alrypa; junior homonym and junior synonym of (Planatrypa) Struve; each author based his generic name on the same group species]

(Planalrypa) Struve, 1966:143 [subgenus of Alrypa]

Planicardinza Savage, 1968a:628

* Planidorsa Schuchert and Cooper, 1931:244 [= Cyrtonotella]

Planirhynchia Sučić-Protić, 1969:19

Planispina Stehli, 1954:331

Planodouvillina Harper and Boucot, 1978b: 145

Planoharknessella Havlícek, 1950:42

Planoproduclus Stainbrook, 1947:310

Planovatirostrum Sartenaer, 1970:16

Plalıdia Costa, 1852:48

Platilites Rafinesque, 1831 [incertae sedis]

Platyconcha Waterhouse, 1975:8

Plalyglossariorhynchus Sartenaer, 1970:8

Platymena Cooper, 1956c:879
Platymerella Foerste, 1909b:70

Platyorthis Schuchert and Cooper, 1931:246

* Platyrachella Fenton and Fenton, 1924:158 [= Spinocyrtia]

Platyselma Gordon, 1966:575

Platyspirifer Grabau, 1931 a:355

Platystrophia King, 1850:106

Platylerorhynchus Sartenaer, 1970:5

Platythyris Middlemiss, 1959:109

Platyloechia Neuman, 1964:E20

* Playfairia Reed, 1917:866 [= Rafinesquina]

Plebejochonetes Boucot and Harper, 1968:159

Plectambonites Pander, 1830:90

Pleclatrypa Schuchert and Cooper, 1930:279

Plectelasma Cooper and Grant, 1969:17

Pleclella Lamansky, 1901 [nomen nudum]; 1905:22

Plectocamara Cooper, 1956c:596

Plecloconcha Cooper, 1942:233

Plectodonta Kozlowski, 1929:29

Plectodontella Havlícek, 1953:8

Plecloglossa Cooper, 1956c:222

Plectoidothyris Buckman, 1914 [nomen nudum]; 1917:122

Plectorhynchella Cooper and Muir-Wood, 1951:195 [new name for Montzcola]

Plectorthis Hall and Clarke, 1892:194

Plectospira Cooper, 1942:228 [new name for Plychospira]

* Plectospirifer Grabau, 1931 a:380 [= Adolfia]

Plectothyrella Temple, 1965:412

Pleclothyris Buckman, 1914 [nomen nudum]; 1917:121

**Pleclothyris Rollier, 1918 [junior homonym and junior objective synonym of Plectothyris Buckman, 1917]

Plectotreta Ulrich and Cooper, 1936a:339

Plectotrophia Ulrich and Cooper, 1936b:627

Pleiopleurina Schmidt, 1964:506

Plekonella Campbell, 1953:17

Plesicarinatina Mizens, 1977a:89

Plesiothyris Douvillé, 1879:275

Plethorhyncha Hall and Clarke, 1893:191

Plethorhynchus._Hall and Clarke, 1893 [misspelling of Plethorhyncha]

Pleuranisis Rafinesque, 1831 [incertae sedis]

Pleurelasma Cooper and Grant, 1976b:2922

Pleurinea._Dall, 1877 [misspelling of Pleurinia]

Pleurinia Rafinesque, 1820 [nomen nudum]

(Pleurochonetes) Isaacson, 1977a:175 [subgenus of Chonetes]

Pleurocornu Havlíček, 1961a:46

Pleurodium Wang, 1955:345

* Pleurohorridonia Dunbar, 1955:90 [= Horridonia]

Pleuropugnoides Ferguson, 1966:354

Pleurorthis Cooper, 1956c:329

Pleurothyrella Boucot, Caster, Ives, and Talent, 1963:89

$\dagger$ Pleurolhyris Cloud, 1942 [replaced by Cloudella]

Plicanoplia Boucot and Harper, 1968:169

†Plicanoplia Havlícek, 1973 [replaced by Plicanoplites] 
Plicanoplites Havlícek, 1974:170 [new name for Plicanoplia Havlícek, 1973]

Plicarostrum Burri, 1953:281

*Plicathyris Khalfin, 1946 [= Anathyris]

Plicatifera Chao, 1927:25

Plicalocyrtia Gauri, 1965:55

Plicatoderbya Thomas, 1937:14

Plicatolingula Liu, 1979:509

Plicalosyrinx Minato, 1952:168

Plicigera Bittner, 1890 [group term, no generic validity]

Plicirhynchia Allan, 1947a:493

Plicochonetes Paeckelmann, 1930:222

Plicocoelina Boucot and Johnson, 1966b: 1038

Plicocyrtia Boucot, 1963:704

Plicocyrtina Havlícek, 1956:608

Plicodevonaria Boucot and Harper, 1968: 162

(Plicogypa) Rzonsnitzkaja, 1975:27 [subgenus of Gypidula]

Plicoplasia Boucot, 1959a: 19

Plicoproduclus Ljaschenko, 1969a:14

Plicostricklandia Boucot and Ehlers, 1963:55

Plicostropheodonta Sokolskaja, 1960:214

Plicotorynifer Abramov and Solomina, 1970, in Menner et al., 1970:111; in Abramov, 1970:153

Plionoptycherhynchus Sartenaer, 1979a:537

(Pocockia) Lazarev, 1976:112 [subgenus of Schizophoria]

Podichnus Bromley and Surlyk, 1973 [ichnogenus]

Podolella Kozlowski, 1929:31

Podtsheremıa Kalashnikov, 1965 [nomen nudum]; 1966:50

Poikilosakos Watson, 1917:215

Polymorpharia Cooper and Grant, 1975:1143

Polyloechia Hall and Clarke, 1892:239

Pomatospirella Bittner, 1892:26

Pomatotrema Ulrich and Cooper, 1932, in Schuchert and Cooper, 1932: 109

Pomeromena Mitchell, 1977:112

Pompeckium Havlícek, 1970a:290

Pondospirifer Waterhouse, 1978:93

Pontielasma Waterhouse and Piyasin, 1970:163

Pontısia Cooper and Grant, 1969:13

Poramboniles Pander, 1830:95

Poramboniloides Xu, 1978a:315

Poramborthts Havliček, 1949a: 107

Porostictia Cooper, 1955a:62

Portneufia Hoover, 1979:7

Portranella Wright, 1964:169

Posteptthyris Makridin, 1960, in Licharew et al., 1960:294

Posllobothyrella Tchorszhewsky, 1971 [nomen nudum]

Postrhapidothyris Tchorszhewsky, 1973 [nomen nudum]

Pradoia Comte, 1938:44

Praecubanothyris Dagys, 1974:187

Praecyclothyris Makridin, 1955:84

Praegnantenia Havlícek, 1961a:99

Praegoniolhyris Ovtsharenko, 1977 [nomen nudum]
Praehorridonia Ustritsky, 1962:57; 1963, in Ustritsky and Tschernjak, 1963:94

Praelacazella Smirnova, 1969a:81

Praelacunosella Wiśniewska-Żelichowska, 1978:109

Praeleiorhynchus Rzonsnitzkaja, 1973 [nomen nudum]

Praelongithyris Middlemiss, 1959:134

Praemagas Fischer and Oehlert, 1892 [nomen nudum]

Praeneothyris Katz, 1962:143

Praenucleala Tchorszhewsky, 1973 [nomen nudum]

Praewaagenoconcha Sokolskaja, 1948:132

Prantlina Havlicek, 1949b:250

Priambonites._Fischer de Waldheim, 1834 [misspelling of Plectambonites]

Primorewia Licharew and Kotljar, 1978:71

Prionites._Fischer de Waldheim, 1834 [misspelling of Pronites]

Prionorhynchia Buckman, 1914 [nomen nudum]; 1917:62

Prionolhyris Cloud, 1942:66

*(Prisca) Gregorio, 1930a:31 [subgenus of Lytlonia; = Leptodus]

Proatribonium Gratsianova, 1967:92

Probolarina Cooper, 1959:37

Probolionia Cooper, 1957b:27

Proboscidella Oehlert, 1887, in Fischer, 1887:1277

Proboscidina Branisa, 1965; Boucot, 1975 [nomina nuda; = Proboscidina Isaacson]

Proboscidina Isaacson, 1977b: 192

Procerulina Andronov, 1961:76

Prochlidonophora Thomson and Owen, 1979:28

Prochoristitella Legrand-Blain, [1969]:220

Proconchidium Sapelnikov, 1969, in Nikolayev and Sapelnikov, 1969:15

Prodavidsonia Havlícek, 1956:564

Producla.-Conybeare and Phillips, 1822; Sowerby, 1825 [misspelling of Productus]

Produclella Hall, 1867b: 154

Productellana Stainbrook, 1950:374

Productellina Reed, 1943:99

Produclina Sutton, 1938:551

Productorthis Kozlowski, 1927:9

Productus Sowerby, 1814:153

Progonamboniles Öpik, 1934:138

*Prokeyserlingina Fredericks, 1916 [hypothetical genus; = Poikilosakos]

Prokopia Havlícek, 1953:5

Promarginifera Shiells, 1966:428

(Pronalivkinia) Rukavischnikova, 1977:134 [subgenus of Nalivkinia]

**Pronites Pander, 1830 [= Clitambonites]

* Propatella Grubb, 1939:558 [= Philhedra]

Propygope Bittner, 1890:210

Prorensselaeria Raymond, 1923:468

Proreticularia Havlícek, 1957a:247

Prorichthofenia King, [1931]:97 
Proschizophoria Maillieux, 1912:177

Prospira Maxwell, 1954:35

Prosserella Grabau, 1910, in Grabau and Sherzer, 1910:139

Prostricklandia Rukavischnikova and Sapelnikov, 1973:106

*Prosyringothyris Fredericks, 1916:51 [= Syringothyris]

Protambonites Havliček, 1972, in Havlícek and Josopait, 1972:348

Protanidanthus Liao, 1979:537

Protathyris Kozlowski, 1929:30

Protatrypa Boucot, Johnson, and Staton, 1964:809

Proteguliferina Licharew, 1960, in Sarytcheva et al., 1960: 236

Proteorthis Havlíček, 1974:168

Protobolus Liu, 1979:507

Protochonetes Muir-Wood, 1962:50

Protocortezorthis Johnson and Talent, 1967a:154

(Protocymostrophia) Harper and Boucot, 1978b:127 [subgenus of Mesodouvillina]

Protodouvillina Harper and Boucot, 1978b: 138

Protogusarella Perry and Chatterton, 1979:317

Protoleptostrophia Caster, 1939:75

(Protomegastrophia) Caster, 1939:36 [subgenus of Megastrophia]

Protomendacella Havlícek, 1970b:31

Protonia._Link, 1830 [misspelling of Productus]

Protoniella Bell, 1929:110

Protophragmapora Alekseeva, 1967:7

Protorhyncha Hall and Clarke, 1893:180

Protorthis Hall and Clarke, 1892:232

(Protoshaleria) Harper and Boucot, $1978 \mathrm{~b}: 162$ [subgenus of Shaleria]

*Protosiphon Matthew, 1897:70 [= Trematobolus]

Protoskenidioides Williams, 1974:83

Protosyphon.-Gorjansky, 1960 [misspelling of Protosiphon]

Protosyringothyris. - Fredericks, 1918 [misspelling of Prosyringothyris]

Prototreta Bell, 1938:405

Protozeuga Twenhofel, 1913, in Savage, 1913:51

Protozyga Hall and Clarke, 1893:149

Psamathopalass Liu, 1979:510

Psebajithyris Tchorszhewsky, 1974:48

Pseudoanisopleurella Xu, 1978:222 [spelled Pseudoanisopeurella in paper]

Pseudoatrypa Copper, 1973:492

Pseudobolus Cooper, 1956c: 194

Pseudobornharditina Yang, 1977:460

Pseudocamarophoria Wedekind, 1926:197

Pseudocamarotoechia Kulkov, 1974, in Ivanovskii and Kulkov, 1974:53

Pseudochonetes Su, 1976:183

Pseudoconchidium Nikiforova and Sapelnikov, 1971:52

Pseudocrania McCoy, 1851:389

Pseudocyrtina Dagys, 1962a:54

Pseudoderbyia Licharew, 1934a:213
Pseudodicellomus Bell, 1962, in Bell and Ellinwood, 1962: 407

* Pseudodicoelosia Boucot and Amsden, 1958:162 [= Rhipidomella]

Pseudodielasma Brill, 1940:317

* Pseudodouvillina Stainbrook, 1945:27 [= Nervostrophia]

Pseudogibbirhynchia Ager, 1962:108

Pseudoglossinoloechia Tcherkesova, 1967:9

Pseudoglossothyris Buckman, 1901:240

Pseudogruenewaldtia Rzonsnitzkaja, 1960:46

Pseudohalorella Dagys, 1965:66

Pseudoharttina Licharew, 1934a:212

Pseudohomeospira Nikiforova, 1970:139

*Pseudokeyserlingina Fredericks, 1916 [hypothetical genus; = Cardinocrania]

Pseudokingena Böse and Schlosser, 1900:177

Pseudolabaia Ching and Ye, 1979:130

Pseudolaballa Dagys, 1974:145

*Pseudoleiorhynchus Rozman, 1962:122 [= Trifidorostellum]

Pseudolepismatina Ching and Sun, 1976, in Ching et al., 1976:321

*Pseudoleptaena Miloradovich, 1947:96 [= Leptagonia]

**Pseudoleptaena Williams, 1965 [invalid new name for Leptaenella Sokolskaja, 1952, which is a junior objective synonym of Leptagonia]

Pseudoleptodus Stehli, 1956:311

Pseudolingula Mickwitz, 1909:771

Pseudomagas Makridin and Katz, 1965 [nomen nudum]

Pseudomarginifera Stepanov, 1934:56

**Pseudomartinia Leidhold, 1928 [new name proposed for Martinia Waagen, citation unknown; = Martinia $]$

Pseudometoptoma Huene, 1899:211

Pseudonudirostra Rozman, 1960 [nomen nudum]

*Pseudoorthotetes Sokolskaja, 1963 in Sarytcheva et al., 1963: 97 [= Orthoietes]

Pseudoparazyga Johnson, 1970b:181

* Pseudopentagonia Besnossova, 1963, in Sarytcheva et al., 1963:315 [= Composita]

Pseudopholidops Bekker, 1921:64

Pseudoporambonites Zeng, 1977:51

$\ddagger$ Pseudoprotathyris Modzalevskaja, 1979:59

Pseudopugnax Licharew, 1956, in Kiparisova et al., 1956:56

Pseudopygoides Xu, 1978b:303

Pseudorugitela Dagys, 1959b:100

Pseudosieberella Godefroid, 1972:3

Pseudospiriferina Yang and $\mathrm{Xu}, 1966: 41$

Pseudostrophalosia Clarke, 1970:987

Pseudostrophomena Rōōmusoks, 1963:237

Pseudosyringothyris Fredericks, 1916:51

Pseudosyrinx Weller, 1914:405

Pseudotubithyris Almeras, 1971:361

Pseudouncinulus Rzonsnitzkaja, 1968, in Markowskii, 1968: 111

Pseudowattonithyris Almeras, 1971:393 
Pseudowellerella Licharew, 1956, in Kiparisova et al., 1956: 58

Pseudoyunnanella Chen, 1978:336

Psilocamara Cooper, 1956a:523

†Psilonotus Stehli, 1954 [replaced by Elliottella]

Psiloria Cooper, 1976:282

Psilothyris Cooper, 1955c:10

Psioidea Hector, 1879:538

Psioidiella Campbell, 1968:33

Pterophloeus.-Bittner, 1890 [misspelling of Pterophloius]

Pterophloios. - Zugmayer, 1880 [misspelling of Pterophloius]

**Pterophloius Guembel, 1861 [= Bactrynium]

Pteroplecta Waterhouse, 1978:56

Pterospirifer Dunbar, 1955:128

Ptilorhynchia Crickmay, 1933:877

Plilotorhynchus Cooper and Grant, 1976a:2011

Ptychoglyptus Willard, 1928:283

Plychomaletoechia Sartenaer, 1961a:7

Ptychopeltis Perner, 1903:42

Ptychopleurella Schuchert and Cooper, 1931:244

$\dagger$ Ptychospira Hall and Clarke, 1894 [replaced by Plectospira]

Plyctorhynchia Buckman, 1914 [nomen nudum]; 1917:47

Plyctothyris Buckman, 1914 [nomen nudum]; 1917:101

Ptygmactrum Cooper and Grant, 1976a: 1939

Puanatrypa Xian, 1978a:302

Puanospirifer Jiang, 1978:329

Pugilis Sarytcheva, 1949:104 [replacement by Pugilus invalid, Pugilis not preoccupied]

**Pugilus Sarytcheva, 1952 [invalid new name for Pugilis, Pugilis not preoccupied]

Pugites DeHaan, 1838 [nomen nudum]

Pugnacina Zyong and Rzonsnitzkaja, 1968, in Zyong et al., 1968:?

Pugnax Hall and Clarke, 1893:203

Pugnoides Weller, 1910:512

Pulchratia Muir-Wood and Cooper, 1960:250

*Pulchrithyris Sahni, 1925:361 [= Carneilhyris]

(Pulsia) Ivanov, 1925:114 [subgenus of Schellwienella]

Pumilus Atkins, 1958:560

Punclatrypa Havlícek, 1953:8

Punctocyrtella Plodowski, 1968:252

Punctolira Ulrich and Cooper, 1936b:628

*Punctopalella Grubbs, 1939:559 [= Petrocrania]

Punctospirella Dagys, 1974:136

Punctospirifer North, 1920:213

Punctothyris Hyde, 1953:288

Punctspinatrypa Rzonsnitzkaja, 1975:116

Purdonella Reed, 1944:218 [new name for Munella]

Pustula Thomas, 1914:259

Pustulatia Cooper, 1956b:769 [new name for Pustulina]

†Puslulina Cooper, 1942 [invalid new name for Vilulina, Pustulina preoccupied; replaced by Pustulatia]

Pustuloplica Waterhouse, 1968a:45

Pygites Buckman, 1906a:449 [new name for Pugites]
Pygmaella Baranov, 1977:80

Pygmochonetes Jing and $\mathrm{Hu}, 1978: 111$

Pygope Link, 1830:435

Pyraeneica Sučić-Protić, 1971:23

* Pyramidalia Nalivkin, 1947:124 [= Cyrtinaella]

Pyramina Ljaschenko, 1969a:23

Qiansispirifer Yang, 1977:423

Qilianoconcha Ching, Sun, and Ye, 1979:166

Qilianotryma Xu, 1979b:97

Qingyenia Yang and $\mathrm{Xu}, 1966: 50$

** Quadraneles Sadlick, 1963 [= Neochonetes]

Quadratia Muir-Wood and Cooper, 1960:162

Quadratirhynchia Buckman, 1914 [nomen nudum]; 1917:42

*Quadrifarius Fuchs, 1923:854; 1929:195 [= Delthyris]

(Quadrikentron) Boucot and Gauri, 1966:1023 [subgenus of Strophochonetes)

Quadrisonia Rowell and Henderson, 1978:6

Quadrilhyrina Havlíček, 1959a:244

Quadrithyris Havlíček, 1957b:440

Quadrochonetes Stehli, 1954:309

Quangyuania Sheng, 1975:81

(Quasiavonia) Brunton, 1966:220 [subgenus of Avonia]

Quasimarinia Havlíček, 1957 [nomen nudum]; 1959a:257

(Quasistrophonella) Harper and Boucot, 1978a:98 [subgenus of Strophonella]

Quasithambonia Bednarczyk and Biernat, 1978:303

*Quebecia Walcott, 1905:320 [= Yorkia]

Quinquenella Waterhouse, 1975:2

Quizhouspirifer Xian, 1979:116

(Radiatrypa) Copper, 1978:293 [subgenus of Variatrypa]

Radiomena Havlícek, 1962:471

Rafinesquina Hall and Clarke, 1892:282

Rallacosta Cooper and Grant, 1976a:2278

Ramavectus Stehli, 1954:327

Ranorthis Öpik, 1939:119

Rastelligera Hector, 1879:538

Ratburia Yanagida, 1970:85

*Rauffia Schulz, 1914:371 [= Bornhardina]

†Rauna Öpik, 1932 [replaced by Raunites]

Rauniles Öpik, 1939:135 [new name for Rauna]

Ravozetina Havlíček, 1974: 169

Rawdonia Peou, 1979:190

Raymondella Bancroft, 1933 [nomen nudum]

†Raymondella Whittington, 1938 [replaced by Bancrofina]

Rectirhynchia Buckman, 1917:74

Rectithyris Sahni, 1929:9

Rectotrophia Bates, 1968:176

Redlichella Walcott, 1908:90

(Reedoconcha) Kotljar, 1964:124 [subgenus of Productus]

Reeftonella Boucot, 1959c: 768

Reeftonia Allan, 1947b:436

Reflexia Rotai, 1931:24 
*Regelia Crickmay, 1952:2 [= Cyrlospirifer]

Reinversella Bates, 1968:169

Remnevitoechia Gratsianova, 1970, in Alekseeva et al., 1970: 97

(Renaudia) Racheboeuf, 1976:71 [subgenus of Eodevonaria] Rensselaeria Hall, 1859b:38

Rensselaerina Dunbar, 1917:469

Rensselandia Hall, 1867a:385

Resserella Bancroft, 1928b:54

Retaria Muir-Wood and Cooper, 1960:231

Retichonetes Muir-Wood, 1962:63

Reticularia McCoy, 1844:143

Reticulariina Fredericks, 1916:16

Reticulanopsis Fredericks, 1916:17

Reticulatia Muir-Wood and Cooper, 1960:284

Reticulatochonetes Bublichenko, 1956:97

Reticulatrypa Savage, 1970:663

Retimarginifera Waterhouse, 1970:123

(Retrorsirostra) Schuchert and Cooper, 1931:244 [subgenus of Plaesiomys]

Retzia King, 1850:137

*Retziella Nikiforova, 1937:83 [= Rhynchosperina]

Retzispirifer Kulkov, 1960:929

Reuschella Bancroft, 1928a:180

Reushella.-Alichova, 1960 [misspelling of Reuschella]

Reveroides Sapelnikov, 1976:3

Rhactomena Mitchell, 1977:111

Rhactorhynchia Buckman, 1914 [nomen nudum]; 1917:50

Rhactorthis Williams, 1963:372

Rhaetina Waagen, 1882:334

Rhaetinopsis Yang and Xu, 1966:80

Rhamnaria Muir-Wood and Cooper, 1960:119

Rhapidothyris Tuluweit, 1965:72

Rhenorensselaeria Kegel, 1913:127

(Rhenospirifer) Mittmeyer, 1972:99 [subgenus of Euryspirifer]

(Rhenostrophia) Boucot, 1960a:483 [subgenus of Strophodonta]

Rhenothyris Struve, 1970:460

Rhinobolus.-Hall and Clarke, 1892 [misspelling of Rhynobolus]

Rhipidium Schuchert and Cooper, 1931:249

Rhipidomella Oehlert, 1890:372 [new name for Rhipidomys]

*Rhipidomelloides Boucot and Amsden, 1958:165 [= Rhipidomella]

Rhipidomena Cooper, 1956c:867

†Rhipidomys Oehlert, 1887 [replaced by Rhipidomella]

Rhepedothyris Cooper and Williams, 1935:847

Rhizolhyris Thomson, 1915d:399

Rhombothyris Middlemiss, 1959:99

*Rhynchatrypa Siehl, 1962:199 [= Dubaria]

*Rhynchocamara Schuchert and Cooper, 1931:248 [= Camerella]

Rhynchoferella Spriestersbach, 1942:199
Rhynchonella Fischer de Waldheim, 1809:35

Rhynchonellina Gemmellaro, 1871:102

Rhynchonellis.-Keferstein, 1828 [misspelling of Rhynchonella]

Rhynchonelloidea Buckman, 1914 [nomen nudum]; 1917:38

Rhynchonelloidella Muir-Wood, 1936a:49

†Rhynchonellopsis Böse, 1894 [replaced by Capillirostra]

†Rhynchonellopsis Gregorio, 1930 [replaced by Sulcirostra]

Rhynchonellopsis Vincent, 1893:50

Rhynchopora King, 1865:124 [replacement by Rhynchoporina invalid, Rhynchopora not preoccupied]

** Rhynchoporina Oehlert, 1887 [invalid new name for Rhynchopora, Rhynchopora not preoccupied]

Rhynchora Dalman, 1828:136

Rhynchorina Oehlert, 1887, in Fischer, 1887:1326

Rhynchorthis Bates, 1968:160

$\dagger$ Rhynchospira Hall, 1859 [replaced by Rhynchospirina]

*Rhynchosprifer Paulus, 1957:51 [= Prosserella]

Rhynchospirina Schuchert and LeVene, 1929:121 [new name for Rhynchospira]

Rhynchotetra Weller, 1910:506

Rhynchotrema Hall, 1860:68

Rhynchotreta Hall, 1879:166

Rhynchotretina Khalfin, 1948:175

Rhynchura.-Agassiz, 1848 [misspelling of Rhynchora]

Rhyncospira.-Hall, 1859 [misspelling of Rhynchospira]

Rhynobolus Hall, 1871a:5

Rhyselasma Yadrenkina, 1972:177

Rhysostrophia Ulrich and Cooper, 1936b:630

Rhysotreta Cooper, 1956c:259

Rhyssochoneles Johnson, 1970a:2095

Rhyliophora Muir-Wood and Cooper, 1960:192

Rhylirhynchia Cooper, 1957a:8

Rhytisia Cooper and Grant, 1975:967

*Rhytistrophia Caster, 1939:86 |= Leptostrophia $]$

Richthofenia Kayser, 1881:351

Rictia Gregorio, 1930a:27

Rigbyella Stehli, 1956:310

Rimirhynchia Buckman, 1914 [nomen nudum]; 1917:60

Rimirhynchopsis Dagys, 1963:71

Rograndella Kobayashi, 1937:422

Rionirhynchia Kvakhadze, 1971 [nomen nudum]

Rioultina Pajaud, 1966c:631

Ripidiorhynchus Sartenaer, 1966a:2

Robertorthis Havlícek, 1977a:51

Robinsonella Moisseiev, 1936:45

Robustirhynchia Seifert, 1963:174

Rochatorhynchia Katz, 1962:132

Rocheithyris Almeras, 1971:345

Roemerella Hall and Clarke, 1890:137

Romingerina Hall and Clarke, 1893:271

Rorespirifer Waterhouse and Piyasin, 1970:156

Rosella Andreeva, 1972:52

$\nmid$ Rossella Jones, 1978 [replaced by Nanukidium] 
Rossirhynchus Gaetani, 1964:638

Rostranteris Gemmellaro, 1898 [nomen nudum]; 1899:104

Rostricellula Ulrich and Cooper, 1942:626

Rostrirhynchia Sučić-Protić, 1969:47

* Rostrospirifer Grabau, 1931c:407 [= Euryspirifer]

Rotaia Rzonsnitzkaja, 1959:30 |new name for Welleria Rotai, 1941]

Rotundostrophia Gratsianova, 1960, in Khalfin, 1960b:439

Rouillieria Makridin, 1960, in Licharew et al., 1960:295

Rowellella Wright, 1963:233

* Rowleyella Weller, 1911:448 [= Camarospira]

Rozmanaria Weyer, 1972:85

**Rudinia Muir-Wood and Cooper, 1960 [= Desmoinesia]

Rudirhynchia Buckman, 1914 [nomen nudum]; 1917:44

Ruegenella Owen, 1977:224

Rugaltarostrum Sartenaer, 1961a:6

Rugaria Cooper and Grant, 1969:5

Rugatia Muir-Wood and Cooper, 1960:286

Rugauris Muir-Wood and Cooper, 1960:193

Rugia Steinich, 1963:735

Rugicostella Muir-Wood and Cooper, 1960:167

Rugitela Muir-Wood, 1936a:121

Rugithyris Buckman, 1915 [nomen nudum]; 1917:127

Rugivestis Muir-Wood and Cooper, 1960:235

Rugoclostus Easton, 1962:59

Rugoconcha Ching and Sun, 1979 [nomen nudum]

Rugoleplaena Havlíček, 1956:558

Rugosatrypa Rzonsnitzkaja, 1975:98

Rugosochonetes Sokolskaja, 1950:23

(Rugosowerbyella) Mitchell, 1977:83 [subgenus of Sowerbyella]

Rugostrophia Neuman, 1971:119

* Rugulatia Sokolskaja, 1952, in Sarytcheva and Sokolskaja, 1952: 187 [= Licharewia]

Russtella Makridin, 1964:288

Russirhynchia Buckman, 1914 [nomen nudum]; 1917:52

*Rustella Walcott, 1905:311 [= Kutorgina]

**Ruthenia Fredericks, 1928 [= Waagenoconcha]

Rzonsnickiana Mamedov, 1976:121

Saccogonum Havlícek, 1971c:27

Saccorhynchia Ching, Sun, and Ye, 1979:154

Sacothyris Ching, Sun, and Ye, 1979:214

Sagueresia Mohanti, 1972:172

Sajakella Nasikanova, 1968, in Sarytcheva, 1968:141

Sakawairhynchia Tokuyama, 1957b: 126

Salacorthis Williams, 1974:79

Salairina Alekseeva, 1970, in Alekseeva et al., 1970:131

Salairotoechia Rzonsnitzkaja, 1968, in Markowskii, 1968: 123

Salgirella Moisseiev, 1936:48

Salonia Cooper and Whitcomb, 1933:496

Salopia Williams, 1955, in Whittington and Williams, 1955:409
Salopina Boucot, 1960b:3

Sampo Öpik, 1933a:35

Sandia Sutherland and Harlow, 1973:41

Sanjuania Amos, 1958:841

Sanqiaothyris Yang and Xu, 1966:83

Sapelnikovia Boucot, 1975 [nomen nudum; = Sapelnikovna

Boucot and Johnson, 1979]

**Sapelnikovia Boucot and Johnson, 1979:118 [= Reveroides]

Sardope Dieni et al., 1973:196

Sarganostega Cooper and Grant, 1969:15

Saucrorthis Xu, Rong, and Liu, 1974:151

*Saughina Bancroft, 1949a:7 [= Fardenia]

Saukrodictya Wright, 1964:219

(Savagema) Boucot, 1975:355 [subgenus of Anastrophia]

Scacchinella Gemmellaro, 1891:23

Scalpellirhynchia Muir-Wood, 1936b:477

Scambocris Liu, 1979:507

Scamnomena Bassett, 1977:134

Scapharina Cooper and Grant, 1975:895

Scaphelasma Cooper, 1956c:260

Scaphiocoelia Whitfield, 1891:107

Scaphorthis Cooper, 1956c:502

Sceletonia Cooper and Grant, 1974:406

Scenesia Cooper and Grant, 1976b:2757

Scenidium. - Oehlert, 1887 [misspelling of Skenidium]

Schachriomonia Nikiforova, 1978:118

Schedophyla Neuman, 1971:122

Schegullania Andronov, 1961 [nomen nudum]

Schellwienella Thomas, 1910:92

Schistochonetes Roberts, 1971:54

Schizambon Walcott, 1884:70

Schizambonia.-Oehlert, 1887 [improper emendation of Schizambon]

Schizobolus Ulrich, 1886:26

Schizocrania Hall and Whitfield, 1875:71

†Schizonema Foerste, 1909 [replaced by Schizoramma]

Schizopholis Waagen, 1885:753

Schizophorella Reed, 1917:858

Schizophoria King, 1850:106

Schizoramma Foerste, 1912:139 [new name for Schizonema]

Schizospirifer Grabau, 1931 a:353

Schizotreta Kutorga, 1848:274

Schizotretoides Termier and Monod, 1978:151

†Schmidtia Volborth, 1869 [replaced by Schmidtites]

Schmidtites Schuchert and LeVene, 1929:121 [new name for Schmidtia]

Schnurella Schmidt, 1964:505

Schnuria Clarke, 1889 [nomen nudum]

Schrenkiella Barchatova, 1970 [nomen nudum]; 1973:97

Schuchertella Girty, 1904:734

Schuchertellopsis Maillieux, 1939:5

†Schuchertia Fredericks, 1926 [replaced by Striispirifer]

Schuchertina Walcott, 1905:323

Schwagerispira Dagys, 1972a:101 
Scoloconcha Gordon, 1966:583

Scumulus Steinich, 1968a: 199

Scutepustula Sarytcheva, 1963, in Sarytcheva et al., 1963: 165

Sedenticellula Cooper, 1942:231

Selenella Hall and Clarke, 1893:271

Sellithyris Middlemiss, 1959:113

Selloproductus Termier, Termier, Lapparent, and Marin, 1974:143

Semenewia Paeckelmann, 1930:224

Semibrachythyrina Yang and Ting, 1962, in Yang et al., 1962:104

Semicostella Muir-Wood and Cooper, 1960:195

* Seminula McCoy, 1844:158 [= Composita]

Semiotoechia Ljaschenko, 1973:44

Semiplanella Sarytcheva and Legrand-Blain, 1977:79

Semiplanus Sarytcheva, 1952, in Sarytcheva and Sokolskaja, 1952: 119

Semiproductus Bublichenko, 1956:99

Semitreta Biernat, 1973:75

Sendaithyris Hatai, 1940:253

Senokosica Sučić-Protić, 1971:28

Sentolunia Havliček, 1967a:53

Sentosia Muir-Wood and Cooper, 1960: 196

Septacamera Stepanov, 1937: 146

Septacalazyga Distler, 1972 [nomen nudum]

Septachonetes Chatterton, 1973:76

Seplalaria Leidhold, 1928:38

Septalariopsis Chen, 1978:331

Septaliphoria Leidhold, 1921:354

Septaliphorioidea Yang and Xu, 1966:29

Septamphiclina Jin and Fang, 1977:57

Septaparmella Su, 1976:185

Seplarinia Muir-Wood and Cooper, 1960:252

Septasteges Waterhouse and Piyasin, 1970:120

Septathyris Boucot, Johnson, and Staton, 1964:819

Septatoechia Lobatscheva and Titova, 1977:102

Septatrypa Kozlowski, 1929:30

Septemirostellum Roberts, 1971:132

Septiconcha Termier, Termier, Lapparent, and Marin, 1974: 125

Septirhynchia Muir-Wood, 1935:106

Septocamera.-Licharew, 1960 [misspelling of Septacamera]

Septocrurallia Makridin, 1954 [nomen nudum]

Seplocrurella Wiśniewska, 1932:65

Septocrurethyris Askerov, 1964; 1965; 1967 [nomina nuda]

Septocyclothyris Xu, 1978b:282

* Seploproductus Frech, 1911:132 [= Tschernyschewia]

Septorthis Hints, 1973:251

Septospirifer Waterhouse, 1971, in Bamber and Waterhouse, 1971:222

Septospirigerella Grunt, 1965, in Ruzhentsev and Sarytcheva, 1965:237

Seplosyringothyris Vandercammen, 1955:2
Septothyris Cooper and Williams, 1935:849

Septulirhynchia Almeras, 1966:110

(Seratrypa) Copper, 1967a:132 [subgenus of Desquamatia]

Serbiothyris Sučić-Protić, 1971:25

Sergospirifer Ivanova, 1952, in Sarytcheva and Sokolskaja, 1952: 190

Sergunkovia Nalivkin, 1979:129

Sericoidea Lindström, 1953:134

Serratocrisla Brunton, 1968:39

Serrulatrypa Havlíček, 1977b:299

Sestropoma Cooper and Grant, 1969:8

† Seligerella Girty, 1938 [replaced by Seligerites]

Setigerites Girty, 1939:141 [new name for Setigerella]

Seltedabania Abramov, 1970:148

(Severella) Sapelnikov, 1963:15 [subgenus of Brooksina]

Shagamella Boucot and Harper, 1968:167

Shaleria Caster, 1939:34

Shaleriella Harper and Boucot, 1978b: 160

Shiqianella Xian, 1978a: 272

* Shiragia Kobayashi, 1935:70 [= Bohemella]

Shizotreta._Davidson, 1856 [misspelling of Schizotreta]

Shrockia Boucot and Smith, 1978:268

Shroshaerhynchia Ovtsharenko, 1977 [nomen nudum]

Shumardella Weller, 1910:512

Siberiothyris Dagys, 1968:128

Siberistrophia Astashkina, 1970:131

Sibiratrypa Rzonsnitzkaja, 1975:100

Sibina Gorjansky, 1977, in Pelman, 1977:47; in Gorjansky, 1977:99

Sibirrhynchia Rzonsnitzkaja, 1978 [nomen nudum]

Sibirispira Alekseeva, 1968: 198

Siburtoechia Alekseeva, 1966:1147

Sicelia Gortani and Merla, 1934:284 [new name for Gemmellaria]

Sichuanrhynchus Tong, 1978:243

Sicorhyncha Havlícek, 1961 a:28

Sieberella Oehlert, 1887, in Fischer, 1887:1311

** Sieberina Andronov, 1961 [= Gypidulina]

Sikasella Nalivkin, 1979:156

Silesiathyris Brügge, 1977:664

Simplicarina Cooper and Grant, 1975:966

Simplicilhyris Zezina, 1976:101

* Sinocyrtiopsis Gatinaud, 1949:491 [= Cyrtiopsis ]

Sinoproduclella Wang, 1955:350

**Sinoproductus Chan, 1962 [= Monticulifera]

Sinorhynchia Yang and Xu, 1966:32

Sinorthis Wang, 1955:328

Sinoshaleria Xian, 1978a:268

* Sinospirifer Grabau, 1931a:231 [= Cyrlospirifer]

Sinostrophia Hamada, 1968 [nomen nudum]; 1971:52

Sinotectirostrum Sartenaer, 1961a:3

* Sinothyris Minato, 1953:68 [= Reticularia]

Sinuatella Muir-Wood, 1928:37

Sinucosla Dagys, 1963:104 
Sinuplicorhynchia Dagys, 1965:56

Siphonosia Cooper and Grant, 1975:1188

Siphonotreta Verneuil, 1845:286

Skelidorygma Carter, 1974:692

Skenidioides Schuchert and Cooper, 1931:243

Skenidium Hall, 1860:70

Slovenirhynchia Siblik, 1967:159

Smeathenella Bancroft, 1928a: 177

Socraticum Gregorio, 1930a:25

Solidipontirostrum Sartenaer, 1970:22

Somalirhynchia Weir, 1925:80

Somalitela Muir-Wood, 1935:140

Somalithyris Muir-Wood, 1935:124

*Soudleyella Bancroft, 1945:209 [= Onniella]

* Sowerbina Fredericks, [1928]:778 [= Horridonia]

Sowerbyella Jones, 1928:384

Sowerbyites Teichert, 1937:66

Spanodonta Prendergast, 1935:13

Spasskothyris Smirnova, 1975c:74

Sphaerirhynchia Cooper and Muir-Wood, 1951:195 [new name for Wilsonella]

*Sphaerobolus Matthew, 1895:263 [= Lingulobolus]

Sphaeroidothyris Buckman, 1914 [nomen nudum]; 1917:115

Sphenalosia Muir-Wood and Cooper, 1960:88

Sphenarina Cooper, 1959:63

Sphenophragmus Imbrie, 1959:365

Sphenorhynchia Buckman, 1914 [nomen nudum]; 1917:30

Sphenorthis Grubbs, 1939:555

Sphenospira Cooper, 1954:330

Sphenosteges Muir-Wood and Cooper, 1960:108

Sphenotreta Cooper, 1956c:663

Spinarella Cooper and Grant, 1975:1059

Spinatrypa Stainbrook, 1951:196 [new name for Hystricina]

Spinatrypina Rzonsnitzkaja, 1964:101

Spinauris Roberts, 1971:107

Spinella Talent, 1956:21

Spinifrons Stehli, 1954:318

Spinilingula Cooper, 1956c:210

Spinocarinifera Roberts, 1971:100

Spinochonetes Rong, Xu, and Yang, 1974:204

Spinocyrtia Fredericks, 1916:18

*Spinocyrtina Fredericks, 1916:18 [= Cyrtina]

Spinolepismatina Dagys, 1974:147

Spinolyttonia Sarytcheva, 1964:69

Spinomarginifera Huang, 1932:19

Spinomartinia Waterhouse, 1968a:53

Spinoplasia Boucot, 1959a:18

Spinorthis Wright, 1964:185

Spinorugifera Roberts, 1976:50

†Spinospirifer Biernat, 1966:117

Spinospirifer Martynova, 1961:107

Spinostrophia Jiang, 1978:267

Spinulicosta Nalivkin, 1937:49

Spinuliplica Campbell, 1961:442
Spinulothele Rowell, 1977:76

Spinulothyris Antostschenko, 1973:112

Spirelytha Fredericks, [1924a]:304

Spiridiophora.-Sarytcheva, 1960 [misspelling of Spyridiophora]

Spirifer Sowerby, 1818:41

Spirifera.-Murray, 1831; Sowerby, 1835 [misspelling of Spirifer]

Spiriferella Tschernyschew, 1902:121

Spiriferellina Fredericks, [1924a]:299

Spiriferina Orbigny, 1847:268

Spiriferinaella Fredericks, 1926:407

Spiriferinoides Tokuyama, 1957a:101

Spiriferus._Blainville, 1827 [misspelling of Spirifer]

Spirigera Orbigny, 1847 [invalid new name for Athyris, Athyris not preoccupied]

Spirigerella Waagen, 1883:452

Spirigerellina Dagys, 1974:160

Spirigerina Orbigny, 1847:268

Spirinella Johnston, 1941:161

Spirisosium Gregorio, 1930a:23

*Spirithyris Quenstedt, 1868 [nomen nudum; = Athyris]

Spiropunctifera Ivanova, 1971:120

Spitzbergenza Kotljar, 1977:155

Spondilobolus._Gorjansky, 1960 [misspelling of Spondylobolus]

Spondylobolus McCoy, 1851:408

Spondylobus._Davidson, 1853; Dall, 1870 [misspelling of Spondylobolus]

Spondylopyxis Johnson, Boucot, and Murphy, 1976:47

Spondylospira Cooper, 1942:232

(Spondylospiriferina) Dagys, 1972c:38 [subgenus of Pennospiriferina]

Spondylostrophia Kulkov, 1967:33

Spondylotreta Cooper, 1956c:255

Spuriosa Cooper and Grant, 1975:898

Spurispirifer Havlǐček, 1971a:27

Spyridiophora Cooper and Stehli, 1955:471

Squamaria Muir-Wood and Cooper, 1960:288

Squamatina Havliček and Racheboeuf, 1979:109

Squamilingulella Xu, 1978a:285

Squamiplana Sučić-Protić, 1971:8

Squamirhynchia Buckman, 1914 [nomen nudum]; 1917:63

*Squamularia Gemmellaro, 1899:192 [= Phricodothyris]

Squamulariina Fredericks, 1916:19

Stegacanthia Muir-Wood and Cooper, 1960:198

Stegerhynchops Amsden, 1978:27

Stegerhynchus Foerste, 1909b:97

Stegocomu Dürkoop, 1970:185

Stegorhynchella Rzonsnitzkaja, 1959:27

Steinhagella Goldring, 1957:223

Stelckia Crickmay, 1963:21

Stenaulacorhynchus Sartenaer, 1968a:3

Stenocamara Cooper, 1956c:602 
Stenochisma._Schuchert, 1897; Grabau and Shimer, 1909 [improper emendation of Stenoscisma]

Stenocisma.-Hall, 1847 [improper emendation of Stenoscisma ]

Stenoglossariorhynchus Sartenaer, 1970:6

(Stenopentamerus) Boucot, 1975 [nomen nudum; = (Stenopentamerus) Boucot and Johnson, 1979]

(Stenopentamerus) Boucot and Johnson, 1979:111 [subgenus of Harpidium]

Stenosarina Cooper, 1977:95

Stenoschisma._Oehlert, 1887; Hall and Clarke, 1894 [improper emendation of Stenoscisma]

Stenoscisma Conrad, 1839:59

*Stepanoviella Zavodowsky, 1960:336 [= Globiella]

Slepanoviina Zavodowsky, 1968, in Markowskii, 1968:170

Stereochia Grant, 1976:150

Stethothyris Thomson, 1918:23

Stichotrophia Cooper, 1948:473

Stictozoster Grant, 1976:96

Stiphrothyris Buckman, 1915 [nomen nudum]; 1917:109

Stipulina Muir-Wood and Cooper, 1960:200

*(Stita) Gregorio, 1930a:32 [subgenus of Lyttonia; = Adriana ]

Stolmorhynchia Buckman, 1914 [nomen nudum]; 1917:46

Stolzenburgiella Bittner, 1903:508

Straelenia Maillieux, 1935:12

Streptaria Cooper, 1959:39

Streptis Davidson, 1881a:150

Streptopomum Havlíček, 1967a:207

Streptorhynchus King, 1850:107

Stranna Cooper, 1973a:7

Striatifera Chao, 1927:94

Strialochoneles Mikrjukov, 1968, in Markowskii, 1968:90

Striatoproduclella Krylova, 1962:55

*Stratoproductus Nalivkin, 1947:75 [= Devonoproductus]

Striatopugnax Chen, 1978:322

Striatospica Waterhouse, 1975:11

Stricklandia Billings, 1859a:134 [replacement by Stricklandinia invalid, Stricklandia not preoccupied]

Stricklandiella Sapelnikov and Rukavischnikova, 1973:36

** Stricklandinia Billings, 1863 [invalid, new name for Stricklandia, Stricklandia not preoccupied]

Stricklandistrophia Sapelnikov and Rukavischnikova, 1975b:96

Strigirhynchia Cooper and Grant, 1969:14

Strigocephalus._DeFrance, 1827 [misspelling of Sirygocephale ]

Strigospina Liao, 1979:536

Striirhynchia Buckman, 1914 [nomen nudum]; 1917:68

Striispirifer Cooper and Muir-Wood, 1951:195 [new name for Schucherlia]

Striilhyris Muir-Wood, 1935:129

Stringocephalus Defrance, 1825, in Blainville, 1825:511;
Sandberger, 1842 [misspelling of Strygocephale; however, ICZN opinion 807 validated spelling]

(Stringomimus) Struve, 1965b:461 [subgenus of Geranocephalus ]

Striochonetes Waterhouse and Piyasin, 1970:106

*Strixella Boucot and Amsden, 1958:170 [= Rhipidomella]

Strophalosia King, 1844:313

Strophalosiella Licharew, 1935:372

Strophalosiina Licharew, 1935:369

Stropheodonta._Hall, 1852 [misspelling of Strophodonta]

Stropheria Rafinesque, 1820 [nomen nudum]

Strophesia Rafinesque, 1831 [nomen nudum]

Strophiodonia._Paetel, 1875 [misspelling of Strophodonta]

Strophochonetes Muir-Wood, 1962:40

Strophodonta Hall, 1850:348

Stropholosia. - Gray, 1848 [misspelling of Strophalosia]

Strophomena Rafinesque, 1824, in Blainville, 1824:302

Strophomene._Bayle, 1878 [misspelling of Strophomenes]

Strophomenes Rafinesque, 1820 [nomen nudum]

Strophonella Hall, 1879:154

Strophonellites Havlícek, 1967a:184

Strophonelloides Caster, 1939:106

Strophopleura Stainbrook, 1947:324

*Sirophoprion Twenhofel, 1914:25 [= Sirophonella]

Strophoproductus Nalivkin, 1937:46

Strophorichthofenia Termier, Termier, Lapparent, and Marin, 1974:123

Stroudithyris Buckman, 1915 [nomen nudum]; 1917:111

Struveina Boucot, 1975:364

Strygocephale Defrance, 1824 [nomen nudum]; 1825 [ICZN opinion 807 validated spelling as Stringocephalus]

Strygocephalus. -Keferstein, 1828 [misspelling of Strygocephale ]

*Stuartella Belanski, 1928:24 [= Hamburgia]

Sturtella Savage, 1971:417

Siyriasis Rafinesque, 1831 [nomen nudum]

Subansiria Sahni and Srivastava, 1956:212

Subcuspidella Mittmeyer, 1965:81

Subglobosochoneles Afanasjeva, 1976:67

Subrensselandia Cloud, 1942:92

Subriana Sapelnikov, 1960b:111

Substriatifera Kotljar, 1964:123

Suessia Deslongchamps, 1855:539

Suiaella Moisseiev, 1956, in Kiparisova et al., 1956:61

Sulaella Barchatova, 1970 [nomen nudum]

Sulcalaria Cooper and Grant, 1969:5

Sulcathyris Dürkoop, 1970:190

Sulcatina Schmidt, 1964:506

Sulcatinella Dagys, 1974:177

(Sulcalospira) Xu, 1979b:109 [subgenus of Zygospira]

Sulcatospirifer Maxwell, 1954:11

* Sulcatostrophia Caster, 1939:81 [= Nervostrophia]

Sulcatothyris Dagys, 1974:176

Sulciplica Waterhouse, 1968a:25 
Sulcirhynchia Burri, 1953:271

Sulcirosira Cooper and Muir-Wood, 1951:195 fnew name for Rhynchonellopsis Gregorio, 1930]

Sulcorhynchia Dagys, 1974:103

(Superirilobus) Boucot, 1975 |nomen nudum; = (Supertrilobus) Boucot and Johnson, 1979]

(Superirilobus) Boucot and Johnson, 1979:100 [subgenus of Penlamerus];

Surugalhyris Yabe and Hatai, 1934:588

$\ddagger$ Svalbardia Barchatova, 1970 [nomen nudum]; Afanasjeva, 1977a:26

Svaljavithyris Tchorszhewsky, 1974:55

Svobodaina Havlíček, 1950:38

Svobodiana.-Alichova, 1960 [misspelling of Svobodaina]

Swaicoelia Hamada, 1968a:6

Swalbardoproductus Ustritsky, 1971 [nomen nudum]

Sivantonia Walcott, 1905:296

Symmatrypa Mizens and Sapelnikov, 1975:43

Symphythyris Smirnova, 1966:37

(Synatrypa) Copper, 1966b:10 [subgenus of Desquamatia]

Syndielasma Cooper, 1956c:743

Syntrielasma.-Meek and Worthen, 1866 [misspelling of Syntrilasma]

* Syntrilasma Meek and Worthen, 1865, in Meek, 1865:277 [= Enteletes ]

Syntrophia Hall and Clarke, 1892 [nomen nudum]; 1893: 216

Syntrophina Ulrich, 1928, in Weller and St. Clair, 1928:74

Syntrophinella Ulrich and Cooper, 1934 in Kobayashi, 1934: 164

Syntrophioides Schuchert and Cooper, 1931:247

Syntrophopsis Ulrich and Cooper, 1936b:630

Syphonotrela. - Rouiller and Vossinsky, 1847 [misspelling of Siphonotreta]

* Syringopleura Schuchert, 1910:224 [= Syringothyris]

Syringospira Kindle, 1909:29

Syringothyris Winchell, 1863:7

Szajnochiella Tchorszhewsky, 1973 [nomen nudum]

Tadschikia Nikiforova, 1937:35

Taemosirophia Chatterton, 1973:43

Taeniothaerus Whitehouse, 1928:281

Taffia Ulrich, 1926, in Butts, 1926:100

Tafilallia Havliček, 1970b: 17

Taimyrella Ustritsky, 1963, in Ustritsky and Tschernjak, 1963: 115

Taimyropsis Ustritsky, 1963, in Ustritsky and Tschernjak, 1963:69

Taimyrolhyris Dagys, 1968:118

Tainotoechia Tcherkesova, 1967:5

Taleolepiaena Havlíček, 1967a:121

Tamarella Owen, 1965:57

Tanakura Hatai, 1936:322

Tanerhynchia Allan, 1947b:442

Tangkouella Grabau, 1931 [nomen nudum]
Tangshanella Chao, 1929:57

Tangxiangia Xu, 1977:68

Tannuspirifer Ivanova, 1960:267

Tapajotia Dresser, 1954:33

Taphrodonta Cooper, 1956c:740

Taphrorthis Cooper, 1956c:326

Taphrosestria Cooper and Grant, 1975:954

Tarandrospirifer Simakov, 1970:88

Tarfaya Havlícek, $1971 \mathrm{c}: 32$

Taslaria Havliček, 1965b:6

Tauromenia Seguenza, 1885:253

Taurolhyris Kyansep, 1961:27

Taulosia Cooper and Grant, 1969:14

Tazzarinia Havlíček, 1971c:39

Tchegemilhyres Tchorszhewsky, 1972:38

Tcherskidium Nikolayev and Sapelnikov, 1969:12

Tecnocyrtina Johnson and Norris, 1972:566

Teclarea Licharew, 1928:268

Tectorthis Maillieux, 1940 [nomen nudum]

†Tegulifera Schellwien, 1898 [replaced by Teguliferina]

Teguliferina Schuchert and LeVene, 1929:121 [new name for Tegulifera]

Tegulithyris Buckman, 1915 [nomen nudum]; 1917:123

Tegulorhynchia Chapman and Crespin, 1923:175

Teichertina Veevers, 1959:37

Teichostrophia Harper, Johnson, and Boucot, 1967:420

(Telaeoshaleria) Williams, 1950:281 [subgenus of Shaleria]

Tenellodermis Havlíček, $1971 \mathrm{a}: 11$

Tenisia Martynova, 1970:68

Tenticospirifer Tien, 1938:113

Tenuiatrypa Rzonsnitzkaja, 1975:91

Tenuichonetes Jing and Hu, 1978: 109

Tenuicostella Mittmeyer and Geib, 1967:40

Tenuisınurostrum Sartenaer, 1967:2

Teratelasma Cooper, 1956c:823

Terebratalia Beecher, 1893:382

Terebrataliopsis Smirnova, 1962:98

Terebratella Orbigny, 1847:269

Terebratula Müller, 1776:249

Terebralularius Duméril, 1806 [nomen nudum]

Terebralulier Duméril, 1806 [vernacular; = Terebratularius]

Terebratulina Orbigny, 1847:268

Terebraluliles Martini, 1769 [nomen nudum]

Terebratuloidea Waagen, 1883:415

*Terebralulopsis Gregorio, 1930b:8 [= Rhynchonellina ]

Terebrirostra Orbigny, 1847:269

Terrakea Booker, 1931:66

Tesuquea Sutherland and Harlow, 1973:53

$\ddagger$ Tethorotes Manankov, 1979:50

Tetracamera Weller, 1910:503

Tetractinella Bittner, 1890:300

(Telragonetes) Cooper and Grant, 1975:1246 [subgenus of Dyoros ]

Tetralobula Ulrich and Cooper, 1936b:628 
Tetraodontella Jaanusson, 1962:1

Tetraphalerella Wang, 1949:29

Tetrarhynchia Buckman, 1914 [nomen nudum]; 1917:41

Tetratomea Schmidt, 1941:13

Texarina Cooper and Grant, 1970:579 [new name for Texasia]

$†$ Texasia Cooper and Grant, 1969 [replaced by Texarina]

Texathyris Carter, 1972:730

*Thaerodonta Wang, 1949:19 [= Eoplectodonta]

Thamnosia Cooper and Grant, 1969:10

Thaumatosia Cooper, 1973a:15

Thaumatrophia Wang, 1955:343

Thebesia Amsden, 1974:69

Thecedea.-Orbigny, 1847 [misspelling of Thecidea]

Thecidaea._Keferstein, 1828 [misspelling of Thecidea]

Thecidea Defrance, 1822, in Férussac, 1822:xxxviii

Thecidella Munier-Chalmas, 1887, in Fischer, 1887:1331

*Thecidellella Hayasaka, 1938:10 [= Thecidellina]

Thecidellina Thomson, 1915c:462

Thecideum.-Fischer de Waldheim, 1834 [misspelling of Thecidium]

Thecidiopsis Munier-Chalmas, 1887, in Fischer, 1887:1330

Thecidium.-Sowerby, 1823 [misspelling of Thecidea]

Thecocyrtella Bittner, 1892:15 [new name for Cyrtotheca]

Thecocyrtelloidea Yang and Xu, 1966:58

Thecospira Zugmayer, 1880:152

Thecospirella Bit tner, 1900:46

Thecospiropsis Dagys, 1974:75

Thedusia Cooper and Grant, 1976b:2807

Theodossia Nalivkin, 1925:282

Thiemella Williams, 1908:60

Thliborhynchia Lenz, 1967:1188

Thomasaria Stainbrook, 1945:57

Thomasella Fredericks, [1928]:778

$\dagger$ Thomasella Paul, 1942 [invalid new name for Thomasina Paeckelmann, 1931, Thomasella preoccupied; replaced by Argentiproductus]

$\dagger$ Thomasia Fredericks, [1928] [replacement by Thomasina Paeckelmann, 1931, invalid, Thomasina preoccupied; replacement by Thomasella Paul, 1942, invalid, Thomasella preoccupied; replaced by Argentiproductus ]

Thomasina._Bigot, 1927 [misspelling of Tomasina]

$\dagger$ Thomasina Paeckelmann, 1931 [invalid new name for Thomasia Fredericks, 1928, Thomasina preoccupied; replacement by Thomasella Paul, 1942, invalid, Thomasella preoccupied; replaced by Argentiproductus ]

†Thomsonia Jackson, 1916 [replaced by Cacata and Coptothyris]

** Thomsonica Cossman, 1920 [invalid new name for Aetheia, Aetheia not preoccupied]

Thuleproductus Sarytcheva and Waterhouse, 1972:67

Thurmannella Leidhold, 1921:357

Thysanotos Mickwitz, 1896:130

Tianzhushanella Liu, 1979:509
Tibetothyris Ching and Sun, 1976, in Ching et al., 1976: 327

Tichosina Cooper, 1977:62

Timalina Batrukova, 1969:70

Timaniella Barchatova, 1968, in Markowskii, 1968:162

Timanospirifer Ljaschenko, 1973:92

Timorhynchia Ager, 1964:58

Timorina Stehli, 1961b:465

*Tingella Grabau, 1931 la:407 [= Reticulariopsis]

Tipispirifer Grant, 1976:213

Tiramnia Grunt, 1977a:64

(Tisimania) Hatai, 1938:97 [subgenus of Diestothyris]

Tissintia Havlícek, 1970b: 14

Tilanambonites Cooper, 1956c: 717

Titanaria Muir-Wood and Cooper, 1960:334

Tilanomena Bergström, 1968:16

Tityrophoria Waterhouse, 1971, in Bamber and Waterhouse, 1971:214

Togaella Severgina, 1960, in Khalfin, 1960a:409

Tolmatchoffia Fredericks, 1933:29

Tomasina Hall and Clarke, 1892:65

Tomheganella Boucot, 1975 [nomen nudum]

Tomilia Sarytcheva, 1963, in Sarytcheva et al., 1963:220

$\dagger$ Tomiopsis Benediktova, 1956 [preoccupied by Tomiopsis Cope, 1893, mammal]

Tomiproductus Sarytcheva, 1963, in Sarytcheva et al., 1963: 201

Tongzithyris Ching, Liao, and Fang, 1974:313

Toquimaella Johnson, 1967:876

Toquimia Ulrich and Cooper, 1936b:626

Tornquistia Paeckelmann, 1930:218 [replacement by Paeckelmannia invalid, Tornquistia not preoccupied]

Torquirhynchia Childs, 1969:95

Torynechus Cooper and Grant, 1962:1128 [new name for Uncinuloides]

Torynelasma Cooper, 1956c:257

Torynifer Hall and Clarke, 1893, p1. 84

Toryniferella Weyer, 1967:435

Totia Rzonsnitzkaja and Mizens, 1977:92

Toxorthis Temple, 1968:20

Transennatia Waterhouse, 1975:10

Trasgu Martínez, 1979:251

Trautscholdia Ustritsky, 1967:37

Treioria Neuman and Bates, 1978:584

Trematis Sharpe, 1848:68

Trematobolos. - Easton, 1960 [misspelling of Trematobolus]

Trematobolus Matthew, 1893:276

Trematorthis Ulrich and Cooper, 1938:112

Trematosia Cooper, 1976:276

Trematospira Hall, 1859a:207

Tremorthis.-Ulrich and Cooper, 1938 [printer's error for Trematorthis]

Tretorhynchia Brunton, 1971:98

Triadispira Dagys, 1961b:457 
Triadithyris Dagys, 1963:187

Triathyris Comte, 1938:45

Trichochonetes Roberts, 1976:22

Trichorhynchia Buckman, 1914 [nomen nudum]; 1917:58

Trichothyris Buckman, 1915 [nomen nudum]; 1917:125

Tricoria Cooper and Grant, 1976a: 1945

Tridensilis Su, 1976: 193

Trifidarcula Elliott, 1959:147

Trifidorostellum Sartenaer, $1961 \mathrm{a}: 5$

*Trigeria Bayle, 1878, pl. 13 [= Retzia]

†Trigonella Quenstedt, 1868 [replaced by Neotrigonella]

Trigonellina Buckman, 1907:342

Trigonirhynchella Dagys, 1963:41 [new name for Trigonirhynchia Dagys, 1961]

Trigonirhynchia Cooper, 1942:228 [new name for Uncinulina]

†Trigonirhynchia Dagys, 1961 [replaced by Trigonirhynchella]

Trigonithyris Muir-Wood, 1935:131

Trigonoglossa Dunbar and Condra, 1932:35

Trigonosemus Koenig, 1825:3

Trigonotreta Koenig, 1825:3

(Trigonotrophia) Andreeva, 1972:54 [subgenus of Idiostrophia]

Trigorima Rafinesque, 1819; 1831 [nomina nuda]

Trigrammaria Wilson, 1945:140

Trimerella Billings, 1862:166 [replacement by Gotlandia Dall, 1870, later withdrawn by Dall]

Trimurellina Mitchell, 1977:74

*Triplecella Wilson, 1932:400 [= Cyclospira $]$

Triplecia.-Oehlert, 1887; Hall and Clarke, 1892 [incorrect emendation of Triplesia ]

Triplesia Hall, 1859b:44

Triseptalia Makridin, 1954 [nomen nudum]

Triseptata Bondarev, 1965, in Bondarev et al., 1965:34

Triseptothyris Xu, 1978b:314

Tritoechia Ulrich and Cooper, 1936b:625

Trochalocyrtina Wright, 1975:175

Trochifera Ching and Ye, 1979, in Ye and Yang, 1979:68

Trondorthis Neuman, 1974, in Neuman and Bruton, 1974: 77

Tropeothyris Smirnova, 1972:69

Trophisina Cooper and Grant, 1976a:2078

Tropidelasma Cooper and Grant, 1969:3

Tropidoglossa Rowell, 1966:31

Tropidoleptus Hall, 1857:151

Tropidothyris Cooper, 1956c:507

*Tropiorhynchia Buckman, 1914 [nomen nudum]; 1917:33 [= Piarorhynchia $]$

Trotlandella Neuman, 1974, in Neuman and Bruton, 1974: 95

Trucizetina Havlíček, 1974:169

Truncalosia Imbrie, 1959:401

Trunculites Rafinesque, 1831 [nomen nudum]

Tschatkalia Nikiforova, 1964:85

Tschernyschewia Stoyanow, 1910:855
$†$ Tschernyschewiella Fredericks, 1924 [replaced by Kochiproductus]

Tubaria Muir-Wood and Cooper, 1960:236

Tubegatanella Prosorovskaja, 1968:244

Tubersulculus Waterhouse, 1971, in Bamber and Waterhouse, 1971:208

Tubithyris Buckman, 1915 [nomen nudum]; 1917:115

Tubulostrophia Havlícek, 1967a: 139

Tudiaophomena Xian, 1978a:266

Tufoleptina Havlíček, 1961b:447

Tulathyris Grunt, 1976: 78

Tulcumbella Campbell and Engel, 1963:68

Tulipina Smirnova, 1962: 102

Tulungospirifer Ching and Sun, 1976, in Ching et al., 1976: 316

Tumarinia Solomina and Grigorjewa, 1973, in Grigorjewa and Solomina, 1973:35

$\dagger$ Tunaria Hoek, 1912 [replaced by Tunarites]

*Tunarites Cooper and Muir-Wood, 1951:196 [new name for Tunaria; = Bistramia $]$

(Tungussotoechia) Lopushinskaja, 1976:60 [subgenus of Stegerhynchus]

Tunisiglossa Massa, Havlícek, and Bonnefous, 1977:13

Turkmenithyris Prosorovskaja, 1962:109

Turriculum Gregorio, 1930a:26

Tuvaella Tschernyschew, 1937:11

Twenhofelia Boucot and Smith, 1978:272

Tyersella Philip, 1962:197

Tyloplecta Muir-Wood and Cooper, 1960:291

Tylospiriferina Xu, 1978b:297

Tylothyris North, 1920:197

**Tylotoma Grabau, 1934 [= Spiriferellina]

Tyrganiella Kulkov, 1970, in Alekseeva et al., 1970:167

Tyronella Mitchell, 1977:64

*Tyrothyris Öpik, 1953:15 [= Meifodia]

$\ddagger$ Tythothryis Zezina, 1979:224

Uchtella Ljaschenko, 1973:50

*Uchtospirifer Ljaschenko, 1957:885 [= Cyrtiopsis]

Ujandinella Baranov, 1977:75

Ukoa Öpik, 1932:33

Ulbospirifer Gretchischnikova, 1965:33

Uldziathyris Grunt, 1977b:82

Umboanctus Waterhouse, 1971, in Bamber and Waterhouse, 1971:212

Uncinella Waagen, 1883:494

†Uncinulina Bayle, 1878 [replaced by Trigonirhynchia Cooper, 1942]

†Uncinuloides King, 1931 [replaced by Torynechus]

Uncinulus Bayle, 1878, p1. 11

Uncinunellina Grabau, 1932:100

Uncisteges Jing and $\mathrm{Hu}, 1978: 117$

Uncites Defrance, 1825, in Blainville, 1825:630

Undaria Muir-Wood and Cooper, 1960:318 
(Undatrypa) Copper, 1978:302 [subgenus of Punctatrypa]

Undellaria Cooper and Grant, 1975:1158

Undiferina Cooper, 1956c:262

Undispirifer Havlícek, 1957b:439

Undispiriferondes Xian, 1978a:330

Undithyrella Havliček, 1971c:78

Undulella Cooper and Grant, 1969:5

*Ungula Pander, 1830:59 [= Obolus]

Ungulites._Bronn, 1848 [misspelling of Ungula]

Unispirfer Campbell, 1957:67

Uralella Makridin, 1960, in Licharew et al., 1960:295

†Uralia Licharew, 1925 [replaced by Uralina]

*Uralina Schuchert and LeVene, 1929:122 [new name for Uralıa; = Eolyttonia $]$

$\ddagger$ Uraloproductus Ustritsky, 1971:21

Uralorhynchia Dagys, 1968:54

Uralospira Mizens, 1977a:82

Uralospirifer Havlícek, 1959a:246

Uralotoechia Sapelnikov, 1963:17

Urbanirhynchia Katz, 1974:252

Urbimena Havlícek, 1976:367

Urella Rzonsnitzkaja, 1960, in Markowskii, 1960:403

**Urticites Defrance, 1828 [= Hysterolutes]

Urushtenia Licharew, 1935:370

Urushtenoidea Jing and $\mathrm{Hu}, 1978: 116$

Ushkolia Martynova and Sverbilova, 1969:96

**Ussovia Khalfin, 1955 [= Wilsoniella]

Ussuricamara Koczyrkevicz, 1969:11

Ussurirhynchia Koczyrkevicz, 1976a:80

Vaga Sapelnikov and Rukavischnikova, 1973:33

(Vagranella) Sapelnikov, 1960b: 116 [subgenus of Subriana] Vagrania Alekseeva, 1959:389

Valcourea Raymond, 1911:240

Valdaria Bassett and Cocks, 1974:18

Valdiviathyris Helmcke, 1940:237

Vallomyonia Johnson, 1966a: 158

Vandercammenina Boucot, 1975:363

*Vandergrachiella Crickmay, 1953a:7 [= Theodossia]

Vandobiella Pojariskaja, 1966:22

Vaniella Kvakhadze, 1974:493

(Variatrypa) Copper, 1966b:12 [subgenus of Desquamatia]

Vectella Owen, 1965:51

Vediproductus Sarytcheva, 1965, in Ruzhentsev and Sarytcheva, 1965:219

Veghirhynchia Dagys, 1974:84

Velciella Havlícek and Snajdr, 1952 [nomen nudum]

Veliseptum Popov, 1976, in Nazarov and Popov, 1976:38

Vellamo Öpik, 1930:212

Velostrophia Havliček, 1965b:7

Venezuelia Weisbord, 1926:22

Verchojania Abramov, 1970:112

*Verkhotomia Sokolskaja, 1963, in Sarytcheva et al., 1963: 280 [= Pseudosyrinx]

Vermiculothecidea Elliott, 1953:694

Vemeuilia Hall and Clarke, 1893:57

Vex Hoover, 1979:9
Viallithyris Vörös, 1978:62

Viarhynchia Calzada Badía 1974b: 170

Victorithyris Allan, 1940:289

Viligella Dagys, 1965:116

Viligothyris Dagys, 1968:89

Villicundella Levy and Nullo, 1974a: 178

* (Vincia) Gregorio, 1930a:31 [subgenus of Lyttonia; $=$ Leptodus ]

Virbium Gregorio, 1930a:25

Virgiana Twenhofel, 1914:27

Virgianella Nikiforova and Sapelnikov, 1971:49

Virginiata Amsden, 1968:56

Virgoria Neuman, 1977:23

(Viruella) Rōōmusoks, 1959:14 [subgenus of Sowerbyella]

Visbyella Walmsley, Boucot, Harper, and Savage, 1968: 306

Vitiliproductus Ching and Liao, 1974:278

†Vitulina Hall, 1860 [nomen nudum]; 1867 [replacement by Pustulina invalid, Pustulina preoccupied, replaced by Pustulatia]

*Vltavothyris Havlíček, 1956:621 [= Meganteris]

Voiseyella Roberts, 1964:187

Volborthia Möller, 1870:409

Volirhynchia Dagys, 1974:104

Vosmiverstum Breivel and Breivel, 1970a:54

Waagenia Hall, 1889 [nomen nudum; = Polytoechia]

Waagenttes Paeckelmann, 1930:223

Waagenoconcha Chao, 1927:85

* Waagenopora Noetling, 1902 [invalid new name for Oldhamina]

Waconella Owen, 1970:74

Waiparia Thomson, 1920:380

Wairakiella Waterhouse, 1967a:87

Waisiuthyrna Beets, 1943:342

Walcottina Cobbold, 1921:336

†Waldheimia King, 1850 [replaced by Magellanza]

** Waldheimiathyris Helmcke, 1939 [invalid new name for Macandreva, Macandrevia not preoccupied]

Walkeria Haas, 1890 [nomen nudum]

Walkerithyris Cox and Middlemiss, 1978:424

Wallonia Davidson, 1850a:475

Wardakia Termier, Termier, Lapparent, and Marin, 1974: 94

Warrenella Crickmay, 1953b:596

Warsawia Carter, 1974:684

Wattonithyris Muir-Wood, 1936a:91

*Wattsella Bancroft, 1928b:55 [= Dalmanella]

Weberithyris Smirnova, 1969b: 144

Weiningia Ching and Liao, 1974:282

Weldonithyris Muir-Wood, 1952:130

Wellerella Dunbar and Condra, 1932:287

* Welleria Maillieux, 1931 [invalid new name for Delthyris in sensu Weller, 1914 (not Delthyris Dalman, 1828), Welleria preoccupicd and nomen nudum; = Tylothyris]

†Welleria Rotai, 1938 [nomen nudum]; 1941 [replaced by Rotaial 
Werneckeella Lenz, 1971:844

* Werriea Campbell, 1957:44 [= Orthotetes]

Westonia Walcott, 1901:683

Westralicrania Cockbain, 1967: 119

Whidbornella Reed, 1943:71

Whitfieldella Hall and Clarke, 1893:58

*Whitfieldia Davidson, 1882:107 [= Meristina ]

Whitspakia Stehli, 1964:610 [new name for Pakistania]

Whitlardia Williams, 1974:52

Wilberrya Yancey, 1978:301

† Wilsonella Nikiforova, 1937 [invalid new name for Wilsonia,

Wilsonella preoccupied; replaced by Sphaerirhynchia]

†Wilsonia Kayser, 1871 [replacement by Wilsonella invalid,

Wilsonella preoccupied; replaced by Sphaerirhynchia]

Wilsoniella Khalfin, 1939:83

Wimanella Walcott, 1908:99

*Winterfeldia Spriestersbach, 1942:198 [= Uncites]

Wittenburgella Dagys, 1959a:30

Worobievella Dagys, 1959a:33

†Worthenella Girty, 1938 [replaced by Labriproductus]

Wyatkina Fredericks, 1931:211

Wyella Khodalevich, 1939:21

Wyndhamia Booker, 1930:25

Wynnia Walcott, 1908 [nomen nudum]; 1912:771

Wysogorskiella Hints, 1975:61

$X$, Pajaud, 1966 [un-named; = Konstantia]

Xana Garcia-Alcalde, 1972a:5

Xenamboniles Cooper, 1956c:814

Xenelasma Ulrich and Cooper, 1936b:631

Xenelasmella Rozman, 1964, in Chugaeva et al., 1964:137

Xenelasmopsis Rozman, 1969 [nomen nudum]

Xenizostrophia Su, 1976:181

Xenomartinia Havlíček, 1953:6

Xenorthis Ulrich and Cooper, 1936b:620

Xenosaria Cooper and Grant, 1976a:2171

Xenospirifer Hou and Xian, 1975:69

Xenosteges Muir-Wood and Cooper, 1960:111

Xenostrophia Wang, 1974, in Wang et al., 1974:38

Xenothyris Ching, Rong, and Sun, 1976, in Ching et al., 1976:337

Xerospirifer Havliček, 1978:103

Xerxespirifer Cocks, 1979b:40

Xestosia Cooper and Grant, 1975:1063

Xestotrema Cooper and Grant, 1969:16

Xiangzhounia $\mathrm{Ni}$ and Yang, 1977:464

Xinanorthis Xu, Rong, and Liu, 1974:145

Xinanospirifer Rong, Xu, and Yang, 1974:206

Xinshaoella Zhao, 1977:391

Xizangostrophia Rong, 1976, in Liu, 1976:150

Xystostrophia Havlicek, 1965a:292

Yabeithyris Hatai, 1948:498

Yagonia Roberts, 1976, in Roberts et al., 1976:210
Yakovlevia Fredericks, 1925:7

Yakutijaella Baranov, 1977:77

Yanbianella Tong, 1978:239

Yangkongia Xu and Liu, 1979 [nomen nudum]

Yangtzeella Kolarova, 1925:219

Yanguania Yang, 1977:346; 1978a:107; 1978b:249

Yanishewskiella Licharew, 1957:138

Yanospira Dagys, 1977a:11

Yarirhynchia Ching and Sun, 1979 [nomen nudum]

* Yatsengina Semichatova, 1936:216 [= Choristites]

* Yavorskiella Lazutkin, 1940 [nomen nudum; = Lazutkinia]

Yeosinella Reed, 1933:193

* Ygera Havlícek, 1961 b:449 [= Plectodontella]

Ygerodiscus Havlíček, 1967a:62

Yidunella Ching, Sun, and Ye, 1979:148

Yingtangella Bai and Ying, 1977 [nomen nudum]; 1978 in Yoh and Bai, 1978:54

Yingwuspirifer Rong, $\mathrm{Xu}$, and Yang, 1974:201

Yochelsonia Stehli, 1961a:454

Yorkia Walcott, 1897:714

Ypsilorhynchus Sartenaer, 1970:9

* Yrctospirifer Gatinaud, 1949:488 [= Cyrtospirifer]

Yuezhuella Ching and Ye, 1979, in Ye and Yang, 1979:69

Yunnanella Grabau, 1923:195

†Yunnanella Grabau, 1931 [replaced by Nayunnella]

** Yunnanellina Grabau, 1931 [= Yunnanella Grabau, 1923]

Zaissania Sokolskaja, 1968, in Sarytcheva, 1968:197

Zanclorhyncha Xu, 1979b:96

Zdimir Barrande, 1881:171

Zdimirella Tcherkesova, 1973:32

Zeilleria Bayle, 1878, pl. 9

Zeillerina Kyansep, 1959:119

Zejszneria Siemiradzki, 1922:172

Zellania Moore, 1855:111

Zeravshanotoechia Rzonsnitzkaja, 1977:127

Zeuschneria Smirnova, 1975a:137

Zhidothyris Ching, Sun, and Ye, 1979:216

Zhonghuacoelia Chen, 1978:361

Zia Sutherland and Harlow, 1973:59

Ziganella Nalivkin, 1960, in Ivanova, 1960:280

*Zigania Nalivkin, 1937 [nomen nudum; = Dzieduszyckia]

Zilımia Nalivkin, 1937 [nomen nudum]; 1947:98

Zittelina Rollier, 1919:368

Zlichopyramis Havliček, 1975:233

Zlichorhynchus Havlícek, 1963a:403

Zonarites Rafinesque, 1831? [incertae sedis]

Zophostrophia Veevers, 1959:63

Zugmayerella Dagys, 1963:99

Zugmayeria Waagen, 1882:334 [originally spelled Zugmeyeria by Waagen, corrected to Zugmayeria ]

Zygatrypa Copper, 1977:307

Zygospira Hall, 1862:155

Zygospiraella Nikiforova, 1961, in Nikiforova and Andreeva, 1961:237 


\section{Computerized List of Valid Genera and Subgenera}

(Including subjective synonyms)

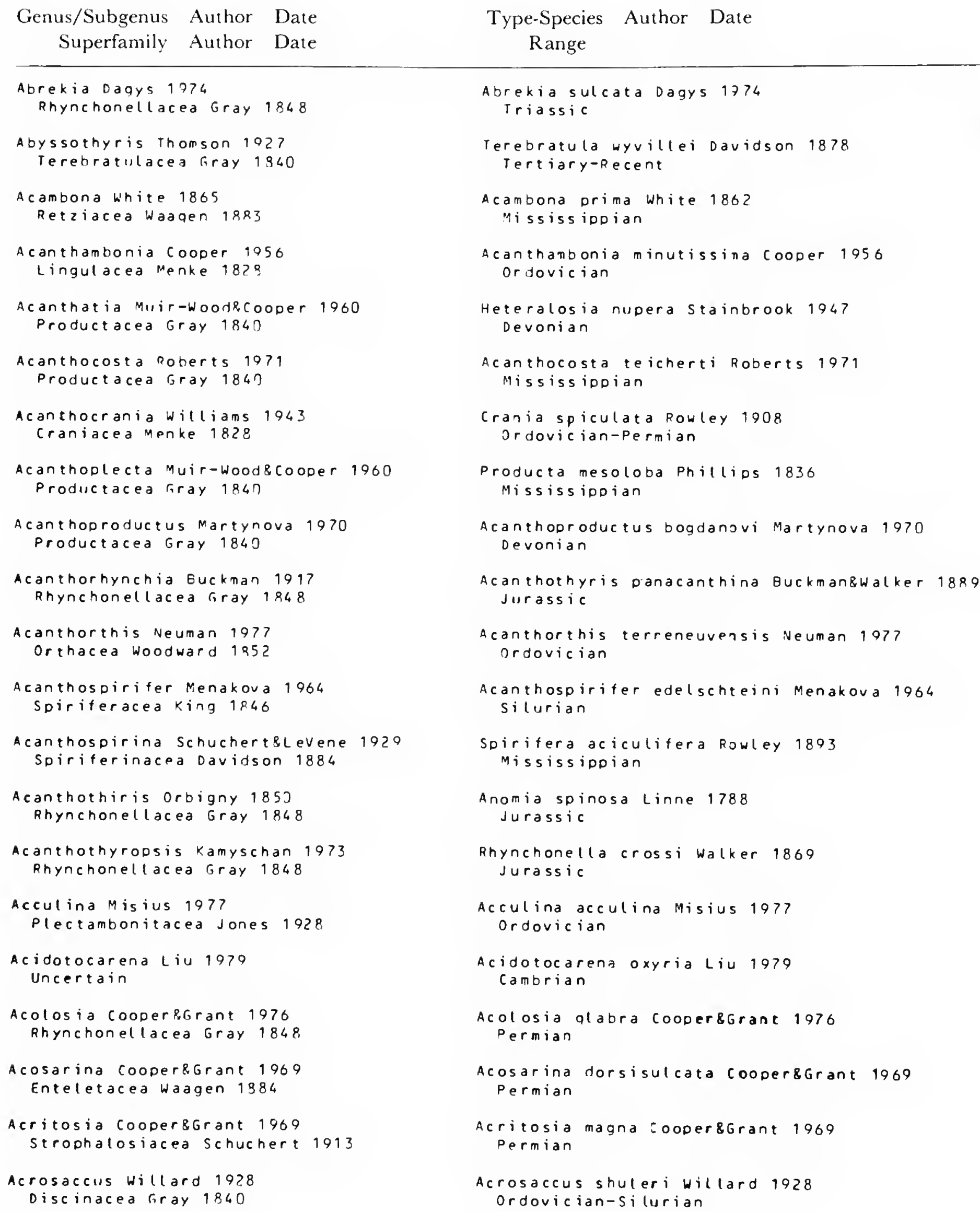


Acrospirifer Helmbrecht\&wedek ind 1923 Spiriferacea King 1846

Acrothele Linnarsson 1876 Acrotretacea schuchert 1893

Acrothyra Mat thew 1901 Acrotretacea Schuchert 1893

Acrothyris Hou 1963 Strinqocephal acea King 1850

Acrotreta Kutorqa 1848 Acrotretace 7 Schuchert 1893

Acrotretella Ireland 1961 Acrotretacea schwchert 1893

Actinoconchus Mecoy 1844 Athyridacea McCoy 1844

Actinomena opik 1930 strophomenacea $k$ ing 1846

Acuminothyris Roberts 9963 Spiriferacea King 1846

Acutatheca stainbrook 1945 Spiriferacea King 1846

Acutella Ljaschenko 1073 Spiriferacea kina 1846

Acutilineolus Amsden 1978 Cyrtiacea Fredericks 1924

Adiaphragma xian 1978 Plectambonitacea Jones 1928

Adminiculoria waterhouse\&Gupta 1978 Delthyridacea Phillips 1841

Admixtella Rozman 1978

Clit ambonitacea Winchell \&schuchert 1893

Adoltia Gurich 1909

Spiriferacea King 1846

Adolfispirifer Krylova 1962 Spiriferacea Kina 1846

Adrenia chatterton 1973 Stringocephalacea King 1850

Adriana Greqorio 1930 Lyttoniacea Wagen 1883

Adygella Dagys 1959

Dielasmat acea Schuchert 1913

Adygelloides Dagys 1959 Dielasmatacea Schuchert 1913

Aegiria Opik 1933 Plectambonitacea Jones 1928

Aegiromena Havlicek 1961 Plectambonitacea Jones 1928

Aegironetes Havlicek 1967 Plectambonitacea Jones 1928

Aemula Steinich 1968 Terebratellacea King 1850

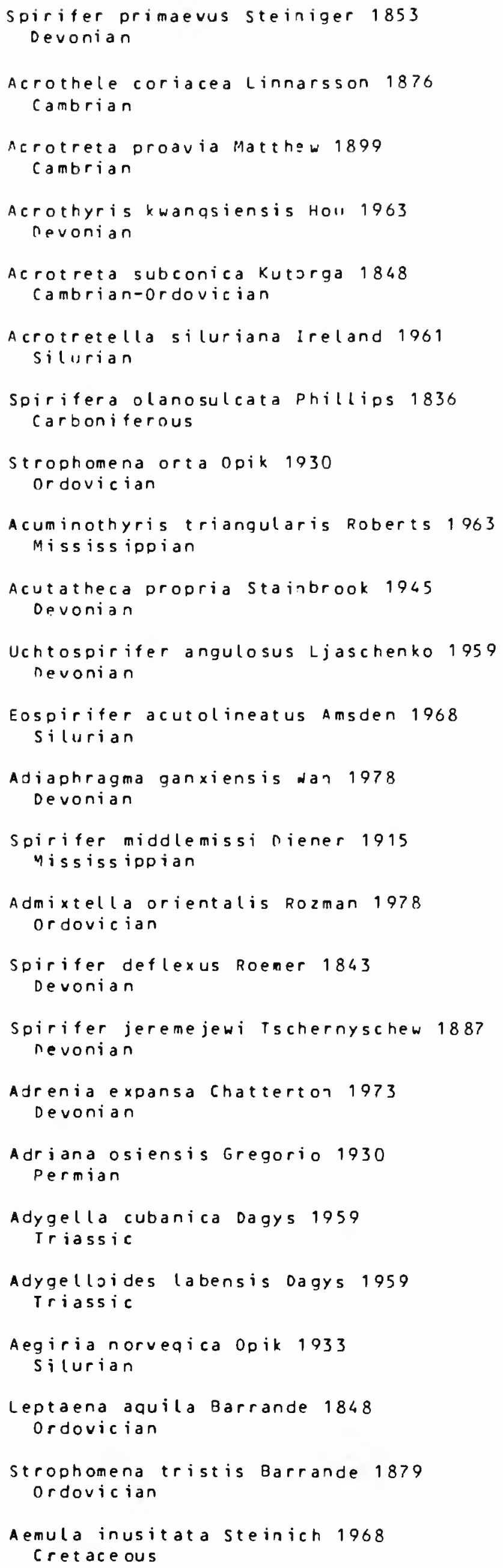




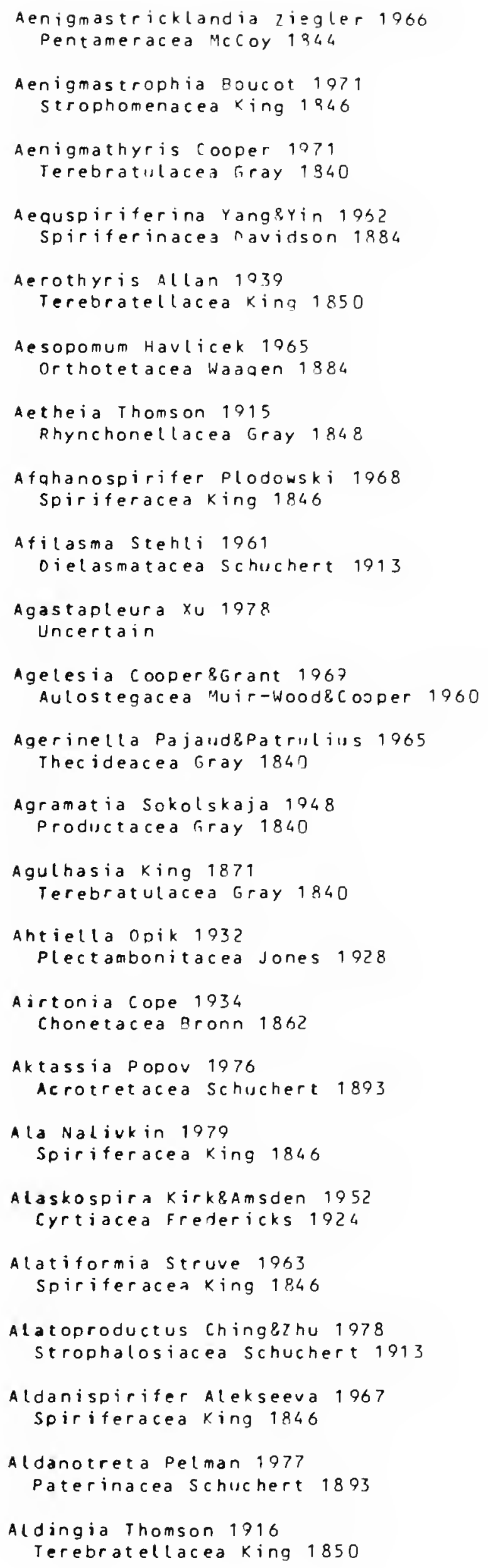

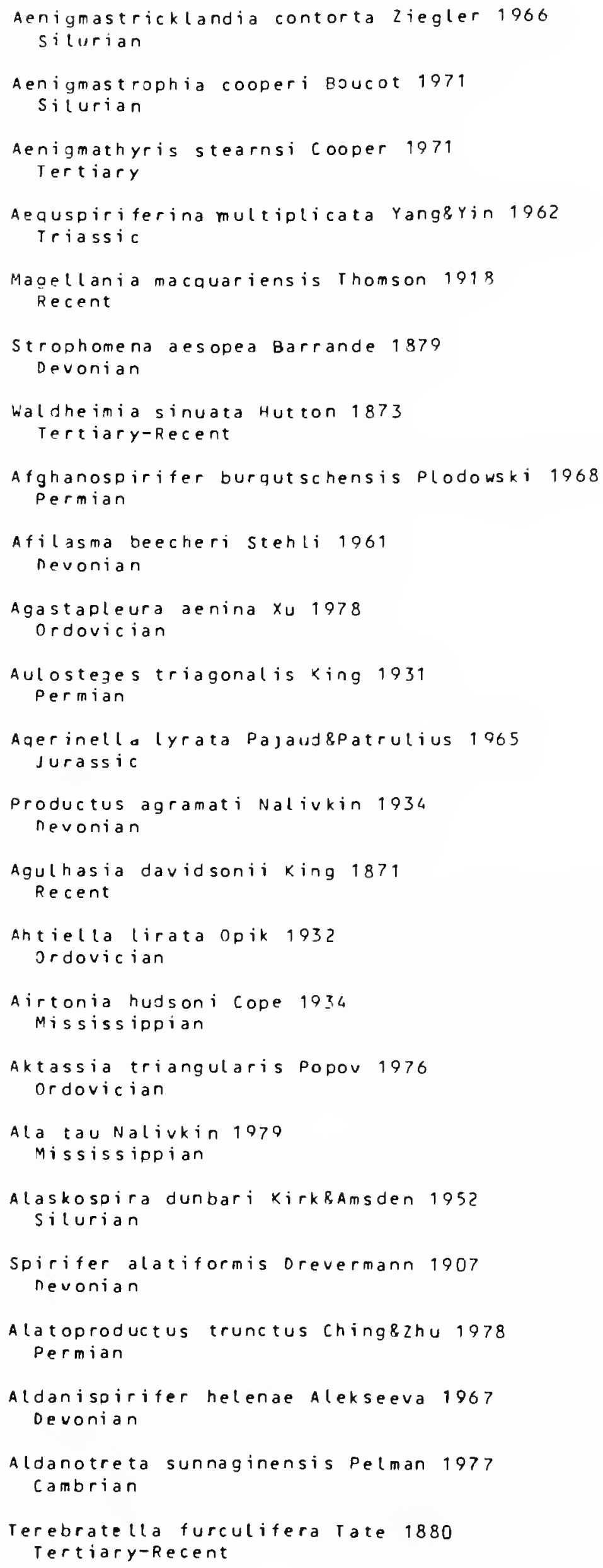


Alexenia I vanova 1935

Productacea riray 1843

Alichovia Gorjansky 1969 Siphonotretacea Kutorga 1848

Aliconchidium St.Joseph 1042

Pentameracea McCoy 1344

Alimbella Andreeva 1967 Porambonitacea Davidson 1853

Alipunctifera waterhouse 1975 Spiriferinacea Davitson 1984

Alisina Rowell 196 ? obolellacea walcottsschuchert 1003

Alispira Nikiforova 1961 Atrypacea Gill 1871

Alispirifer ramphell 1961 Spiriferacea kina 1946

Alitaria Cooperimuir-Wood 1967 Productacea Gray 1840

Allanella Crickmay 1953 spiriferacea king 1846

Allanetes 9oucotljohnson 1967 chonetacea aronn 1862

Allorhynchoides Savaqe et al 1978 Rhynchonel lacea Gray 1848

Allorhynchus weller 1910 Rhynchonel lacea riray 1848

Almerarhynchia Calzada 1974 Rhynchonel lacea Gray 1848

Alphachoristites 6atinaud 1749 Spiriferacea king 1.946

Alphacyrtiopsis Gatinaud 1949 Spiriferacea king 1846

Alphaneospirifer riatinaud 1949 Spjriferacea king 1846

Altaestrophia gublichenko 1956 Strophomenacea King 1346

Altaethyrella severgina 1978 Atrypacea Gill 1871

Altajella Kulkov 196 ? Spiriferacea king 1846

Altiplecus Stehli 1954 Spiriferinacea Davidson 1884

Altorthis Andreeva 1767 orthacea Woodward 125?

Alwynella spjeldnaes 1957 Plectambonitacea Jones 1928

Alwynia Stehli 1961 Dielasmatacea Schuchert 1913

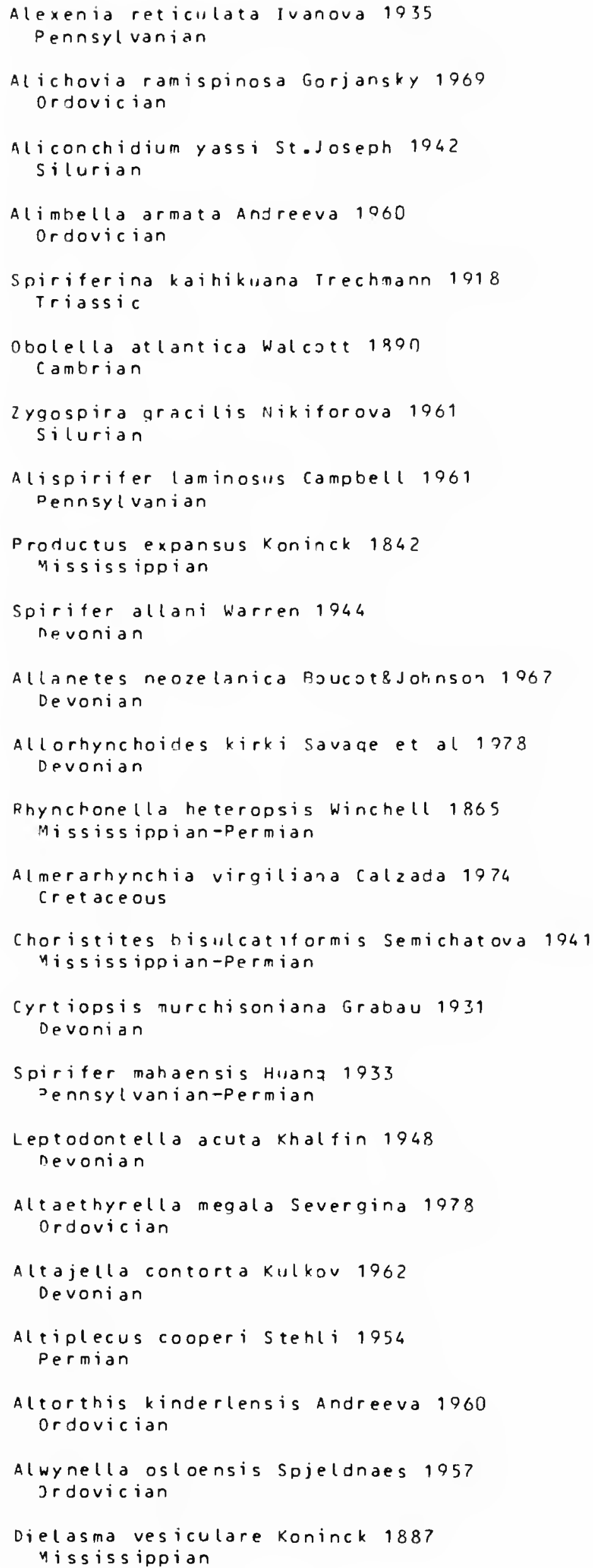




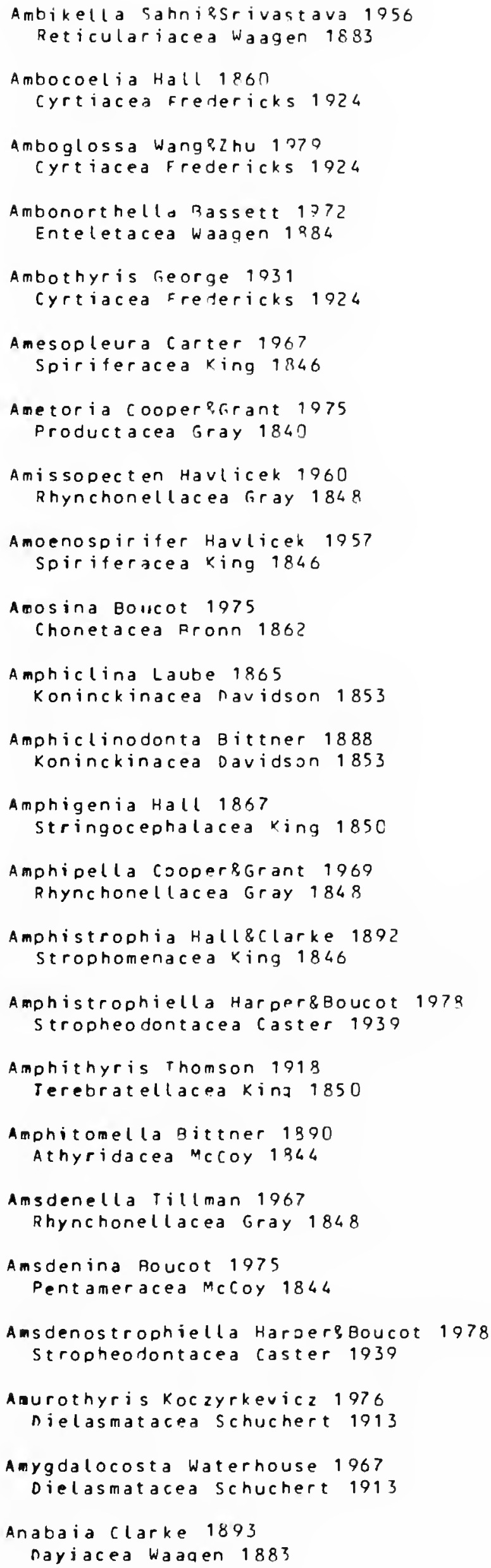

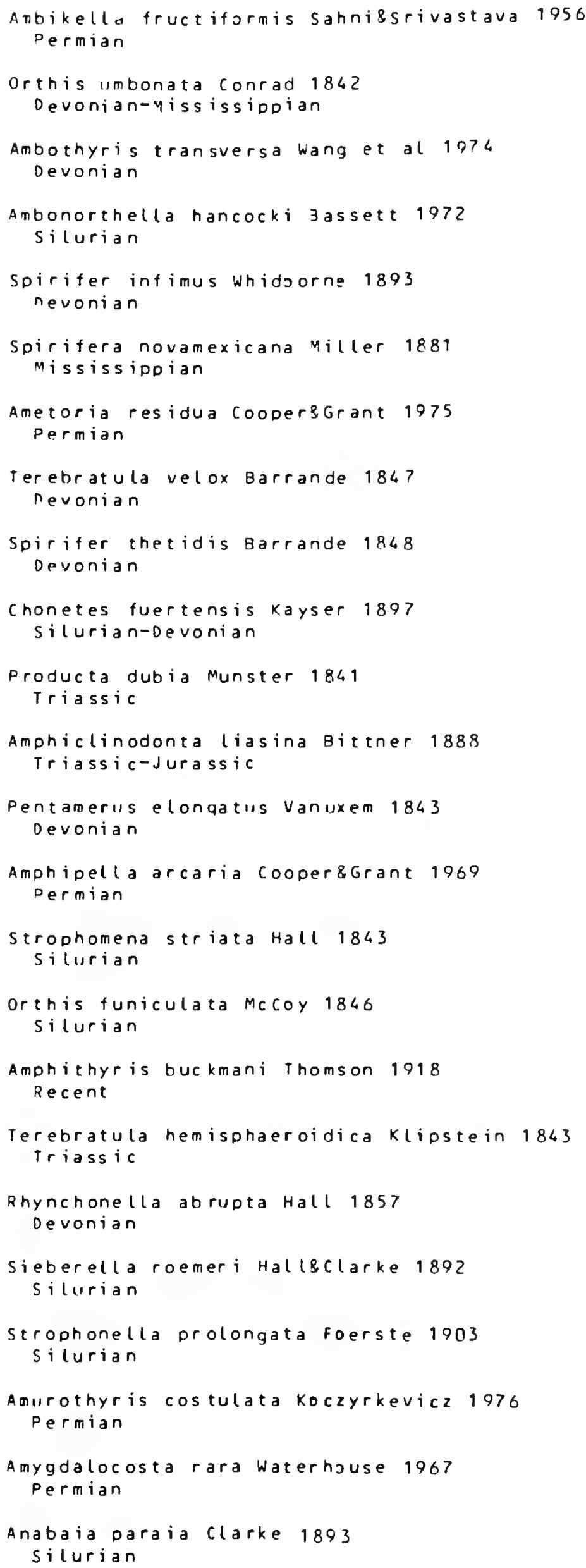




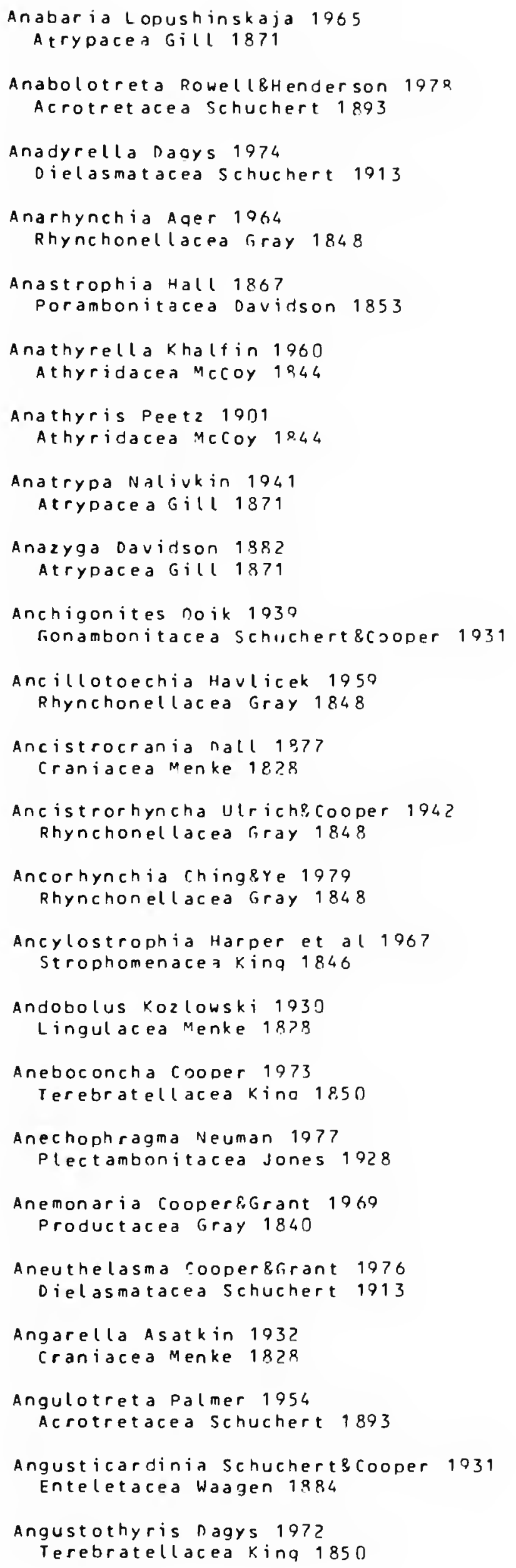

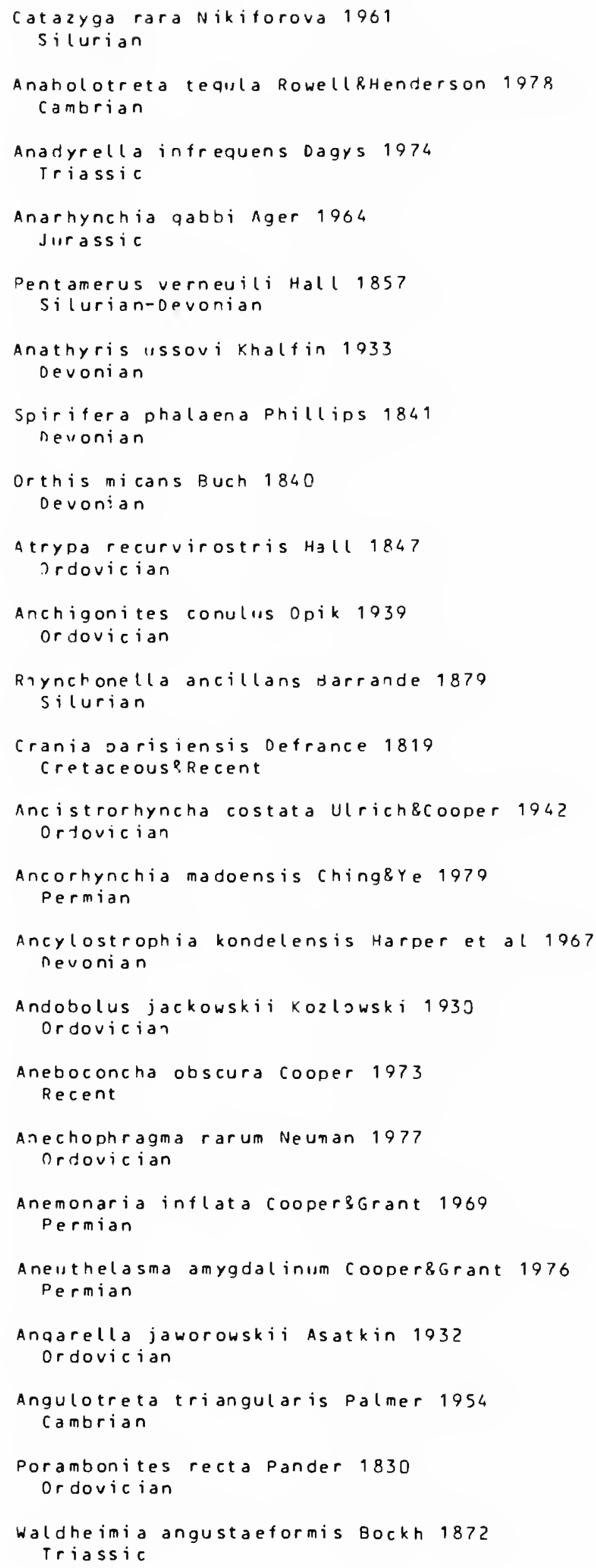




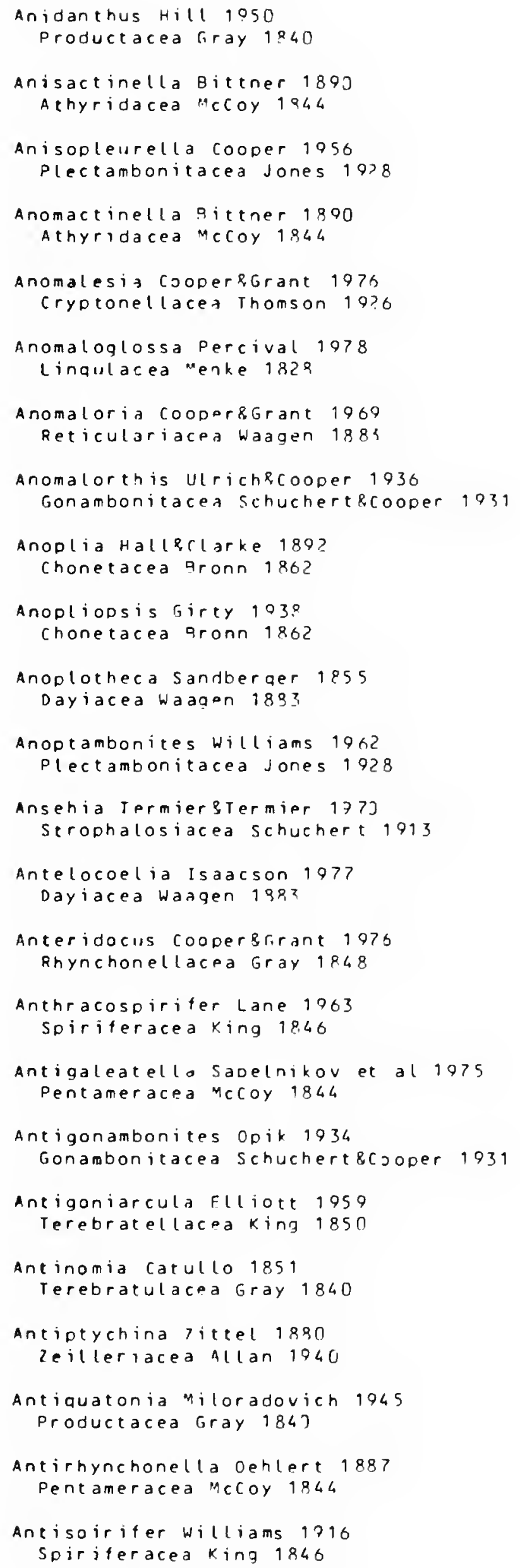

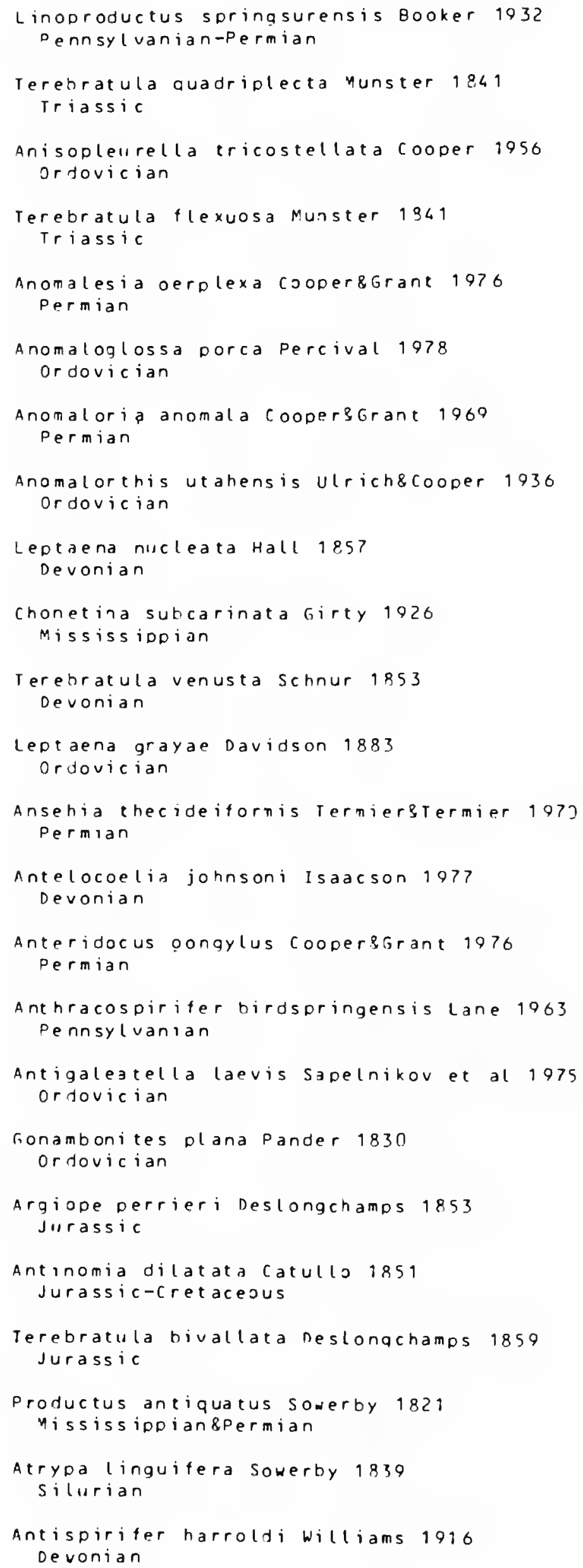




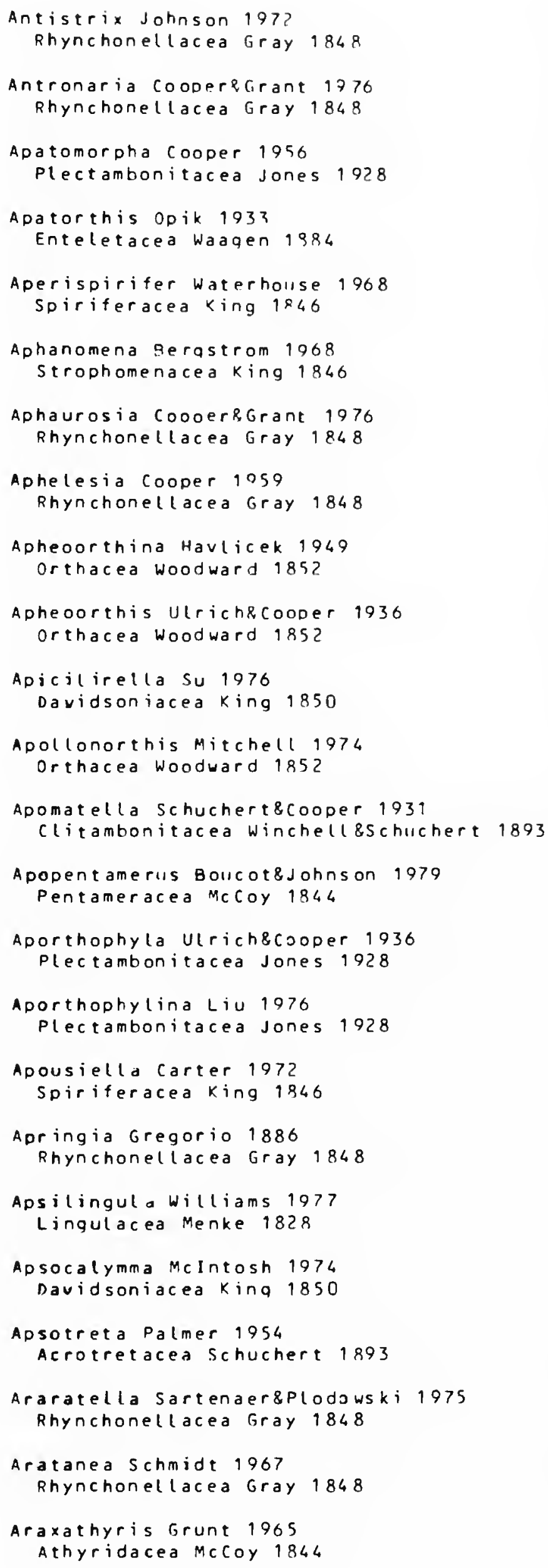

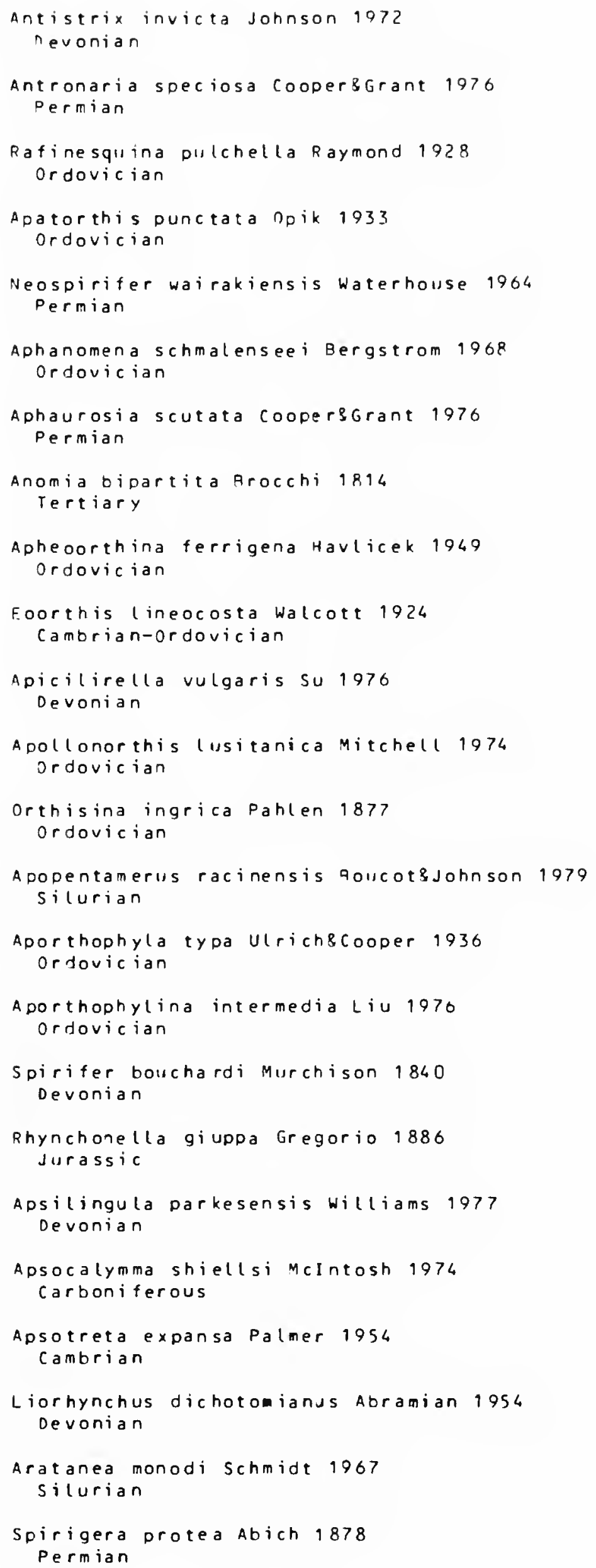




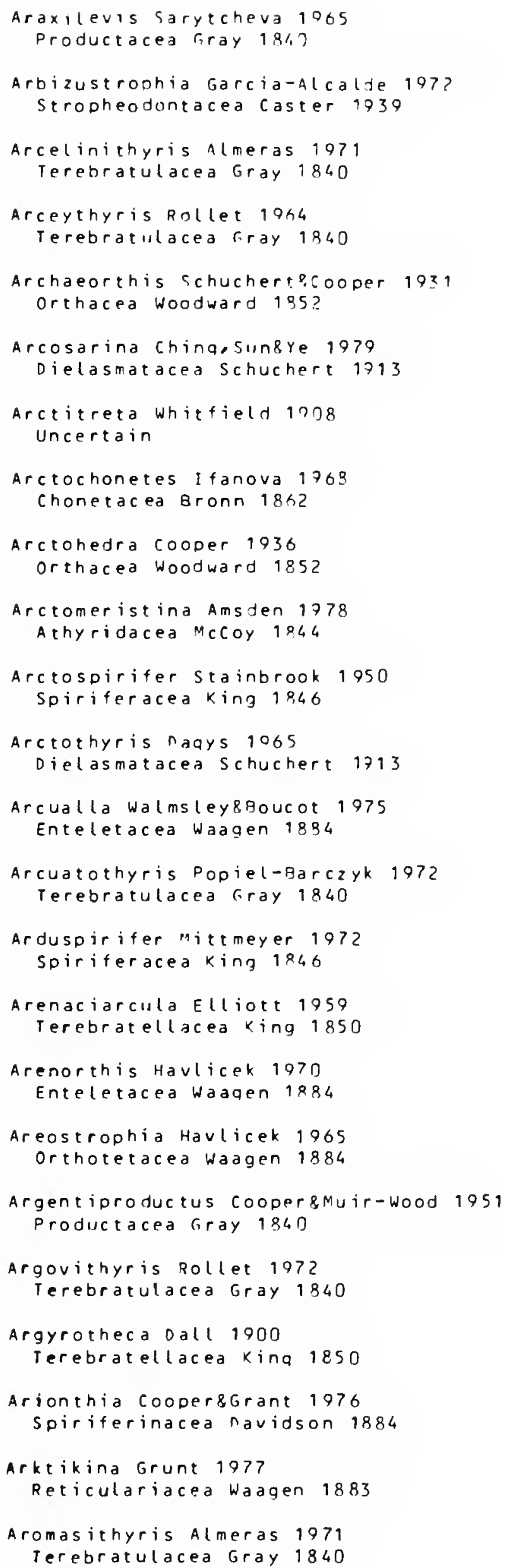

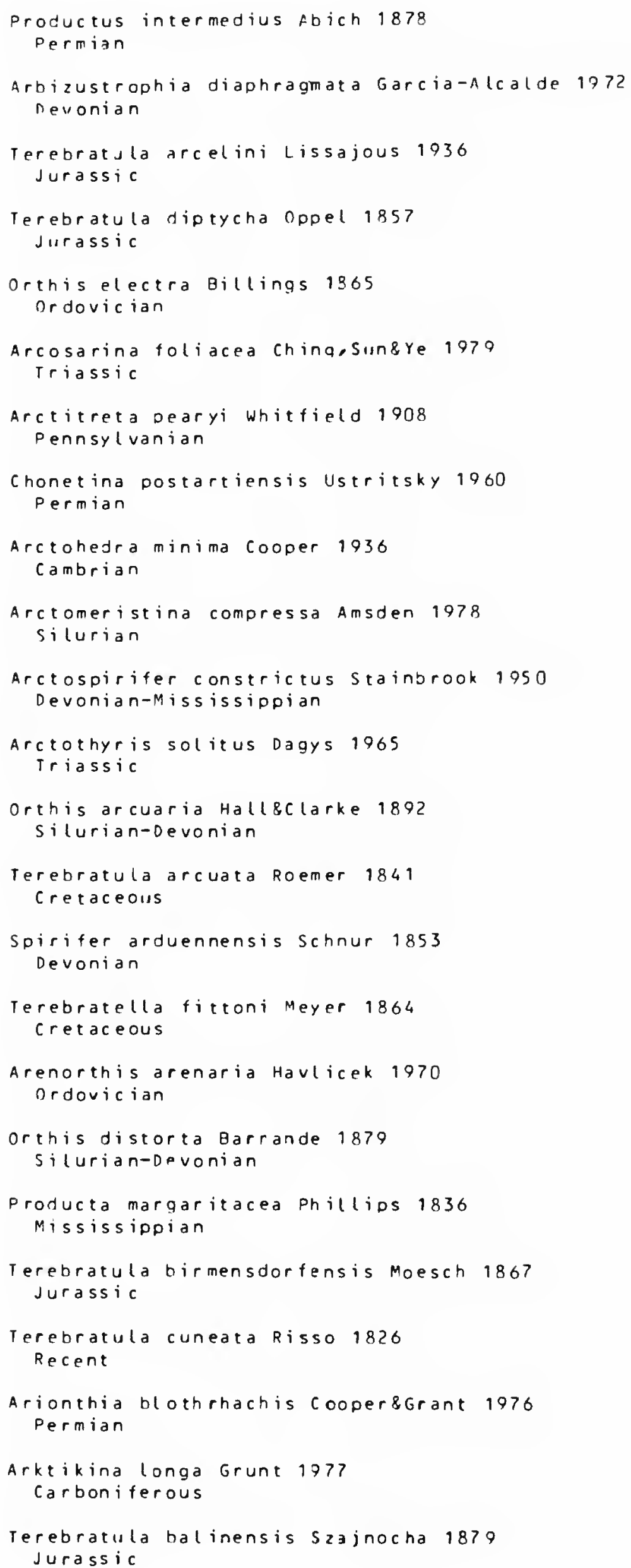




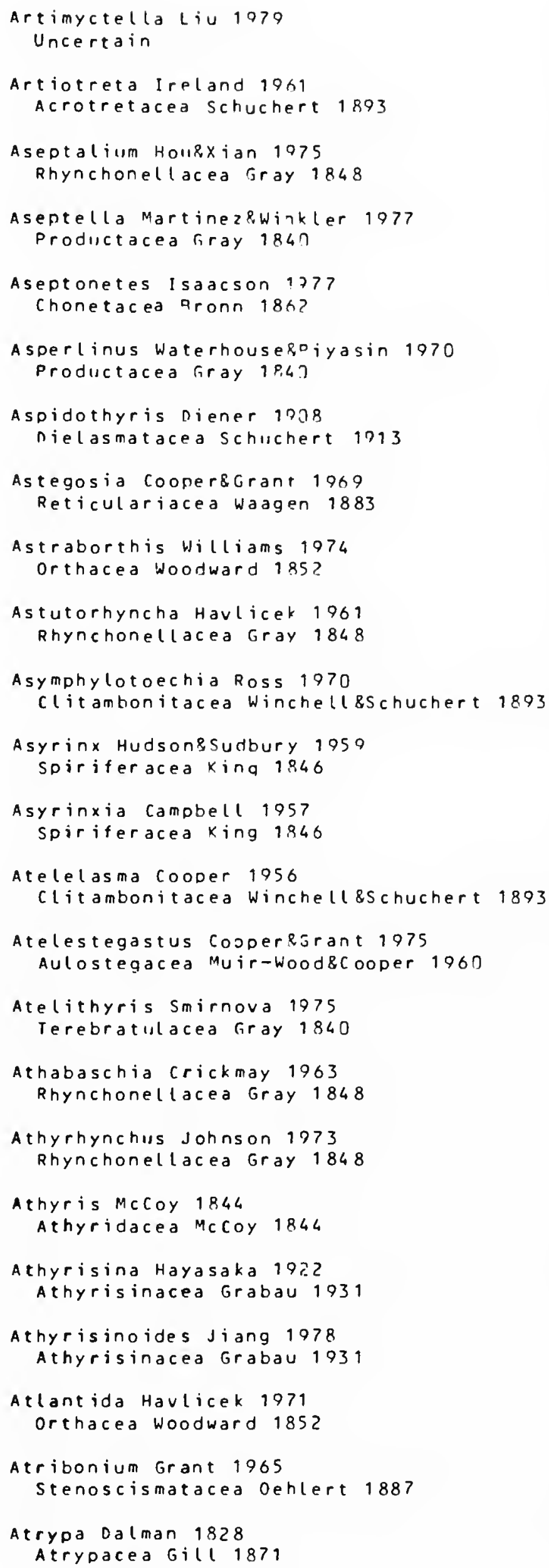

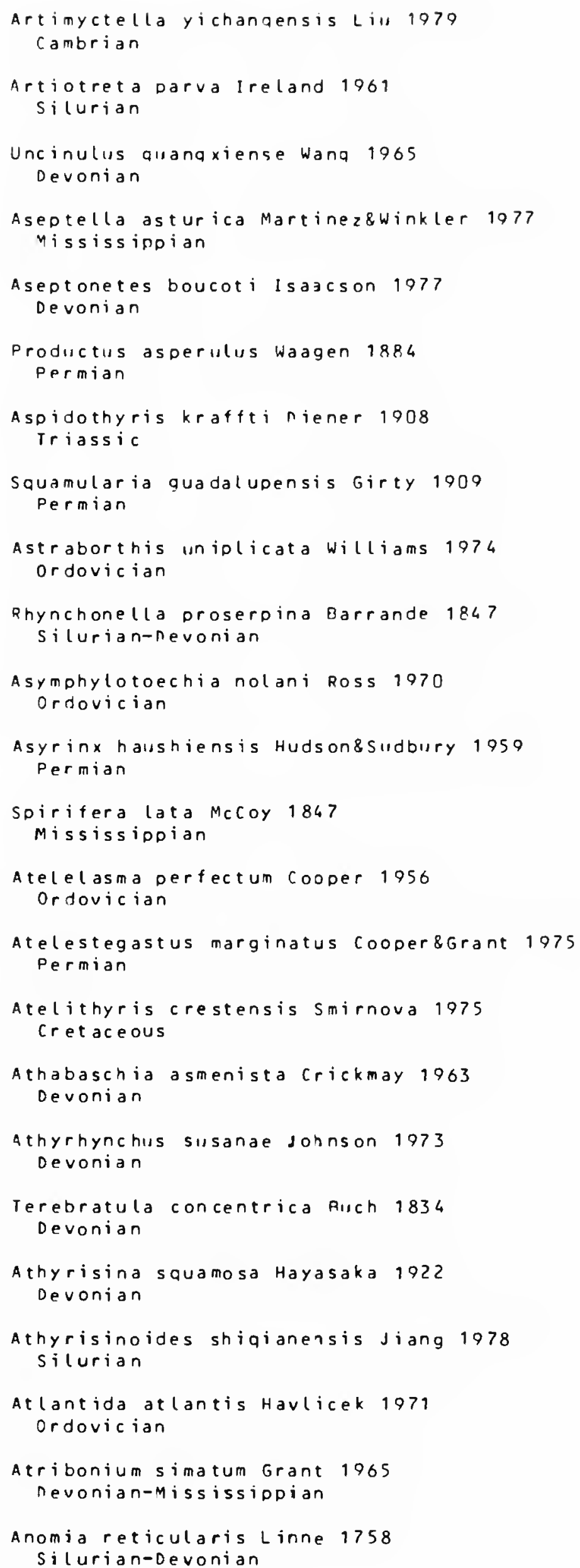




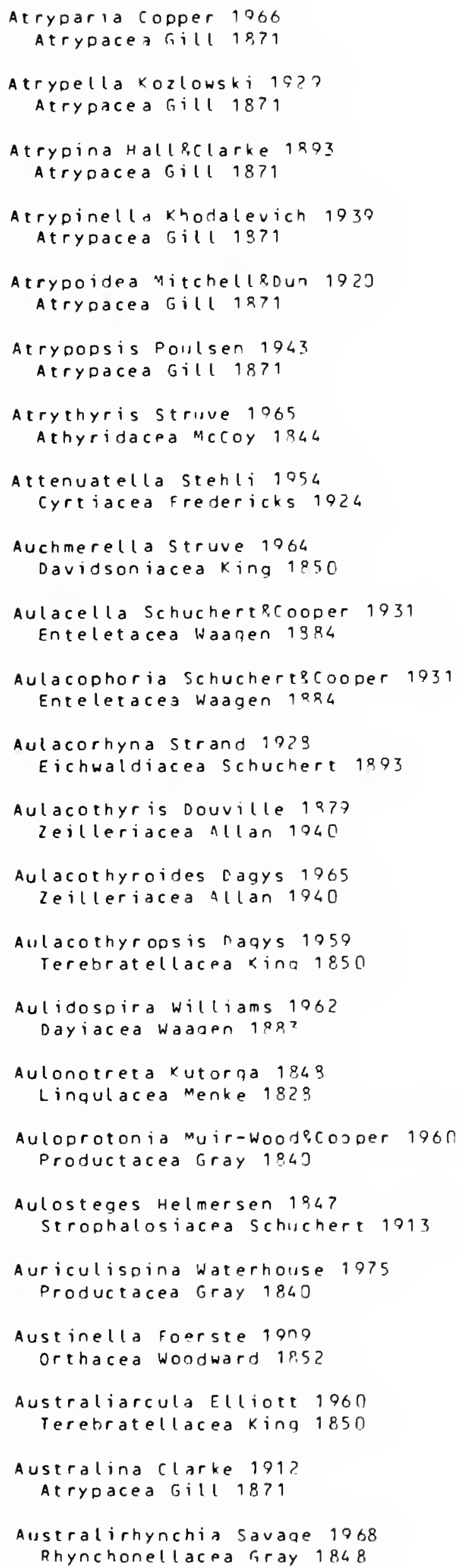

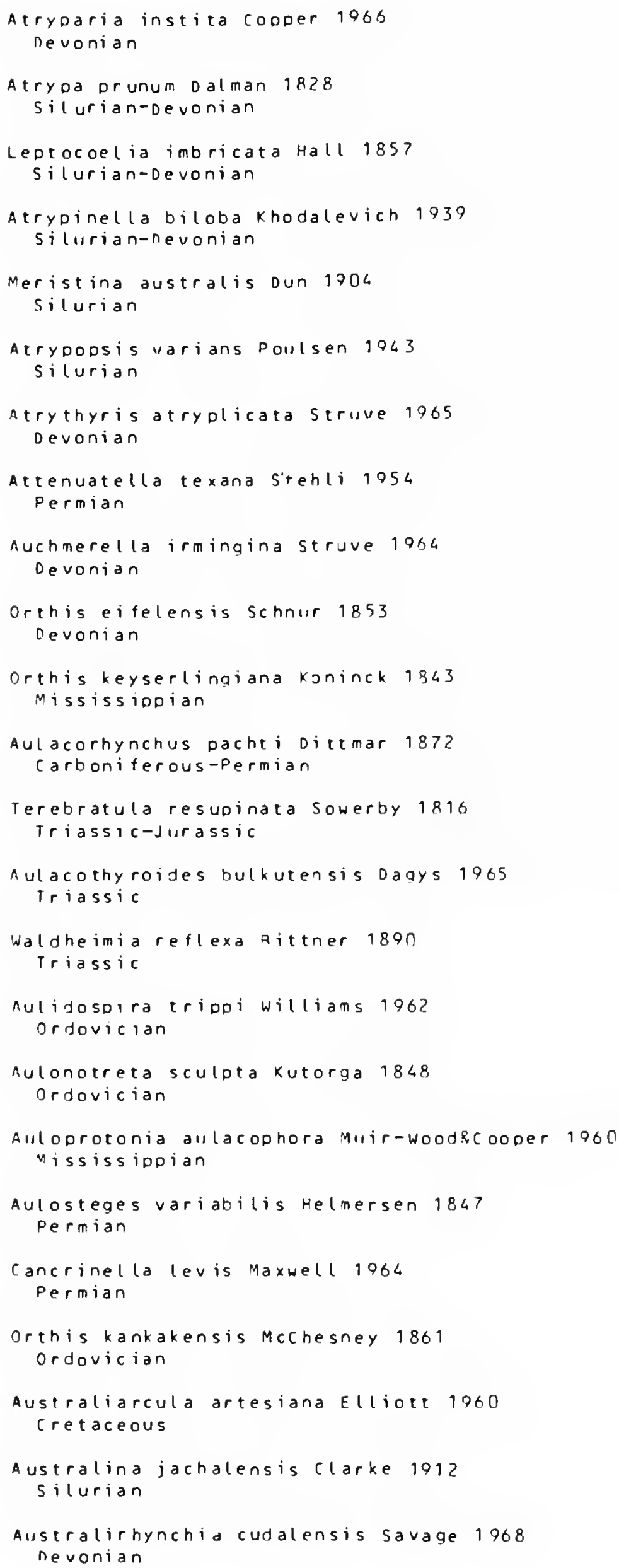




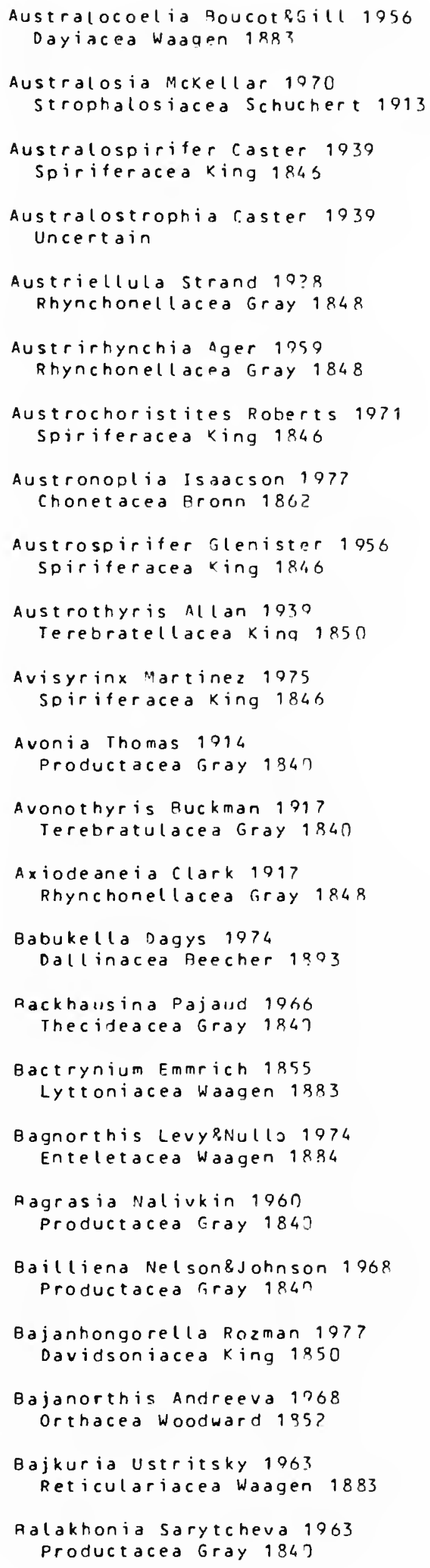

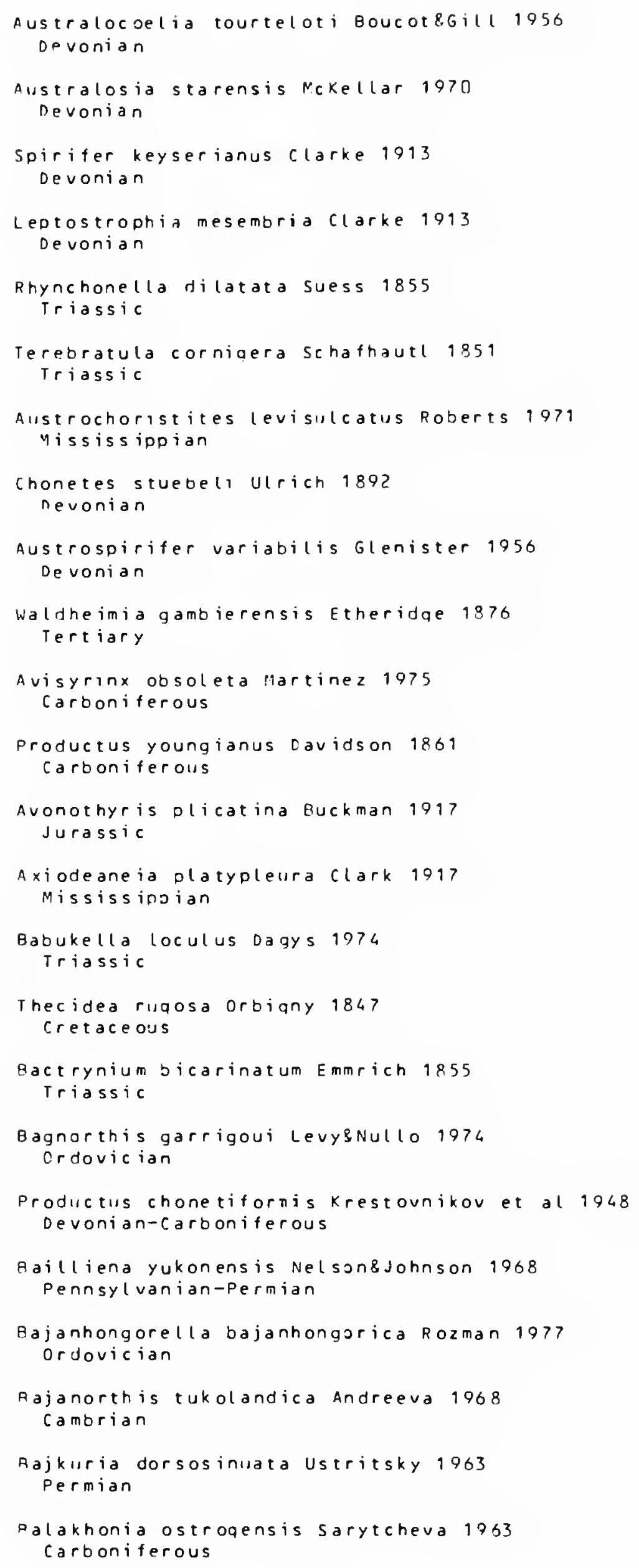




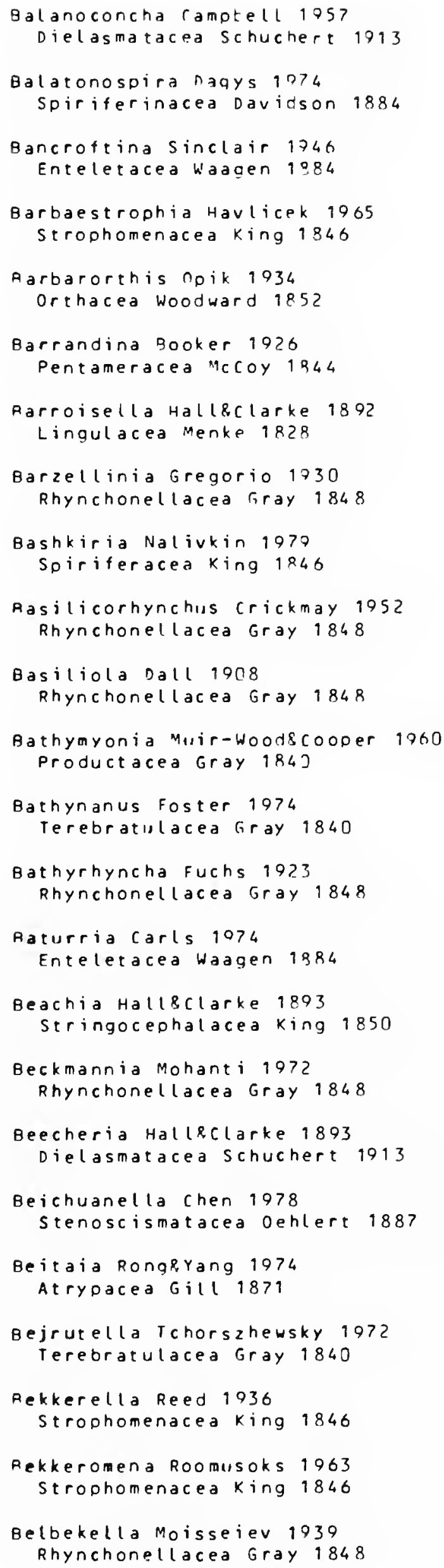

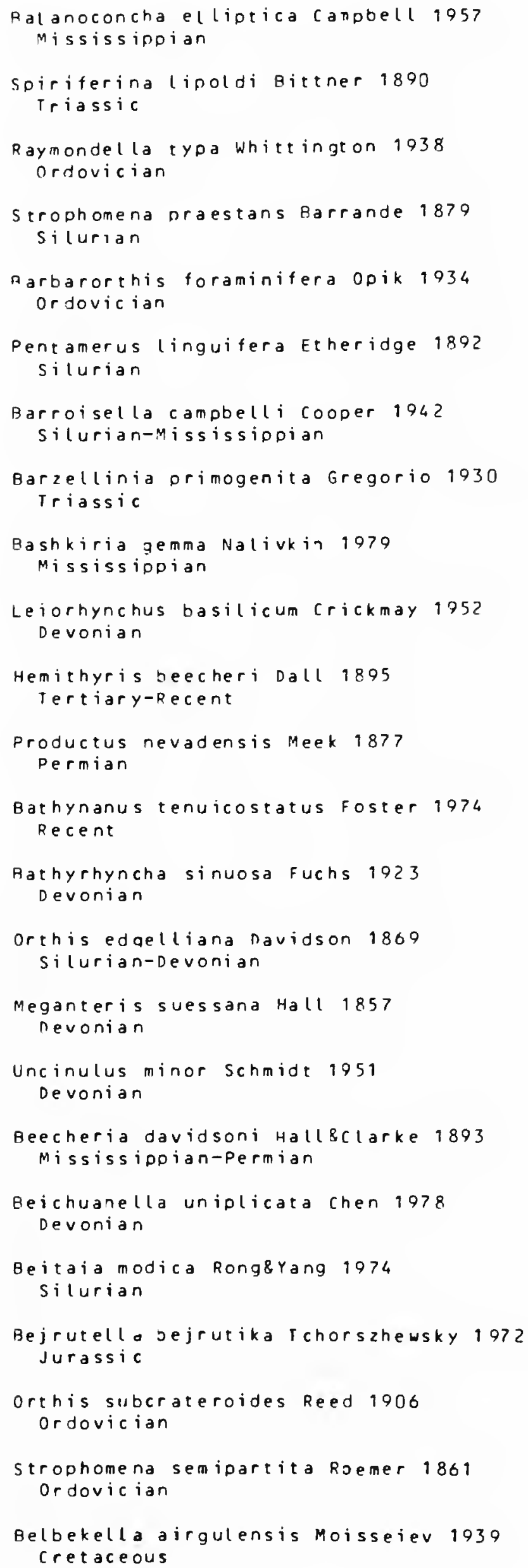


Beleutella Litvinovich 1967

Productacea firay 1943 ]

Bellaclathrus winters 1963

Productacea Gray 1840

Rellimurina cooper 1956 Strophomenacea king 1846

Belothyris Smirnova 1040 Terebratellacea kino 1850

Benignites Havlicek 1952

$P l e c t a m b o n i t a c e a$ Jones 1928

Bergalaria Schmidt 1975

Rhynchonel lacea Gray 1848

Reschevella Poletaev 1775

Reticulariacea Wagen 1883

Betachoristites 6 at inaud 1949

Spiriferacea king 1846

Betacyrtionsis ratinaud 1949

spiriferacea king 1846

Betaneospirifer Gatinaud 1949

spiriferacea kinq 1846

Biarea Torbakova 1959

Uncertain

Aibatiola frant 1976

Chonetacea Aronn 186?

Bicarinatina Aatrukova 1969

Linqulacea Menke 1828

Bicia Walcott 1001

Obolel lacea Walcott\$schuchert 1908

Riconostrophia Havlicek 1956

Davidsoniacea $x$ ing 1850

Bicusoina Havlicek 1950

Triplesiacea Schuchert 1913

Bidentatus Khodalevich\&8re ivel 1972

Cyrtiacea Fredericks 1924

Riernatelld Balinski 1977

Dayiacea Waagen 1883

Biernatium Havlicek 1975

Enteletacea Waggen 1884

Bifida Davidson 1882

Dayiacea Wagen 1883

Rifolium Elliott 1948

Thec ideacea fray 184 J

Bihendulirhynchia Muir-Wood 1935

Rhynchonellacea Gray 1848

Bihenithyris Muir-Wood 1935

Terebratulacea Gray 1840

Bilaminel la Rabanova 1964

Rhynchonellacea Gray 1848

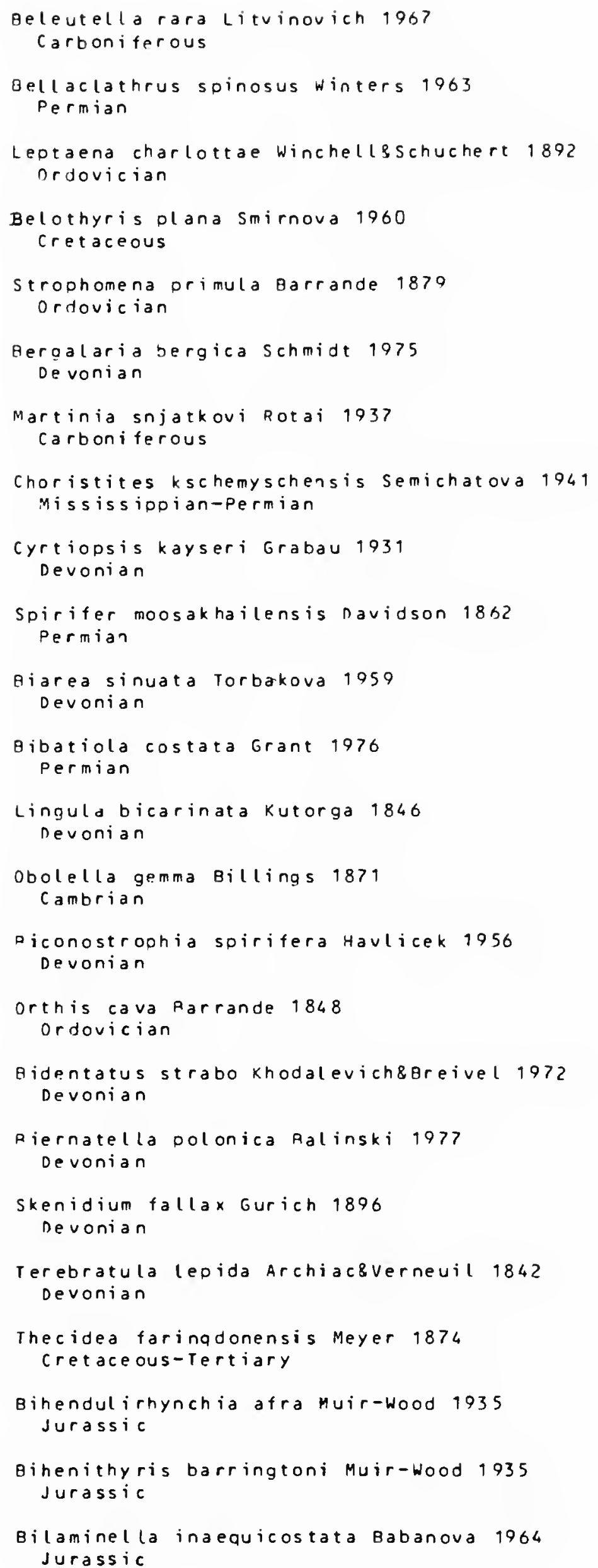


Billinasella Hall $5 C$ larke 1892 Billingsellacea schuchert 1893

Bilobia cooper 1056

Plectambonitacea Jonas 1078

Bilotina peed 1046 Strophalosiacea Schuchert 1913

Bimuria ulrich:cooper 194? Plectambonitacea jones 10 ?

Biparetis Amsden 1974 Strophomenacea king 1846

Biseptum Khoralevich89reivel 1957 Pentameracea Mecoy 19.44

Bisinocoel ia Havlicek 1053 Cyrtiacea Fredericks 1924

8 ispinoproductus Stainbrook 1047 Productacea riray 1840

Bistramia Hoek 1912 Lingulacea Nenke 1829

Aisulcata Aoucot\&Johnson 1979

Pentameracea McCoy 9944

Bisulcina Titova 1977

Terebratulacea roray 1840

Bittnerel.l a Dagys 1974 Thecospiracea Eittner 1890

Bittnerula Hall?.Clarke $189 \%$ Suessiacea waagen 18.93

Blasispirifer kulikov 1050 Spiriferacea king 1046

Blochmanella Leidhold 1721 Rhynchonellacea Gray 184 :

Blyskavomena Havlicek 1076 Strophomenacea vina 19,46

Bobinella Androeva 1968 Porambonitacoa navidson 1853

Bodrakella Moisseiev 1736 Rhynchonel lacea Gray 1849

Bohemiella schuchert orthacea wodward 105 ?

Bohemirhynchia lekvasilova 1773 Rhynchonel lacea Gray 1848

Boicinetes HavlicekPPachejoeuf 1079 Chonetacea Aronn $18 k$ ?

Bojarinovia obsarina 1278 . Uncertain

Bojodouvillina Havlicel 1067 Strophomenacea ring $19,4 \theta$

Bojothyris Havlicek 125? spiriferacea king 1046

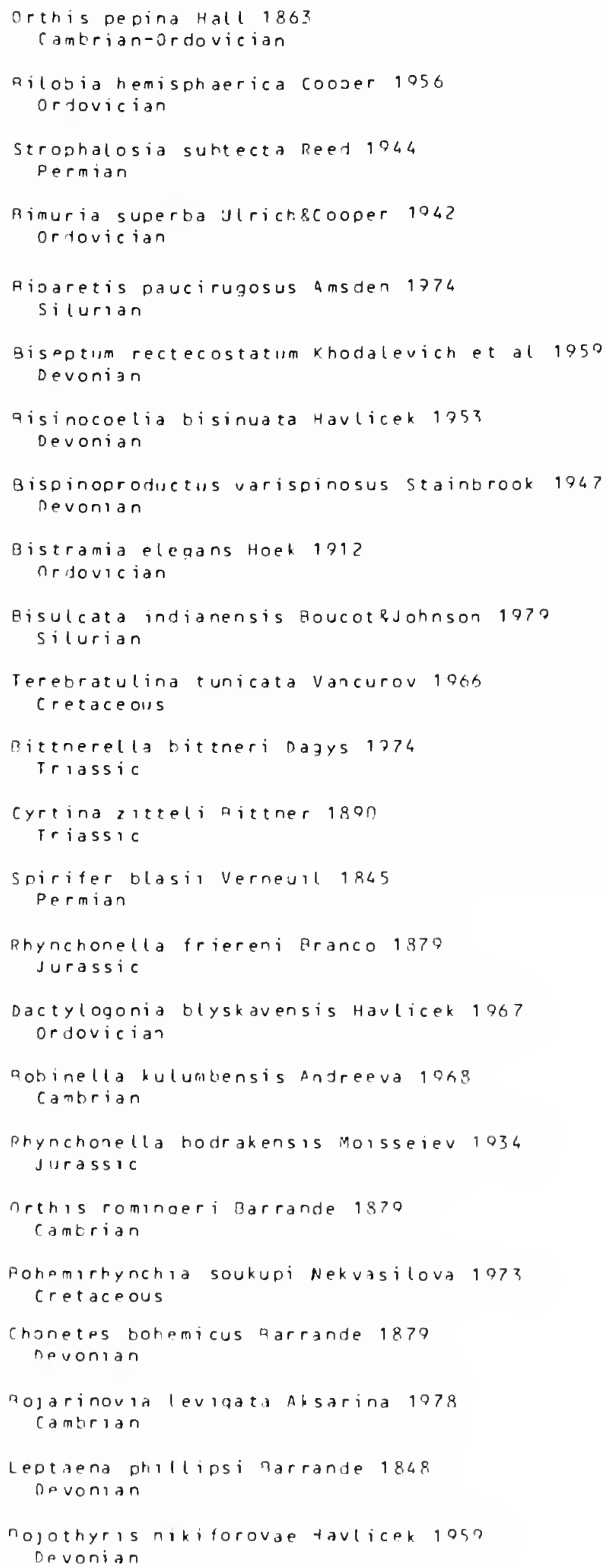




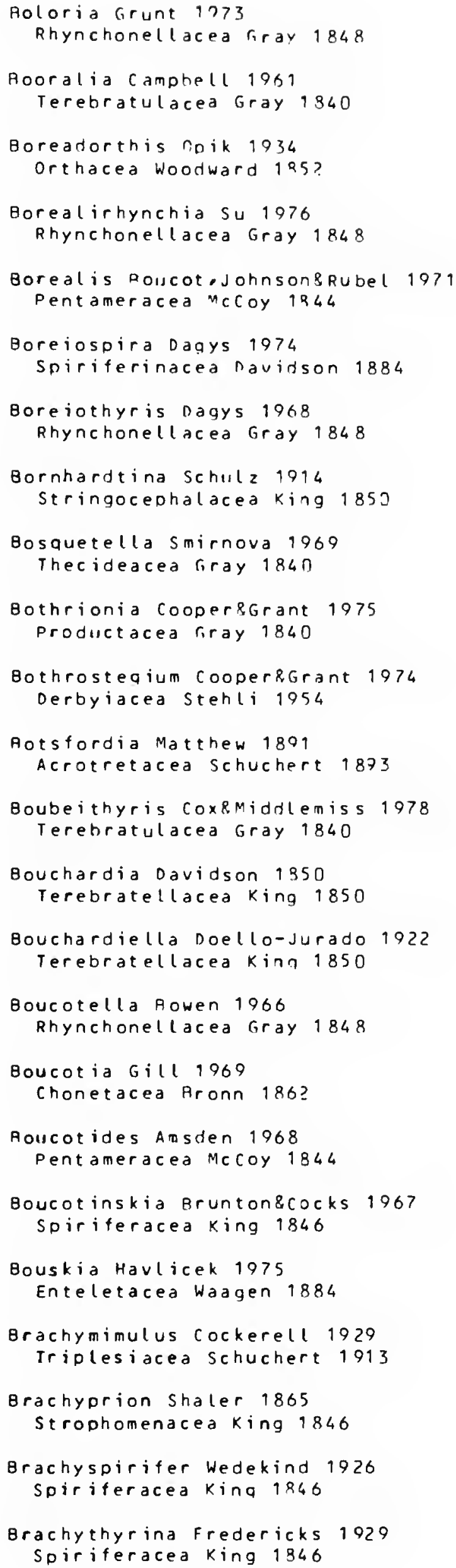

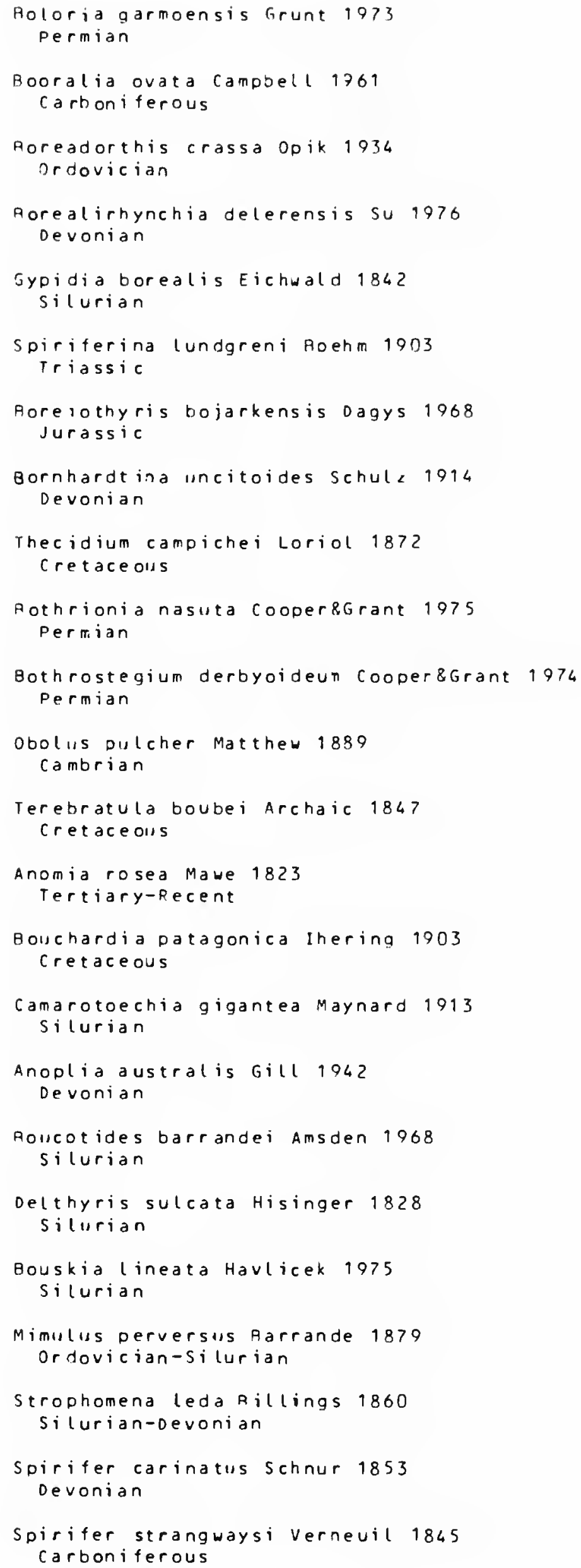




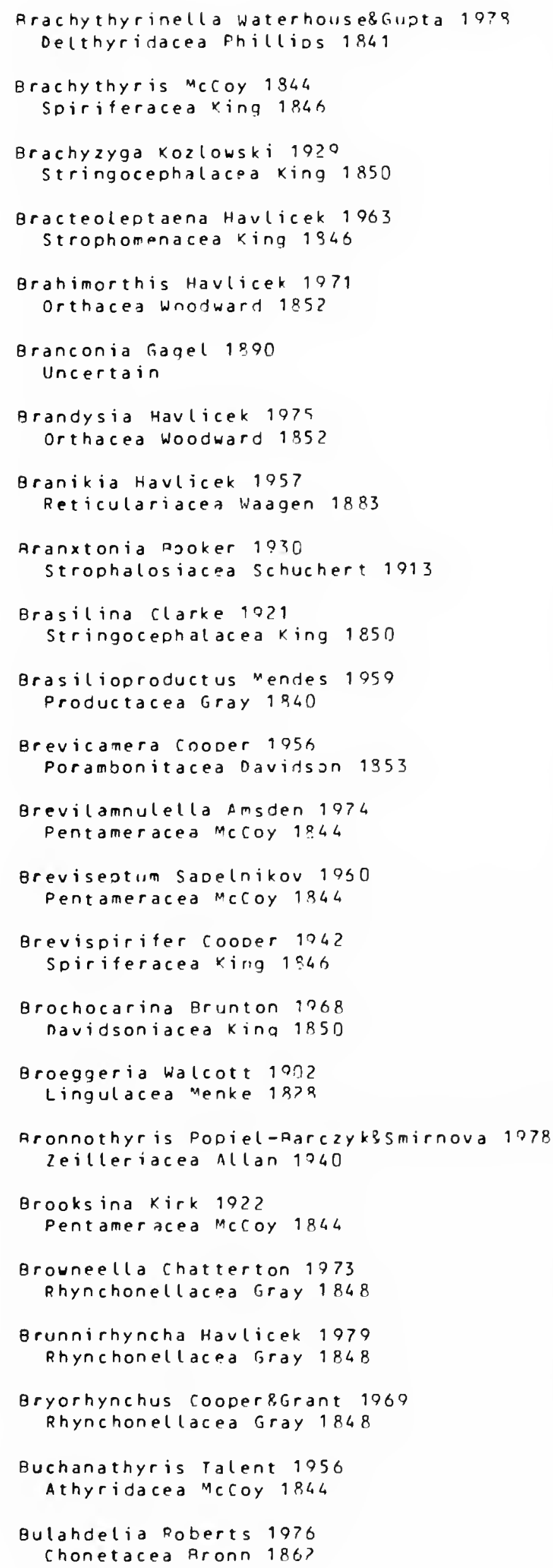

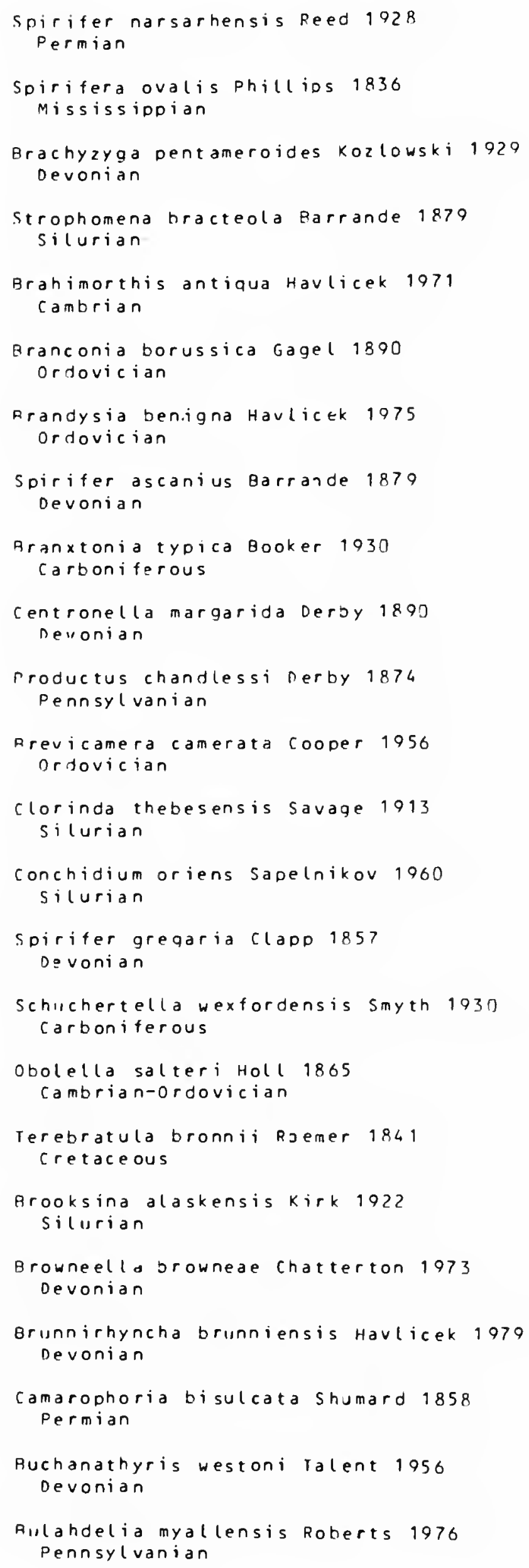


Bullothyris Sucic-protic 1971 Loboidothyridacea Makridin 1964

Rurmirhynchia Ruckman 1017

Rhynchonellacea Gray 184 R

Burrirhynchia owen 1962

Rhynchonel Lacea Gray 1848

Buxtonia Thomas 1914

Productacea riray 1840

Buxtonioides Mendes 1059

Prodictacea fray 1840

Bystromena williams 1974

Strophomenacea King 1846

Cacemia Mitchell 1774

Orthacea Wondward 185?

Cactosteges Cooper 8 cirant 1975

Aulostegacea Muir-Wood\&cooper 1060

Cadomella Munier-chalmas 1887

Cadomellacea schuchert 1893

Caenanoplia Carter 1969

chonetacea pronn 1852

Caenotreta Cocks 1079

Acrotretacea schuchert 1893

Caeroplecia williams 1974

Triolesiacea Schuchert 1913

Calcirhynchia Buckman 1917

Rhynchonellacea Gray 1848

Callaiapsida Grant 1971 stenoscismatacea oehlert 1887

Callicalyptella Boucot?Johnson 1972 Chonetacea aronn 196 ?

Calliglypha cloud 1048 Porambonitacea Davidson 18.53

Calliomarginatia Ching 1976

Productacea firay 184 ?

Callipentamerus Boucot 1964 pentameracea McCoy 1944

Callipleura cooper 194? Rhynchonel lacea Gray 1848

Calliprotonia Muir-WoodeCooper 1960 Productacea Gray 1840

Callispirina Cooper\&Muir-Wood 1951 Spiriferinacea Davidson 1884

Callosoiriferina Rousselle 1977 Spiriferinacea Davidson 1884

Calvinaria Stainbrook 1945 Rhynchonellacea Gray 1848

Calyptolepta Neuman 1977 Plectambonitacea Jones 1928

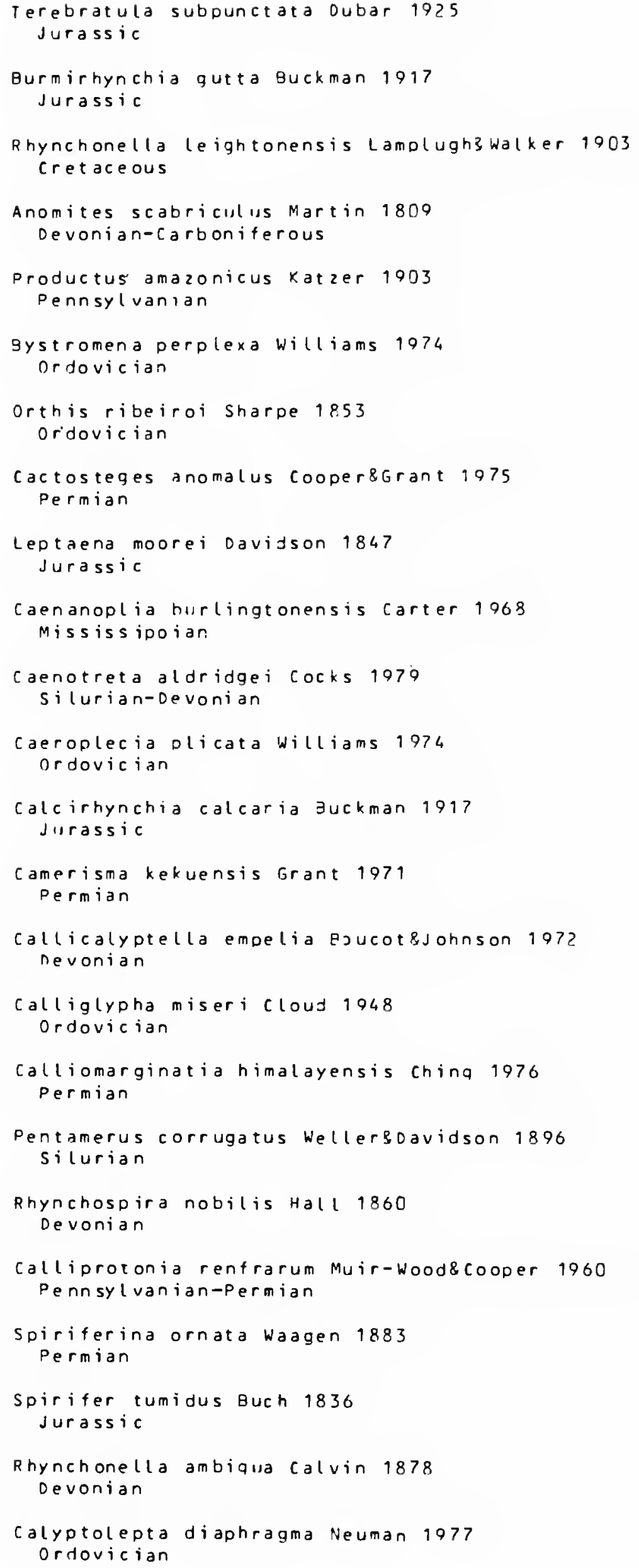




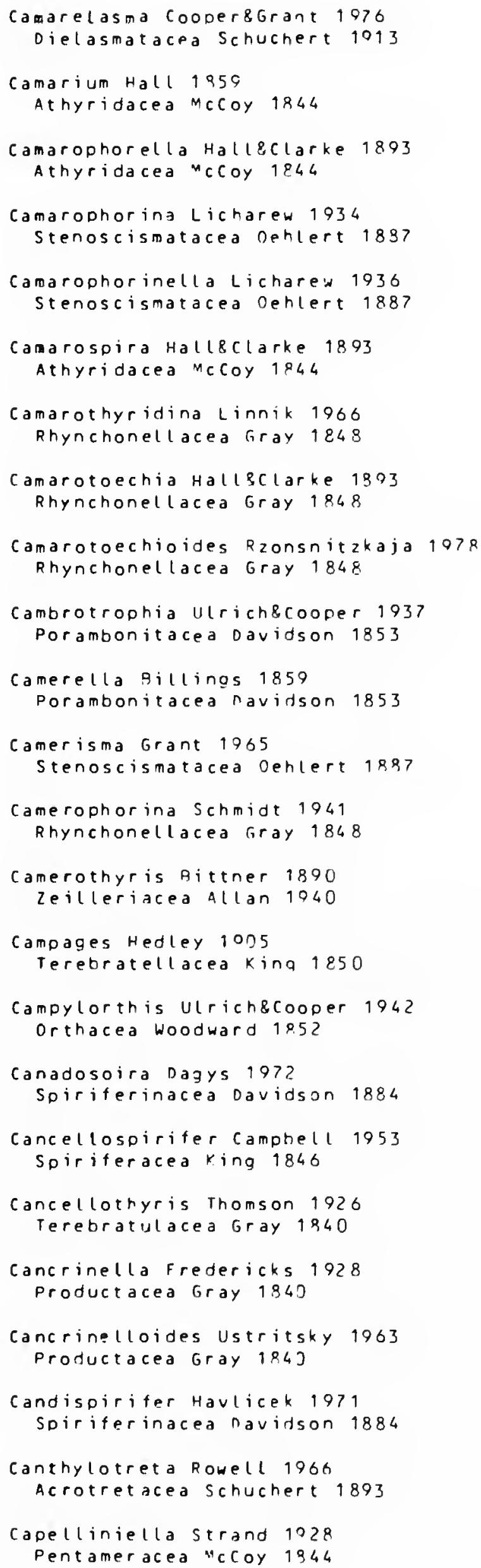


Capillirhynchia Buckman 1917

Rhynchonellacea Gray 1848

Capill irostra Coopersmuir-wood 1951

Rhynchonellacea Gray 1848

Capillithyris Katz 1974 Terebratulacea Gray 1840

Capillonia waterhouse 1973 Chonetacea Aronn 18t?

Caplinoplia Havlicek R Racheboeut 1979 Chonetacea Pronn 9862

Carapezzia Toml in 193 ? Rhynchonellacea Gray 1848

Carbocyrtina Ivanova 1775 Cyrtinacea Fredericks 1912

Cardiarina Cooper $195 \mathrm{~A}$ Rhynchonel lacea Gray 1848

Cardinirhynchia Ruckman 1917 Rhynchonellacea Gray 1848

Cardinocrania Wagen 1935 Lyttoniacea Waagen 1983

Cardiothyris Roherts 1971 Athyridacea MCCOY 1944

Caricula Grant 1976 Productacea Gray 1840

Carinaqypa Johnson\&luduiqsen 1972 Pentameracea McCOY 1844

Carinastrophia fir atsianova 1975 Davidsoniacea King 1850

Carinatina Nalivkin 1030 Atrypacea rill 1871

Carinatinella Grats ianova 1967 Atrypacea Gill 1871

Carinatrypa Copper 1973 Atrypacea Gill 1871

Cariniferella schuchert\&cooper 1931 Enteletacea Hagen 1994

Carinokoninckina Jinsfana 1977 Koninckinacea Davidson 1853

Carlinia fiordon 1971 Productacea Gray 1843

Carlopsina Reed 1954 navidsoniacea king 1950

Carneithyris Sahni 1925 Terebratulacea Ciray 1840

Carpatothyris smirnova 1975 Terebratellacea Kirig 1850

Cartorhium cooper 8 Grant 1976 Spiriferacea King 1946

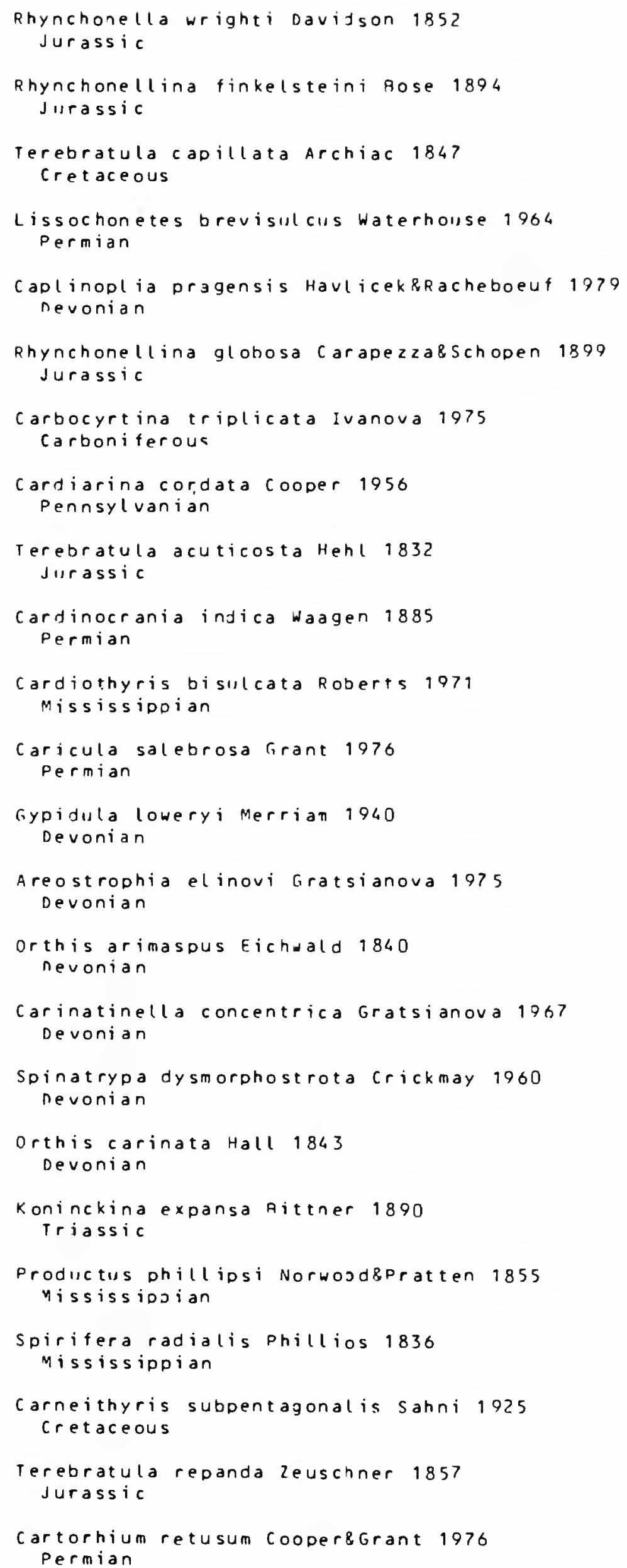




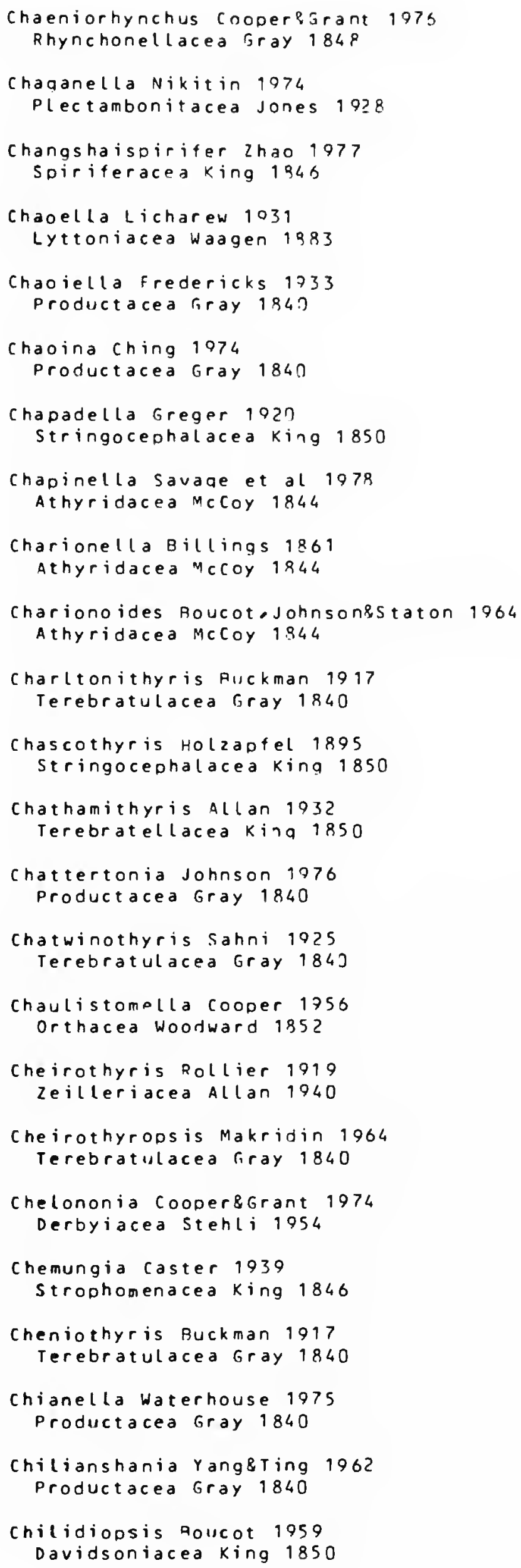

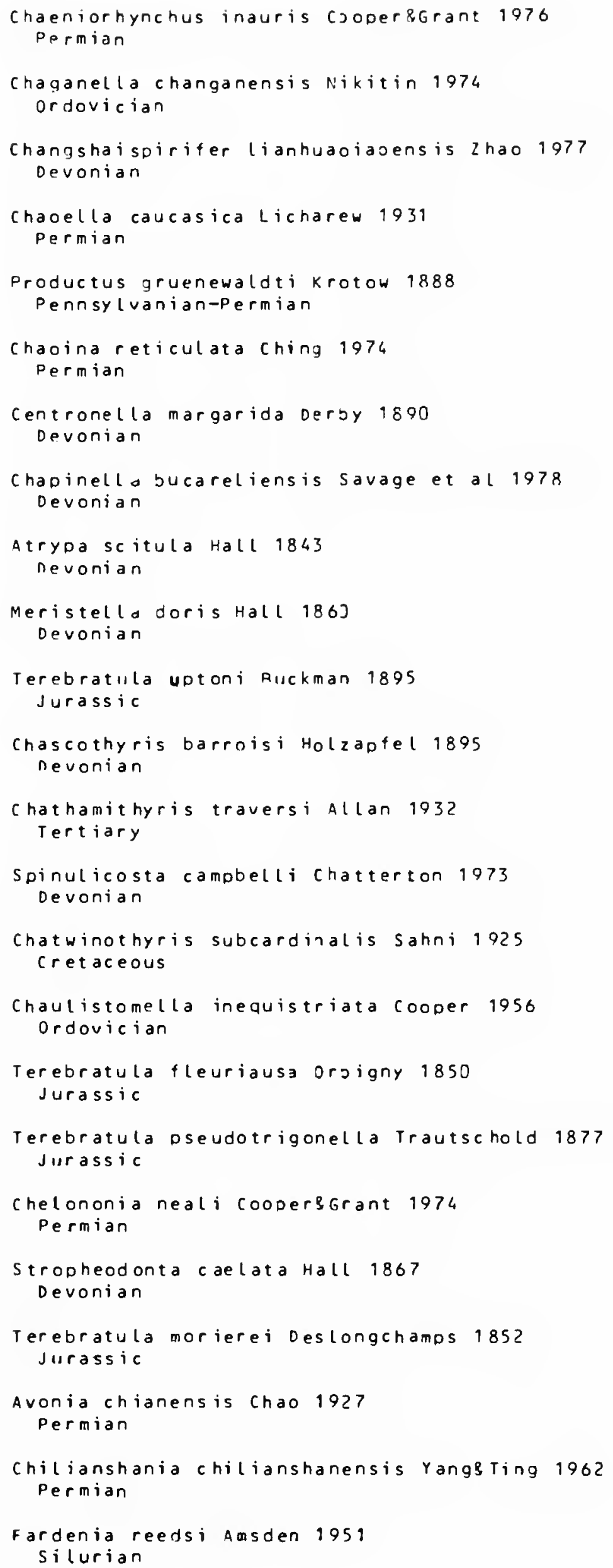




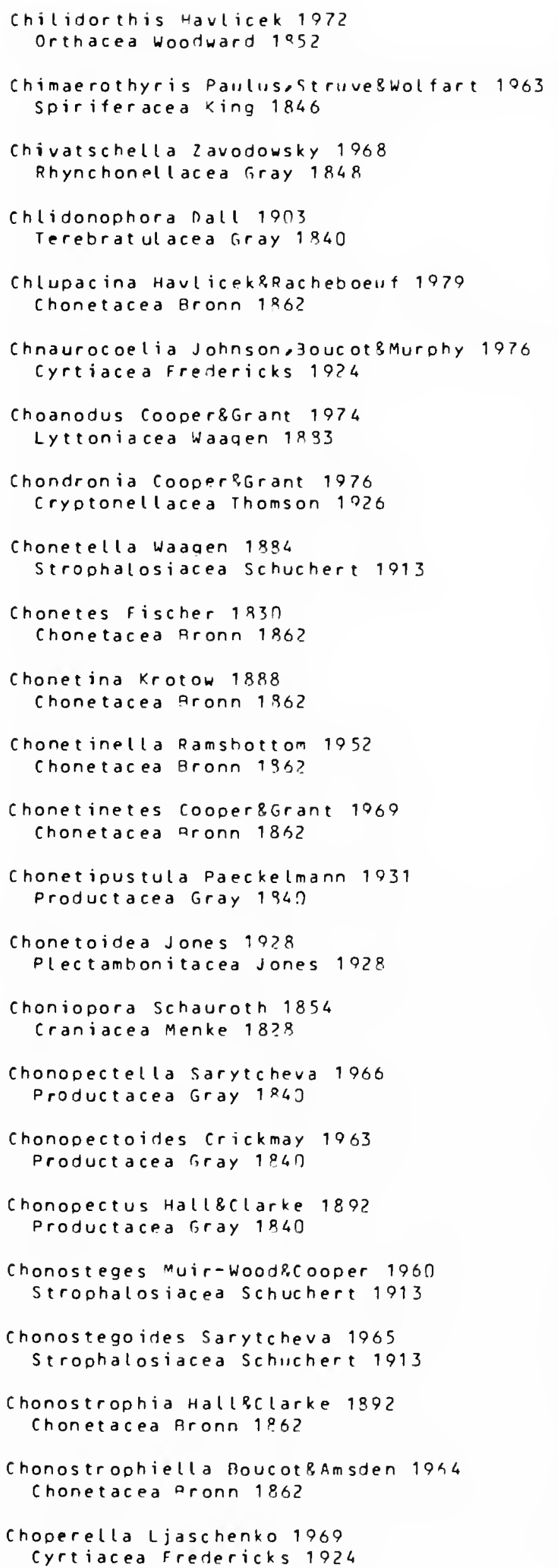

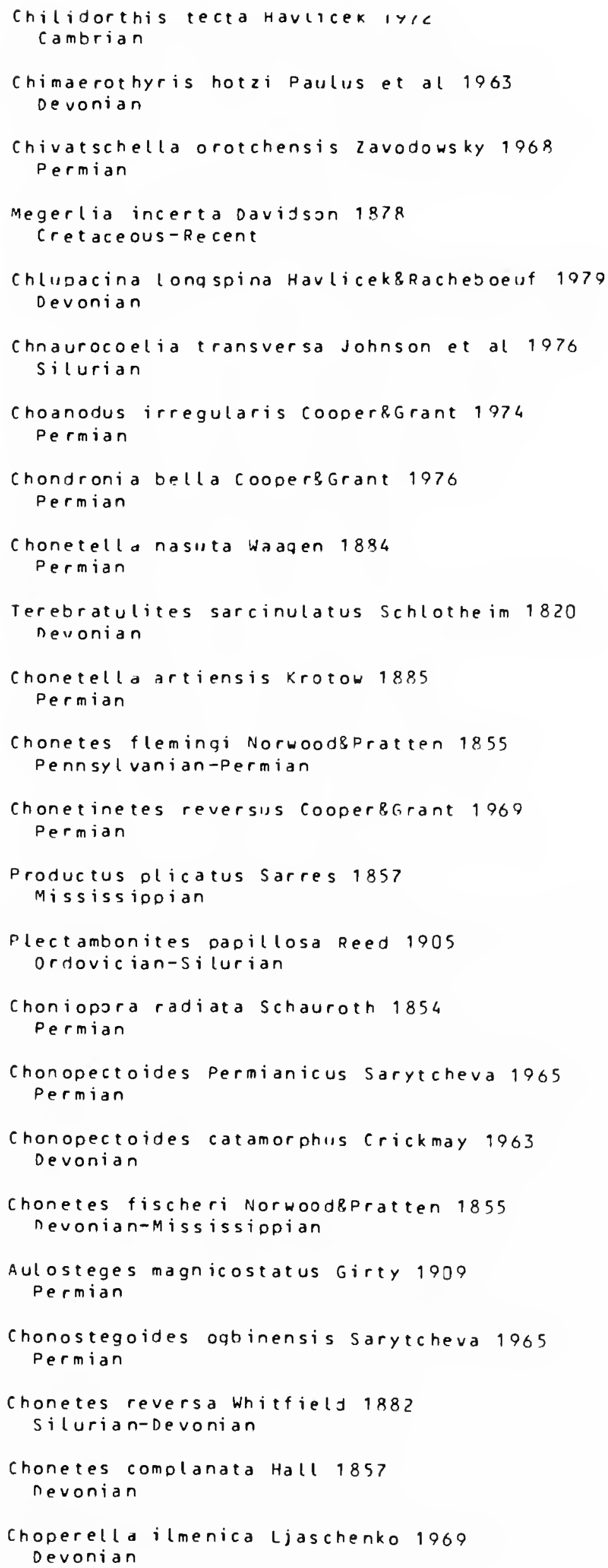


Choristitella I vanov\&Ivanova 1937 Spiriferacea King 1846

Choristites Fischer 1825 Spiriferacea King 1846

Choristothyris cooper 1942 Terebratellacea Kina 1850

Christiania Hall $8 \mathrm{Clarke} 1892$ Strophomenacea king 1846

Chrustenopora Havlicek 1958 Enteletacea Waagen 1984

Chynistrophia Havlicek 1977 Stropheodontacea Caster 1939

Cilinella Havlicek 1970 Enteletacea Waacen 1894

Cimicinella schmidt 1943 strinaocephal acea Kina 1850

Cimicinoides Anjerson, pouc ot RJohnson 1969 Stringocephal acea King 1850

Cincta Quenstedt 1868 zeilleriacea Allan 1240

Cinctifera Muir-Wood\&cooper 1960 Productacea firay 1840

Cinerorthis Havlicek 1774 Orthacea Woorward 1952

Cinqulodermis Havlicek 1771 Reticulariacea Wagen 1883

Cirpa Gregorio 1930 Rhynchonellacea foray 1848

Cistellarcula Elliott 1754 Terebratellacea king 1850

Clarkeia Kozlowski 1923 Rhynchonel lacea Gray 1848

Clarkella walcott 1903 Porambonitacea Davidson 1853

Clathrithyris Snirnova 1974 Dallinacea Recher 1993

Clavigera Hector 1979 Reticulariacea Waagen 1883

Clavodalej ina Havlicek 1977 Enteletacea wagen 1884

Cleiothyridellina Waterhouse 1978 Athyridacea Mccoy 1944

Cleiothyridina Buckman 1906 Athyridacea McCoy 1844

Cliftonia foerste 1900 Triplesiacea Schuchert 1913

Clinambon Schuchert\&cooper 1932 Clitambonitacea winchell \&schuchert 1893

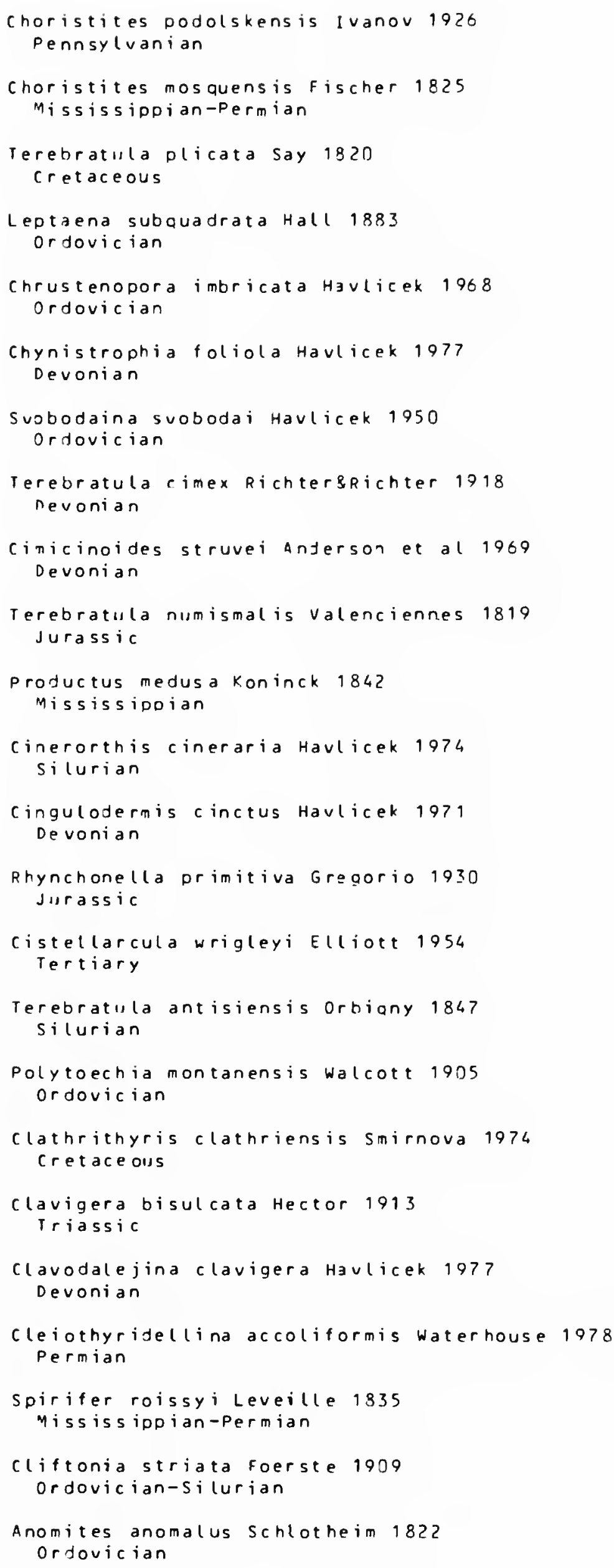




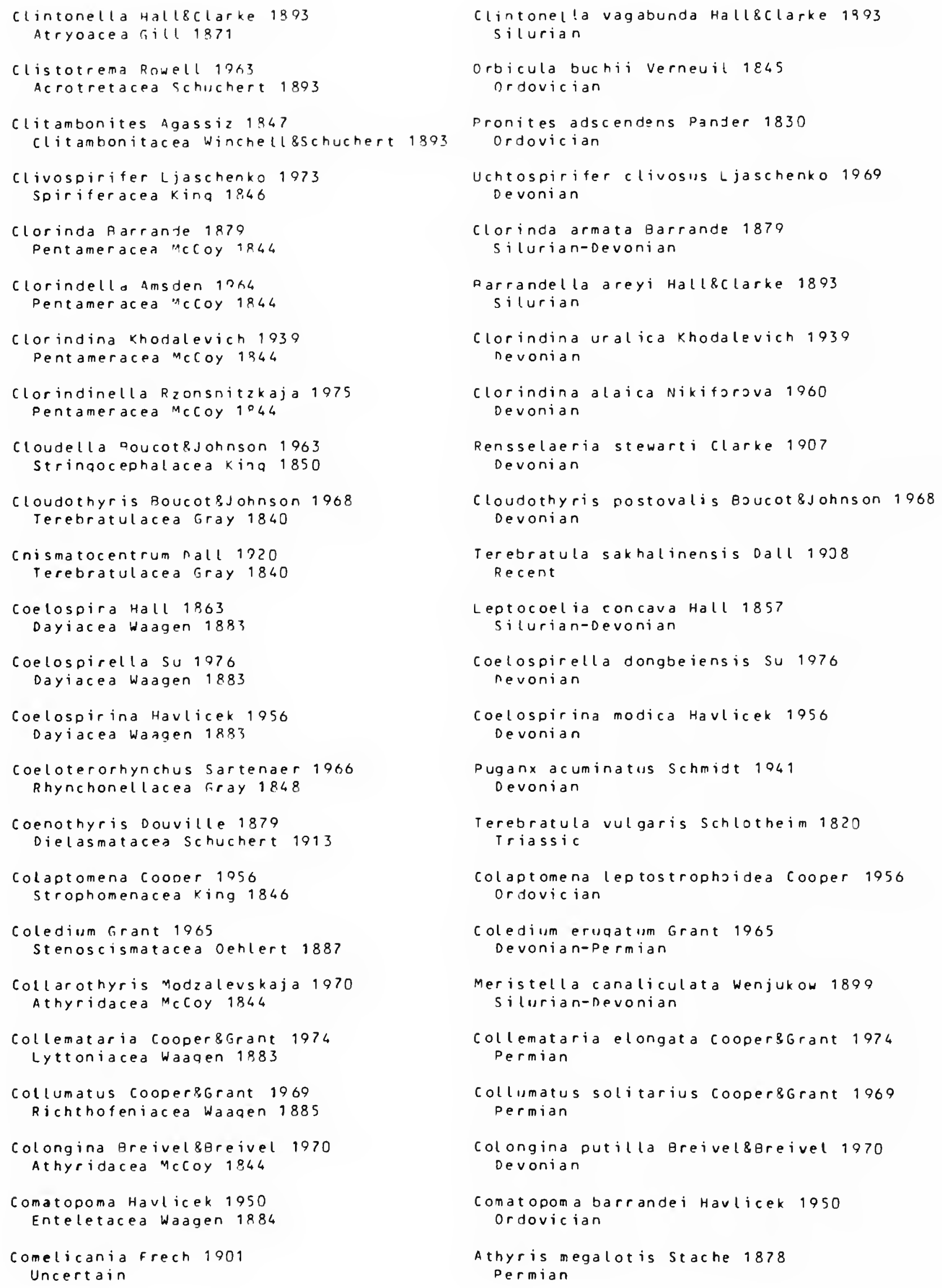




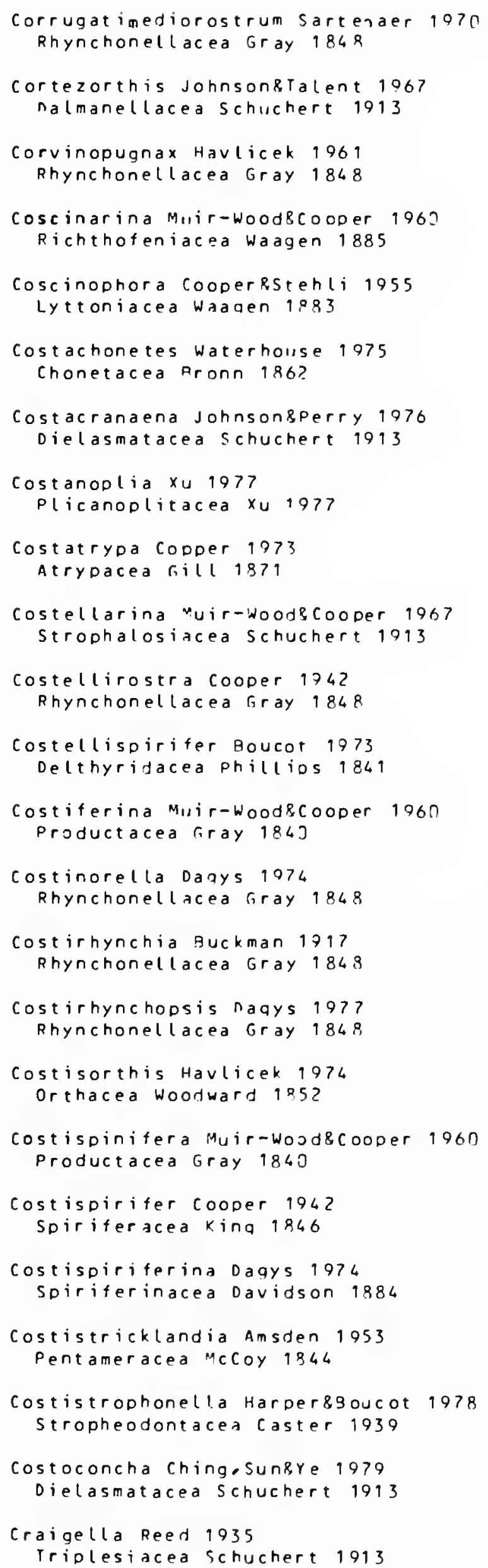

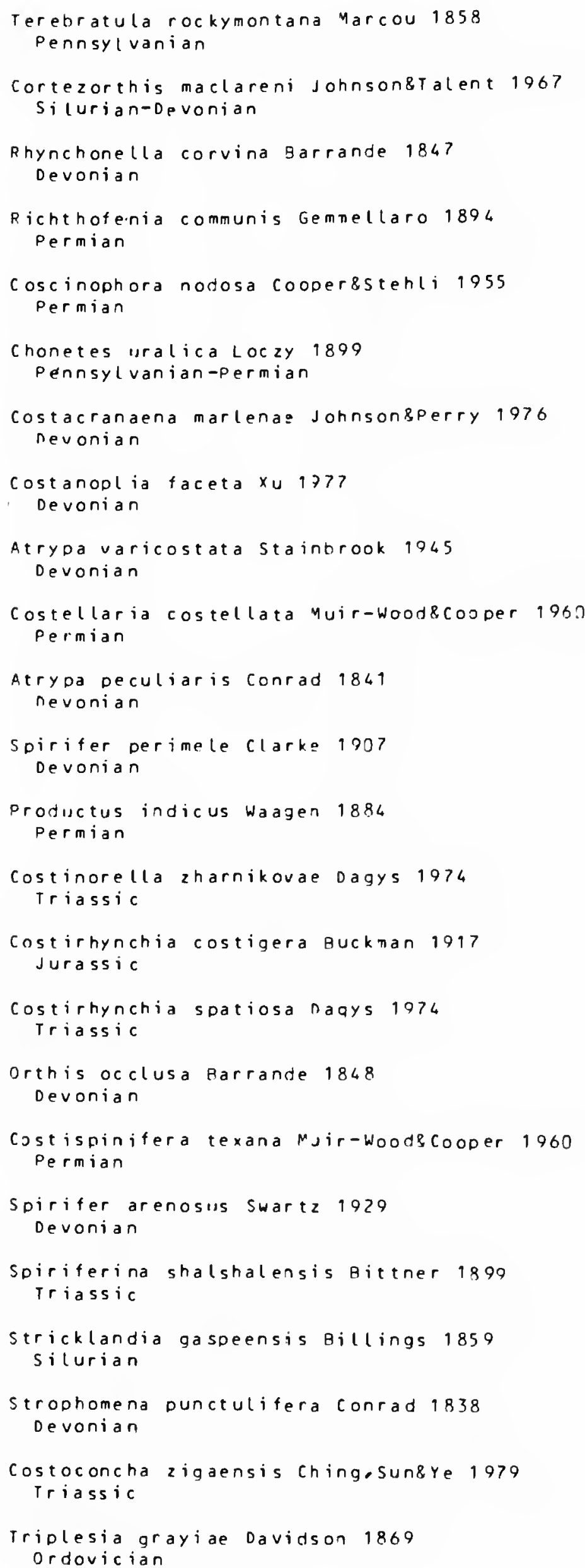




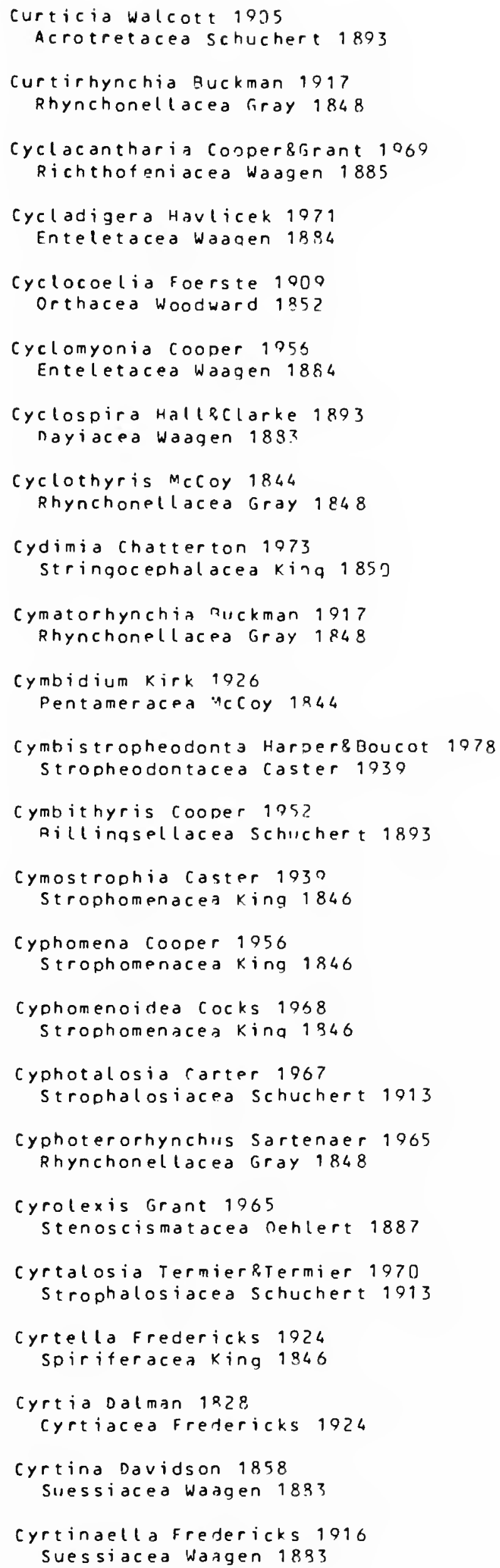

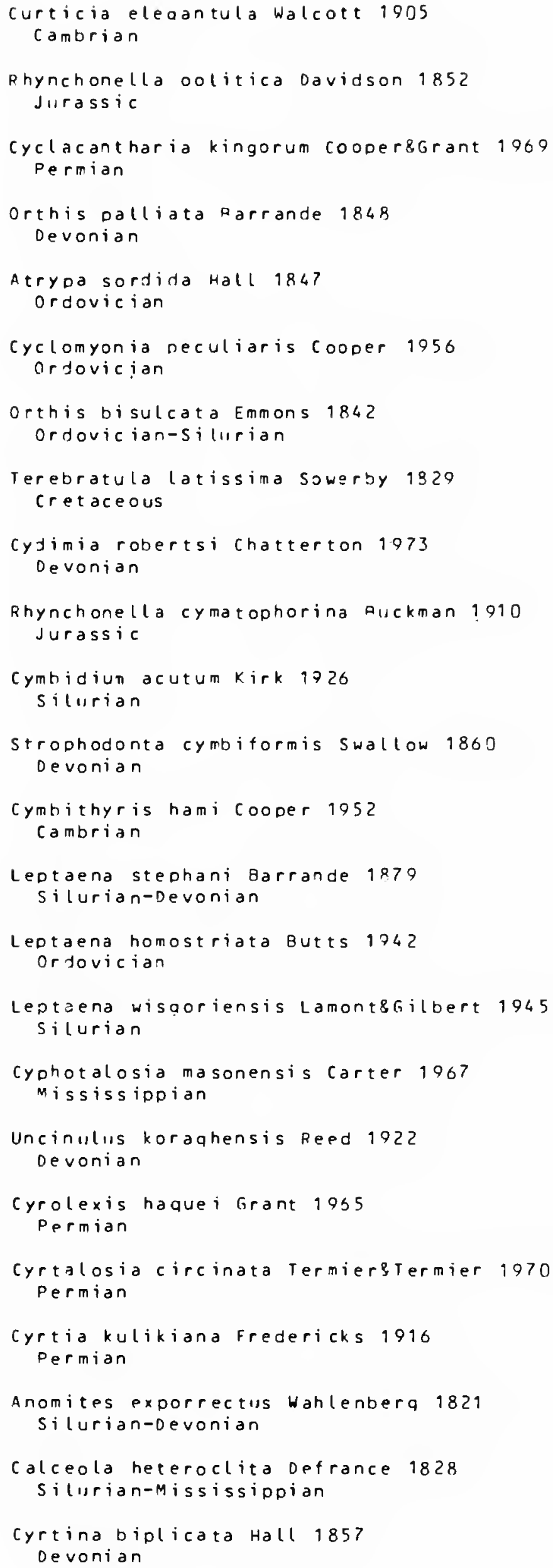




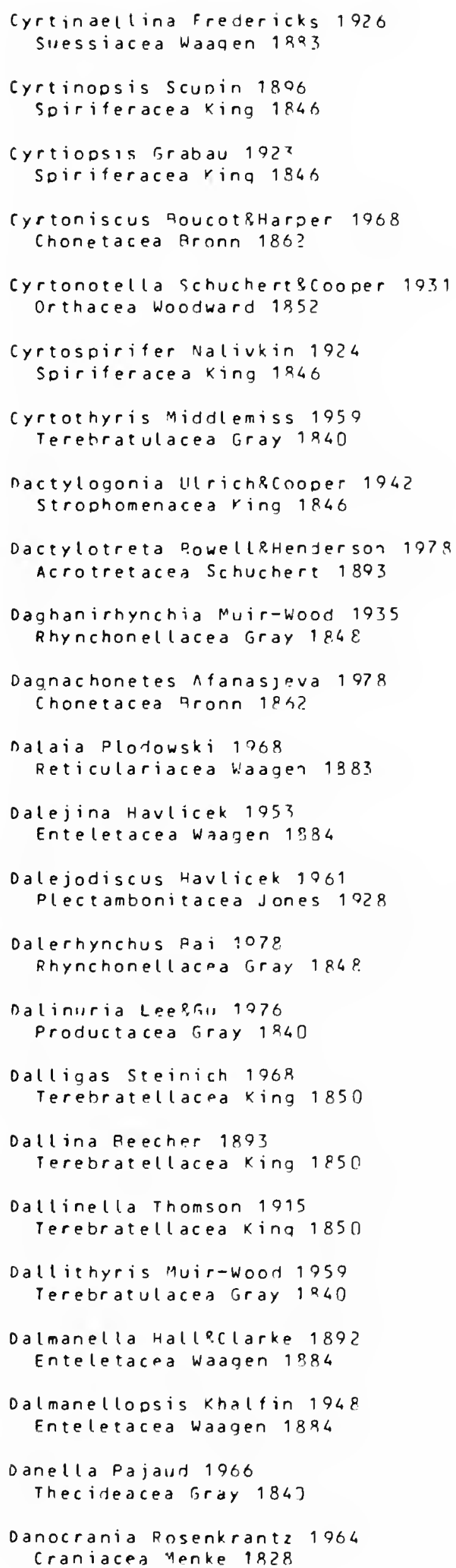

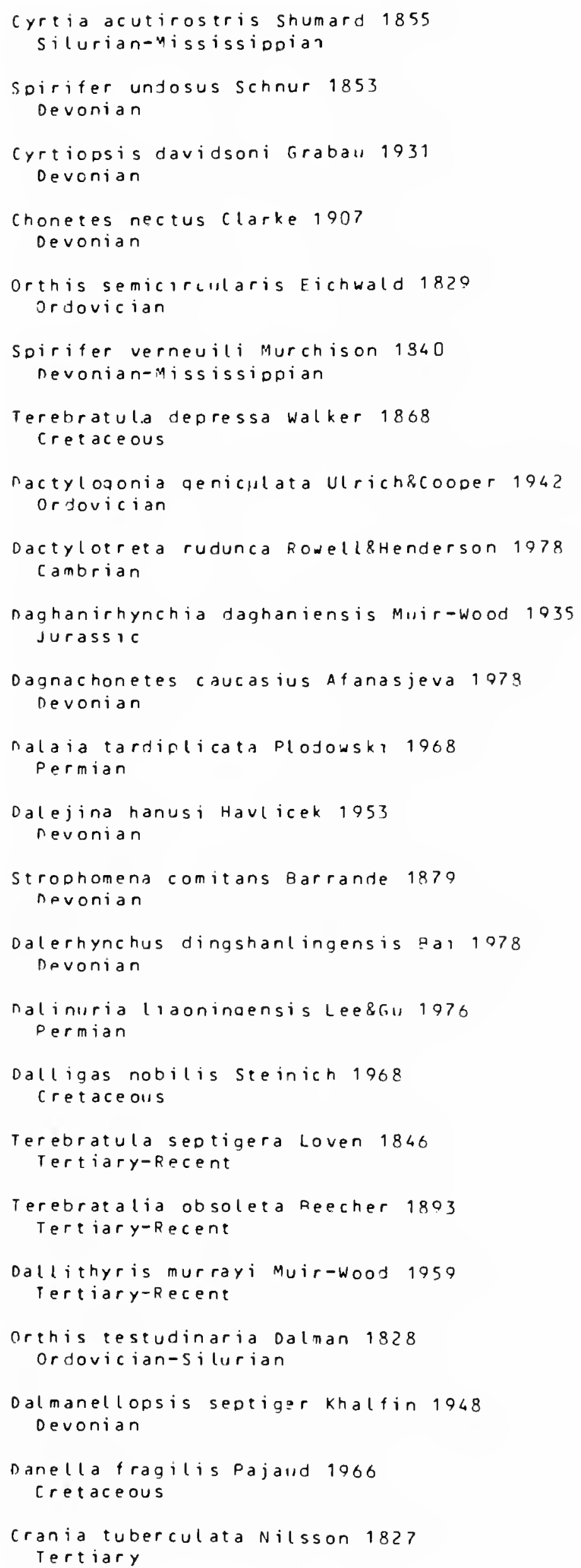




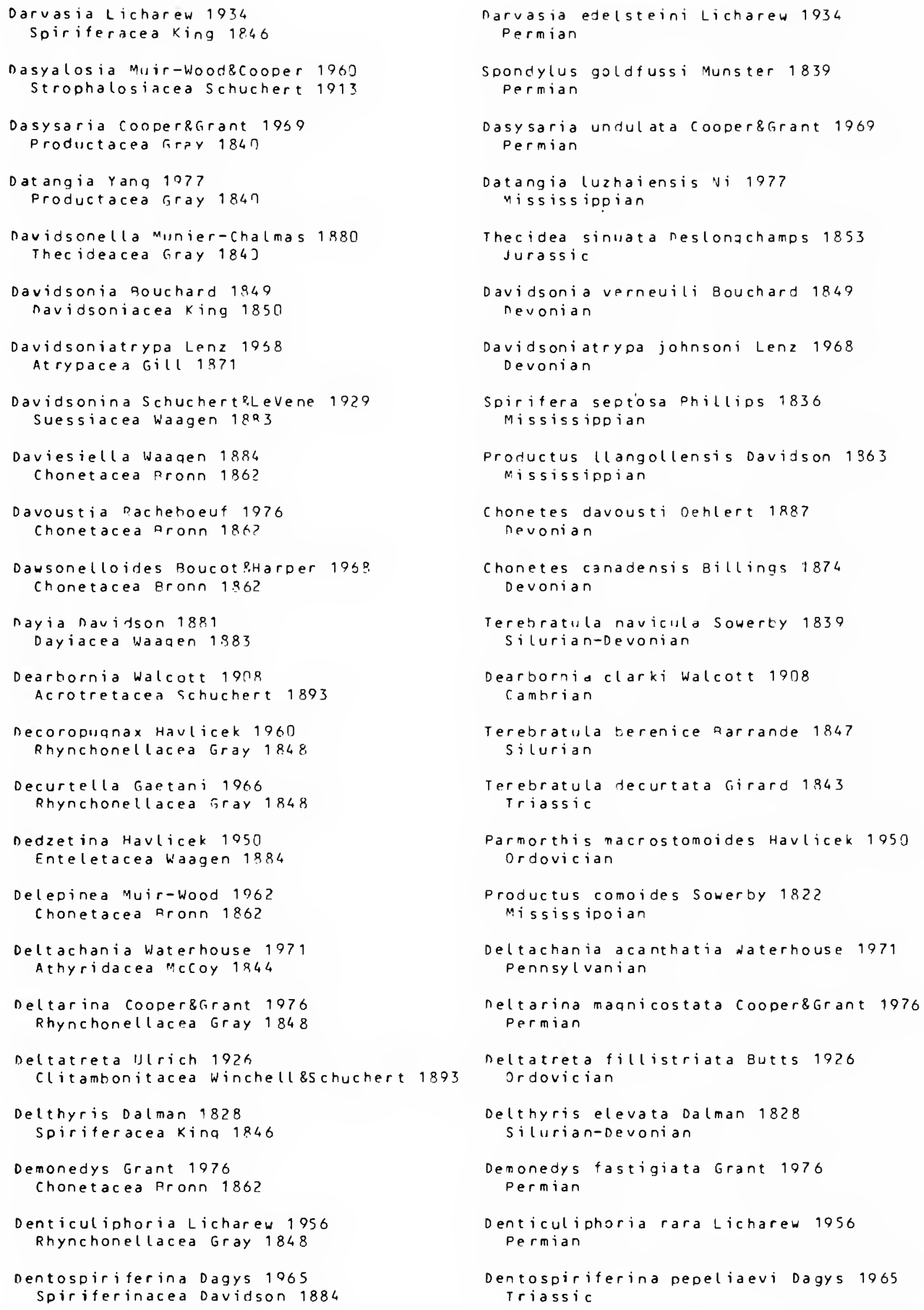




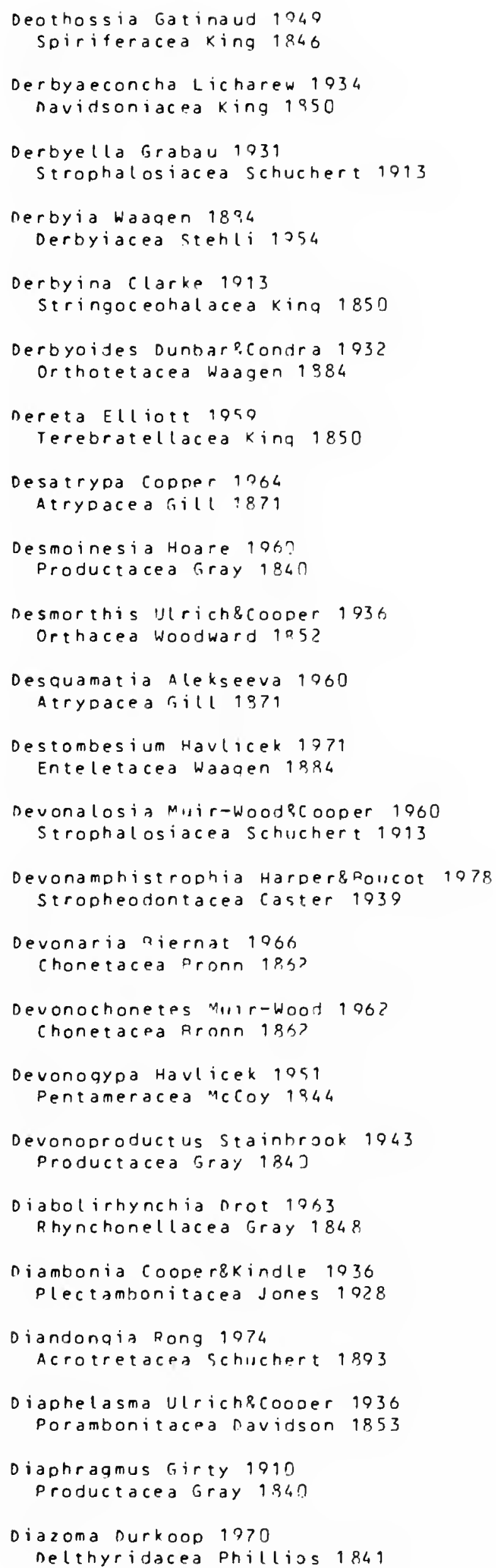

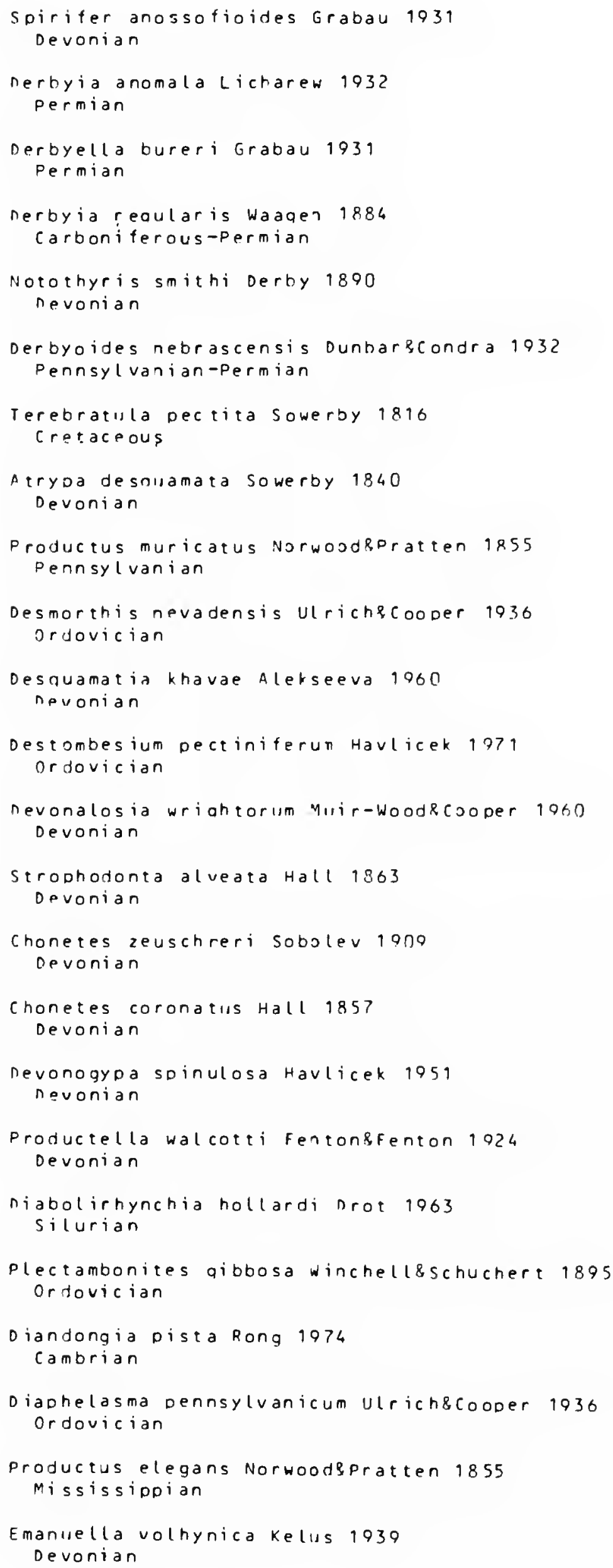




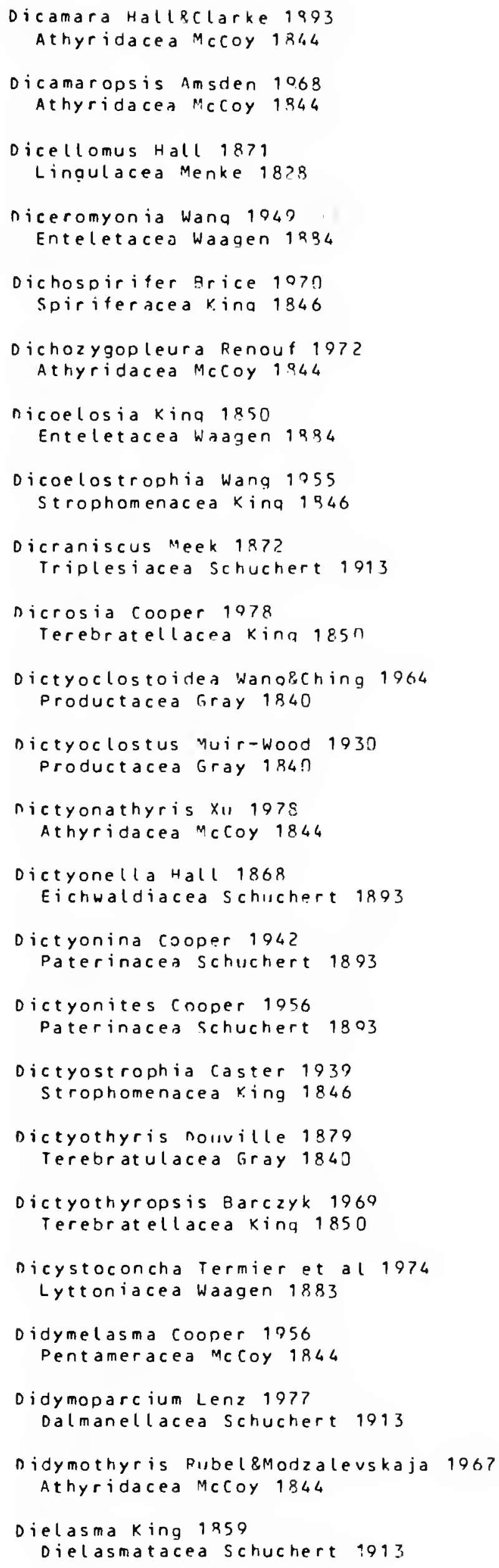

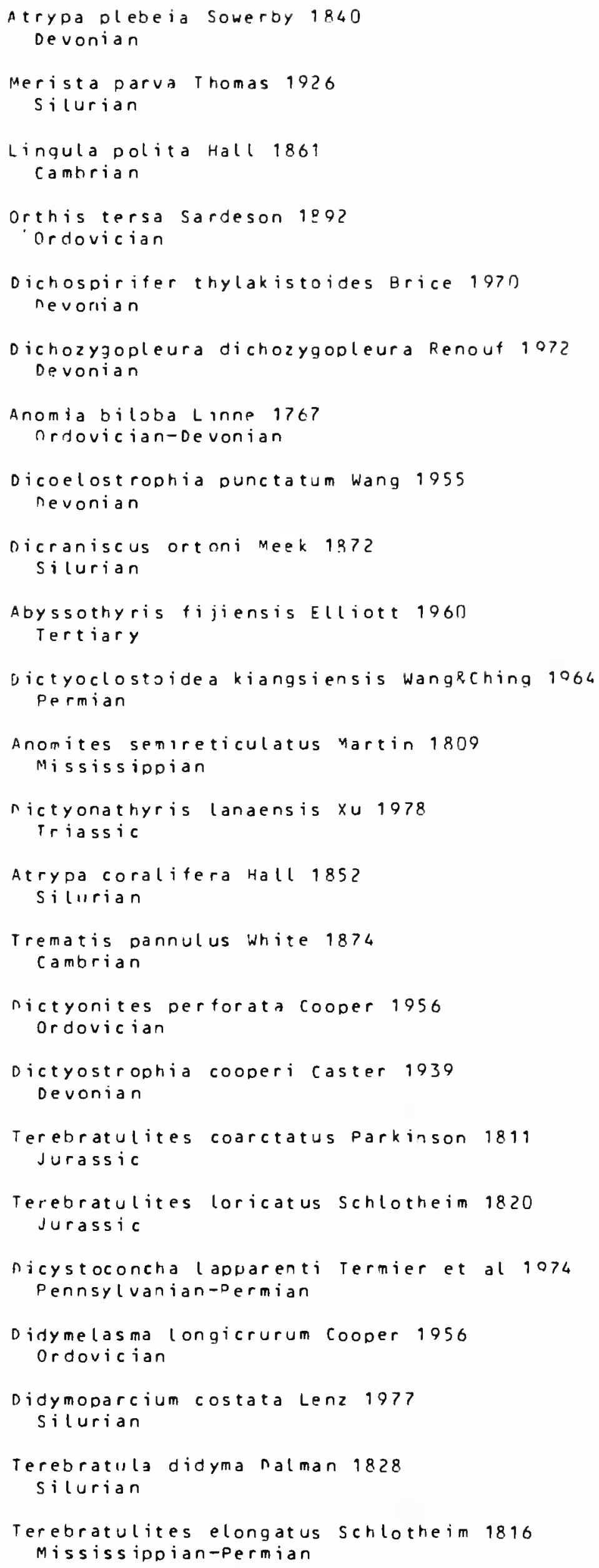




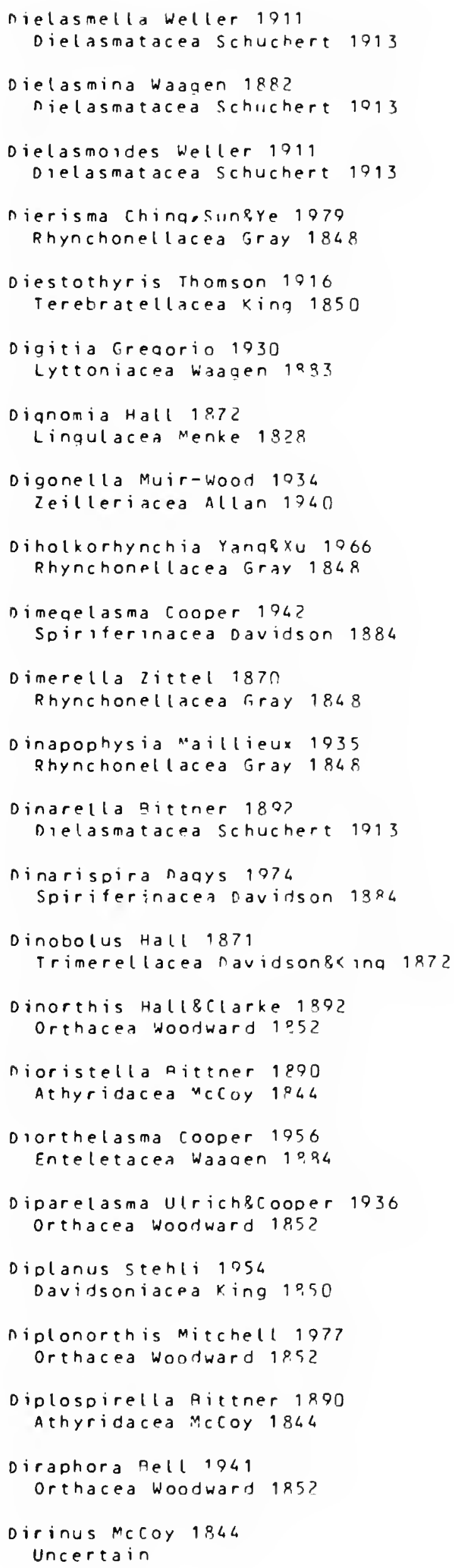

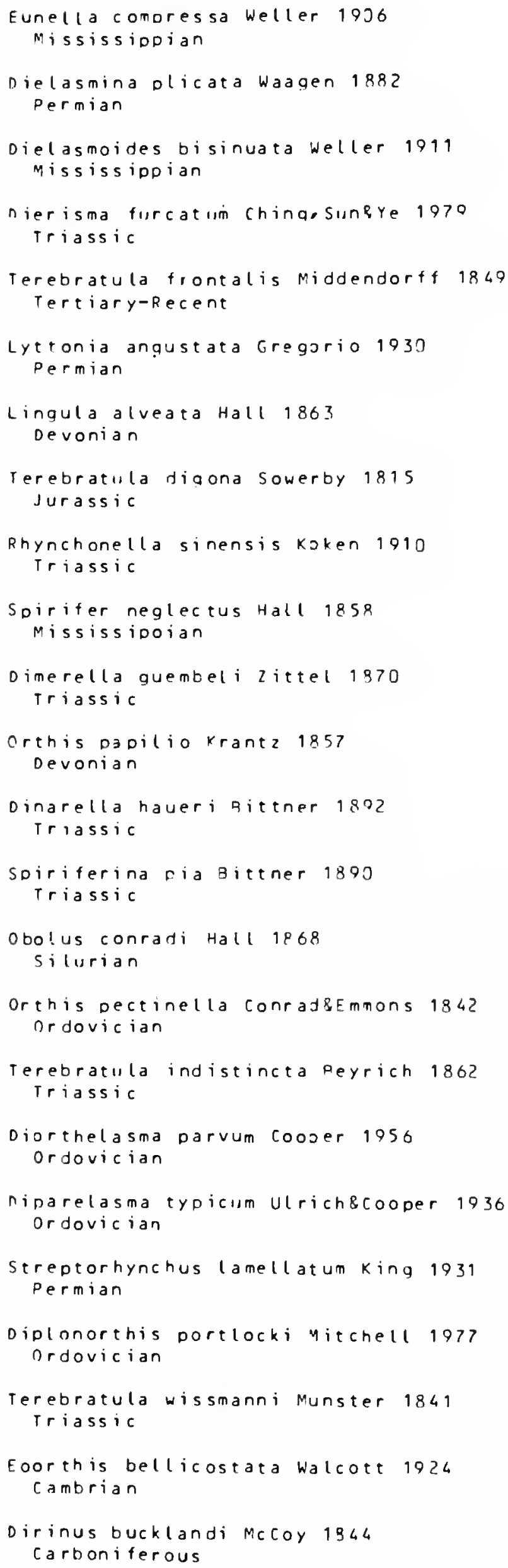


Discina Lamarck 1819 Discinacea riray 1840

Discinisca Dall 1971

Discinacea Gray 1840

Discinolepis wagen 1885

Acrotretacea Schuchert 1893

Discinopsis Matthew 1892 Acrotretacea Schuchert 1893

niscomyorthis Johnson 1070

Enteletacea Wagen 1884

Discotreta Ulrich\&Cooper 1936 Acrotretacea Schuchert 1893

Discradisca Stenzel 1964

Discinacea fray 1840

nisculina neslonqchamps 1884

Terebratulacea Gray 1847

Dispiriferina siblik 1965

Spiriferinacea navidson 1884

Ditreta Biernat 1973

Acrotretacea schuchert 1893

nivaricosta Coopersfirant 1969

Rhynchonel lacea Gray 1248

nmitria sitiachenko 1961 Spiriferacea king $184 \mathrm{~h}$

Dmitrispirifer Ljaschenko 1973 Spiriferacea king 1946

Dnestrina NikiforovaSModzalevskaja 1968 Atrypacea Gill 1971

noleroides cooper 1930

orthacea woodward 1852

Dolerorthis Schuchert.eCooper 1931

Orthacea woodward 18.52

Doloresella Sando 1957

Porambonitacea Davidson 1853

Donella Rotai 1031

Rhynchonellacea Gray 1848

Dongbaella Ching\&ye 1979 nielasmatacea Schuchert 1913

Dorashamia Sarytcheva 1965

Productacea Gray 184 ?

Dorsisinus Sanders 1958

Rhynchonellacea Gray 1848

Dorsoplicathyris Almeras 1971

Terebratellacea King 1850

Dorsoscyphus Roberts 1971

Davidsoniacea King 1850

Dorytreta Cooper 1956

Rhynchonel lacea fray 1848

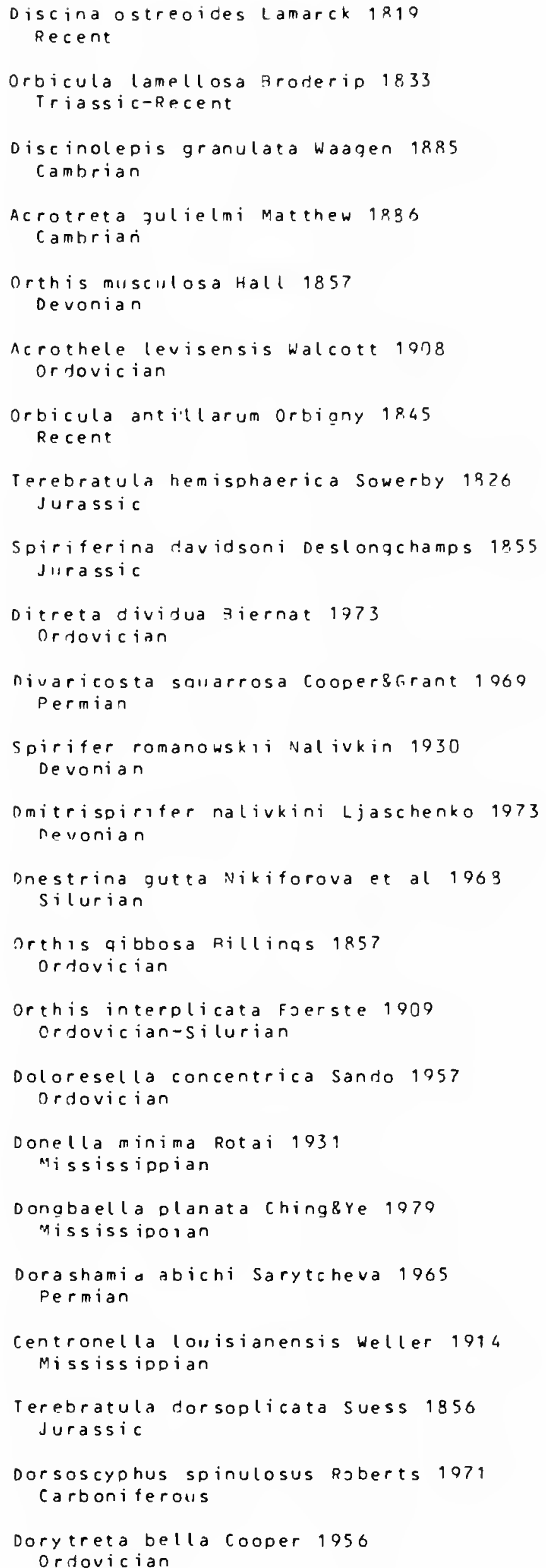




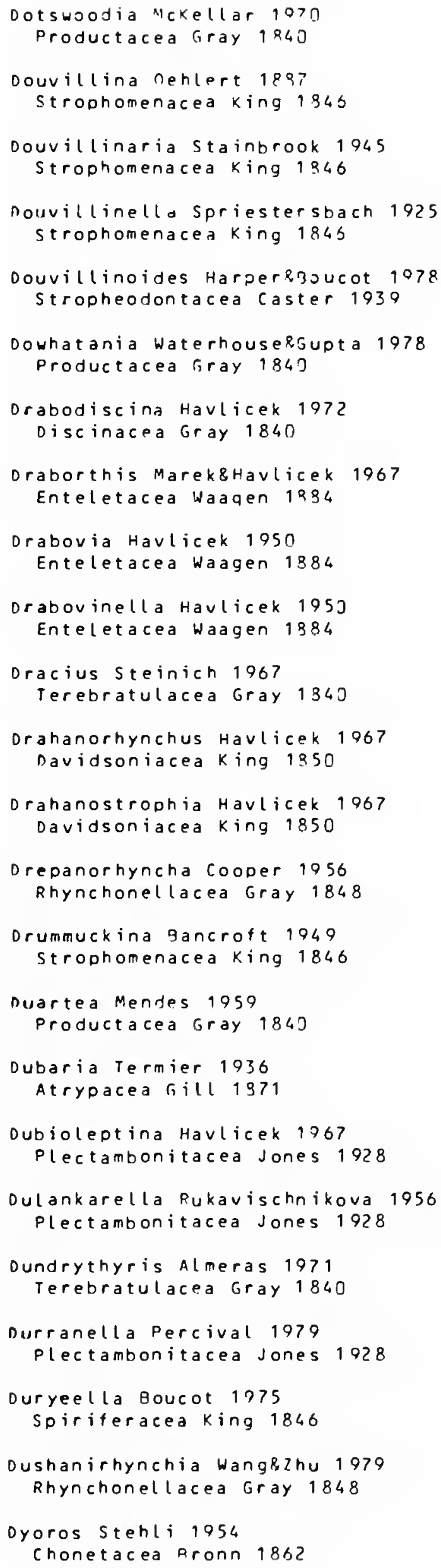

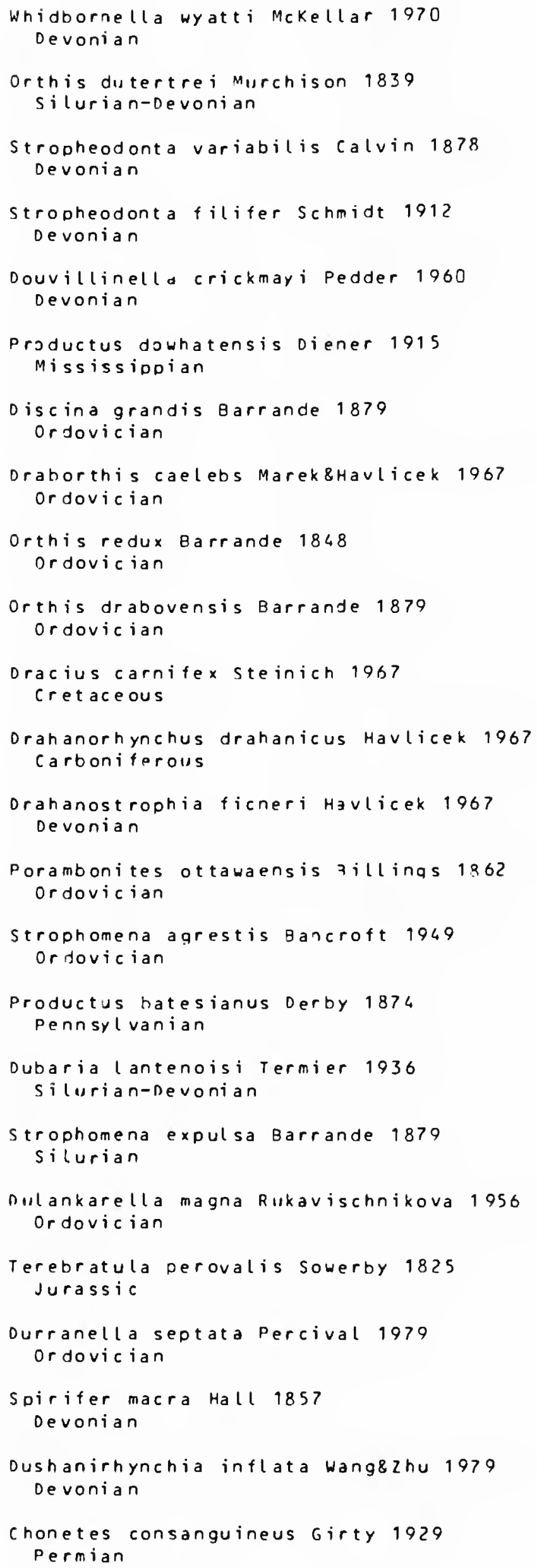


Dyschrestia Grant 1976

Productacea Gray 1840

Dyscolia Fischer\&oehlert 1870

Terebratulacea riray 9840

Dyscritothyris cooper 1979

Terebratulacea firay 1840

nysoristis Rell 9944

Siphonotretacea Kutor $\exists a 1348$

nyticospirifer Johnson 1966

Delthyridacea Phillios 1841

Dzieduszyckia Siemiradzki 1909

Athyrisinacea Grabau 1939

Dzirulina Nutsubidze 9945

Terebratulacea Gray 1340

Eatonia Hall 18.57

Rhynchonel Lacea Gray 1848

Eatonioides MCLearn 9919

Rhynchonel lacea Gray 9848

Eccentricosta Berdan 1963

Chonetacea Pronn 1862

Echinalosia Waterhouse 1967 Strophalosiacea Schuchert 1913

Echinaria Muir-Wood 8 cooper 1960

Productacea Gray 184 ?

Echinauris Muir-Wood\&Cooper 1960 Productacea Gray 1840

Echinirhynchia childs 1969 Rhynchonel lacea Gray 9848

Echinocoelia Cooper\&williams 1935 cyrtiacea Fredericks 1924

Echinocoeliopsis Hamada 1968 Cyrtiacea Fredericks 1924

Echinoconchus Weller 1994

Productacea Gray 1840

Echinospirifer Ljaschenko 1973 Spiriferacea King 1846

Echinosteges Muir-WoodRCooper 996 Aulosteqacea Muir-Wood\&Cooper 9960

Ecnomiosa cooper 9977 Terebratellacea King 1850

Ectenoqlossa Sinclair 1945 Lingulacea Menke 1828

Ectochoristites Campbel 1957 Spiriferacea kina 1846

Ectoposia cooper $86 r a n t 9976$ Dielasmatacea Schuchert 1913

Ectorenssel andia Johnson 1973 Stringocephal acea King 1850

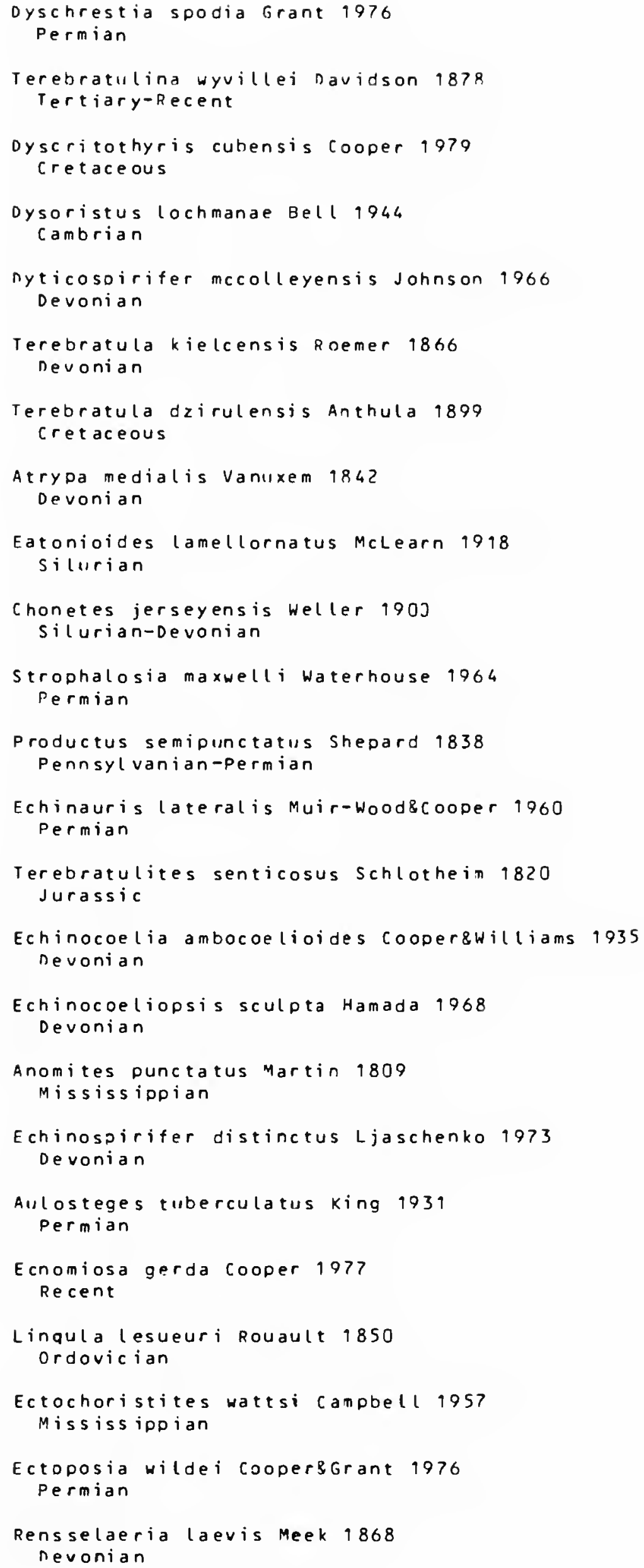




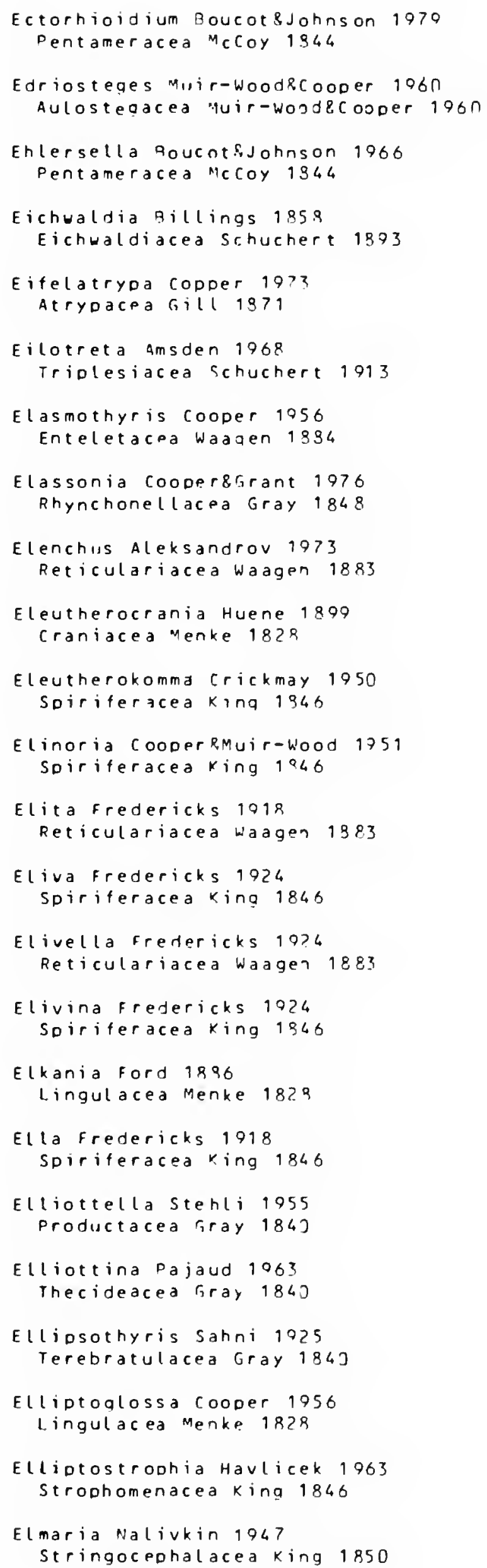

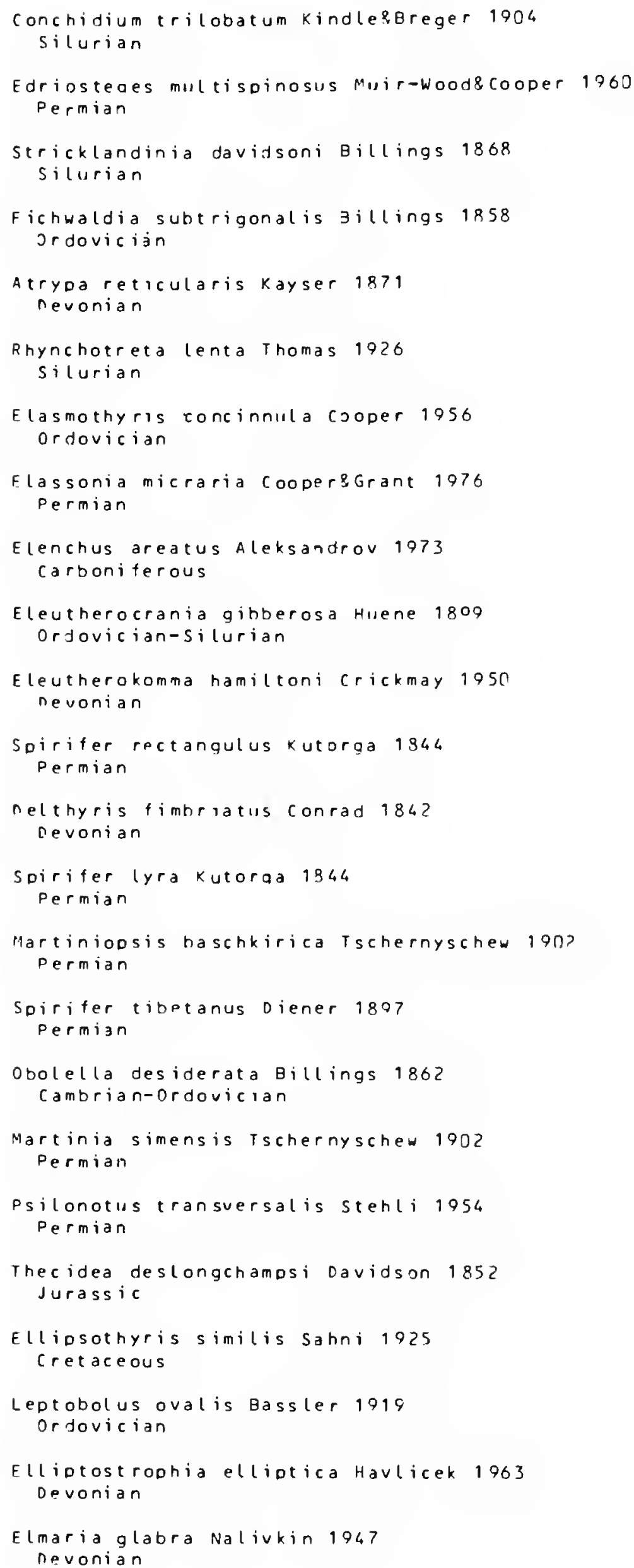


Elsaella Alichova 1960

Enteletacea waraen 9884

Elymospirifer wang 1074

Spiriferacea kina 1846

Elythyna Rzonsnitzkaja 1952

Reticulariacea Waagen 1883

Emanuella Grabau 1923

Cyrtiacea Fredericks 1924

Emeithyr is $x u 10>8$

Dielasmatacea Schuchert 1913

Enallosia cooper 8 rirant 1976

Cryptonellacea Thomson 1926

Enantiosphen whidborne 1893

Pentameracea McCoy 1344

Enantiosphenell a Johnson 1974

Pentameracea McCOY 1844

Enchondrospiriter Rrice 1970 Spiriferacea king 1846

Enigmalosia czarniecki 1950 strophalosiacea schuchert 1913

Enteletella Licharew 1926

Enteletacea wagen 1884

Enteletes Fischer 1825

Enteletacea wagen 9984

Enteletina Schuchert\&cooper 1031 Enteletacea wagen 1994

Enteletoides Stuckenberg 1935 Enteletacea wagen 18.84

Eoamphistrophia Harper\$goucot 9778 Stropheodontacea Caster 1939

Eoanastrophia Nikiforova et a 1973 Camerellacea Halleclarke 1894

Eobrachythyris 9rice 1970 Spiriferacea king 1846

Eochonetes Reed 1917 Plect ambonitacea Jones 1928

Eochoristites chu 1933 Spiriferacea king 1846

Eocoelia Nikiforova 1961 Dayiacea Waagen 9893

Eoconcha coooer 1959 Rillingsellacea Schuchert 1893

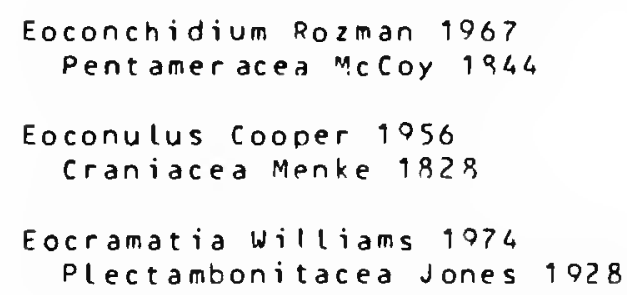

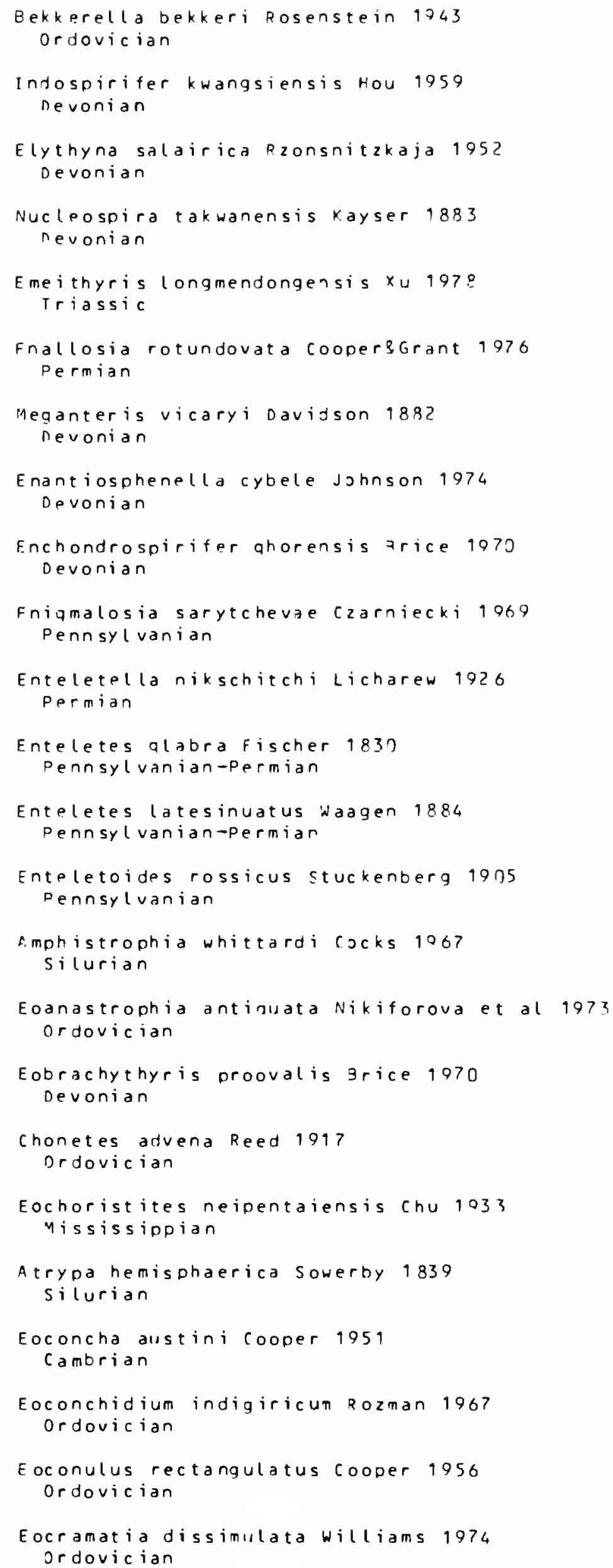




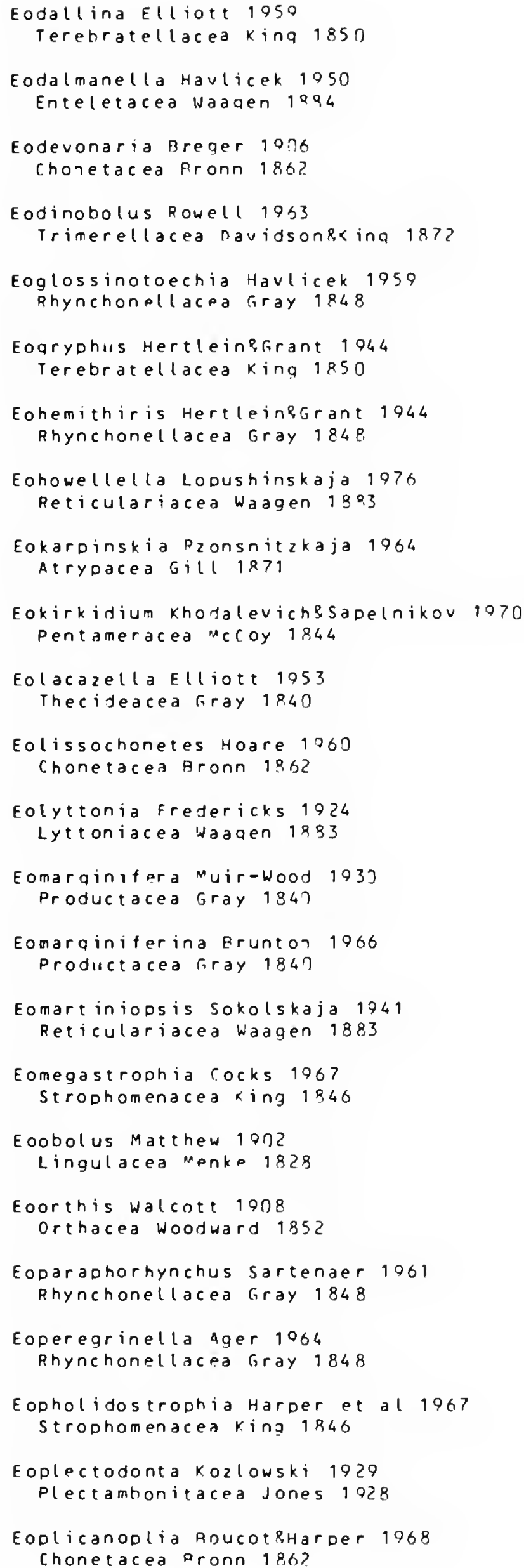

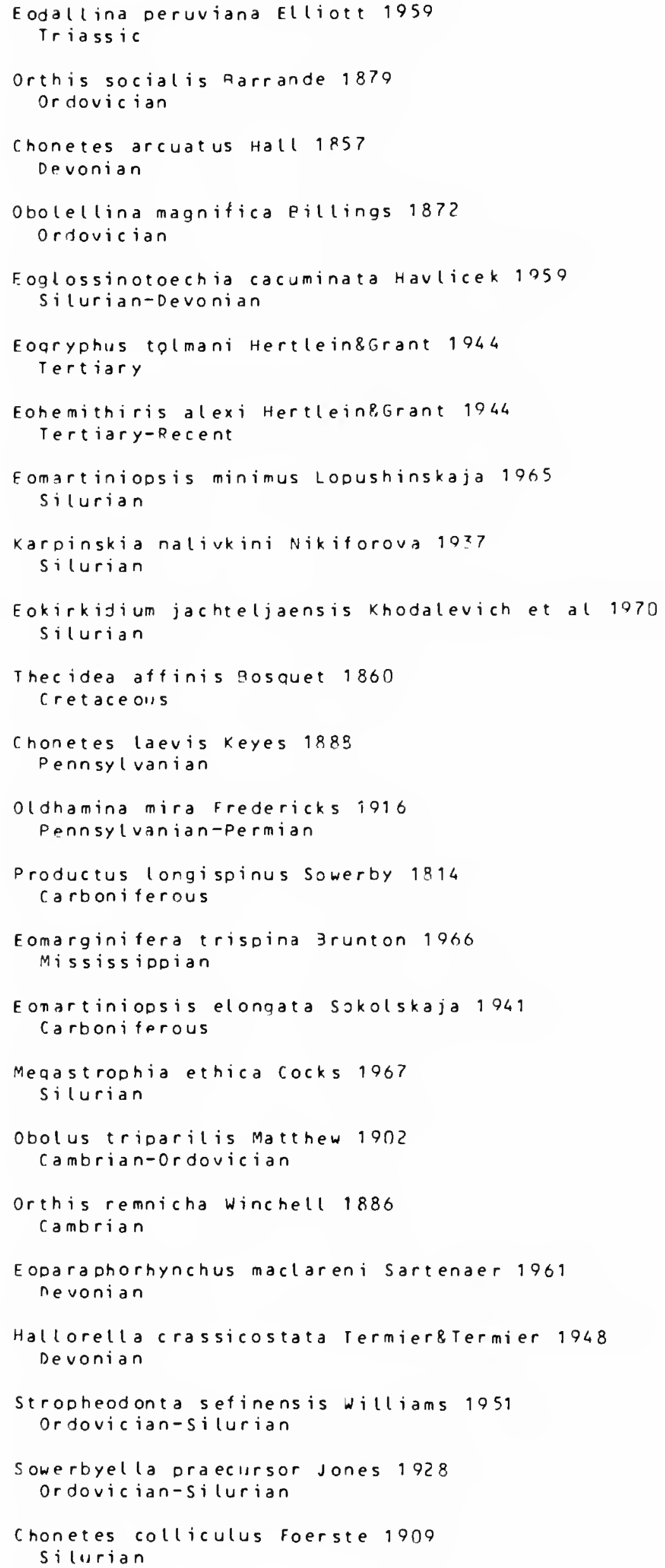


Eoreticularia Nalivkin 1924

Reticulariacea la agen 1883

Eorhipidomella Hints 1971

Enteletacea waagen 1984

Eoschuchertella Gratsianova 1974

navidsoniacea king 1950

Eoseptaliphoria Ching 8 sun 1776

Rhynchonellacea Gray 1848

Eosophragmophora wang 1974

Enteletacea waagen 1394

Eosotrematorthis wang 1955

Billingsellacea Schuchert 1993

Eospinatryoa ropper 1273

Atrypacea Gill 1s?1

Eospirifer schuchert 1013

cyrtiacea fretericks $10 \geq 4$

Eospiriferina hrabau 1931

Spiriferacea king 1946

Eospirigerina Boucot\& Johns on 1967 Atrypacea rill 1871

Eostrophalosia Stainbrook 1743 Strophalosiacea Schuchert 1913

Eostropheodonta Pancroft 9949 Strophomenacea $\mathrm{king} 1946$

Eostrophomena walcott 1305 Orthacea Woodward 125?

Eostrophonella williams 1950 Strophomenacea $k$ ing 1946

Eosyringothyris stainbrook 1943 Spiriteracea king 1946

Eousella Dvtsharenko 1976 zeilleriacea Allan 1940

Ephippelasma cooper 1056 Acrotretacea Schuchert 1893

Epicelia rirant 1972

Strophalosiacea Schuchert 1913

Epicyrta Deslongchamps 1884 Zeilleriacea Allan 1040

Epithyris Dhillips 1841 Terebratulacea Gray 1840

Epithyroides $\mathrm{xu} 1978$ Dielasmatacea Schuchert 1713

Epitomyonia wright 1968 Enteletacea Wagen 1884

Equirostra Coopersmuir-wood 1951 Porambonitacea Davidson 1853

Erectocephalus xian 1978 Stringocephal acea king 1850

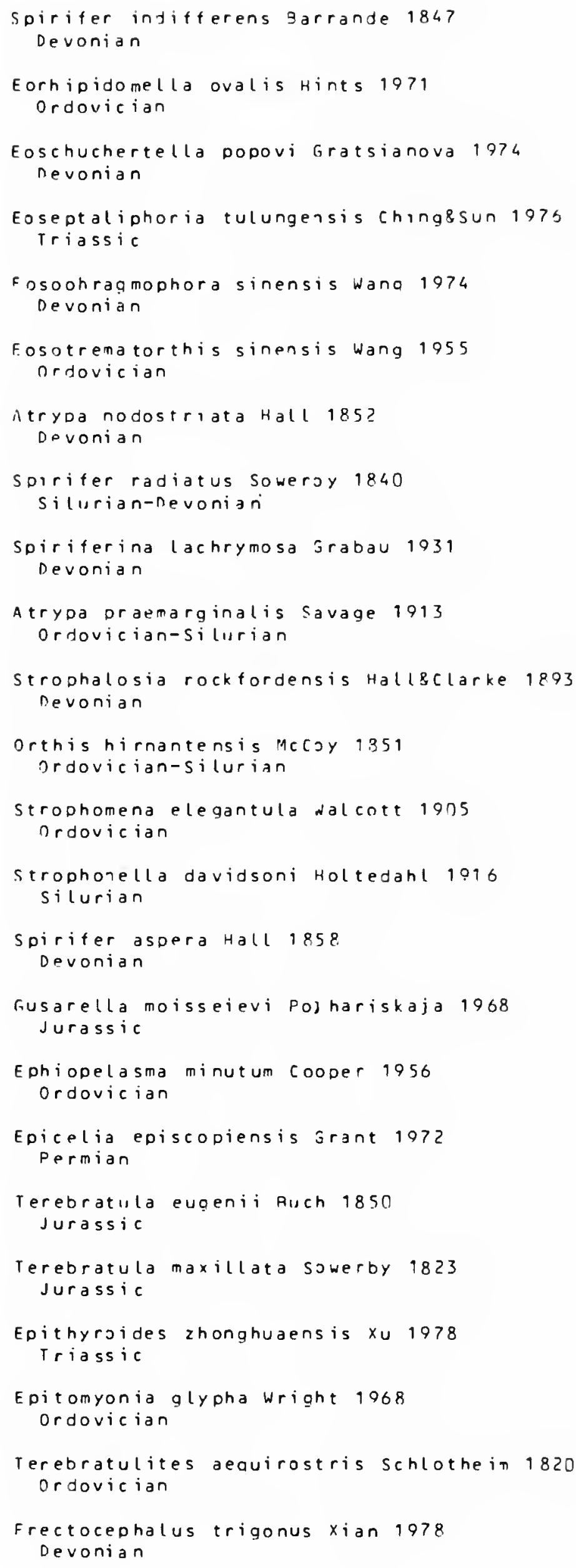




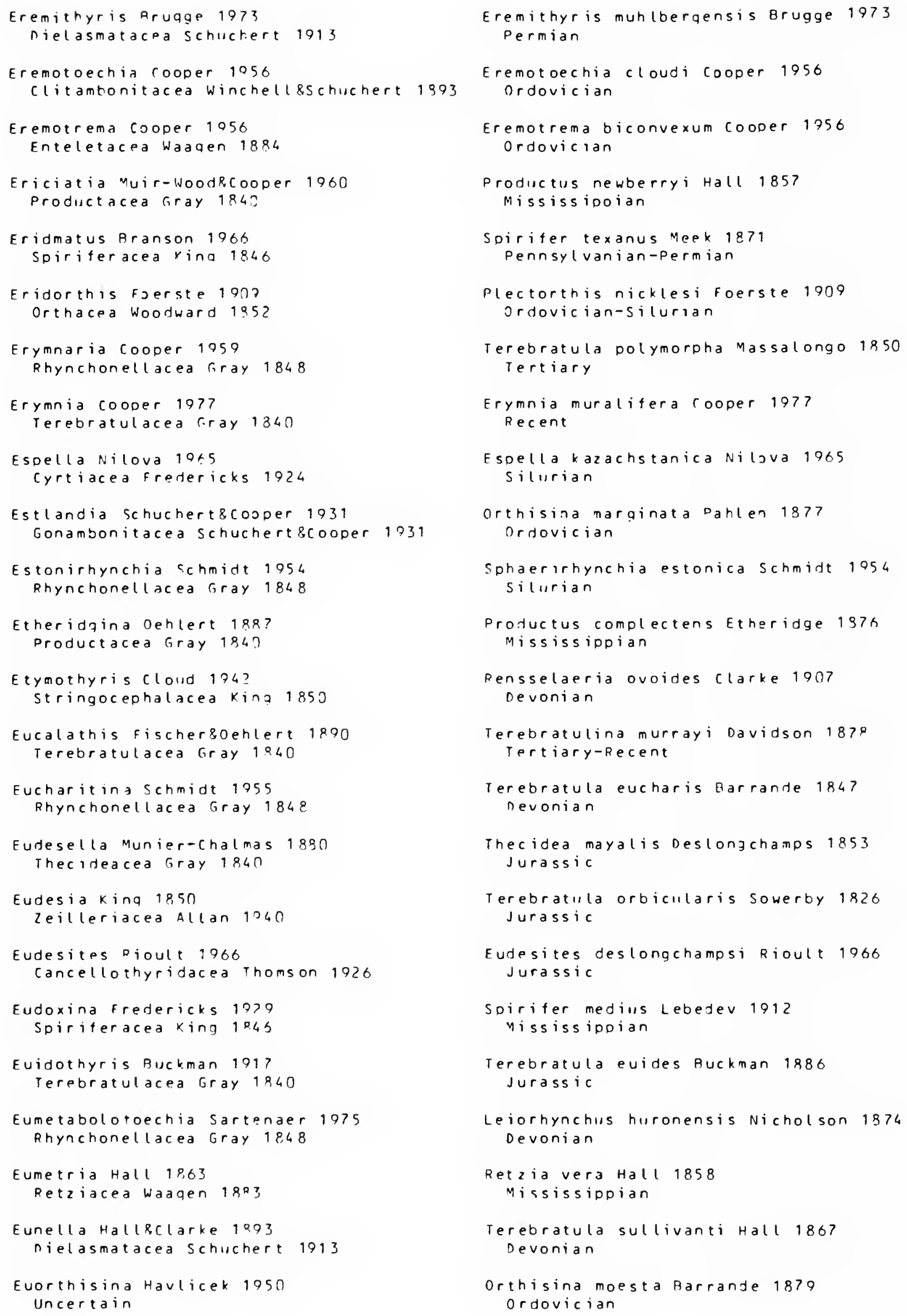


Euractinella Rittner 1990 Athyridacea McCoy 1846

Eurekaspirifer Johnson 1966 Delthyridacea Phillips 1841

Eurycoloorhynchus Sartenaer 1968 Rhynchonel lacea fray 1848

Euryspirifer wedekind 1026 Spiriferacea King 1846

Eurytatospirifer Gat inaud 1949 spiriferacea king 1846

Eurythyris Cloud 1942 Stringocephalacea King 1850

Eurytreta Rowell 9966 Acrotretacea Schuchert 1893

Euxinella Moisspiev 9036 Rhynchonellacea foray 1948

Evanescirostrum Sartenaer 1965 Rhynchonel lacea Gray 9868

Evenkina Andreeva 9061 Orthacea woodward 1852

Evenkinorthis Yadrenkina 1977 Orthacea Woodward 18,5 ?

Evenkorhynchia Rozman 1969 Rhynchonellacea Gray 1848

Exatrypa Copoer 1967 Atryoacea filll 1871

Excavatorhynchia Jin\&fana 197 ? Rhynchonellacea firay 1848

Exceptothyris Sucic-Protic 1971 Loboidothyridacea Makridin 1964

Faksethyris Asaard 1071 Terebratulacea Gray 1840

Falafer Grant 1072 Strophalosiacea Schuchert 1913

Fallax Atkins 1960 Terebratellacea King 1850

Fallaxispirifer su 1976 Reticulariacea Wagen 1883

Falsatrypa Havlicek 1956 Atrypacea rill 1871

Famatinorthis Levy\&nullo 1973 orthacea Woodward 1852

Fardenia Lamont 19035 Davidsoniacea King 1850

Fascicosta Stehli 1955 Rhynchonel lacea Gray 1868

Fascicostella Schuchert \& cooper 1931 Enteletacea Wagen 1984

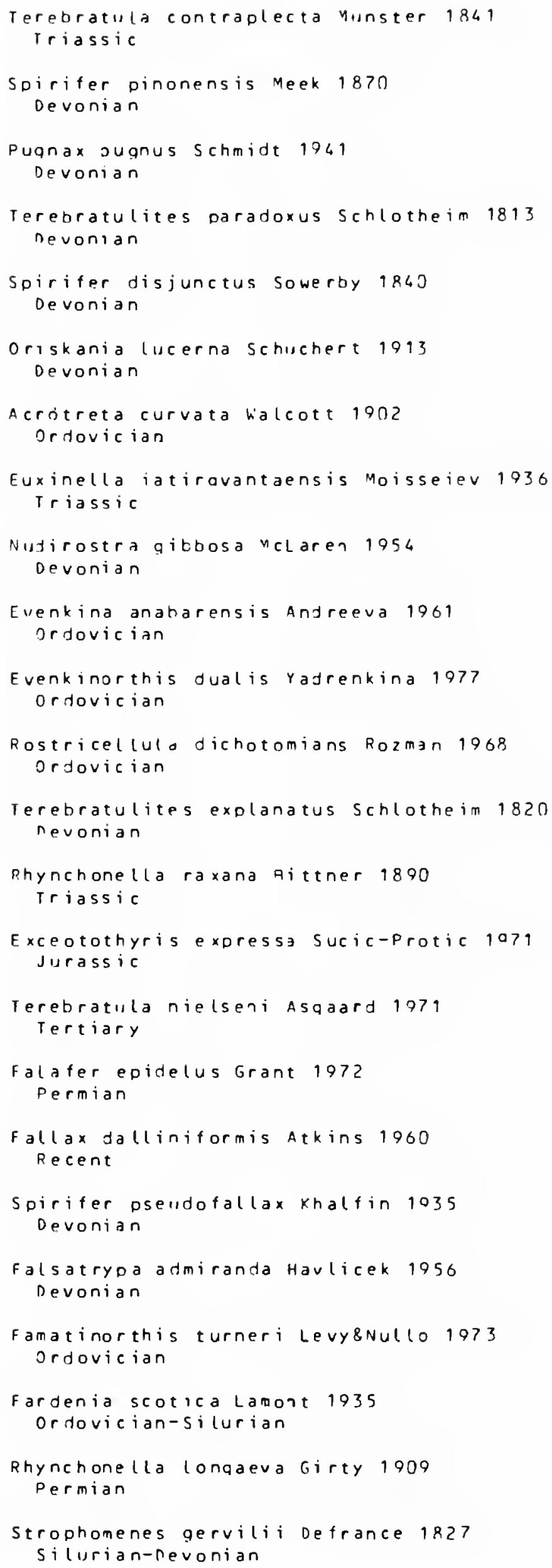




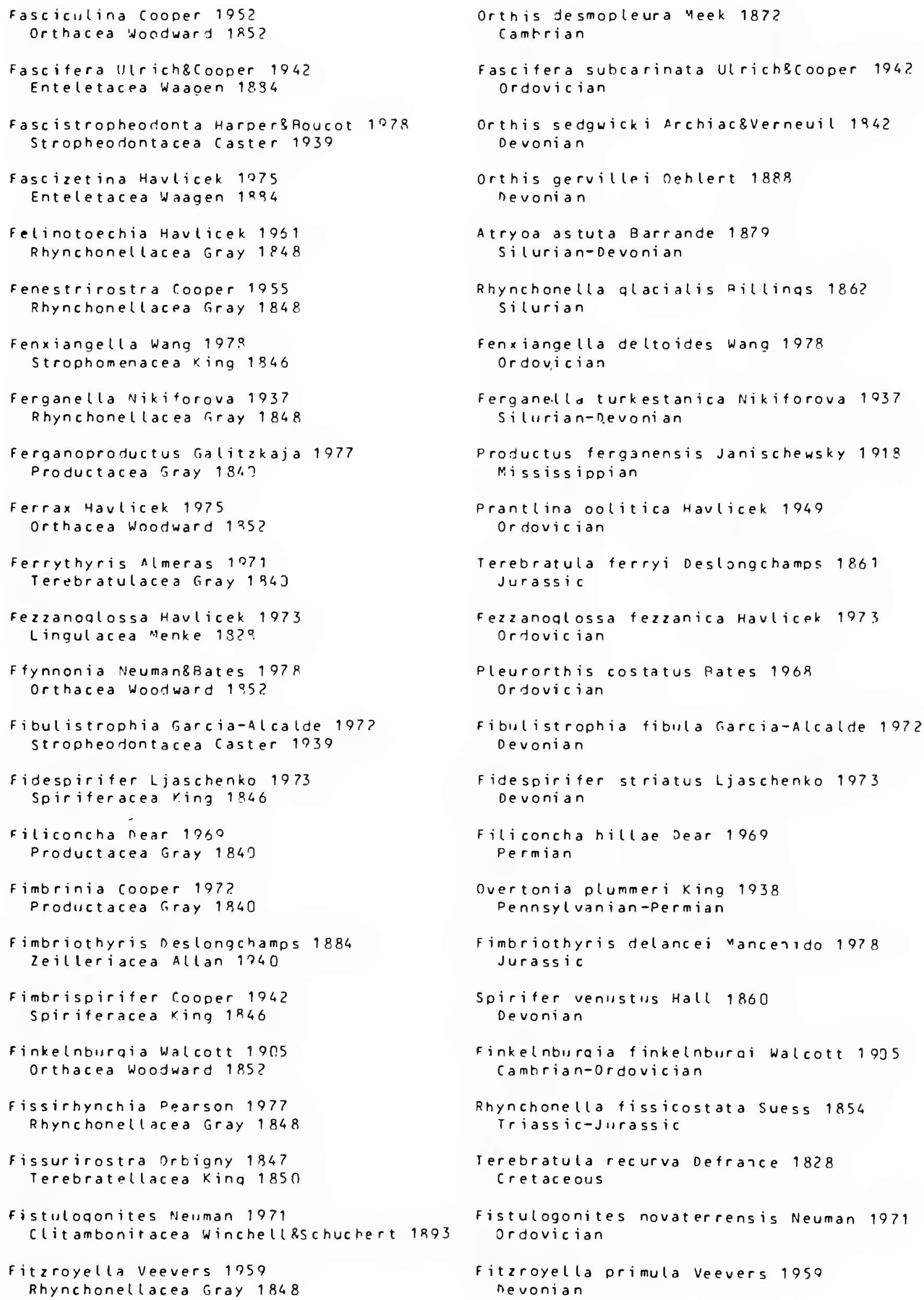




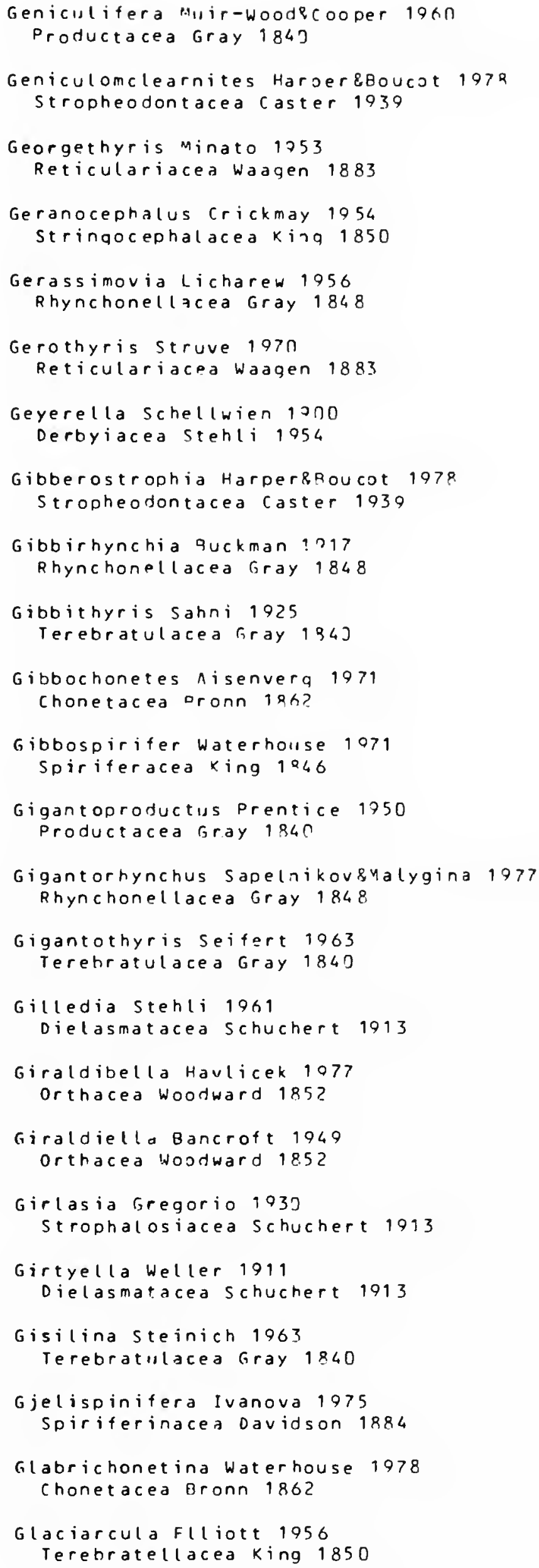

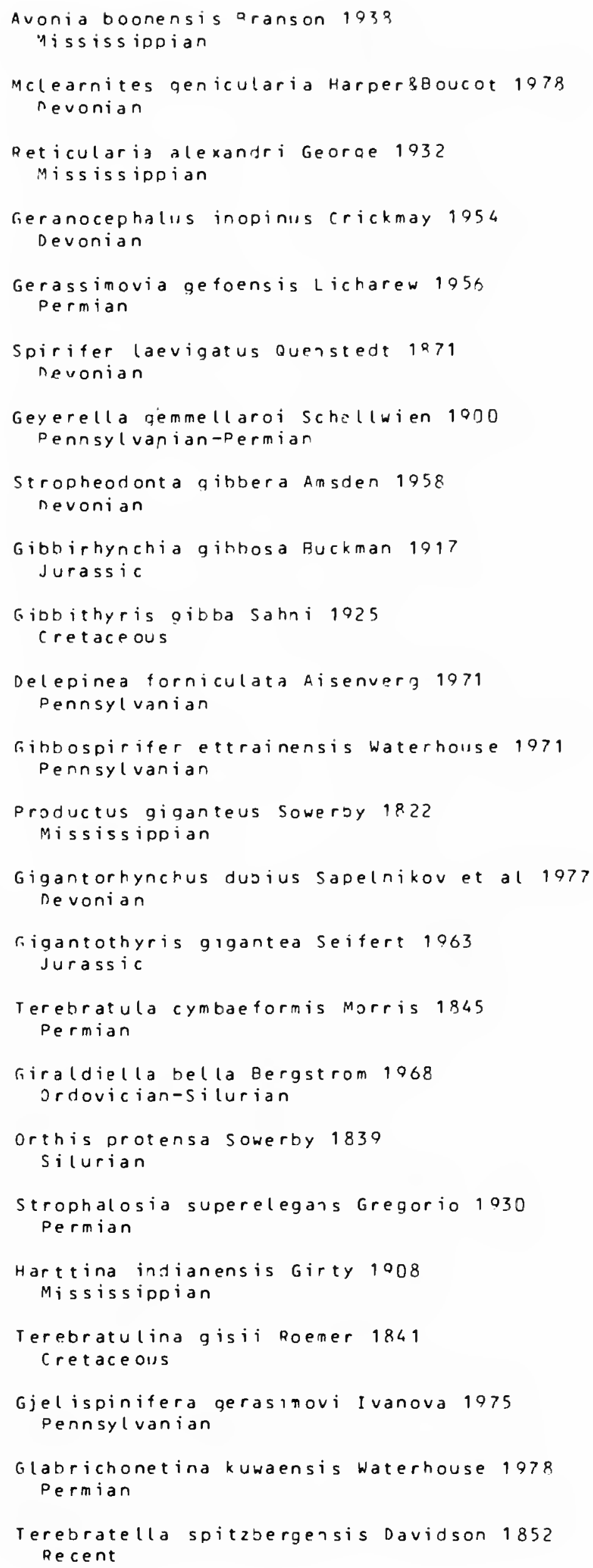




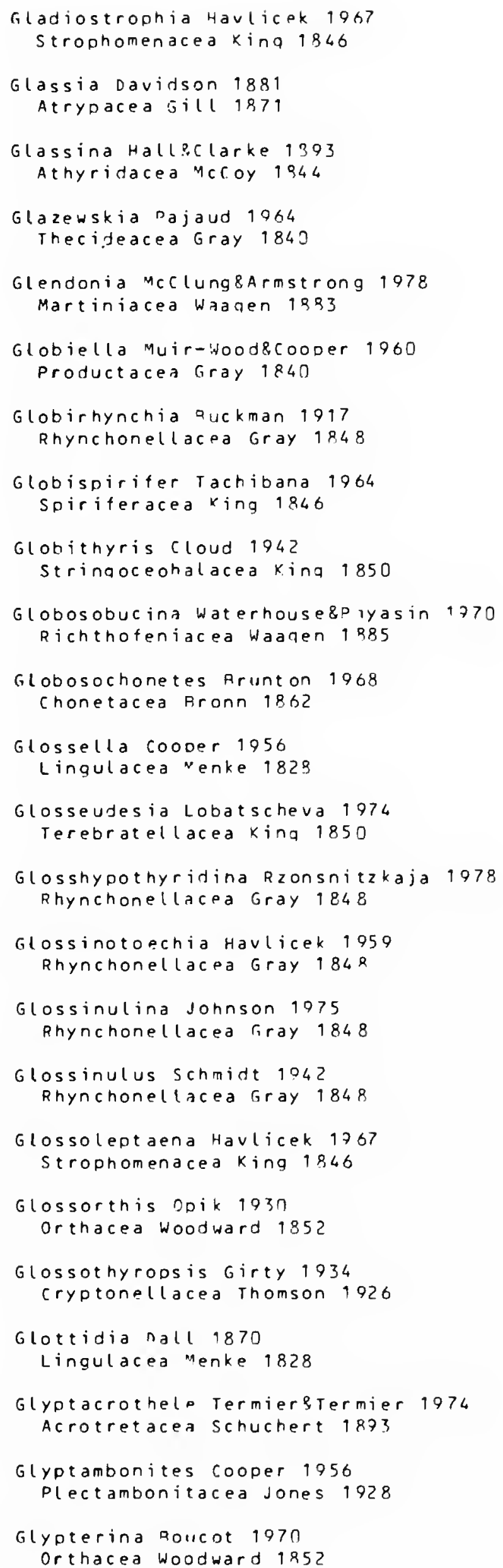

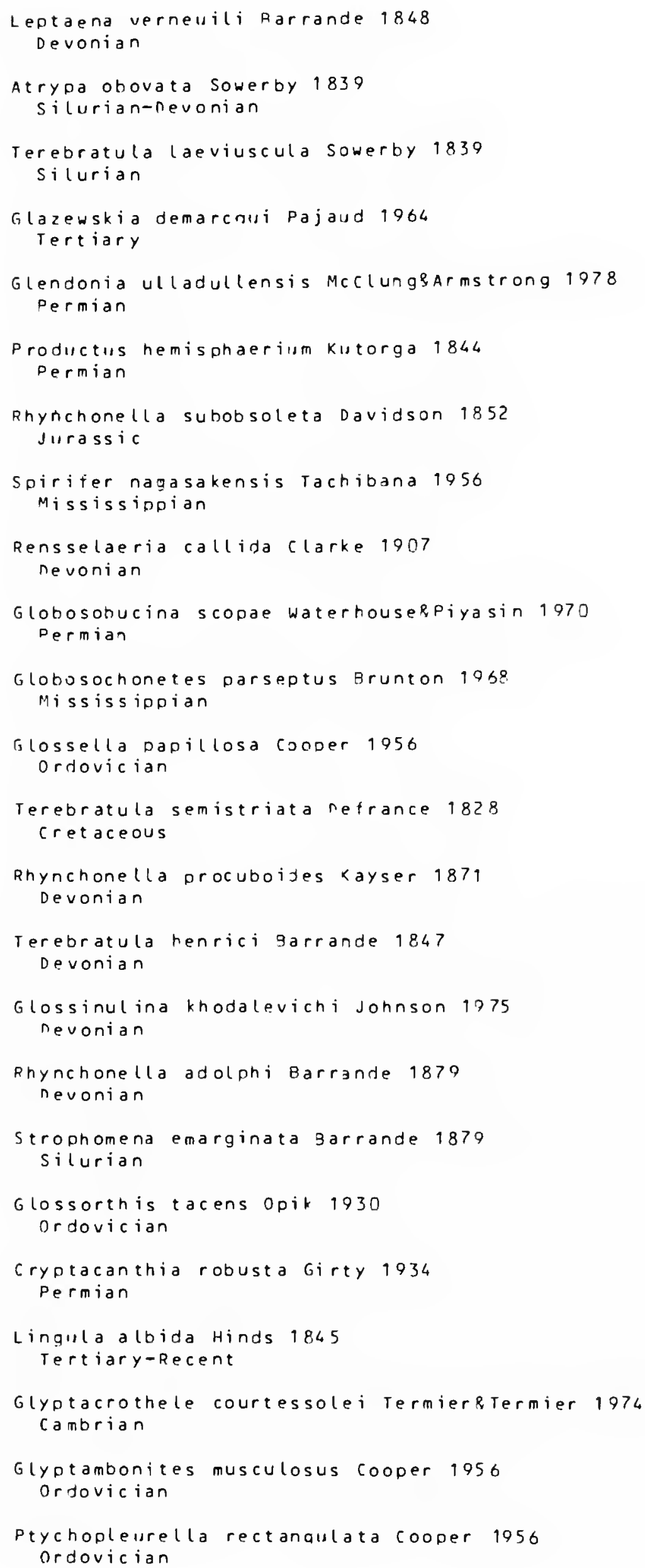




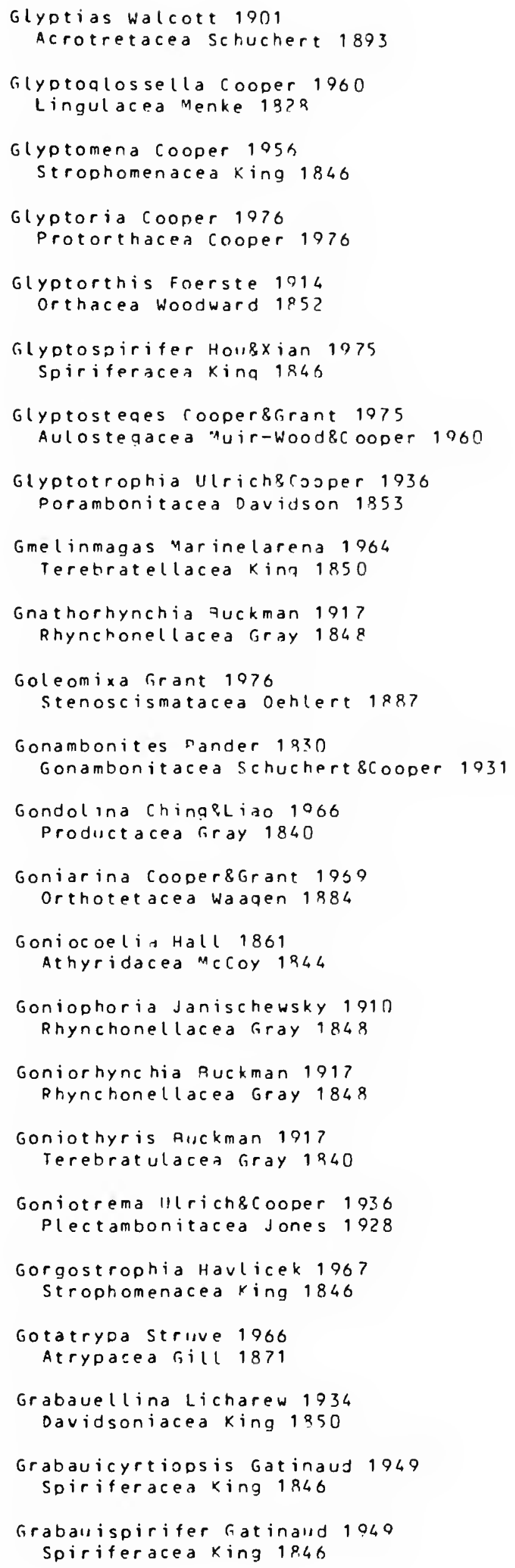

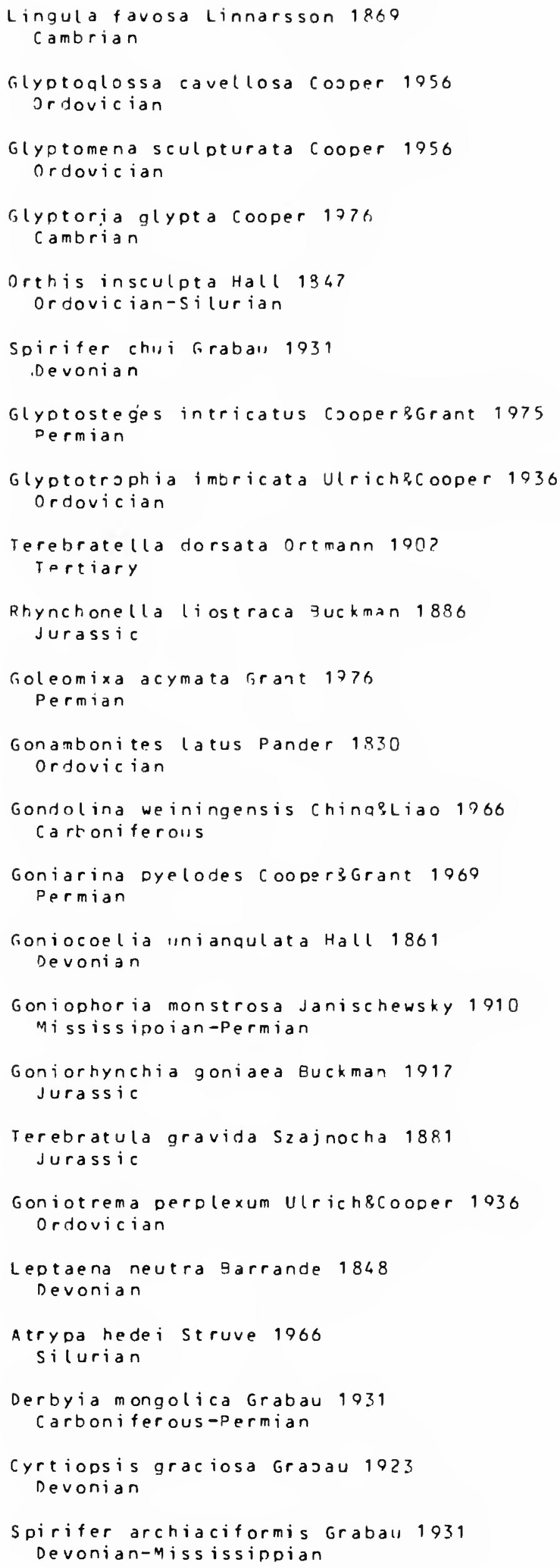




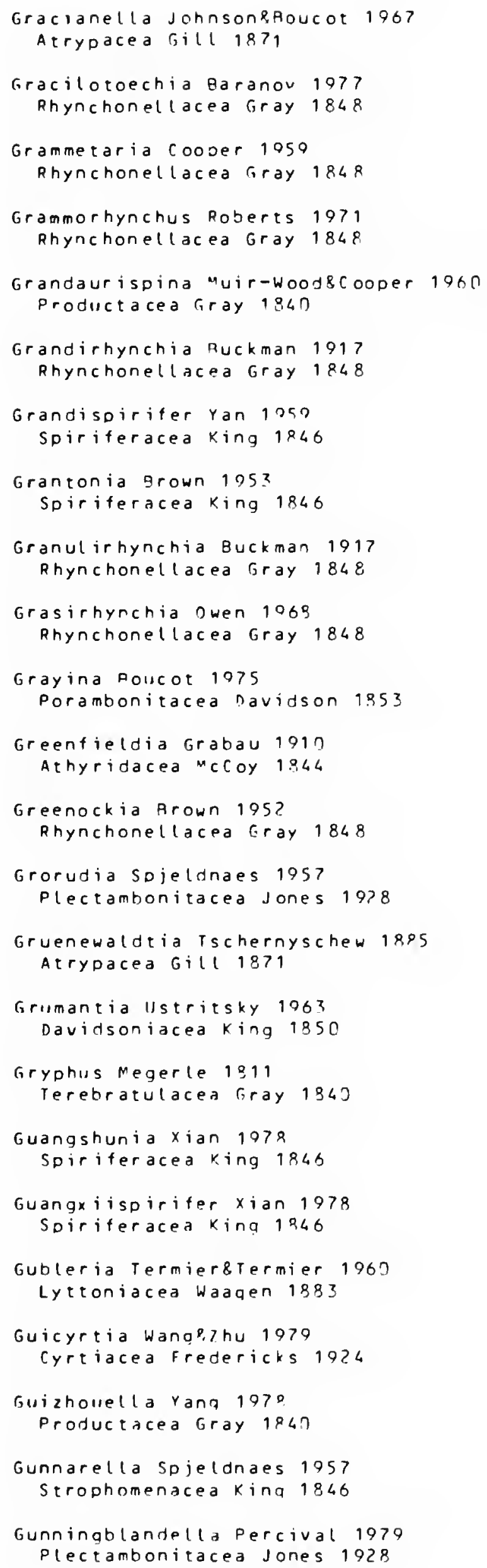

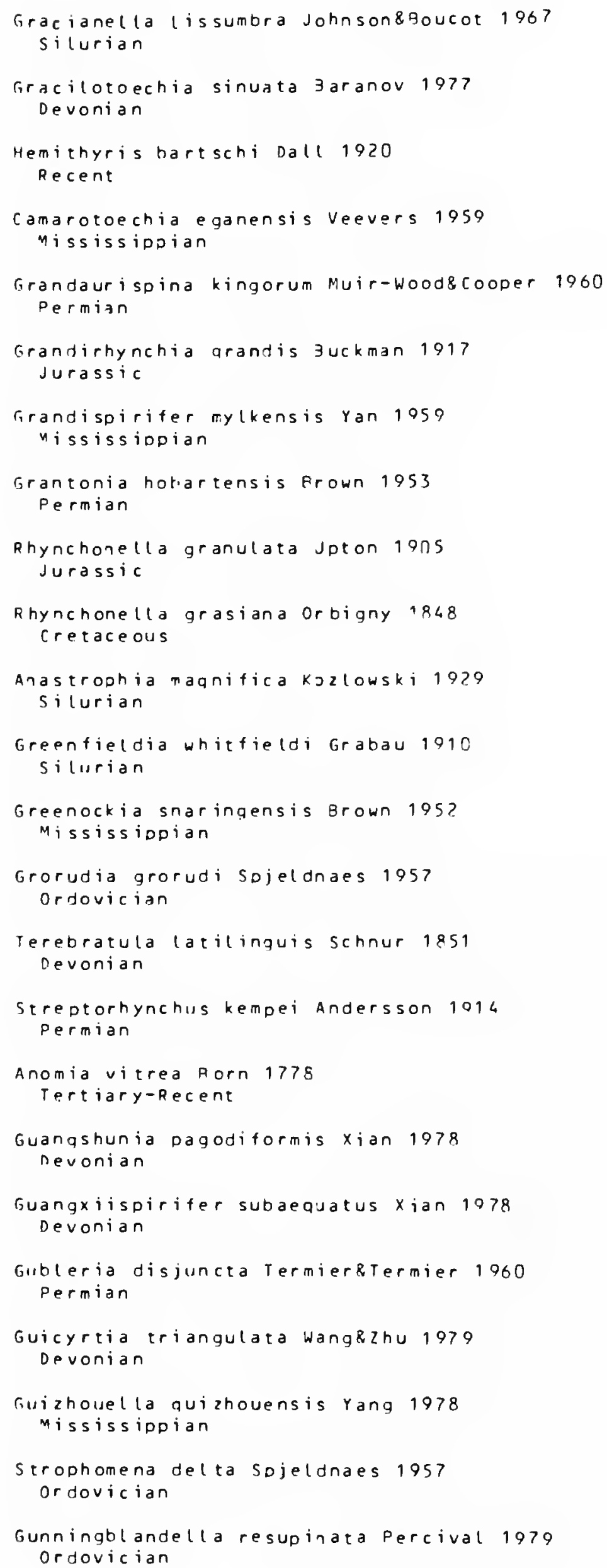




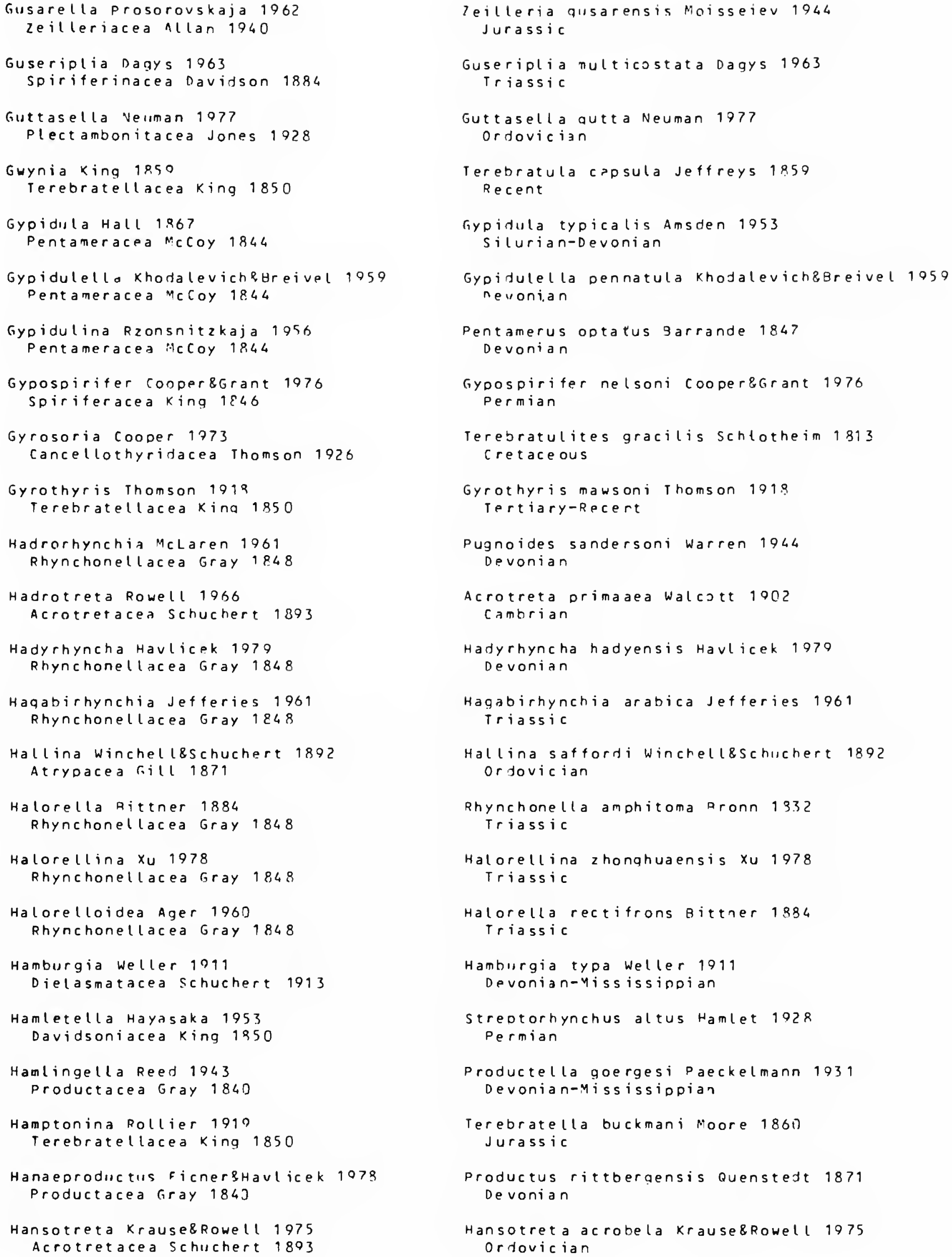




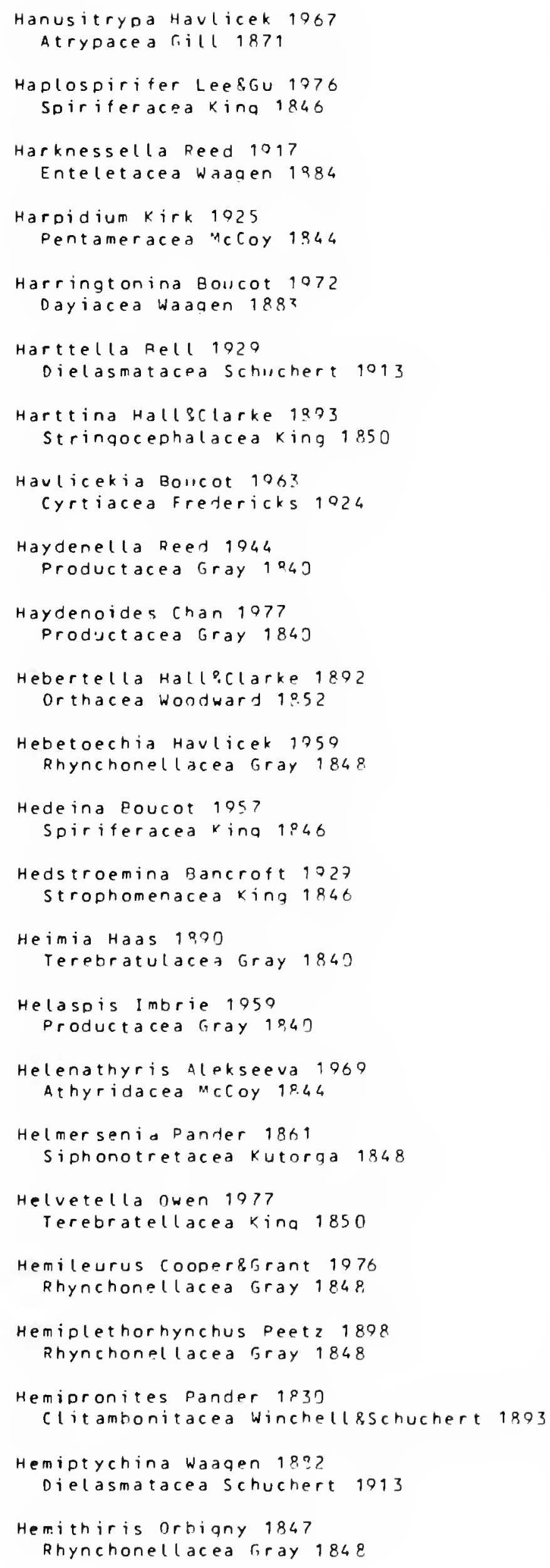

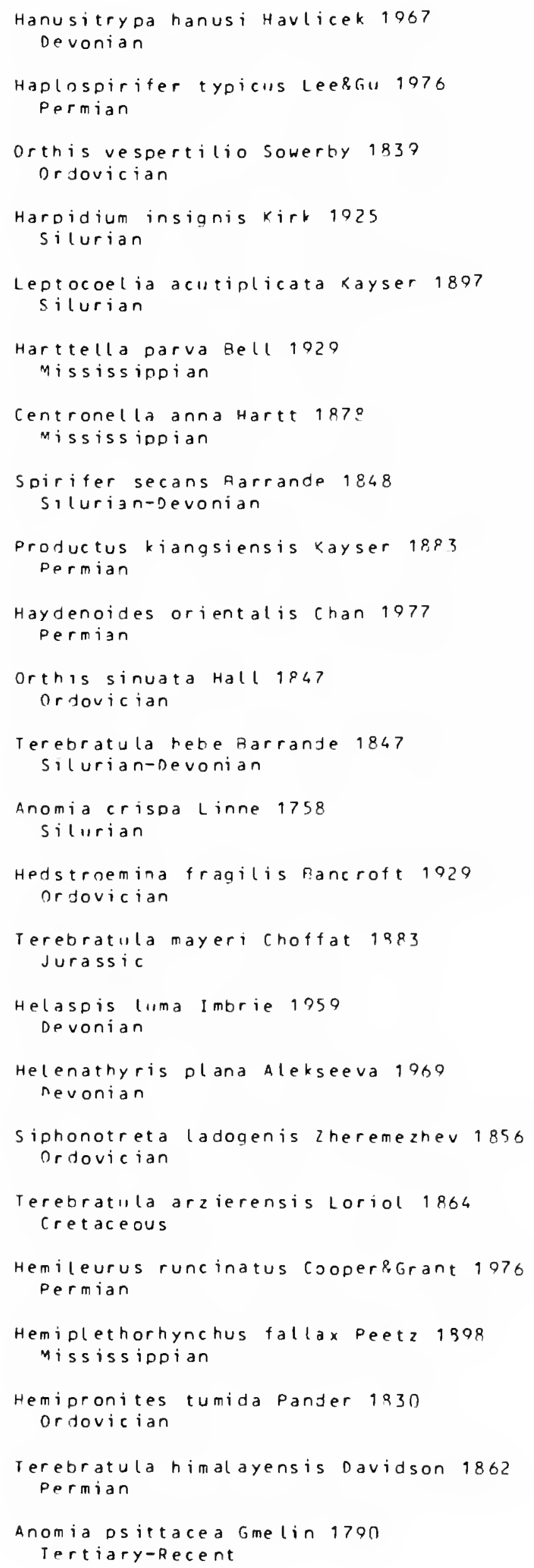


Hemithyropsis Katz 1974

Rhynchonel lacea Gray 1849

Hemitoechia Nikiforova 1970

Rhynchonel lacea Gray 1848

Hercosestria Cooper 8 Grant 1969

Richthofeniacea wa aen 1885

Hercosia Cooper 8 Gant 1969

Richthofeniacea wagen 1885

Hercostroohia williams 1950

strophomenacpa $\mathrm{Kina} 1846$

Hercothyris rooper 1979 Dallinacea geacher 1993

Hesperinia cooper 1056

strophomenacea king 1846

Hesperithyris Dubar 1042

Terebratulacea Gray 1840

Hesperomena cooper 1956

$P$ lectambonitacea Jones 1928

Hesperonomia Ull rich\&Conper 1936 orthacea Woorward 1852

Hesperonomiella Ulrichscooper $193 \mathrm{~A}$ Orthacea Woorward 1852

Hesperorhynchia Warren 1937 Rhynchonel lacea Gray 1848

Hesperorthis Schuchert\&Cooper 1931 Orthacea Noortward 195?

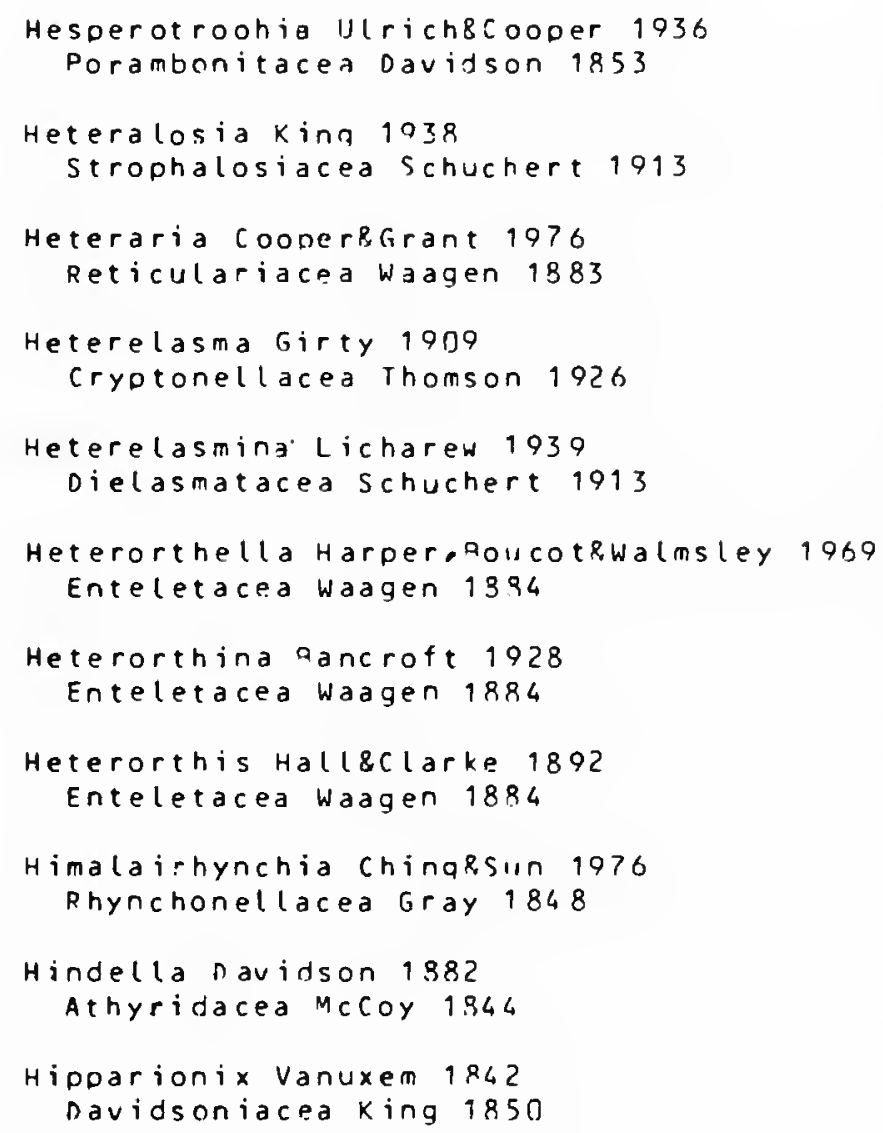

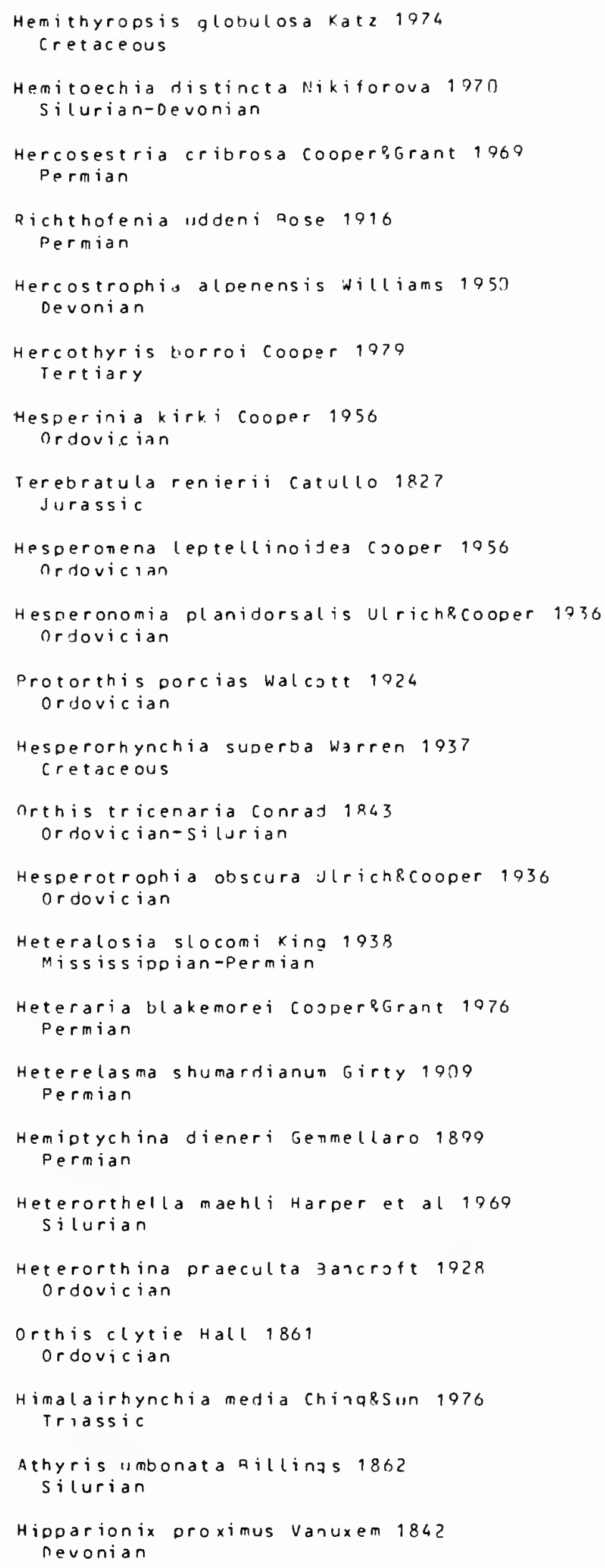




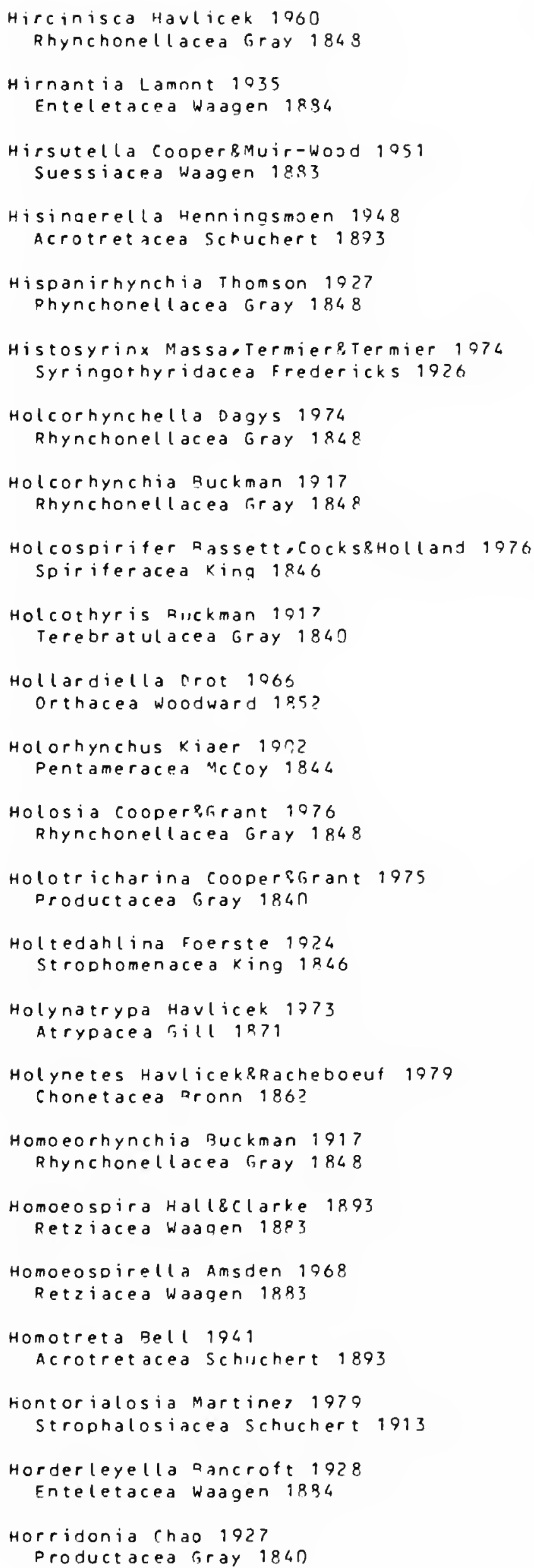

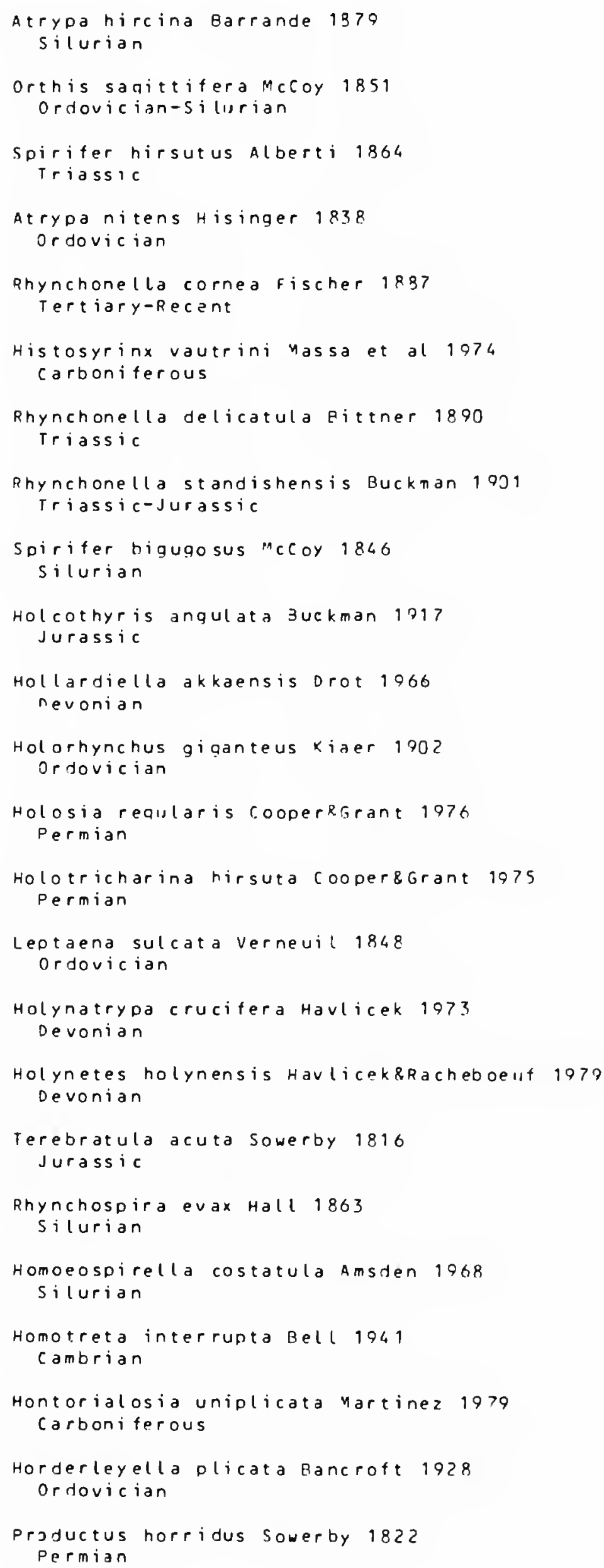




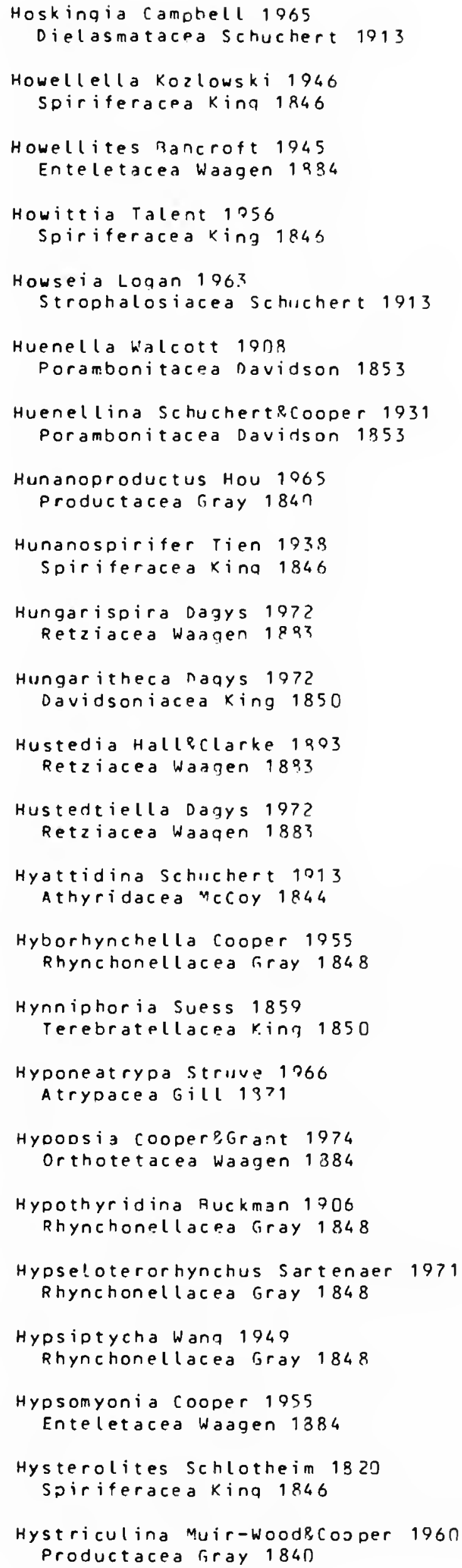

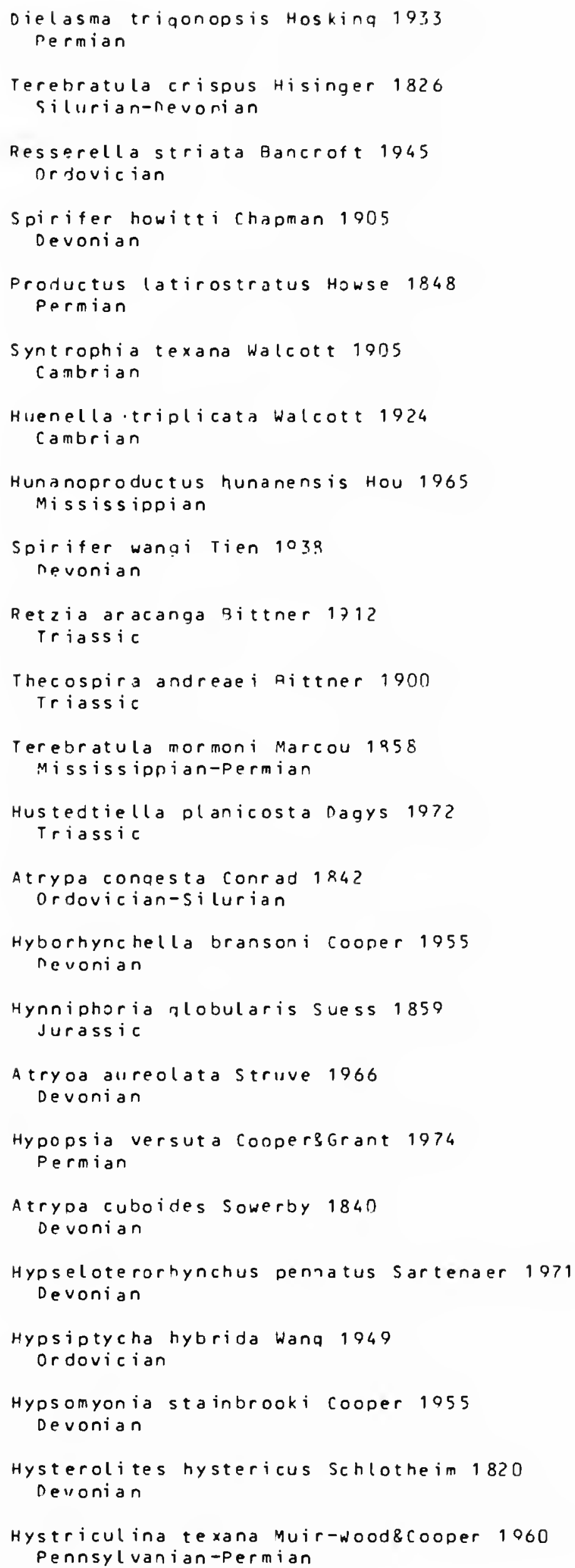




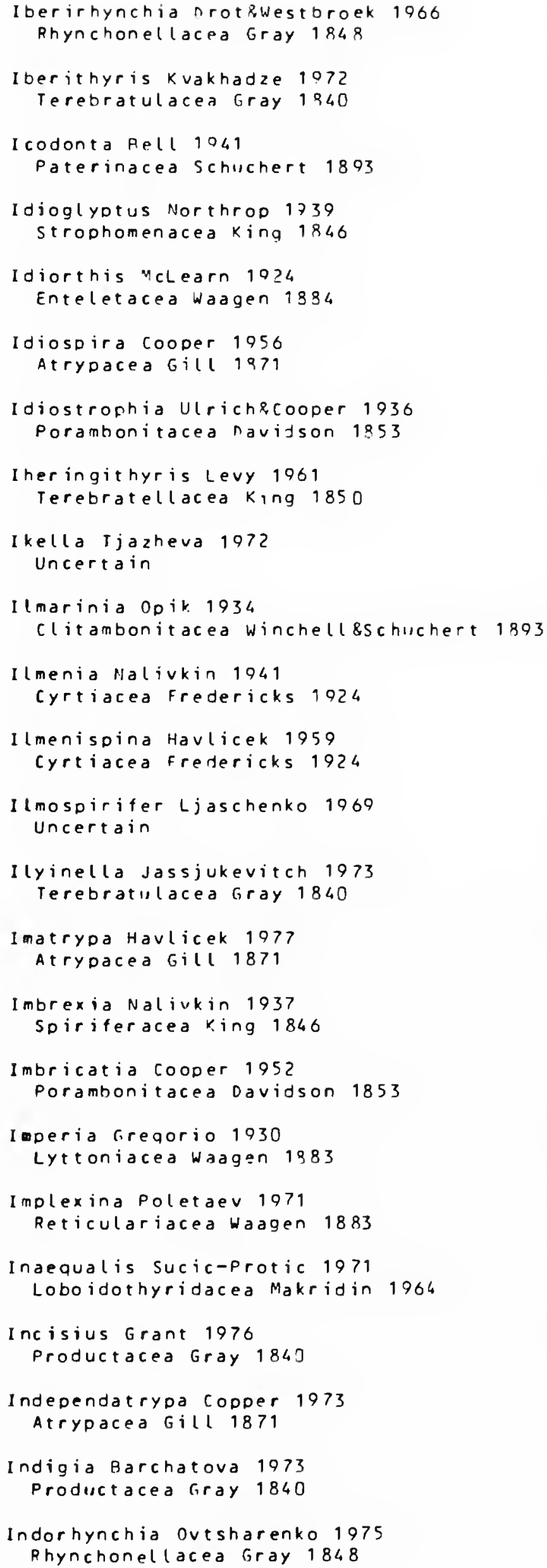

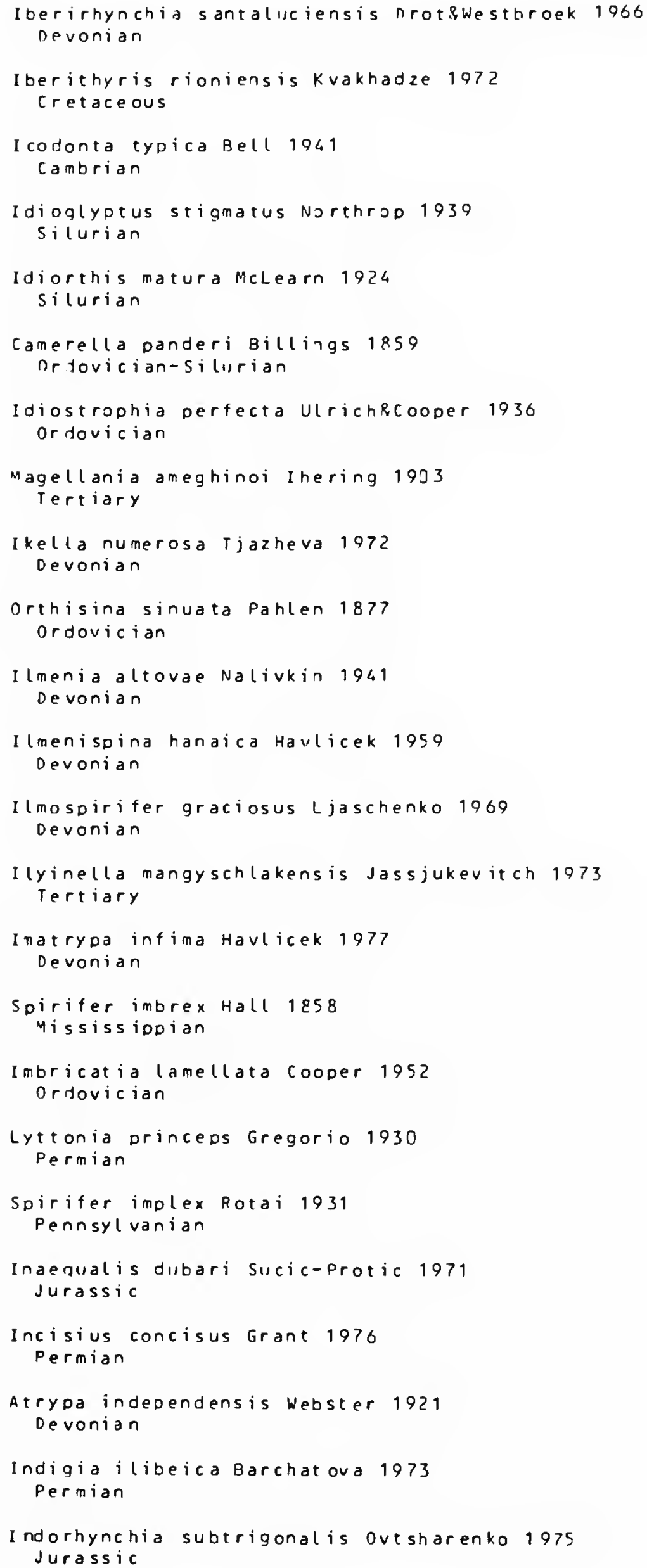


Indosoirifer Grabau 1731 Spiriferacea king 1846

Inflatia Muir-wondecooper 1060 Productacea Gray 184 )

Ingelarella camnbell 1959 Reticulariacea waqen 1883

Ingria opik 1030

Plectambonitacea Jones 1928

Iniathyris gesnossova 1063 Athyridacea McCOy 1844

Innuitella Crickmay 1968 Rhynchonellacea Gray 9848

Inopinatarcula Flliott 1952 Terebratulacea foray 1849

Institella cooper 1942

Aulosteqacea Muir-Wood\&Cooper 1060

Institifera Muir-WoodPooper 1960

Productacea Gray 184?

Institina Muir-WoodRCooper 1930

Productacea riray 184 n

Inversella Doik 1933

Plectambonitacea Jones 1028

Inversithyris nagys 1968

Terebratulacea Gray 1340

Invertryoa Struve 1961

Atryoacea fill 1971

Iotina Cooper\&Grant 1975

Rhynchonellacea Gray 184 ?

Iowatryoa Copder 1973 Atrypacea Gill 1871

Iphidella walcott 1905 Paterinacea Schuchert 1803

Irboskites Aekker 1926

Davidsoniace $\mathrm{K}$ ing 1850

Irenothyris Pojariskaja 1966

Terebratellacea kina 1850

Iraislella Tjazheva 1972 Rhynchonel lacea Gray 1848

Irhirea Havlicek 1971 orthacea woodward 1852

Irjdistrophia Havlicek 1965 Orthotet acea waagen 1884

Irma Gregorio 1930 Lyttoniacea wagen 1883

Iru Opik 1934

Clit ambonitacea Winchell \&Schuchert 1803

Ishimia Nikitin 1974

Plectambonitacea Jones 1928

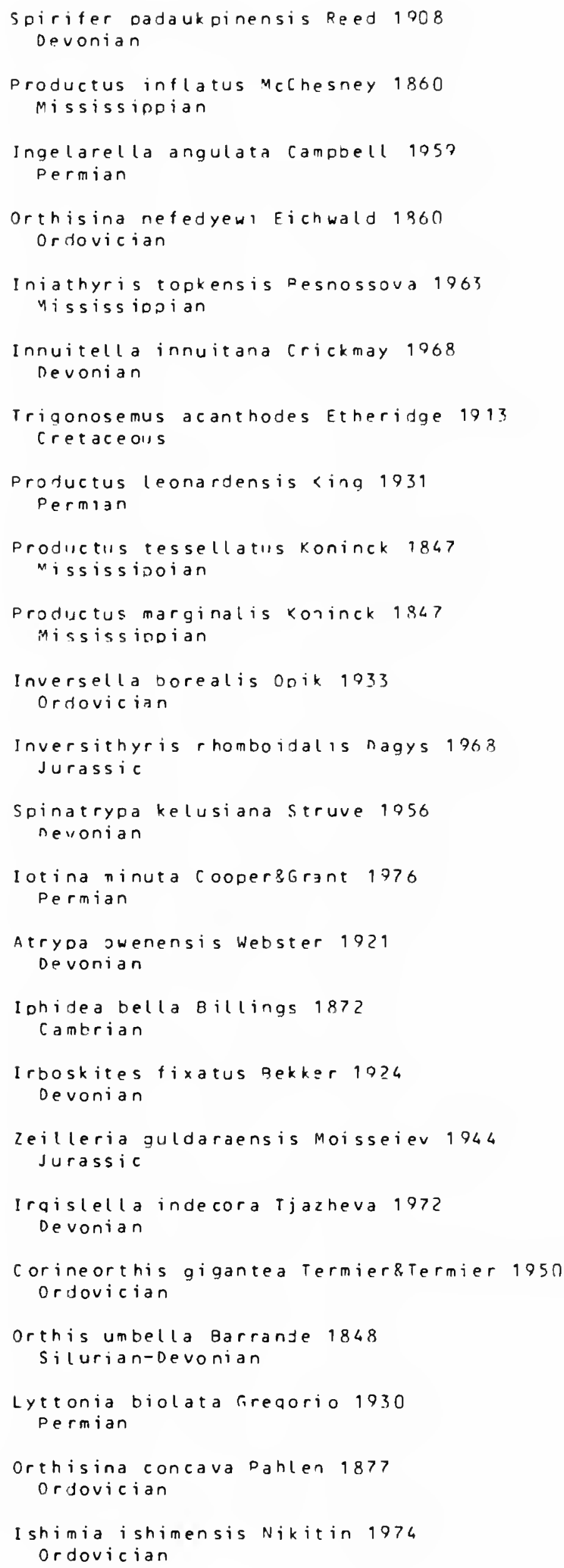




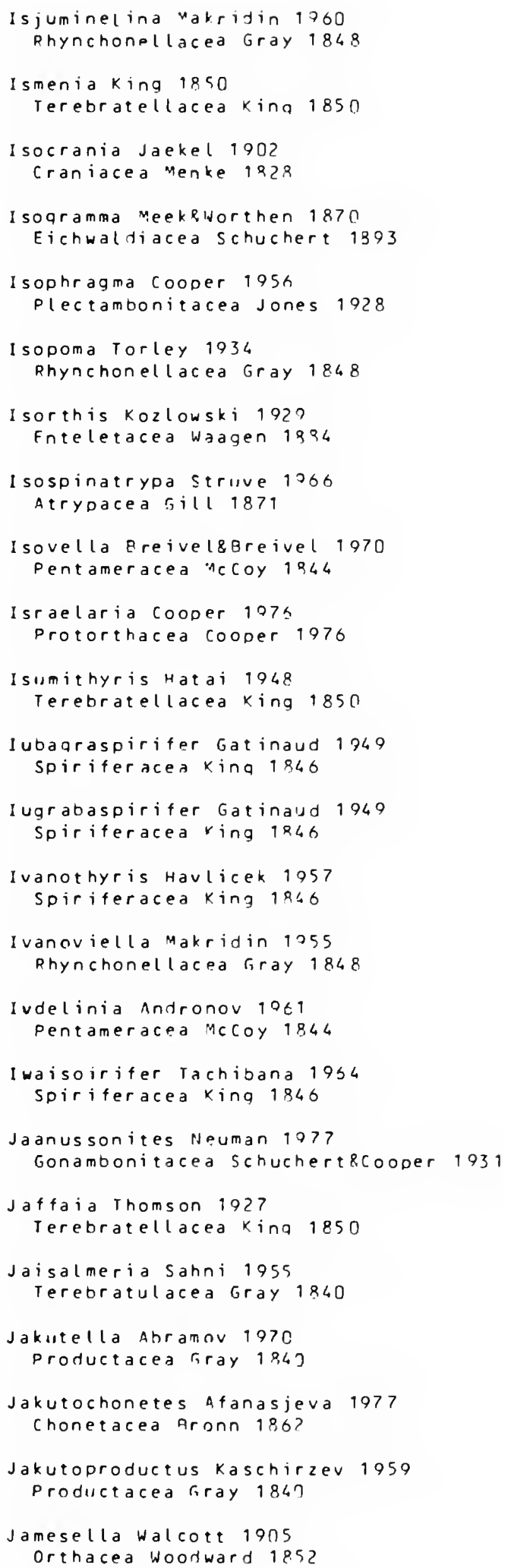

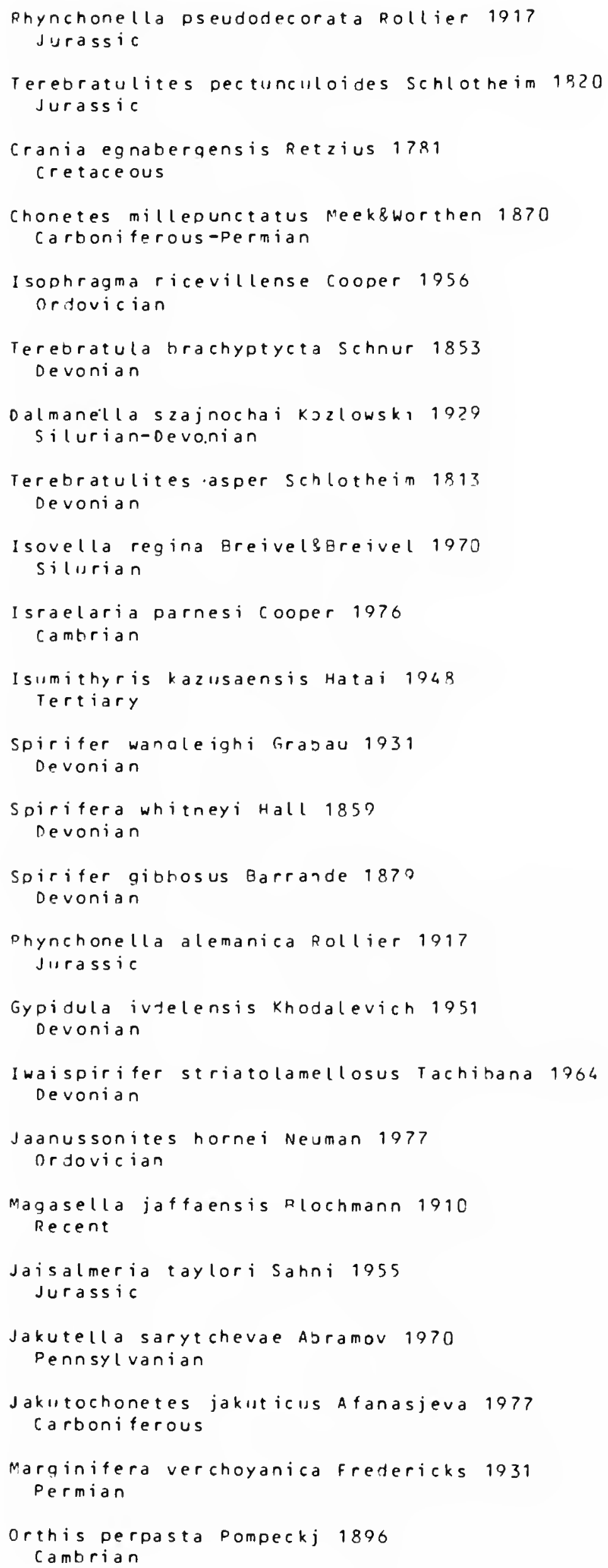




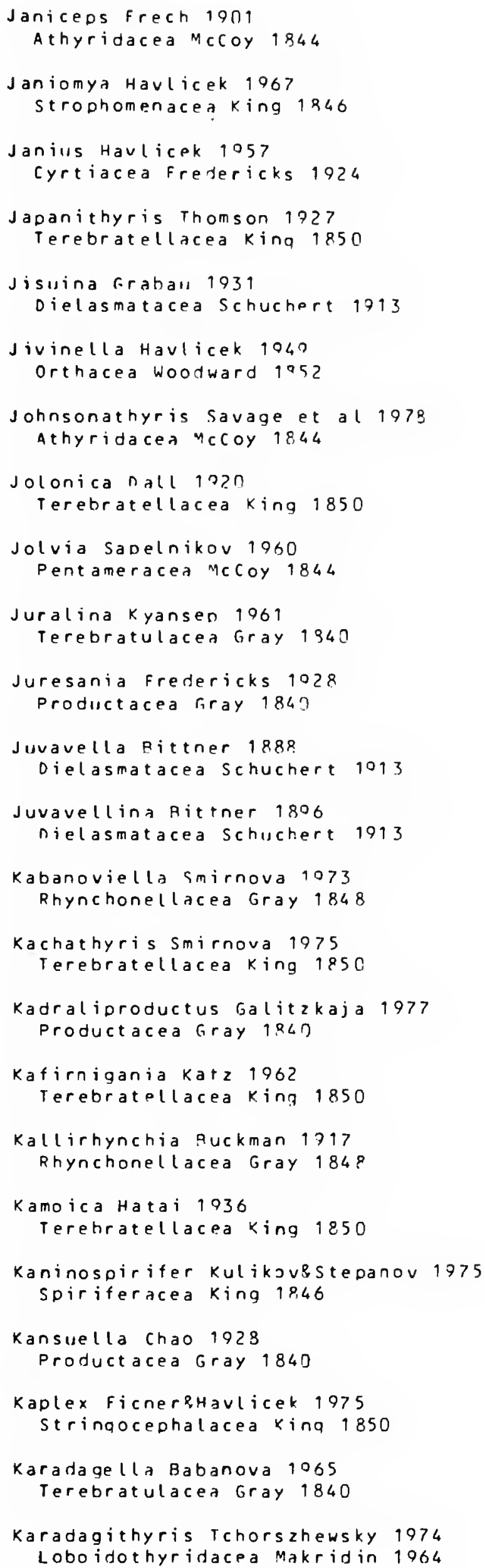

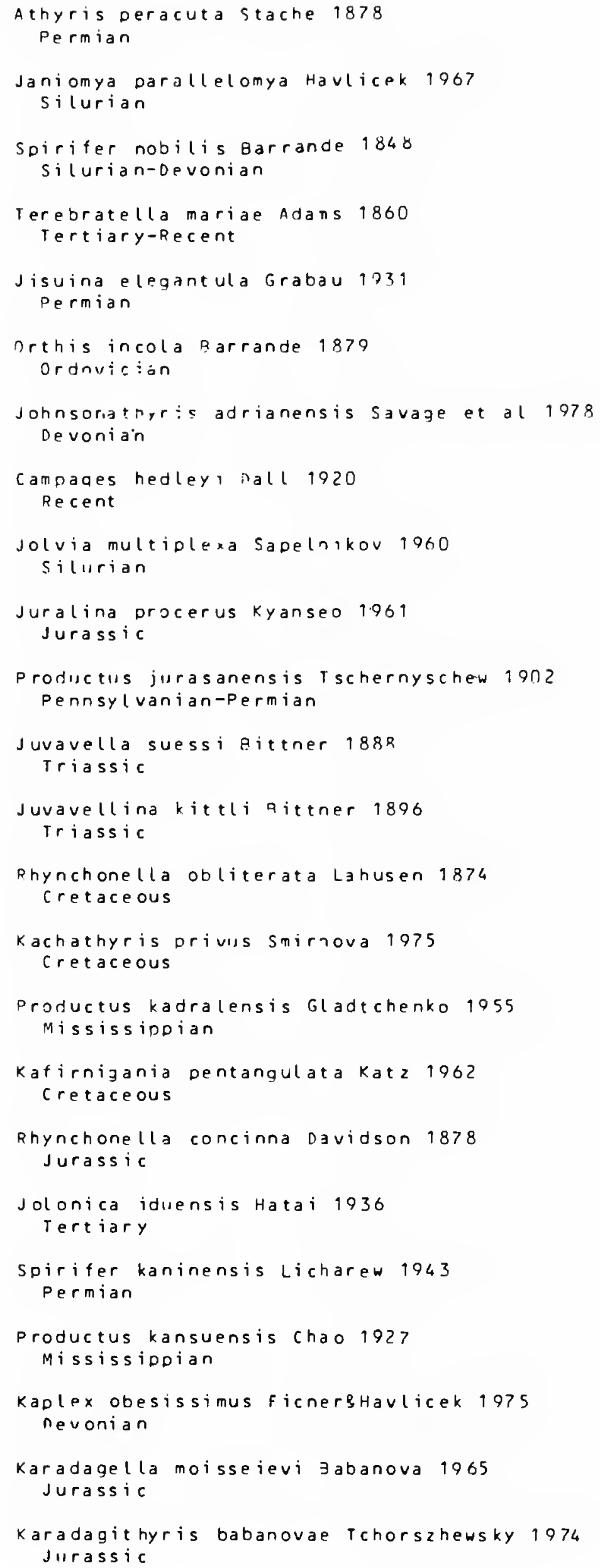


Kingenella Pooiel-Aarczyk 1968 Terebratellacea kinq 1850

Kinghiria Litvinovich 1966 Spiriferacea king 1846

Kinnel la Rergstrom 1068 Enteletacea Wagen 1884

Kirkidium Amsden,Roucot\&Johnson 1967 Pent ameracea "Ac coy $1 \varepsilon 44$

Kirkina salmon 1042

strophomenacea kina 19.40

Kisilia Nalivkin 1979 Spiriferacea King 1846

Kitakamithyris Minato 1951 Reticulariacea wagen 1383

Kjaerina qancroft 1929 Strophomenacea King 1846

Kjerulfina Rancroft $10>9$ Strophomenacea king 1846

KLipsteinella Dagys 1974 suessiacea Waqen 1093

Kochiproductus Nunbar 1955 Productacea roray 19,40

Koeveskallina Dagys 1965 Spiriferinacea navidson 1884

Kolhidaella Moisseiev 1939 Rhyrichonellacea firay 1848

Kolymithyris ranys 1965 Zeilleriacea Allan 1740

Komispirifer Ljaschento 1973 Soiriferacea Kirig 1846

Koninckella Munier-chalmas 1880 Koninckinacea Davidson 1853

Koninckina Suess 1853 Koninckinacea Davidson 1853

Koninckodonta pittner 1893 Koninckinacea Davidson 1853

Konstantia Pajaud 1970 Thecideacea riray 1840

Kosirium FicnerkHavlicek 1975 Cyrtiacea fredericks 1924

Kotujella Andreeva 1962 Billingsellacea Schuchert 1893

Kozlowskia Fredericks 1933 Productacea firay 1840

Kozlowskiellina Rourcot 1958 Spiriferacea king 1846

Kozlowskites Havlicek 1952 Plectamonitacea Jones 1928

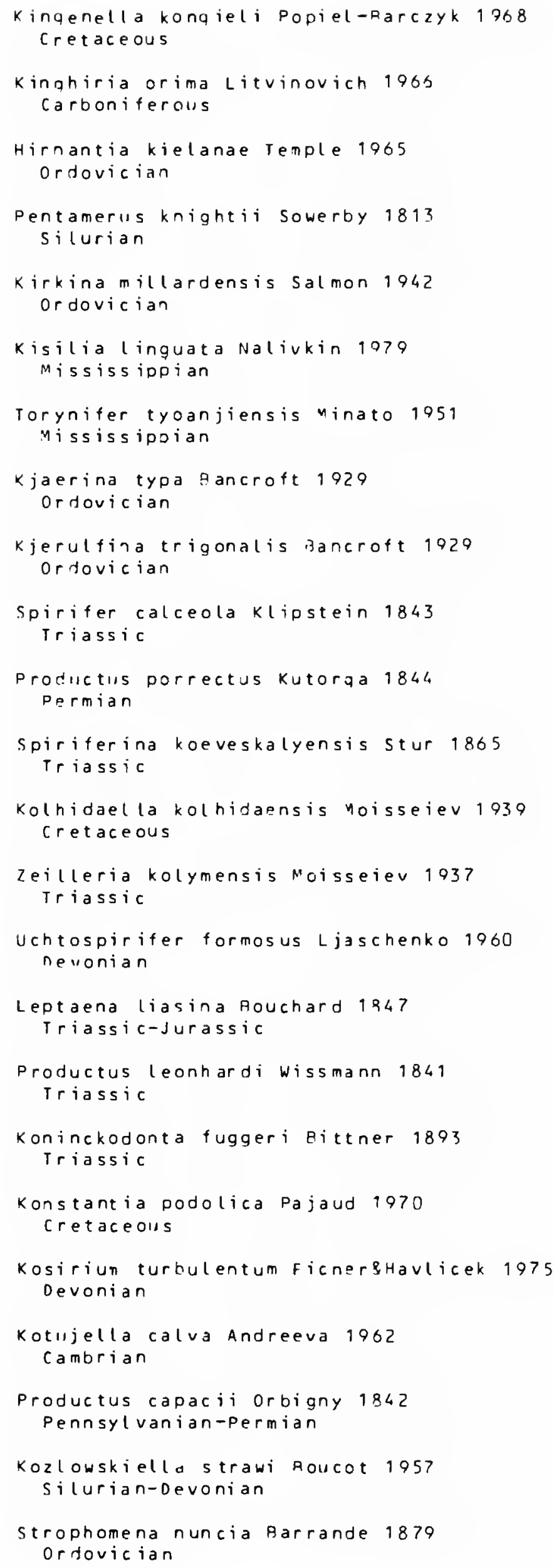




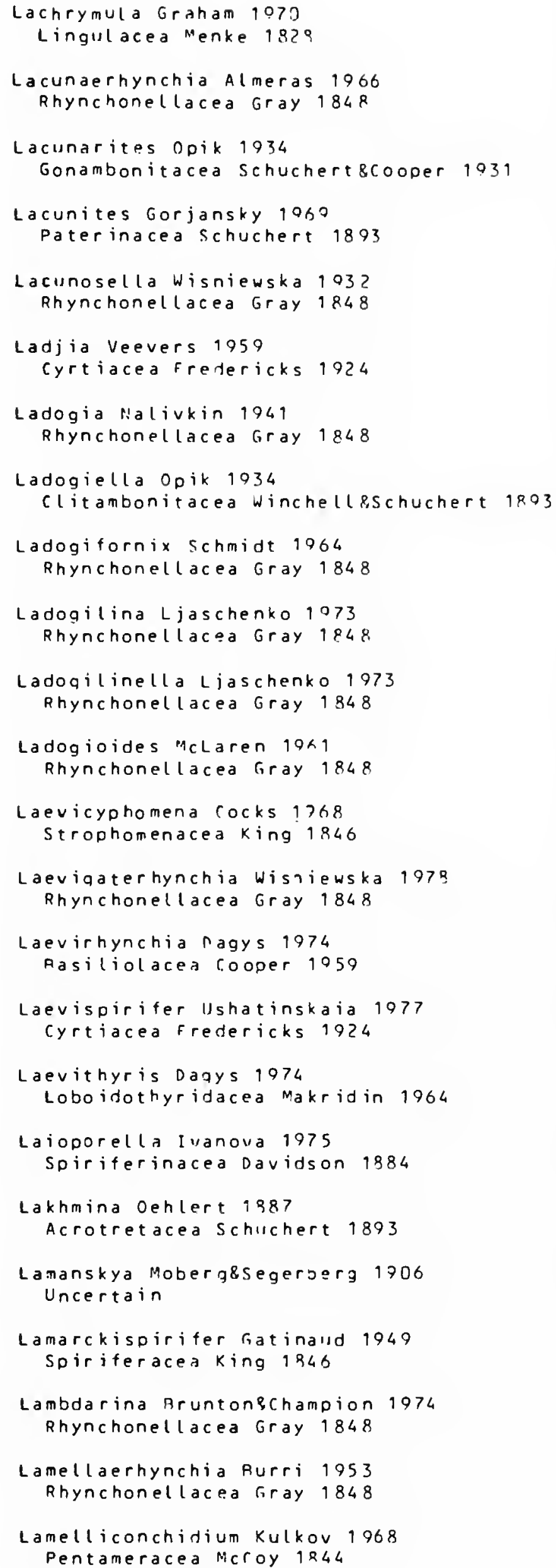

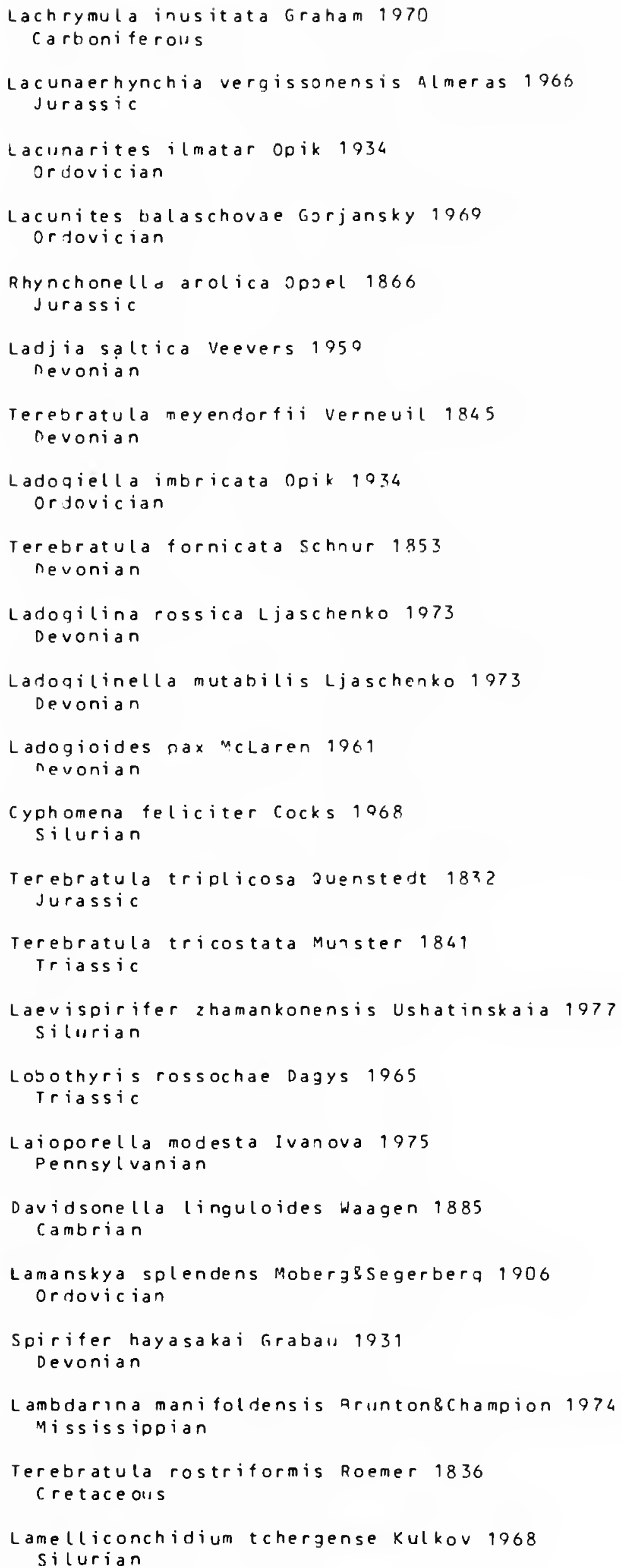


Lamellokoninckina Jinkfang 1977 Koninckinacea Davidson 1853

Lamellosia CooperRGrant 1975 Chonetacea Aronn 1.86?

Laminatia M11ir-NoodRcosoer 1960 Productacea Gray 1840

Lamnaspina waterhouse 1976 Spiriferinacea Davidson 9884

Lamnimargus watarhouse 1975 Productacea firay 184 ?

Lancangjiangia Jinf Fang 1777 Spiriferinacea Davidson 1984

Lanceomyonia Havlicek 1060 Rhynchonellacea Gray $184 \%$

Langella Mendes 1061

Lingulacea Menke 122 ?

Langkawia Hamada 1960

Rhynchonellacea Gray 1848

Laosia Mansuy 1213

Rhynchonel lacea Gray $18: 8$

Lanueus rall aroc

Terebratellacea king 1850

Larium Gregorio 1930

Uncertain

Lateralatirostrum Sartenaer 1979

Rhynchonel lacea Gray 1848

Lathamella Liu 1970 uncertain

Laticrura Cooner 1056 Enteletacea wagen 9884

Latiplecus LeeRGu 1976 Reticulariacea wagen 1883

Latiproductus carytcheva et al 1077 Productacea firay 184 ?

Latonotoechia Havlicek 1960 Rhynchonellacea Gray 1848

Lazutkinia Rzonsnitzkaja 1952 Spiriferacea king 1946

Leanqella npik 1933 Plectambonitacea Jones 1928

Leiochonetes poberts 1976 Chonetacea Aronn 1862

Leiolepismatina Yang $\& \times u \quad 1966$ Spiriferinacea Davidson 1884

Leioproductus stainbrook 1947 productacea firay 184 ?

Leiorhynchoidea Cloud 9944 Rhynchonel lacea Gray 1848

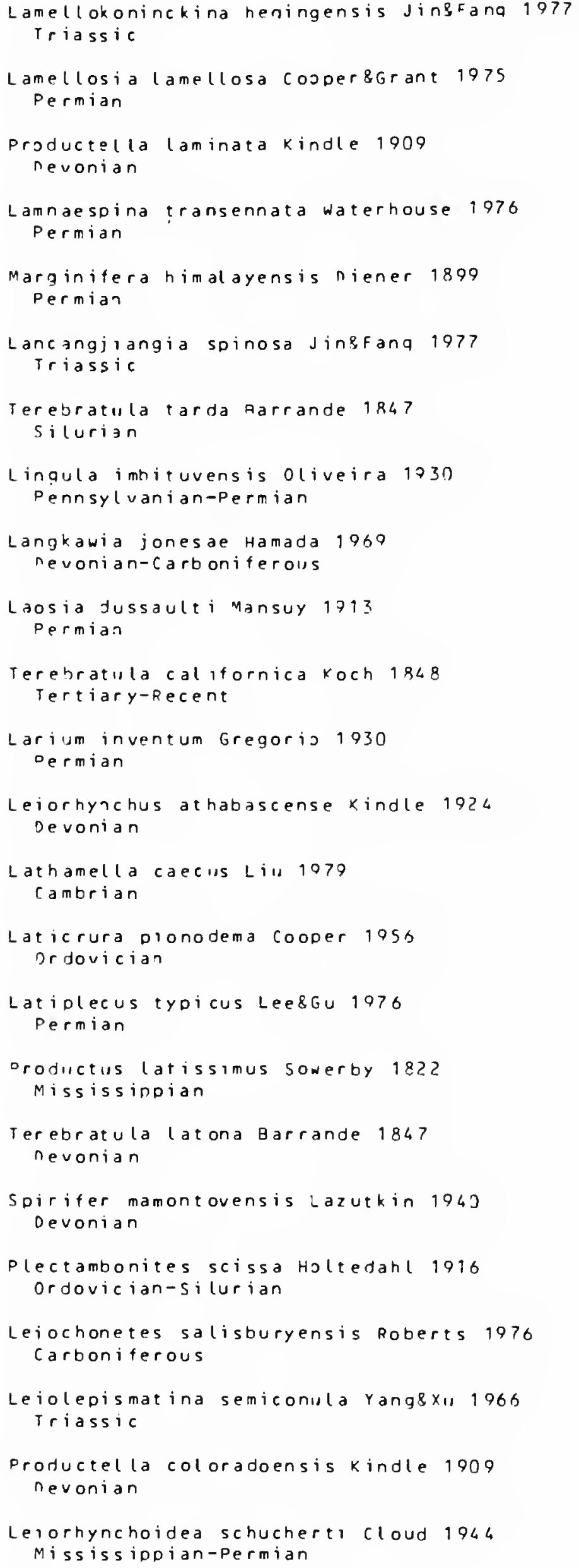


Leiorhynchoides novgal 1953 Rhynchonel lacea Gray 1848

Leiorhynchus Hall 1860 Rhynchonel lacea firay 1848

Leioria Cooper 1976 orthacea Woodward 105?

Leioseptathyris Wu 1974 Stringocephalacea King 1850

Lenatoechia Nikiforova 1970 Rhynchonellacea Gray 1848

Lenorthis Andreeva 1755 Orthacea Noodward 19.52

Lenothyris Daqys 1969 Loboidothyridacea Makridin 1964

Lepidocrania Cooper\&Grant 1974 Craniacea Menke 1,9?

Lepidocycloides Nikiforova 1961 Rhynchonellacea Gray 1848

Lepidocyclus wang $194^{\circ}$ Rhynchonel lacea Gray 1848

Lepidoleptaena Havlicek 1963 Strophomenacea Kina 1846

Lepidorhynchia Aurri 1056 Rhynchonellacea Gray 1848

Lepidorthis Wang 1955 Orthacea woodward 1852

Lepidospirifer Conper?Grant 1969 Soir iferacea $\mathrm{king} 1846$

Leoismatina wang 1955 Suessiacea iaacen 1883

Leptaena Dalman $18.2 \%$ Stroohomenacea king 1846

Leptaenella Fredericks 1918 strophomenacea king 1846

Leptaenisca Reecher 1 Rु? Strophomenacea king 1946

Leptaenoidea Hedstrom 1917 Strophomenacea King 1846

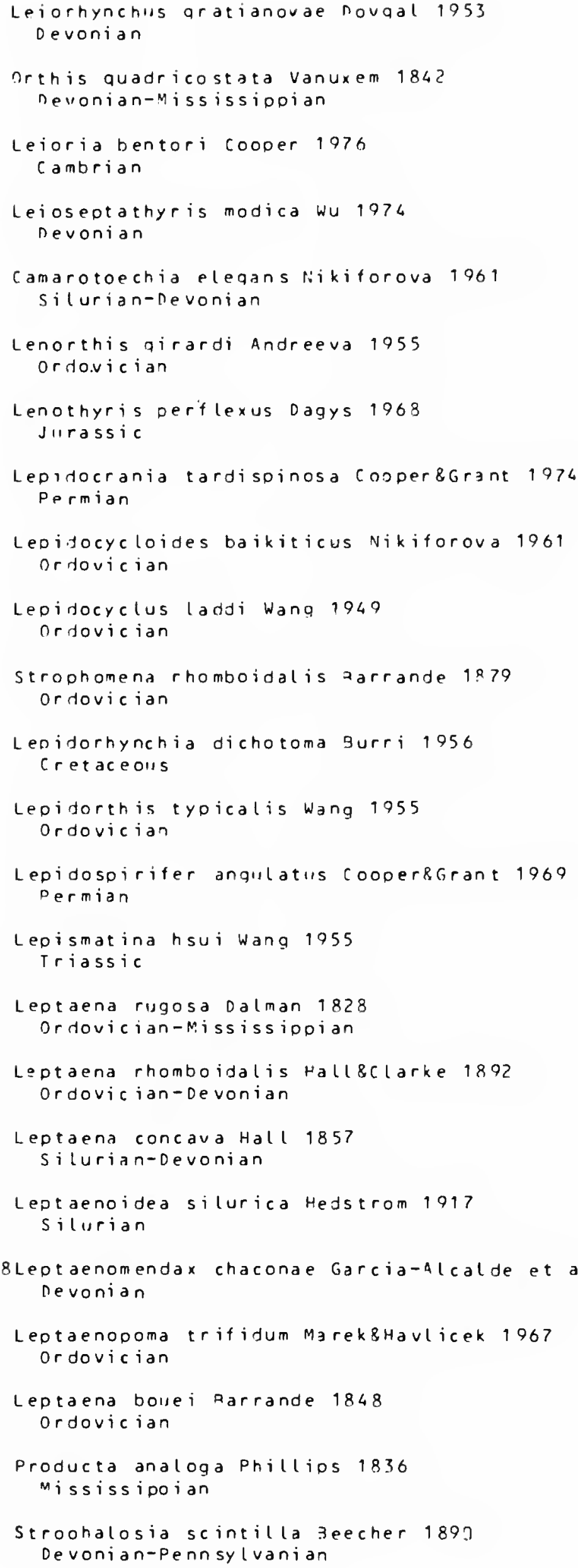




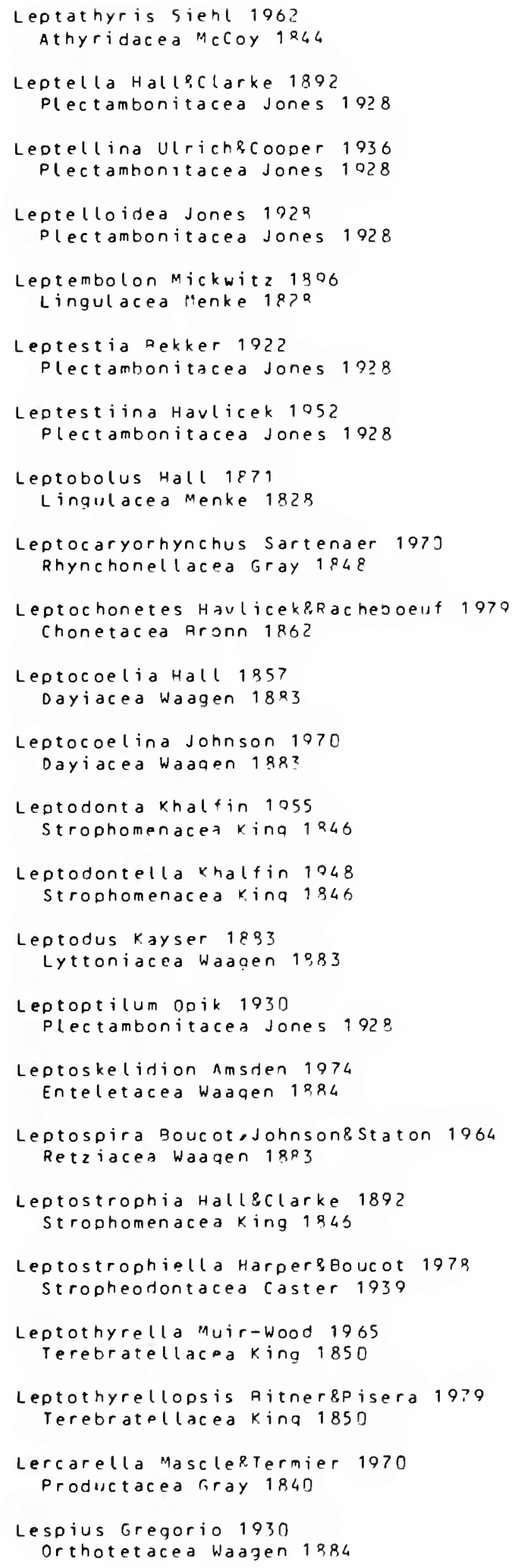

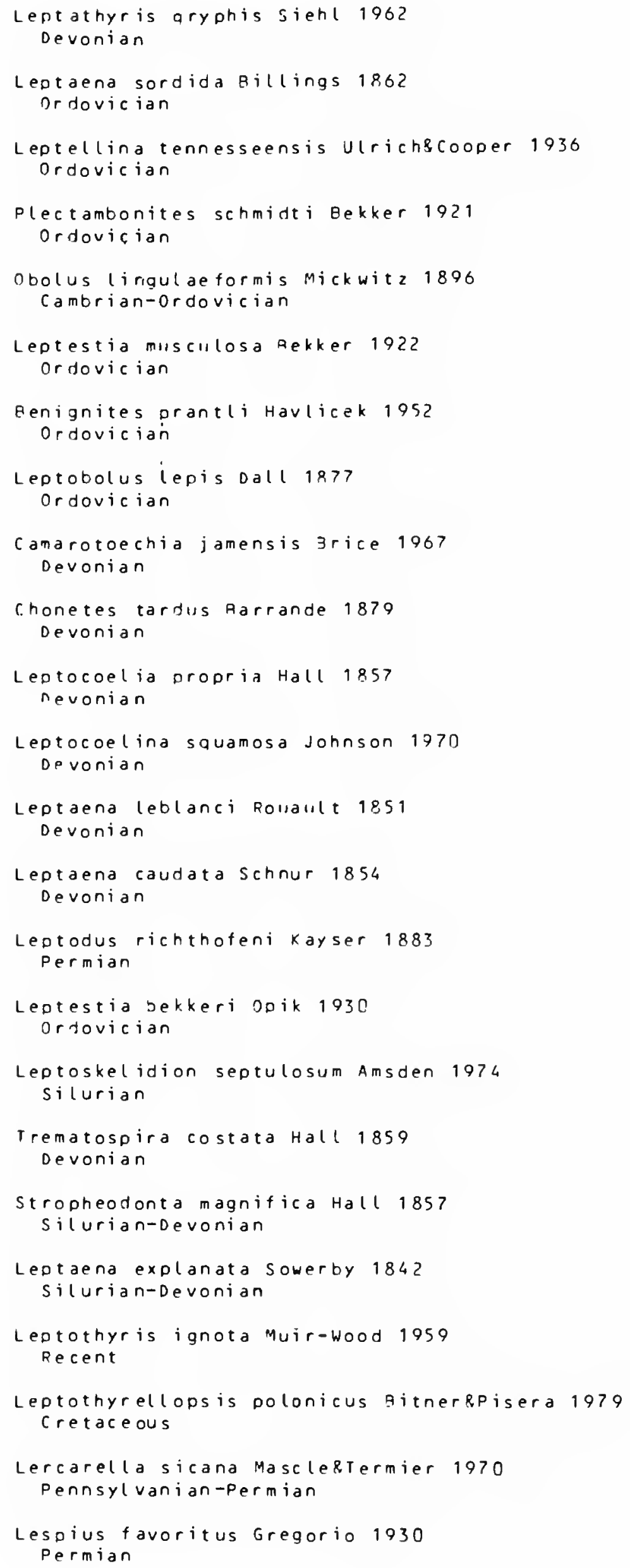




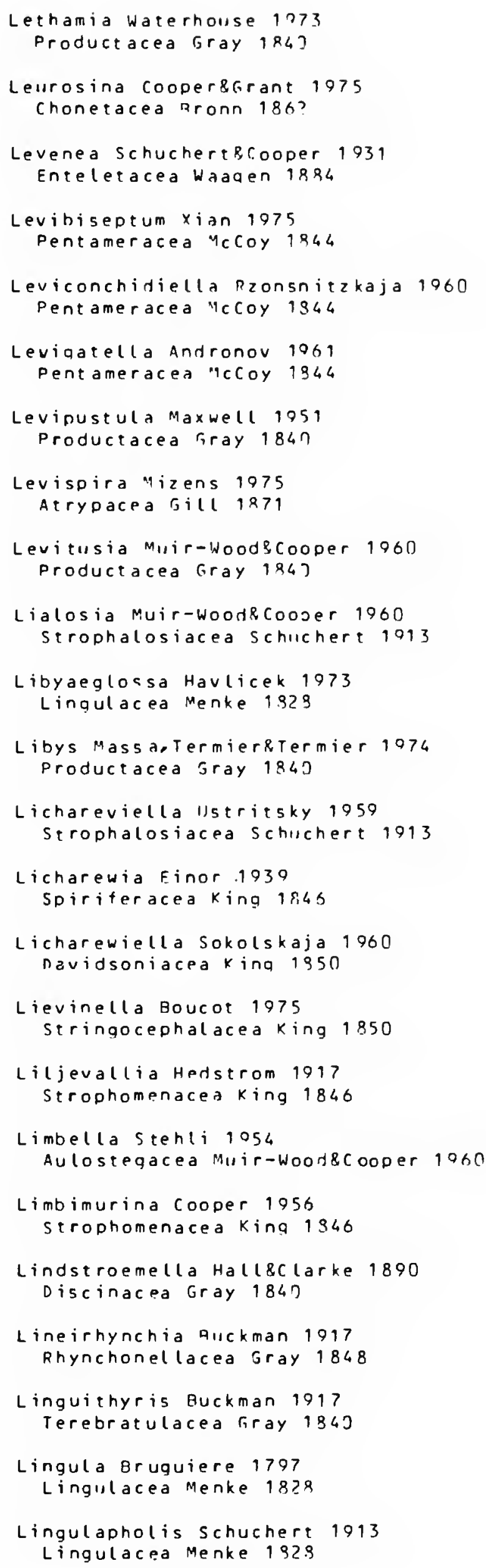

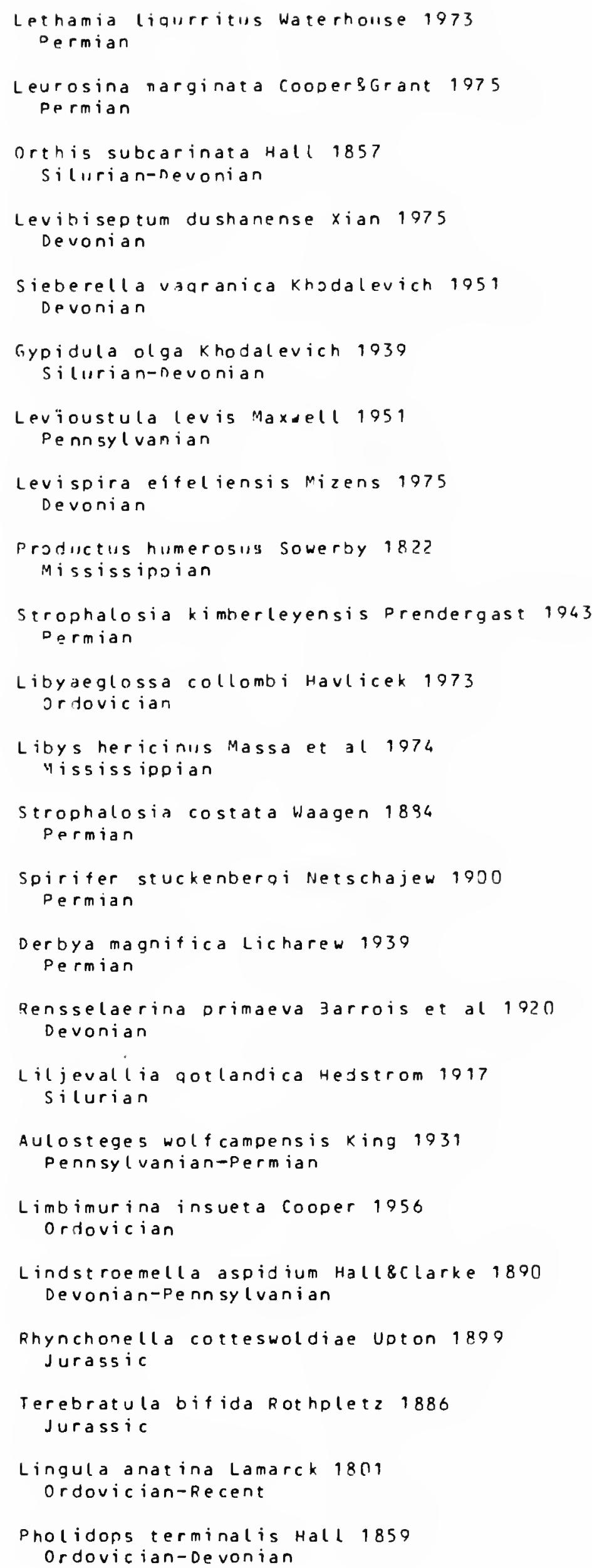




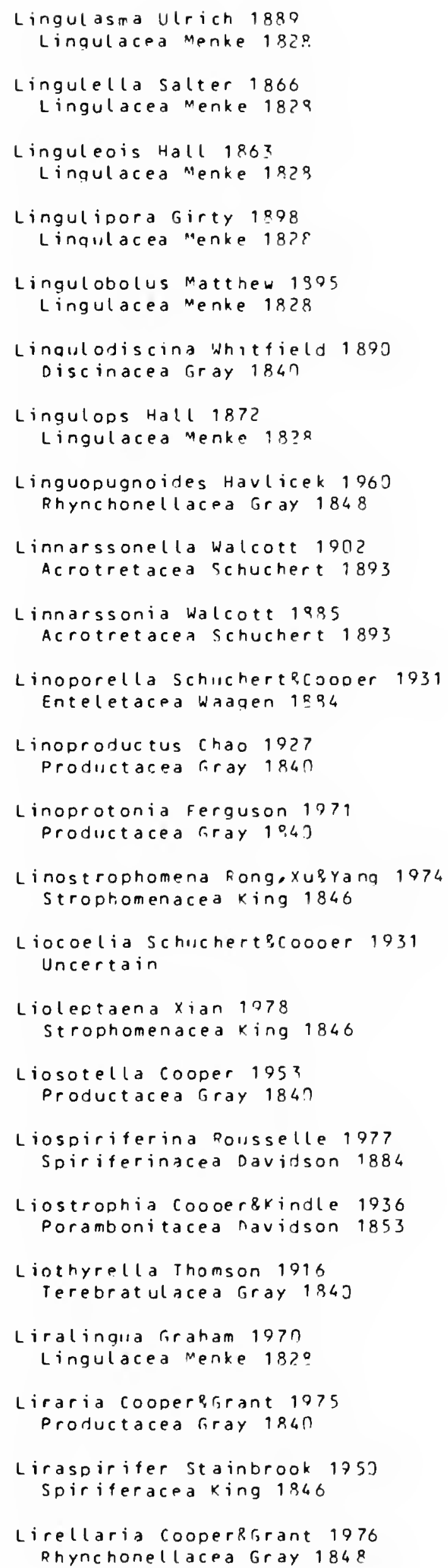

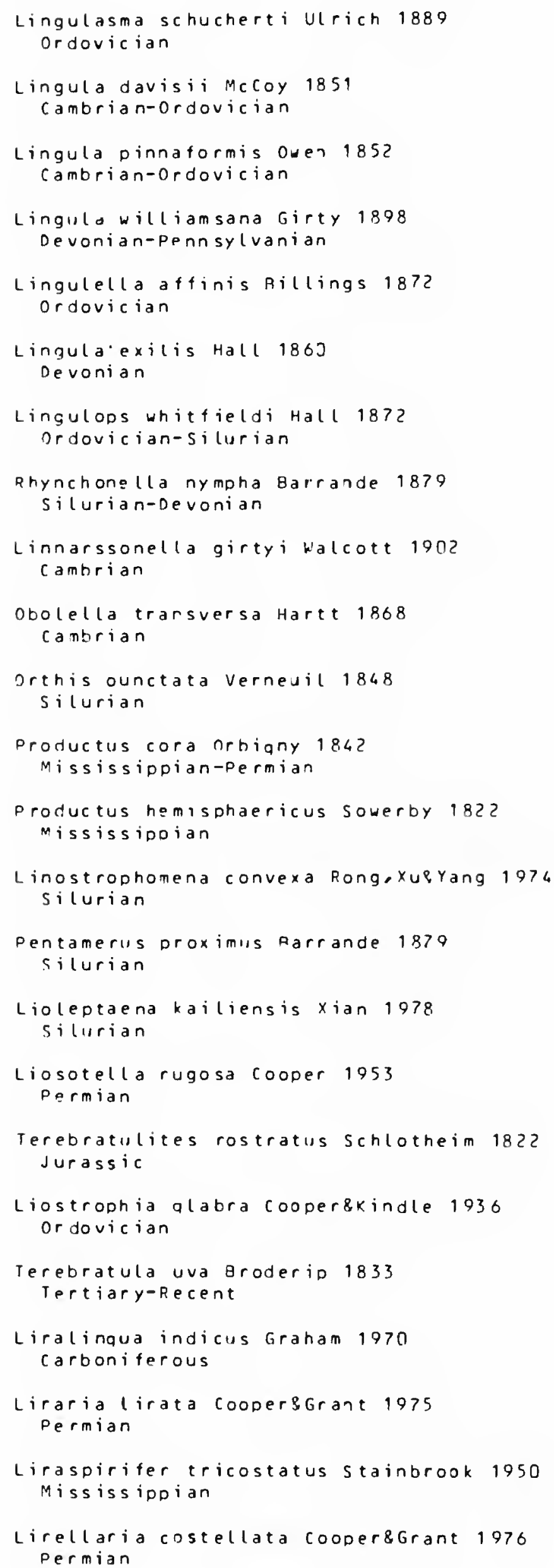


Liricamera cooper 1956

Porambonitacea Davidson 1.853

Liriplica Campbell 1961

Spiriferinacea Davidson 1884

Lissajousithyris Almeras 1971

Terehratulacea Gray 1840

Lissatrypa Twenhofel 1714 Atrypacea Gill 1871

Lissatrypoidea Moucot?4msden 1958 Atryoacea filll 1971

Lissella campbell 1961

Rhynchonellacea Gray 1848

Lissochonetes Dunbar\&Condra 1932 chonetacea aronn 1862

Lissocoelina schuchert 3 Cooper 1931 Pentameracea McCoy 1944

Lissocrania Williams 1043 Craniacea Menke 18 ? ?

Lissomarginifera Lane 1962 productacea firay 1840

Lissopleura whitfield 1906 Rhynchonel lacea Gray 1848

Lissorhynchia Yanql $x_{11} 1066$ Rhynchonel lacea Gray 1848

Lissosia Cooperecrant 1975 chonetacea aronn 1962

Lissostrophia Amsden 1049 Strophomenacea king 1846

Lissotreta Amsden $196^{\circ}$. Trioles iacea Schuchert 1913

Litocothia Grant 1976 Lyt

Litothyris Roberts 1971 Spiriferacea king 19.46

Ljaschenkovia Ratrukova 1969 Discinacea Gray 184 ?

Ljudmilispirifer Tcherkesova 1976 Cyrtiacea Fredericks 1924

Llanoella Boucot 1975 Porambonitacea navidson 1853

Loboidothyris 9uckman 1917 Terebratulacea Gray 1840

Loboidothyropsis sucic-Protic 1971 Loboidothyridacea Makrifin 1964

Lobothyris Risckman 1917

Terebratulacea Gray 1840

Lobothyroides $x u 1978$ Terebratulacea Gray 1940

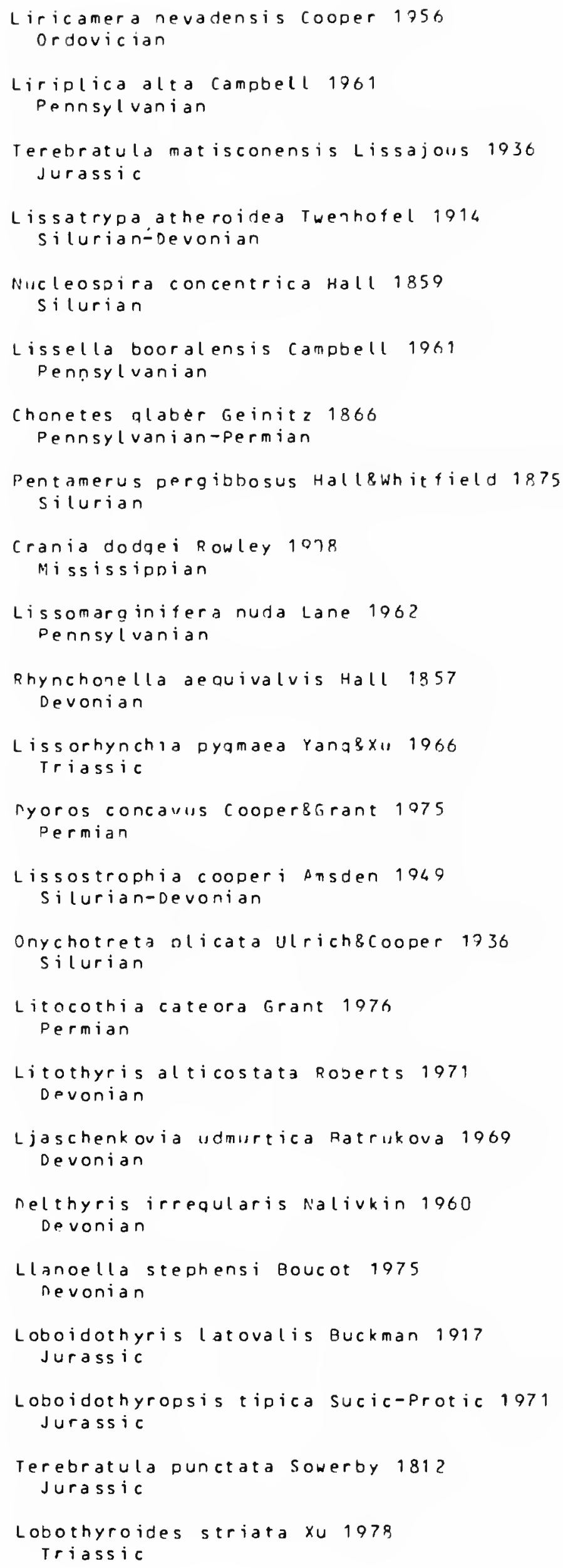




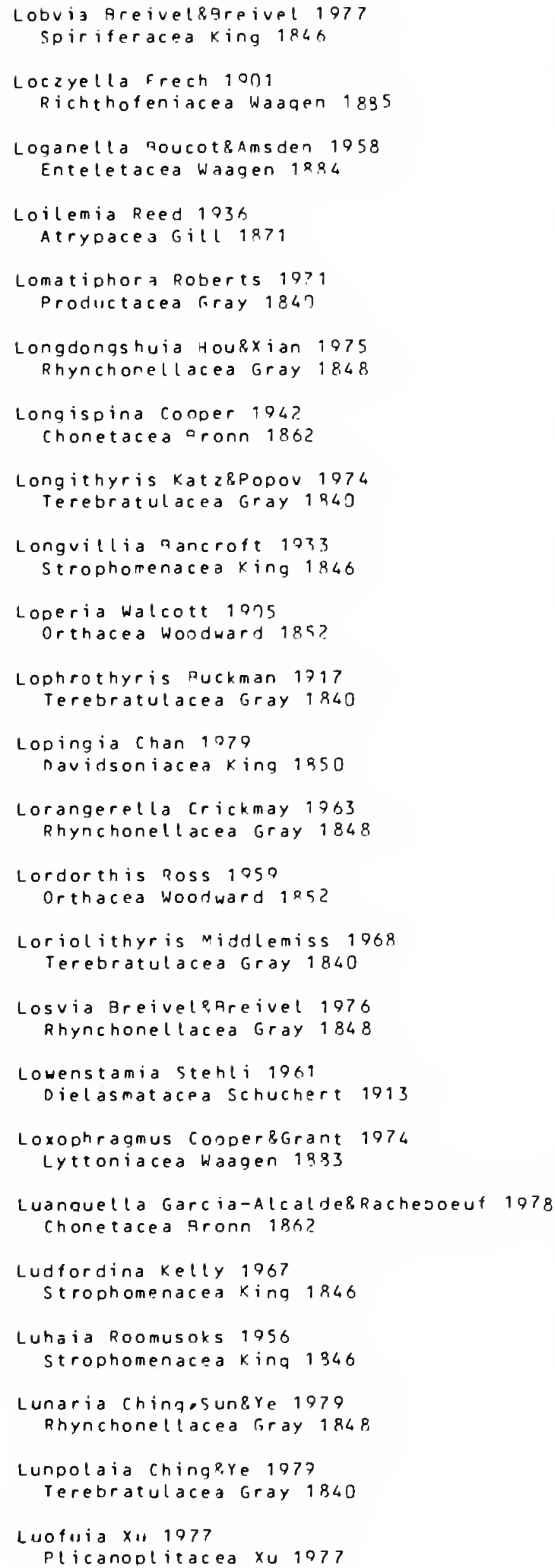

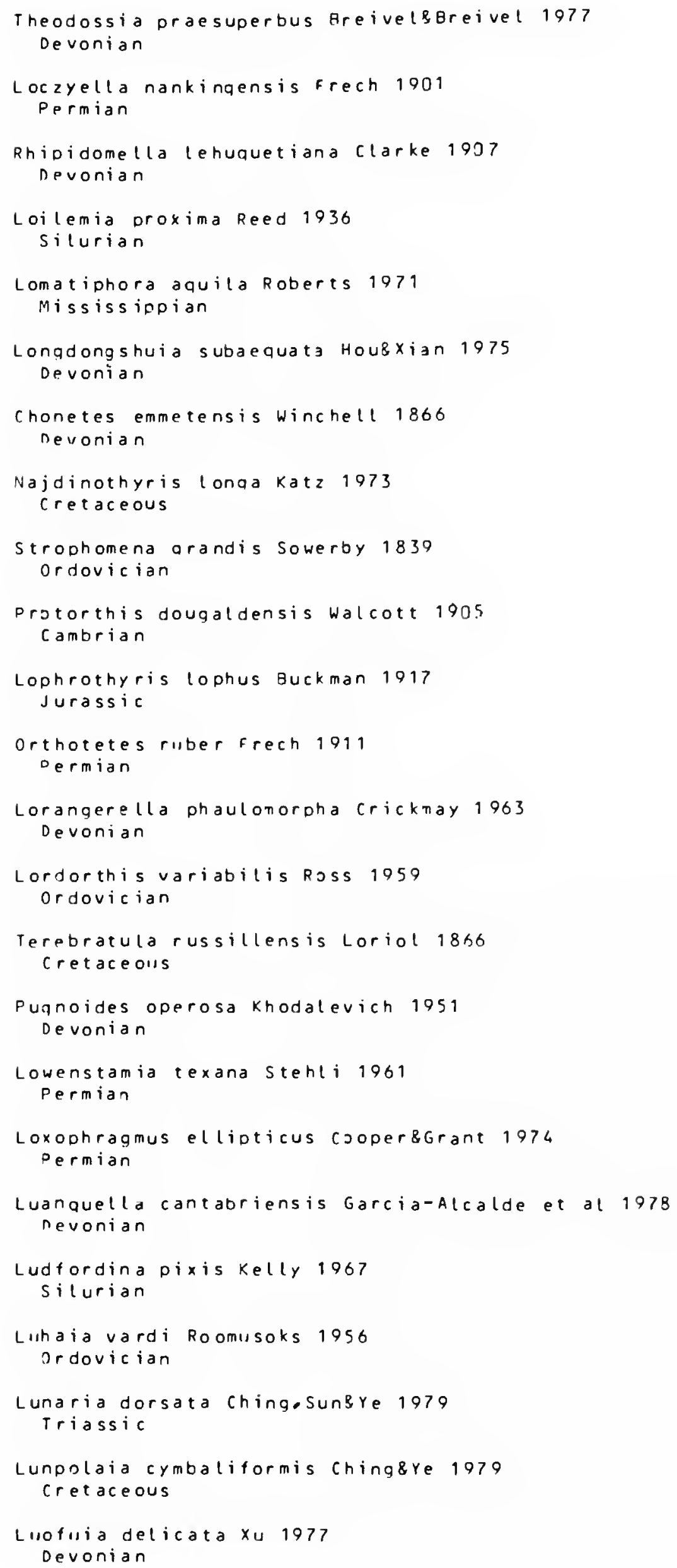


Lutetiarcula El liott 1054

Terebratellacea Kina 1850

Lycophoria Lahusen 19,96

Porambonitacea Davidson 1853

Lyra Cumberland 1918

Terebratellacea King 1850

Lytha Fredericks 1974

Spiriferacea King 1846

Lyttonia wagen 1883

Lyttoniacea Wagen 1993

Makina Andreeva 1061

Strophomenacea $\mathrm{King} 1846$

Macandrevia $\mathrm{King} 1859$

Terebratel Lacea Kinq 1850

Machaeraria Cooner 1955

Rhynchonel lacea firay 1848

Mackerrovia Cocks 1968 Strophomenacea King 1946

Maclarenella stehli 1955

Dielasmatacea Schuchert 1713

Macrocoelia Cooper 1956 Strophomenacea kina 1846

Macroplectane cossmann 1909 Stringocephal acea King 1850

Macropleura Roucot 1963 Cyrtiacea Fredericks 1924

Macropotamorhynchus Sartenaer 1970 Rhynchonellacea Gray 1848

Madarosia Coooer \&6rant 1976 Rhynchonel lacea firay 184 \%

Magadania Ganel in 1077 Productacea Gray 184 ?

Magadina Thomson 1915 Terebratellacea King 1850

Magadinella Thomson 1915 Terebratellacea Kina 1850

Magas Sowerby 1918 Terebratel lacea King 1850

Magasella nall 1870

Terebratellacea King 1850

Magella Thomson 1015 Terebratellacea King 1850

Maqellania Rayle 1880 Terebratellacea King 1850

Magharithyris Farag\&Gat inaud 1960 Terebratulacea Gray 1840

Magnicanalis Rowell 1962

Obolellacea Walcott \& S chuchert 1908

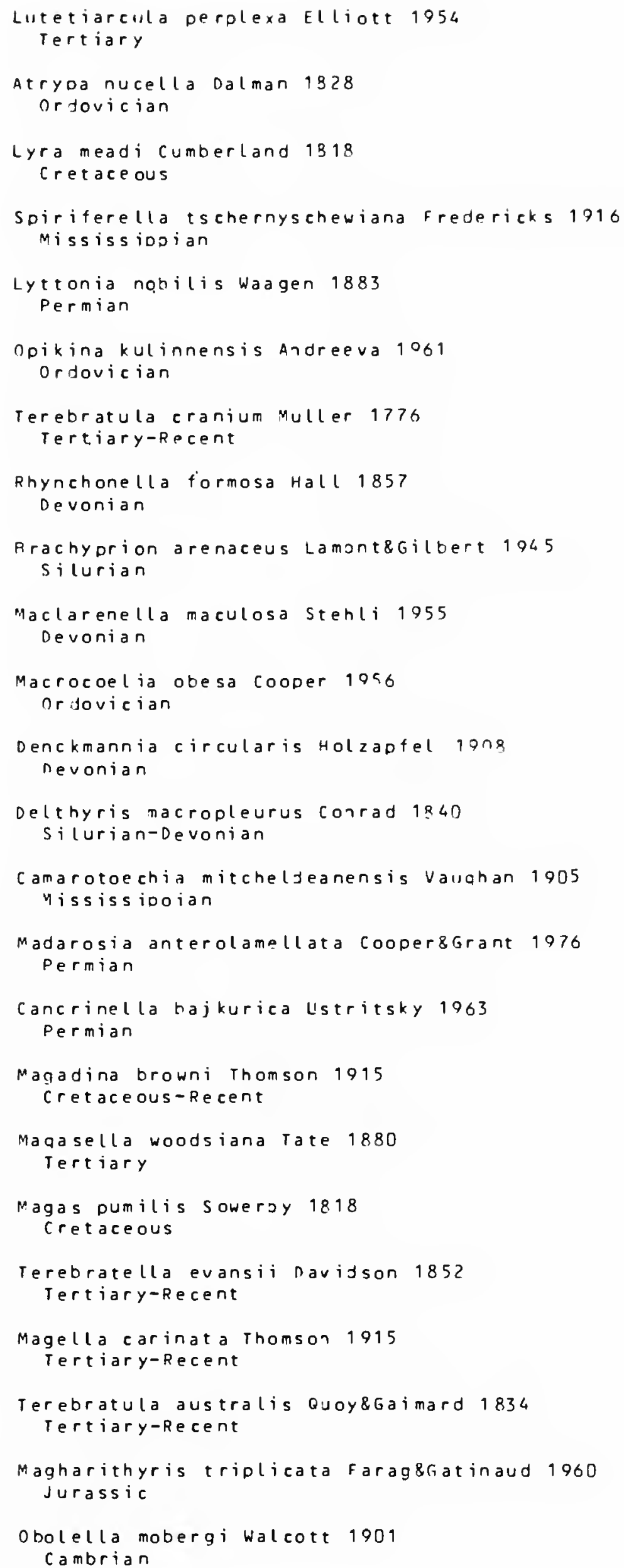




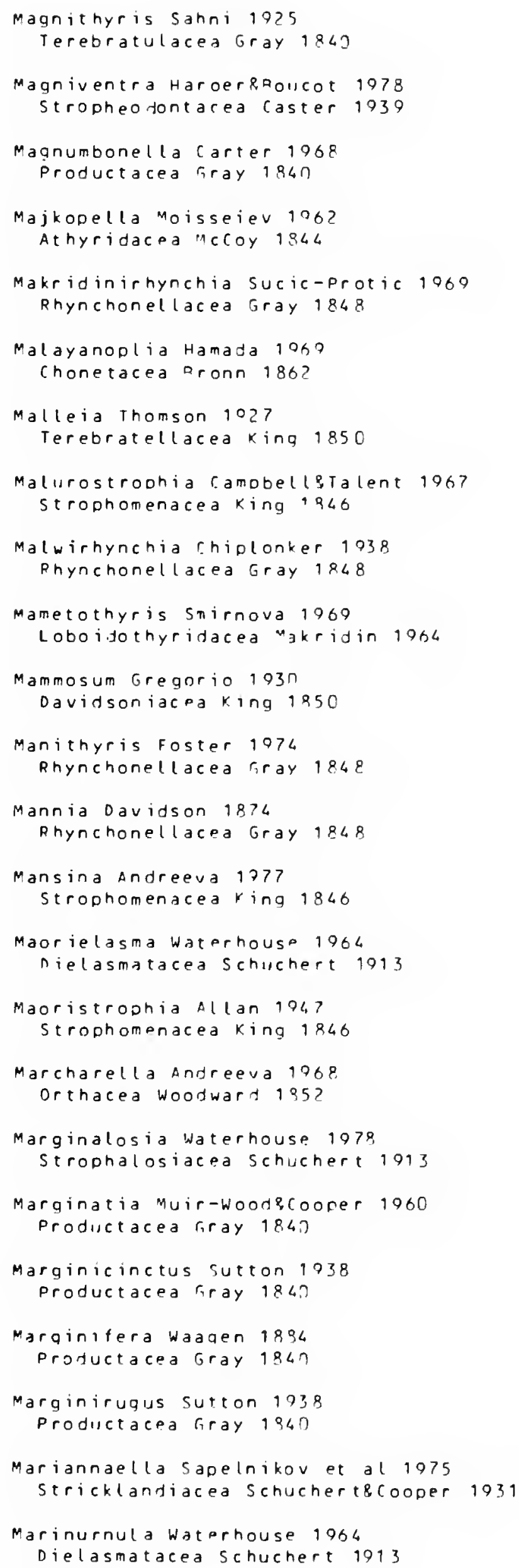

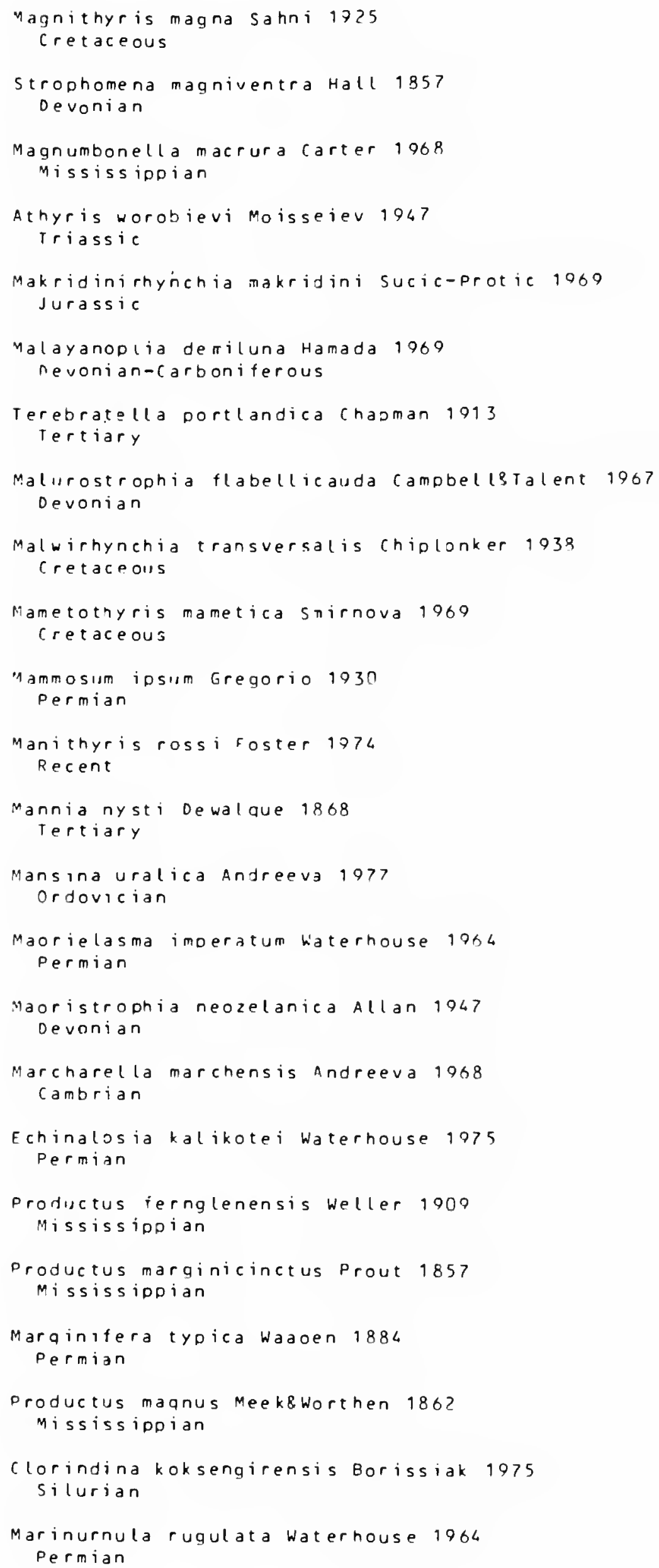




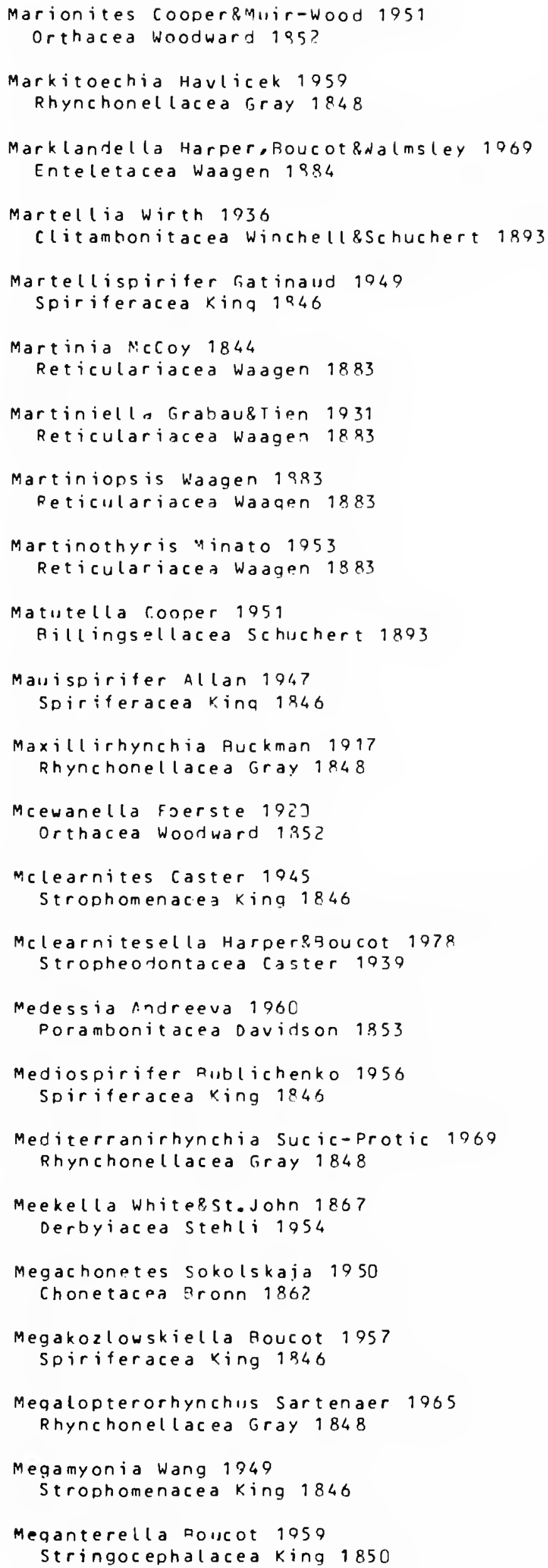

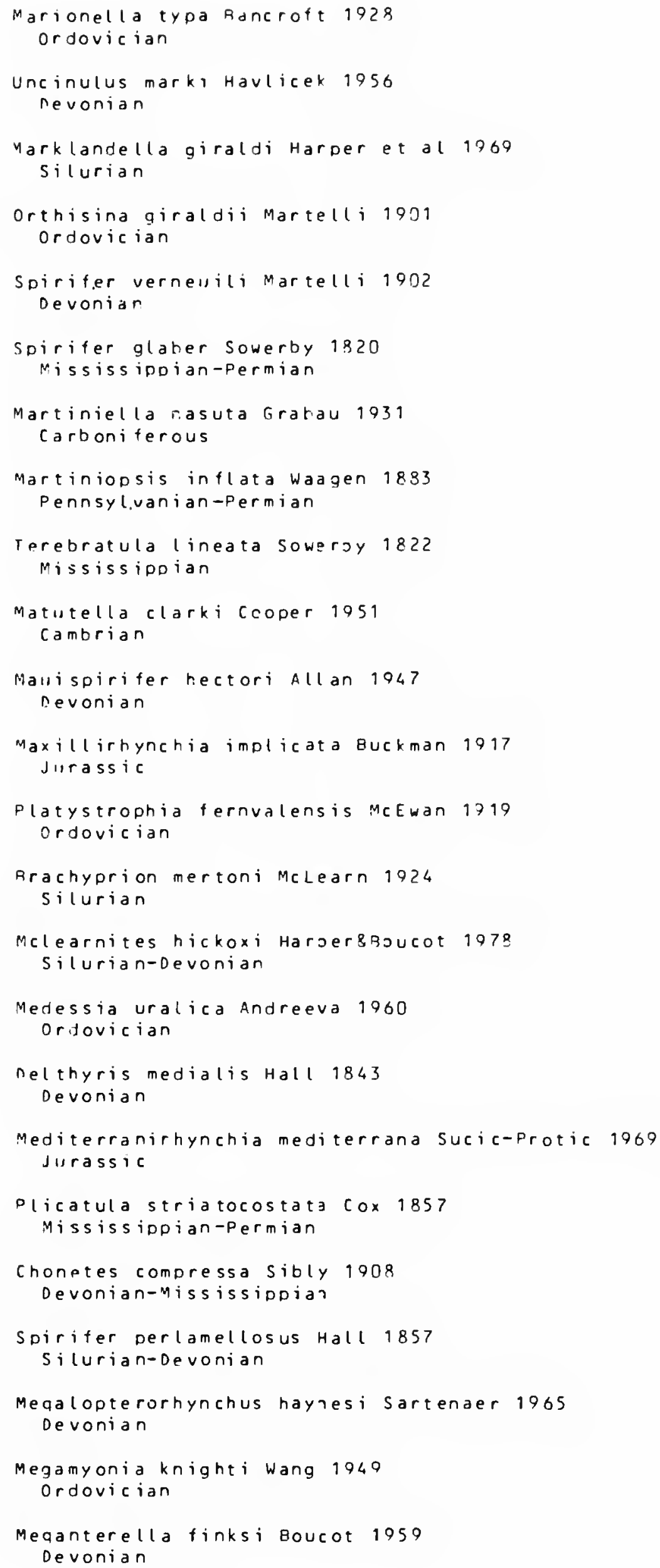




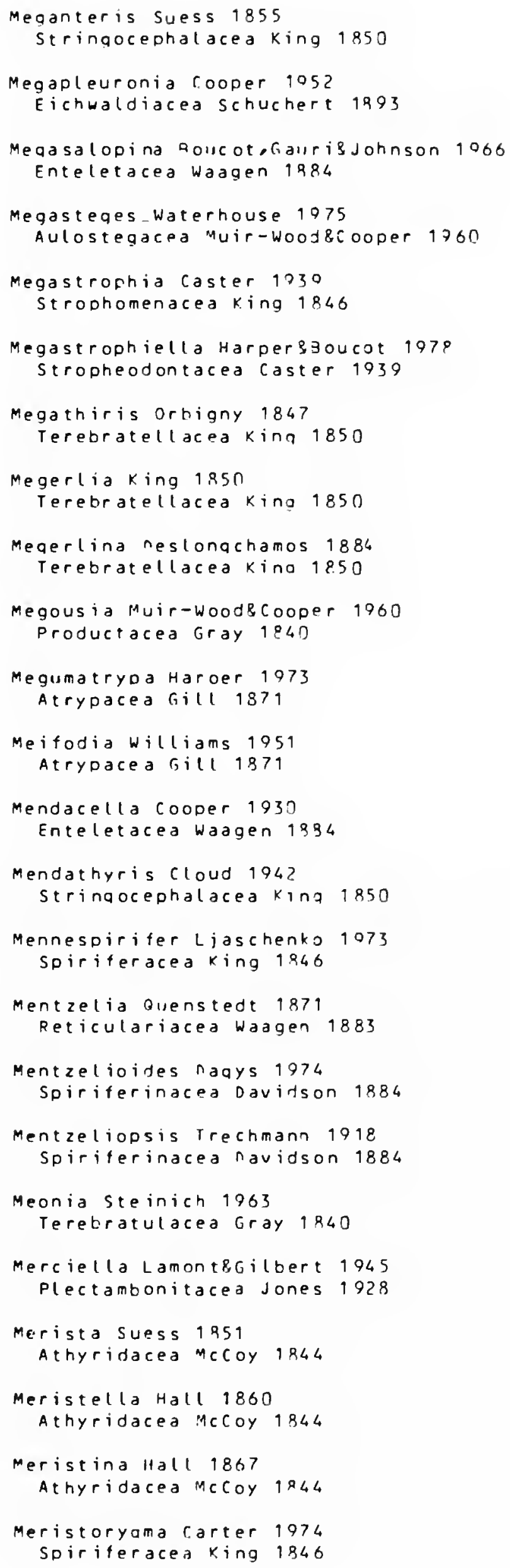

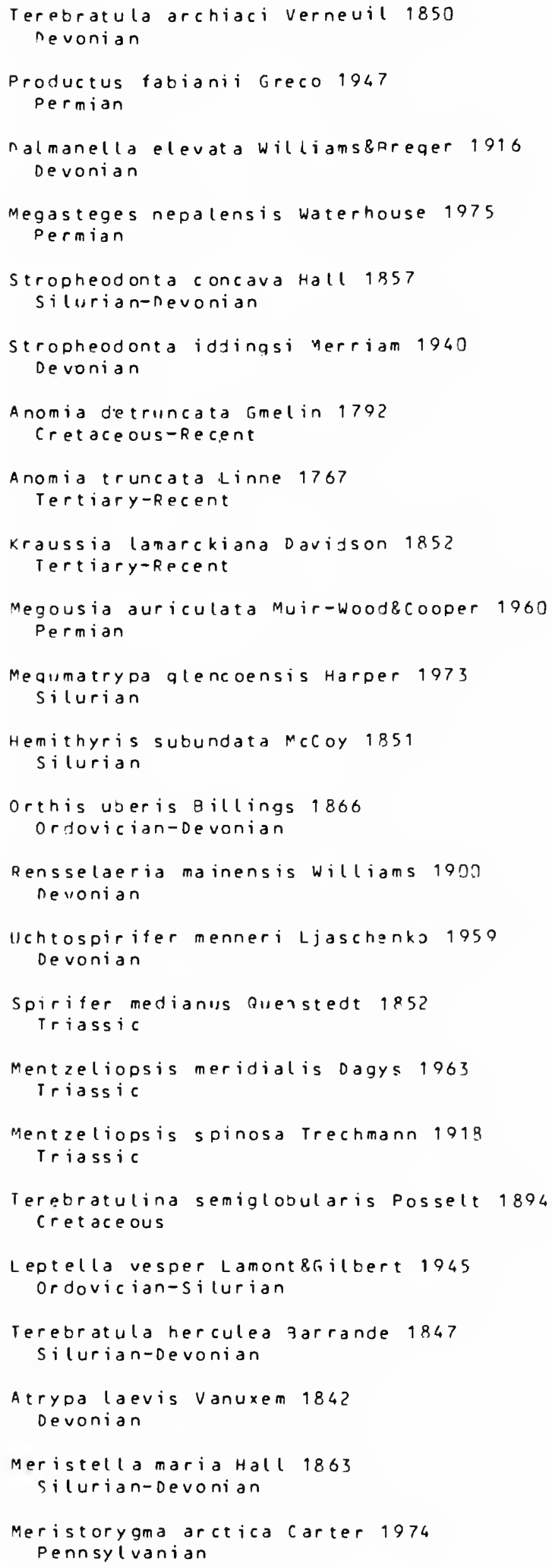




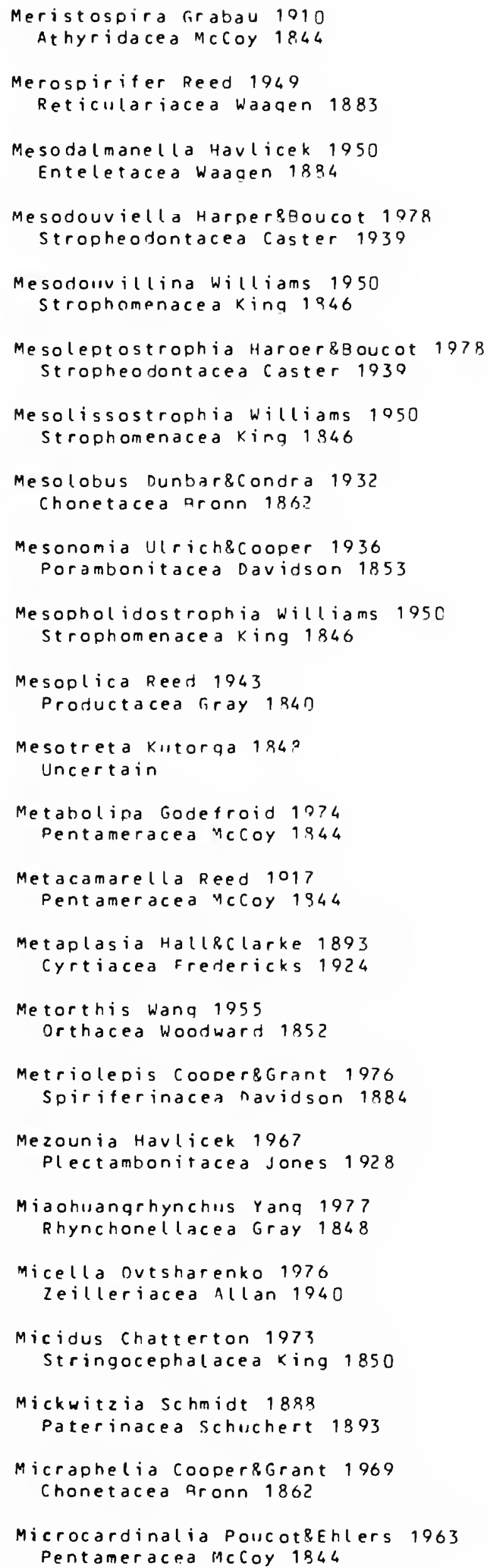

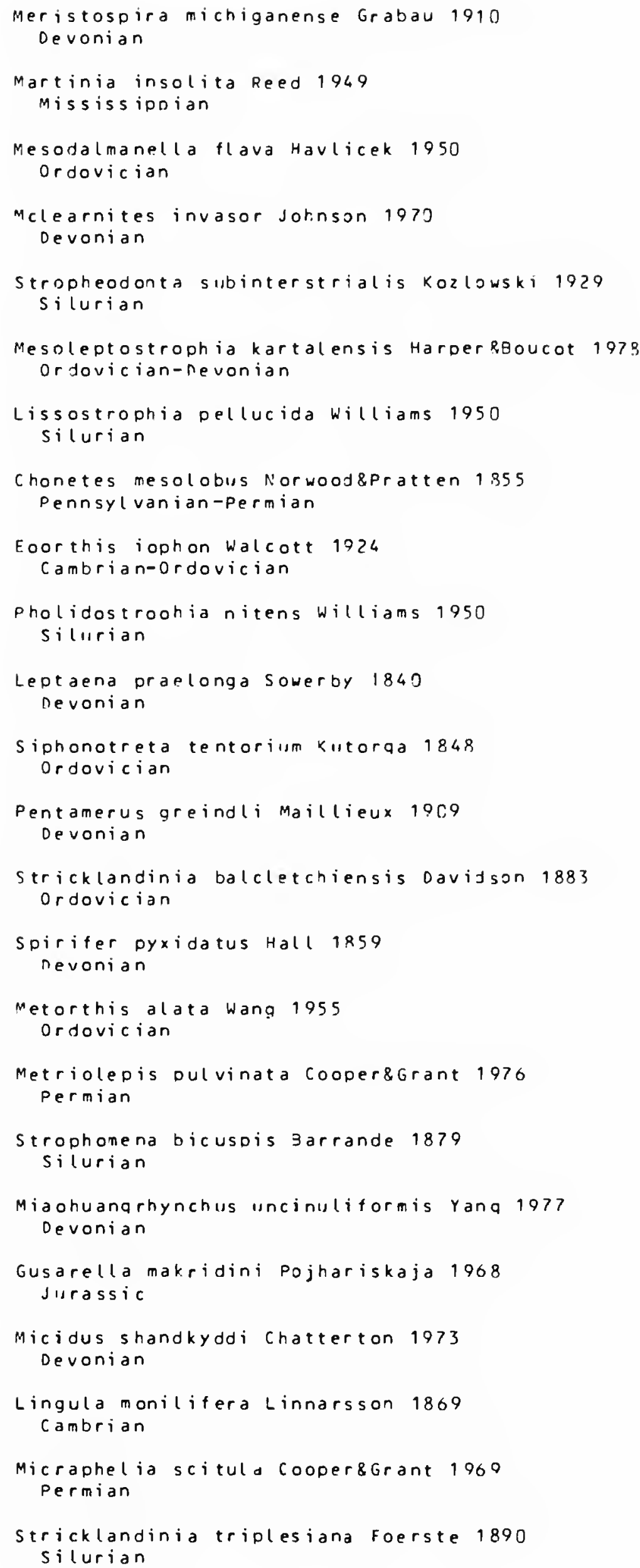




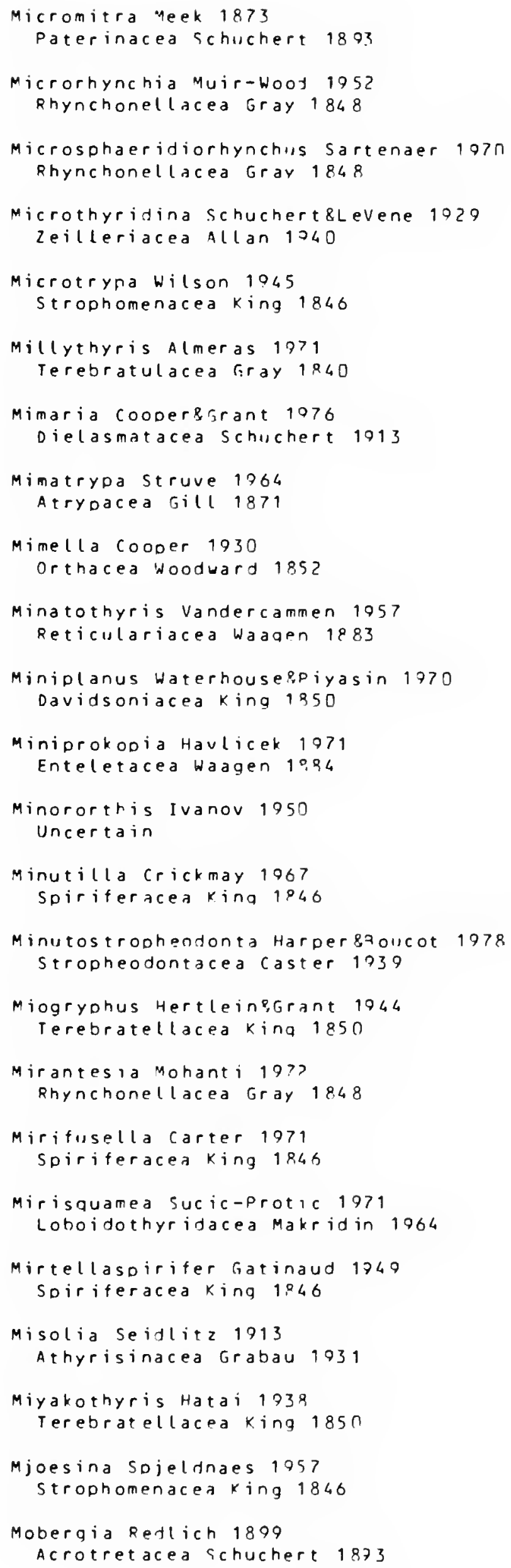

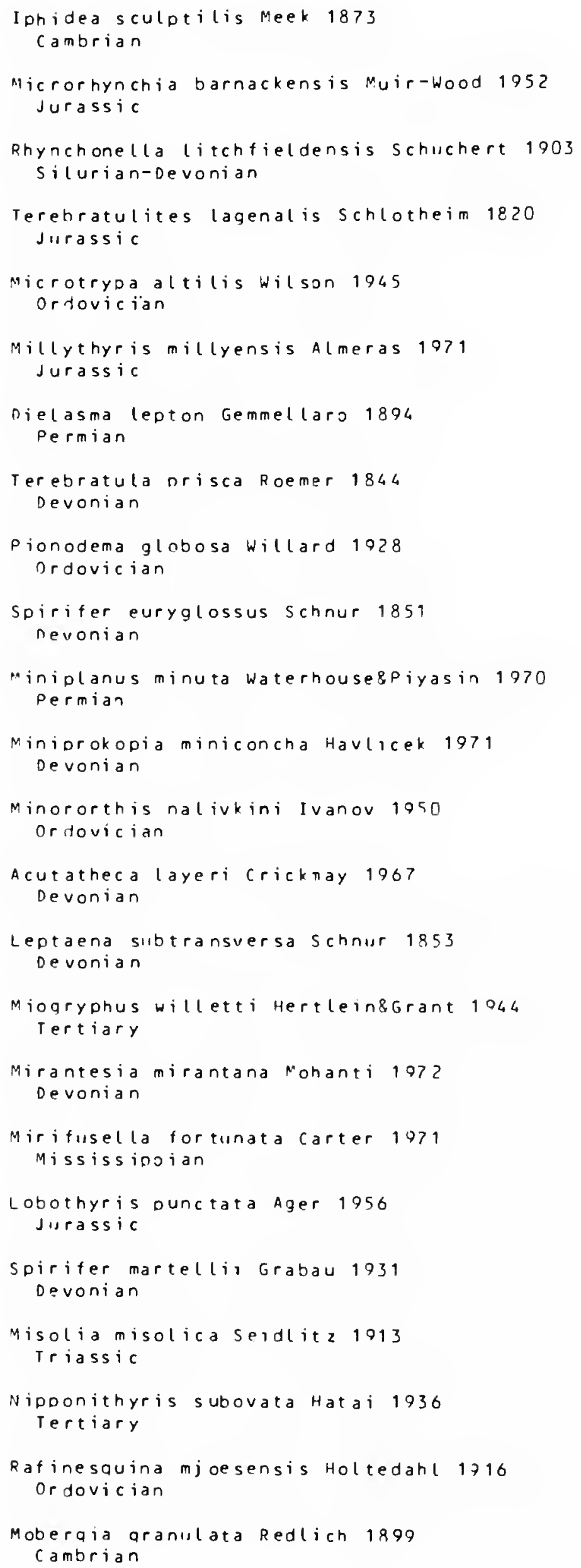




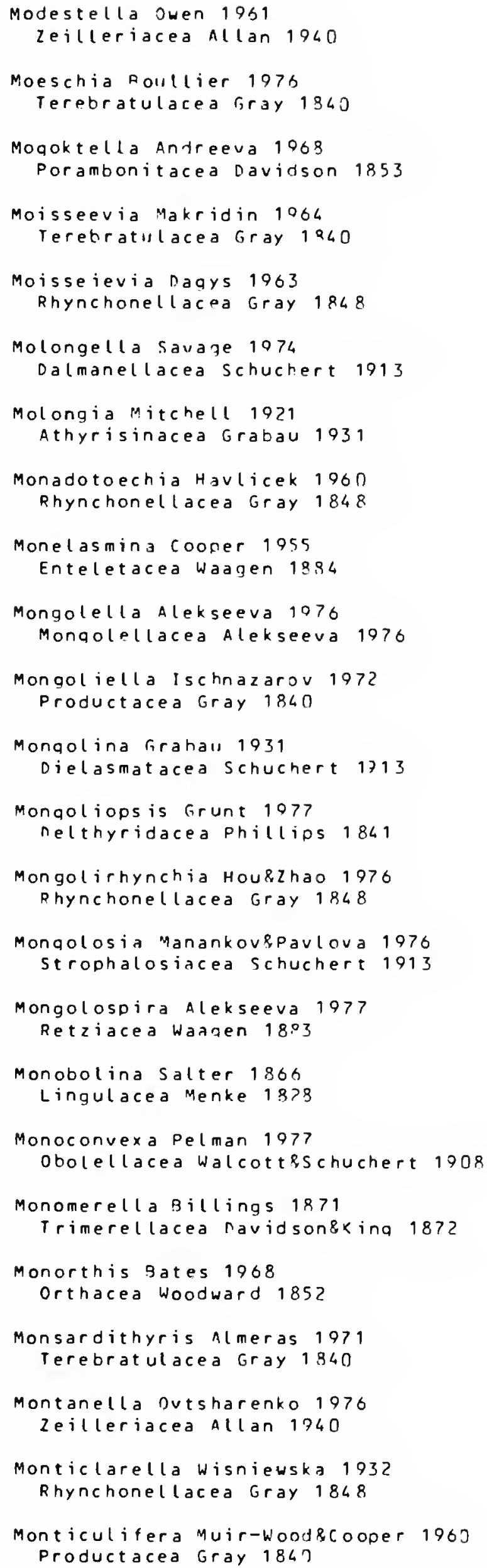

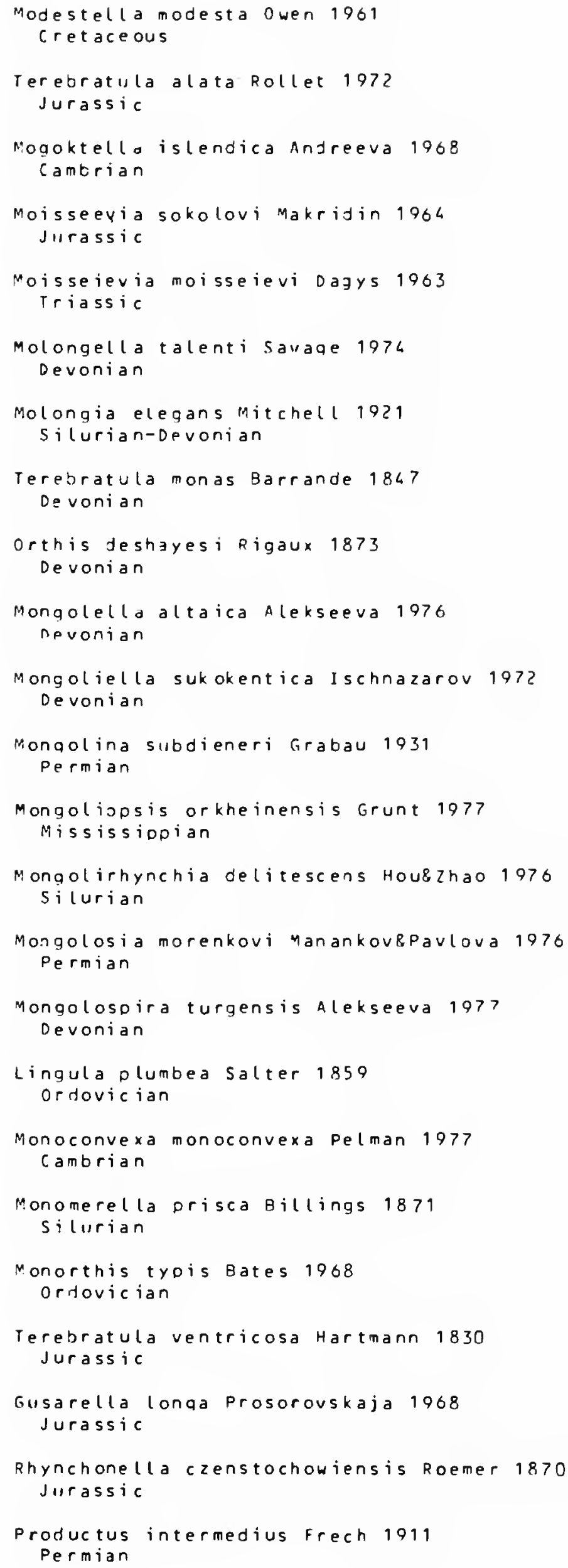




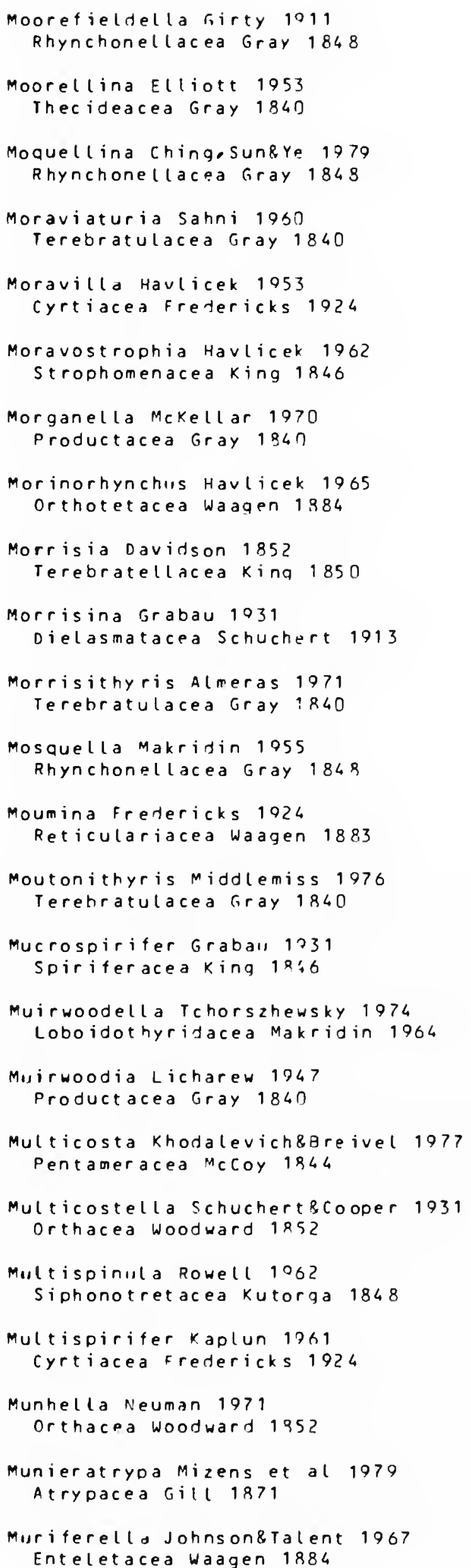

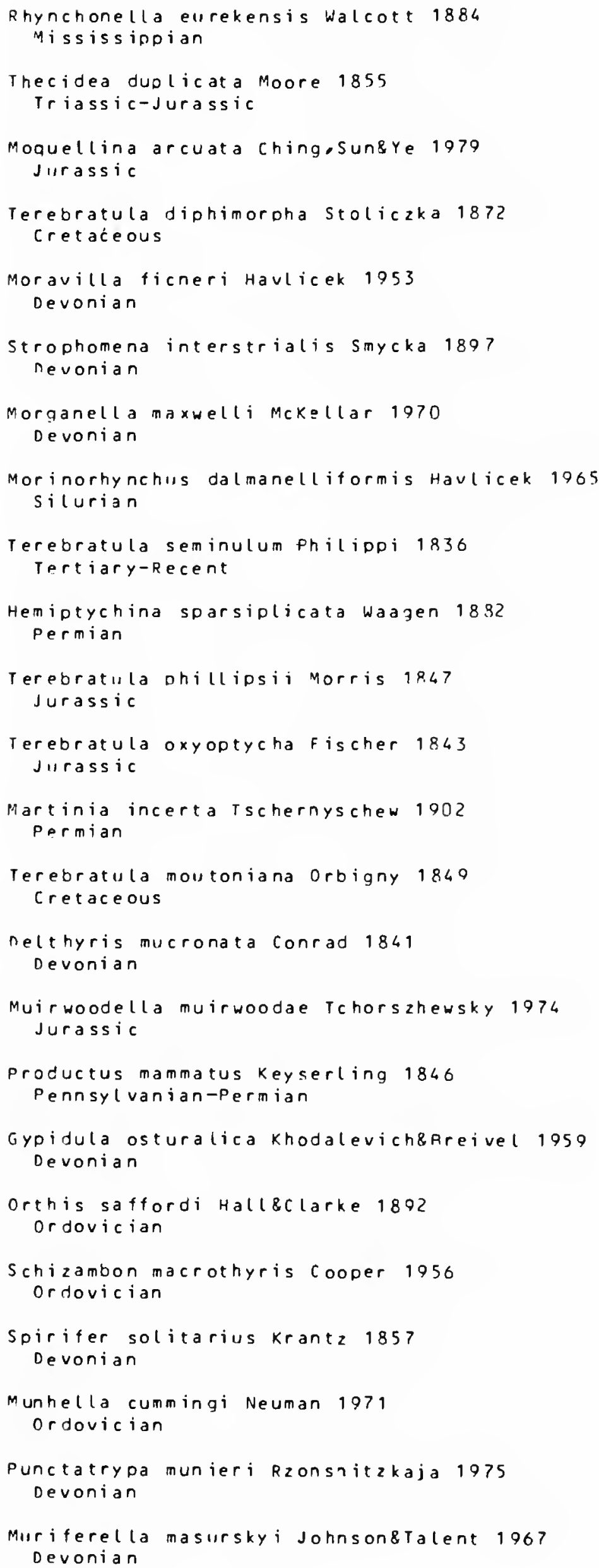




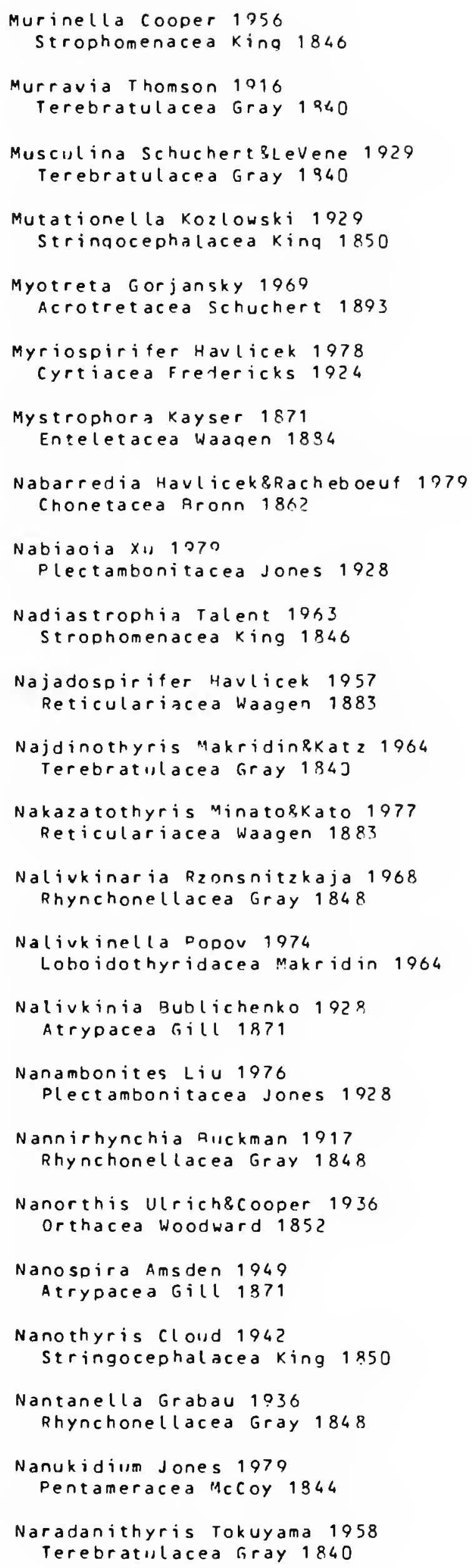

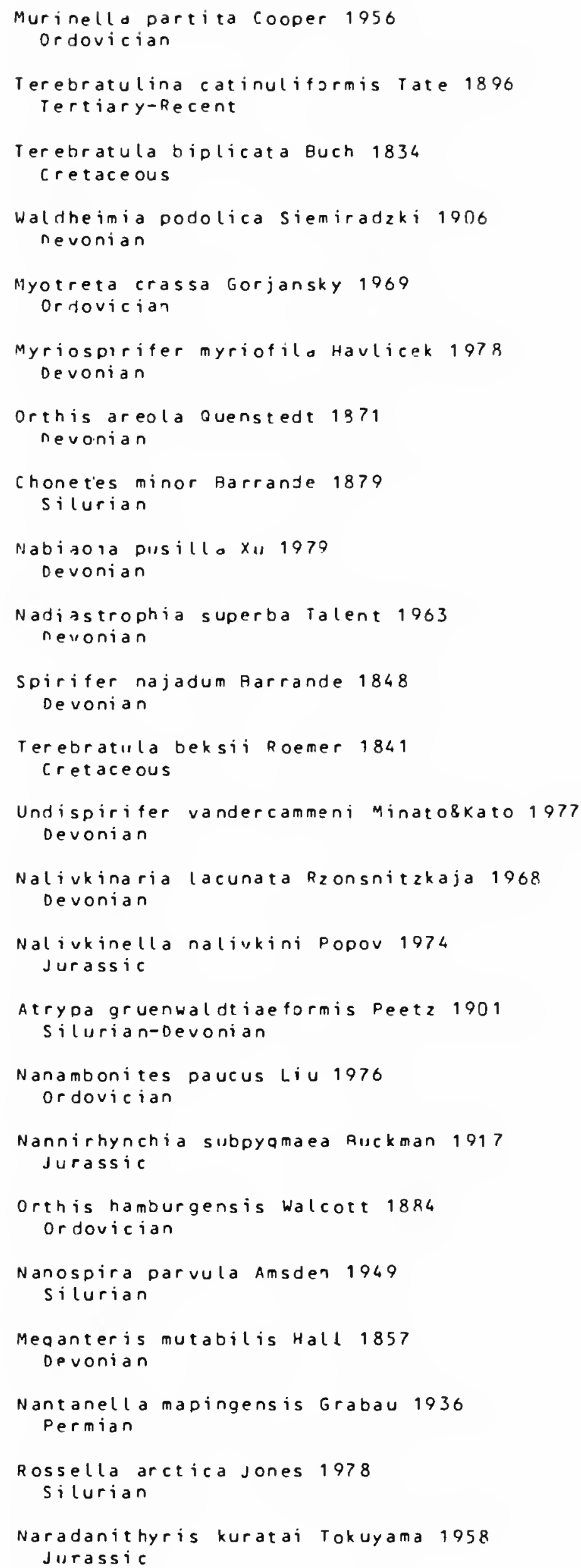




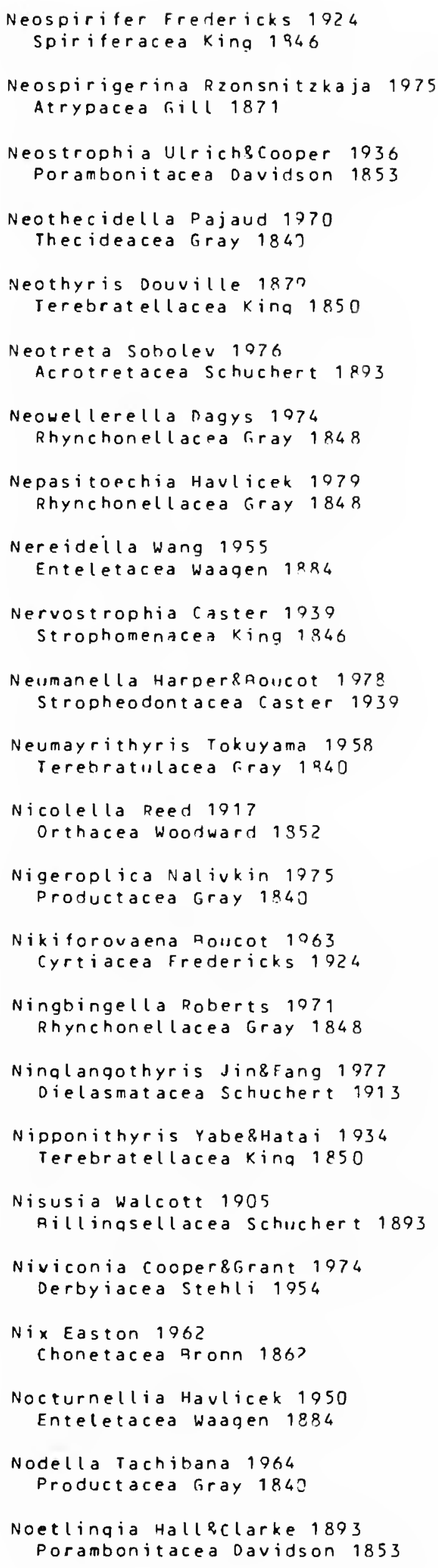

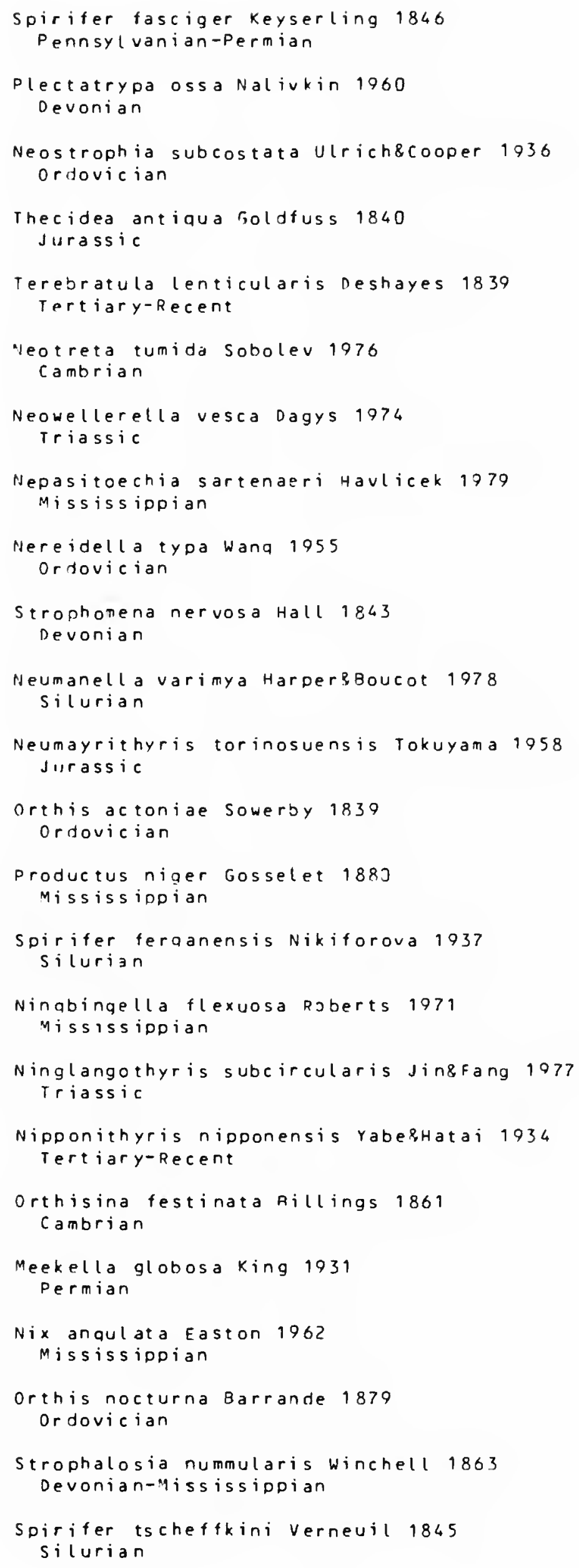




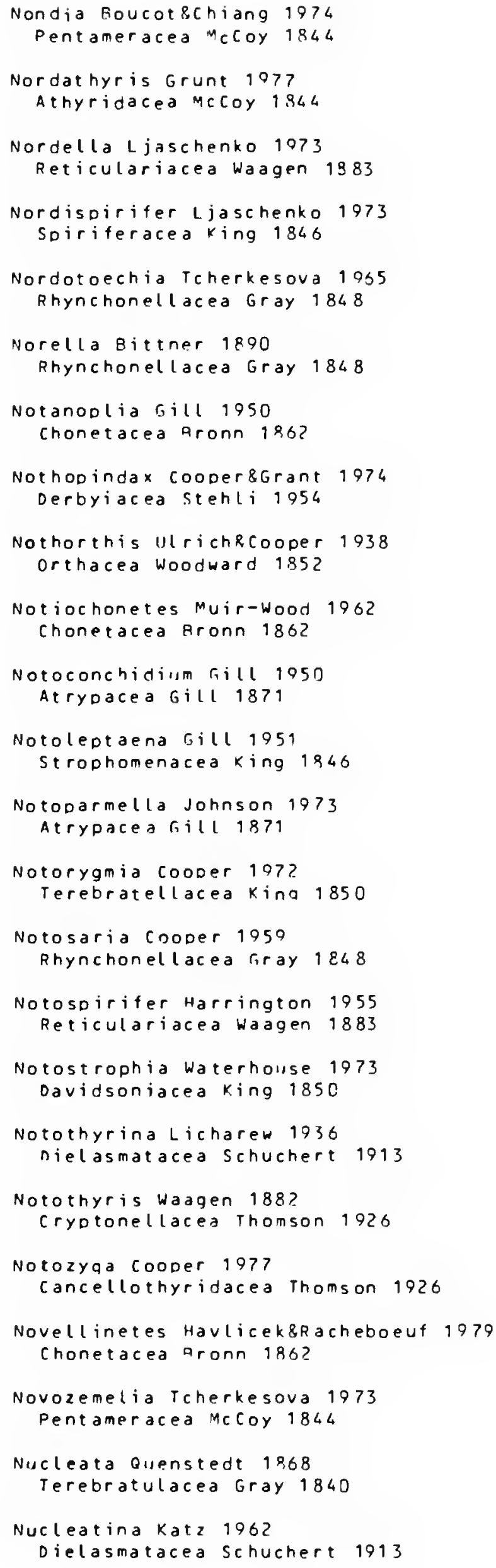

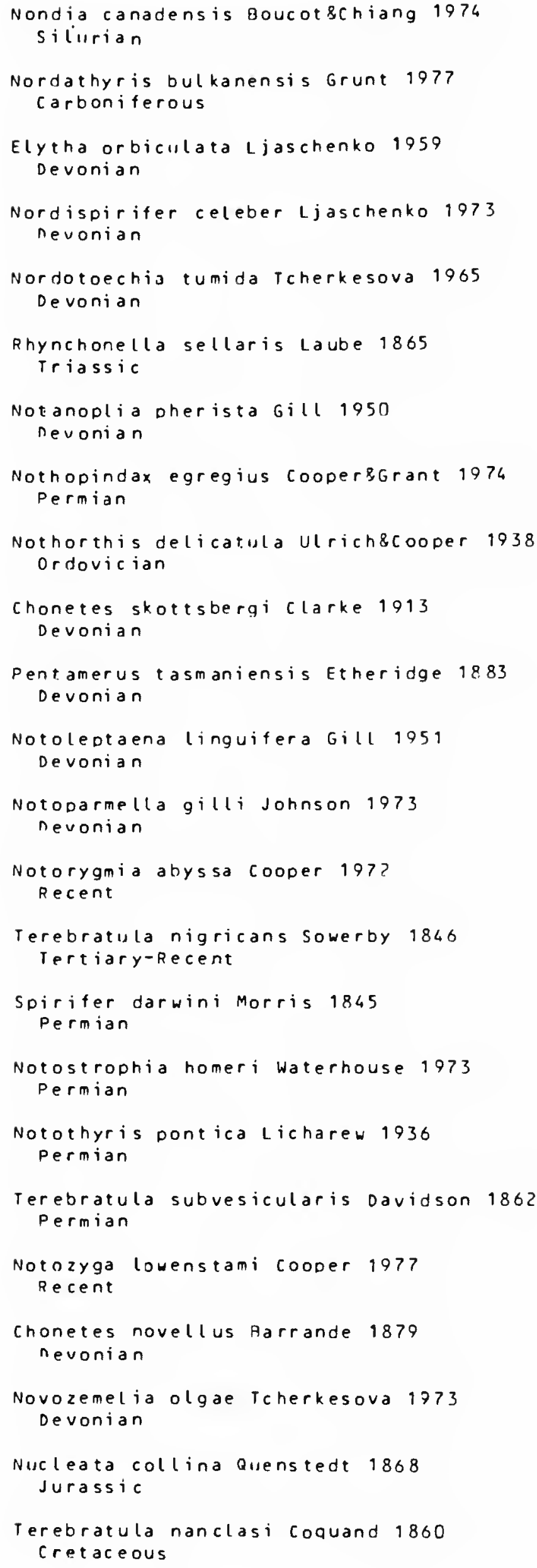




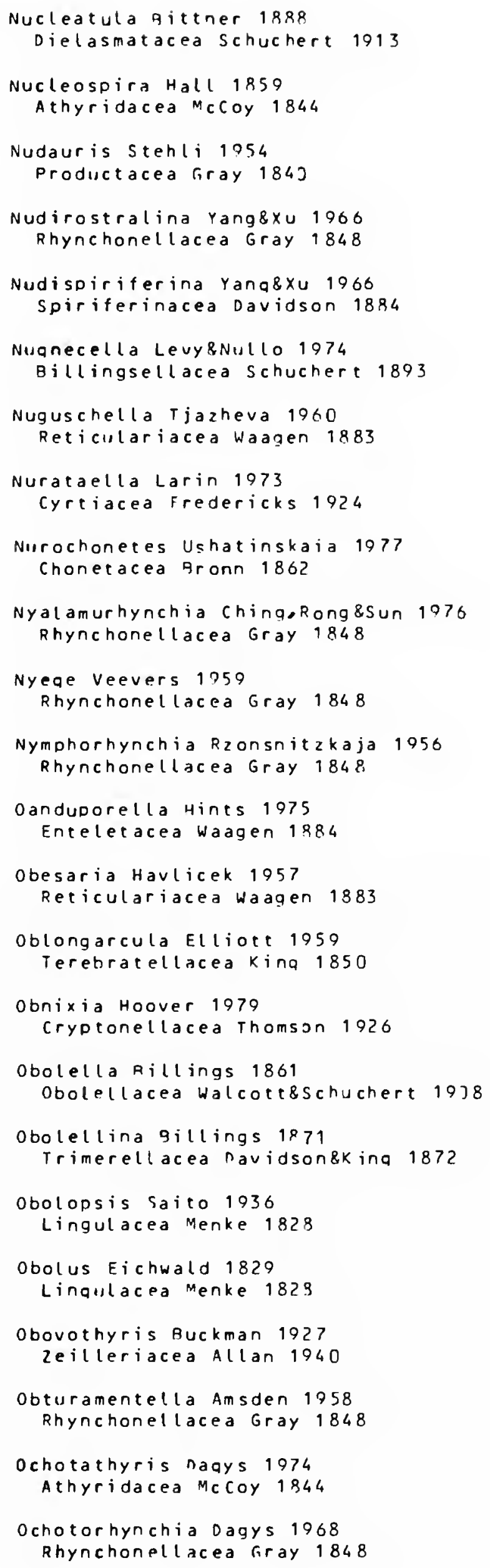

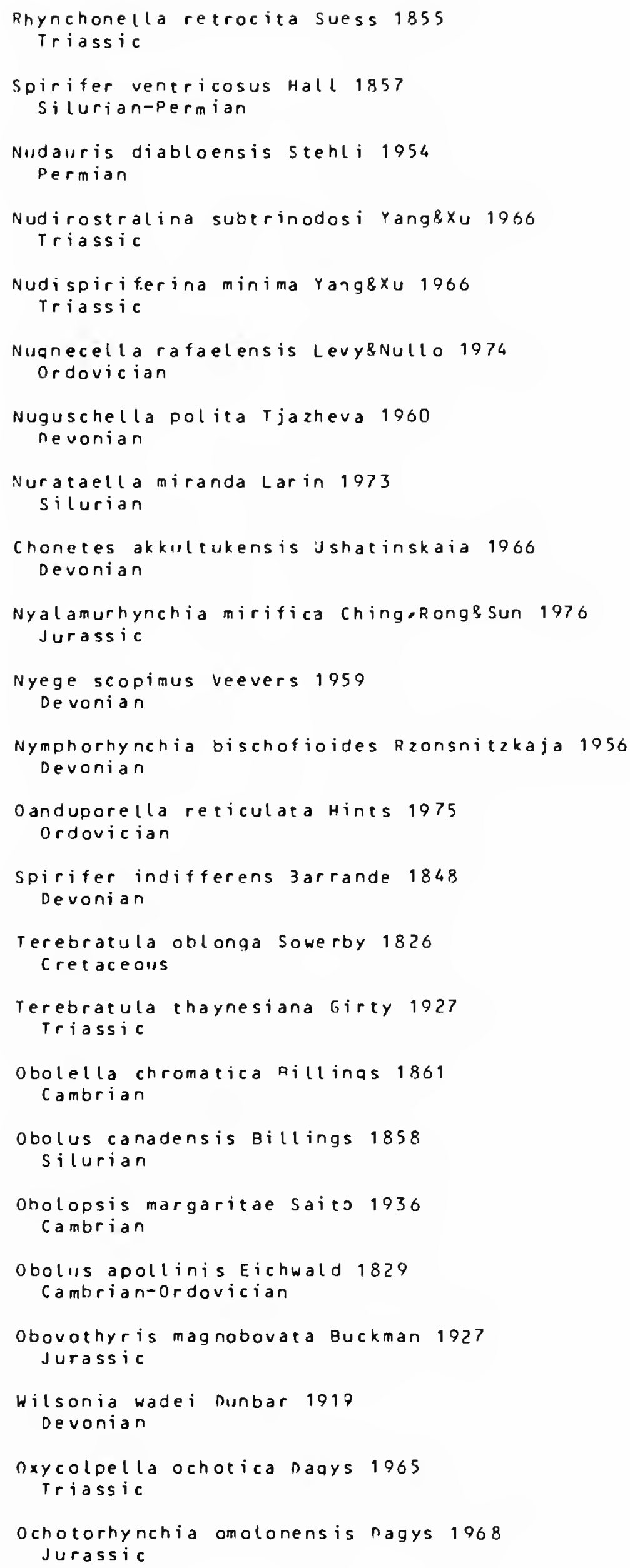




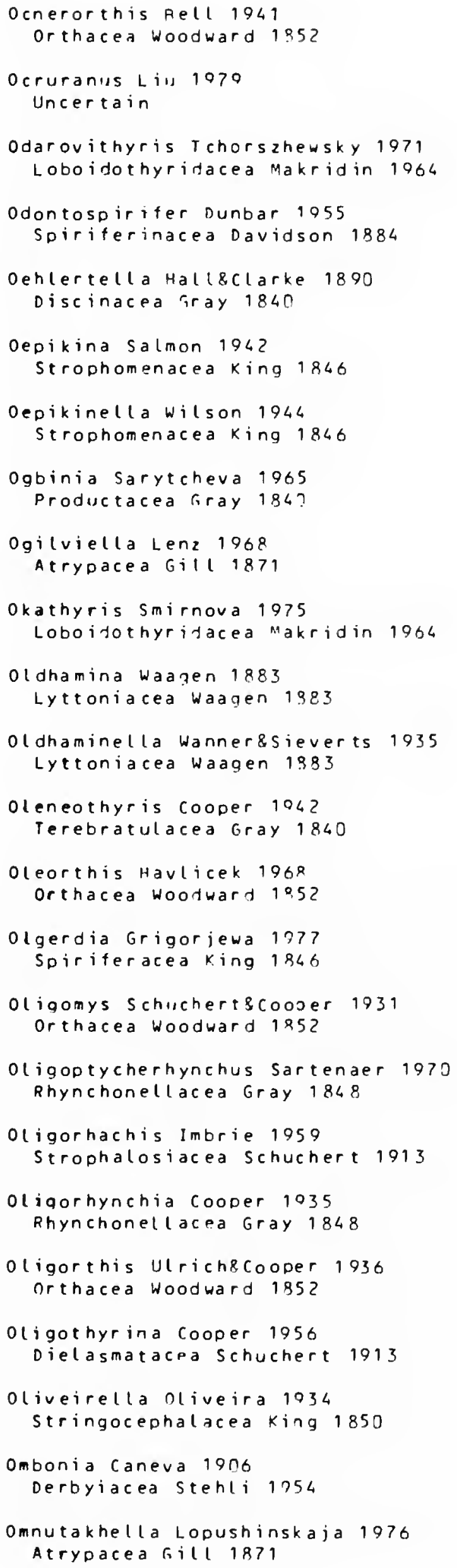

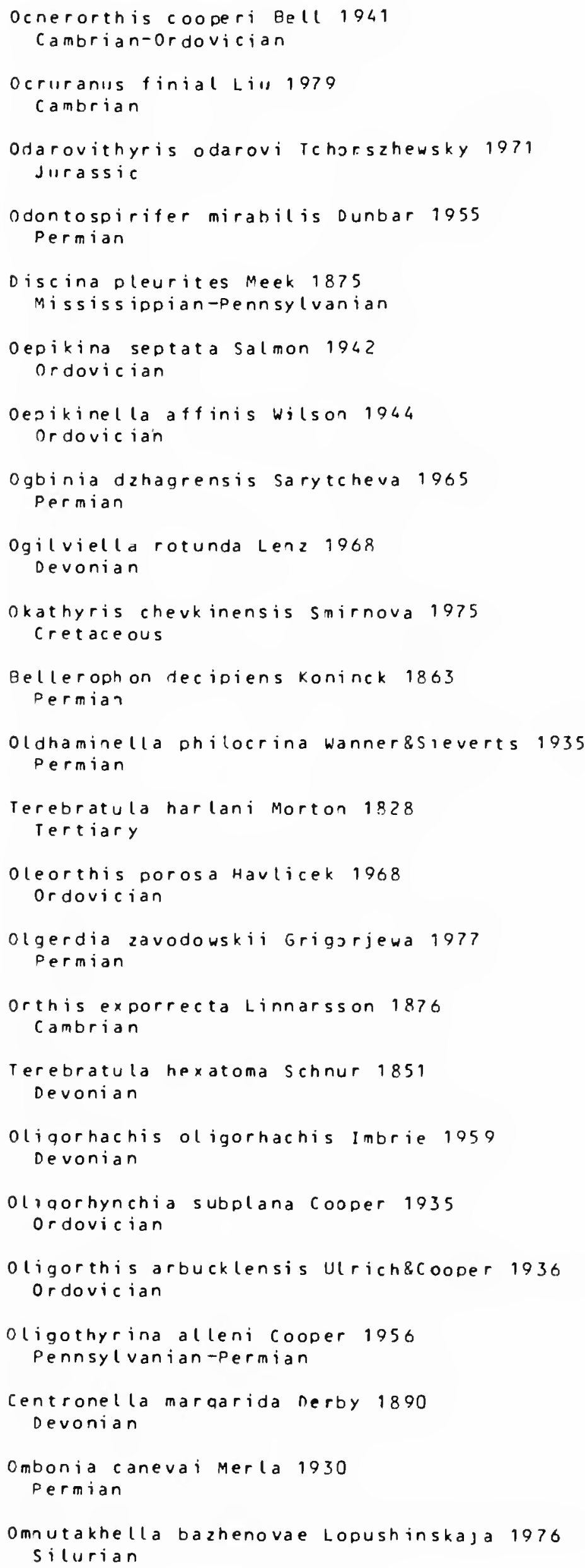


Omolonella Moisseiev 1936

Rhynchonel lacea Gray 1848

Omolonia Alekseeva 1967 Stringocephalacea Kinq 1850

Omolonothyris Dagys 1968 Loboidothyridacea Makridin 1964

Oncosarina cooper 8 firant 1969

Productacea Gray 1840

Onniella Aancroft 1028

Enteletacea wagen 1884

Onnizetina Havlicek 1974

Enteletacea Wagen 1884

Onopordumaria Waterhouse 1071

Productacea Gray 1840

Onychoplecia rooper 1956

Triolesiacea Schuchert 1913

Onychotret a Ulrich\&cooper 1936

Triolesiacea schuchert 1913

opikella Amsden 1968

Plectambonitacea Jones 1928

Opisthotreta Palmer 1954

Acrotretacea Schuchert 1893

Opsiconidion Ludvigsen 1974

Acrotretacea $5 c h u c h e r t 1893$

Orbicoelia waterhouseRPiyasin 1970

Cyrtiacea Fredericks 1924

Orbicula cuvier 1798

Craniacea Menke 1828

Orbiculoidea orbigny 1847

Discinacea Gray $184 \pi$

Orbiculopora Datriskova 1969

Discinacea fir ay 1840

Orbiculothyris wolfart 1968

Discinacea firay 1840

Orbinaria Muir-Wood\&Cooper 1960 Productacea Gray 1840

Orbirhynchia Pettitt 1954 Rhynchonel lacea Gray 1848

Orbithele Sdzuy 1955 Acrotretacea schuchert 1893

Crenburgel la Pavlova 1969 Reticulariacea Wagen 1883

orientospira naqys 1965

Spiriferinacea Davidson 1884

Orientospirifer Hou\&xian 1975 Spiriferacea king 1846

Orientothyris KatzRPopor 1974 Terebratulacea Gray 1840

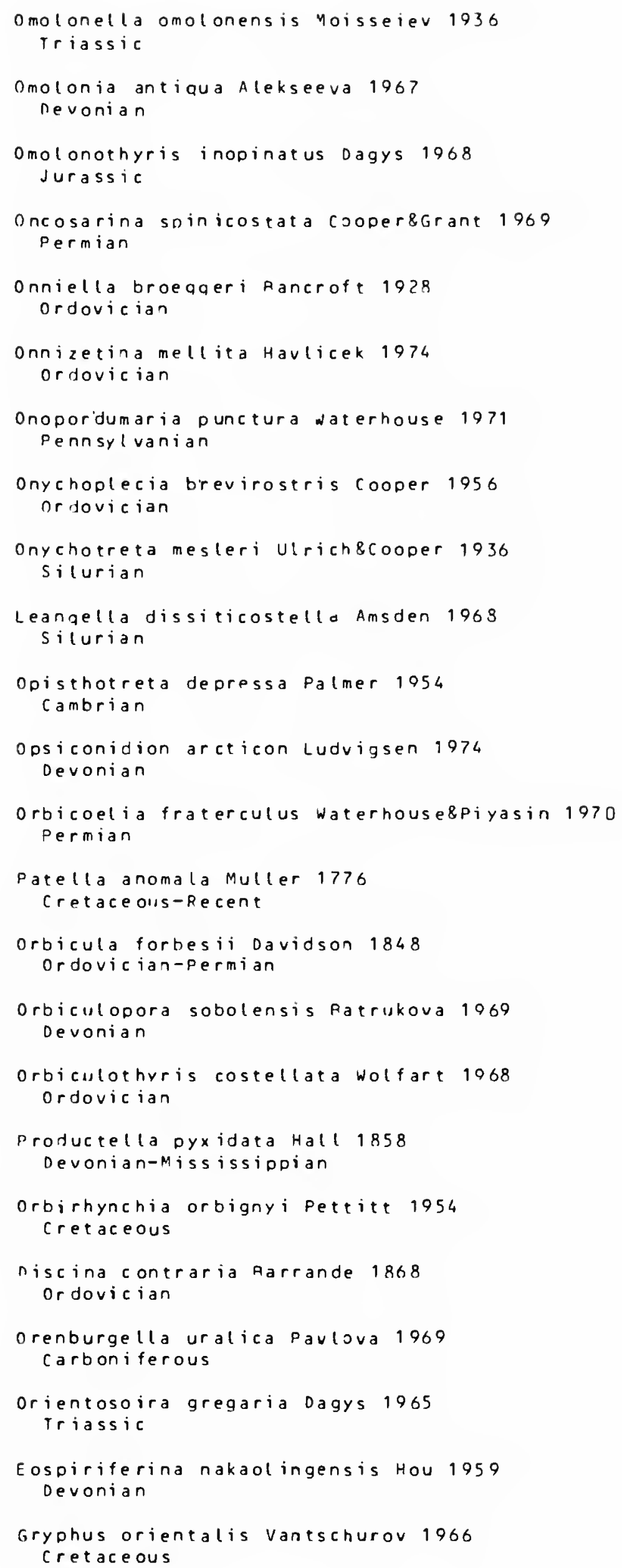


Orthothrix Geinitz 1847 Strophalosiacea schuchert 1913

orthothyris cooper 1955

Terebratulacea Gray 1940

Orthotichia Hall $8 \mathrm{Clarke} 1392$

Enteletacea Wagen 1884

Orthotoma Quenstedt 1869

Terebratulacea Gray 194,0

Orthotropia Halliclarke 1803

Enteletacea Wagen 1884

Orulgania solomina\&tschernjak 1961

Spiriferacea kina 19.96

Orusia Walcott 1905

orthacea woodward 1952

Osloqonites opik 1939

Gonambonitacea Schuchert 8 Conper 1931

Oslomena sojeldnaes 1957

Strophomenacea King 1846

Osmarella Pearson 1977

Rhynchonellacea fray 1848

Otariella Waterhouse 1978

Productacea Gray 1840

Otospirifer Housxian 1975

Spiriferacea King 1846

Otusia wal cott 1905

Orthacea Woodward 1852

Ovalella walmsley8goucot 1975

Enteletacea Wagen 1884

Ovatia Muir-Wood\&Cooder 1960

Productacea raray 1840

Overtonia Thomas 1914

Productacea Gray 1840

Overtonitina firunt 1973

Productacea Gray $184 \mathrm{~J}$

oxlosia Ulrichecooper 1936

Discinacea fir ay 1840

Oxoplecia Wilson 1913

Triplesiacea Schuchert 1913

Oxycolpella naqys 1962

Athyridacea Mccoy 1844

Oxypleurorhynchia Plodowski 1973

Rhynchonellacea Gray 1848

Pachyglossella cooper 198:0

Lingulacea Menke 1828

Pachymagas Ihering 1903

Terebratellacea King 1850

Pachythyris Boullier 1976

Terebratulacea Gray 1840

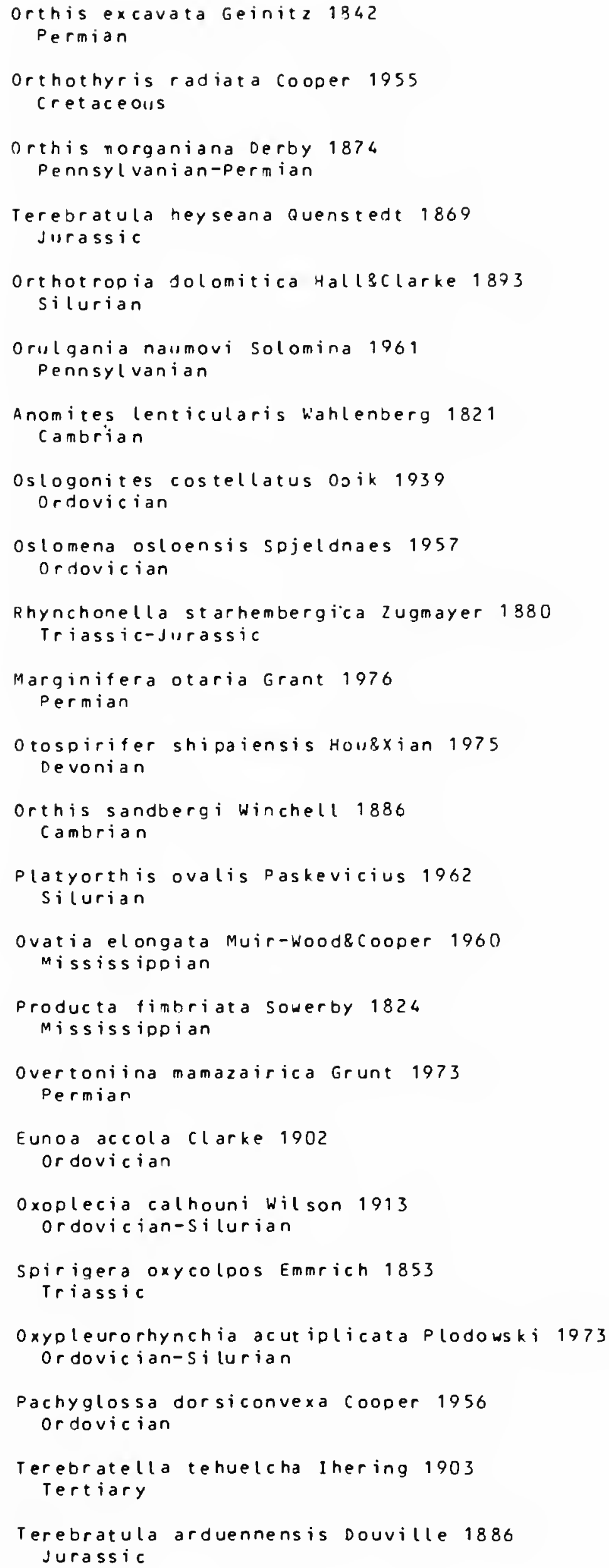




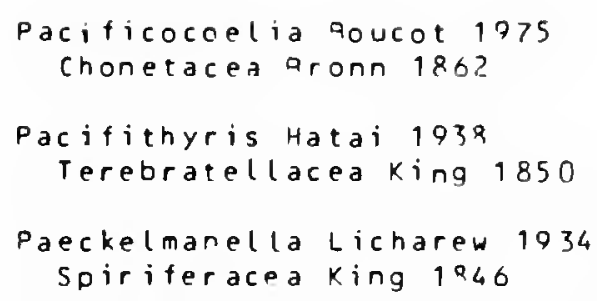

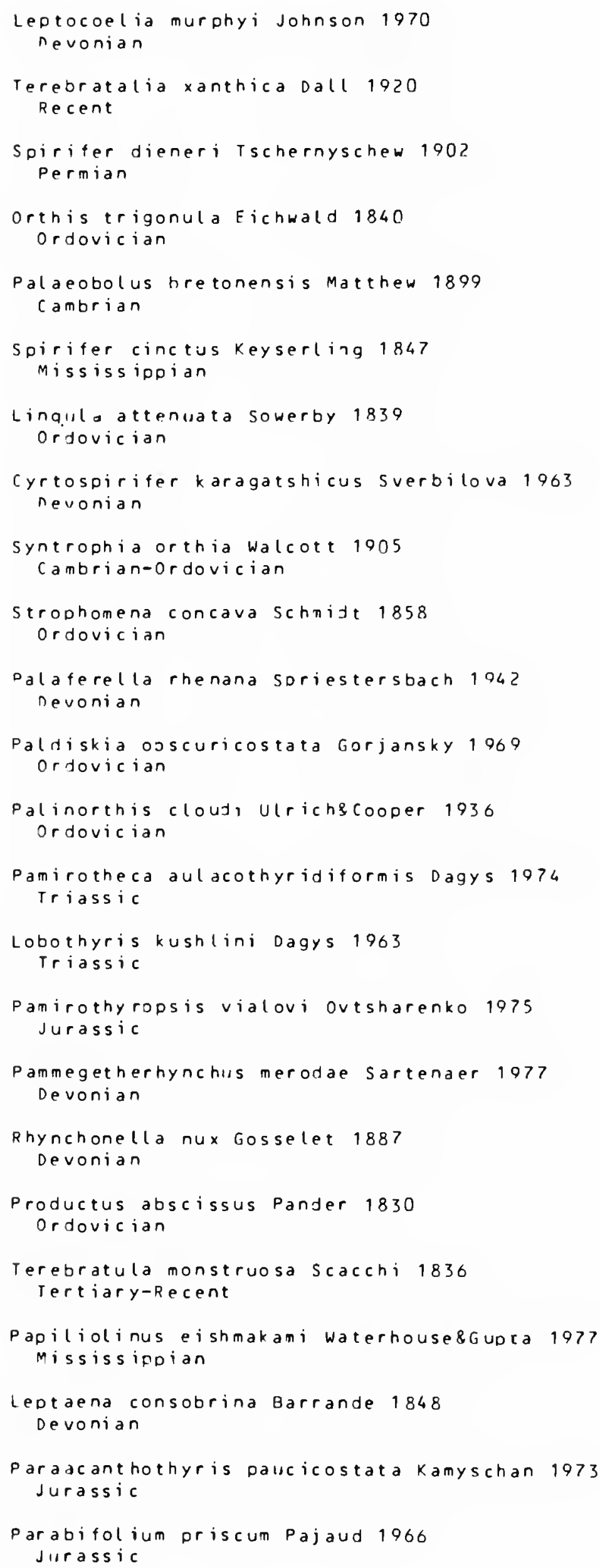


Parabornhardt ina Sun\&HOU 1964 Stringoceohalacea King 1850

Paracapillithyris Katz\&Popov 1974 Terebratulacea roray 1840

Parachonetes Johnson 1966 Chonetacea aronn 186?

Parachoristites Barchatova 1968 Spiriferacea Kina 1846

Paraconchidium Rong\&Yana 1974 Pentameracea McCoy 1844

Paracostanoplia $\times u \quad 1977$ $p l i c a n o p l i t a c e a x u 1977$

Paracraniops williams 1963 Linqulacea Menke 1828

Paracrothyris wu 1974 Stringocephalacea king 1850

Paracyrtiopsis Gatinaud 1949 spiriferacea king 1846

Paradoxothyris xu 197.8 nielasmatacea Schuchert 1913

Paradygella Liao\&Sun 1974 Diel asmatacea Schuchert 1913

Paraemanuel la Yana 1977 Cyrtiacea fredericks 1924

Parakeyser Lingina Fredericks 1916 Lyt toniacea Waaen 1383

Paralazut kinia Jiang 1978 Spiriferacea king 1.346

Paraldingia Richardson 1973 Terebratellacea kina 1850

Paralepismatina Yang\&Xu 1966 Suessiacea Waggen 1833

Paraleptostrophia Harper\&Boucot 1978 Stropheodontacea Caster 1939

Parallelelasma cooper 1956 Pentameracea $1 \mathrm{cCOY} 1844$

Parallelora Carter 1974 Spiriferacea Kinq 1846

Paralyttonia Wanner\&sieverts 1935 Lyttoniacea Wagen 1883

Paramarginatia Yang 1978 Productacea Gray 1840

Paramarainifera Fredericks 1916 Productacea Gray 1840

Paramartinia Reed 1949 Reticulariacea Wagen 1883

Paramentzelia $x u 1978$ Reticulariacea Wagen 1883

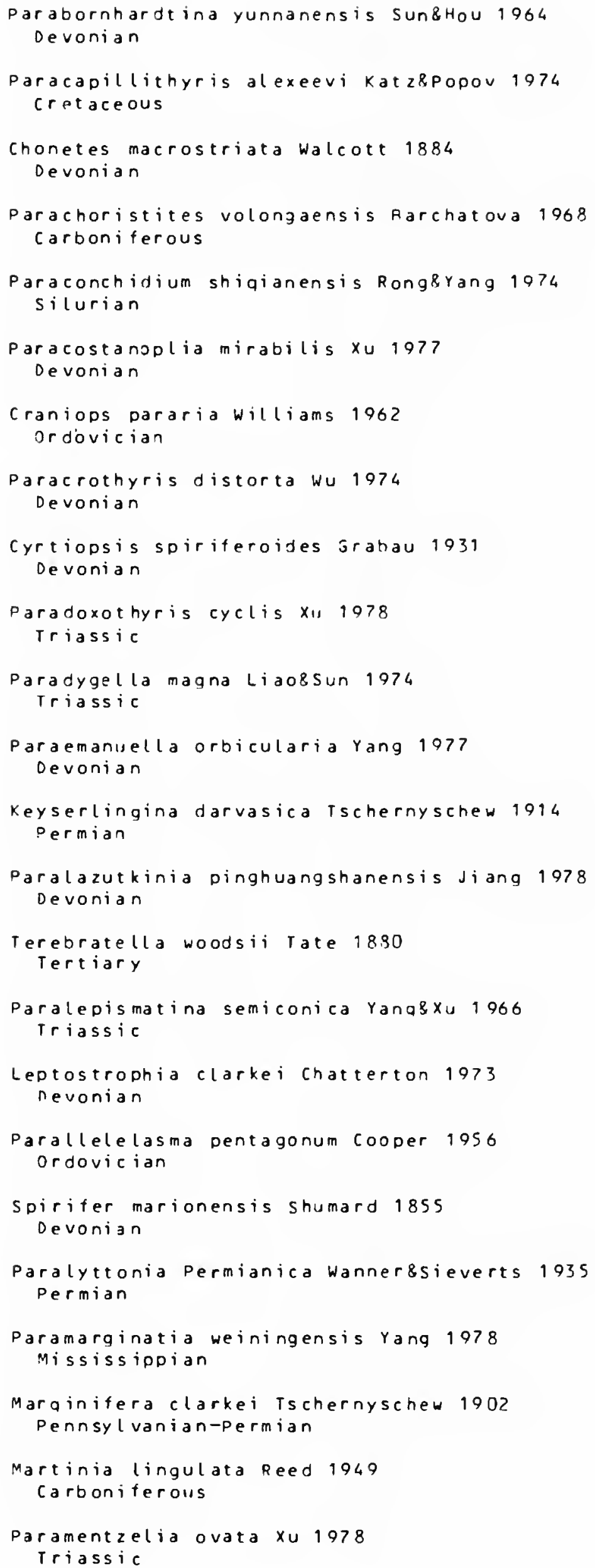




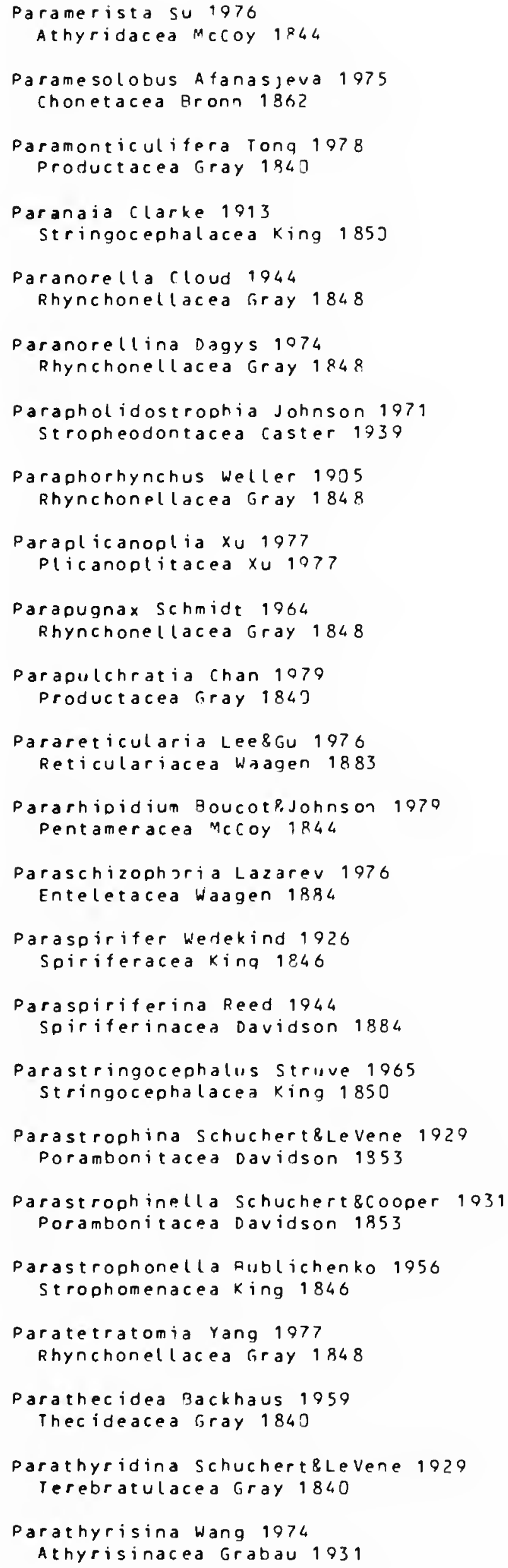

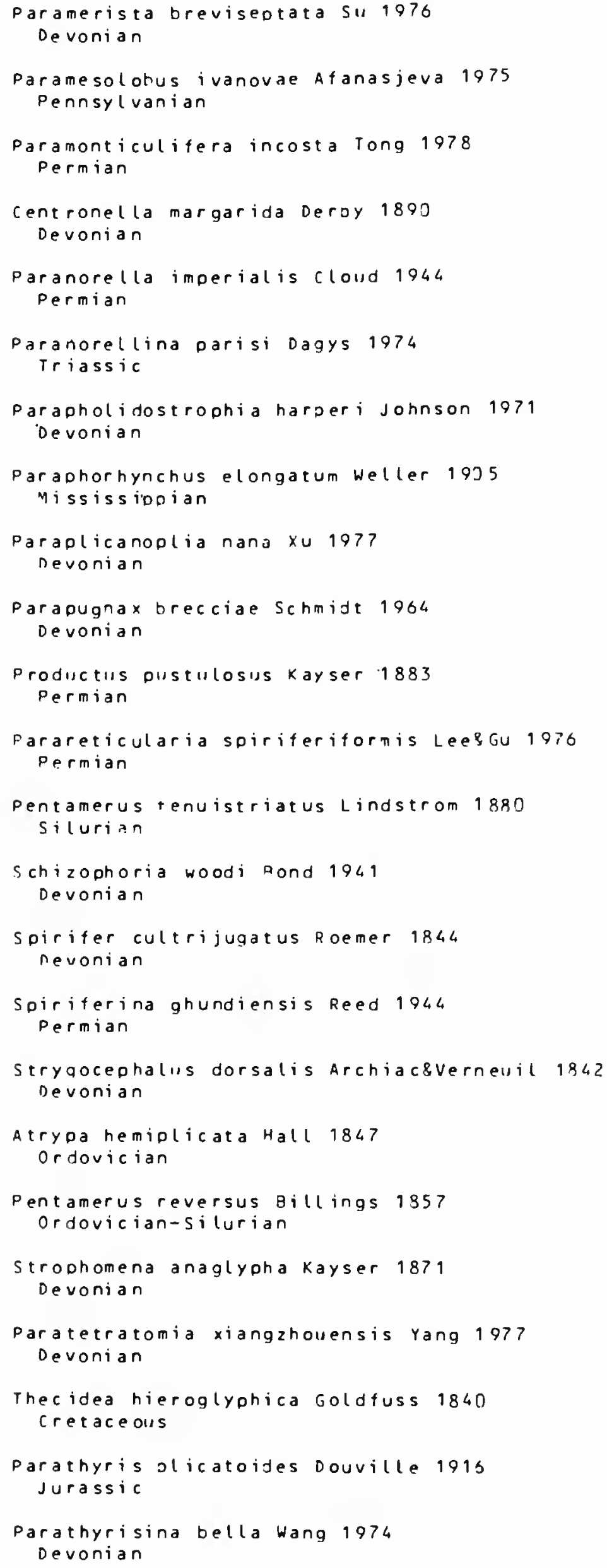




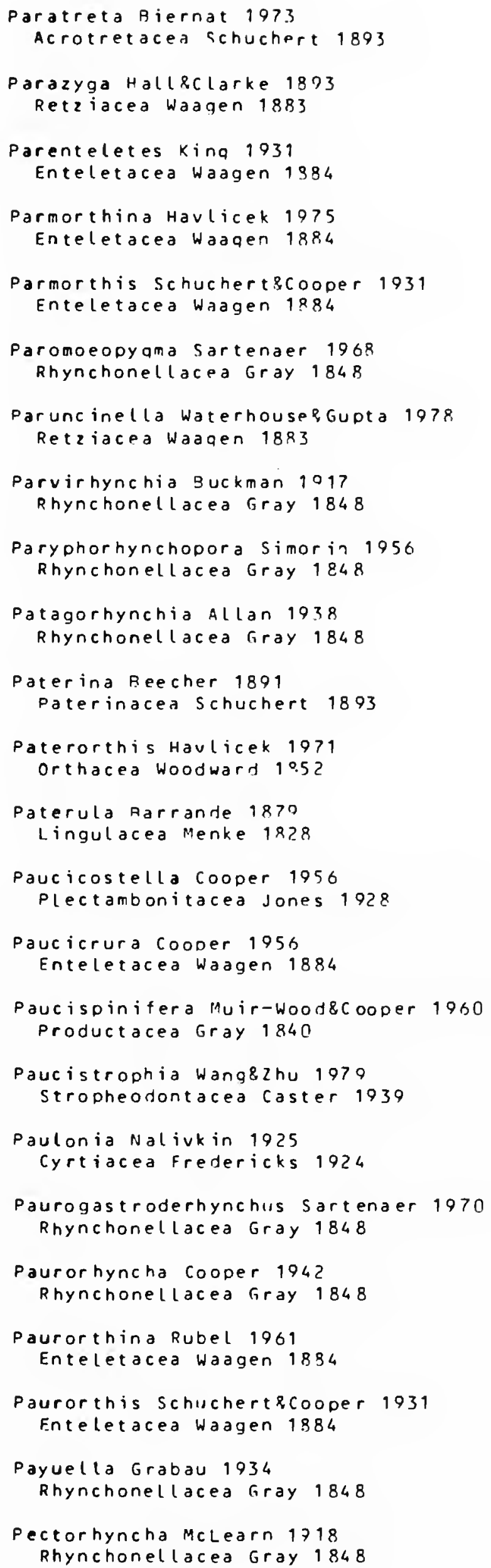

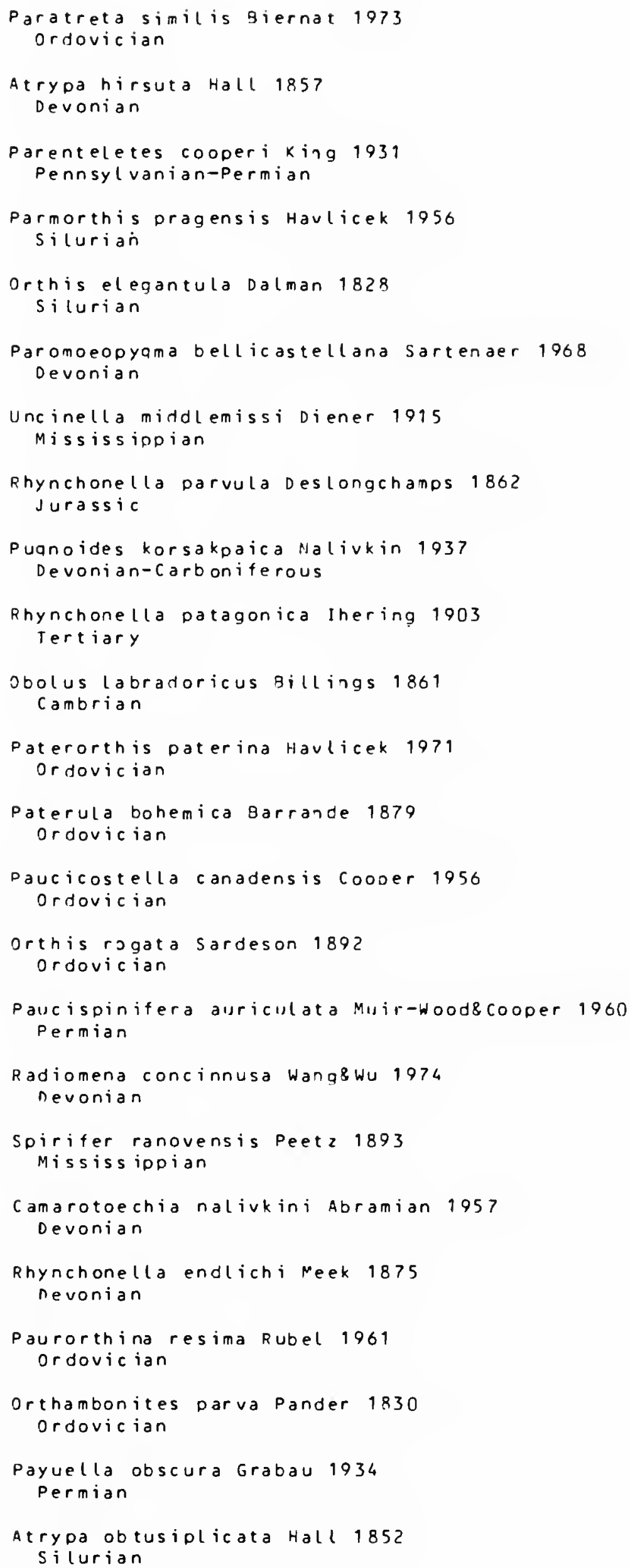




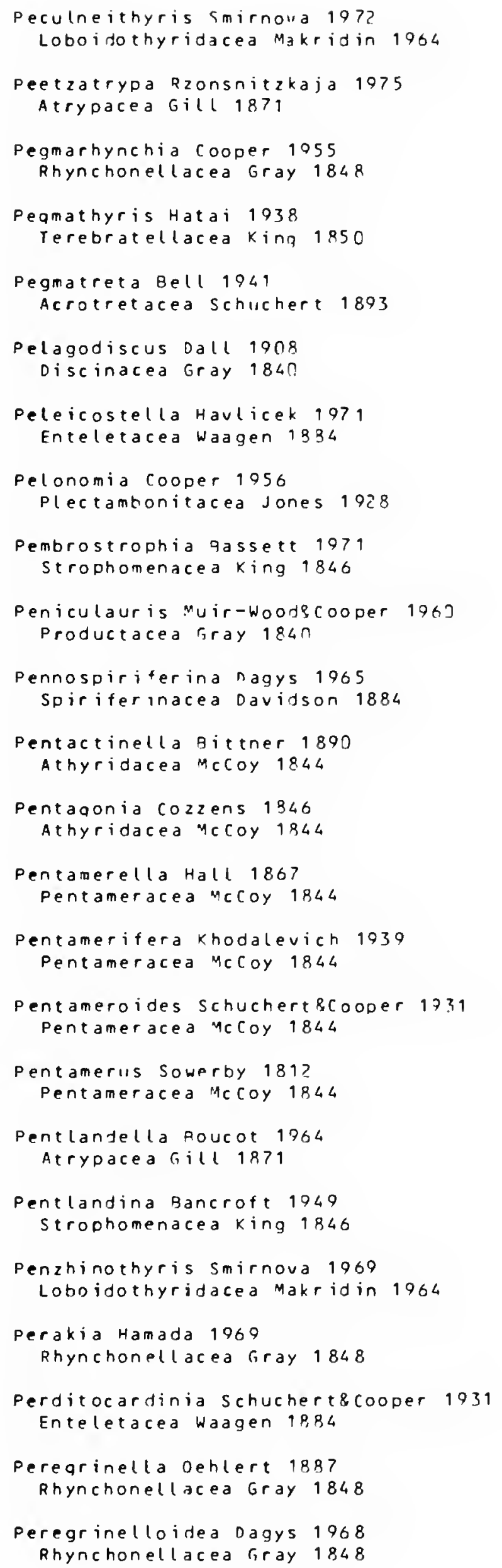

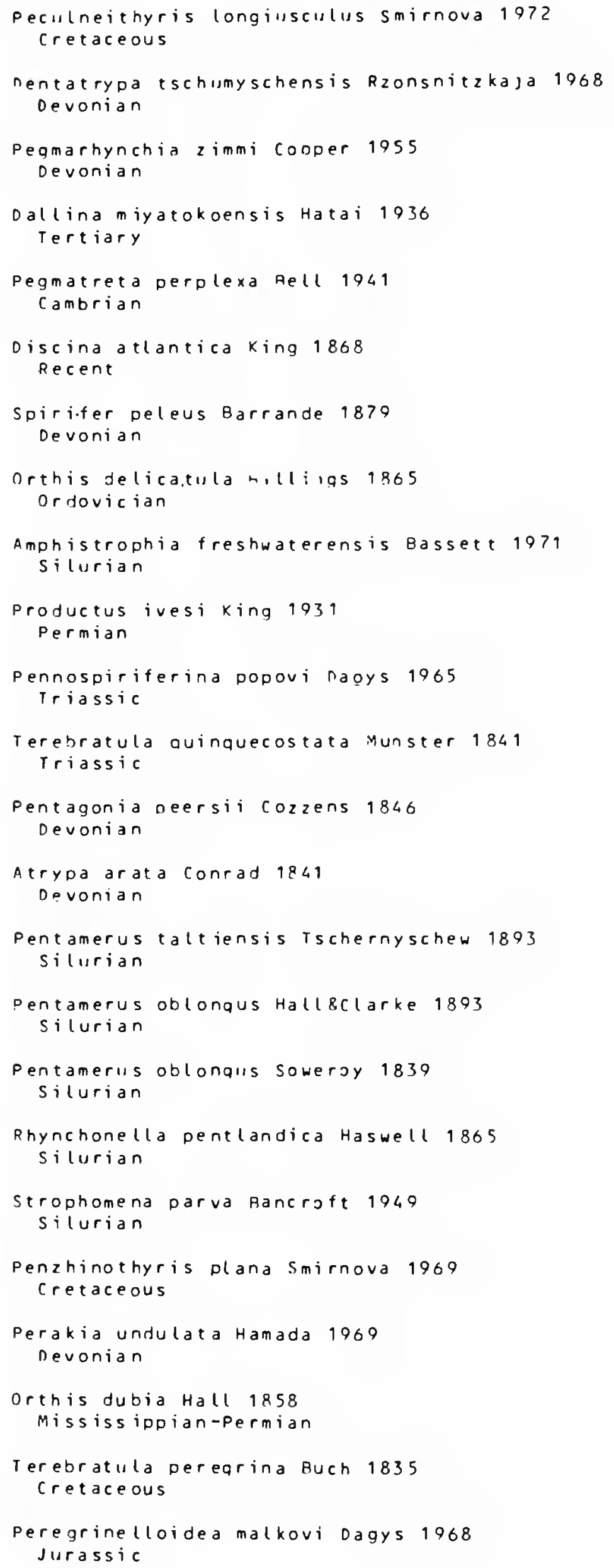


Periallus Hoover 1079

Dallinacea Reecher 1893

Perichonetes $x u 1979$

Chonetacea Aronn 1862

Peridalejina Havlicek 1073

Enteletacea Wagen 1884

Perigeyerella wang 1055 Davidsoniacea King 1850

Perimecocoelia cooper 1956 Porambonitacea Davidson 1853

Perissothyris Carter 1967

spiriferacea King 1846

Peritritoechia Xu, Rong\&L iu 1974

clitambonitacea winchell 8 schuchert 1893

Permianel la He\&Zhu 1979

Permianellacea He\&zhu 1979

Permicola Koczyrkevicz 1976

Dielasmatacea Schuchert 1913

Permochonetes Afanasjeva 1977

Chonetacea aronn 1862

Permophricodothyr is Pavlova 1965 Reticulariacea Wagen 1883

Permorthotetes Thomas 1958 Davidsoniacea King 1250

Permospirifer kulikov 1950 Spiriferacea $k$ ing 1946

Permundaria Nakamura,kato $\&$ Choi 1970 Productacea Gray 1840

Perrierithyris Almeras 1971

Terebratulacea Gray 1840

Peshiatrypa $x$ ian 1978 Atrypacea rill 1871

Pesterevatrypa Rzonsnitzkaja 1975 Atrypacea Gill 1971

Petasmaia Cooper 8 Grant 1959 Lyttoniacea wagen 1883

Hetasmatherus Cooper 8 Grant 1969 Rhynchonellacea Gray 1848

Petria Mendes 1961

Dielasmatacea Schuchert 1913

Petrocrania Raymond 1911

Craniacea Menke 1828

Petroria Wilson 1026 Plectambonitacea jones 1928

Pexidella Rittner 1890 Athyridacea McCoy 1844

Phapsirhynchia Pajaud 1976 Rhynchonel lacea Gray 1848

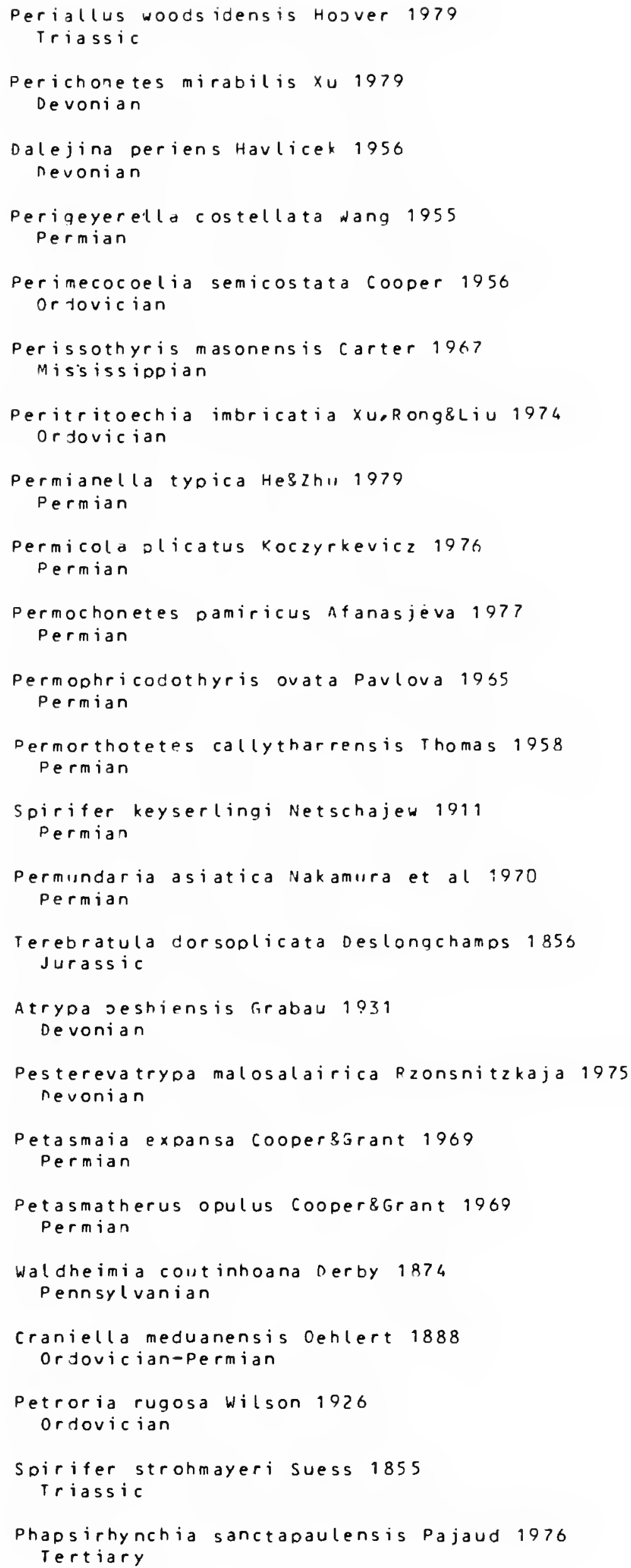


Pinguispirifer Havlicek 1957 Spiriferacea Kinq 1946

Pionodema foerste 1912 Enteletacea Wagen 1.894

Pionomena Cooper 1956 Strophomenacea King 1846

Pionorthis Schuchert\&cooper 1931 Orthacea woodward 1852

Pirgulia CooperkMuir-wood 1951 Lyttoniacea Wagen 1883

Pirothyris Thomson 1927 Terebratellacea king 1850

Pirotothyris Sucic-Protic 1971 Loboidothyridacea Makritin 1964

Pisirhynchia nuckman 1017 Rhynchonel lacea Gray 1848

Pizarroa Hoek 1912

Lingulacea Menke 1828

Placothyris westphal 1970

Terebratulacea firay 1840

Placotriplesia Amsden 1968 Triplesiacea Schuchert 1913

Plaesiomys Hall\&Clarke 1892 Orthacea Woodward 1.852

Plagiorhyncha McLearn 1918 Rhynchonel lacea Gray 1848

Planalvus Carter 1971 Athyridacea MCCOy 1844

Planatrypa Copper 1067 Atrypacea fill 1871

Planatrypa Struve 1966 Atrypacea Gill 1871

Planicardinia Savaqe 1968 Dalmanellacea Schuchert 1713

Planidorsa Schuchert\&Cooper 1931 orthacea woodward 1852

Planirhynchia Sucic-Protic 1969 Rhynchonel lacea Gray 1848

Planispina Stehli 1954 Strophalosiacea Schuchert 1913

Planodouvillina Harper $880 u \cot 1978$ Stropheodontacea Caster 1939

Planoharknessel la Havlicek 1950 Enteletacea wagen 1884

Planoproductus stainbrook 1947 Productacea Gray 1840

Planovatirostrum Sartenaer 1970 Rhynchonellacea Tray 1848

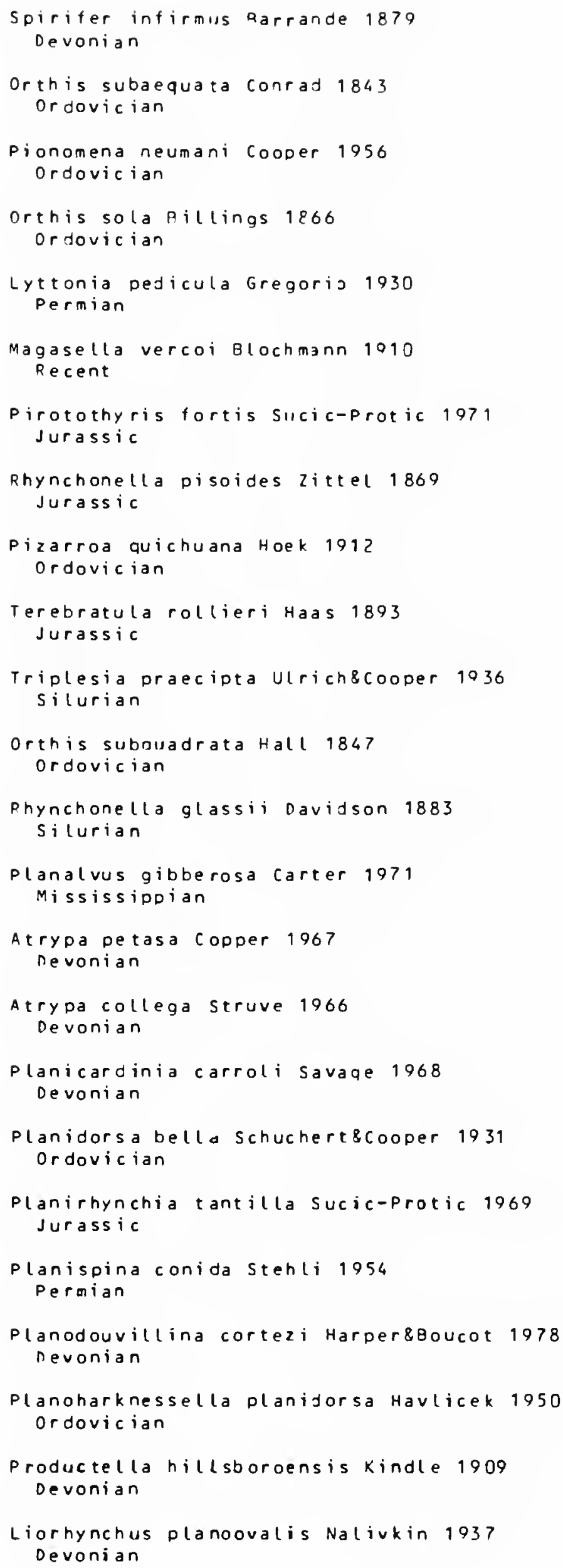




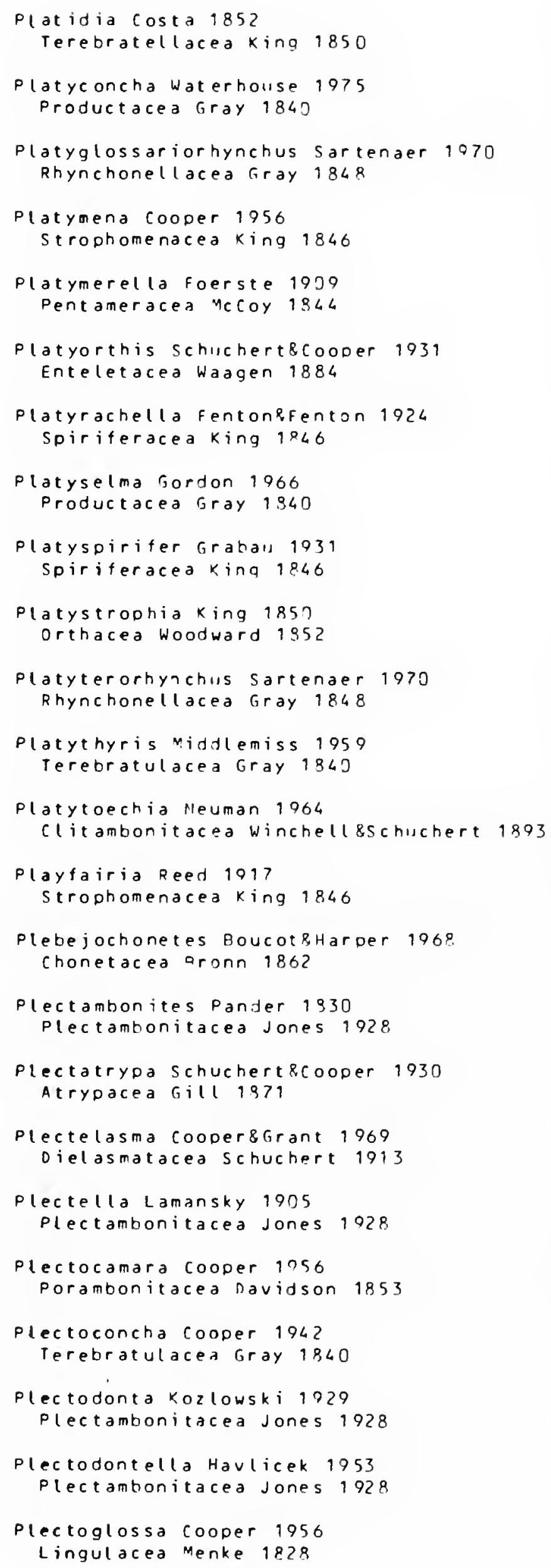

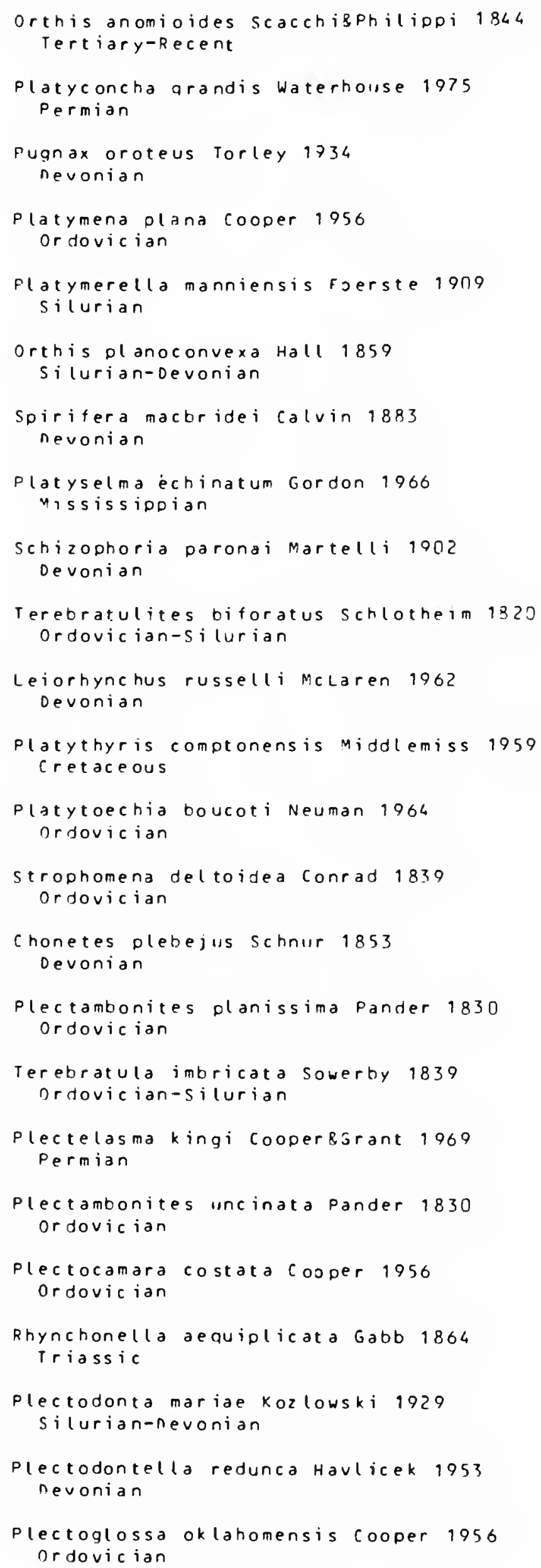


Plectoidothyris Buckman 1917 Terebratulacea firay 1940

Plectorhynchella CooperkMuir-Wood 1051 Rhynchonellacea Gray 1848

Plectorthis Hall $8 \mathrm{Cl}$ arke 1892 orthacea woodward 1852

Plectospira Cooper 194? Retziacea Wagen 1883

Plectospirifer Grabau 1931 Spiriferacea King 1846

Plectothyrella Temple 9065 Athyridacea McCoy 1844

Plectothyris Buckman 1917 Terebratulacea fray 1840

Plectotreta Ulrich\&cooper 1736 Iriplesiacea schuchert 1913

Plectotrophia Ulrich\&cooper 1936 Porambonitacea Davidson 1853

Pleiopleurina Schmidt 1964 Rhynchonel lacea Gray 1848

Plekonella campbell 1953 Rhynchonel lacea Gray 1848

Plesicarinatina M1zens 1977 Atrypacea Gill 1871

Plesiothyris Douville 1879 zeilleriacea Allan 1040

Plethorhyncha Hal 8 Clarke 1893 Rhynchonel lacea Gray 1848

Pleurelasma cooper\&Grant 1976 Dielasmatacea Schuchert 1913

Pleurochonetes Is a acson 1977 chonetacea Bronn $\$ 862$

Pleurocornu Havlicek 1961 Rhynchonel lacea Gray 1848

Pleurodium Wang 1955

Pentameracea McCoy 1844

Pleurohorridonia nunbar 1955

Productacea Gray 1840

Pleuropugnoides Ferguson 9966 Rhynchonel lacea Gray 1848

Pleurorthis Cooper 1956 orthacea woodward 1852

Pleurothyrella Roucot et al 1963 Stringocephal acea King 1850

Plicanoplia Boncot\&Harper 1968 Chonetacea Bronn 1862

Plicanoplites Havlicek 1974 Chonetacea Bronn 1862

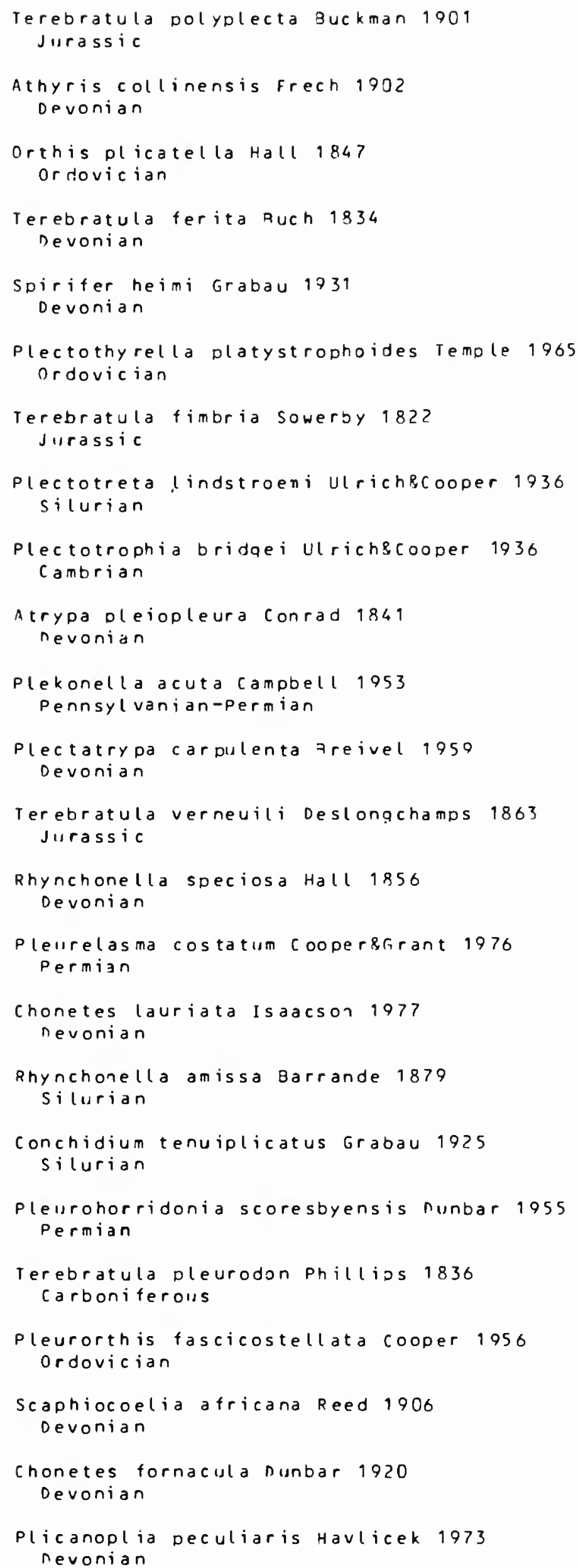




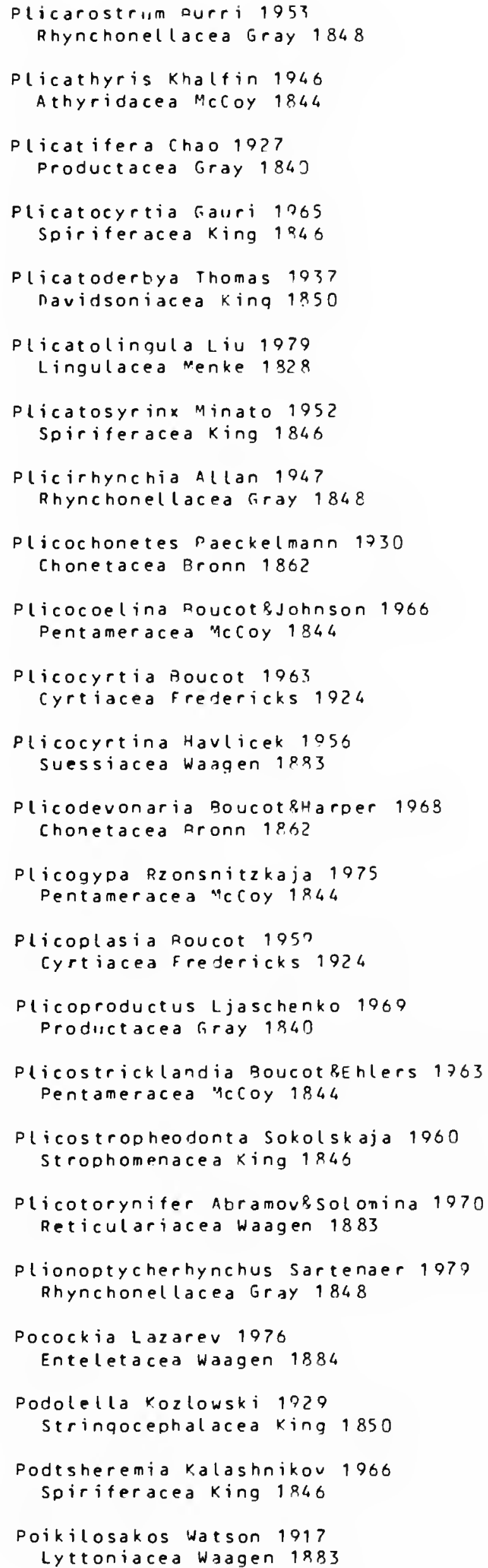

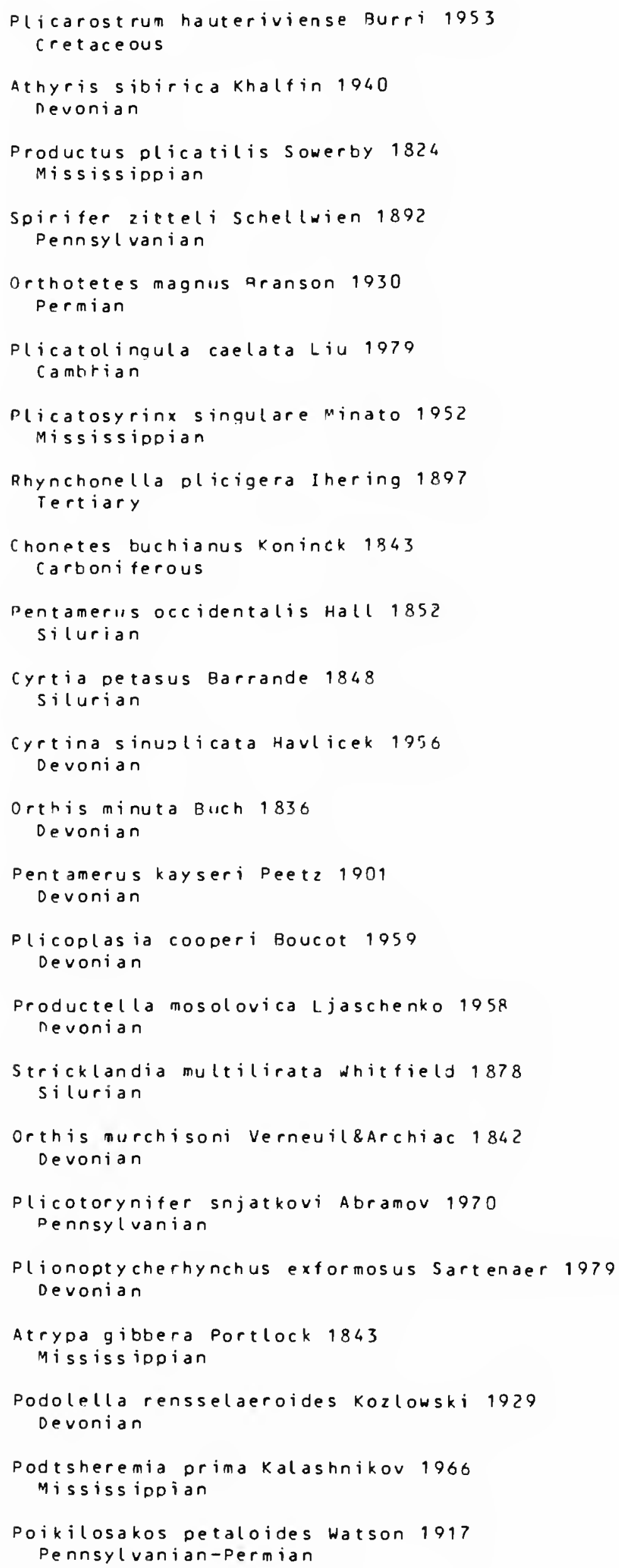




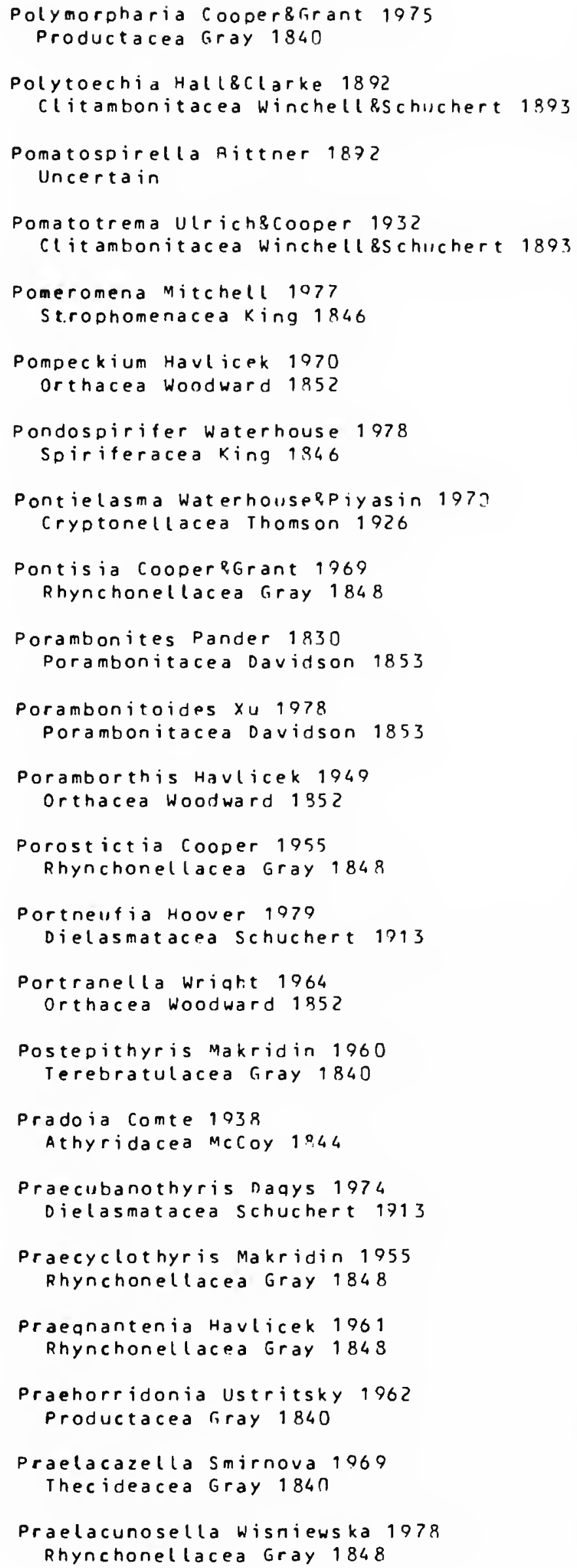

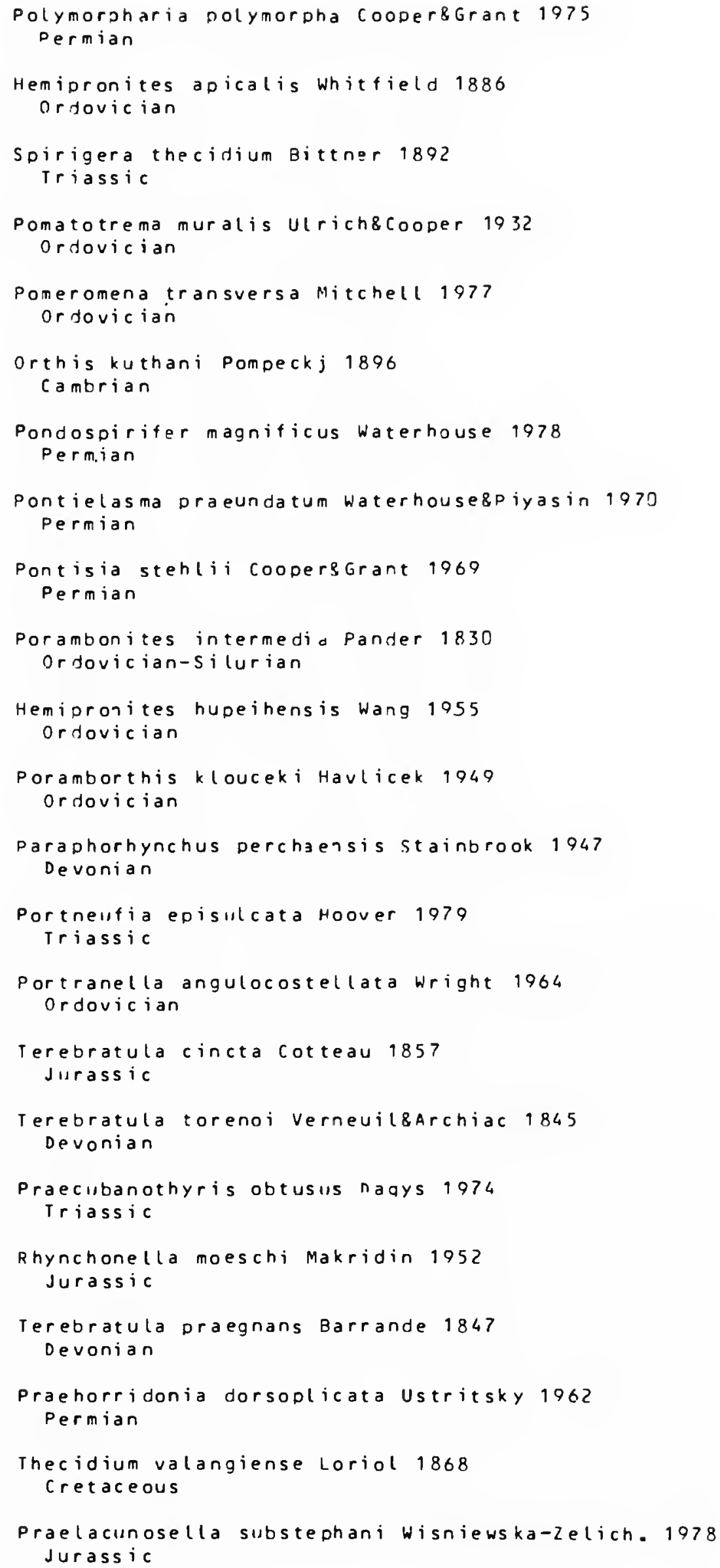


Proqonambonites 0 pik 1934 Gonambonitacea Schuchert \&Cooper 1931

Prokopia Havlicek 1953 Enteletacea wagen 1884

Promarginifera Shiells 1966 Productacea Gray 1340

Pronalivkinia Rukavischnikova 1977 Atrypacea Gill 1871

Propatella Grubbs 1939 Craniacea Menke 1828

Propyqope aittner 1890 Diel asmatacea Schuchert $1 \geqslant 13$

Prorensselaeria Raymond 1723 Stringocephalacea King 1850

Proreticularia Havlicek 1957 Reticulariacea wagen 1883

Prorichthofenia Kina 1931 Richthofeniacea Wagen 1885

Proschizophoria Maillieux 1912 Enteletacea Waggen 19.94

Prospira Maxuell 1954 Spiriferacea King 1846

Prosserella Grahau 1910 cyrtiacea fredericks 1924

Prostrickl andia Rukavischnikova et al 1973 Pentameracea McCoy 1844

Prosyringothyris fredericks 1916 Soiriferacea king 1846

Protambonites Havlicek 1972 clit ambonitacea winchell \&schuchert 1.893

Protanidanthus Liao 1979 Productacea Gray $184 \mathrm{~J}$

Protathyris Kozlowski 1929 Athyridacea McCOY 1944

Protatrypa Boucot, Johnsonsstaton 1964 Atrypacea Gill 1871

Proteguliferina Licharew 1960 strophalosiacea Schuchert 1913

Proteorthis Havlicek 1074 Orthacea Woodward 1952

Protobolus Liu 1979 Obolellacea Walcott\&schuchert 1908

Protochonetes Muir-Wood 1962 Chonetacea Rronn 1862

Protocortezorthis Johnsons Talent 1967 Dalmanellacea Schuchert 1713

Protocymostroohia Harper 8 a oucot 1978 Stropheodontacea Caster 1939

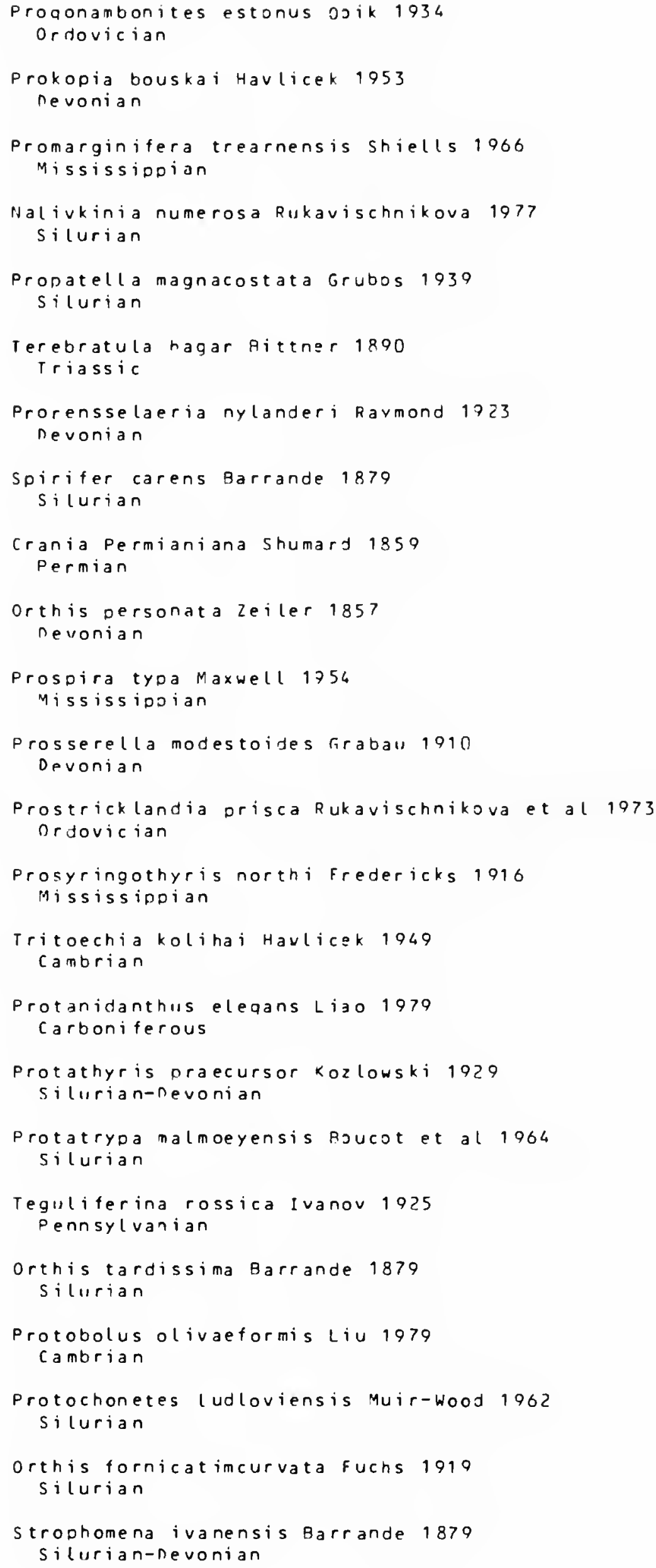




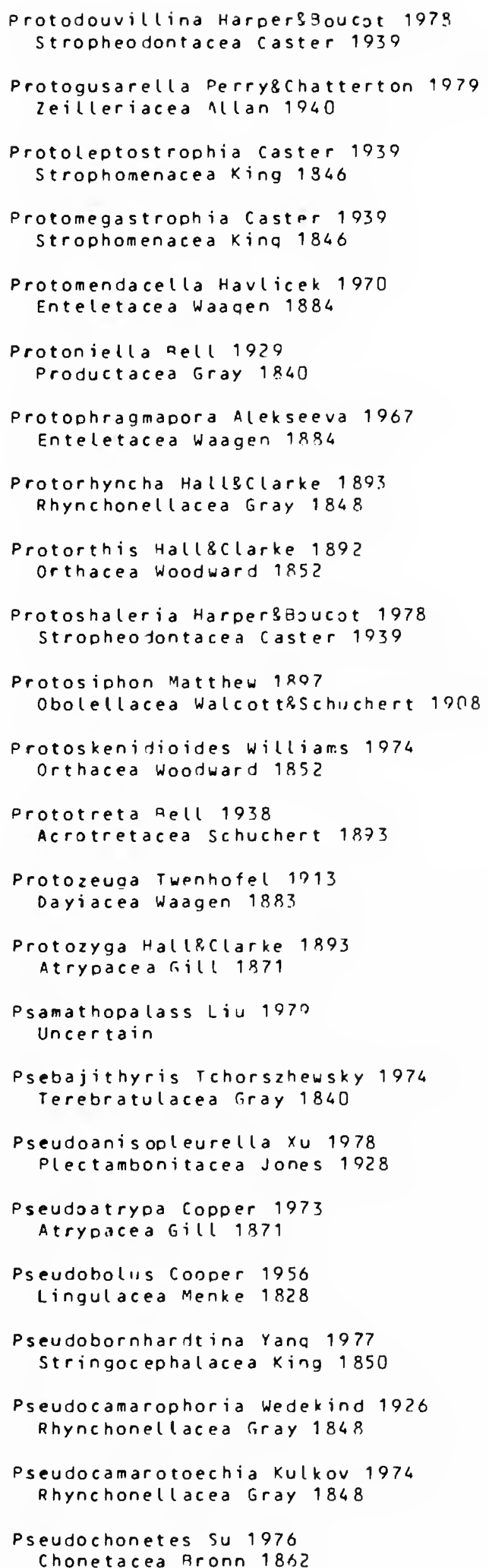




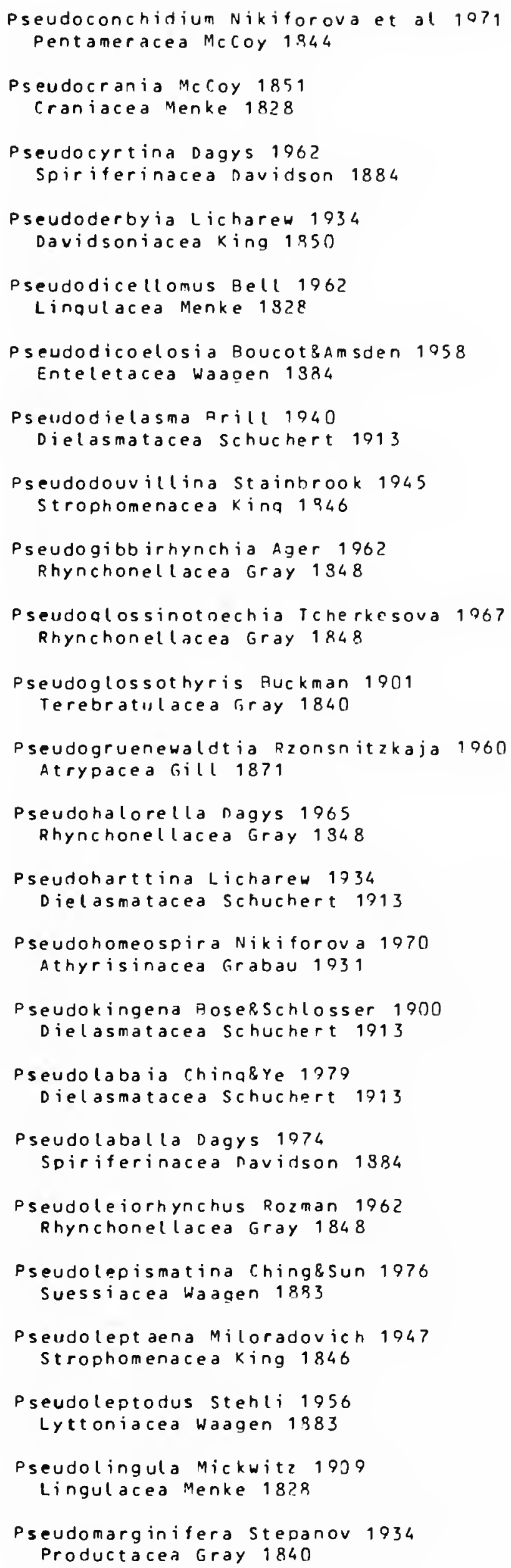

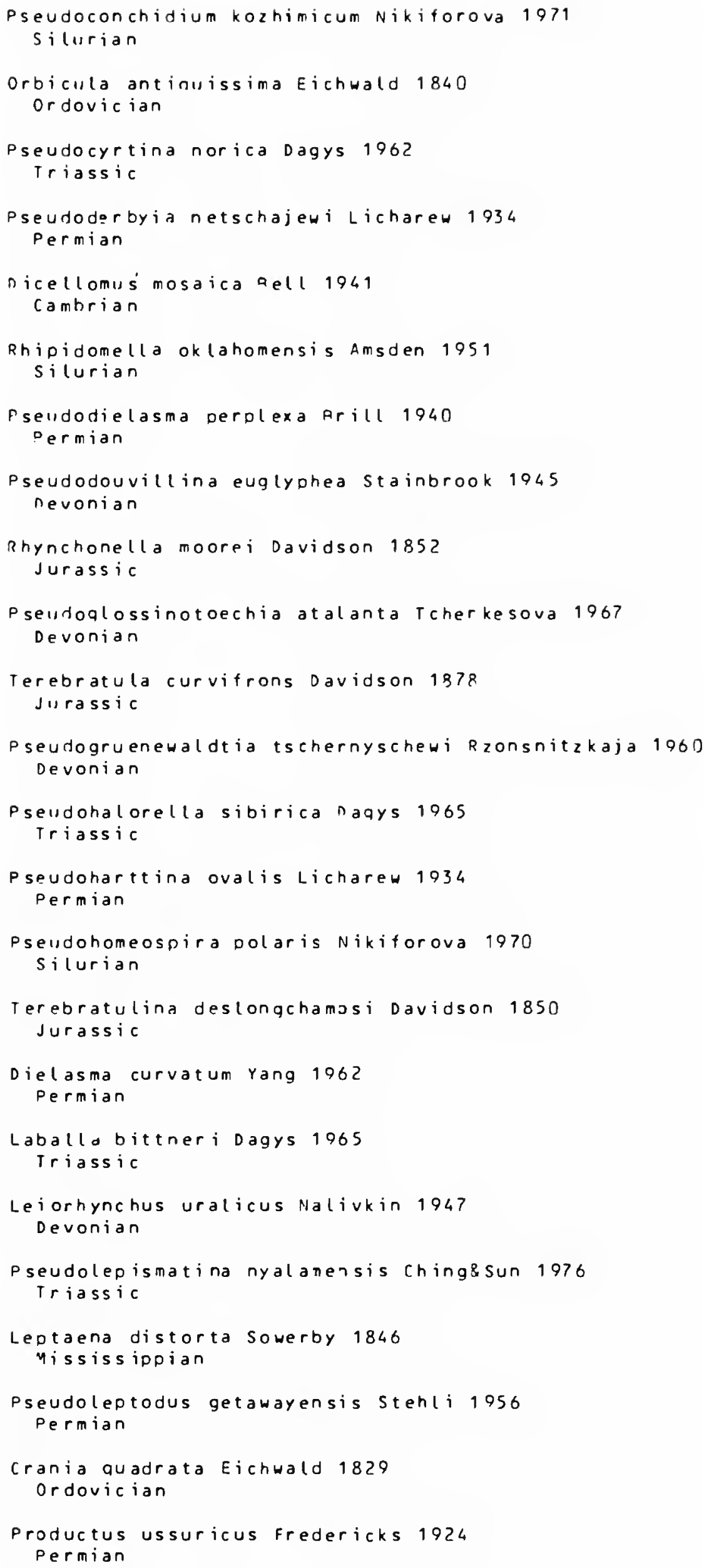




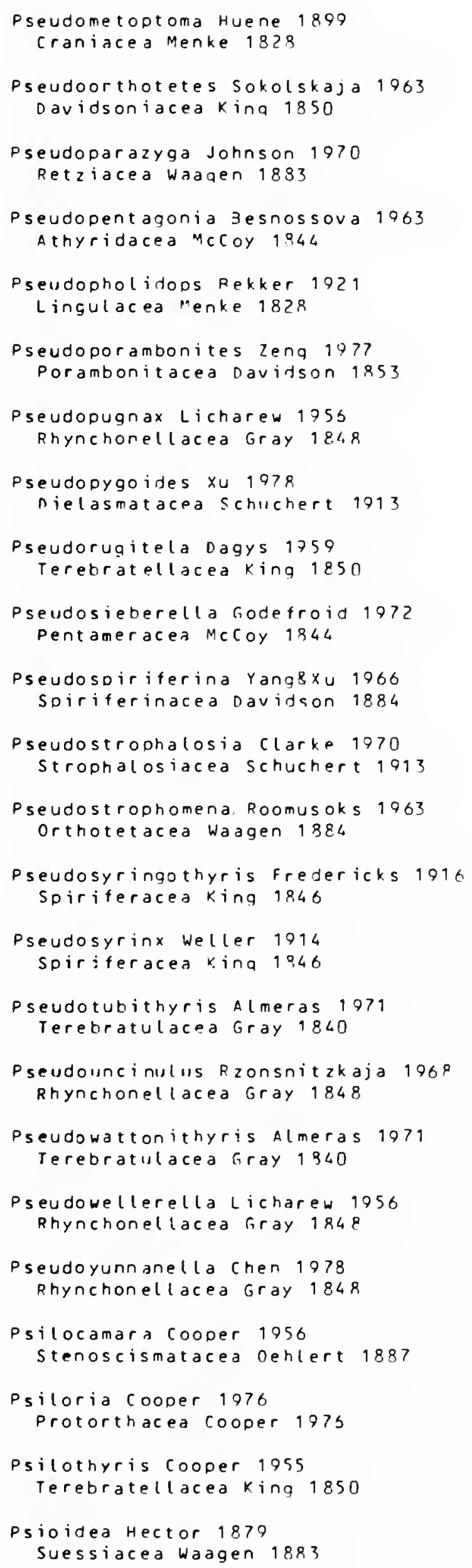

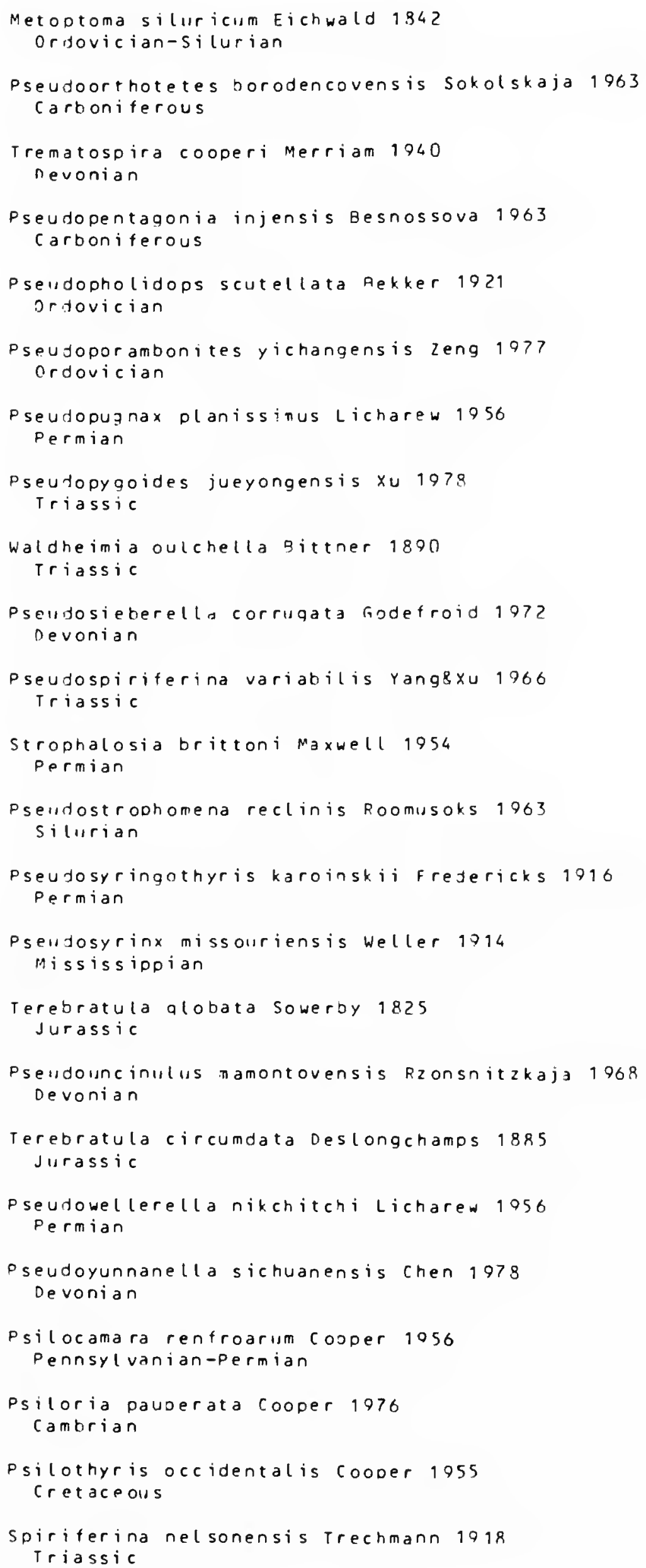


Psioidiella rampbell 1968 Spiriferinacea Davidson 1884

Pteroplecta waterhouse 1978 Delthyridacea Phillips 1841

Pterospirjter Dunbar 1955 Spiriferacea king 1846

Ptilorhynchia Crickmay 1933

Rhynchonellacea riray 1848

Ptilotorhynchus CooperRGrant 1976 Rhynchonellacea Gray 1848

Ptychoqlyptus willard 1028 Plectambonitacea Jones 1928

Ptychomaletoechia Sartenaer 1961 Rhynchonel lacea Gray 1848

Ptychopelt is Derner 1003 paterinacea Schuchert 1893

Ptychopleurella Schuchert 8 Cooper 1031 orthacea Woodward 1952

Ptyctorhynchia 3uckman 1917 Rhynchonel lacea Gray 1848

Ptyctothyris Auckman 1017 Terebratulacea Gray 1840

Ptygmactrum Cooper \&Grant 1976 Rhynchonel lacea Gray 1848

Puanatrypa xian 1978 Atrypacea fill 1871

Puanospirifer Jiang 1979 Reticulariacea Waagen 1983

Pugilis Sarytcheva :949 Productacea Gray $184 \mathrm{~J}$

Pugnax Hal L 8 Clarke 1893 Rhynchonellacea Gray 1848

Pugnoides weller 1910 Rhynchonel Iacea Gray 1848

Pulchratia Muir-wood\&Eooper 1960 Productacea Gray 1840

Pulchrithyris Sahni 1025 Terebratulacea Gray 1843

Pulsia Ivanov 1925 Davidsoniacea king 1850

Pumilus Atkins 1958 Terebratellacea King 1850

Punctatrypa Havlicek 1953 Atrypacea Gill 1871

Punctocyrtella Plodowski 1968 Spiriferacea king 1846

Punctolira Ulrich\&Cooper 1936 Porambonitacea Davidson 1853

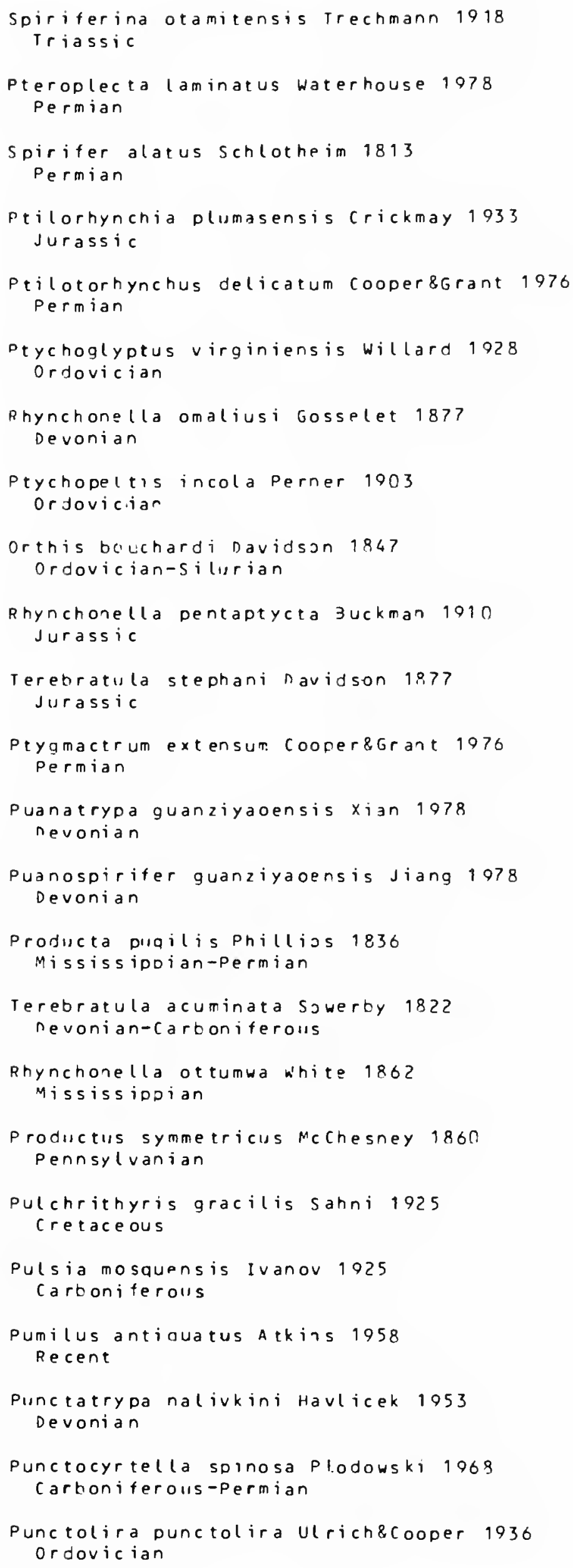

Punctolira punctolira Ulrich\&cooper 1936 ordovician 


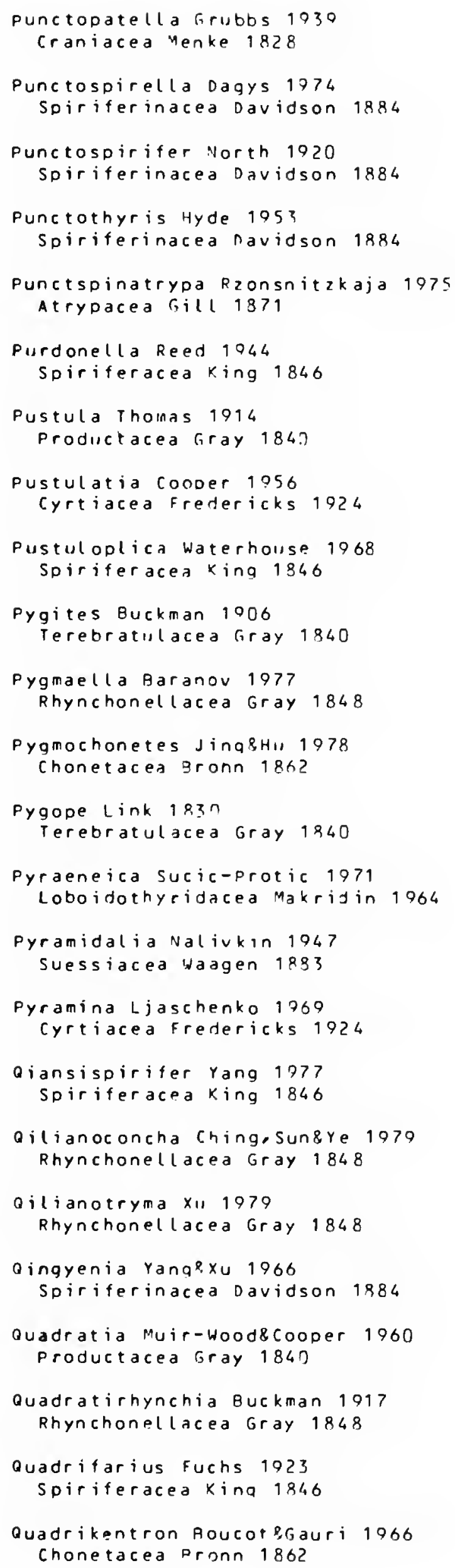

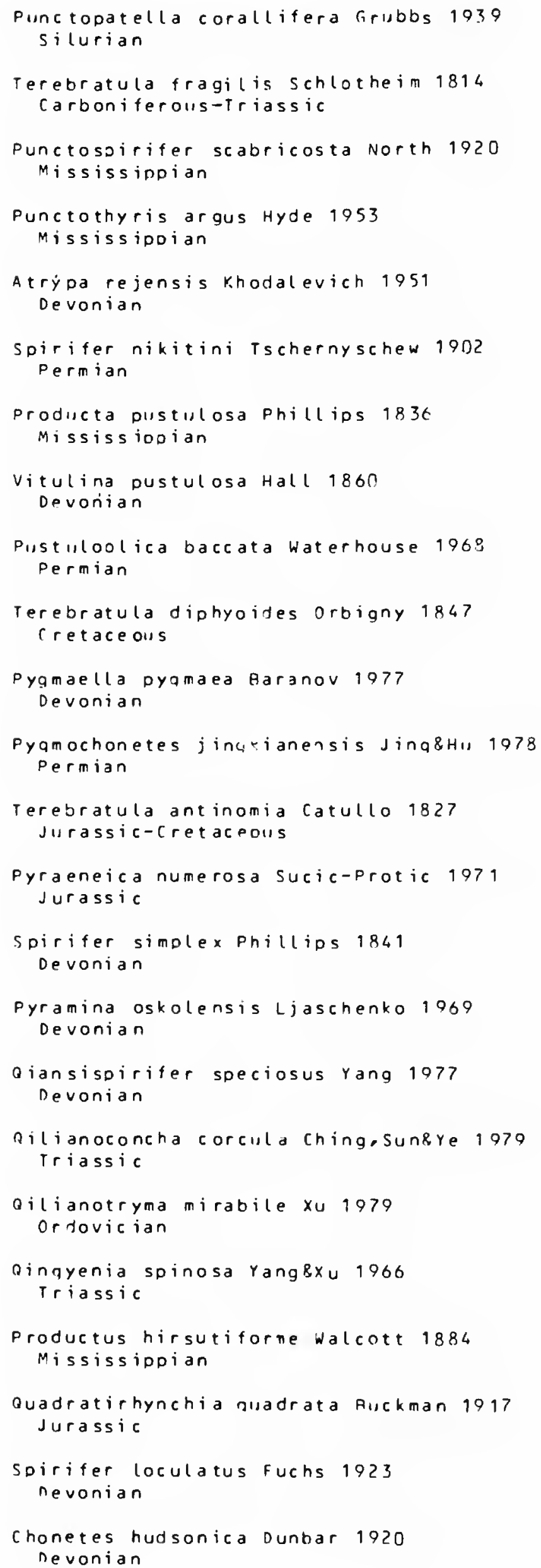


Quadrisonia Powel 18 Henders on 1978 Acrotret acea Schuchert 1893

Quadrithyrina Havlicek 1959 Reticulariacea wagen 1883

Quadrithyris Havlicek 1957 Reticulariacea Wagen 1.983

Quadrochonetes Stehli 1954 Chonetacea Aronn 1862

Quangyuania sheng 1975

Atrypacea Gill 1871

Quasiavonia Brunton 1966 Productacea Gray 1840

Quasimartinia Havlicek 1959 cyrtiacea Fredericks 1924

Quasistrophonel la Harper 89011 ct 1978 Stropheodontacea Caster 1939

Quasithambonia Rednarczyks Biernat 1978 Lingulacea Menke 13?

Quebecia Walcott 9905 Kutorqinacea schuchert 1893

Quinquenel la waterhouse 1975 chonetacea aronn 1862

Quizhouspirifer Xian 1979 spiriferacea $k$ ing 1846

Radiatrypa Copper 197R Atrypacea Gill 1871

Radiomena Havlicek 1962 strophomenacea king 1846

Rafinesquina $\mathrm{Hall}$ \& $\mathrm{Cl}$ arke 1892 Strophomenacea king 1846

Rallacosta cooper \&6rant 1975 Reticulariacea Wagen 1883

Ramavectus Stehli 1954 Aulosteqacea Muir-Wood\&cooper 1960

Ranorthis Opik 1939

orthacea wcodward 18.52

Rastelligera Hector 1879 Spiriterinacea Davidson 1884

Ratburia Yanagida 1970 Chonetacea Bronn 186 ?

Rauffia Schulz 1914 stringocephalacea King 1850

Raunites opik 1739

Gonambonitacea Schuchert\&Cooper 1931

Ravozetina Havlicek 1974

Enteletacea wagen 1884

Rawdonia Peou 1979 Athyridacea MeCoy 1844

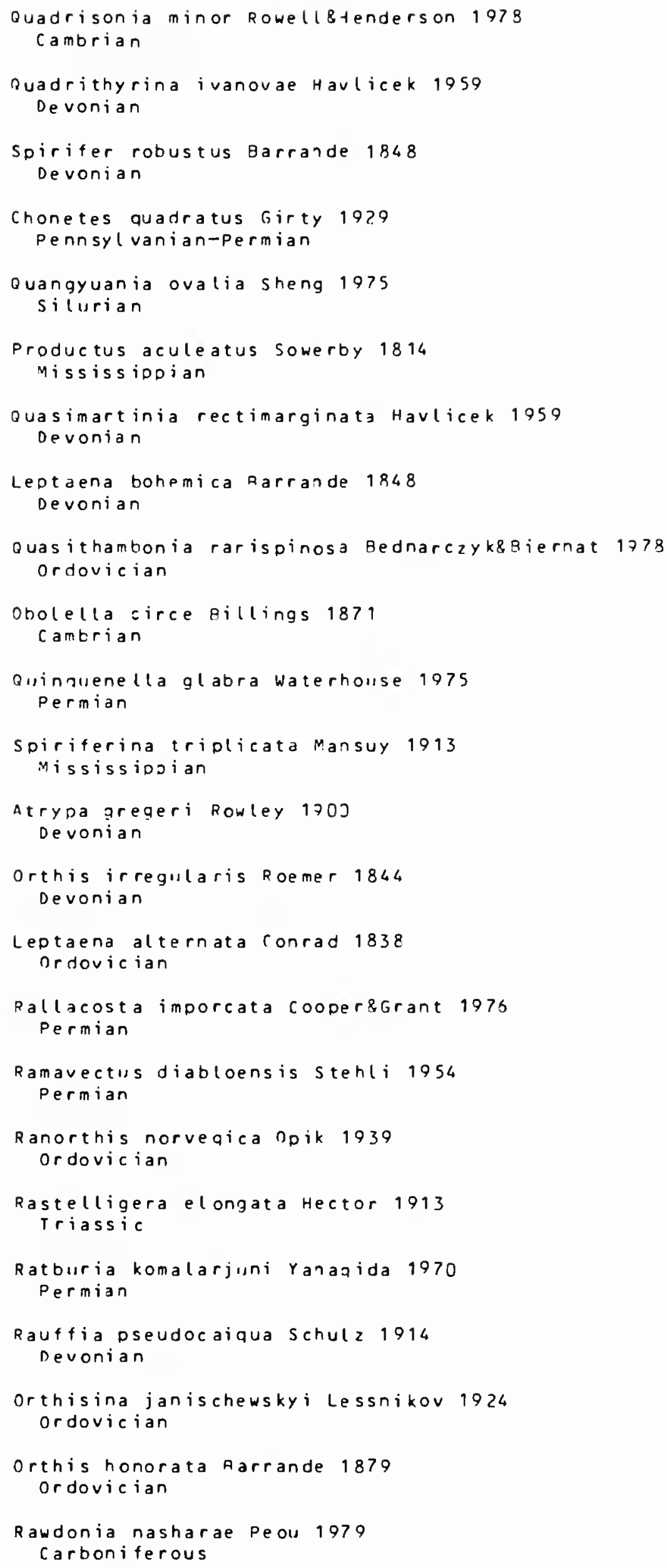




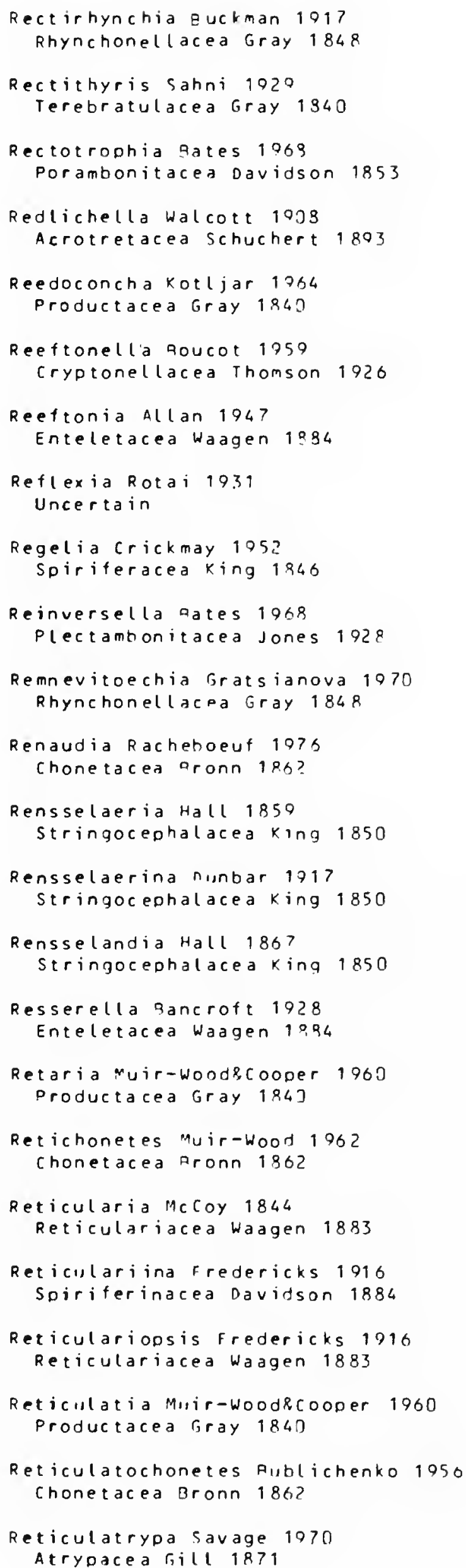

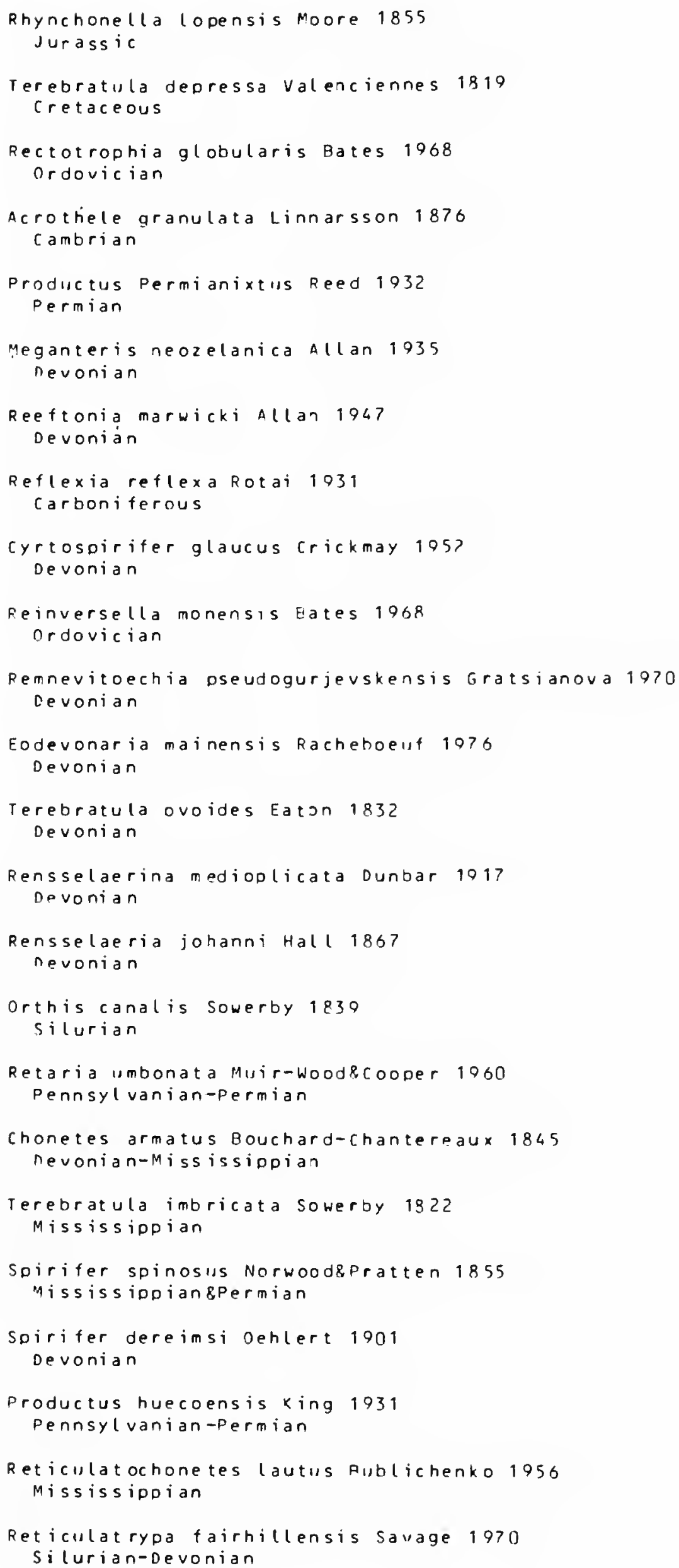




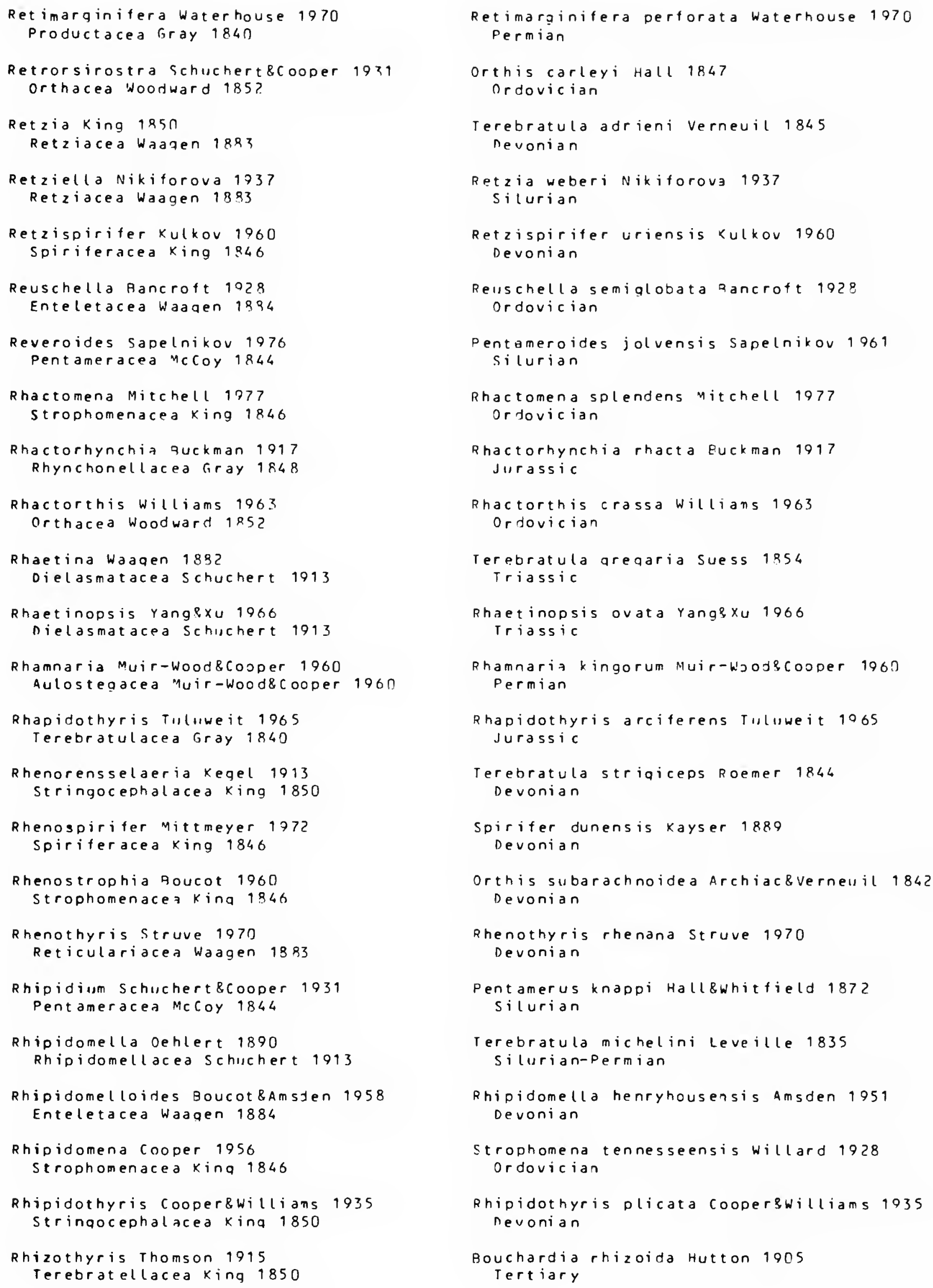




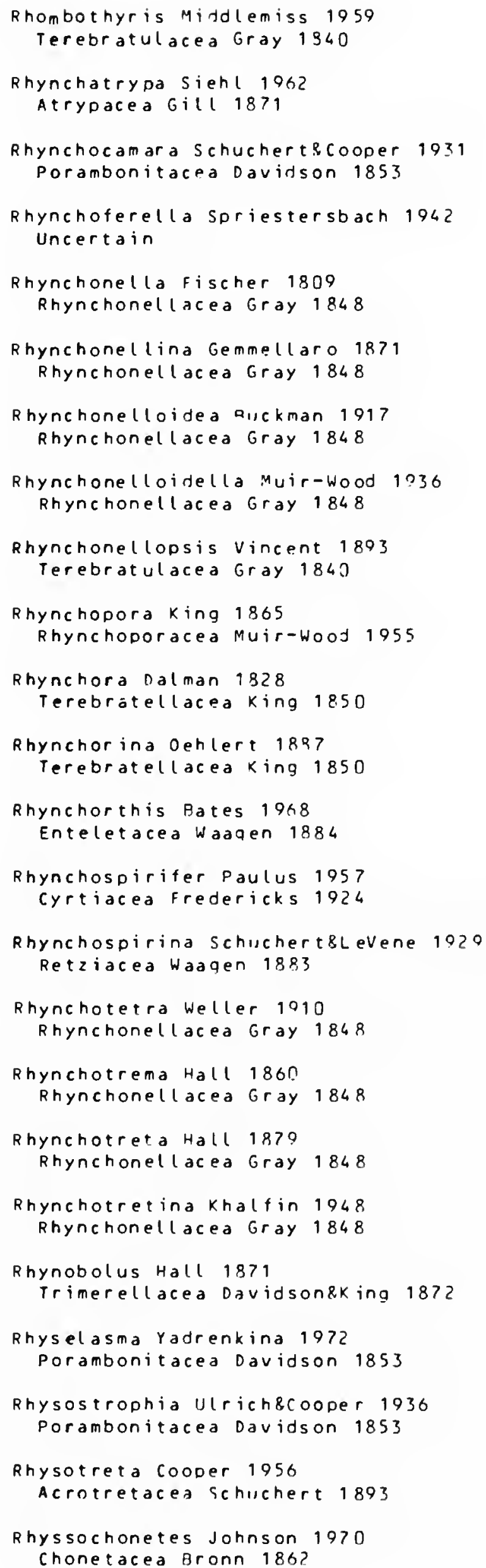

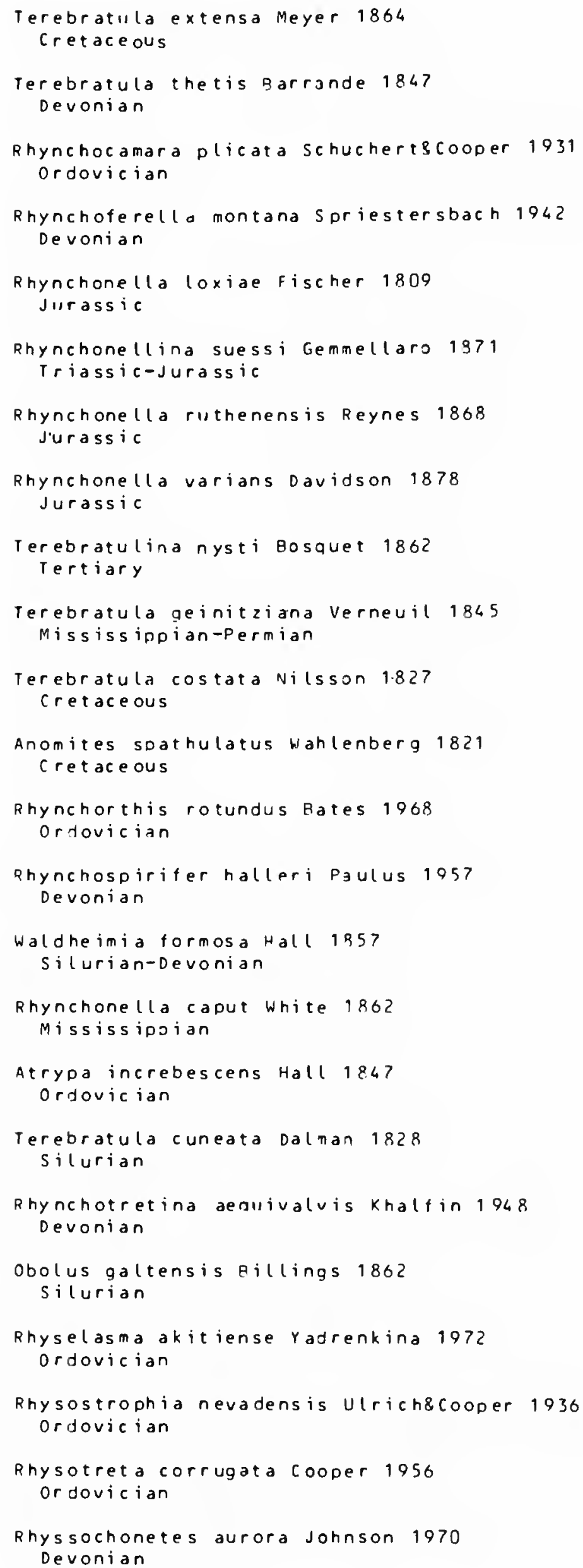


Rhytiophora Muir-Wood\&Cooper 1960 Productacea Gray 1840

Rhytirhynchia Cooper 1057 Rhynchonellacea Gray 1848

Rhyt is ia Cooperstarant 1975 Productacea Gray 1840

Rhyt istrophia caster 1939 Strophomenacea King 1946

Richthofenia Kayser 1881 Richthofeniacea Wagen $188 \mathrm{~S}$

Rictia Gresorio 1930 Uncertain

Rigbyella Stehli 1956 Lyttoniacea Wagen 1833

Rimirhynchia Auckman 1917 Rhynchonellacea Gray 1848

Rimirhynchops is Dagys 1963 Rhynchonellacea Gray 1848

Rioarandella Kobayashi 1937 Orthacea Woodward 185 ?

Rioultina Pajaud 1966 Thecideacea riray 1840

Ripidiorhynchus Sartenaer 1766 Rhynchonellacea Gray 1848

Robertorthis Havlicek 1977 orthacea Woodward 1852

Robinsonella Moisseiev 1936 Rhynchonel lacea fray 1848

Robust irhynchia seifert 1763 Rhynchone 1 lacea Gray 1848

Rochatorhynchia Katz 1752 Rhynchonellacea Gray 1848

Rocheithyris Almeras 1771 Terebratulacea Gray 1840

Roemerella Hall $8 \mathrm{Cl}$ larke 1890 Discinacea riray 1840

Romingerina Hal $18 \mathrm{Clarke} 1893$ Stringocephalacea $\mathrm{K}$ ing 1850

Rorespirifer waterhouse\&piyas in 1970 Reticislariacea Waaen 1883

Rosella Andreeva 1972 Porambonitacea Davidson 1853

Rossirhynchus Gaetani 1964 Rhynchonellacea Gray 1848

Rostranteris riemmellaro 1899 Dielasmatacea Schuchert 1913

Rostricellula Ulrichscooper 1942 Rhynchonel lacea Gray 1848

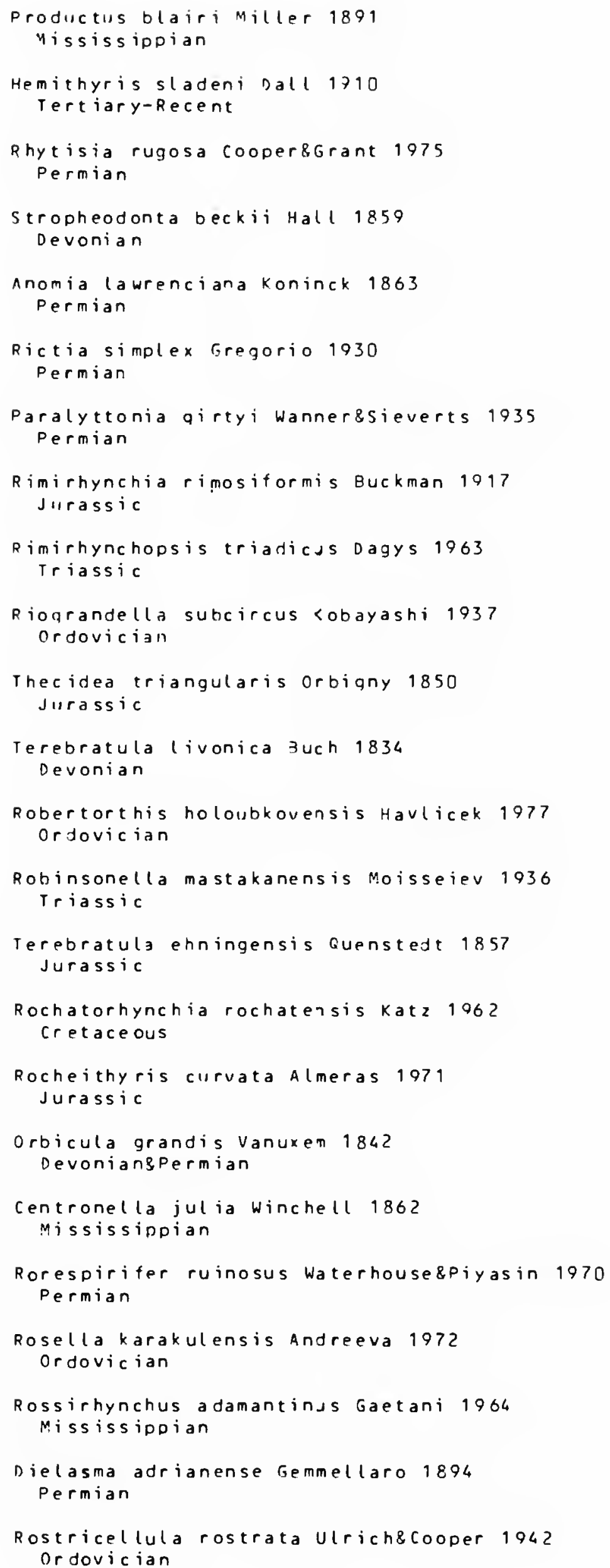


Rostrirhynchia sucic-Protic 1969 Rhynchonellacea Gray 1848

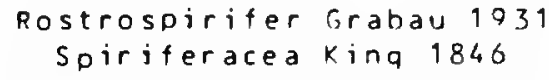

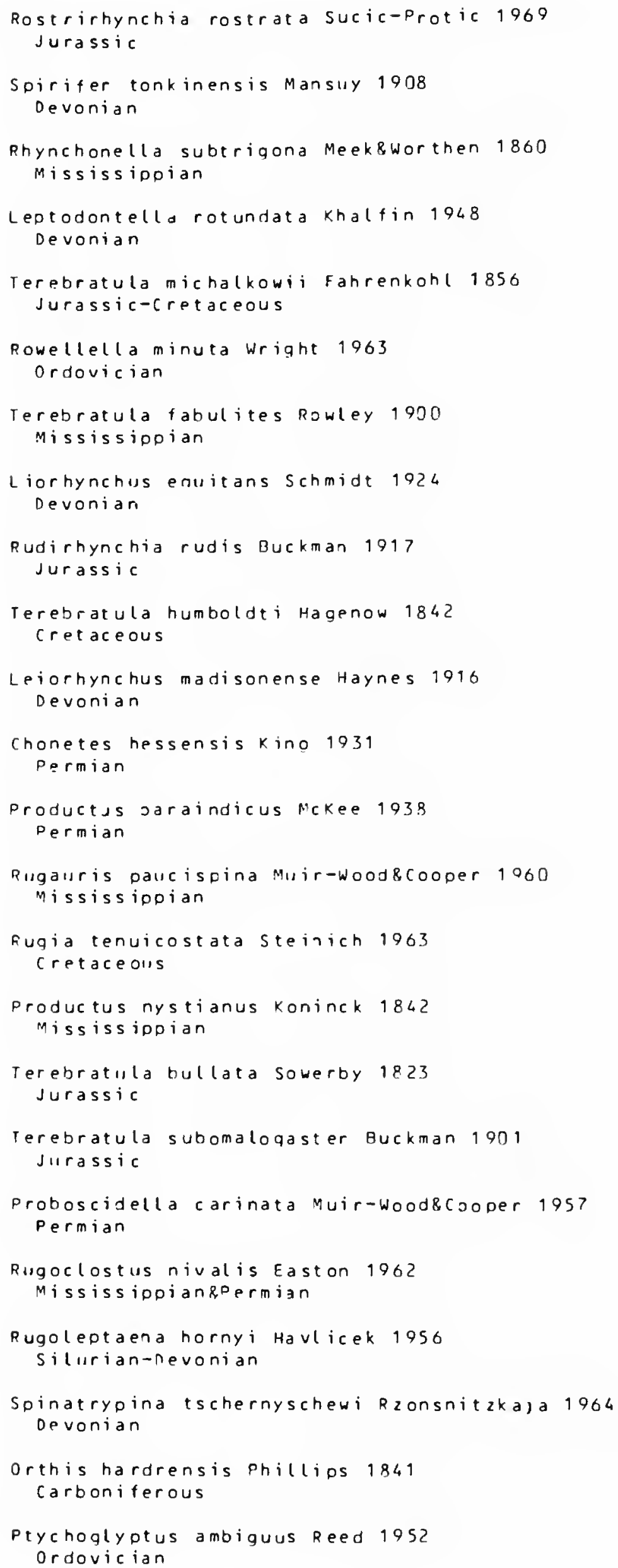




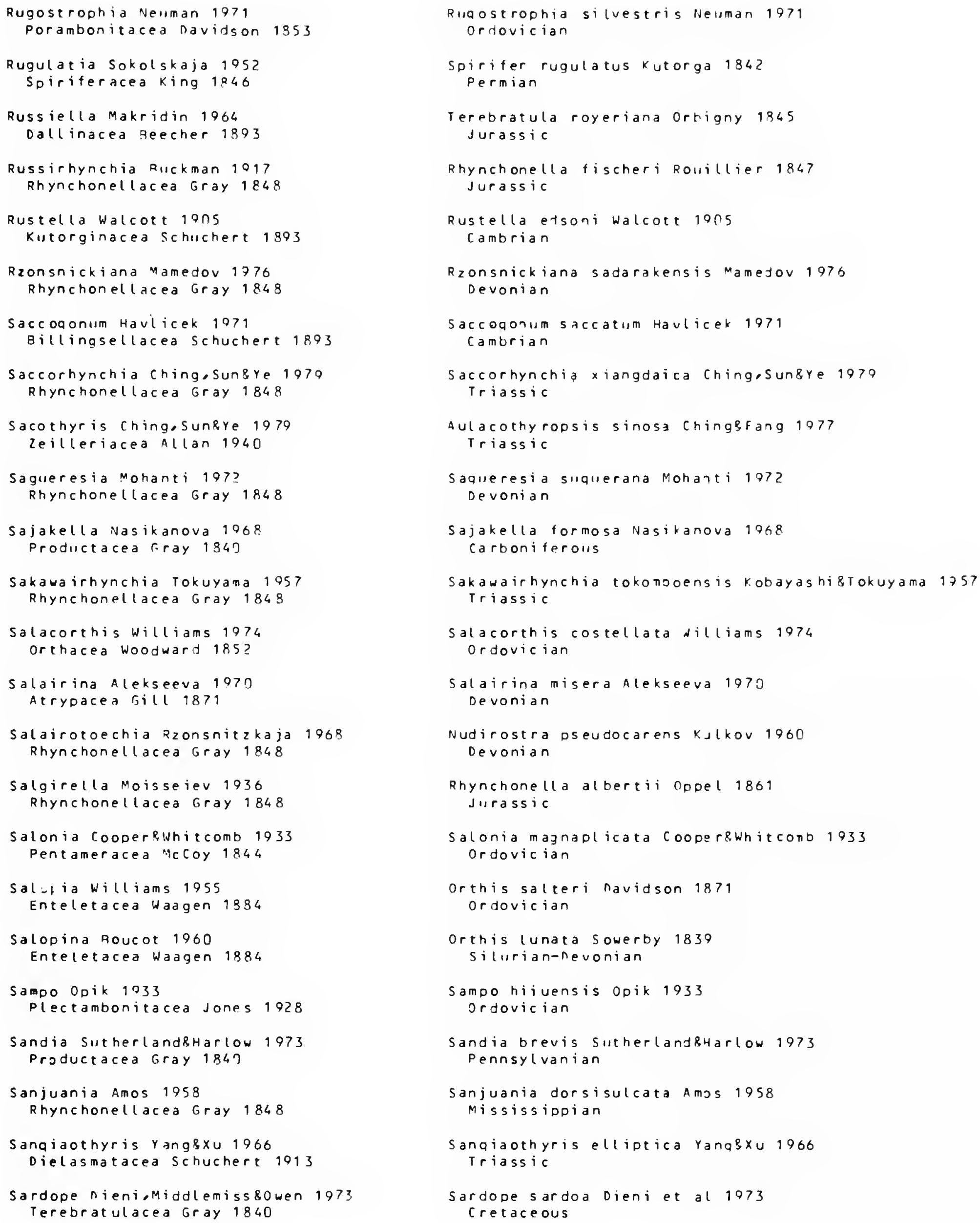




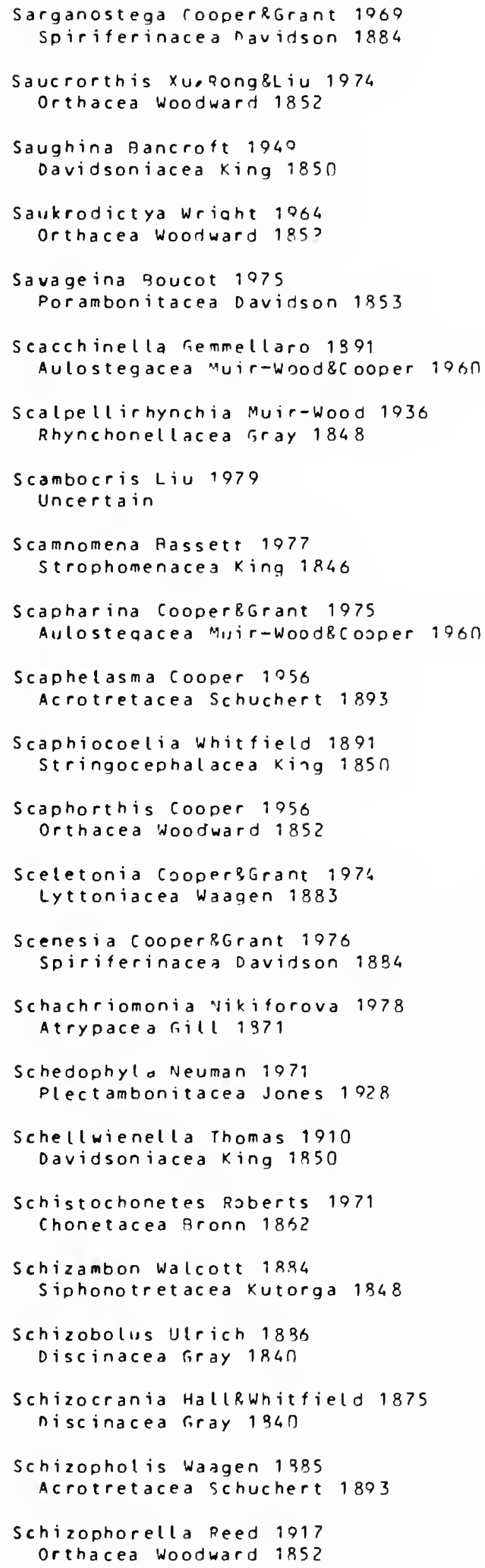

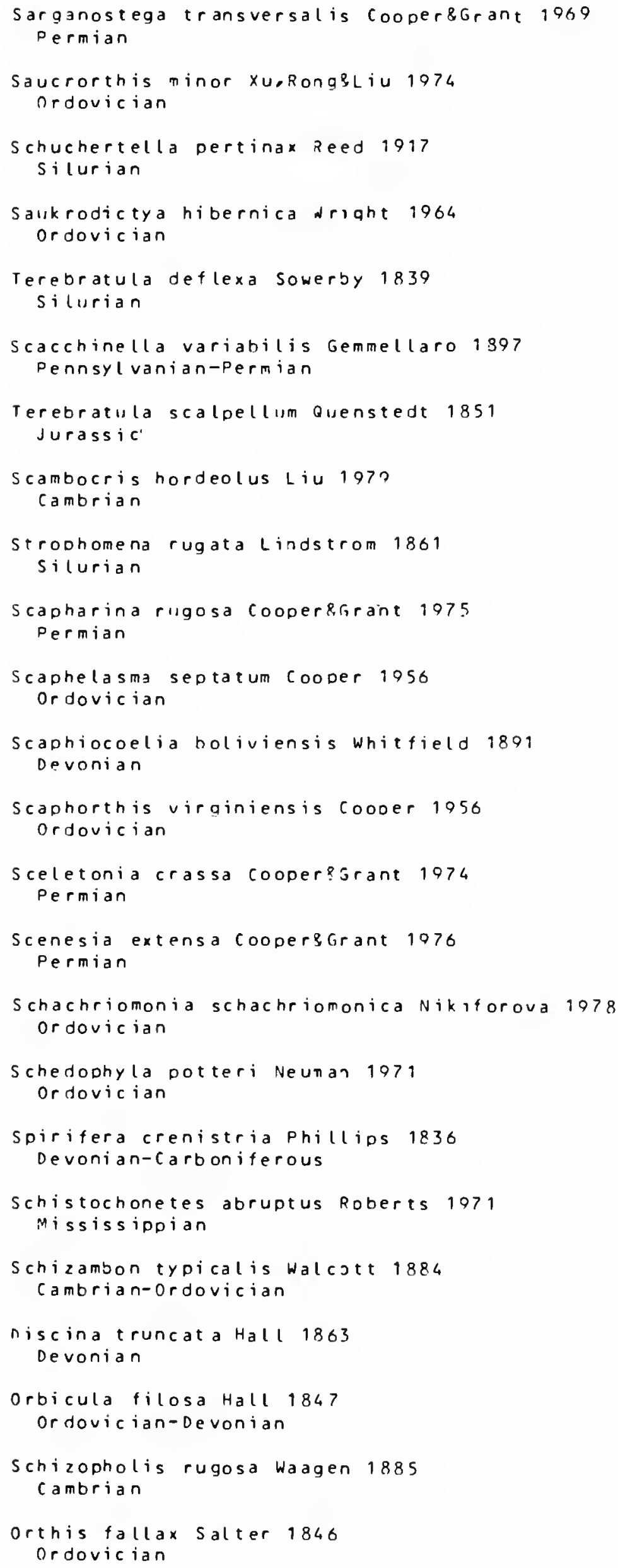


Schizophoria King 1850

Enteletacea Waagen 1884

Schizoramma Foerste 1912 Orthacea woodward 1852

Schizospirifer frabau 1931 Spiriferacea King 1846

Schizotreta Kutorga 1848 Discinacea firay 1840

Schizotretoides Termier\&Monod 1978 Acrotretacea Schuchert 1893

Schmidtites SchuchertRlevene 1929

Linqulacea Menke 1878

Schnurella Schmidt 1964 Rhynchone I lacea Gray 1848

Schrenkiella Barchatova 1973 Productacea Gray 1840

Schuchertella Girty 1904 Orthotetacea wagen 1884

Schuchertellopsis Maillieux 1939 Davidsoniacea King 1950

Schuchertina walcote 1705 Kutorginacea Schuchert 1893

Schwagerispira Dagys 1972 Retziacea Waagen 1833

Scoloconcha Gordon 1966 Productacea riray 1840

Scumulus steinich 1968 Terebratellacea King 1850

Scutepustula Sarytcheva 1963 Productacea fray 1840

Sedenticellula Cooper 1942 stenoscismatacea Oehlert 1887

Selenella Hall\&clarke 1893 Uncertain

Sellithyris Middlemiss 1959 Terebratulacea Gray 1840

Selloproductus Termier et al 1974 Productacea Gray $184 \pi$

Semenewia Paeckelmann 1930 Chonetacea Sronn 1862

Semibrachythyrina Yang\&Ting 1962 Spiriferacea King 1846

Semicostella Muir-wood\&Cooper 1963 Productacea Gray 1840

Seninula McCoy 1844 Athyridacea McCoy 1844

Semiotoechia Ljaschenko 1973 Rhynchonel lacea firay 1848

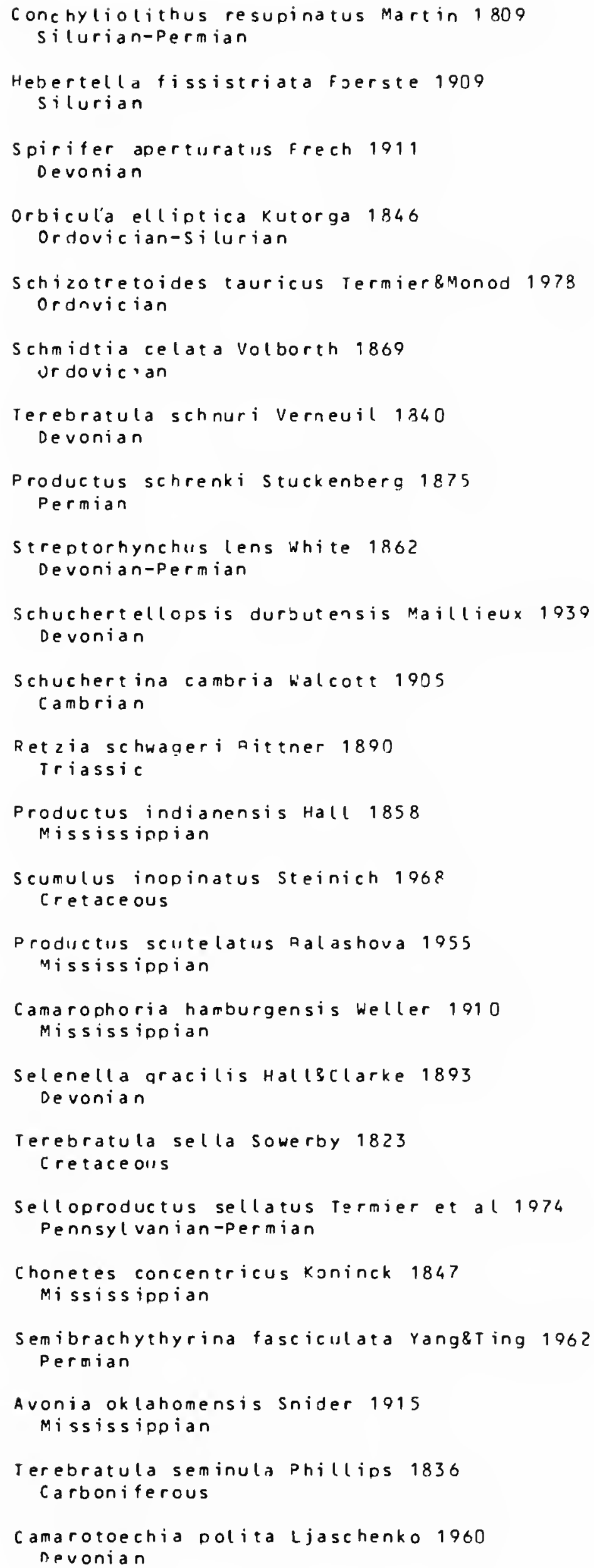




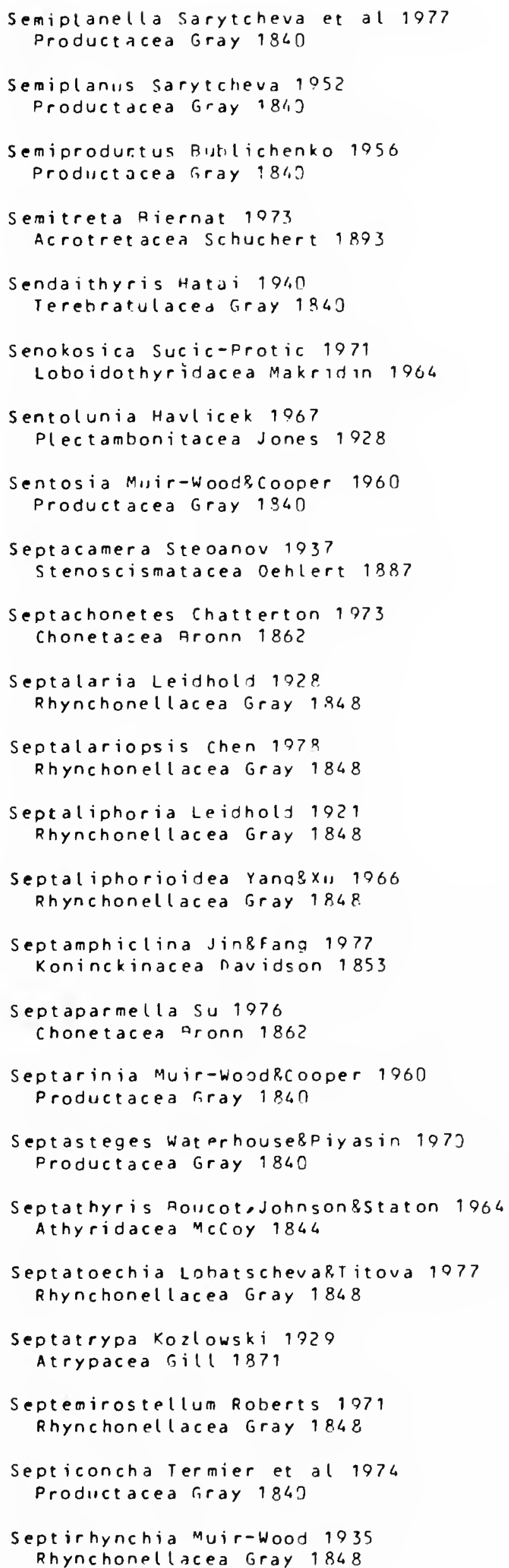

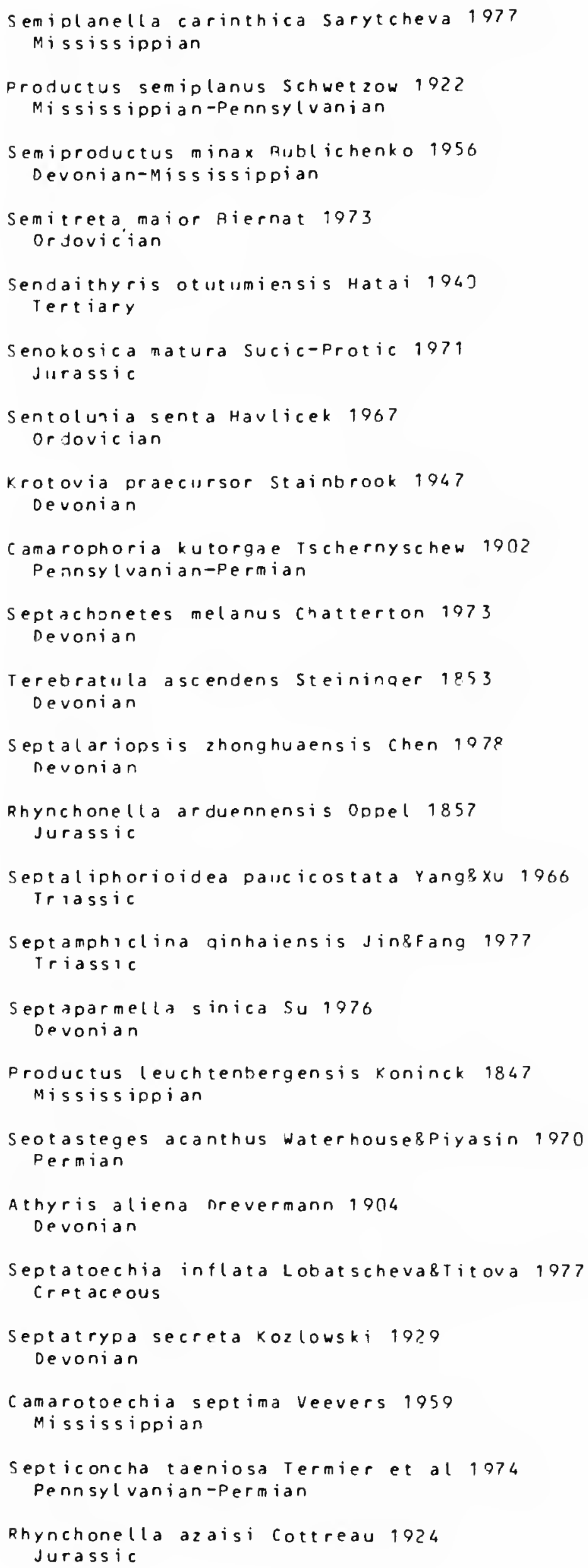




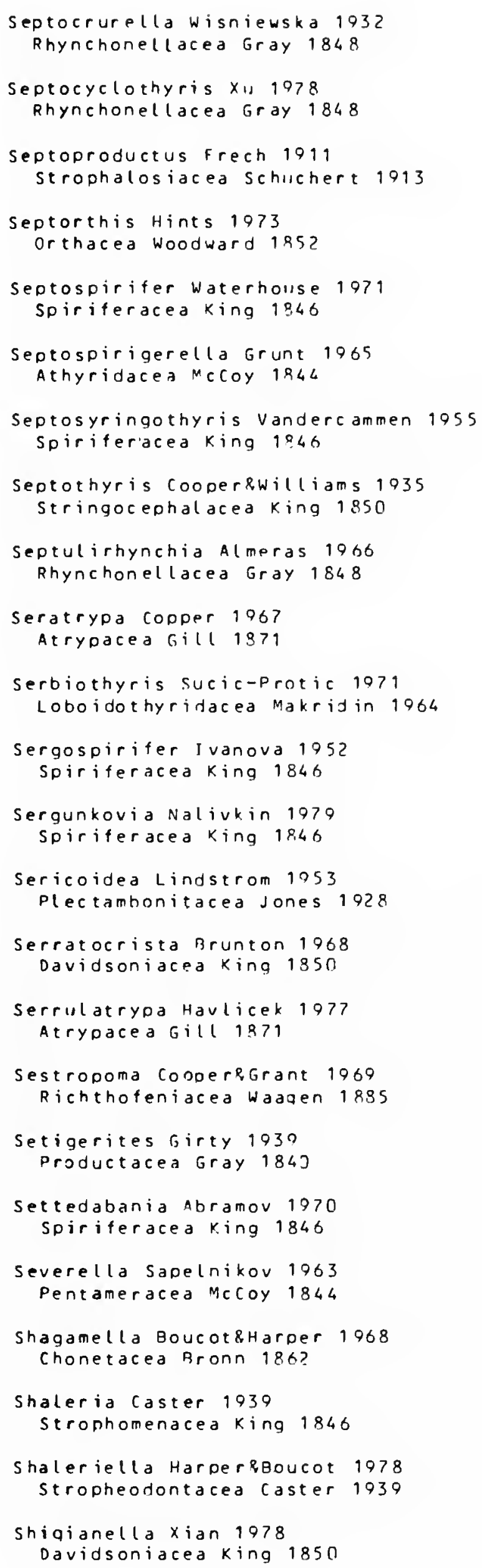

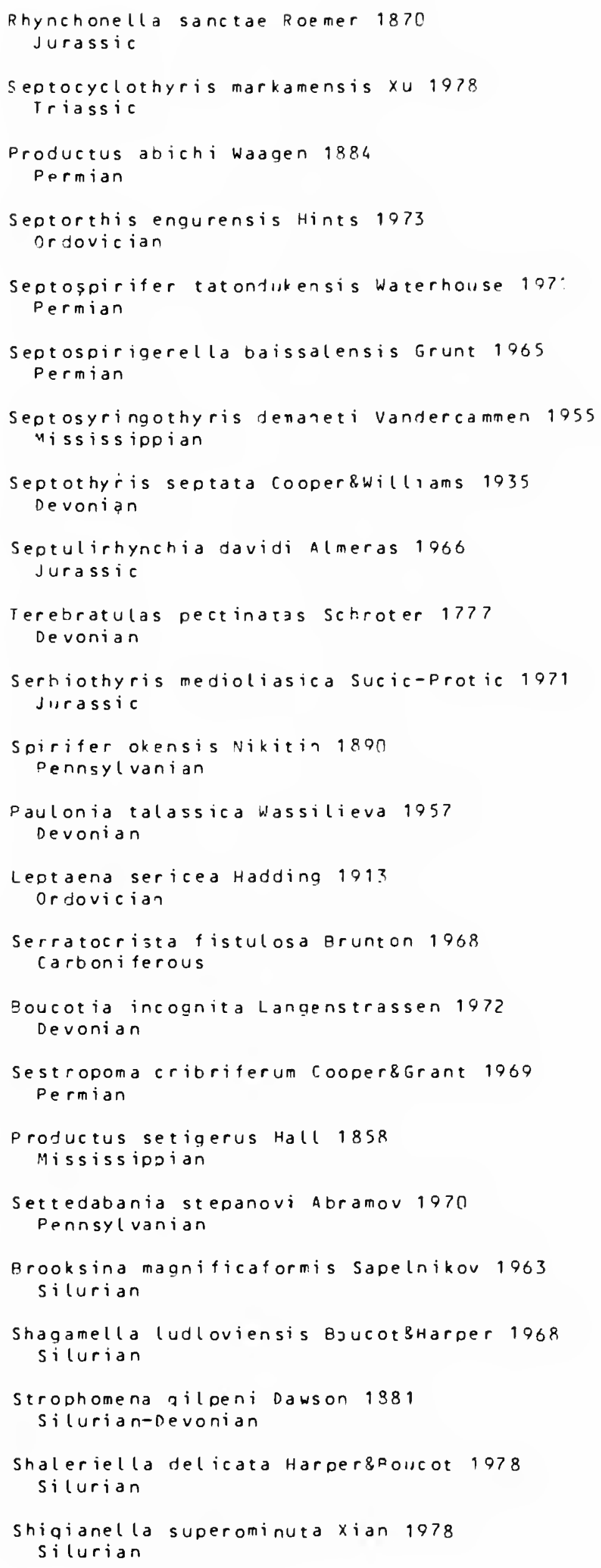




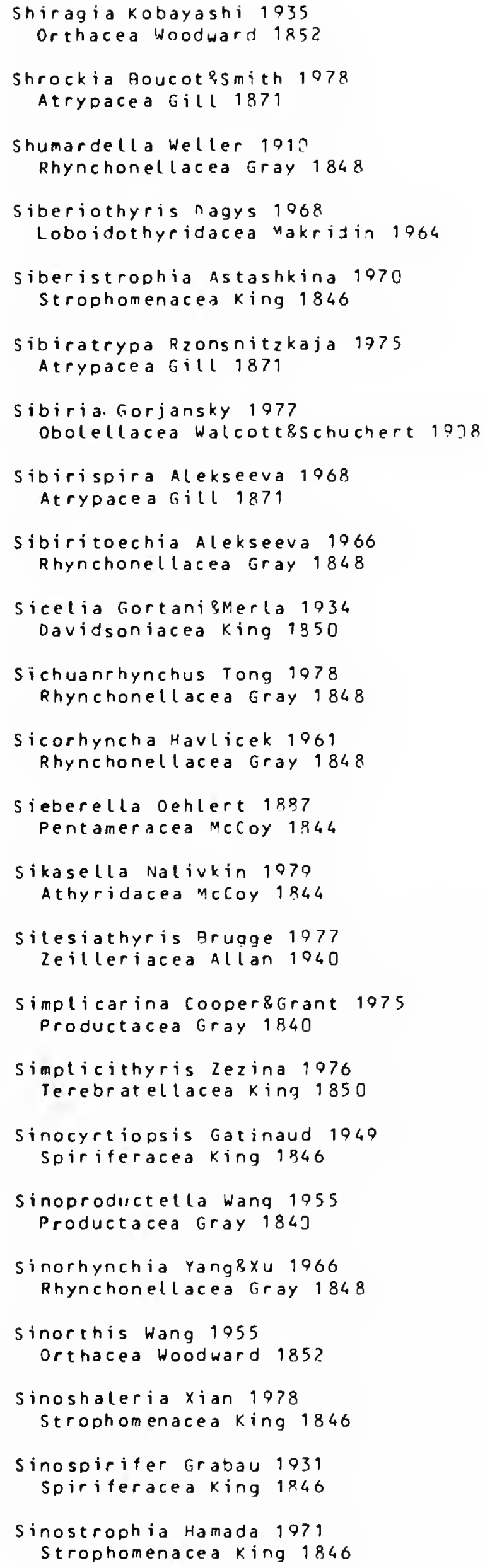

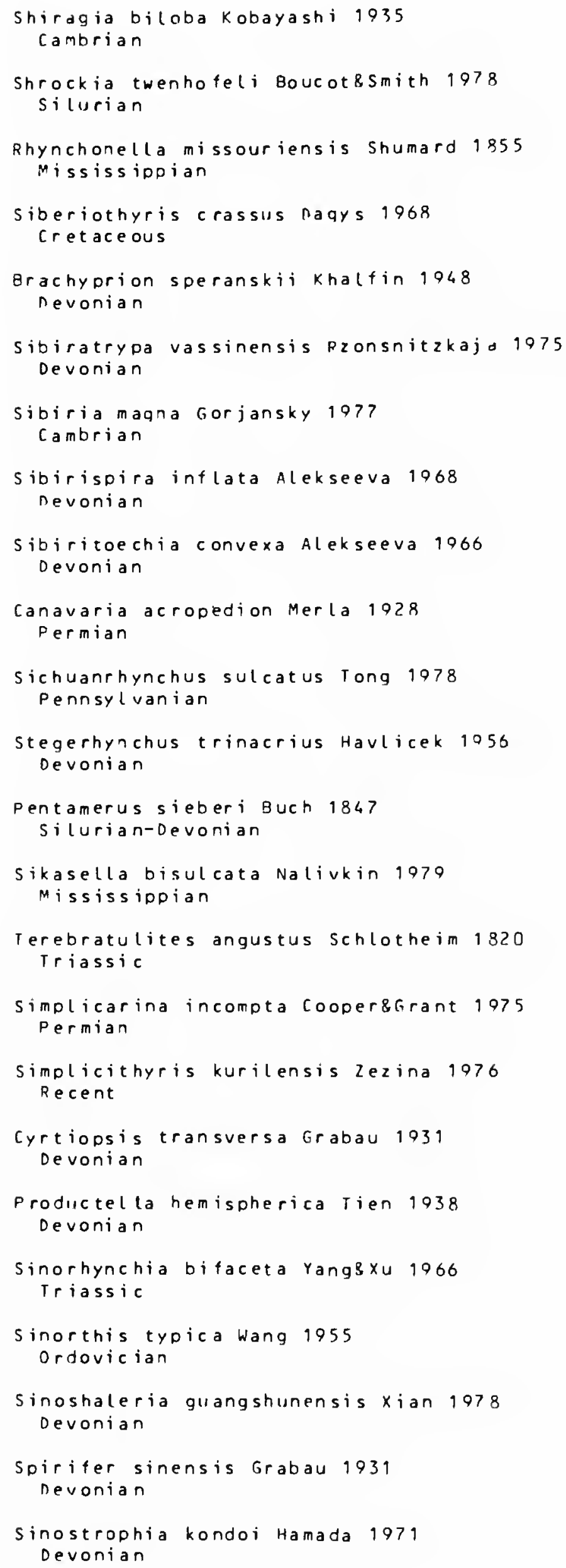


Sinotectirostrum Sartenaer 1961

Rhynchonel lacea Gray 1848

Sinothyris Minato 1953

Reticulariacea Wagen 1883 .

Sinuatella Muir-Wood 1928

Strophalosiacea Schuchert 1913

Sinucosta Dagys 1063

Spiriferinacea ravidson 1884

Sinuplicorhynchia Dagys 1965

Rhynchonellacea Gray $184 \mathrm{f}$

Siphonosia cooper 8 Grant 1975

Productacea Gray 184 ?

Siphonotreta Verneuil $\$ 945$

Siphonotretacea Kutorga 1848

Skel idorygma Carter 1974

Spiriferacea king 1846

Skenidioides schuchert\&cooper 1931

orthacea Woodward 1852

Skenidium Hall 1860

Orthacea Woodward 1852

Slovenirhynchia siblik 1957

Rhynchonel lacea Gray 1848

Sweathenel la Pancroft 1928

Enteletacea wagen 1984

Socraticum Gregorio 1930 Uncertain

Solidipontirostrum sartenaer 1970 Rhynchonel lacea Gray 1848

Somalirhynchia weir 1925

Rhynchonellacea Gray 1848

Somalitela Muir-Wood 1935

Zeilleriacea Allan 1940

Somalithyris Muir-wood 1935

Terebratulacea Gray 1840

Soudleyella pancroft 1945 Enteletacea wagen 1984

Sowerbina Fredericks 1928

productacea firay 1840

Sowerbyella Jones 1928

$P l e c t a m b o n i t a c e a$ Jones 1928

Sowerbyites Teichert 1937

Plectambonitacea Jones 1928

Spanodonta Prendergast 1935

Plectambonitacea Jones 1928

Spasskothyris Smirnova 1975

Loboidothyridacea Makridin 1064

Sphaerirhynchia Cooper\&Muir-wood 1951

Rhynchonellacea Gray 1848

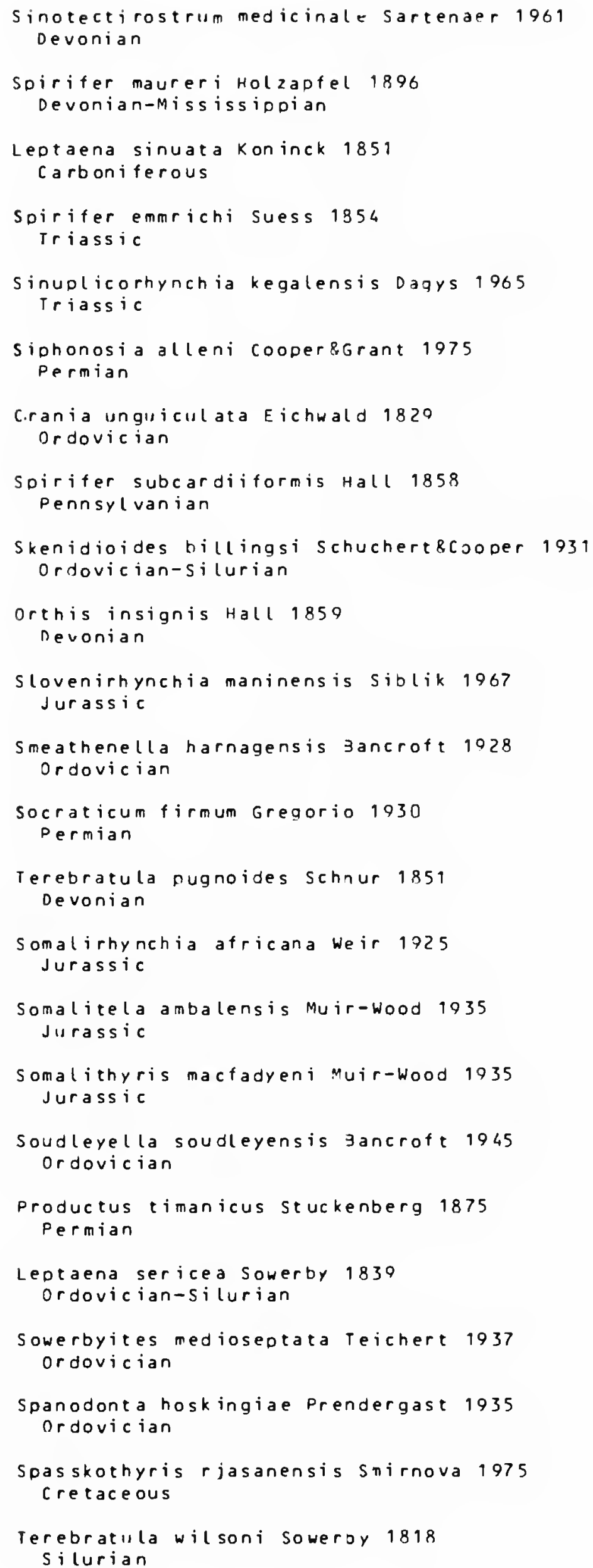




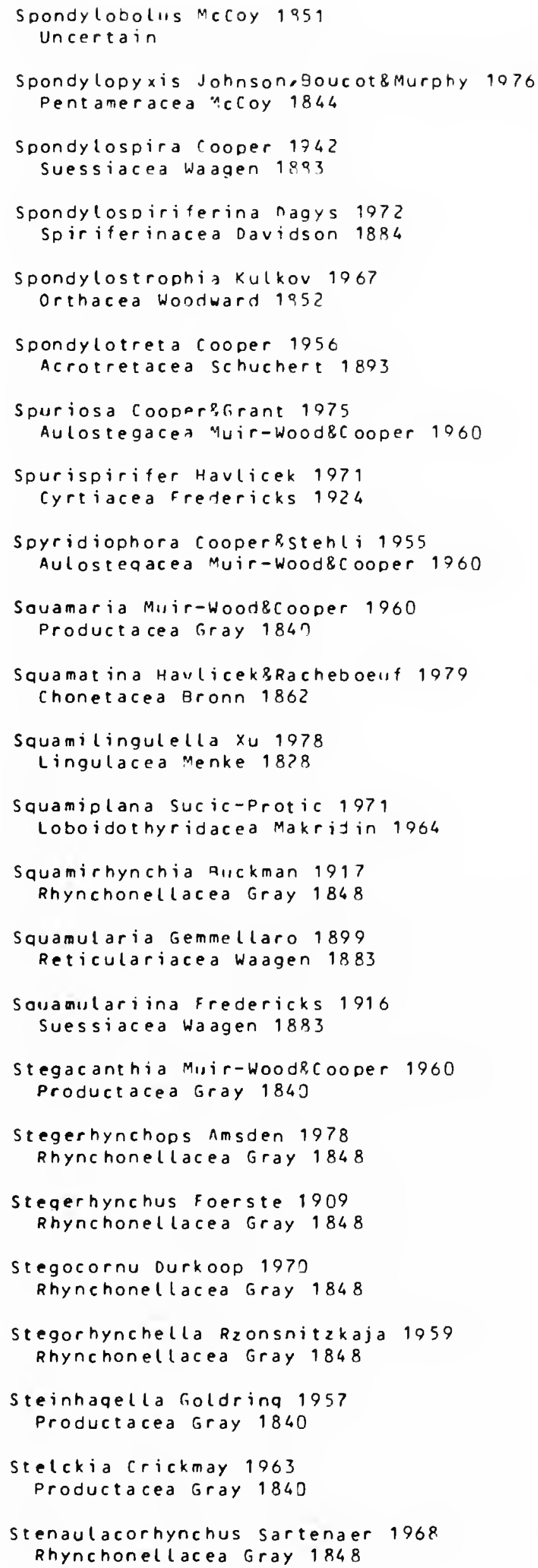

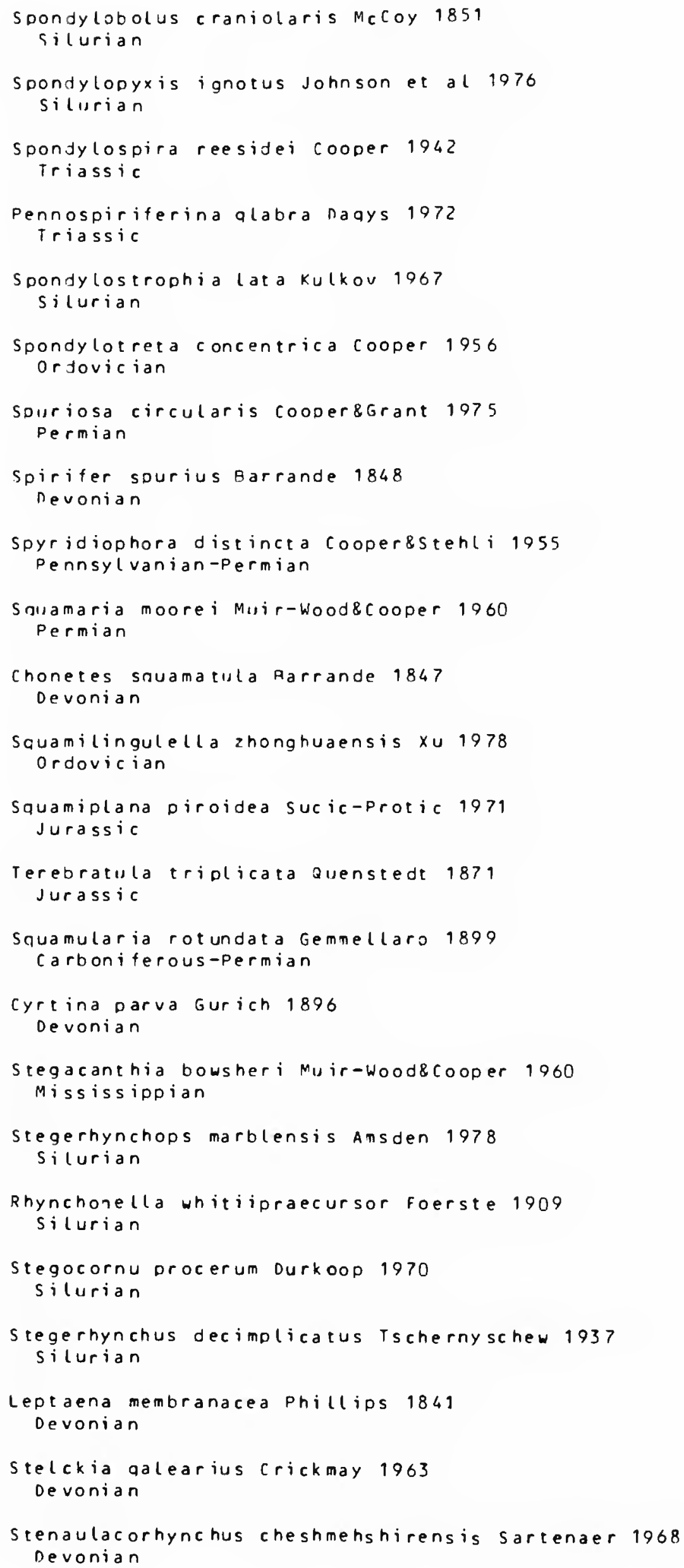




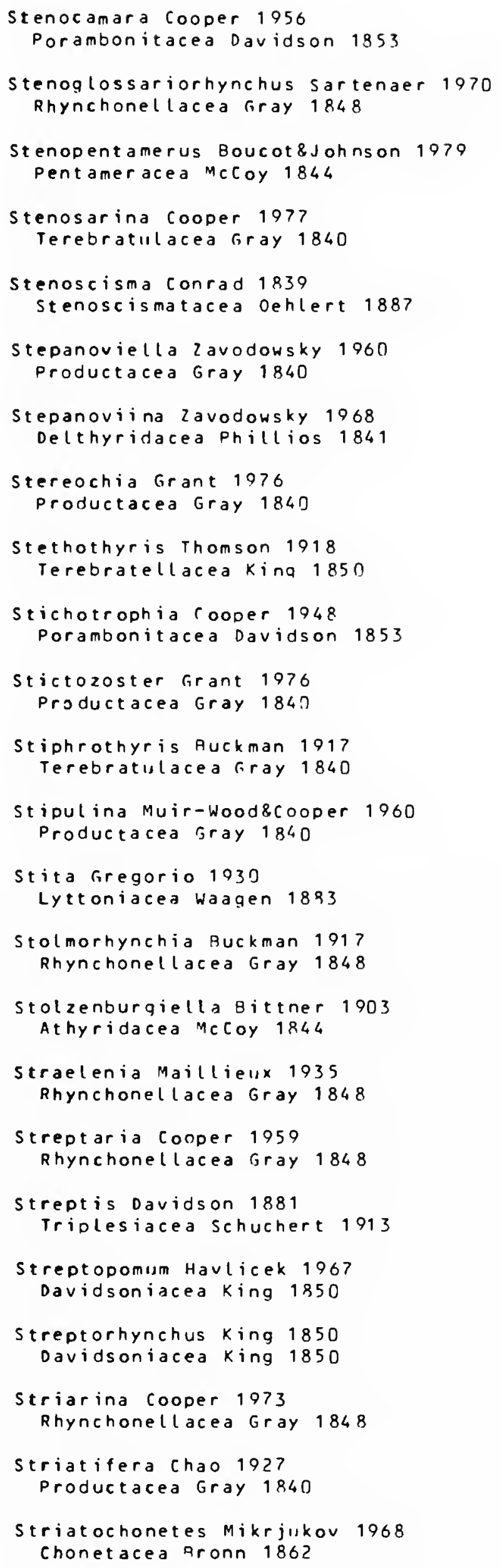

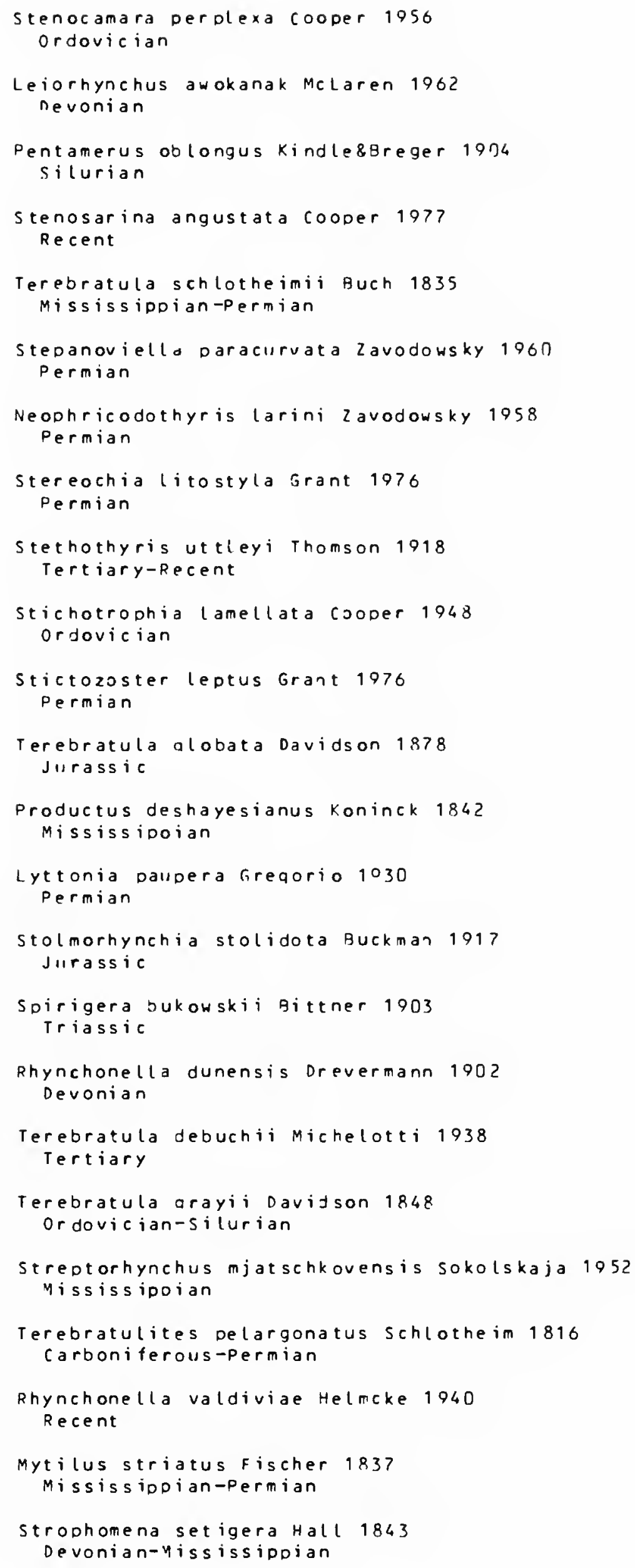




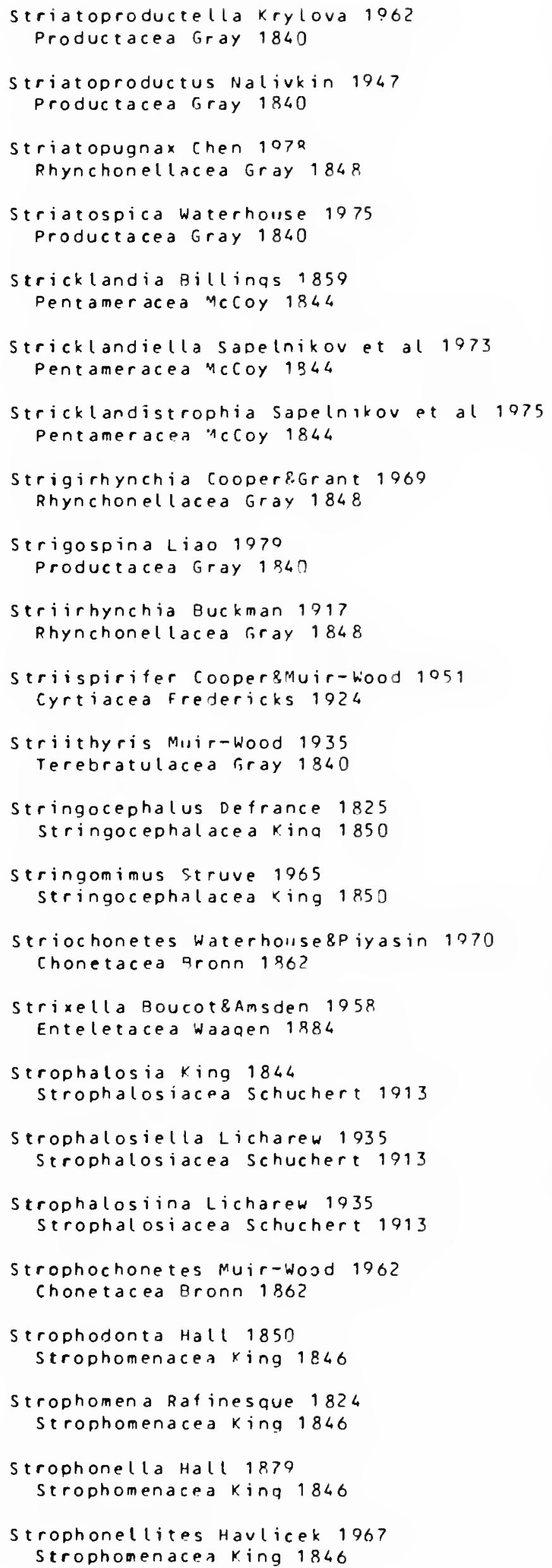

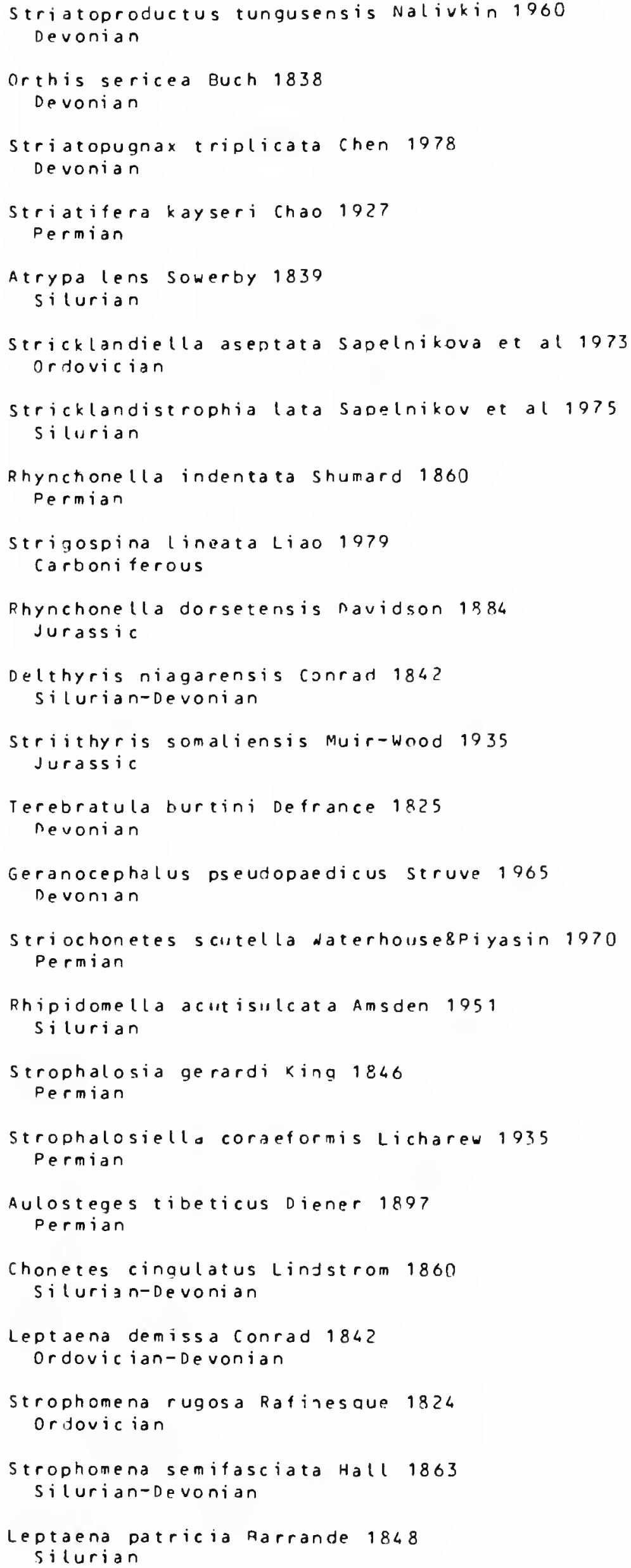


Sulcatothyris Dagys 1074 Dielasmatacea Schuchert 1913

sulciplica waterhouse 1968

Spiriferacea $k$ ing 1846

Sulcirhynchia Burri 1053

Rhynchonellacea Gray 1848

Sulcirostra Cooder\&Mnir-wood 1951

Rhynchonel acea Gray 1848

Sulcorhynchia Dagys 1074 Rhynchonellacea foray 1848

Supertrilobus Roucot RJohns on 1979 Pentameracea McCoy 1844

Suruqathyris Yabe\&Hatai 1934 Terebratulacea Gray 1340

Svaljavithyris Tehorszhewsky 1974 Loboidothyridacea Makrijin 1064

Svobodaina Havlicek 1050 Enteletacea wagen 1984

Swaicoelia Hamada 1968 Cyrtiacea Fretericks 1924

Swantonia walcott 1905 Uncertain

Symmatrypa Mizens 95 apelnikov 1075 Atrypacea fill 1971

Symphythyris $5 \pi$ irnova 1966 Terebratulacea Gray 1840

Synatrypa Copper 1966 Atrypacea Gill 1871

Syndielasma cooper 1956 Plectambonitacea Jones 1928

Syntrilasma Meek 8 Worthen 1865 Enteletacea Wagen 1884

Syntrophia Hall 8 Clarke 1893

Porambonitacea Davidson 1853

Syntrophina Jlrich 1928

Porambonitacea navidson 1853

Syntrophinella IJlrichscooper 1934 Porambonitacea Davidson 1853

Syntrophioides Schuchert \&Cooper 1931 uncertain

Syntrophopsis Ulrich\&cooper 1936 Porambonitacea Davidson 1853

Syringopleura Schuchert 1910 Spirifer acea king 1846

Syringospira kindle 1909 Spiriferacea king 1846

Syringothyris winchell 1863 spiriferacea king 1846

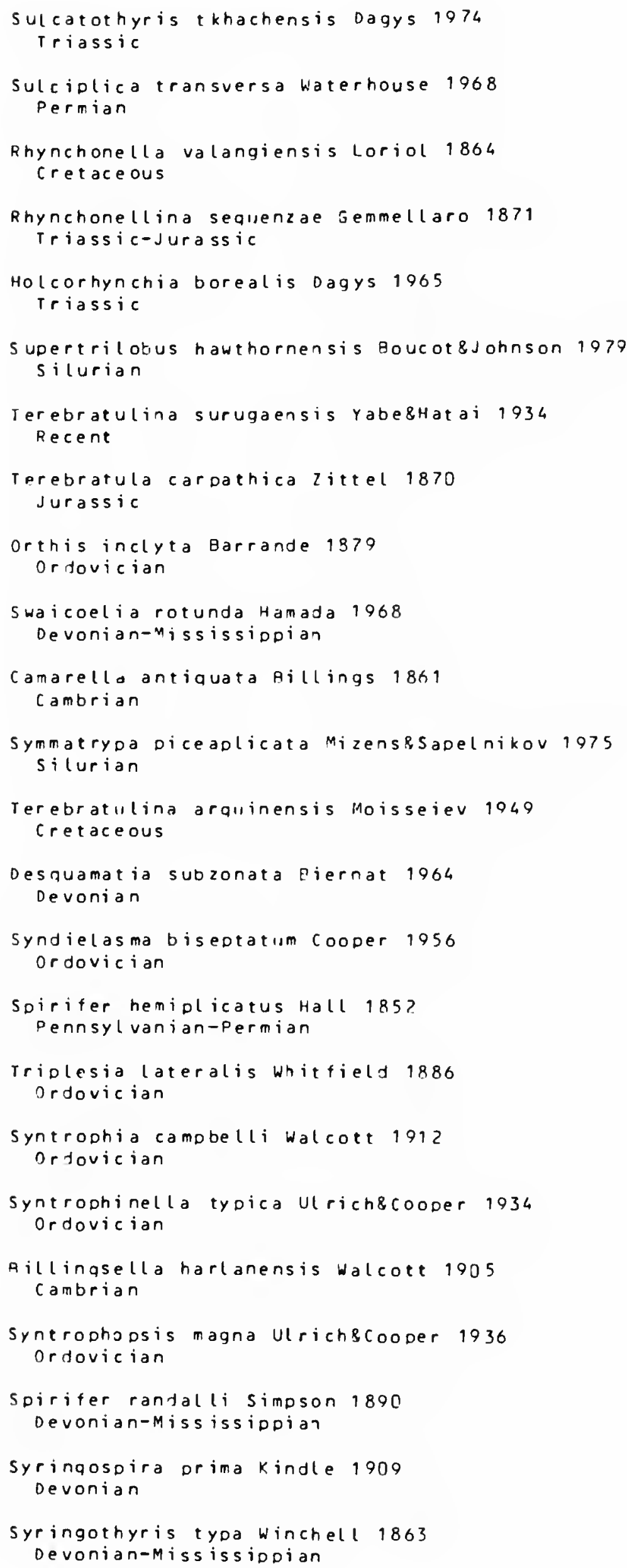




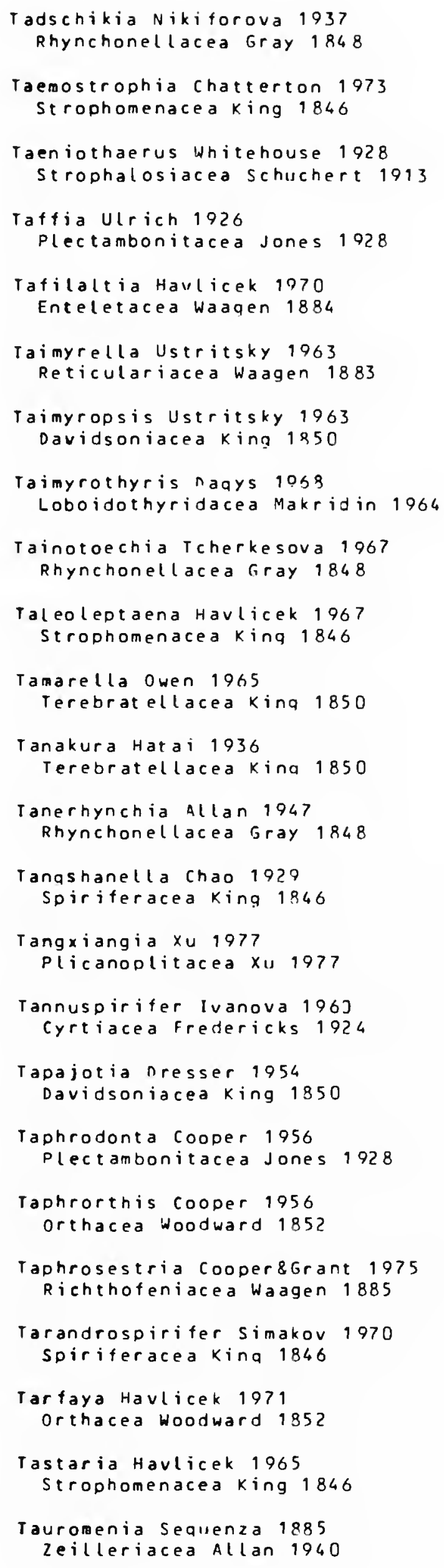

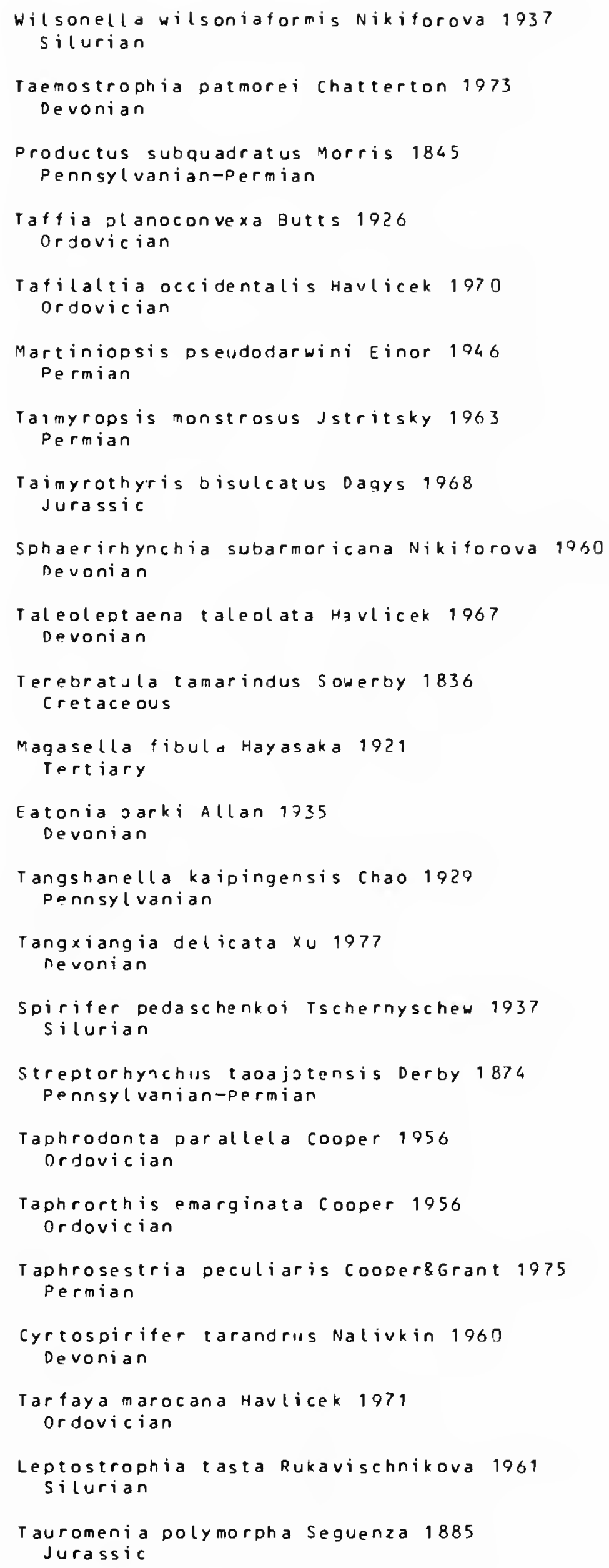




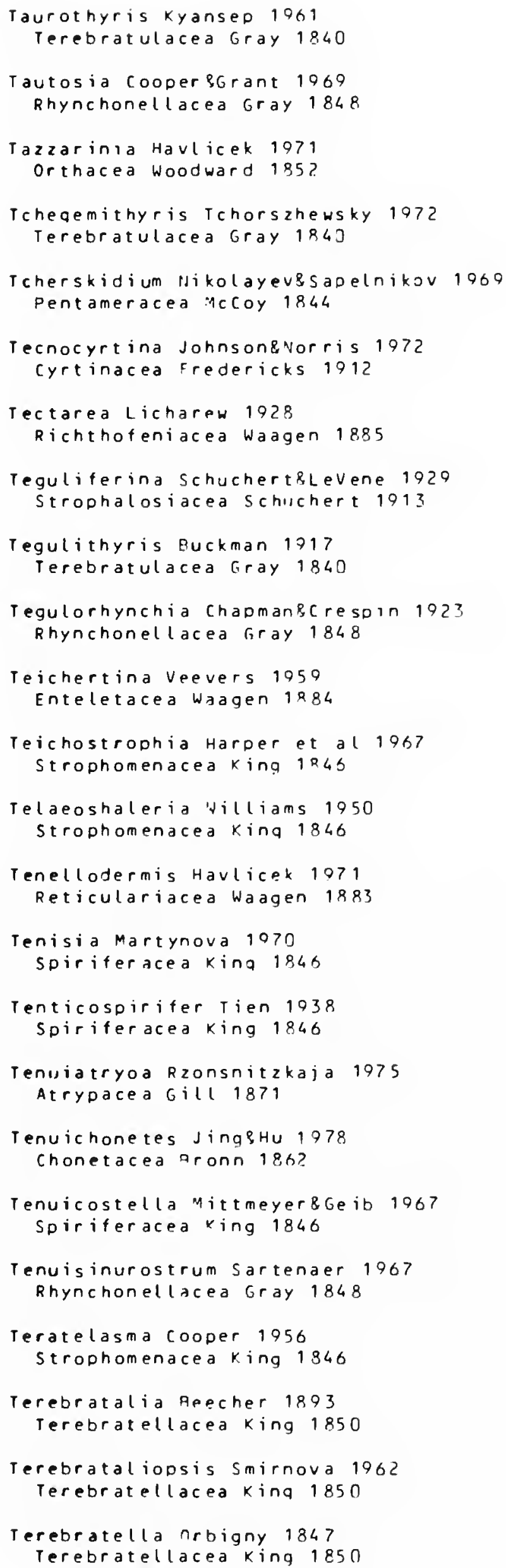

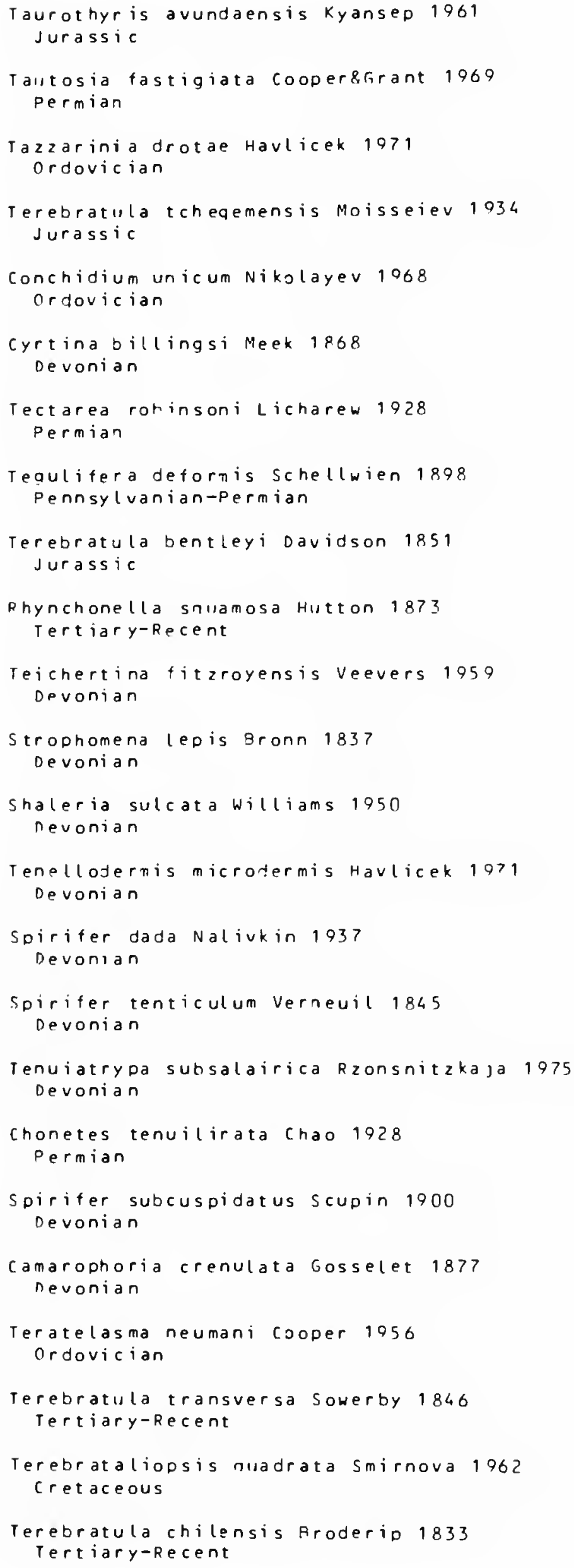


Terebratula Muller 1776

Terebratulacea Gray 1840

Terebratulina orbigny 1.347

Terebratulacea Gray 19.40

Terebratul oidea Waagen 1883

Rhynchonellacea Gray 1848

Terebratulopsis Gregorio 1930

Rhynchonellacea Gray 1848

Terebrirostra orbigny 1947

Terebratellacea King 1850

Terrakea Booker 1931

Productacea Gray 1840

Tesuquea Sutherland?.Harlow 1973

Productacea Gray 1940

Tetracamera weller 1910 Rhynchonel lacea Gray 1848

Tetractinella 9 ittner 1890 Athyridacea McCoy 1844

Tetraqonetes Cooper\&firant 1975 Chonetacea Rronn 1862

Tetralobula Ulrich\&cooper 1936 Porambonitacea Davidson 1853

Tetraodontella Jaanusson 1962 $P$ lectambonitacea Jones 1928

Tetraphalerella wana 1949 Strophomenacea King 1846

Tetrarhynchia Buckman 1917 Rhynchonel lacea Gray 1848

Tetratomia Schmidt 1949 Rhynchone l lacea Gray 1848

Texarina cooper 8 Grant 1970 cryptonellacea Thomson 1926

Texathyris carter 1072 Spiriferacea king 1846

Thaerodonta wang 1949 Plectambonitacea Jones 1928

Thamosia Cooperifrant 1969 Productacea Gray 1840

Thaumatosia Cooper 1973

Terebratellacea King 1850

Thaumatrophia Wang 1955 Porambonitacea Davidson 1853

Thebesia Amsden 1974 Rhynchonel lacea Gray 1848

Thecidea Defrance 1822

Thec ideacea Gray 1840

Thecidella Munier-Chalmas 1887

Thecideacea Gray 1840

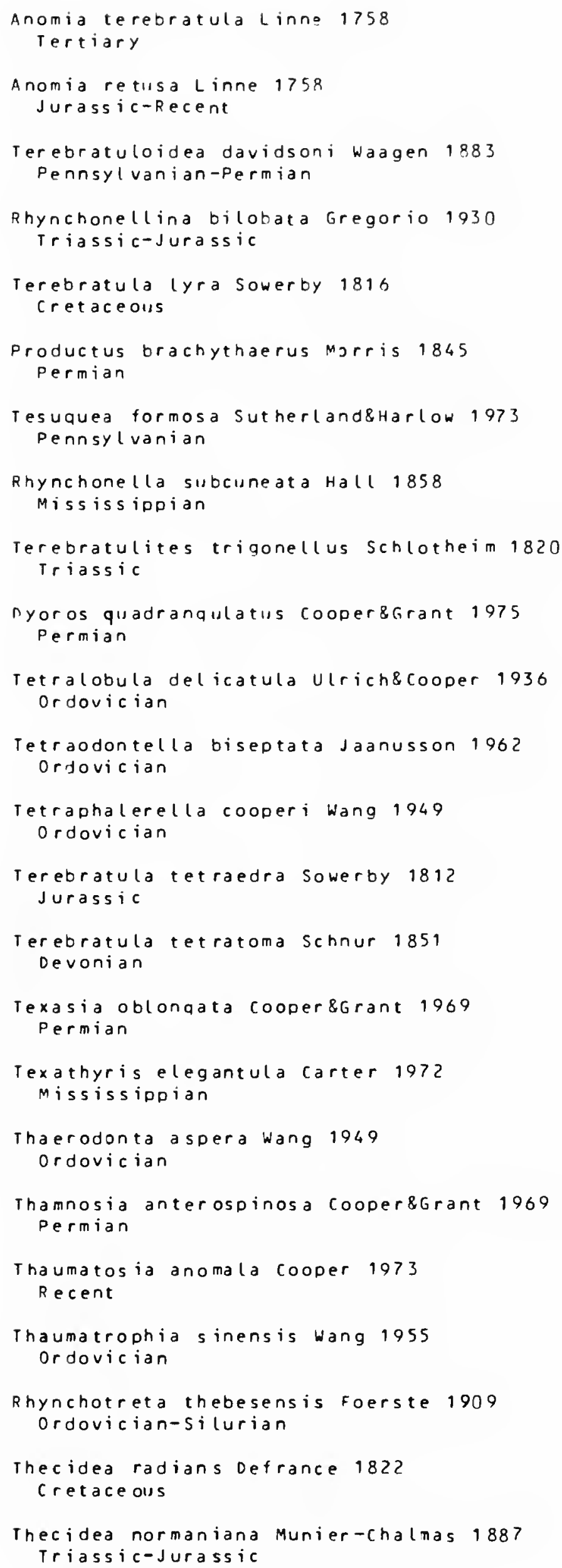




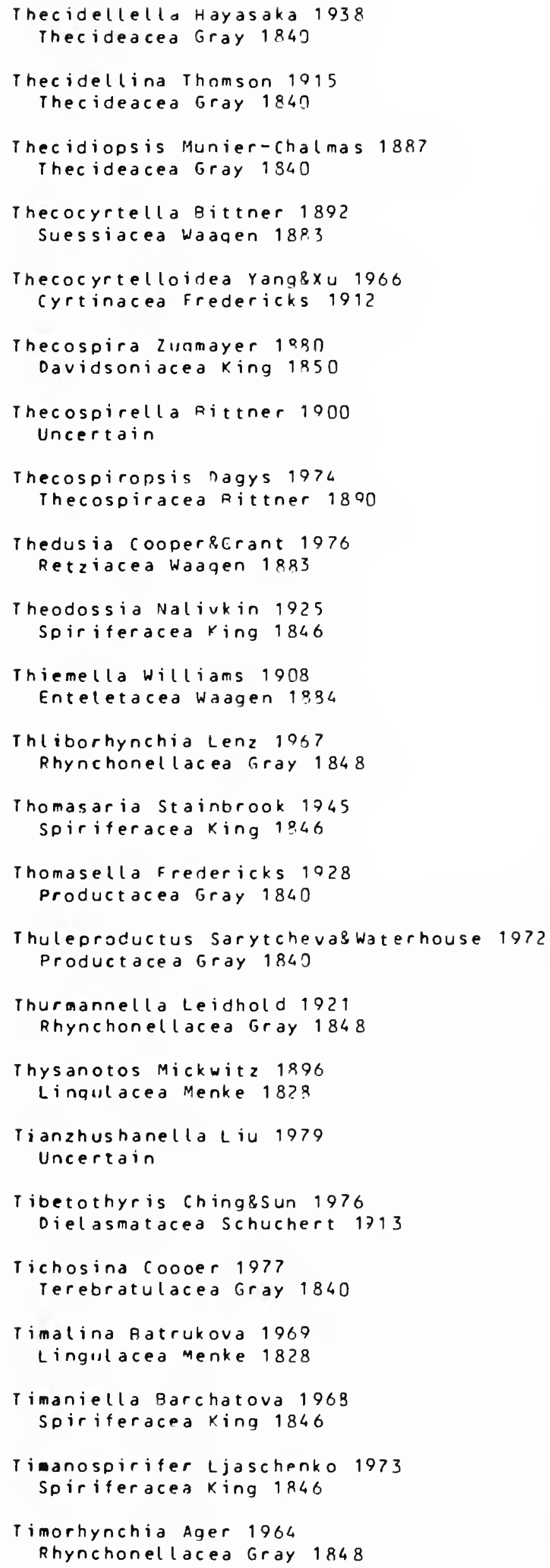

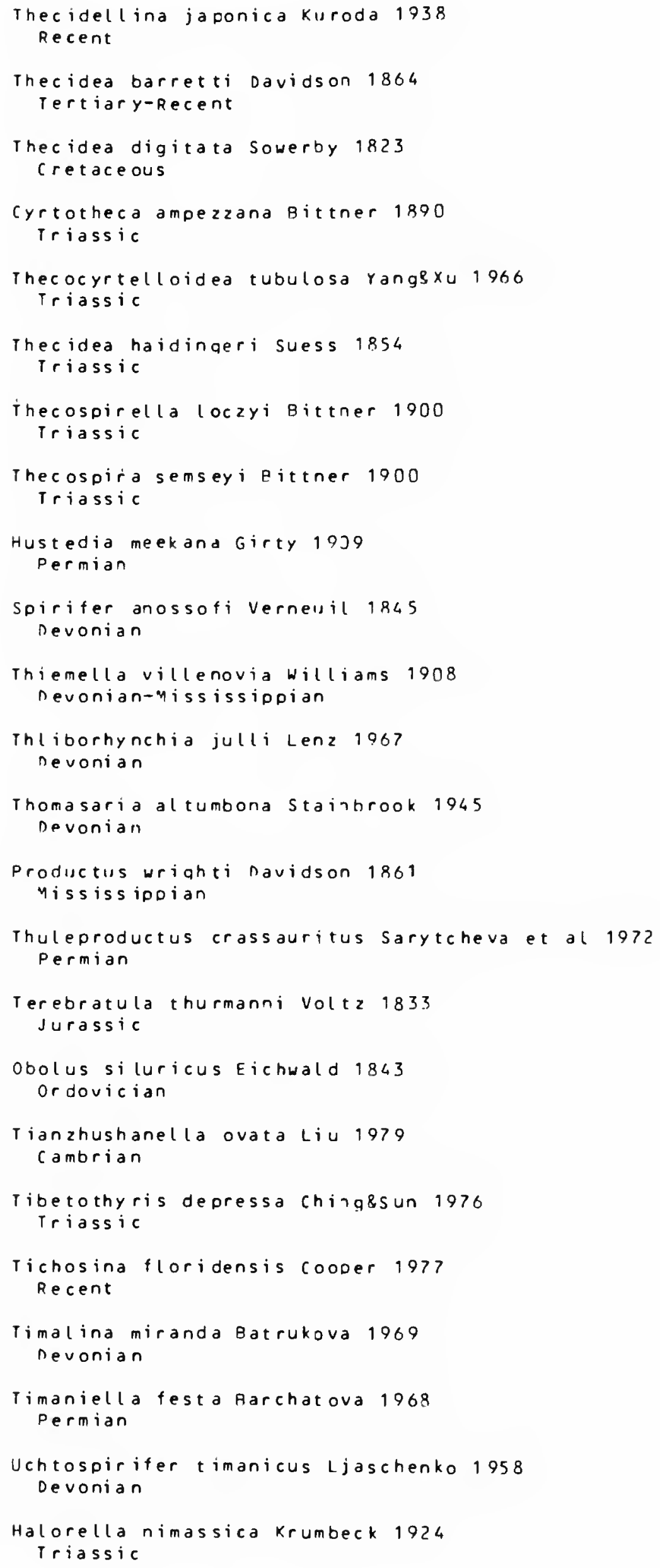




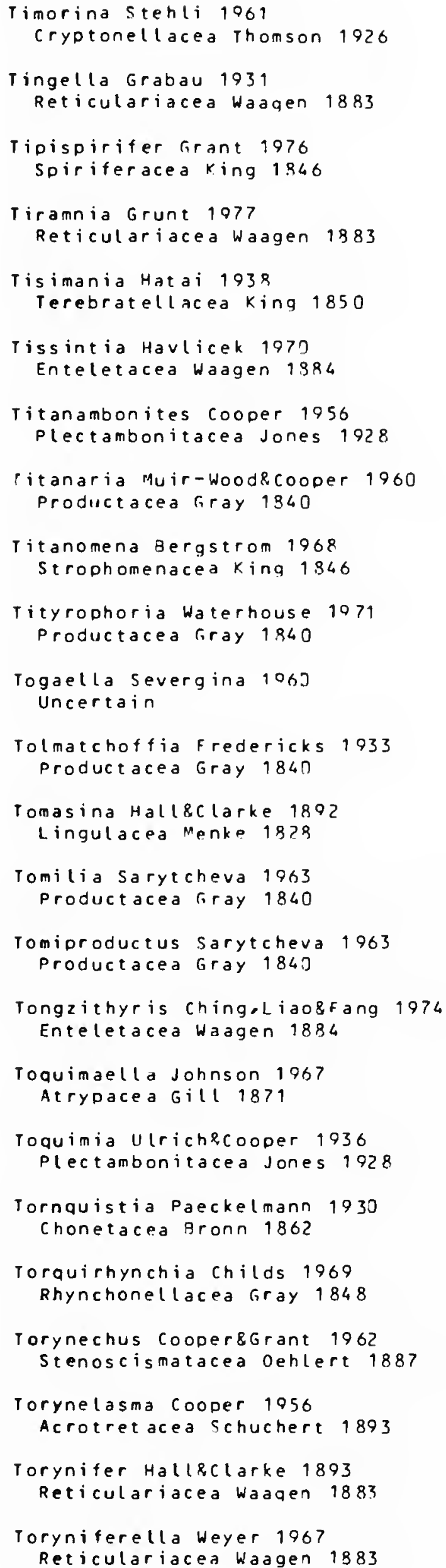

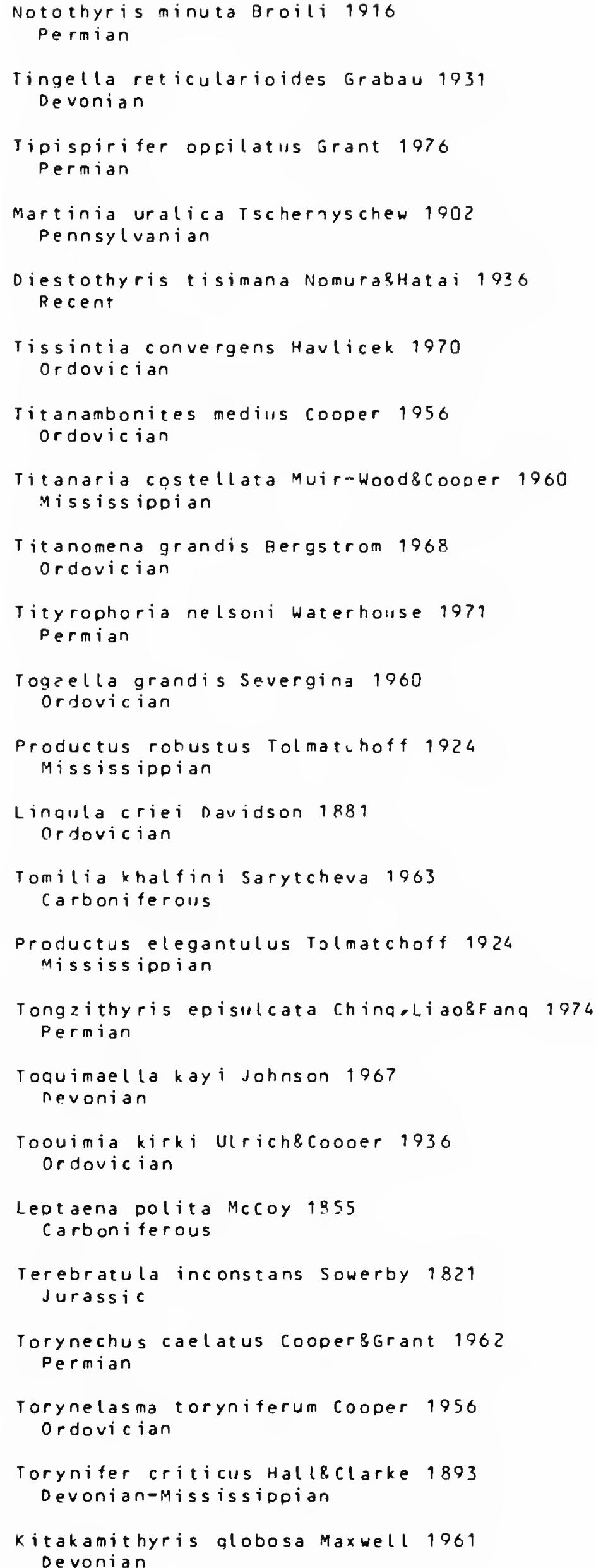


Trigonirhynchella Dagys 1763 Rhynchonel lacea Gray 1848

Trigonirhynchia Cooper 1942 Rhynchonellacea Gray 1848

Trigonithyris Muir-Wood 1935

Terebratulacea Gray 1840

Triqonoqlossa nunhar 8 condra 1932 Lingulacea Menke 182 ?

Trigonosemus koenig 1825 Terebratellacea King 1850

Trigonotreta koenig 1825 Spiriferacea king 1846

Trigonotrophia Andreeva 1972

Porambonitacea navidson 1853

Trigrammaria Wilson 1045 strophomenacea King 1846

Trimerella Rillinas 1862 Trimerellacea Davidson 8 king 1872

Trimurellina Mitchell 1977 $p$ lectambonitacea Jones 1928

Triplecella wilson 1932

Dayiacea wagen 1883

Triplesia Hall 1859

Triplesiacea Schuchert 1913

Triseptata Bondarev 1965

Porambonitacea Davidson 1853

Triseptothyris Xu 1978

zeilleriacea Allan 1940

Tritoechia Uleich\&cooper 1936

Clit ambonitacea Winchel $(85$ chuchert 1893

Trochalocyrtina Wright 1975

Suessiacea waagen 1883

Trochifera ching 8 ye 1979

cancellothyridacea Thomson 1926

Trondorthis Neuman 1974

Orthacea Woodward 1852

Tropeothyris Smirnova 1972

Terebratulacea Gray 1840

Trophisina cooper\&Grant 1976

Rhynchonel lacea Gray 1848

Tropidelasma Cooper \&Grant 1969

Derbyiacea Stehli 1954

Tropidoglossa Rowell 1966

Lingulacea Menke 1828

Tropidoleptus Hall 1857

Enteletacea Wagen 1884

Tropidothyris cooper 1956 Orthacea Woodward 1852

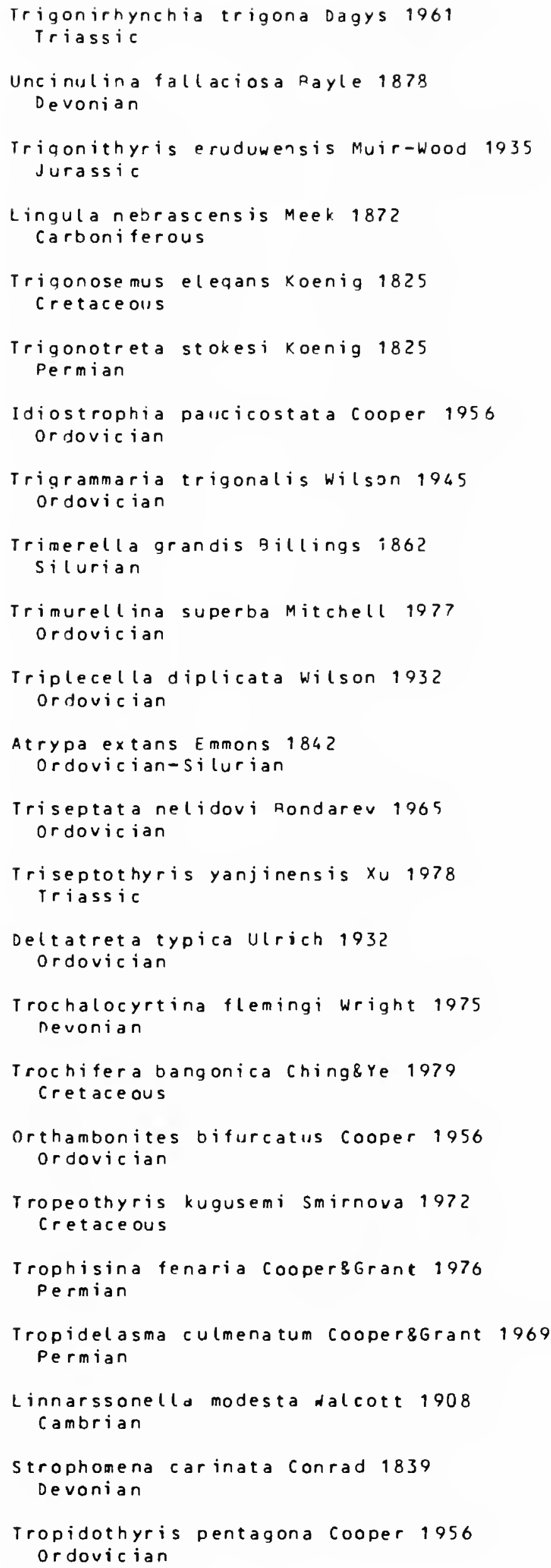




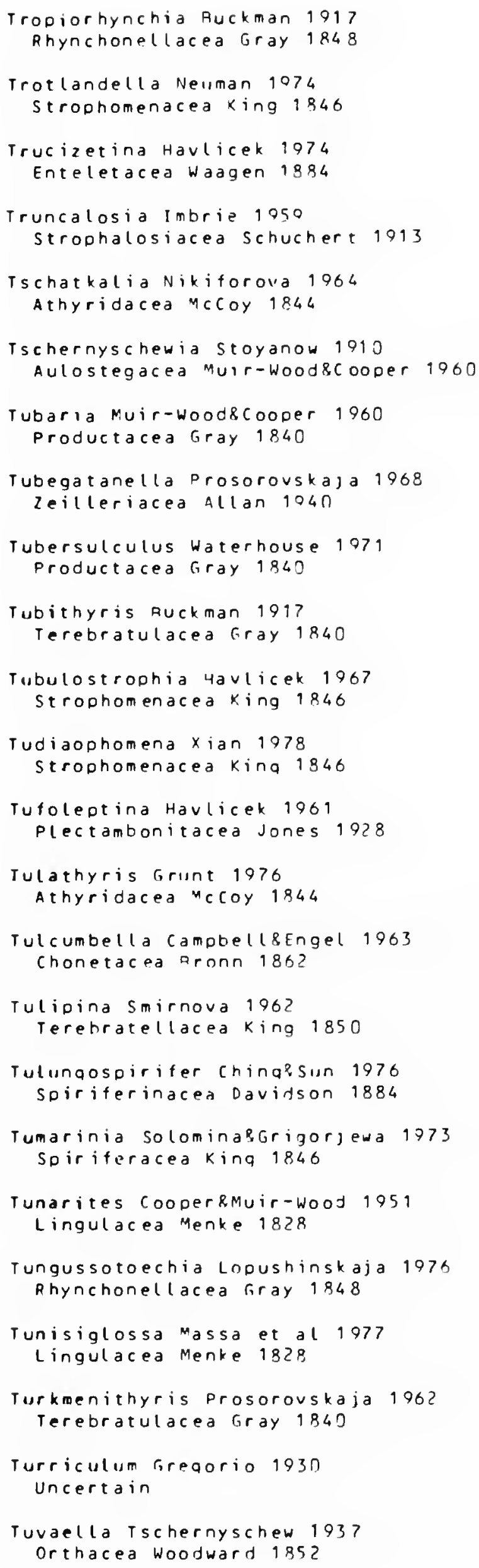

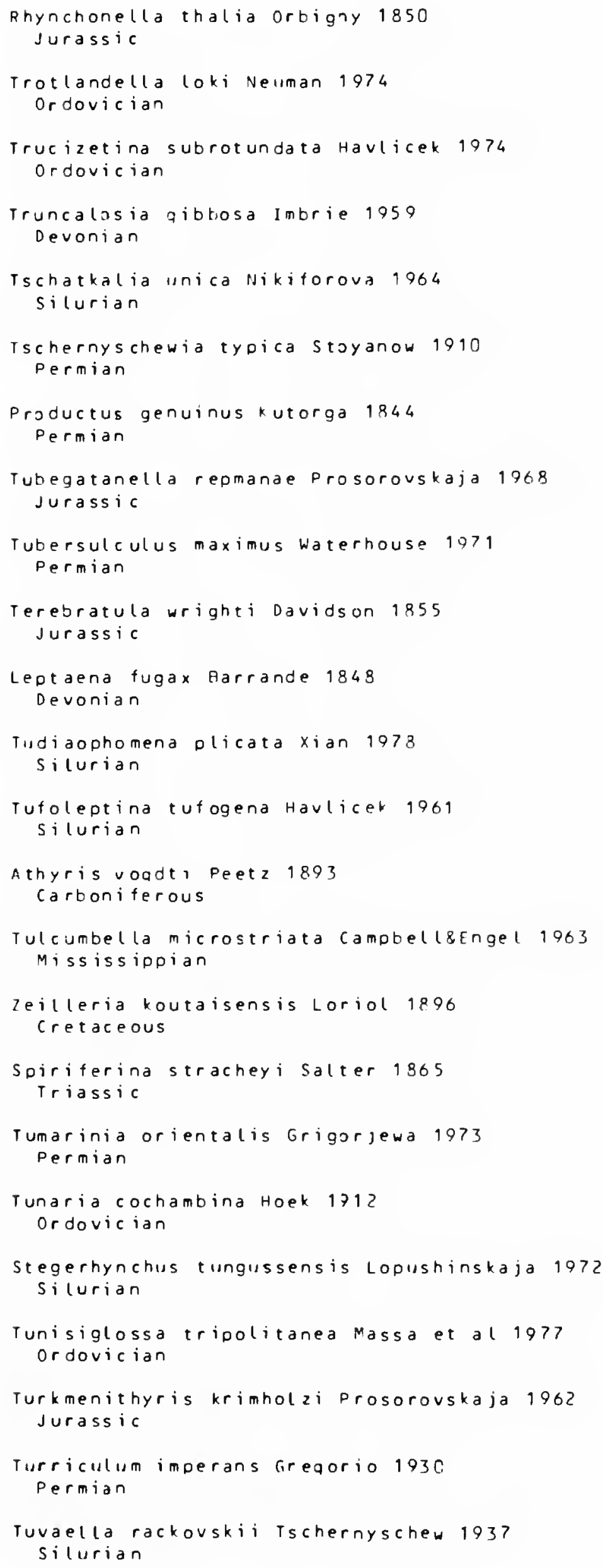




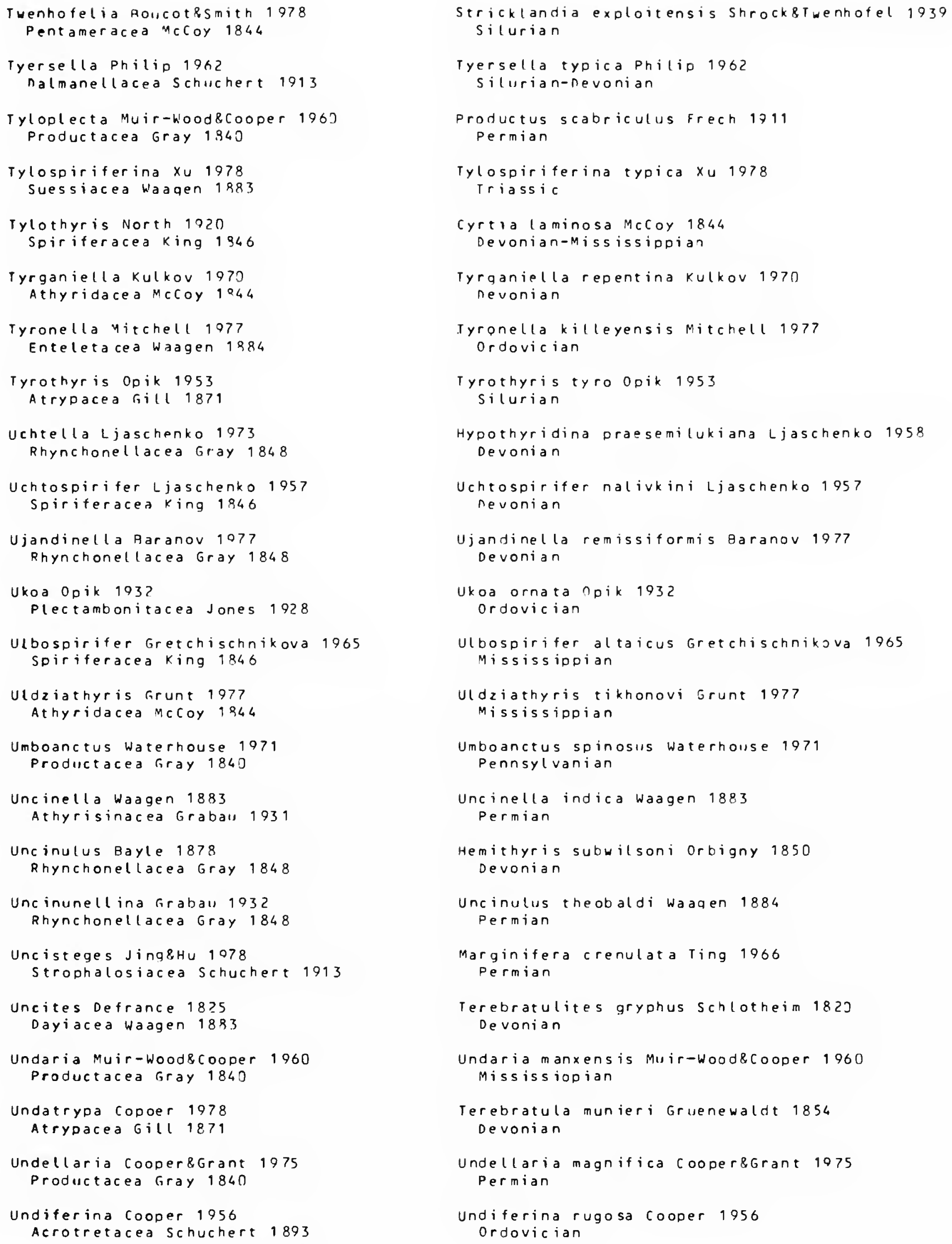




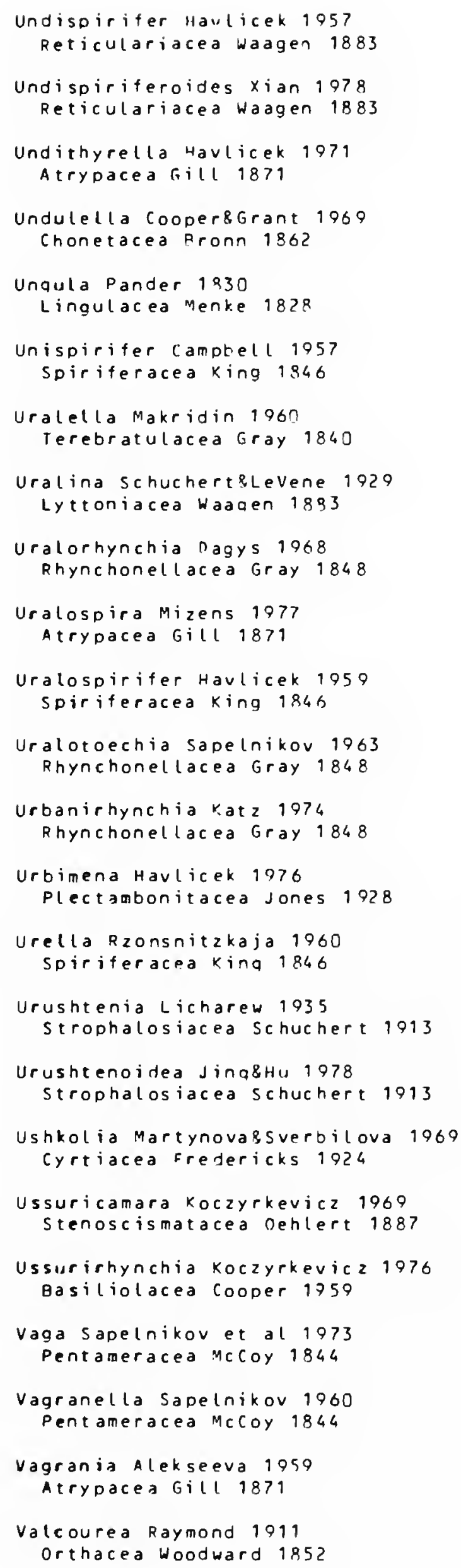

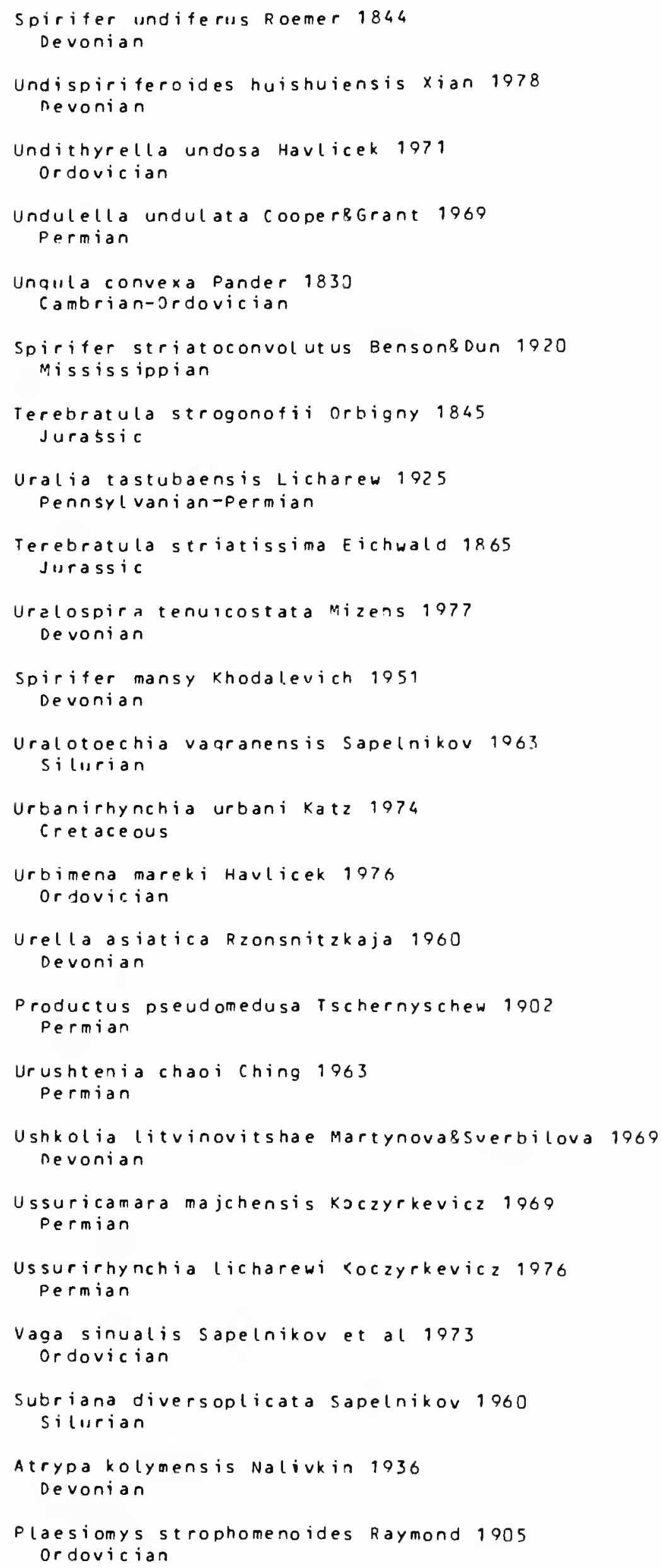


Valdaria RassettRCocks 1974 Davidsoniacea King 1850

Valdiviathyris Helmcke 1940 craniacea Menke 18.28

Vallomyonia Johnson 1366 Enteletacea Wagen 1884

Vandercammenina Boucot 1975 Suessiacea wagen 1883

Vandergrachtella Crickmay 1953 Spiriferacea King 1846

Vandobiella Pojariskaja 1966 Terebratellacea King 1850

Vaniella Kvakhadze 1974 Terebratellacea King 1850

Variatrypa Copper 1966 Atrypacea fill 1871

Vectella owen 1965 zeilleriacea 4 llan 1940

Vediproductus Sarytcheva 1965 Productacea Gray 1840

Veghirhynchia nagys 1974 Basiliolacea Cooper 1959

Veliseptum Popov 1976 Acrotretacea Schuchert 1893

Vellamo Opik 1930

clitambonitacea winchell $85 \mathrm{schuchert} 1893$

Velostrophia Havlicek 1965 Strophomenacea Kina 1846

Venezuelia Weisbord 1926 uncertain

Verchojania Abramov 1970 Productacea Gray 1840

Verkhotomia Sokolskaja 1963 spirjferacea king 1846

Vermiculothecidea Elliott 1953 Thecideacea Gray 1840

Verneuilia Hall RClarke 1893 Reticulariacea Wagen 1883

Vex Hoover 1979 Terebratulacea Gray 1840

Viallithyris Voros 1978 Terebratulacea Gray 1840

Viarhynchia Calzada 1974 Rhynchonel lacea Gray 1848

Victorithyris Allan 1940 Terebratellacea King 1850

Viligella Daqys 1965 Spiriferinacea Davidson 1884

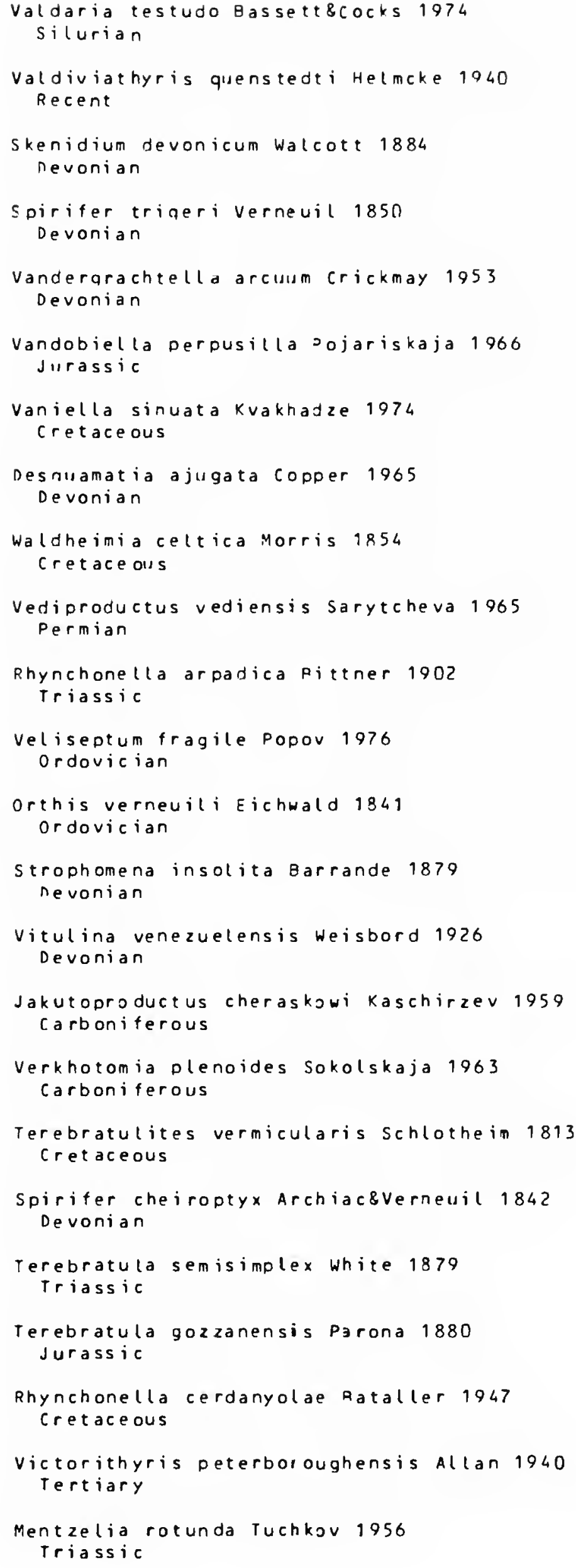




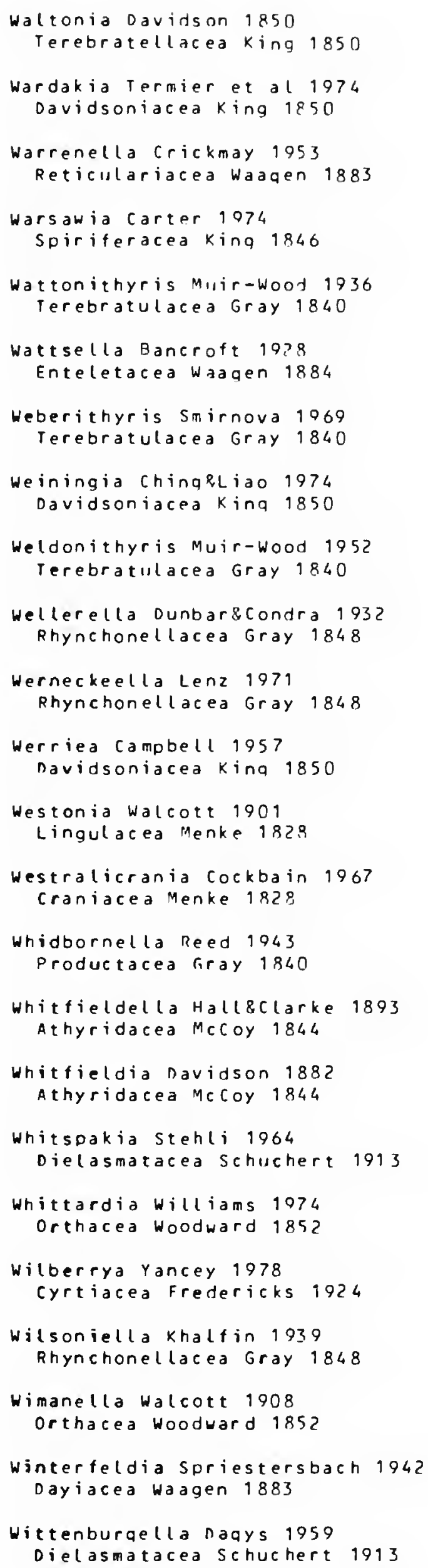

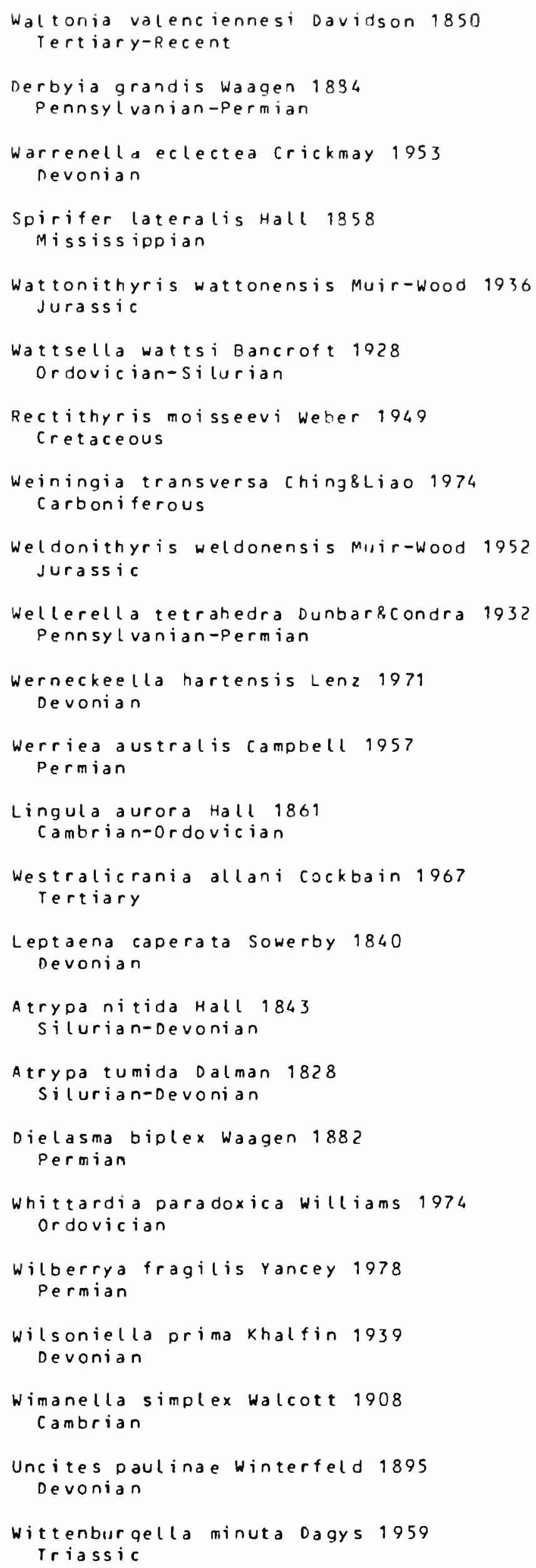




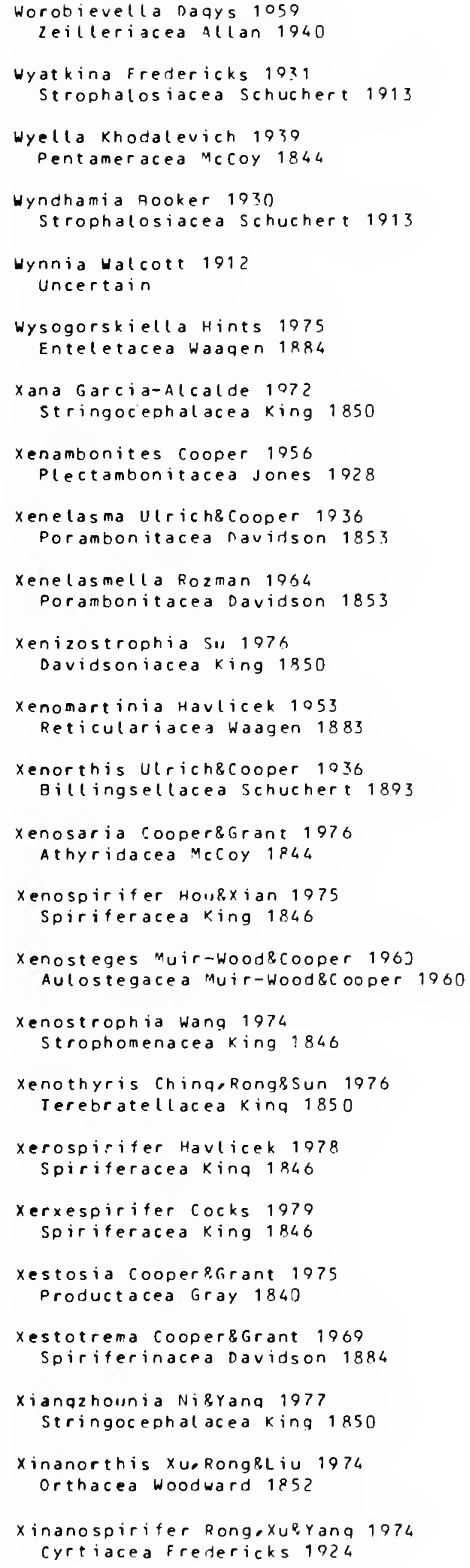

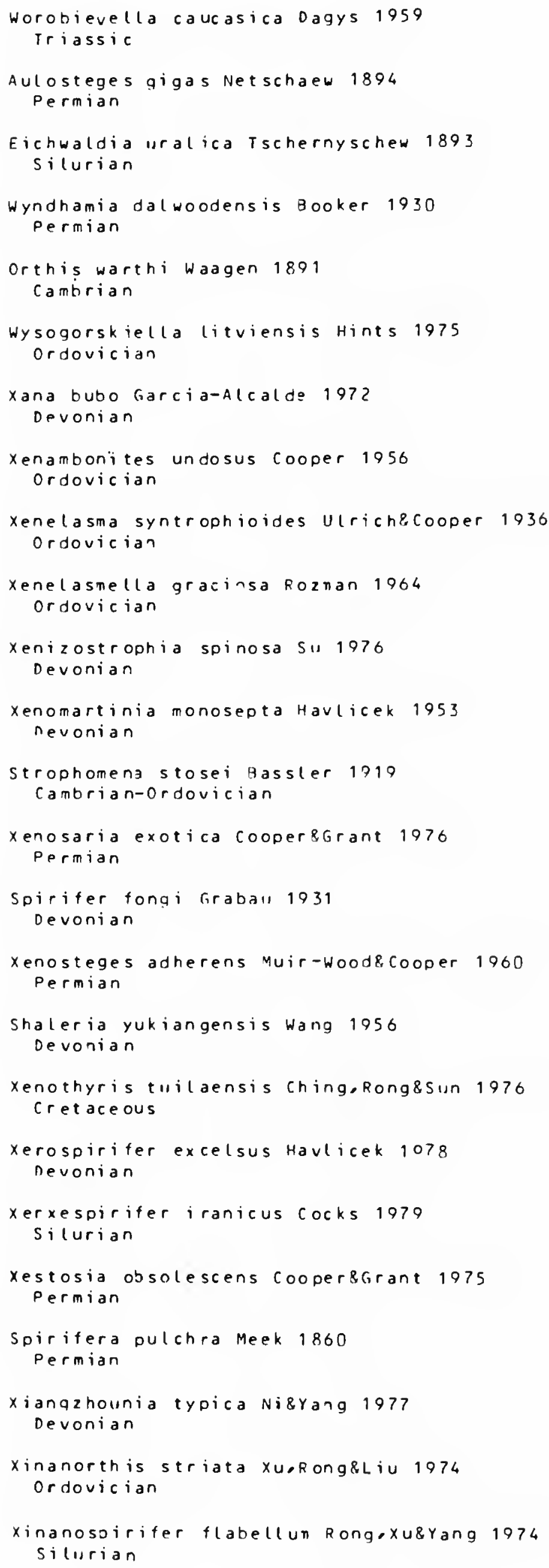


Xinshaoella 2 hao 1977

Rhynchonel lacea Gray 1848

Xizangostrophia Rona 1976

Porambonitacea navidson 1853

Xystostrophia Havlicek 1965

Orthotet acea Wagen 1884

Yabe ithyris Hat ai 1948

Terebratellacea King 1850

Yagonia Roberts 1976

Chonetacea Rronn 1862

Yakovlevia Fredericks 1925

Productacea Gray 1840

Yakutijaella Baranov 1977

Rhynchonel lacea Gray 1848

Yanbianella Tong 1978

Rhynchonel lacea Gray 1848

Yangtzeella Kolarova 1925

Porambonitacea Davidson 1853

Yanguania Yang 1977

Productacea Gray 1840

Yanishewskiella Licharew 1957 Rhynchonellacea Gray 1848

Yanospira naqys 1977

Spiriferinacea Davidson 1884

Yatsengina Semichatova 1936 Spiriferacea King 1846

Yeosinella Reed 1933 Uncertain

Ygera Havlicek 1961 Plectambonitacea Jones 1928

Ygerodiscus Havlicek 1967 Plectambonitacea Jones 1928

Yidunella China, Sun\&Ye 1979 Rhynchonellacea Gray 1848

Yingtangella Bai\&ying 1978 Stringocephalacea King 1850

Yingwuspirifer Rong, Xu\&Yang 1974 uncertain

Yochelsonia Stehli 1961 Dielasmatacea Schuchert 1913

Yorkia Walcott 1897

Kutorginacea Schuchert 1893

Ypsilorhynchus Sartenaer 1970 Rhynchonel Lacea Gray 1848

Yretospirifer Gatinaud 1949 Spiriferacea King 1846

Yuezhuella Chinasye 1979 Terebratellacea King 1850

Yunnanella frabau 1023 Rhynchonel lacea Gray 1848

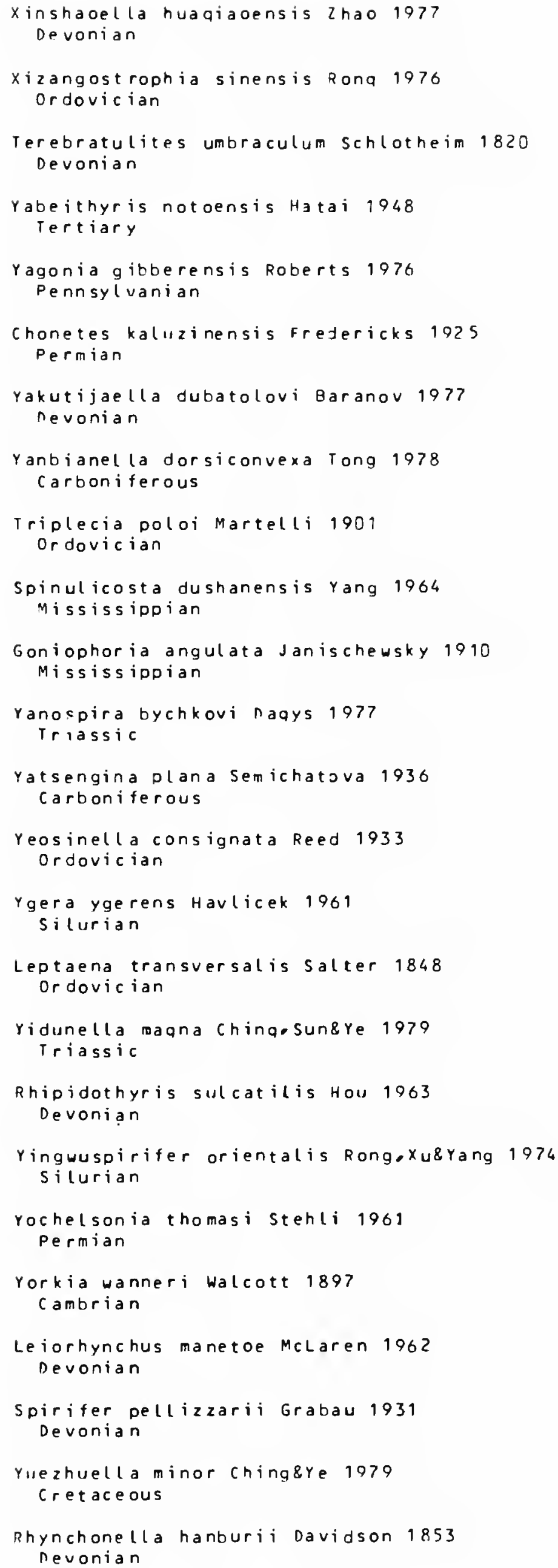




\section{Literature Gited}

Abramov, B. S.

1970. Biostratigrafiia Kamennougolnykh Otlozhenii Sette-Dabana. 178 pages, 38 plates. Moscow: Akademiia Nauk SSSR, Iakutsk Filial Sibirskogo Otdeleniia Institut Geologii.

Afanasjeva, G. A. (= G. A. Besnossova)

1975. Chonetacea (Brachiopoda) Srednego i Pozdnego Karbona Russkoi Platformy. Paleontologicheskii Zhumal, 1975(2):96-113, 8 figures.

1976. Chonetacea (Brachiopoda) Rannego Karbona Russkoi Platformy. Paleontologicheskii Zhurnal, 1976(3):58-70, 4 figures, 1 plate.

1977a. In T. G. Sarytcheva, editor, Pozdnepaleozoiskie Produktidy Sibiri Arktiki. Akademiza Nauk SSSR, Paleontologicheskii Institut, Trudy, 161:1-219.

1977b. Permochonetes n. gen. (Brachiopoda) iz Nizhnei Permi Pamira. Paleontologicheskii Zhurnal, 1977(1): 147-151, 2 figures.

1978. Novye Khonetatsei iz Devona Nakhichevanskoi ASSR. Paleontologicheskii Zhurnal, 1978(3):64-71, 1 figure, 1 plate.

Agassiz, L.

1847. Nomenclatoris zoologici, index universalis. In $\mathrm{No}$ menclator Zoologicus, 2: 393 pages. Soloduri: Jent and Grassman.

Ager, D. V.

1959. The Classification of the Mesozoic Rhynchonelloidea. Journal of Paleontology, 33(2):324-332, 1 plate.

1960. Nomenclatural Problems in the Mesozoic Rhynchonelloidea. Geological Magazine, 97:157-162.

1962. A Monograph of the British Liassic Rhynchonellidae, Part III. Paleontographical Society Monograph, 1962:85-136, 4 plates.

1964. The Supposedly Ubiquitous Tethyan Brachiopod Halorella and Its Relations. Journal of the Palaeontology Society of India, 5-9(1960-1964):54-70.

Aisenverg, D. E.

1971. In D. E. Aisenverg, editor, Atlas fauny turneiskikh otlozhenii Donetškogo basseina [Atlas of the Tournaisian Fauna in the Donets Basin (with Description of the New Species)], pages 70-73, 1 plate. Kiev: Akademiia Nauk Ukrainskoi SSR, Institut Geologicheskikh Nauk.

Aksarina, N. A., and J. L. Pelman

1978. [Cambrian Brachiopods and Bivalve Mollusks of Siberia.] Akademiia Nauk SSSR, Sibirskoe Otdelenie,
Institut Geologii i Geofiziki, Trudy, 362:1-147, 20 plates. [In Russian.]

Aleksandrov, V. A., and R. V. Solomina

1973. Brachiopoda. In O. L. Einor, editor, Stratigrafiya $i$ Fauna Kamenno Ugol'nykh Otlozhenii Reki Shartym (Yuzhnyi Ural), pages 86-131, 23 figures, 9 plates. Kiev: Uralskoe Geologicheskoe Upravlenie, Kievskii Ordena Lenina, Gosudarstvennyi Universitet.

Alekseeva, R. E.

1959. [A New Genus of the Family Atrypidae Gill (Brachiopoda).] Akademiia Nauk SSSR, Doklady, 126(2):389-391. [In Russian.]

1960. [A New Subgenus Atrypa (Desquamatia) Subgen. of the Family Atrypidae Gill (Brachiopoda).] Akademiia Nauk SSSR, Doklady, 131(2):421-424. [In Russian.]

1966. [Sibirotechia, a New Genus of the Order Rhynchonellida.] Akademiia Nauk SSSR, Doklady, 167(5): 1147-1149. [In Russian.]

1967. Brakhiopody i Stratigrafiia Nizhnego Devona Severo-Vostoka SSSR. 160 pages, 16 plates. Moscow: Akademiia Nauk SSSR, Sibirskoe Otdelenie, Institut Geologii i Geofiziki.

1968. [Sibirispira-a New Genus of the Atrypida Order.] Akademiia Nauk SSSR, Doklady, 179(1):198-201. [In Russian.]

1969. Novye Svoyeobraznyye Nizhnedivonskiye Spiriferidy (Podotryad Athyrididina). Akademiia Nauk SSSR, Doklady, 187(5):1157-1159.

1976. [New Early Devonian Terebratulids from Mongolia.] In N." N. Kramarenko, editor, Paleontologiia i Biostratigrafiia Mongolii. Sovmestnaia Sovetsko-Mongol'skaia Paleontologicheskaia Ekspeditsiia, Trudy, 3:1-383. [In Russian.]

1977. [A New Genus of Retziid Brachiopod.] Bespozvonochnye Paleozoia Mongolii: Soumestnaya Sovetsko-Mongol'skaya Paleontologicheskaya Ekspeditsiia, Trudy, 5: 63-65. [In Russian.]

Alekseeva, R. E., R. T. Gratsianova, E. A. Elkin, and N. I. Kulkov

1970. [Stratigraphy and Brachiopods of the Lower Devonian of N.-E. Salair.] Akademiia Nauk SSSR, Sibirskoe Otdelenie, Institut Geologii i Geofiziki, Trudy, 72:1-188, 100 figures, 22 plates. [In Russian.]

Alekseeva, R. E., and N. A. Nuzhdina

1967. [Middle Devonian Terebratulids from the Omolon Massif (North-Eastern Territory of USSR).] 
Paleontologicheskii Zhurnal, 1967(1):137-143, 3 figures, 1 plate. [In Russian.]

Alichova, T. N.

1960. Mshanki, Brakhiopody. In T. G. Sarytcheva, editor, Otryad Orthida. In Yu. A. Orlov, editor, Osnovy Paleontologii, pages 183-197, plates 7-19.

Allan, R. S.

1932. [Tertiary Brachiopoda from the Chatham Islands, New Zealand. New Zealand Institute, Transactions and Proceedings, 63(1):11-23, 3 plates.

1938. A New Genus of Tertiary Rhynchonellids (Brachiopoda) from Patagonia. Canterbury Museum, Records, 4(4):199-201.

1939. Studies on the Recent and Tertiary Brachiopoda of Australia and New Zealand. Canterbury Museum, Records, 4(5):231-248, plates 29-31.

1940. Studies on the Recent and Tertiary Brachiopoda of Australia and New Zealand. Canterbury Museum, Records, 4(6):277-297, plates 35-37.

1947a. Plicirhynchia, a New Genus of Tertiary Brachiopoda from Patagonia. Journal of Paleonlology, 21 (5):493-494.

1947b. A Revision of the Brachiopoda of the Lower Devonian Strata of Reefton, New Zealand. Journal of Paleontology, 21(5):436-452, plates 61-63.

Almeras, $Y$.

1966. Les Rhynchonellides du Bajocien Moyen de Ronzevaux pres Davaye (Saône-et-Loire): Genres $C y$ matorhynchia S. Buckman, Lacunaerhynchia nov. et Seplulirhynchia nov. Travaux du Laboraloire de Geologie de la Faculte des Sczences de l'Universite de Lyon, 13:31-119.

1971. Les Terebratulidae du Dogger dans le Maconnais, le Mont d'Or Lyonnais et le Jura Meridional. Documents des Laboratoires de Géologie de la Faculté des Sciences de Lyon, 39:1-690, 451 figures, 211 plates.

Amos, A. J.

1958. Some Lower Carboniferous Brachiopods from the Volcan Formation, San Juan, Argentina. Journal of Paleonlology, 32(5):838-845, 2 plates.

1972. Silurian of Argentina. In W. B. N. Berry and A. J. Boucot, Correlation of the South American Silurian Rocks. Geological Society of America, Special Paper, 133:1-19.

Amsden, T. W.

1949. Two New Genera of Brachiopods from the Henryhouse Formation (Silurian) of Oklahoma. Washington Academy of Sciences, Journal, 39(6):202-203.

1953. Some Notes on the Pentameracea, Including a Description of One New Genus and One New Subfamily. Washington Academy of Sciences, Journal, 43(5): 137-147, 7 figures.

1958. Stratigraphy and Paleontology of the Hunton Group in the Arbuckle Mountain Region, Part II:
Haragan Articulate Brachiopods. Oklahoma Geological Survey Bulletin, 78(2-3):9-157, 39 figures, 14 plates.

1964. Brachial Plate Structure in the Brachiopod Family Pentameridae. Palaeonlology, 7(2):220-239, plates 40-43.

1968. Articulate Brachiopods of the St. Clair Limestone (Silurian), Arkansas, and the Clarita Formation (Silurian), Oklahoma. Journal of Paleonlology, 42(3), supplement:1-117, 83 figures, 20 plates.

1974. Late Ordovician and Early Silurian Articulate Brachiopods from Oklahoma, Southwestern Illinois, and Eastern Missouri. Oklahoma Geological Survey Bulletin, 119:1-154, 51 figures, 28 plates.

1978. Articulate Brachiopods of the Quarry Mountain Formation (Silurian), Eastern Oklahoma. Oklahoma Geological Survey Bulletin, 125:1-75.

Amsden, T. W., A. J. Boucot, and J. G. Johnson

1967. Conchidium and Its Separation from the Subfamily Pentamerinae. Journal of Paleonlology, (41)4:861867.

Anderson, M. M., A. J. Boucot, and .J. G. Johnson

1969. Eifelian Brachiopods from Padaukpin, Northern Shan States, Burma. British Museum (Natural History), Bulletin (Geology), 18:105-163, 10 figures, 10 plates.

Andreeva, O. N.

1955. Polevoi Allas Ordovikskoi z Silurizskoi Fauny Sibirskoi Platformy. 268 pages, 62 plates. Moscow: Vsesoiuznyi Nauchno-Issledovatelskii Geologicheskii Institut (VSEGEI), Minist. Geol. i Okhrany Nedr.

1962. [Some Cambrian Brachiopods from Siberia and Central Asia.] Paleontologicheskii Zhurnal, 1962(2): 87-96, 1 plate. [In Russian.]

1972. [Brachiopods of the Ordovician Kuragan Suite of the South Urals.] Paleonlologicheskii Zhumal, 1972(1):45-56, 2 plates. [In Russian.]

1977. [New Ordovician Strophomenids of the Polar Urals.] In G. A. Stukalina, editor, Novye Vidy Drevnikh Raslenii i Bespozvonochynkh SSSR, 4:116-117. Moscow: Akademiia Nauk SSSR, Paleontologicheskii Institut. [In Russian.]

Andronov, S. M.

1961. [Some Representatives of the Family Pentameridae from the Devonian Deposits of the Northern Urals.] Akademiia Nauk SSSR, Institut Geologicheskovo, Trudy, 55:1-136, 32 plates. [In Russian.]

Antostschenko, Z. A.

1973. On the New Genus Spinulothyris Antostschenko Genus Novum (Brachiopoda).] Byulleten Moskovskogo Obschestva Ispilatelei Prirody (Otdel Geologicheskii), 48:109-116. [In Russian.]

Asatkin, B. P.

1932. [Ecardines from the Lower Silurian of the Siberian 
Platform.] Izvestiya Vsesoyuznogo Geologo-Razvednochnogo Obedineniya, 51:483-495, 2 plates. [In Russian.]

Asgaard, V.

1971. Faksethyris nielsent n.g. et n.sp. (Terebratulacea) from the Middle Danian of Denmark. Geological Society of Denmark Bulletin, 20:385-389, 6 figures, 9 plates.

Astashkina, V. F.

1970. [New Genus of the Family Stropheodontidae Caster (Brachiopoda).] Sibirskii Nauchno-Issledovatelskii Institut Geologii, Geofiziki i Mineralogii Syr'ia (SNIIGGIMS), Trudy, 110:131-134, 1 plate. [In Russian.]

Atkins, $\mathrm{D}$.

1958. A New Species and Genus of Kraussinidae (Brachiopoda) with a Note on Feeding. Zoological Society of London, Proceedings, 131(4):559-581, 14 figures, 1 plate.

1960. A New Species and Genus of Brachiopoda from the Western Approaches and the Growth Stages of the Lophophore. Marine Biological Association of the United Kingdom, Journal, 39:71-89, 14 figures, 1 plate.

Babanova, L. I.

1964. [New Data on Jurassic Brachiopods.] Paleontologicheskii Zhurnal, 1964(1):63-70, 1 plate. [In Russian.]

1965. [A New Genus of Terebratulid Brachiopods from the Middle Jurassic of the Crimean Range.] Paleontologicheskii Zhurnal, 1965(4):94-97, 3 figures. [In Russian.]

Backhaus, E.

1959. Monographie der cretacischen Thecideidae (Brach.). Hamburg Geologisches Staatsinstitut, Mitteilungen, 28:5-90, 7 plates.

Balinski, A.

1977. Biernatella-A New Devonian Double-Spired Brachiopod. Acta Palaeontologica Polonica, 22(2): 175-186.

Bamber, E. W., and J. B. Waterhouse

1971. Carboniferous and Permian Stratigraphy and Paleontology, Northern Yukon Territory, Canada. Bulletin of Canadian Petroleum Geology, 19(1):29-250, 19 figures, 27 plates.

Bancroft, B. B.

1928a. The Harknessellinae. Manchester Literary and Philosophical Society, Memoirs and Proceedings, 72:173-196, 2 plates.

1928b. On the Notational Representation of the Rib-System in Orthacea. Manchester Literary and Philosophical Society, Memoirs and Proceedings, 72:53-90, 3 plates.

1929. Some New Genera and Species of Strophomenacea from the Upper Ordovician of Shropshire. Manchester Literary and Philosophical Society, Memoirs and Proceedings, 73(4):33-65, 2 plates.

1933. Correlation Tables of the Stages Costonian-Onnian in England and Wales. 4 pages, 3 tables. Printed and published by the author at Blakeney, England.

1945. The Brachiopod Zonal Indices of the Stages Costonian-Onnian in Britain. Journal of Paleontology, 19(3):181-252, plates 22-38.

1949a. Welsh Valentian Brachiopods. In Quarry Managers' Journal, pages 2-10, 2 plates.

1949b. The Strophomena antiquata Group of Fossil Brachiopods. In Quarry Managers'Journal, pages 11-16, 1 plate.

Baranov, V. V.

1977. [New Early Devonian Rhynchonellids in the Northeast of the USSR.] Paleontologicheskii Zhurnal, 1977(3):75-82. [In Russian.]

Barchatova, V. P.

1970. Biostratigraphy of the Carboniferous and Lower Permian of Northern Timan. Trudy Vsesoyuznogo Nefiianogo Nauchno-Issledovatel'skogo Geologorazvedochnogo Instituta (VNIGRI), 283:1-228, 21 plates. [In Russian.]

1973. In Novye Vidy Drevnikh Rastenii i Bespozvonochnykh. Vsesoiuznyi Nauchno-Issledovatelskii GeologoRazvedochnyi Neftianoi Institut (VNIGRI), Trudy, 318 1-179, 34 plates.

Barczyk, W.

1969. Upper Jurassic Terebratulids from the Mesozoic Border of the Holy Cross Mountains in Poland. Prace Muzeum Ziemi, 14:3-82, 73 figures, 18 plates.

Barrande, J.

1879. Systême silurien du centre de la Bohême, partie I: Recherches paléontologiques, 5. 226 pages, 153 plates.

1881. Systême silurien du centre de la Bohême..., 6. 342 pages, 361 plates.

Bassett, M. G.

1971. Wenlock Stropheodontidae (Silurian Brachiopoda) from the Welsh Borderland and South Wales. Palaeontology, 14(2):303-337, 6 plates.

1972. The Articulate Brachiopods from the Wenlock Series of the Welsh Borderland and South Wales, Part 2. Palaeontographical Society Monograph, 532:2778, plates 4-17.

1977. The Articulate Brachiopods from the Wenlock Series of the Welsh Borderland and South Wales, Part 4. Palaeontographical Society Monograph, 547: 123-176, plates 33-47.

Bassett, M. G., and L.R.M. Cocks

1974. A Review of Silurian Brachiopods from Gotland. Fossils and Strata, 3:1-56, 11 plates.

Bassett, M. G., L.R.M. Cocks, and C. H. Holland 1976. The Affinities of Two Endemic Silurian Brachio- 
pods from the Dingle Peninsula, Ireland. Palaeontology, 19(4):615-625.

Bates, D.E.B.

1968. The Lower Palaeozoic Brachiopod and Trilobite Faunas of Anglesey. British Museum (Natural History), Bulletin (Geology), 16(4):127-199.

Batrukova, L. S.

1969. [New Devonian Lingulids and Discinids from the Russian Platform.] Vsesoiuzny' Nauchno-Issledovalelskui Geologo-Razvedochnyi Neftianoi Instilut (VNIGNI), Trudy, 93:59-77, 4 plates. [In Russian.]

Bayle, E.

1878. Fossiles principaux des terrains de la France. In Explication de la Carte Géologique de la France, 4(1): 158 plates.

1880. Liste rectificative de quelques noms de genres et d'espèces. Journal de Conchyliologıe, 28(3):240-251.

Bednarczyk, W., and G. Biernat

1978. Inarticulate Brachiopods from the Lower Ordovician of the Holy Cross Mountains, Poland. Acta Palaeontologica Polonica, 23(3):223-407, 59 plates.

Beecher, C. E.

1890. On Leplaenısca, a New Genus of Brachiopod from the Lower Helderberg Group. American Journal of Science, series 3, 40:238-240, 5 figures, 9 plates.

1891. Development of the Brachiopoda, Part I: Introduction. American Journal of Science, series 3, 41:343357, 1 plate.

1893. Revision of the Families of Loop-Bearing Brachiopoda. Conneclicul Academy of Arts and Sciences, Transactions, 9:376-391, 2 plates.

Beets, C.

1943. On Waisiuthyrina, a New Articulate Brachiopod Genus from the Upper Oligocene of Buton (S. E. Celebes), Dutch East Indies. Leidsche Geologische Mededeelingen, 13(1):341-347.

Bekker, $\mathrm{H}$.

1921. The Kuckers Stage of the Ordovician Rocks of N. E. Estonia. Acla el Commentalzones Universitatis Tarluensis (Dorpat), series A, 2: 92 pages, 15 figures, 12 plates.

1922. A New Brachiopod (Leptestia) from the Kuckers Stage in Estonia. Geological Magazine, 59:361-365, 4 figures.

1924. The Devonian Rocks of the Irboska District (S. E. Estonia) with the Description of a New Cemented Brachiopod Irboskiles. Nalurkunde Esllands Naturforscher Gesellschaft, Universily Tariu (Dorpat), 10(1): $1-55,6$ plates.

Belanski, C. H.

1928. Terebratulacea of the Devonian of Northern Iowa. Iowa Unwersity, Studies in Natural History, new series, 12(8):29 pages, 15 figures, 4 plates.
Bell, W. A.

1929. Horton-Windsor District, Nova Scotia. Canada, Geological Survey, Department of Mines and Resources, Memoir, 155: 268 pages, 36 plates.

Bell, W. C.

1938. Prololrela, a New Genus of Brachiopod from the Middle Cambrian of Montana. Michigan Academy of Science, Arts and Letters, Papers, 23:403-408, 1 plate.

1941. Cambrian Brachiopoda from Montana. Journal of Paleontology, 15(3): 193-255, plates 28-37.

Bell, W. G., and H. L. Ellinwood

1962. Upper Franconian and Lower Trempealeauan Cambrian Trilobites and Brachiopods, Wilberns Formation, Central Texas. Journal of Paleonlology, $36(3): 385-423,14$ plates.

Berdan, J. M.

1963. Eccentricosta, a New Upper Silurian Brachiopod Genus. Journal of Paleontology, 37(1):254-256, 1 figure.

Bergström, J

1968. Upper Ordovician Brachiopods from Vastergotland, Sweden. Geologica el Palaeontologica, 2:1-35.

Biernat, G.

1966. Middle Devonian Brachiopods of the Bodzentyn Syncline (Holy Cross Mountains, Poland). Palaeontologia Polonica, 17:1-162, 32 plates.

1973. Ordovician Inarticulate Brachiopods from Poland and Estonia. Palaeontologia Polonica, 28:1-120, 40 figures, 40 plates.

Billings, E.

1858. Report for the Year 1857 of E. Billings, Esq., Palaeontologist. In Canada, Geological Suncy, Report of Progress for the Year 1857, pages 147-192, 24 figures.

1859a. On Some New Genera and Species of Brachiopoda from the Silurian and Devonian Rocks of Canada. Canadian Naluralest and Geologist, 4:131-135.

1859b. Description of a New Genus of Brachiopoda and on the Genus Cyrtodonta. Canadian Naturalist and Geologist, 4:301-303.

1861a. On Some New or Little Known Species of Lower Silurian Fossils from the Potsdam Group (Primordial Zone). In Geological Survey of Canada, Paleozoic Fossils, 1:1-18.

1861b. Note on a New Genus of Paleozoic Brachiopoda. Canadian Journal of Industry, Science, and Art, 6:148.

1862. New Species of Fossils from Different Parts of the Lower, Middle and Upper Silurian Rocks of Canada. In Geological Survey of Canada, Paleozoic Fossils, 1:96- 185 .

1871. On Some New Species of Palaeozoic Fossils. Canadian Naluralist, 6:213-222. 
Bitner, M. A., and A. Pisera

1979. Brachiopods from the Upper Cretaceous Chalk of Mielnik (Eastern Poland). Acta Geologica Polonica, 29(1):67-88, 7 plates.

Bittner, A.

1884. Aus den Salzburger Kalkhochgebirgen. zur Stellung der Hallstatte Kalke. Geologische Reichsanstalt, Verhandlungen, 6:99-113.

1888a. Ueber Koninckiniden des Alpinen Lias. Grologische Reichsanstalt, Jahrbuch, 37:281-293.

1888b. Ueber das Aufreten von Terebrateln aus der Subfamilie der Centronellinen in der Alpinen Trias. Geologische Reichsanstalt, Verhandlungen, 5: $125-128$.

1890. Brachiopoden der Alpinen Trias. Geologische Reichsanstalt, Abhandlungen, 14:1-325, 41 plates.

1892. Brachiopoden der Alpinen Trias, Nachtrag I. Geologische Reichsanstalt, Abhandlungen, 17(2):1-40, 4 plates.

1893. Neue Koninckiniden des Alpinen Lias. Geologische Reichsanstalt. Jahrbuch, 43(1):133-144.

1896. Eine Neue Form der Triadischen Terebratulidengruppe der Neocentronellinen Oder Juvavellinen. Geologische Reichsanstalt, Verhandlungen, 4:131-132.

1900. Brachiopoden aus der Trias des Bakonyer Waldes. In Resultate der Wissenschaftlichen Erforschung des Balatonsees, Palacontologischer Anhang, 2(1):1-58.

1903. Brachiopoden und Lamellibranchiaten aus der Trias von Bosnien, Dalmatien und Venetian. Geologische Reichsanstalt, Jahrbuch, 52(3,4):495-843.

Blainville, H.M.D. de

1824. Mollusques and Palliobranches. In Dictionnaire des Sciences Naturelles, 32:298-302.

1825. Manuel de malacologie et de conchyliologie. 647 pages. Paris.

Bolten, J. F.

1798. Museum Boltenianum... Pars secunda .... viii +199 pages.

Bondarev, V. I., A. Z. Burskii, K. N. Koloskov, and A. B. Nekhorosheva

1965. Ranneordovikskaia Fauna Iuga Novoi Zemli i Severa Pai-Khoia i ee Stratigraficheskoe Znachenie. Uchenyi Zapiski, Nauchno-Issledovatelskii Institut Geologï Arktiki, Paleontologiia i Biostratigrafiia, 10: 15-63, 10 plates.

Booker, F. W.

1926. The Internal Structure of Some of the Pentameridae of New South Wales. Royal Society of New South Wales, Journal, 60:130-145, plates 5-8.

1930. Preliminary Note on New Subgenera of Productus and Strophalosia from the Branxton District. Royal Society of New South Wales, Journal, 63:24-32, 3 plates.

1931. A Review of Some of the Permo-Carboniferous
Productidae of New South Wales, with a Tentative Reclassification. Royal Society of Nere South V'ales, Journal, 64:65-77, 3 plates.

Böse, E., and M. Schlosser

1900. Ueber die Mittelliasische Brachiopodenfauna von Südtyrol. Palacontographica, 46:177-178, plate 17, figures $1,2$.

Bouchard-Chantereaux, N. R.

1849. Mémoire sur un nouveau genre de brachiopode formant le passage des formes articulées à celles qui ne sont pas. Annales des Sciences Naturelles, series 3, 12:84-93, 1 plate.

Boucot, A. J.

1957. Revision of Some Silurian and Early Devonian Spiriferid Genera and Erection of Kozlowskiellinae, New Subfamily. Senckenbergiana Lethaea, 38(56):311-334, 3 plates.

1958. Kozlowskiellina, New Name for Kozlowskiella Boucot, 1957. Journal of Paleontology, 32(5):1031.

1959a. Early Devonian Ambocoeliinae (Brachiopoda). Journal of Paleontology, 33(1):16-24, plates 1, 2.

1959b. A New Family and Genus of Silurian Orthotetacid Brachiopods. Journal of Paleontology, 33(1):25-28, figures 1, 2, plate 3 .

1959c. Brachiopods of the Lower Devonian Rocks at Highland Mills, New York. Journal of Paleontology, 33(5):727-769, 14 plates.

1960a. A New Lower Devonian Stropheodontid Brachiopod. Journal of Paleontology, 34(3):483-485, 1 plate.

1960b. A Late Silurian Fauna from the Sutherland River Formation, Devon Island, Canadian Arctic Archipelago. Canada, Geological Survey Bulletin, 65:1-51, plates $1-10$.

1963. The Eospiriferidae. Palaeontology, 5(4):682-711, plates 97-104.

1964a. Callipentamerus, a New Genus of Brachiopoda from the Silurian of Iowa. Journal of Paleontology, 38(5): $887-888,2$ plates.

1964b. A New Genus of Silurian Atrypacean Brachiopod. In Reconnaissance Bedrock Geology of the Presque Isle Quadrangle, Maine. Quadrangle Mapping Series, 2(Appendix):104-112, 2 plates. Augusta, Maine: Maine Geological Survey, Department of Economic Development.

1970. Glypterina, a New Genus, the Ordovician Ptychopleurellid. Journal of Paleontology, 44(5):984-985.

1971. Aentgmastrophia, New Genus, a Difficult Silurian Brachiopod. In J. T. Dutro, Jr., editor, Paleozoic Perspectives: A Paleontological Tribute to G. Arthur Cooper. Smithsonian Contributons to Paleobrology, 3:155-158.

1973. Early Paleozoic Brachiopods of the Moose River Synclinorium, Maine. U.S. Geological Suney Professional Paper, 784:1-81, 23 plates. 
1975. Evolution and Extinction Rate Controls. 426 pages, 44 figures, 4 plates. New York: Elsevier.

Boucot, A. J., and T. W. Amsden

1958. New Genera of Brachiopods. Oklahoma Geological Survey Bulletin, 78(4):159-170, figures 40-42, plate 14.

1964. Chonostrophiella, a New Genus of Chonostrophid Brachiopod. Journal of Paleontology, 38(5):881-884, 1 plate.

Boucot, A. J., K. E. Caster, D. Ives, and J. A. Talent

1963. Relationships of a New Lower Devonian Terebratuloid (Brachiopoda) from Antarctica. Bulletins of American Paleontology, 46(207):77-151, plates 1641.

Boucot, A. J., and K. K. Chiang

1974. Two New Lower Silurian Virgianid (Family Pentameridae) from the Nonda Formation, Northern British Columbia. Journal of Paleontology, 48(1):63 73.

Boucot, A. J., and G. M. Ehlers

1963. Two New Genera of Stricklandid Brachiopods. Untversity of Michigan, Museum of Paleoniology', Contributions, 18:47-66, 5 plates.

Boucot, A. J., and K. L. Gauri

1966. Quadrikentron (Brachiopoda) a New Subgenus of Sirophochonetes. Journal of Paleontology, 40(5):10231026, 1 plate.

Boucot, A. J., K. L. Gauri, and J. G. Johnson

1966. New Subfamily Proschizophoriinae of Dalmanellid Brachiopods. Palaeontologische Zeitschrift, 40(34):155-172, 1 figure, 4 plates.

Boucot, A. J. and E. D. Gill

1956. Australocoelia, a New Lower Devonian Brachiopod from South Africa, South America, and Australia. Journal of Paleontology, 30 (5):1173-1178, plate 126.

Boucot, A. J., and C. W. Harper

1968. Silurian to Lower Middle Devonian Chonetacea. Journal of Paleontology, 42(1):143-176, 4 plates.

Boucot, A. J., and J. G. Johnson

1966a. Ehlersella, a New Silurian Stricklandiid Brachiopod Genus. Journal of Paleontology, 40(3):569-572.

1966b. Lissocoelina and its Homeomorph Plicocoelina n. gen. (Silurian, Pentameracea). Journal of Paleontology, 40(5): 1037-1042, 4 plates.

1967a. Silurian and Upper Ordovician Atrypids of the Genera Plectatrypa and Spirigerina. Norsk Geologisk Tidsskrift, 47(1):79-101.

1967b. Allanetes, a New Lower Devonian Chonetid Brachiopod Genus. Royal Society of Victoria, Proceedings, 80(1): 141-145.

1968. Brachiopods of the Bois Blanc Formation in New York. U.S. Geological Survey Professional Paper, 584 B: 27 pages.

1972. Callicalyptella, a New Genus of Notanopliid
Brachiopod from the Devonian of Nevada. Joumal of Paleontology, 46(2):299-302, 2 plates.

1979. Pentamerinae (Silurian Brachiopoda). Palaeontographica, series A, 163(4-6):87-129, 15 plates.

Boucot, A. J., J. G. Johnson, and M. Rubel

1971. Descriptions of Brachiopod Genera of Subfamily Virgianinae Boucot and Amsden, 1963. Eesti NSV Teaduste Akadeemia Toimetised, Keemia Geoloogia, 20: 271-280, 6 plates.

Boucot, A. J., J. G. Johnson, and R. D. Staton

1964. On Some Atrypoid, Retzioid and Athyridoid Brachiopoda. Journal of Paleontology, 38(5):805-822, 4 plates.

Boucot, A. J., and R. E. Smith

1978. First Upper Silurian Fossils from Northern Newfoundland. Journal of Paleontology, 52(2):267-274.

Boullier, A. (= A. Rollet)

1976. Les Terebratulides de l'Oxfordien du Jura et de la bordure sud du bassin de Paris. Annales Scientifiques de l'Universite de Besançon, series 3 (Géologie), 27: 1457, 212 figures, 25 plates.

Bowen, Z. P.

1966. Boucotella, a New Silurian Rhynchonelloid Brachiopod Genus. Journal of Paleontology, 40(1): 186-189, 2 plates.

Branson, C. C.

1966. New Genus of Spiriferid Brachiopod from Oklahoma and Texas. Oklahoma Geological Survey Notes, 26(3):74-78, 1 plate.

Breger, C. L.

1906. On Eodevonaria, a New Sub-Genus of Chonetes. American Journal of Science, series 4, 22:534-536.

Breivel, I. A., and M. G. Breivel

1970a. Dva Novykh Siluriiskikh Roda iz Nadsemeistva Pentameracea. In Materialy po Paleontologii Urala, pages 52-56, 4 plates. Sverdlovsk: Akademiia Nauk SSSR, Uralskii Filial Institut Geologii i Geokhimi.

1970b. Novye Vidy Gipiduli i Roda Colongina iz Pogranichnykh Siluro-Devonskikh Otlozhenii Vostochnogo Sklona Urala. In Materialy po Paleontologii Urala, pages 57-62, 1 plate. Sverdlovsk: Akademiia Nauk SSSR, Uralskii Filial Institut Geologii i Geokhimi.

1977. Brakhiopody. In T. A. Gorokhova, editor, Biostratigrafiya i Fauna Rannego Devona Vostochnogo Sklona Urala, 309:1-246, 59 plates. Moscow: Ministerstvo Geologii RSFSR Ural'skoe Territorial'noe Geologicheskoe Upravlenie.

Brice, D.

1970. Étude paleontologique et stratigraphique du Devonien de l'Afghanistan: Contribution a la connaissance des brachiopodes et des polypiers 
rugueux. Notes et Memoires sur le Moyen-Orient, 11: 1-364, 59 figures, 20 plates.

Brill, K. G.

1940. Brachiopods of the Whitehorse Sandstone. In N. D. Newell, The Invertebrate Fauna of the Late Permian Whitehorse Sandstone. Geological Society of America, Bulletin, 51(2):316-319.

Brown, I. A.

1953. Permian Spirifers from Tasmania. Royal Sociely of New South Wales, Journal and Proceedings, 86:55-63, 2 plates.

Brown, R.A.C.

1952. Carboniferous Stratigraphy and Palaeontology in the Mount Greenock Area, Alberta. Geological Survey of Canada, Memoir, 264:1-119, 2 plates.

Brown, $\mathrm{T}$.

1849. Illustrations of the Fossil Conchology of Great Britain and Ireland, with Descriptions and Localities of all Species. 273 pages, 98 plates.

Brügge, N.

1973. Eremilhyris n. gen. (Brachiopoda, Dielasmatidae)ein neues Leitfossil aus den Hauptdolomit des germanischen Zechsteins. Zeitschrift für Geologische Wissenschaflen (Berlin), 1(2):195-213, 9 figures, 2 plates.

1977. Silesiathyris n. gen. (Brachiopoda, Zeilleriidae)ein neues Genus aus dem Unteren Muschelkalk von Gorny Slask (VR Polen). Zeitschrift für Geologische Wissenschaften, 5(5):663-675, 3 plates.

Bruguière, J. G.

1797. Vers, coquilles, mollusques et polypiers, volume 2, plates 190-286. In Tableau encyclopédique el méthodique des trois règnes de la nature. Paris: Agasse.

Brunton, C.H.C.

1966. Silicified Productoids from the Visean of County Fermanagh. British Museum (Natural History), Bulletin (Geology), 12(5):173-243, 11 figures, 19 plates.

1968. Silicified Brachiopods from the Visean of County Fermanagh (II). British Museum (Natural History), Bulletin (Geology), 16(1):70 pages, 9 plates.

1971. An Endopunctate Rhynchonellid Brachiopod from the Visean of Belgium and Britain. Palaeontology, 14(1):95-106, 2 plates.

Brunton, C.H.C., and C. Champion

1974. A Lower Carboniferous Brachiopod Fauna from the Manifold Valley, Staffordshire. Palaeontology, 17(4):811-840, 7 figures, 5 plates.

Brunton, C.H.C., and L.R.M. Cocks

1967. Brachiopods in the Linnaean Collection. Proceedings, Linnaean Society of London, 178(2):161-183.

Bublichenko, N. I.

1928. Die Brachiopodenfauna des unteren Palaeozoicum aus der Umgegend des Dorfes Sara-Tschu- mysch aus dem Kohlenbassin von Kusnetzk. Comité Geologique, Bulletin, 46(8):979-1008, plates 4950 .

1956. Nekotorye Novye Predstaviteli Brakhiopod Devona i Karbona Rudnogo Altaya i Sary-Arka. Akademiia Nauk Kazakhskoi SSR, Izvestiya, 23:93104, 4 figures, 1 plate.

Buckman, S. S.

1901. Homoeomorphy among Jurassic Brachiopoda. Proceedings, Colteswold Naturalists Field Club, 13(4): 231-290, plates 12-13.

1906a. Brachiopod Homoeomorphy: Pygope, Antinomia, Pygites. Geological Society of London, Quarterly Journal, $62: 433-455$, plate 41

1906b. Brachiopod Nomenclature. Annals and Magazine of Natural History (London), series 7, 18:321-327.

1907. Brachiopod Morphology: Cincla, Eudesia, and the Development of Ribs. Geological Society of London, Quarterly Journal, 63:338-343.

1917. The Brachiopoda of the Namyau Beds, Northern Shan States, Burma. Palaeontologia Indica, new series, 3(2): 299 pages, 21 plates.

1927. Jurassic Chronology, III: Some Faunal Horizons in Cornbrash. Geological Society of London, Quarterly Journal, 83:1-37, 1 plate.

Burri, F.

1953. Beiträge zur Systematik der Brachiopoden aus der untersten Kreide im westschweizerischen Juragebirge. Ecologae Geologicae Helvetiae, 46:269-285, plate 15.

1956. Die Rhynchonelliden der unteren Kreide (Valanginien-Barremien) im westschweizerischen Juragebirge. Ecologae Geologicae Helvetiae, 49:599-701, 38 figures, 15 plates.

Butts, C.

1926. The Palaeozoic Rocks, in the Geology of Alabama. Special Report, Geological Survey of Alabama, 14:41230.

Calzada Badía, S.

1974a. Almerarhynchia n. gen. virgiliana n. sp. del Maastrichtiense de Figols, Prepirineo catalán. Acta Geológica Hispánica, 9(3):92-97, 2 plates.

1974b. Rhynchonella sardanyolae $=$ Viarhynchia $\mathrm{n}$. gen. cerdanyolae (Bataller, 1947) del Cretácico superior (Prepirineo catalán). Acta Geológica Hispánica, 9(5):169_ 173, 2 plates.

Campbell, J. D.

1968. Raslelligera (Brachiopoda) of the Upper Triassic of New Zealand. Transactions of the Royal Society of New Zealand (Geology), 6(3):23-37, 4 plates.

Campbell, K.S.W.

1953. The Fauna of the Permo-Carboniferous Ingelara Beds of Queensland. Queensland University, Department of Geology, Paper, 4(3):1-44, 7 plates. 
1957. A Lower Carboniferous Brachiopod-Coral Fauna from New South Wales. Journal of Paleontology, 31 (1):34-98, 27 figures, 7 plates.

1959. The Martiniopsis-like Spiriferids of the Queensland Permian. Palaeontology, 1(4):333-350, 2 plates.

1961. Carboniferous Fossils from the Kuttung Rocks of New South Wales. Palaeontology, 4(3):428-474, plates 53-63.

1965. Australian Permian Terebratuloids. Australia, Bureau of Mineral Resources, Geology and Geophysics, Bulletin, 68: 114 pages, 17 plates.

Campbell, K.S.W., and B. A. Engel

1963. The Faunas of the Tournaisian Tulcumba Sandstone and Its Members in the Werrie and Belvue Synclines, New South Wales. Geological Society of Australia, Journal, 10(1):55-122, 9 plates.

Campbell, K.S.W., and J. A. Talent

1967. Malurostrophia, a New Genus of Stropheodontid Brachiopod from the Devonian of Australia. Royal Society of Australia, Proceedings, 80(2):309-330, 4 plates.

Caneva, G.

1906. Ueber die Bellerophonkalkfauna zur Frage der Perm-Triasgrenzen. Neues Jahrbuch für Mineralogie, Geologie und Paläontologie, Abhandlungen, 1:52-60.

Carls, $\mathrm{P}$.

1974. Die Proschizophoriinae (Brachiopoda; SiluriumDevon) der Östlichen Iberischen Ketten (Spanien). Senckenbergiana Lethaea, 55(1-5):153-227, 4 figures, 13 plates.

Carter, J. L.

1967a. Mississippian Brachiopods from the Chappel Limestone of Central Texas. Bulletin of American Paleontology, 53:249-488.

1967b. An Unusual Spiriferacean Brachiopod from the Barnett Formation (Mississippian) of Central Texas. Journal of Paleontology, 41(3):587-594, 1 plate.

1968. New Genera and Species of Early Mississippian Brachiopods from the Burlington Limestone.Journal of Paleontology, 42(5): 1140-1152, 4 plates.

1971. New Early Mississippian Silicified Brachiopods from Central Iowa. In J. T. Dutro, Jr., editor, Paleozoic Perspectives: A Paleontological Tribute to G. Arthur Cooper. Smithsonian Contributions to Paleobiology, 3:245-255, 2 figures, 2 plates.

1972. Two New Genera of Lamellose Spiriferacean Brachiopods. Journal of Paleontology, 46(5):729-734.

1974. New Genera of Spiriferid and Brachythyridid Brachiopods. Journal of Paleontology, 48(4):674-696, 4 figures, 4 plates.

Casey, R.

1961. The Stratigraphical Paleontology of the Lower
Greensand. Palaeontology, 3(4):487-621, 14 figures, plates 77-84.

Caster, K. E.

1939. A Devonian Fauna from Colombia. Bulletin of American Paleontology, 24(83):1-218, 14 plates.

1945. New Names for two Homonyms. Journal of Paleontology, 19(3):319.

Catullo, T. A.

1851. On the Epiolitic Rocks of the Venetian Alps. Geological Sociely of London, Quarterly Journal, 7:6676.

Chan, Li-Pei (= Zhan Li-pei)

1977. In Zhong Nan Di Qu Gu Sheng Wu Tu Ce, Er, Wan Gu Sheng Dai Bu Feng [Paleontological Atlas of Central-South China, Volume 2 (Late Paleozoic)]. 856 pages, 253 plates. Peking. [In Chinese.]

Chao, Y. T.

1927. Productidae of China, Part I: Producti. China, Geological Survey, Palaeontologia Sinica, series B, 5(2): 1-192, 7 figures, 16 plates.

1928. Productidae of China, II: Chonetinae, Productinae and Richthofeniinae. China, Geological Survey, Palaeontologia Sinica, series B, 5(3):1-103, 6 plates.

1929. Carboniferous and Permian Spiriferids of China. China, Geological Survey, Palaeontologia Sinica, series B, 11(1):1-101, 20 figures, 11 plates.

Chapman, F., and I. Crespin

1923. The Austral Rhynchonellacea of the "Nigricans Series," with a Description of the New Genus Tegulorhynchia. Royal Society of Victoria, Proceedings, 35:170-193, plates 11-13.

Chatterton, B.D.E.

1973. Brachiopods of the Murrumbidgee Group, Taemas, New South Wales. Australia, Bureau of Mineral Resources, Geology and Geophysics, Bulletin, 137:1-146, 49 figures, 35 plates.

Chen, Yuan-ren

1978. In Xi Nan Di Qu Gu Sheng Wu Tu Ce, Si-Chuan Feng Ce, Yi Zhen Dan Ji Zhi Ni Peng Ji [Paleontological Atlas of Southwestern China, Sichuan Province, Volume 1 (Sinian-Devonian)]. 617 pages, 185 plates. Peking. [In Chinese.]

Childs, A.

1969. Upper Jurassic Rhynchonellid Brachiopods from Northwestern Europe. British Museum (Natural Hislory), Bulletin (Geology), 6(Suppl.):1-119, 36 figures.

Ching, Yu-kan (= Jing Yu-kan and Jin Yu-gan)

1974. In Xi Nan Di Qu Di Ceng Gu Sheng Wu Shou Ce [A Handbook of the Stratigraphy and Paleontology in Southwest China]. 454 pages, 202 plates, Peking. [In Chinese.]

Ching, Yu-kan, and Zhuo-ting Liao

1974. In Xi Nan Di Qu Di Ceng Gu Sheng Wu Shou Ce [A 
Handbook of the Stratigraphy and Paleontology in Southwest China]. 454 pages, 202 plates. Peking. [In Chinesc.]

Ching, Yu-kan, Zhuo-ting Liao, and Bing-xing Fang

1974. In Xi Nan Di Qu Di Ceng Gu Sheng Wu Shou Ce [A Handbook of the Stratigraphy and Paleontology in Southwest China]. 454 pages, 202 plates. Peking. [In Chinese.]

Ching, Yu-kan, Dong-li Sun, and Jia-yu Rong

1976. [Mesozoic and Cenozoic Brachiopods from the Mount Jolmo Lungma Region]. In A Report of Scientific Expedition in the Mount Jolmo Lungma Region (1966-1968), Paleontology, 2:271-357, 10 plates. [In Chinese.]

Ching, Yu-kan, Dong-li Sun, and Song-ling Ye

1979. In $\mathrm{Xi} B e i \mathrm{D} l Q u$ Gu Sheng Wu Tu Ce, Qing-hai Feng $\mathrm{Ce}, \mathrm{Yi}$ [Paleontological Atlas of Northwestern China, Qinghai Province, Volume 1 (Lower Paleozoic-Cenozoic)]. 393 pages, 96 plates. Peking. [In Chinese.]

Ching, Yu-kan, and Song-ling Ye

1979. In Xi Bei Di Qu Gu Sheng Wu Tu Ce, Qing-han Feng $\mathrm{Ce}$, Yi [Paleontological Atlas of Northwestern China, Qinghai Province, Volume 1 (Lower Paleozoic-Cenozoic)]. 393 pages, 96 plates. Peking. [In Chinese.]

Chiplonker, C. W.

1938. Rhynchonellids from the Bagh Beds. Indian Academy of Sciences, Proceedings, 7(6):300-316, 7 figures, plate 30.

Chorowicz, J., and G. Termier

1975. Une Faunule silificée nouvelle dans le trias moyen de la Svilaja (Yougoslavie). Annales Societe Geologique Nord, 95(4):231-242, 11 figures, 2 plates.

Chu, S.

1933. Corals and Brachiopods of the Kinling Limestone. National Research Institute of Geology, Academia Sinica, Monographs, series A, 2:1-73, 5 plates.

Chugaeva, M. N., Kh. S. Rozman, and V. A. Ivanova

1964. [Comparative Biostratigraphy of Ordovician Deposits in the North-east of the USSR.] Akademiia Nauk SSSR, Geologicheskii Institut, Trudy, 106:1-236, 35 figures, 25 plates. [In Russian.]

Clark, T. H.

1917. New Blastoids and Brachiopods from the Rocky Mountains. Harvard University, Museum of Comparative Zoology, Bulletin, 61(9):361-380, 5 figures, 2 plates.

Clarke, J. M.

1907. Some New Devonic Fossils. New York State Museum, Bulletin, 107:153-291.

1912. El Devoniano de la Argentina Occidental. Annales Ministerio de Agricultura Argentina, Sección Geologia, 8(2): 1-19. 1913a. Ninth Report of the Director of the Science Division, Including the 66th Report of the Statc Muscum, the 32nd Report of the State Geologist, and the Report of the State Paleontologist for 1912 New York Stale Museum, Bulletin, 164:140-214, plate 21

1913b. Fosseis Devonianos do Paraná. Monographras do Serviqgo Geologico e Mineralogıco do Brasil, 1:1-353, 27 plates.

1921. The Heavenly Twins. Nautilus, 34:138-139.

Clarke, M. J.

1970. A New Permian Strophalosiid Brachiopod Genus from Eastern Australia. Journal of Paleonlology, 44(5):986-987.

Cloud, R. E., Jr.

1942. Terebratuloid Brachiopoda of the Silurian and Devonian. Geological Society of America, Special Paper, 38:1-182, 26 plates.

1948. Brachiopods from the Lower Ordovician of Texas. Harvard University, Museum of Comparative Zoology, Bulletin, 100(5):451-472.

Cobbold, E. S.

1921. The Cambrian Horizons of Comley (Shropshire) and Their Brachiopoda, Pteropoda, Gasteropoda, etc. Geological Society of London, Quarterly Journal, 76: 325-386, plates 21-24.

Cockbain, A. E.

1967. A New Craniacean Brachiopod from the Tertiary of Western Australia. Geological Survey of Western Australia Report, 1966:119-120, 1 plate.

Cockerell, T.D.A.

1911. The Name Glossina. Nautilus, 25:96.

1929. The Brachiopod Called Mimulus. Nautilus, 42:105.

Cocks, L.R.M

1967. Llandovery Stropheodontids from the Welsh Borderland. Palaeontology, 10(2):245-265, 3 figures, 3 plates.

1968. Some Strophomenacean Brachiopods from the British Lower Silurian. British Museum (Natural History), Bulletin (Geology), 15(6):285-324, 14 plates.

1979a. New Acrotretacean Brachiopods from the Palaeozoic of Britain and Austria. Palaeontology, 22(1): 93-100, 2 plates.

1979b. A Silicified Brachiopod Fauna from the Silurian of Iran. British Museum (Natural History), Bulletin (Geology), 32(1):25-42, 4 plates.

Comte, $\mathrm{P}$.

1938. Brachiopodes Devoniens des Gisements de Ferrones (Asturies) et de Sabero (Leon). Annales of Paleontologie, 27:39-88.

Conrad, T. A

1839. Second Annual Report on the Paleontological De- 
partment of the Survey. New York Geological Survey, Annual Report, 3:57-66.

Cooper, G. A.

1930. The Brachiopod Genus Pionodema and Its Homoeomorphs. Journal of Paleontology, 4(4):369-382, plates 35-37.

1935. Oligorhynchia, a New Ordovician (Chazy) Brachiopod. American Journal of Science, series 5, 29(169): 48-53, 1 plate.

1936. New Cambrian Brachiopods from Alaska. Journal of Paleontology, 10(3):210-214, plate 26.

1942. New Genera of North American Brachiopods. Washington Academy of Sciences, Journal, 32(8):228235.

1948. A New Genus of Brachiopoda from the Longview Limestone of Virginia. Harvard University, Museum of Comparative Zoology, Bulletin, 100(6):473-474, 1 plate.

1951. New Brachiopods from the Lower Cambrian of Virginia. Washington Academy of Sciences, Journal, 41(1):4-8, 1 plate.

1952a. Unusual Specimens of the Brachiopod Family Isogrammidae. Journal of Paleonlology, 26(1):113119 , plates 21-23.

1952b. New and Unusual Species of Brachiopods from the Arbuckle Group in Oklahoma. Smithsonian Miscellaneous Collections, 117(14):1-35, plates 1-4.

1953. Permian Fauna at El Antimonio, Western Sonora, Mexico. Smithsonian Miscellaneous Collections, 119(2): 21-77, plates 4-23.

1954. Unusual Devonian Brachiopods. Journal of Paleontology, 28(3):325-332, 5 figures, plates 36-37.

1955a. New Genera of Middle Paleozoic Brachiopods. Journal of Paleontology, 29(1):45-63, plates 11-14.

1955b. New Brachiopods from Cuba. Journal of Paleontology, 29(1):64-70, plate 15 .

1955c. New Cretaceous Brachiopoda from Arizona. Smithsonian Miscellaneous Collections, 131(4):1-18, 4 plates.

1956a. New Pennsylvanian Brachiopods. Journal of Paleontology, 30(3):521-530, 1 figure, plate 61 .

1956b. Pustulatia, New Name for the Devonian Brachiopod Pustulina, Preoccupied. Journal of Paleontology, 30(3): 769 .

1956c. Chazyan and Related Brachiopods. Smithsonian Miscellaneous Collections, 127(1):1-1024; 127(2): 1025-1245, 269 plates.

1957a. Tertiary and Pleistocene Brachiopods of Okinawa, Ryukyu Islands. U.S.G.S. Professional Paper, 314-A: $1-20,5$ plates.

1957b. Permian Brachiopods from Central Oregon. Smithsonian Miscellaneous Collections, 134(12):1-79, 12 plates.

1959. Genera of Tertiary and Recent Rhynchonelloid
Brachiopods. Smithsonian Miscellaneous Collections, 139(5): 1-90, 22 plates.

1960. Correction of Brachiopod Names. Journal of Paleontology, 34(3):601.

1971. Eocene Brachiopods from Eua, Tonga. U.S.G.S. Professional Paper, 640-F:1-8, 1 plate.

1972a. Correction of a Brachiopod Name. Journal of Paleontology, 46(3):450.

1972b. Homeomorphy in Recent Deep-Sea Brachiopods. Smithsonian Contributions to Paleobiology, 11:1-16, plates 19-25.

1973a. New Brachiopoda from the Indian Ocean. Smithsonian Contributions to Paleobiology, 16:1-43, 8 plates.

1973b. Vema's Brachipoda (Recent). Smithsonian Contributions to Paleobiology, 17:1-51, 5 figures, 9 plates.

1973c. Fossil and Recent Cancellothyridacea (Brachiopoda). Science Reports of Tohoku University (Geology), special volume 6:371-390, plates 42-46.

1976. Lower Cambrian Brachiopods from the Rift Valley (Israel and Jordan). Journal of Paleontology, 50(2):269-289, 3 plates.

1977. Brachiopods from the Caribbean Sea and Adjacent Waters. Studies in Tropical Oceanography, 14:1212, 35 plates.

1978. Tertiary and Quaternary Brachiopods from the Southwest Pacific. Smithsonian Contributions to Paleobiology, 38:1-23, 4 figures, 2 plates.

1979. Tertiary and Cretaceous Brachiopods from Cuba and the Caribbean. Smithsonian Contributions to $\mathrm{Pa}$ leobiology, 37:1-45, 7 plates.

Cooper, G. A., and R. E. Grant

1962. Torynechus, New Name for Permian Brachiopod Uncinuloides King. Journal of Paleontology, 36(5): 1128-1129.

1969. New Permian Brachiopods from West Texas. Smithsonian Contributions to Paleobiology, 1:1-20, 5 plates.

1970. New Name for Brachiopod Homonym and Citation of Types. Journal of Paleontology, 44(3):579.

1974. Permian Brachiopods of West Texas, II. Smithsonian Contributions to Paleobiology, 15:233-793, plates 24-191.

1975. Permian Brachiopods of West Texas, III. Smithsonian Contributions to Paleobiology, 19:795-1921, plates 192-502.

1976a. Permian Brachiopods of West Texas, IV. Smithsonian Contributions to Paleobiology, 21:1923-2607, plates 503-662.

1976b. Permian Brachiopods of West Texas, V. Smithsonian Contributions to Paleobiology, 24:2609-3159, plates 663-780.

Cooper, G. A., and C. H. Kindle

1936. New Brachiopods and Trilobites from the Upper 
Ordovician of Percé, Quebec. Journal of Paleontology, 10(5):348-372, plates 51-53.

Cooper, G. A., and H. M. Muir-Wood

1951. Brachiopod Homonyms. Washington Academy of Sciences, Journal, 41 (6):195-196.

1967. New Names for Brachiopod Homonyms. Journal of Paleontology, 41(3):808.

Cooper, G. A., and F. G. Stehli

1955. New Genera of Permian Brachiopods from West Texas. Journal of Paleontology, 29(3):469-474, plates 52-54.

Cooper, G. A., and L. Whitcomb

1933. Salonia, a New Ordovician Brachiopod Genus. Washington Academy of Sciences, Journal, 23(11):496503, 23 figures.

Cooper, G. A., and J. S. Williams

1935. Tully Formation of New York. Geological Society of America, Bulletin, 46(5):781-868, 3 plates.

Cope, F. W.

1934. Airtonia, a New Brachiopod Genus from the Lower Carboniferous of Yorkshire. Annals and Magazine of Natural History, series 10, 14:273-289, 1 plate.

Copper, $\mathrm{P}$

1964. European Mid-Devonian Correlations. Nature, 204(4956):363-364.

1966a. European Mid-Devonian Correlations. Nature, 209(5027):982-984.

1966b. The Atrypa zonata Brachiopod Group in the Eifel, Germany. Senckenbergiana Lethaea, 47(1):1-55, 7 plates.

1967a. Frasnian Atrypidae (Bergisches Land, Germany). Palaeontographica, series A, 126(3-6):116-140.

1967b. Atrypa (Planatrypa) a New Devonian Brachiopod Species Group. Neues Jahrbuch für Geologie und Paläontologie, Abhandlungen, 128(3):229-243.

1973. New Siluro-Devonian Atrypoid Brachiopods. Journal of Paleontology, 47(3):484-500, 3 figures, 3 plates.

1977. Zygospira and Some Related Ordovician and Silurian Atrypoid Brachiopods. Palaeontology, 20(2): 295-335, plates 37-40.

1978. Devonian Atrypoids from Western and Northern Canada. Geological Association of Canada, Special Paper, 18 (Percival Sydney Warren Memorial Volume):289-331.

Cossmann, $\mathrm{M}$.

1898. Errata et rectifications. Revue Critique de Paléozoologie, 1898(2):76-77.

1909. Organe trimestriel. Revue Critique de Paléozoologie, $1909(4): 215$.

Costa, O. G.

1852. Class V, Brachiopodi. In Fauna del Regno di Napoli, 10:1-60, 9 plates. Naples.

Cox, M. M., and F. A. Middlemiss

1978. Terebratulacea from the Cretaceous Shenley
Limestone. Palaeontology, 21(2):411-441, plates 4042.

Cozzens, I.

1846. Description of Three New Fossils from the Falls of Ohio. Annals of the Lyceum of Natural History, New York, 4:157-159.

Crickmay, C. H.

1933. Attempt to Zone the North American Jurassic on the Basis of Its Brachiopods. Geological Society of America, Bulletin, 44(5):871-893, plates 19-22.

1950. Some Devonian Spiriferidae from Alberta. Journal of Paleontology, 24(2):219-225, plates 36-37.

1952. Nomenclature of Certain Devonian Brachiopods. 2 pages. Calgary: published by the author.

1953a. New Spiriferidae from the Devonian of Western Canada. 11 pages, 6 plates. Calgary: published by the author.

1953b. Warrenella, a New Genus of Devonian Brachiopod. Journal of Paleontology, 27(4):596-600, 25 figures.

1954. Paleontological Correlation of Elk Point and Equivalents. In Western Canada Sedimentary Basin: A Symposium of the Alberta Society of Petroleum Geologists (Ralph Leslie Rutherford Memorial Volume), pages 143-158. Tulsa: American Association of Petroleum Geologists.

1963. Significant New Devonian Brachiopods from Western Canada. 63 pages, 16 plates. Calgary: Evelyn de Mille Books.

1967. The Method of Indivisible Aggregates in Studies of the Devonian. 22 pages. Calgary: Evelyn de Mille Books.

1968. Discoveries in the Devonian of Westem Canada. 12 pages, 17 plates. Calgary: published by the author.

Cuvier, G.L.C.F.D.

[1798.] Tableau elementaire de l'histoire naturelle des animaux. xvi +710 pages, 14 plates.

Czarniecki, S.

1969. Sedimentary Environment and Stratigraphical Position of the Treskelodden Beds (Vestspitsbergen). Prace Muzeum Ziemi, 16:201-336, 12 figures, 34 plates.

Dagys, A. S.

1959a. [New Genera of Triassic Terebratulida.] Lietuvos TSR Mokslu, Akademija Geologijos ir Geografijos Institutas, Moksliniai Pranesimai, SSR, Trudy, series B, 9: 23-41, 1 plate. [In Russian.]

1959b. [New Triassic Terebratellacea.] Akademiia Nauk Litovskoi SSR, Trudy, series B, 3(19):97-102, 2 figures. [In Russian.]

1961a. [Two New Genera of Triassic Rhynchonellids.] Paleontologicheskii Zhurnal, 1961(4):93-99. [In Russian.]

1961b. [New Genus of Triassic Spiriferid, Triadispira, gen. 
nov.] Akademiia Nauk SSSR, Doklady, 141(2):457460. [In Russian.]

1962a. [New Late Triassic Spiriferinacea from the Northwestern Caucasus.] Paleontologicheskii Zhurnal, 1962(3):47-56, 1 plate. [In Russian.]

1962b. [Some New and Little Known Upper Triassic Athyrids.] Lietuvos TSR Geologijos ir Geografijos Institutas, Mokslmiai Pranesimai, SSR, Trudy, 14(1):5978, 4 plates. [In Russian.]

1963. Verkhnetriasouye Brakhiopody Yuga S.S.S.R. 248 pages, 31 plates. Moscow: Akademiia Nauk SSSR, Sibirskoe Otdelenie.

1965. Triasovye Brakhiopody Sibrr. 186 pages, 66 figures, 26 plates. Moscow: Akademiia Nauk SSSR, Sibirskoe Otdelenie, Institut Geologii i Geofiziki.

1968. Yurskie i Rannemelovye Brakhiopody Severa Sibiri. Akademiia Nauk SSSR, Sibirskoe Otdelenie, Institut Geologii i Geofizlki, Trudy, 41:1-167, 26 plates.

1972a. [Morphology and Systematics of Mesozoic Retzioid Brachiopods.] In Morfologicheskie i Filogeneticheskie Voprosy Paleontologii. Akademiia Nauk SSSR, Sibirskoe Otdelente, Institut Geologii i Geofiziki, Trudy, 112:94-105. [In Russian.]

1972b. [Ultrastructure of Thecospirid Shells and Their Position in Brachiopod Systematics.] Paleonlologicheskiz Zhurnal, 1972(3):87-98, 5 figures, 2 plates. [In Russian.]

1972c. [The Occurrence of a Metachoresa in Triassic Spiriferina.] In Problemy Paleozoogeografii Mezozoia Sibiri. Akademuia Nauk SSSR, Siburskoe Otdelenee, Instılut Geologii i Geofiziki, Trudy, 111:34-44, 4 figures. [In Russian.]

1972d. [The Postembryonic Development of the Brachidia in Late Paleozoic and Early Mesozoic Terebratulida.] In Morfologicheskie i Filogeneticheskie Voprosy Paleontologii. Akademiia Nauk SSSR, Sibirskoe Otdelenie, Institut Geologii i Geofiziki, Trudy, 112: 22-58, 27 figures. [In Russian.]

1974. Triasovye Brakhiopody (Morfologia, Sistema, Filogenii, Stratigraficheskoe Znachenie i Biogeografiia). Akademiia Nauk SSSR, Sibirskoe Otdelenie, Institut Geologii Geofiziki, Trudy, 214:1-386, 49 plates.

1977a. [New Triassic Brachiopods from the North-East of the USSR.] In Stratigrafiia i Fauna Boreal'nogo Triasa. Akademiia Nauk SSSR, Sibirskoe Otdelenie, Instilut Geologii i Geofiziki, Trudy, 344:5-23. |In Russian.]

1977b. [A New Name for a Triassic Rhynchonellid Genus.] Paleontologicheskii Zhurnal, 1977(3):139. [In Russian.]

Dall, W. H.

1870. A Revision of the Terebratulidae and Lingulidae, with Remarks and Descriptions of some Recent
Forms. American Journal of Conchology, 6:88-168, plates 6-8.

1871a. A New Genus of Brachiopods. American Naturalist, 5:55.

1871b. Report on the Brachiopoda Obtained by the United States Coast Survey Expedition in Charge of L.F. de Pourtales, with a Revision of the Craniidae and Discinidae. Harvard University, Museum of Comparative Zoology, Bulletin, 3(1):1-45, 2 plates.

1877. Index to the Names Which Have Been Applied to the Subdivisions of the Class Brachiopoda. United States National Museum, Bulletin, 8:1-88.

1895. Report on Mollusca and Brachiopoda Dredged in Deep Water, Chiefly near the Hawaiian Islands, with Illustrations of Hitherto Unfigured Species from Northwest America. United States National Museum, Proceedings, 17:675-733, plates 23-32.

1900. Some Names Which Must be Discarded. Nautilus, 14:44-45.

1903. Contributions to the Tertiary Fauna of Florida. Wagner Free Institute of Science of Philadelphia, Bulletin, 3(6):1219-1620, plates 48-60.

1908. The Mollusca and the Brachiopoda. Harvard University, Museum of Comparative Zoology, Bulletin, 43: 205-487, 22 plates.

1919. New Shells from the North-west Coast. Biological Society of Washington, Proceedings, 32:249-251.

1920. Annotated List of the Recent Brachiopoda in the Collection of the United States National Museum, with Descriptions of 33 New Forms. Uniled States National Museum, Proceedings, 57(2314):261-377.

Dalman, J. W.

1828. Uppställning och Beskrifning af de i sverige funne Terebratuliter. Kongl. Sivenska Vetenskapsakademien, Handlingar, 1827:85-155, plates 1-6.

Davidson, $T$.

1850a. On the Genus Waltonza. Annals and Magazine of Natural Hislory, series 2, 5:474-476.

1850b. Sur quelques Brachiopodes nouveaux ou peu Connus. Société Géologique de France, Bulletin, series 2, 8: $62-74$.

1852a. A Monograph of the British Fossil Brachiopoda [volume 1, part 2]. Palaeontolographical Society [Monographs], 1852-1855:1-117, plates 1-12.

1852b. Notes and Descriptions of a Few Brachiopoda; Including a Monograph of the French Liasic Spirifers. Annals and Magazine of Nalural History, series 2, 9:249-27, plates 13-15.

1852c. Sketch of a Classification of Recent Brachiopoda Based Upon Internal Organization. Annals and Magazine of Natural History, series 2, 9:361-377.

1853. A Monograph of the British Fossil Brachiopoda [volume 1, Introduction]. Palaeontographical Society [Monographs], 1853:1-136, plates 1-9. 
1858. A Monograph of the British Fossil Brachiopoda [volume 2, part 5]. Palaeontographical Society [Monographs], 1858-1863:1-280, plates 1-55.

1874. On the Tertiary Brachiopoda of Belgium. Geological Magazine, 1:150-159, plates 7-8.

1881a. Description of New Upper Silurian Brachiopoda from Shropshire. Geological Magazine, 8:145-156, plate 5 .

1881b. On Genera and Species of Spiral-Bearing Brachiopoda, from Specimens Developed by the Rev. Norman Glass. Geological Magazine, 8:1-13, 15 figures.

1881c. On the Genera Merista, Suess, 1851, and Dayia, Davidson, 1881. Geological Magazine, 8:289-293.

1882. A Monograph of the British Fossil Brachiopoda [volume 5, part 1]. Palaeontographica Society [Monographs], 1882:1-134, plates 1-7.

Dear, J. F.

1969. A New Queensland Permian Linoproductid with Arctic Affinities. In K.S.W. Campbell, editor, Stratigraphy and Paleontology: Essays in Honour of Dorothy Hill, pages 299-306, 1 plate. Canberra: Australian National Press.

de Férussac, Andre. See Férussac, Andre (Baron de)

Deslongchamps, E. Eudes-

1855. Notice sur un genre nouveau de brachiopodes. Annuaire de l'Institut des Provinces, 1855:529-553, 1 plate.

1884. Notes sur les modification a apporter a la classification des Terebratulidae. Bulletin de la Société Linnéenne de Normandie, 3rd series, 8:161-388, plates 13-28.

Diener, C.

1908. Ladinic, Carnic and Noric Faunae of Spiti. Palaeontologia Indica, series 15, 5(3):1-157, plates 124.

Dieni, I., F. A. Middlemiss, and E. F. Owen

1973. The Lower Cretaceous Brachiopods of East-Central Sardinia. Bollettino della Società Paleontologica Italiana, 12(2):166-216, 18 figures, 7 plates.

Doello-Jurado, M.

1922. Nota preliminar sobre braquiópodos fósiles de la Argentina referidos al genero Bouchardia y sobre la posición del horizonte Salamanquense. Anales de la Sociedad Cientifica Argentina, 94:197-207.

Donakova, L. M.

1974. [On the Genus Connectoproductus gen. nov. and the Phylogeny of Early Carboniferous Linoproductids (Brachiopoda).] In Biostratigraficheskii Sbornik. Vsesoiuznyi Nauchno-Issledovatelskii Geologicheskii Institut, Trudy, new series, 182(5):172-177. [In Russian.]

Douvillé, $\mathrm{H}$. $\mathrm{H}$.

1879. Sur quelques genres de brachiopodes Terebratu- lidae et Waldheimiidae. Société Géologique de France, Bulletin, series 3, 7:251-277, 19 figures.

Dovgal, V. N.

1953. [Leiorhynchoides - a New Subgenus of Brachiopod from the Middle Devonian of Gorny Altai.] Akademiia Nauk SSSR, Zapadno-Sibirski Filial, Gorno-Geologicheskii Institul, Trudy, 13:139-141. [In Russian.]

Dresser, $\mathrm{H}$.

1954. Notes on Some Brachiopods from the Itaituba Formation (Pennsylvanian) of the Tapajos River, Brazil. Bulletin of American Paleontology, 35:15-84, 8 plates.

Drot, J.

1963. Un Nouveau Genre de Rhynchonelloidea dans le Ludlow du Maroc présaharien. Maroc Service Géologique, Notes, 23(172):111-116.

1966. Nouvelles Observations sur le brachiopodes devoniens du Maroc présaharien. Société Géologique de France, Bulletin, 8(6):877-883.

Drot, J., and P. Westbroek

1966. Iberirhynchia santaluciensis, nouveau Rhynchonellacea du dévonien de Leon (Espagne). Letdsche Geologische Mededeelingen, 38:165-169.

Dubar, G.

1942. Études paléontologiques sur le Lias du Maroc: Brachiopodes térébratules et zeilléries multiplissées. Maroc Service Géologique du Division des Mines el de la Géologie, Notes et Memoires, 57:1-103, plates 110.

Dunbar, C. O.

1917. Rensselaerina, a New Genus of Lower Devonian Brachiopods. American Journal of Science, series 4, 43: 466-470, plate 2.

1955. Permian Brachiopod Faunas of Central East Greenland. Meddelelser om Grønland, 1 10(3):1-169, 32 plates.

Dunbar, C. O., and G. E. Condra

1932. Brachiopoda of the Pennsylvanian System in Nebraska. Nebraska Geological Survey Bulletin, series 2, 5:1-377, 25 figures, 44 plates.

Dürkoop, A.

1970. Brachiopoden aus dem Silur, Devon und Karbon in Afghanistan. Palaeontographica, series A, 134: 153-225, 59 figures, 6 plates.

Easton, W. H.

1962. Carboniferous Formations and Faunas of Central Montana. U.S.G.S. Professional Paper, 348:1-126, 13 plates.

Easton, W. H., J. E. Sanders, J. B. Knight, and A. K. Miller 1958. Mississippian Faunas in Northwestern Sonora, Mexico: Brachiopoda and Pelecypoda. Smithsonian Miscellaneous Collections, 119(3):41-72, plates 3-7.

Eichwald, E. von

1829. Zoologia specialis, quam expositis animalibus tum vivis, 
tum fossilibus polıssium Rossiae in universum el Poloniae in specie... . Volume 1, 314 pages, 5 plates.

Einor, O. L.

1939. [Lower Permian Brachiopods of Taimyr.] Arkicheskogo Nauchno-Issledovalel'skogo Instilula, Trudy, 135:1-150, 15 plates. [In Russian.]

Elliott, G. F.

1947. The Development of a British Aptian Brachiopod. Geologists' Association, Proceedings, 58(2):144-159, 2 plates.

1948. Palingenesis in Thecidea (Brachiopoda). Annals and Magazine of Nalural History, series 12, 1:1-30, plates 1-2.

1952. The Internal Structure of Some Western Australian Cretaceous Brachiopods. Royal Society of Western Australia, Journal, 36:1-21, 2 plates.

1953. The Classification of the Thecidean Brachiopods. Annals and Magazine of Nalural History, series 12, 6 : 693-701, plate 18.

1954. New Brachiopoda from the Eocene of England, France and Africa. Annals and Magazine of Nalural Hislory, series 12, 7:721-728, plate 15 .

1956. On Tertiary Transarctic Brachiopod Migration. Annals and Magazine of Nalural History, series 2, 9: 280-286, 1 figure.

1959. Six New Genera of Mesozoic Brachiopoda. Geological Magazine, 96:146-148.

1960. A New Mesozoic Terebratellid Brachiopod. Geologists' Associalıon, Proceedings, 71:25-30, plate 2.

Emmrich, H.

1855. Notiz über den Alpenkalk der Lienzer Gegend. Jahrbuch Geologischen Reichsanslall, 6:444-450.

Farag, I.A.M., and W. Gatinaud

1960. Un Nouveau Genre de Térébralulidés dans le Bathonien d'Égypte. Journal of Geology United Arab Republic, 4(1):77-79, 1 plate.

Fenton, C. L., and M. A. Fenton

1924. The Stratigraphy and Fauna of the Hackberry Stage of the Upper Devonian. Michigan University, Museum of Geology, Contributions, 1:1-260.

Ferguson, J.

1966. Variation in Two Species of the Carboniferous Brachiopod Pleuropugnoides. Yorkshire Geological Sociely, Proceedings, 35:353-374, 12 figures, 1 plate.

1971. Linoprolonia, a New Genus of Lower Carboniferous Productoid. Yorkshire Geological Society, Proceedings, 38(4):549-564.

Férussac, Andre (Baron de)

1822. Tableaux systémaliques des animaux mollusques. Pages i-xlvii.

Ficner, F, and V. Havlíček

1975. Two Thick-shelled Brachiopods from the Middle Devonian of Moravia. Vèsinik Ústredniho Ústavu Geologického, 50(6):361-363, 2 plates.
1978. Middle Devonian Brachiopods from Čelechovice, Moravia. Sbornik Geologických Véd [Journal of Geological Sciences], Paleonlologie, 21:49-106, 2 figures, 16 plates.

Fischer, P.

1887. Manuel de conchyliologie el de paléontologie conchyliologique, ou histoire nalurelle des mollusques vivants el fossiles. Part 11:1189-1334. Paris.

Fischer, P., and D. P. Oehlert

1890. Diagnoses de nouveaux brachiopodes. Journal de Conchyliologie, series 3, 30:70-74.

Fischer de Waldheim, G.

1809. Notice des fossiles du gouvernement de Moscou. 35 pages, 3 plates. Moscow.

1825. Notice sur la Choristite, programme d'invitation à la Soctélé Impériale des Naturalistes de Moscou. 12 pages, 1 plate. Moscow.

1829. Quelques fossiles du gouvernement de Moscou. Sociélé Impériale des Naluralisles de Moscou, Bulletin, $1(12): 375-376$

1830. Oryctographie du gouvernement de Moscou. 1st edition, pages ix + 26, plates 1-60. Moscow.

Foerste, A. F.

1909a. Preliminary Notes on Cincinnatian Fossils. Denison University, Scientific Laboratories, Bulletin, 14:209228.

1909b. Fossils from the Silurian Formations of Tennessee, Indiana, and Illinois. Denison University, Scientific Laboratories, Bulletin, 14:61-116, plates 1-4.

1912. Strophomena and Other Fossils from Cincinnatian and Mohawkian Horizons, Chiefly in Ohio, Indiana, and Kentucky. Denison University, Scientific Laboratories, Bulletin, 17:17-173, plates 1-8.

1914. Notes on the Lorraine Faunas of New York and the Province of Quebec. Denison University, Scientific Laboratories, Bulletin, 17:247-328, plates 1-5.

1920. The Kimmswick and Plattin Limestones of Northeastern Missouri. Denison University, Scientific Laboratories, Bulletin, 19:175-224, 3 plates.

1924. Upper Ordovician Faunas of Ontario and Quebec. Geological Survey of Canada, Memoir, 138:1-255, plates 10-15.

Ford, S. W.

1886. Note on the Recently Proposed Genus Billingsia. American Joumal of Science, series 3, 32:325.

Foster, M. W.

1974. Recent Antarctic and Subantarctic Brachiopods. Anlarclic Research Series, 21:1-189, 39 figures, 25 plates.

Frech, F.

1901. Die Dyas. In C. F. von Roemer, Lethaea geognostica, $1(2): 435-578$.

1911. Die Dyas. In F, von Richthofen, China, 5:103-202, plates 19-28. 
Fredericks, G.

1916. [Paleontological Notes on Some Upper Paleozoic Brachiopoda of Eurasia.] Comilé Géologique, Mémoires, 156:1-87, 5 plates. [In Russian.]

1918. [Concerning the Application of the Subdivision of the Apical Apparatus to Brachiopod Classification.] Russkoe Paleontologicheskoe Obshchestvo, 2(1917):85-91. [In Russian.]

1919 [1924a]. [On Upper Carboniferous Spiriferids from the Urals.] Geologicheskogo Komiteta, Izvestiia, 38(3): 295-324. [In Russian.]

1923 [1924b]. [Upper Paleozoic of the Ussuriland, I: Brachiopoda.] Materialy po Geologii i Poleznym Iskopaemym Dal'nego Vostoka, 28: 52 pages. [In Russian.]

1924 [1925]. [Upper Paleozoicum of the Ussuriland, II: Permian Brachiopoda of Cape Kalouzin.] Geologicheskii Komiteta Dal'nego Vostoka, Materialy po Geologii i Poleznym Iskopaemym Dal'nego Vostoka, 40:128, 4 plates.[In Russian.]

1926. [Table for Determination of the Genera of the Family Spiriferidae King.] Akademiia Nauk SSSR, Izvestiya, series 6, 20:393-423. [In Russian.]

1927 [1928]. [Contribution to the Classification of the Genus Productus.] Geologicheskogo Komiteta, Izvestiya (Leningrad), 46(7):773-792. [In Russian.]

1929. [The Fauna of the Kyn Limestone of the Urals.] Geologicheskogo Komiteta, Izvestiya (Leningrad), 48 (3):369-413, plates 20-21. [In Russian.]

1931. Faune paléozoique supérieure des monts Kharaoulakh. Akademïa Nauk SSSR, Izvestiya, series 7, 2: 199-228, 4 plates.

1933. [Palaeontological Notes, 4: On Some Upper Paleozoic Brachiopods of Eurasia.] Tsentralnogo Nauchno-Issledovatelskii Geologo-Razvedochnii Institut (TSNIGRI), Trudy (Leningrad), 2:24-33. [In Russian.]

Friren, A.

1876. Mélanges paléontologiques, article 1: Orthoidea, Straparolus, Ammonites Aulacoceras, and Tisoa siphonalis du Lias Moyen. Bulletin de la Société d'Histoire Naturelle de Metz, 14:1-22.

Fuchs, A.

1923. Über die Beziehungen des sauerländischen Faciesgebietes zur belgischen Nord- und Südfacies und ihre Bedeutung für das Alter des Verseschichten. Jahrbuch der Geologischen Preussische Landesanstalt, 42(2):839-859, 1 plate.

1929. Beitrag zur Kenntnis der Unteren Gedinnefauna. Jahrbuch der Preussischen Geologischen Landesanstalt, 50(1):194-201, 3 plates.

Gaetani, M.

1964. Rossirhynchus adamantinus gen. n., sp. n. from the Tournaisian of Central Elburz, Iran (Rhyncho- nellida). Rivista Italiana di Paleontologia e Stratigrafia, 70(4):637-648, 3 figures, 1 plate.

1966. Decurtella, nuovo genere triassico di Rhynchonellida. Rivista Italiana di Paleontologia e Stratigrafia, 72(2):343-356, 3 figures, 1 plate.

Gagel, C.

1890. Die Brachiopoden der Cambrischen und Silurischen Geschiebe im Diluvium der Provinzen Ostund Westpreussen. Beiträge zur Nalurkunde Preussens hg. von Physikalisch-Oekonomischen Gesellshaft zu Königsberg, 6:1-81, 4 plates.

Galitzkaja, A. I.

1977. Ranne-i Srednekamennougol'nye Produktidy Severnoi Kirgizii. 298 pages, 64 plates. Frunze: Akademiia Nauk Kirgizkoi SSR, Ordena Trudovogo Krasnogo Znameni, Institut Geologii.

Ganelin, V. G.

1977. In T. G. Sarytcheva, editor, [Late Paleozoic Productids of Siberia and the Arctic.] Akademiia Nauk SSSR, Paleontologicheskii Instilut, Trudy, 161:1-219, 27 plates. [In Russian.]

Garcia-Alcalde, J. L.

1972a. Braquiopodos devonicos de la cordillera cantabrica, 2: Genero Xana Garcia-Alcalde, n. gen. (Terebratulida, Stringocephalacea). Breviora Geologica Astúrica, 16(1):4-12, 5 figures, 1 plate.

1972b. Braquiopodos devonicos de la cordillera cantabrica, 3: Fibulistrophia n.gen. (Strophomenida, Strophodontacea). Breviora Geologica Astúrica, 16(3): 42-48, 2 figures, 1 plate.

1972c. Braquiopodos devonicos de la cordillera cantabrica, 4: Arbizustrophia n.gen. (Strophomenida, Strophodontacea). Breviora Geologica Astürica, 16(4): 56-63, 4 figures, 1 plate.

Garcia-Alcalde, J. L., and M. L. Martínez-Chacón

1978. Cementacion en braquipodos articulados. Revista de la Faculiad de Ciencias, Universidad de Oviedo, 17 19:251-258, 1 plate.

Garcia-Alcalde, J. L., and P. R. Racheboeuf

1978. Nouveaux brachiopodes Chonetacea du devonien de la cordillere cantabrique (nord-ouest de l'Espagne). Geobios (Paleotologie, Stratigraphie, $\mathrm{Pa}$ leoecologie), 11(6):835-865.

Gatinaud, G.

1949. Contributions à l'étude des brachiopodes Spiriferidae, 1: Exposé d'une nouvelle méthode d'etude de la morphologie externe des Spiriferidae à sinus plissé. Muséum National d'Histoire Naturelle, Bulletin, series 2, 21(1):153-159; $21(2): 300-307 ; 21(3): 408$ $413 ; 21(4): 487-492$.

Gauri, K. L.

1965. Uralian Stratigraphy, Trilobites and Brachiopods of the Western Carnic Alps (Austria). Jahrbuch der 
Geologischen Bundesanslall Sonderband, 11:1-94, 26 figures, 17 plates.

Geinitz, H. B.

1847. Orthothrix Geinitz. Société Impériale des Naturalistes de Moscou, Bulletin, 20(2):84-86.

Gemmellaro, G. G.

1871. Studi paleontologici sulla fauna del calcare a Terebratula janitor del nord di Sicilia. Giornale di Sczenze Nalurali ed Economiche di Palermo, 7(1):73-108, plates 1-5.

1891. Sopra un nuovo genere di brachiopodi proveniente dei calcari con Fusulina della Provincia di Palermo. Societa di Scienze Nalurali ed Economische di Palermo, Bollellini, 4:22-23.

1899. La fauna dei calcari con Fusulina della valle del Fiume Sosio nella Provincia di Palermo. Giornale di Sczenze Naturali ed Economiche di Palermo, 22:95214, plates 25-36.

George, T. N.

1931. Ambocoelia Hall and Certain Similar British Spiriferidae. Geological Society of London, Quarterly Journal, 87(1):30-61, plates 3-5.

1932. The British Carboniferous Reticulate Spiriferidae. Geological Sociely of London, Quarterly Journal, 88(4): 516-575, 14 figures, plates 31-35.

Gill, E. D.

1950. Palaeontology and Palaeoecology of the Eldon Group. Royal Society of Tasmania, Paper, 1949:231258, I plate.

1951. Two New Brachiopod Genera from Devonian Rocks in Victoria. National Museum of Victoria, Memoir, 17:187-205, 1 plate.

1969. Notanopliidae, a New Family of Paleozoic Brachiopoda from Australia. Journal of Paleontology, 43(5): 1222-1231, 2 plates.

Girty, G. H.

1898. Description of a Fauna Found in the Devonian Black Shale of Eastern Kentucky. American Journal of Science, series 4, 6:384-395, 6 figures.

1904. New Molluscan Genera from the Carboniferous. Uniled States National Museum, Proceedings, 27:721736, plates $16-18$.

1908 [1909]. The Guadalupian Fauna. U.S. Geological Survey, Professional Paper, 58:1-651, 31 plates.

1910. New Genera and Species of Carboniferous Fossils from the Fayetteville Shale of Arkansas. New York Academy of Science, Annals, 20(3)2:189-238.

1911. The Fauna of the Moorefield Shale of Arkansas. U.S. Geological Survey, Bulletin, 439:1-148, 15 plates.

1934. New Carboniferous Invertebrates, IV. Washington Academy of Sciences, Journal, 24(6):249-266.

1938. Descriptions of a New Genus and a New Species of Carboniferous Brachiopods. Washington Academy of Sciences, Journal, 28(6):278-284.
1939. Setigerites Nom. Nov. a Subgenus of Productus. Washington Academy of Sciences, Journal, 29(4): 141.

Glenister, B. F.

1956. Devonian and Carboniferous Spiriferids from the North-West Basin, Western Australia. Royal Society of Western Australia, Journal, 39(2)6:46-71, plates 18.

Godefroid, J.

1972. Pseudosieberella (Pentameridae), nouveau genre couvinien, et genres apparentes. Institut Royal des Sciences Naturelles de Belgique, Bulletins, 48(5):1-41, 7 figures, 5 plates.

1974. Les Gypidulinae des niveaux recifaux du Frasnien de Frasnes et de Boussu-en-Fague. Inslitut Royal des Sciences Naturelles de Belgique, Bulletins, 50(9):1-65, 7 plates.

Goldring, R

1957. The Last Toothed Productellinae in Europe (Brachiopoda, Upper Devonian). Paläontologische Zeitschrifi, 31(3-4):207-228, 3 figures, 1 plate.

Gordon, M., Jr.

1966. New Spinose Early Meramec (Upper Mississippian) Productoid Brachiopods. Journal of Paleontology, 40(3):573-584.

1971. Carlinia, a Late Mississippian Genus of Productidae from the Western United States. In J. T. Dutro, Jr., editor, Paleozoic Perspectives: A Paleontological Tribute to G. Arthur Cooper. Smithsonian Contributions to Paleobiology, 3:257-265.

Gorjansky, V. Yu.

1969. Bezzamkovye Brakhiopody Kembriiskikh i Ordovikskikh Otlozhenii Severo-Zapada Russkoi Platformy. Malerialy po Geologii \& Polezhym Iskolaemym Severo-Zapada RSFSR, 6:1-171, 21 plates.

1977. [New Early Cambrian Obolellids from Eastern Siberia.] In Novye Vidy Drevnikh Rastenii i Bespozvonochnykh SSSR, 4:99-102. Moscow: Akademiia Nauk SSSR, Paleontologicheskii Institut. [In Russian.]

Gortani, M., and G. Merla

1934. Fossili del Paleozoico. In G. Dainelli, editor, Spedizione italiana de Filippi nell'Himalaia, Caracorum, $\ell$ Turchestan cinese (1913-19/4), series 2, 5:323 pages, 27 plates.

Grabau, A. W.

1923. Stratigraphy of China, Part I: Palaeozoic and Older. 528 pages, 6 plates. Peking: China Geological Survey.

193la. Devonian Brachiopoda of China, I: Devonian Brachiopoda from Yunnan and other Districts in South China. China Geological Survey, Palaeontologia Sinica, series B, 3(3): 1-545, 67 figures, 54 plates.

193 lb. The Permian of Mongolia. In Natural History of Central Asia, 4:1-665, 35 plates. New York: American Museum of Natural History. 
1931c. The Brachiopoda, Part 2. In Studies for Students, I: Palaeontology. Science Quarterly, National University of Peking, 2(3):397-422, 21 figures.

1932. Studies of Brachiopoda, III. In Studies for Students. Science Quarterly, National University of Peking, 3(2):75-1 12 .

1934. Early Permian Fossils of China, Part I: Early Permian Brachiopods, Pelecypods, and Gastropods of Kueichow. China Geological Survey, Palaeontologia Sinica, series B, 8(3):1-168, 11 plates.

1936. Early Permian Fossils of China, Part II: Fauna of the Maping Limestone of Kwangsi and Kweichow. China Geological Survey, Palaeontologia Sinaca, series B, 8(4): 1-441, plates 1-31.

Grabau, A. W., and Y. T. Chao

1927. Brachiopod Fauna of the Chihsia Limestone. Geological Society of China, Bulletin, 6(2):83-120.

Grabau, A. W., and W. H. Sherzer

1910. The Monroe Formation of Southern Michigan and Adjoining Regions. Michigan Geological and Biological Survey, Geological Series, series 1, 2: 248 pages, 32 plates.

Graham, D. K.

1970. Scottish Carboniferous Lingulacea. Geological Survey of Great Britain, Bulletin, 31:139-184, 10 figures, 7 plates.

Grant, R. E.

1965. The Brachiopod Superfamily Stenoscismatacea. Smithsonian Miscellaneous Collections, 148(2):1-192, 24 plates.

1971. Taxonomy and Autecology of Two Arctic Permian Rhynchonellid Brachiopods. In J. T. Dutro, Jr., editor, Paleozoic Perspectives: A Paleontological Tribute to G. Arthur Cooper. Smithsonian Contributions to Paleobiology, 3:313-335, 9 figures, 3 plates.

1972. The Lophophore and Feeding Mechanism of the Productidina (Brachiopoda). Journal of Paleontology, 46(2):213-248, 9 plates.

1976. Permian Brachiopods from Southern Thailand. Journal of Paleontology, 50(3)supplement:1-269, 71 plates. [Paleontological Society Memoir, 9]

Gratsianova, R. 'T.

1967. Brakhiopody i Stratigrafiia Nizhnego Devona Gornogo Altaia. 177 pages, 14 plates. Moscow: Akademiia Nauk SSSR, Sibirskoe Otdelenie, Institut Geologii i Geofiziki.

1974. ["Schuchertellas" of the Early and Middle Devonian in the South of Western Siberia.] In Sreda 2 Zhiznv Geologicheskom Proshlom (Paleoekologicheskie Problemy), pages 77-87. Novosibirsk. [In Russian.]

1975. Brakhiopody Rannego i Srednego Devona AltaeSaianskoi Oblasti, Strophomenidy. Akademiia Nauk
SSSR, Sibirskoe Otdelenze, Instutut Geologı i Geofizikı, Trudy, 248: 106 pages, 20 plates.

Greger, D. K.

1920. Notes on Certain Brachiopod Genera. Nautzlus, 34: $69-70$.

Gregorio, A. de

1886. Iconografia della fauna dell'Orizzonte Alpiniano. Annales de Géologie el de Paléontologie, 1:22-23.

1930a. Sul Permiano di Sicilia (Fossili del calcare con Fusulina di Palazzo Adriano non descritti del Prof. G. Gemmellaro conservati nel mio privato gabinetto). Annales de Géologie et de Paléontologie (Palermo), 52:18-32, plates 4-11.

1930b. Fossili triassici della zona a Rhynchonellina bilobata Gemm. dei dintorni di Palermo. Annales de Géologie el de Paléontologie (Palermo), 55:1-18, 4 plates.

Gretchischnikova, I. A.

1965. [Ulbospirifer gen. nov. from the Tournaisian of Rudny Altai.] Paleontologicheskii Zhumal, 1965(4): 33-40, 6 figures, 2 plates. [In Russian].

Grigorjewa, A. D.

1977. [Smooth-Sulcate Spiriferids of the Upper Paleozoic of Siberia and the Artic]. In T. G. Sarytcheva, editor, Brakhiopody Verkhnego Paleozoia Sibiri i Arktiki. Akademiia Nauk SSSR, Paleontologicheskii Institut, Trudy, 162:37-54. [In Russian.]

Grigorjewa, A. D., and R. V. Solomina

1973. [A New Genus of Licharewiinae (Brachiopoda) from the Permian of Verkhoyan'ye and the North East USSR.] Paleontologicheskii Zhurnal, 1973(4): 35-39. [In Russian.]

Grubbs, D. M

1939. Fauna of the Niagaran Nodules of the Chicago Area. Journal of Paleontology, 13(6):543-560, 2 fig. ures, plates 61-62.

Grunt, T. A.

1976. ["Smooth" Athyrids in the Carboniferous Deposits of the Moscow Region.] Paleontologicheskii Zhurnal, 1976(3): 71-86, 6 plates. [In Russian.]

1977a. [Carboniferous Spiriferids of the Family Martiniidae of Siberia and the Arctic.] In T. G. Sarytcheva, editor, Brakhiopody Verkhnego Paleozoia Sibiri i Arktiki. Akademiia Nauk SSSR, Paleontologicheskii Institut, Trudy, 162:63-71. [In Russian.]

1977b. [Athyrids of the Upper Paleozoic of Siberia and the Arctic.] In T. G. Sarytcheva, editor, Brakhiopody Verkhnego Paleozoia Sibiri i Arktiki. Akademiia Nauk SSSR, Paleontologicheskii Institut, Trudy, 162:71-91. [In Russian.]

1977c. [New Martiniids and Athyridids (Brachiopoda) from the Lower Carboniferous of Western Mongolia.] In Bespozvonochnye Paleozoia Mongolii, pages 78-85. Moscow: Sovmestnaia Sovetsko- 
Mongol'skaia Paleontologicheskaia Ekspeditsiia. [In Russian.]

Grunt, T. A., and V. Yu. Dmitriev

1973. [Permian Brachiopoda of the Pamir.] Akademiia Nauk SSSR, Paleonlologicheskii Instilul, Trudy, 136:8209, 16 plates. [In Russian.]

Gürich, G.

1909. Devon. In Leilfossilien, 2:97-199, plates 29-52.

Haas, H. J.

1890. Kritische Beiträge zur Kenntniss der jurassischen Brachiopodenfauna des schweizerischen Juragebirges und seiner angrenzenden Landestheile, Part II. Abhandlungen der Schweizerischen Palaontologischen Gesellschaft, 17:35-102, plates 3-5.

Hall, J.

1850. On the Brachiopoda of the Silurian Period, Particularly the Leptaenidae. American Association for the Advancement of Science, Proceedings, 2:347-351.

1857. Descriptions of Palaeozoic Fossils. New York State Cabinet of Natural History, Annual Report, 10:41-186.

1859a. Containing Descriptions and Figures of the Organic Remains of the Lower Helderberg Group and the Oriskany Sandstone. In Palaeonlology of New York, 3(1):1-532. [Published in part $6(\mathrm{~Pa}-$ laeontology) of Natural Hislory of New York. 30 volumes. New York, 1842-1894.]

1859b. Observations on Genera of Brachiopoda. New York State Cabinet of Natural History Annual Report, 12:8110.

1860. Contributions to Palaeontology. New York State Cabinet of Natural History, Annual Report, 13:53-125

1861. Descriptions of New Species of Fossils from the Upper Helderberg, Hamilton, and Chemung Groups. New York Slate Cabinel of Natural History, Annual Report, 14:99-109.

1862. Contributions to Palaeontology Comprising Descriptions of New Species of Fossils from the Upper Helderberg, Hamilton and Chemung Groups. New York State Cabinet of Natural History, Annual Report, 15:29-197, 11 plates.

1863. Contributions to Palaeontology. Annual Report of the Regents of the University of the State of New York on the Condition of the State Cabinet of Natural History, 16: 3-226.

1867a. Descriptions and Figures of the Fossil Brachiopoda of the Upper Helderberg, Hamilton, Portage and Chemung Groups. In Palaeonlology of New York, 4(1):1-428. [Published in part 6 (Palaeontology) of Nalural History of New York. 30 volumes. New York, 1842-1894.]

1867b. Notice of Volume IV of the Paleontology of New York. New York State Cabinet of Natural History, Annual Report, 20:163.

1868. Note on the Genus Eichwaldia. In Annual Reporl of the Regents of the University of the State of New York on the Condition of the State Cabinet of Natural History, 20: 274-278.

1871a. Notes on Some New or Imperfectly Known Forms among the Brachiopoda. In Preliminary Notice, 23rd Annual Report on the State Cabinet of Natural History (Abstract), pages 1-5.

1871b. Descriptions of Some New Species of Fossils, from the Shales of the Hudson River Group, in the Vicinity of Cincinnati, Ohio. In Advance Copy of the 24 h Report on the State Cabinet of Natural History, pages 1-8, 4 plates.

1872. Notes on Some New or Imperfectly Known Forms among the Brachiopoda. In Preliminary Notice, 23rd Annual Report on the Stale Cabinet of Nalural History, pages 244-247, plate 13 .

1879. The Fauna of the Niagara Group. New York State Museum of Natural History, Report, 28:98-203, plates 3-34.

1883. Brachiopoda, Plates and Explanations. Annual Report of the New York State Geologist, 4(2), plate 36.

Hall, J., and J. M. Clarke

1890. [Extract.] In Palaeontology of New York, 8:120-160.

1892. An Introduction to the Study of the Genera of Palaeozoic Brachiopoda. New York Geological Survey, Palaeontology, 8(1):1-367, 20 plates.

1893. An Introduction to the Study of the Genera of Palaeozoic Brachiopoda. New York Geological Survey, $8(2): 1-317$, plates $21-84$.

1894. An Introduction to the Study of the Brachiopoda Intended as a Handbook for the Use of Students. 13th Annual Report N.Y. State Geologist for the Year 1893, 2:751-943, plates 23-54.

Hall, J., and R. P. Whitfield

1875. Descriptions of Invertebrate Fossils Mainly from the Silurian System: Fossils of the Hudson River Group. Ohio Geological Survey, Report, 2(2):67-110, 4 plates.

Hamada, T.

1968a. Swaicoelia, a New Ambocoeliid Genus (Brachiopoda) from North Thailand. In T. Kobayashi and R. Toriyama, editors, Geology and Palaeontology of Southeast Asia, 5:1-12. Tokyo: University of Tokyo Press.

1968b. Ambocoeliids from Red Beds in the Malayan Peninsula. In T. Kobayashi and R. Toriyama, editors, Geology and Palaeontology of Southeast Asia, 5: 13-25. Tokyo: University of Tokyo Press.

1969a. Late Paleozoic Brachiopods from Red Beds in the Malayan Peninsula. In $\mathrm{T}$. Kobayashi and $\mathbf{R}$. Toriyama, editors, Geology and Palaeonlology of Southeast Asia, 6:251-264, 2 plates. Tokyo: University of Tokyo Press.

1969b. Devonian Brachiopods from Kroh, Upper Perak 
in Malaysia (Malaya). In $\mathrm{T}$. Kobayashi and R. Toriyama, editors, Geology and Palaeontology of Southeasl Asia, 7:1-13. Tokyo: University of Tokyo Press.

1971. Early Devonian Brachiopods from the Lesser Khingan District of Northeast China. Paleontological Society of Japan, Special Paper, 15:1-98, 23 figures, 30 plates.

Harper, C. W., Jr.

1973. Brachiopods of the Arisaig Group (Silurian-Lower Devonian) of Nova Scotia. Geological Survey of Canada, Bulletin, 215:1-163, 3 figures, 28 plates.

Harper, C. W., Jr., and A. J. Boucot

1978a. The Stropheodontacea, Part I (Leptostrophiidae, Eostropheodontidae, and Strophonellidae). $\mathrm{Pa}$ laeontographica, series A, 161(1-3):55-118, 4 figures, 50 plates.

1978b. The Stropheodontacea, Part II (Douvillinidae, Telaeoshaleriidae, Amphistrophiidae, and Shaleriidae). Palaeontographica, series A, 161(4-6):119175, 17 plates.

1978c. The Stropheodontacea, Part III (Stropheodontidae, Pholidostrophiidae, and Lissostrophiidae). Palaeontographica, series A, 162(1-2):1-80, plates $36-50$.

Harper, C. W., Jr., A. J. Boucot, and V. G. Walmsley

1969. The Rhipidomellid Brachiopod Subfamilies Heterorthinae and Platyorthinae (New). Journal of $\mathrm{Pa}$. leontology, 43(1):74-92, 3 plates.

Harper, C. W., Jr., J. G. Johnson, and A. J. Boucot

1967. The Pholidostrophiinae (Brachiopoda; Ordovician, Silurian, Devonian). Senckenbergiana Lethaea, 48(5):403-461, 10 plates.

Harrington, H. J.

1955. The Permian Eurydesma Fauna of Eastern Argentina. Journal of Paleontology, 29(1):112-128, plates 23-26.

Hatai, K. M.

1936. Neogene Brachiopoda from Japan. Japanese Journal of Geology and Geography, 13:283-324, plates 34-35.

1938. The Tertiary and Recent Brachiopoda of Northeast Honsyu, Japan. Saito Ho-on Kai Museum Research Bulletin, 16:89-246, plates 16-20.

1940. The Cenozoic Brachiopoda from Japan. Tohoku Imperial University, Science Reports, series 2 (Geology), 20:1-413, 12 plates.

1946. Kurakithyris, Tertiary Brachiopod Genus from Central Honsyu, Japan. Proceedings of the Japan Academy, 22(4):98-101, 6 figures.

1948. New Tertiary Brachiopoda from Japan. Journal of Paleontology, 22(4):494-499, plate 78.

Havlícek, V.

1949a. [Orthoidea and Clitambonoidea of the Bohemian Tremadoc.] Státniho Geologického Ústavu Ceskoslov- enské Republiky, Sbornik, 16(1):93-144, 5 plates. [In Czech.]

1949b. The Orthacea of the Komarov Beds (Brachiopoda).] Státniho Geologického Ústavu Ceskoslovenské Republiky, Věstnik, 24:249-256. [In Czech.]

1950. [The Ordovician Brachiopoda from Bohemia.] Ústrédniho Úslavu Geologického, Rozpravy, 13:1-72, 13 plates. [In Czech.]

1951. A Paleontological Study of the Devonian of Čelechovice; Brachiopods (Pentameracea, Rhynchonellacea, Spiriferacea). Ústrédniho Ústavu Geologického, Sbornik, 18:1-20, 4 plates.

1952. [On the Ordovician Representatives of the Family Plectambonitidae (Brachiopoda).] Ústrédniho Ústavu Geologického, Sbornik, 19:397-428. [In Czech.]

1953. O několika novyćh ramenonožcích českého a moravského středního devonu. Ústrědniho Ústavu Geologického, Vèstnik, 28:4-9.

1956. [The Brachiopods of the Branik and Hlubocepy Limestones in the Immediate Vicinity of Prague.] Ústrédniho Ústavu Geologického, Sbornik, 22:535-650, 12 plates. [In Czech.]

1957a. O nových rodech českých spiriferidie (Brachiopoda). Ústrédniho Ústavu Geologického, Véstnik, 32(4):245-248.

1957b. Další nove rody čelidi Spiriferidae v Českém Siluru a Devonu. Ústrédniho Ústavu Geologického, Vést$n i k, 32(6): 436-440$.

1959a. [The Spiriferidae of the Silurian and Devonian of Bohemia.] Ústrédniho Ústavu Geologického, Rozpravy, 25:1-275, 101 figures, 28 plates. [In Czech.]

1959b. Rhynchonellacea im böhmischen älteren Paläozoikum (Brachiopoda). Ústrédniho Ústavu Geologického, Véstnik, 34(1):78-82.

1960. Bericht über die Ergebnisse der Revision der böhmischen altpaläozoischen Rhynchonelloidea. Usstrèdniho Ústavu Geologického, Vestnik, 35(3):241-244.

1961a. Rhynchonelloidea des böhmischen alteren Palaözoikums (Brachiopoda). Ústrédniho Ústavu Geologického, Rozpravy, 27:1-211, 27 plates.

1961b. Plectambonitacea im böhmischen Palaözoikum (Brachiopoda). Ústrédniho Ústavu Geologického, Vèstnik, 36(6):447-451.

1962. Oberfamilie Stropheodontacea im mährischen Mitteldevon (Brachiopoda). Ústrédniho Ústavu Geologického, Véstnik, 37(6):471-472.

1963a. Zlichorhynchus hialus n.g. et n. sp., neuer Brachiopode vom Unterdevon Böhmens. Ústrédniho Ustavu Geologického, Vèstník, 38(6):403-404, 1 plate.

1963b. Čeled̆ Leptaenidae (Brachiopoda) v českém Siluru a Devonu. Casopis Národního Muzea, 132(4):220225, 1 plate.

1965a. Superfamily Orthotetacea (Brachiopoda) in the 
Bohemian and Moravian Palaeozoic. Ústrédnitho Ústavu Geologického, Véstnik, 40(4):291-294.

1965b. Family Leptostrophiidae Caster, 1939 (Strophomenida, Brachiopoda) in the Bohemian Paleozoic. Casopis Národniho Muzea, 134(1):6-7.

1967a. Brachiopoda of the Suborder Strophomenidina in Czechoslovakia. Ústrédniho Ústavu Geologického, Rozpravy, 33:1-235, 52 plates.

1967b. Hanusitrypa and Falsatrypa (Atrypacea, Brachiopoda) in the Lower Devonian of Bohemia. Ustrédniho Ústavu Geologického, Vëstník, 42(6):443-444, 2 plates.

1968. New Brachiopods in the Lower Caradoc of Bohemia. U'strédniho Ùstavu Geologického, Véstnik, 43: 123-125.

1970a. Pompeckium and Jamesella (Orthacea, Brachiopoda) in the Middle Cambrian of Bohemia. L'strédniho Üslavu Geologıckého, Véstnik, 45:289-290, 2 plates.

1970b. Heterorthidae (Brachiopoda) in the Mediterranean Province. Geologickyich Vèd, Sbornik (Paleontologie), 12:7-40, 11 plates.

1971a. Non-Costate and Weakly Costate Spiriferidina (Brachiopoda) in the Silurian and Lower Devonian of Bohemia. Geologických Véd, Sbornik (Paleontologie), 14:7-34, 8 plates.

1971b. New Genera of Enteletacean Brachiopods in the Devonian of Bohemia. Ústrédniho Ústavu Geologického, Vésinik, 46:229-232, 18 plates.

1971c. Brachiopodes de l'Ordovicien du Maroc. Maroc Service Geologique du Division des Mines el de la Geologie, Notes et Memoires, 230:1-135, 3 figures, 26 plates.

1972. Life Habit of Some Ordovician Inarticulate Brachiopods. Ústrédniho Ústavu Geologického, Vèstnik, 47(4):229-233, 2 figures, 13 plates.

1973. New Brachiopod Genera in the Devonian of Bohemia. Ústrédntho Ústavu Geologického, Vëstnik, 48: 337-340, 2 plates.

1974. New Genera of Orthidina (Brachiopoda) in the Lower Palaeozoic of Bohemia. Uंstrédniho Ústavu Geologického, Vèsinik, 49(3):167-170, 2 plates.

1975. New Genera and Species of Orthida (Brachiopoda). Ústrédniho Ústavu Geologického, Véstnik, $50(4): 231-235,2$ plates.

1976. Systematic Position of Four Ordovician Brachiopod Species. Ústrédniho Ústavu Geologického, Věstnik, 51:367-370, 2 plates.

1977a. Brachiopods of the Order Orthida in Czechoslovakia. Ústrédniho Ústaun Geologického, Rozpravy, 44: 1-327, 56 plates.

1977b. Minutc Devonian Brachiopods (Notanopliidae, Leptostrophiidae) in Bohcmia. Ústrédnitho Ústavu Geologického, Véstnik, 52:299-302, 2 plates.

1978. New Spiriferids (Brachiopoda) of Pragian Age
(Lower Devonian, Bohemia). Ústrédniho Ústavu Geologického, Véstnik, 53:103-106, 2 plates.

1979. Upper Devonian and Lower Tournaisian Rhynchonellida in Czechoslovakia. Ústrédniho Ústavu Geologického, Vèstník, 54(2):87-101, 4 plates.

Havlíček, V., and V. Josopait

1972. Articulate Brachiopods from the Iberian Chains, Northeast Spain (Middle Cambrian-Upper Cambrian-Tremadoc). Neues Jahrbuch für Geologie und Paläontologie, Abhandlungen, 140(3):328-353, 2 figures, 5 plates.

Havlícek, V., and D. Massa

1973. Brachiopodes de l'Ordovicien Supérieur de Libye Occidentale, Implications Stratigraphiques Régionales. Geobios, 6(4):267-290, 4 plates.

Havlíček, V., and P. Racheboeuf

1979. Chonetacea (Brachiopodes) du Silurien et du Dévonien de Bohême (Tchécoslovaquie). Annales de Paléontologie (Invertébrés), 65(2):69-138, 15 plates.

Hayasaka, I.

1922. Palaeozoic Brachiopoda from Japan, Korea, and China. Tohoku Imperial University, Science Reports, series 2 (Geology), 6(1):1-137, 6 plates.

1938. A New Neotreme Genus of Brachiopoda from Japan. Venus, 8(1):9-13, 2 figures.

1953. Hamletella, a New Permian Genus of Brachiopoda, and a New Species from the Kitakami Mountains, Japan. Paleontological Society of Japan, Transactions and Proceedings, 12:89-95, plate 9.

$\mathrm{He}, \mathrm{X}$, and M. Zhu

1979. [A New Form of Brachiopods and Its Systematical Classification.] Journal of China Institute of Mining Technology, 1979 (4):131-140, 1 figure, 3 plates. [In Chincse.]

Hector, J.

1879. On the Fossil Brachiopoda of New Zealand. New Zealand Institute, Transactons and Proceedings, 11:537539.

Hedley, C.

1905. Mollusca (and Brachiopoda) from One Hundred and Eleven Fathoms, East of Cape Byron, New South Wales. Records of the Australian Museum, 6(2): $41-54$.

Hedström, $\mathrm{H}$.

1917. Ueber einige mit der Schale befestigte Strophomenidae aus dem Obersilur Gotlands. Sveriges Geologiska Undersökning, Arsbok, series C, 276:1-14, 3 plates.

Helmbrecht, W., and R. Wedekind

1923. Versuch einer biost ratigraphischen Gliederung der Siegener Schichten auf Grund von Rensselaerien und Spiriferen. Glückauf, Berg.- und Hüttenmännische Zeitschrift, 59(41):949-953. 
Helmcke, J. G.

1940. Die Brachiopoden der deutschen Tiefsee-Expedition. In Wissenschafliche Ergebnisse der Deulschen Tiefsee-Expedition auf dem Dampfer "Valdivia," 1898 1899, 24(3):217-316.

Helmersen, G. von

1847. Aulosleges variabilis im Zechstein Russlands, ein neucs Brachiopoden-Genus. Neues Jahrbuch für Mineralogie, Geognosie, Geologie, und Pelrefaklen-Kunde, 1847:330-331.

Hertlein, L. G., and U. S. Grant IV

1944. The Cenozoic Brachiopoda of Western North America. University of California Publications in Matheinatics and Physical Sciences, 3:1-236, 21 plates.

Hill, D.

1950. The Productinae of the Artinskian Cracow Fauna of Queensland. University of Queensland, Department of Geology, Papeis, 3(11): 1-36, 9 plates.

Hints, L.

1971. [A New Genus of Rhipidomellid Brachiopods from the Middle Ordovician of the Moscovian Syncline.] Eesti NSV Teaduste Akadeemia Toimetised (Keemia Geoloogia), 20(3):266-270, 1 figure, I plate. [In Russian.]

1973. [New Brachiopods Orthacea from the Middle Ordovician of the Baltic Area and Sweden.] Eesti NSV Teadusle Akadeemia Toimelised (Keemia Geoloogia), 22(3):248-256, 2 figures, 2 plates. [In Russian.]

1975. Brakhiopody Enlelelacea Ordorika Priballiki. 117 pages, 23 plates. Tallin: Eesti NSV Teaduste Akadeemia Geoloogia Instituut.

Hoare, R. D.

1960. New Pennsylvanian Brachiopoda from Southwest Missouri. Journal of Paleontology, 34(2):217-232, plates 1-2.

Holtedahl, O.

1916. The Strophomenidae of the Kristiania Region. Kongelige Norske Videnskabers Selskabs Skrifler, 12:1118,16 plates.

Holzapfel, E.

1895. Die Fauna der Schichten mit Maeneceras terebralum. Königlich Preussischer Geologischen Landesanstall, Abhandlungen, 16:234-237.

Hoover, P. R.

1979. Early Triassic Terebratulid Brachiopods from the Western Interior of the United States. U.S. Geological Survey Professional Paper, 1057:1-21, 4 plates.

Hou, Hung-fei

1963. [Some New Middle Devonian Brachiopods from the Provinces of Guansi and Yunnan.] Acta Palaeonlologica Sinica, 11(3):412-432. [In Chinese.]

1965. [Early Carboniferous Brachiopods from the Mengkungao Formation of Gieling, Central Hunan.]
Professional Paper of Academy of Geological Sciences, Ministry of Geology (Stralıgraphy and Paleonlology), series B, 1:111-146, 6 plates. |In Chinese: English summary.]

Hou, Hung-fci, and Si-yuan Xian

1964. |Brachiopod Fauna of the Nanpanjiang Limestone of Eastern Yunnan and its Geological Age.] Acta Palaeonlologica Sinica, 12(3):411-425, 2 plates. [In Chinese.]

1975. TThe Lower and Middle Devonian Brachiopods from Kwangsi and Kueichow.] Professional Papers of Siralıgraphy and Palaeontology, 1:1-85, 32 plates. [In Chinese].

Hou, Hung-fei, Li-pei Zhan, Bin-wei Chen, et al.

1979. The Coal-bearing Strala and Fossils of Lale Permian from Guanglung. 166 pages, 47 plates. Peking: Geological Publishing House. [In Chinese; Engl ish title page.]

Hou, Hung-fei, and Jin-ke Zhao

1976. In Hua Bei Dl Qu Gu Sheng Wu Tu Ce, Nei Meng Gu Feng Ce, Yi Gu Sheng Dai Bu Feng [Paleontological Atlas of Northern China, Volume 1 (Inner Mongolia)]. 502 pages, 232 plates. Peking. [In Chinese.]

Huang, T. K.

1932. Late Permian Brachiopoda of Southwestern China, Part II. China, Geological Survey, Palaeontologia Sinica, series B, 9(1):1-138, plates 1-9.

Hudson, R.G.S., and R.P.S. Jefferies

1961. Upper Triassic Brachiopods and Lamellibranchs from the Oman Peninsula, Arabia. Palaeontology, 4(1): 1-41, 2 plates.

Hudson, R.G.S., and M. Sudbury

1959. Permian Brachiopoda from South-East Arabia. Muséum Hzsioire Nalurelle, Noles and Mémoires sur le Moyen-Orient, 7:19-55, 12 figures, 6 plates.

Huene, F. von

1899. Die silurischen Craniaden der Ostseeländer mit Ausschluss Gotlands. Russisch-Kaiserlichen Mineralogischen Gesellschafl, Verhandlungen, series 2(36):181359.

Hyde, J. E.

1953. Mississippian Formations of Central and Southern Ohio. Ohio Geological Survey, Bullelin, 51:1-355, plates 1-54.

Ifanova, $\mathrm{V} . \mathrm{V}$.

1968. [Early Permian Chonetidae of the Pechora Basin.] Paleonlologicheskii Zhumal, 1968(3):29-33. [In Russian.]

Ihering, H. von

1903. Les Brachiopodes Tertiares de Patagonie. Anales del Museo Nacional de Buenos Aires, series 3, 2:321349.

Imbrie, J.

1959. Brachiopods of the Traverse Group (Devonian) of 
Michigan. American Museum of Natural History, Bulletin, 116:349-409, 20 plates.

Ireland, H. A.

1961. New Phosphatic Brachiopods from the Silurian of Oklahoma. Journal of Paleontologv, 35(6):1137-1142, plate 137.

Isaacson, P. E.

1977a. Devonian Stratigraphy and Brachiopod Paleontology of Bolivia, Part A: Orthida and Strophomenida. Palaeontographica, series A, 155(5-6):133192, 31 figures, 6 plates.

1977b. Devonian Stratigraphy and Brachiopod Paleontology of Bolivia, Part B: Spiriferida and Terebratulida. Palaeontographica, series A, 156(4-6):168217.

Ischnazarov, N. I.

1972. Novye Dannye o Devonskikh Brakhiopoda Basseina r. Chatkal (Tiań-Shań). In A. S. Masumov and R. N. Abdullaev, editors, Novye Dannye po Faune Paleozoia i Mesozoia Uzbekistana, pages 68-74. Tashkent: Akademiia Nauk Uzbekskoi SSR, Institut Geologii i Geofizikii.

Ivanov, A. P.

1925. Sur la Systématique et la biologie du genre Spirifer et de quelques brachiopodes de $\mathrm{C}_{\mathrm{ii}}$ et $\mathrm{C}_{\mathrm{iii}}$ du gouvernement de Moscou. Société Impériale des Naturalistes de Moscou, Bulletıns, 33:105-123, 6 figures.

1935. [Brachiopods of the Middle and Upper Carboniferous of the Moscow Basin, Part I: Productidae Gray.] Moskouskogo Geologicheskogo Tresta, Trudy, 8: 1-134, 21 figures, 15 plates. [In Russian.]

Ivanov, A. P., and E. A. Ivanova

1937. [Brachiopod Fauna of the Middle and Upper Carboniferous of the Submoscow Basin (Neospirifer, Choristites).] Akademila Nauk SSSR, Paleozoologicheskil Institut, Trudy, 6(2):1-215, plates 1-23. [In Russian.]

Ivanov, A. P., and E. I. Miagkova

1950. Stratigrafiia Nizhnego i Srednego Paleozoia Zapadnogo Sklona Srednego Urala. Gorno-Geologicheskogo Instituta, Trudy, 18:1-32, 19 plates.

Ivanova, E. A.

1951. [New Data on Productid Systematics (Genus Kutorginella).] Akademiia Nauk SSSR, Doklady, 77(2): 329-331. [In Russian.]

1960. Mshanki, Brachiopody. In T. G. Sarytcheva, editor, Otryad Spiriferida. In Yu. A. Orlov, editor, Osnovy Paleontologii, pages 264-280, plates 57-64.

1971. [On a New Spiriferinid Genus.] Paleontologicheskii Zhurnal, 1971(1):120-123, 1 figure. [In Russian.]

1975. [Some Carboniferous Spiriferinidina of the Moscow Basin.] Paleontologicheskii Zhurnal, 1975(2):7988. [In Russian.]
Ivanovskii, A. B., and N. P. Kulkov

1974. [Rugose, Brachiopods and Stratigraphy of the Silurian and Mountainous Altais and Sajan.] Akademiia Nauk.SSSR, Sibirskoe Otdelenie, Institut Geologii i Geofiziki, Trudy, 231:1-96, 25 plates. [In Russian.]

Jaanusson, $\mathrm{V}$.

1962. Two Plectambonitacean Brachiopods from the Dalby Limestone (Ord.) of Sweden. Uppsala University, Geological Institutions, Bulletin, 39:1-8, plate 1.

Jackson, J. W.

1918a. On Terebratula grayi Davidson. Geological Magazine (London), new series, decade 6, 5:479-480.

1918b. Brachiopoda, British Antarctic ("Terra Nova") Expedition (1910). British Museum (Natural History), Report (Zoology), 2(8):177-202, plate 1.

Jaekel, O.M.J.

1902. Ueber verschiedene Wege phylogenetischer Entwickelung. In Verhandlungen des $V$. Internationalen Zoologen-Congresses zu Berlin, 1901, pages 10581117,28 figures.

Janischewsky, M. E.

1910. Fauna Nizhne Kamennougol'nago Izvestniaka okolo poselka Khaharnogo. Tomskago Tekhnologicheskago Instituta, Izvestiia, 17(1):1-305.

Jassjukevitch, L. P.

1973. In Novye Vidy Drevnikh Rastenii i Bespozvonochnykh. Vsesoiuznyı Nauchno-Issledovatelskii GeologoRazvedochnyi Neftianoi Institut (VNIGRI), Trudy, 318: 1-179, 34 plates.

Jeffreys, J. G.

1869. The Deep-Sea Dredgings, Explorations on H.M.S. "Porcupine." Nature, 1:135-137.

Jiang, Zong-long

1978. In Xi Nan Di Qu Gu Sheng Wu Tu Ce, Gui Zhou Feng $\mathrm{Ce}$, Yi Han Wu Ji-Ni Pen Ji [Paleontological Atlas of Southwestern China, Guizhou (Kweichow) Province, Volume 1 (Cambrian-Devonian)]. 843 pages, 214 plates. Peking. [In Chinese.]

Jin, Yu-gan, and Bing-xing Fang

1977. [Upper Triassic Brachiopoda Fauna from the Area in the East of the Hengduan Mountains, Western Yunnan.] In Yun-nan Zhong Sheng Dai Hua Shi, Xia $\mathrm{Ce}$ [Mesozoic Fossils from Yunnan, China]. 2:3971. [In Chinese.]

Jing, Yu-gan, and Shi-zhong Hu

1978. [Brachiopods of the Kuhfeng Formation in South Anhui and Nanking Hills.] Acta Palaeontologica Sinica, 17(2):101-130. [In Chinese.]

Johnson, J. G.

1966a. Middle Devonian Brachiopods from the Roberts Mountains, Central Nevada. Palaeontology, 9(1): 152-181, 6 plates.

1966b. Two New Spiriferid Brachiopod Genera from the 
Lower Devonian of Nevada. Journal of Paleontology, 40(5): 1043-1050, 3 plates.

1966c. Parachoneles, a New Lower and Middle Devonian Brachiopod Genus. Palaeontology, 9(3):365-370, 2 plates.

1967. Toquimaella, a New Genus of Karpinskiinid Brachiopod. Journal of Paleontology, 41(4):874-880, 1 plate.

1970a. Taghanic Onlap and the End of North American Devonian Provinciality. Geological Society of America, Bulletin, 81:2077-2106, 6 figures, 4 plates.

1970b. Great Basin Lower Devonian Brachiopoda. Geological Society of America, Memorr, 121:1-421, 6 figures, 74 plates.

1971. Lower Givetian Brachiopods from Central Nevada. Journal of Paleontology, 45(2):301-326, 2 figures, 10 plates.

1972. The Antistrix Brachiopod Faunule from the Middle Devonian of Central Nevada. Journal of Paleontologv, 46(1):120-124, 3 plates.

1973a. Mid-Lochkovian Brachiopods from the Windmill Limestone of Central Nevada. Journal of Paleontology, 47(6): 1013-1030, 5 plates.

1973b. Some North American Rensselandiid Brachiopods, Part 2. Journal of Paleonlology, 47(6):11021107, 2 plates.

1973c. Late Early Devonian Rhynchonellid Genera from Arctic and Western North America. Journal of Paleontology, 47(3):465-472, 3 plates.

1974. Middle Devonian Givetian Brachiopods from the Leiorhynchus castanea Zone of Nevada. Geologica et Palaeontologica, 8:49-96, 7 figures, 12 plates.

1975. Late Early Devonian Brachiopods from the Disappointment Bay Formation, Lowther Island, Arctic Canada. Journal of Paleontology, 49(6):947978, 9 figures, 10 plates.

1976. Chattertonia N. Gen. (Brachiopoda) the ChonetidProductid Link. Journal of Paleonlology, 50(5):789791.

Johnson, J. G., and A. J. Boucot

1967. Gracianella, a New Late Silurian Genus of Atrypoid Brachiopods. Journal of Paleonlology, 41(4):868-873, 2 plates.

Johnson, J. G., A. J. Boucot, and E. C. Gronberg

1968. A New Genus of Stringocephaloid Brachiopod from the Middle Devonian of Nevada. Journal of Paleontology, 42(2):406-414.

Johnson, J. G., A. J. Boucot, and M. A. Murphy

1976. Wenlockian and Ludlovian Age Brachiopods from the Roberts Mountains Formation of Central Nevada. University of California Publications in Geological Sciences, 115:1-102, 55 plates.

Johnson, J. G., and R. Ludvigsen

1972. Carinagypa, a New Genus of Pentameracean Brach- iopod from the Devonian of Western North America. Journal of Paleontology, 46(1):125-129.

Johnson, J. G., and A. W. Norris

1972. Tecnocyrtına, a New Genus of Devonian Brachiopods. Journal of Paleontology, 46(4):565-572, 2 plates.

Johnson, J. G., and D. G. Perry

1976. Middle Devonian Brachiopods from the Bird Fiord Formation of Bathurst Island, Arctic Canada. Canadian Journal of Earth Sciences, 13(5):615635, 4 plates.

Johnson, J. G., and J. A. Talent

1967a. Cortezorthinae, a New Subfamily of Siluro-Devonian Dalmanellid Brachiopods. Palaeonlology, $10(1): 142-170$.

1967b. Muriferella, a New Genus of Lower Devonian Septate Dalmanellid. Royal Society of Victoria, Proceedings, 80(1):43-50.

Johnston, J.

1941. Studies in Silurian Brachiopoda. Linnean Society of New South Wales, Proceedings, 66(3-4):160-168, plate 7 .

Jones, B.

1979. Nanukidium, a New Name for Rossella Jones, 1978. Journal of Paleontology, 53(5):1261.

Jones, O. T.

1928. Plectambonites and Some Allied Genera. Great Britain, Geological Survey, Memoir, Palaeonlology, 1(5): $367-527,5$ plates.

Kalashnikov, N. V.

1966. Brakhiopody Nizhnego Karbona Verkhnei Pechory na Severnom Urale. In Stratigrafiia i Paleontologiia Severo- Vostoka Evropeiskoi Chasti SSSR, pages 28-61, 12 plates. Moscow: Akademiia Nauk SSSR, Komi Filial, Institut Geologii.

Kamyschan, V. P.

1968. [New Data on the Morphology and Systematics of Mesozoic Striated (Capillate) Rhynchonellidae.] Paleontologicheskii Zhurnal, 1968(3):49-62. [In Russian.]

Kamyschan, V. P., and L. I. Babanova

1973. Sredneiurskie i Pozdneiurskie Brakhiopody Severo-Zapadnogo Kavkaza i Gornogo Kryma. 175 pages, 18 plates. Kharkov: Izdatelskoe Ob'bedinenie Visha Shkola Izlatelstvo Pri Kharkovskom Gosudarstvennom Universitete.

Kaplun, L. I.

1961. Brakhiopody Nizhnego Devona Severnogo Pribalkhashia. In Materialy po Geologiz i Poleznym Iskopaemym Kazakhstana, 1(26):64-114, 2 plates.

Kaschirzev, A. S.

1959. [Jakuloproductus, a New Genus of Brachiopod from the Lower Permian of Eastern Siberia.] Akademiia 
Nauk SSSR, Paleontologicheskii Institul, Trudy, 3:2831, 1 plate. [In Russian.]

Katz, Y.

1962. Novye Rody Pozdnemelovykh Zamkovykh Brakhiopod Tadzhikskoi Depressii i Prilezhashchikh k nei Regionov. Zapiski Geologicheskogo Otdeleniia Kharkhouskogo Gosudarstvennogo Universitela im A.M. Gorkogo, 15:132-154, 2 plates.

1974. In M. Y. Blank, G. Y. Krymholts, D. P. Naidin, and O. V. Savchinskaya, editors, Allas Verkhnemelovoi Fauny Donbassa, pages 240-275. Moscow: Ministerstvo Vysshevo i Srednego Spetsial'nogo Obrazovaniia USSR; Kommunarskii Gornometallurgicheskii Institut.

Katz, Y., and A. M. Popov

1974. Novye Taksony Mezozoiskikh i Kainozoiskikh Petleopornykh Brakhiopod. Vestnik Kharkouskogo Universitela (Geologïa), 108(5):22-32.

Kayser, E.

1871. Die Brachiopoden des Mittel- und Ober-Devon der Eifel. Zeitschrift der Deutschen Geologischen Gesellschaft, 23(3):491-647, plates 9-14.

1881. Mittheilungen über die Fauna des chinesischen Kohlenkalks von Lo-Ping. Zeitschrift der Deutschen Geologischen Gesellschaft, 33:351-352.

1883. Obercarbonische Fauna von Lo-Ping. In F. von Richthofen, China, 4:1-288, 54 plates.

Kegel, W.

1913. Der Taunusquarzit von Katzenelnbogen. Königlich Preussischen Geologischen Landesanslall, Abhandlungen, 76:1-162.

Kelly, F. B.

1967. Silurian Leptaenids (Brachiopoda). Palaeonlology, 10(4):590-602.

Khalfin, L. L.

1939. [On a New Genus of Rhynchonellid.] Sovetskaza Geologiia, 9(12):83. [In Russian.]

1946. O Paleozoiskoi Faune Zap. Sibiri i Zadachakh ee Izucheniya. Izvestiya Zapad. Sibiri Filiala Akademiia Nauk SSSR, Seriya Geologicheskaia, 1. [As cited in Khalfin, 1948:89.]

1948. [Fauna and Stratigraphy of the Devonian Deposits of the Gorny Altay.] Izvestiya Tomskogo Ordena Trudovogo Krasnogo Znameni Polutekhnicheskogo Instiluta, 65(1): 1-464, 36 plates. [In Russian.]

1955. Atlas of Leading Fossil Forms of Fauna and Flora of Western Siberia.] Zapadnoe-Sibirskoe Geologeskoe Upravlenie-Tomskogo Ordena Trudovogo Krasnogo Znameni Politekhnucheskogo Institula, Izvestiya, 1:1-502, 85 plates. [In Russian.]

1960a. Biostratigraphy of the Palaeozoic of the SayanoAltai Mountains, I: Lower Palaeozoic.] Sibirshii Nauchno-Issledovalelskii Instılut Geologii, Geofizikiz i
Mineralogii Syria (SNIIGGIMS), Trudy, 19:1-498, 53 plates. [In Russian.]

1960b. |Biostratigraphy of the Palaeozoic of the SayanoAltai Mountains, II: Lower Palaeozoic.] Sibirskii Nauchno-Issledovatelskii Institut Geologii, Geofizikii $i$ Mineralogii Syria (SNIIGGIMS), Trudy, 20:1-850, 134 plates. [In Russian.]

Khodalevich, A. N.

1939. Upper Silurian Brachiopoda of Eastern Slope of the Urals. 135 pages, 28 plates. Sverdlovsk: Uralskoe Geologischeskoe Upravlenie. [In Russian; English title.]

1975. KKumbella, a New Brachiopod Genus from the Givetian of the Urals.] Paleontologicheskii Zhurnal, 1975(3):137-141. [In Russian.]

Khodalevich, A. N., I. A. Breivel, M. G. Breivel, T. I. Vaganova, A. F. Torbakova, and F. E. Yanet.

1959. Brakhiopody i Korally iz Eyfelskikh Boksilonosnyikh Otlozheniy Vostochnogo Sklona Srednego a Severnogo Urala. 282 pages, 61 plates. Moscow: Ministerstvo Geologii i Okhrany Nedr SSSR-Uralskoe Geologicheskoe Upravlenie.

Khodalevich, A. N., and V. P. Sapelnikov

1970. [Eokirkidium, a New Silurian Pentamerid (Brachiopoda).] Akademiia Nauk SSSR, Doklady, 193(1): 196-198, 4 plates. [In Russian.]

Khu, Bin, V. I. Ustritsky, and A. C. Chan

1959. Stratigrafiya i Fauna Kamennougolnikh i Permskikh Otlozhenii Zapadnogo Kun-Linya. Peking: Ministerstvo Geologii kir Nauchno-Issledovatelskii Geologicheskii Institut.

Kiaer, J.

1902. Studier over den Norske Mellemsilur. Norges Geologiske Undersogelsos Aarbog for 1902, 1:1-112.

Kindle, E. M.

1909. The Devonian Fauna of the Ouray Limestone. U.S. Geological Survey, Bulletin, 391:1-60, 10 plates.

King, R. E.

1930 [1931]. The Geology of the Glass Mountains, Texas, Part II: Faunal Summary and Correlation of the Permian Formations with Descriptions of Brachiopoda. Universily of Texas Bulletin, 3042:1245, 44 plates.

King, R. E., C. O. Dunbar, P. E. Cloud, and A. K. Miller

1944. Geology and Paleontology of the Permian Area of Las Delicias, Southwestern Coahuila, Mexico, Part 3: Brachiopods. Geological Society of America, Special Paper, 52:49-69.

King, R. H.

1938. New Chonetidae and Productidae from Pennsylvanian and Permian Strata of North-Central Texas. Journal of Paleonlology, 12(3):257-279, plates 36-39. 
King, W.

1844. On a New Genus of Palaeozoic Shells. Annals and Magazine of Nalural History (London), 14(92):313.

1850. A Monograph of the Permian Fossils of England. Palaeontographical Society Monograph, 3:1-258, 29 plates.

1859. On Gwynia, Dielasma and Macandrevia, Three New Genera, etc. Dublin Zoological and Botanical Association, Proceedings, 1(3):256-262.

1865. Remarks of the Histology of Two Specimens of Rhynchopora geinilziana deVerneuil, from near the River Oukhla, Province of Archangel. Annals and Magazine of Nalural History (London), series 3, 16: 124-128.

1871. On Agulhasia davidsonii, a New Palliobranchiate Genus and Species. Annals and Magazine of Natural History, series 4, 7:109-112, plate 11 .

Kiparisova, L. D., B. P. Markowski, and G. P. Radchenko

1956. [Material on Palaeontology, New Families and Genera.] Vsesoiuznyi Nauchno-Issledovatelskii Geologicheskii Institut (VSEGEI), Matenaly, new series, 12: 1-266, 53 figures, 43 plates. [In Russian.]

Kirk, E.

1922. Brooksina, a New Pentameroid Genus from the Upper Silurian of Southeastern Alaska. "United Stales National Museum, Proceedings, 60(19):1-8.

1925. Harpidium, a New Pentameroid Brachiopod Genus from Southeastern Alaska. Uniled States National Museum, Proceedings, 66(32):1-7, 2 plates.

1926. Cymbidium, a New Genus of Silurian Pentameroid Brachiopods from Alaska. United States National Museum, Proceedings, 69(23):1-5, 1 plate.

Kirk, E., and T. W. Amsden

1952. Upper Silurian Brachiopods from Southeastern Alaska. United Stales Geological Survey Professional. Paper, 233-C:53-66, plates 7-10.

Kobayashi, T.

1934. A Few Ordovician Brachiopods from Manchuria and Chosen, and a New Species of Conchostraca from the Basal Ordovician of Manchuria. Japanese Journal of Geology and Geography, 11(3-4):161-169.

1935. The Cambro-Ordovician Formation and Faunas of South Chosen, Part 3: Cambrian Faunas of South Chosen. Tokyo Imperial University, Faculty of Science, Journal, series 3, 4(2):49-344, 24 plates.

1937. The Cambro-Ordovician Shelly Faunas of South America. Tokyo Imperial University, Facully of Science, Journal, 4(4):369-522.

Koczyrkevicz, B. V.

1969. K Sistematike Nadsemeistva Stenoscismatacea Oehlert, 1887 (1883) (Brachiopoda). In Iskopaemaia Fauna I Flora Dal'nego Vostoka, I, pages 7-16, 1 plate. Vladivostok: Akademiia Nauk SSSR.

1976a. [A New Genus of Permian Basiliolacea (Brachi- opoda).] In Morfologiya i Sistematika Iskopaemykh Bespozbonochnykh Dal'nego Vostoka. Akademiia Nauk SSSR, Dalnevostochnyi Nauchnyi Tsentr, Biologo-Pochvennogo Institul, Trudy, 42(145):79-82. [In Russian.]

1976b. [Permian Heterelasminidae (Brachiopoda) from South Primorye.] In Morfologia i Sistematika Iskopaemykh Bespozbonochnykh Dal'nego Vostoka. Akademiia Nauk SSSR, Dalnevostochnyi Nauchnyi Tsentr, Biologo-Pochvennogo Institut, Trudy, 42(145): 73-78. [In Russian.]

Koenig, C.

1825. Icones Fossilium Sectiles. 4 pages, 19 plates. London. Koken, E.F.R.

1889. Ueber die Entwickelung der Gastropoden vom Cambrium bis zur Trias. Neues Jahrbuch für Mineralogie, Geologie, und Paläonlologie, 6:305-484, plates $10-14$.

Kolarova, F. N.

1925. The Generic Status of Triplecia poloi. Geological Society of China, Bulletin. 4(3-4):215-219, 1 plate.

Koneva, S. P.

1979. Stenotekoidy i Bezzamkovye Brakhiopody Nizhnego i Nizov Srednego Kembriya Tsentral'nogo Kazakhstana. 124 pages, 31 plates. Alma-Ata: Akademiia Nauk Kazakhskoi SSR, Institut Geologicheskikh Nauk.

Kotljar, G. V.

1964. [New Representatives of the Genus Productus from the Upper Permian of Southern Primoria.] Vsesoyuznyi Nauchno-Issledovatelskii Geologicheskii Institut (VSEGEI), Trudy, 93:123-134, 3 plates. [In Russian.]

1977. In T. G. Sarytcheva, editor, Pozdnepaleozoiskie Producktidy Sibiri i Arktiki. Akademiia Nauk SSSR, Paleontologicheskii Institut, Trudy, 161:1-219.

Kozlowski, R.

1923. Faune devonienne de Bolivie. Annales de Paleontologie (Paris), 12:1-109, plate 9.

1927. Sur Certains Orthides ordoviciens des environs de St. Petersbourg. Bibliotheca Universilatis Libirae Polonae Varsaviae, series A, 17:3-21, 1 plate.

1929. Les Brachiopodes gothlandiens de la Podolie Polonaise. Palaeontologia Polonica, 1:1-254, 95 figures, 12 plates.

1930. Andobolus gen. nov. i kilka innych ramienionogów bezzawiasowych z ordowiku Boliwji. Sprawozdania Polskiego Instytuta Geologicznego, 6(2):293-334.

1946. Howellella, a New Name for Crispella Kozlowski, 1929. Journal of Paleonlology, 20(3):295.

Krause, F. F., and A. J. Rowell

1975. Distribution and Systematics of the Inarticulate Brachiopods of the Ordovician Carbonate Mud Mound of Meiklejohn Peak, Nevada. University of 
Kansas Paleontological Contributions, 61:1-74, 12 plates.

Krotow, P.

1888. Geologische Forschungen am westlichen Ural-Abhange in den Gebieten von Tscherdyn und Ssolikamsk. Comuté Géologique, Mémoires, 6(2):297-563, 2 plates.

Krylova, A. K.

1962. Stratigrafiia i Brakhiopody Devona Sibirskoi Platformy. Vsesoiuzny Nauchno-Issledovalelskii GeologoRazvedochnyi Neftianoi Institut (VNIGRI), Trudy, 200: $1-107,15$ plates.

Kulikov, M. V.

1950. [On the Systematics of Spirifers from the Upper Permian Strata of the European Part of the SSSR.] Vsesoyuznyi Nauchno-Issledovatelskii Geologicheskii Institut (VSEGEI), Trudy, 1:3-7. [Ia Russian.]

Kulkov, N. P.

1960. Novyi Rod Spiriferid iz Eifel'skikh Otlozhenii Severo-Vostochnogo Sklona Salaira. Akademia Nauk SSSR, Doklady, 132:929-931, 2 figures.

1962. Novyi Rod Spiriferid iz Nizhnego Devona Gornogo Altaiia. Akademiia Nauk SSSR, Doklady, 145(3):653-656.

1963. Brakhiopody Solovikhinskikh Sloev Nizhnego Devona Gornogo Allai. 135 pages, 9 plates. Moscow: Akademiia Nauk SSSR.

1967. Brakhiopody z Stratngrafiza Silura Gornogo Altai. 151 pages, 21 plates. Moscow: Akademiia Nauk SSSR, Sibirskoe Otdelenie, Institut Geologii i Geofiziki.

1968. Nekotorye Voprosy Morfologii i Sistematiki Pentamerid. Paleontologicheskii Zhurnal, 1968(3):34-41.

Kutorga, S. S.

1848. Über die Brachiopoden-Familie der Siphonotretaeae. Russische-Kaiserliche Mineralogische Gesellschaft zu St. Petersburg, Verhandlungen, 1847:250-286, plates $6-7$.

Kvakhadze, N. N.

1972. [Iberithyris, â New Brachiopod Genus from Lower Cretaceous Deposits of Western Georgia.] Paleonlologicheskii Zhurnal, 1972(4):75-87. [In Russian.]

1974. [A New Genus of the Lower Cretaceous Kingenidae from Georgia (Brachiopoda).| Soobshcheniia Akademiia Nauk Gruzınskoi SSR, 73(2):493-496. [In Russian.]

1976. [A New Genus of the Family Basiliolidae of the Berriasian of Western Georgia (Brachiopoda).J Soobshcheniia Akademiia Nauk Gruzinskoi SSR, 82(2): 505-508, 2 figures. [In Russian.]

Kyansep, N. P.

1959. Zeillerina gen. nov.-novyi rod iz semeystva Zeiller- idae Rollier. Leningradskogo Universitela, Vestnik, (Geologiia), 18 (3):118-123, 5 figures.

1961. Terebratulidy Luzitanskogo Iarusa i Nizhnego Kimeridzha Iugo-Zapadnogo Kryma. Akademïa Nauk SSSR, Geologicheskii Musei Imeni A. P. Karpinskogo, Trudy, 8:1-101, plates 1-8.

Lahusen, J.

1886. Notiz über die inneren Merkmale einer neuen Untergattung der Strophomeniden. RussischeKaiserliche Mineralogische Gesellschaft zu St. Petersburg, Verhandlungen, series 2, 22:219-222, 1 plate.

Lamansky, V. V.

1905. Die ältesten silurischen Schichten Russlands. Comilé Géologique, Mémoires, new series, 20:1-203.

Lamarck, J.P.B.A. de M. de

1799. Prodrome d'une nouvelle classification des Coquilles. Mémoires de la Société d'Histoire Naturelle de Paris, 1799:63-91.

1819. Histoire naturelle des animaux sans vertêbres. Volume 6, part 1, pages 1-343. Paris.

Lamont, A.

1935. The Drummuck Group, Girvan; A Stratigraphical Revision, with Descriptions of New Fossils from the Lower Part of the Group. Geological Society of Glasgow, Transactions, 19:288-334, plates 7-9.

Lamont, A., and D.L.F. Gilbert

1945. Upper Llandovery Brachiopoda from Coneygore Coppice and Old Storridge Common, near Alfrick, Worcestershire. Annals and Magazine of Natural History, series 11, 12:641-682, plates 3-7.

Lane, B. O

1962. The Fauna of the Ely Group in the Illipah Area of Nevada. Journal of Paleontology, 36(5):888-911, 4 plates.

Lane, N. G.

1963. A Silicified Morrowan Brachiopod Faunule from the Bird Spring Formation, Southern Nevada. Journal of Paleontology, 37(2):379-392, 3 plates.

Larin, N. M.

1973. Novyi Rod Spiriferid iz Verkhnego Venloka Srednei Azii. Paleontologicheskii Zhurnal, 1973(3):134137.

Laube, G. C.

1865. Die Fauna der Schichten von St.-Cassian: Ein Beitrage zur Paläontologie der alpinen Trias. Akademie der Wissenschaften zu Munchen, Denkschriften, 25(2):1-76. 
Lazarev, S. S.

1976. Morfologiia i Razvitie Brakhiopod. Akademiia Nauk SSSR, Paleontologicheskii Institut, Trudy, 154: $1-167,15$ plates.

Lee, $\mathrm{Li}$, and Feng $\mathrm{Gu}$

1976. In Hua Bei Di Qu Gu Sheng Wu Tu Ce, Nei Meng Gu Feng Ce, Yi Gu Sheng Dai Bu Feng [Palaeontological Atlas of Northern China (Inner Mongolia)], 1: 502 pages, 232 plates. Peking: Geological Press. [In Chinese.]

Legrand-Blain, $\mathrm{M}$.

1968 [1969]. Spiriferacea carbonifères et permiens d'Afghanistan central. Museum National d'Histoire Naturelle, Notes et Mémoires sur le Moyen-Orient, 9: 188-253, 4 plates.

Leidhold, C.

1921. Beitrag zur genaueren Kenntnis und Systematik einiger Rhynchonelliden des reichsländischen Jura. Neues Jahrbuch für Mineralogie, Geologie, und Paläontologie, 44:343-368.

1928. Beitrag zur Kenntnis der Fauna des rheinischen Stringocephalenkalkes, insbesondere seiner Brachiopodenfauna. Königlich Preussichen Geologischen Landesanstall, Abhandlungen, new series, 109: 1-99, 7 plates.

Lenz, A. C.

1967. Thliborhynchia, a New Lower Devonian Rhynchonellid from Royal Creek, Yukon, Canada. Journal of Paleontology, 41(5):1188-1191, I plate.

1968. Two New Lower Devonian Atrypid Brachiopods from Royal Creek, Yukon Territory, Canada. Journal of Paleontology, 42(1):180-185, 2 plates.

1971. Werneckeella, à New Lower Devonian Rhynchonellid Brachiopod, Royal Creek, Yukon Territory. Journal of Paleontology, 45(5):844-848, 1 plate.

1973. Quadrithyris Zone (Lower Devonian) Near-Reef Brachiopods from Bathurst Island, Arctic Canada: With a Description of a New Rhynchonellid Brachiopod Franklinella. Canadian Journal of Earth Sciences, 10(9): 1403-1409, 2 plates.

1977. Upper Silurian and Lower Devonian Brachiopods of Royal Creek, Yukon Canada, Part I (Orthida, Strophomenida, Pentamerida, Rhynchonellida). Palaeontographica, series A, 159(1-3):37-109, 5 figures, 22 plates.

Levy, R.

1961. Sobre algunos Terebratellidae de Patagonia (Argentina). Revista de la Asociación Paleontológica Argentina, Ameghiniana, 2(5):79-88, plates 1-4.

Levy, R., and F. Nullo

1973. Braquiópodos ordovícicos de la sierra del Famatina (formación Molles), Provincia de La Rioja. Ameghiniana, 10(2):139-151, 2 plates. 1974a. La Fauna del Ordovicio (Ashgilliano) de Villicun San Juan, Argentina (Brachiopoda). Ameghiniana, 11 (2): 173-194, 2 plates.

1974b. Braquiopodos ordovicicos de Ponon-Trehue, Bloque de San Rafael (Provincia de Mendoza, Argentina). Actas del Primer Congreso Argentino de PaLeontologia y Bioestratigrafia, 1:23-32.

Liao, Zhuo-ting

1979. [Uppermost Carboniferous Brachiopods from Western Guizhou.] Acta Palacontologica Sinica, 18(6):527-544, 4 plates. [In Chinese.]

Liao, Zhuo-ting, and Dong-li Sun

1974. In Xi Nan Di Qu Di Ceng Gu Sheng Wu Shou Ce [Handbook of the Stratigraphy and Paleontology of Southwest China]. 454 pages, 202 plates. Peking. [In Chinese.]

Licharew, B. K.

1926. Un Nouveau Représentant des brachiopodes du paléozoique supérieur de Caucase du Nord. Comité Géologique, Bulletin, 43(6):713-721, plate 5.

1928. Über einige seltene und neue Brachiopoden aus den Unterperm des nördlichen Kaukasus. Palaeontologische Zeitschrift, 10:258-289, plates 3-4.

1931. Über eine problematische Brachiopode aus den unterpermischen Ablagerungen des Nördlichen Kaukasus. Annuaire de la Société Paléontologique de Russie, 9:157-161.

1934a. On Some New Genera of Upper Palaeozoic Brachiopoda. Académie des Sciences URSS, Comptes Rendus, new series, 1(4):210-213.

1934b. The Upper Carboniferous of Fergana and the Question of the Boundary between the Carboniferous and the Permian. Bulletin of the Geological Survey of China, 13(2):155-182.

1935. Bemerkungen über einige oberpaläozoische Brachiopoden. Zentralblatt für Mineralogie, Geologie und Palaeontologie, series B, 9:369-373, 2 figures.

1936a. Über einige oberpaläozoische Gattungen der Terebratulacea aus Eurasien. Problemy Paleontologii, 1(1):263-271, 1 plate.

1936b. The Interior Structure of Camarophoria King. American Journal of Science, 32:55-69, figures 1-6.

1939. Brachiopoda. In B. K. Licharew, editor, Atlas of the Leading Forms of the Fossil Faunas of the U.S.S.R., 6 : 1-270. Tsentralnyi Nauchno-Issledovatelskii Geologi-Razvedochnyi Institut (TSNIGRI). [In Russian; English title.]

1947. [On a New Subgenus, Muirwoodia of the Genus Productus Sow. sens. lat.] Académie des Sciences URSS, Comples Rendus, 57(2):187-190. [In Russian.]

1957. [On the Genus Goniophoria Yanisch. and other Related Genera.] Vsesoyuznoe Paleontologicheskoe Obshchestvo, Ezhegodnik, 16:134-141, 1 plate. [In Russian.] 
Licharew, B. K., and G. V. Kotljar

1978. Permskie Brakhiopody luzhnogo Primoria. In Verkhnii Paleozoi Severo-Vostochnoi Azii, pages 63-75, plates 11-22. Vladivostok: Akademiia Nauk SSSR, Dalnevostochnyi Nauchnyi Tsentr Institut Tektoniki i Geofizikii.

Licharew, B. K., V. P. Makridin, and M. A. Rzonsnitzkaja 1960. Mshanki, Brakhiopody. In T. G. Sarytcheva, editor, Otryad Terebratulida. In Yu. A. Orlov, editor, Osnovy Paleontologii, pages 286-305.

Lindström, $M$.

1953. On the Lower Chasmops Beds in the Fagelsang District (Scania). Geologiska Föreningens, Stockholm, Förhandlingar, 75(2):125-148, 1 plate

Link, H. F.

1830. Handbuch der Physikalischen Erdbeschreibung. Volume 2, part 1, 498 pages. Berlin: Dummler.

Linnarsson, J.G.O.

1876. Brachiopoda of the Paradoxides Beds of Sweden. Bihang till Kongliga Suenska Vetenskapsakademiens Handlingar, 3(12):1-34, 4 plates.

Linnik, L. S.

1966. [New Brachiopods from the Upper Devonian Deposits of Pripjatskaja Depression.] Paleontologiia $i$ Stratigrafiia Pribaltikz i Belorussit, Sbornek, 1(6):127149, 3 plates. Vilnius: Ministry of Geology of the U.S.S.R. [In Russian.]

Litvinovich, N. V.

1966. O Novom Rode Kinghiria gen. nov. iz Nizhnevizeiskih Otlozhenii Tsentralnogo Kazakhstana. Vesinik Moskovskogo Universiteta, 5:98-101.

1967. [Beleutella-a New Genus of Carboniferous Productid.] Paleonlologicheskin Zhurnal, 1967(3):55-61. [In Russian.]

Litvinovich, N. V., G. G. Aksenova, and T. P. Razina

1969. Stratigrafiza i Litologiia Otlozhenii Nizhnego Karbona Zapadnol Chasti Tsentralnogo Kazakhstana. 310 pages, 72 plates. Moscow: Ministerstvo Geologii Kazakhskoi SSR: Tsentral'no-Kazakhstanskoe Geologicheskoe Upravlenie.

Liu, Di-yong

1976. Ordovician Brachiopods from the Mount Jolmo Lungma Region. In A Report of Scientific Expedition in the Mount Jolmo Lungma Region (1966-1968), Palaeontology, 2:139-158. [In Chinese; English title.]

1979. [Earliest Cambrian Brachiopods from Southwest China.] Acta Palaeontologica Sinica, 18(5):505-511, 2 plates. [In Chinese.]

Ljaschenko, A. I.

1957. [A New Genus of Devonian Brachiopods: Uchtospirifer.] Académie des Sciences URSS, Comples Rendus, 117(5):885-888, 1 plate. [In Russian.]

1969a. Novye Devonskie Brakhiopody Tsentral'nykh i Zapadnykh Rainov Russkoi Platformy. Vsesoiuznyz
Nauchno-Issledovatelskii Geologo-Razvedochnyi Neftianoi Institul (VNIGNI), Trudy, 93:9-27.

1969b. [New Devonian Brachiopods from the Volgo-Ural Gas and Oil Bearing Province and from the Urals.] Vsesoiuznyi Nauchno-Issledovatelskii GeologoRazvedochnyi Neftianoi Institut (VNIGNI), Trudy, 93: 32-48. [In Russian.]

1973. Brakhiopody i Stratigrafiia Nizhnefranskikh Otlozhenii Iuzhnogo Timana i Volgo-Ural'skoi Neftegazonosnoi Provintsii. Vsesoiuznyi Nauchno-Issledovalelskii Geologo-Razvedochnyi Neftianoi Instilut (VNIGNI), Trudy, 134:1-279, 58 plates.

Lobatscheva, S. V.

1974. [A New Hautverian Dallinid Genus (Brachiopoda).] Paleontologicheskii Zhurnal, 1974(3):146149. [In Russian.]

Lobatscheva, S. V., and M. V. Titova

1977. [A New Genus of Rhynchonellid Brachiopod from the Cretaceous of Turkmen.] Ezhegodnik Vsesoiuznogo Paleontologicheskogo Obshchestva, 19:102-111. [In Russian.]

Lochman, C., and D. Duncan

1944. Early Upper Cambrian Faunas of Central Montana. Geological Society of America, Special Paper, 54: 144-155, plates 18-19.

Logan, A.

1963. The Brachiopod Genus Howseia from the Permian Magnesian Limestone of Durham. Palaeonlology, 6(4):754-761, plate 108 .

Lopushinskaja, T. V.

1965. [New Brachiopods from the Silurian Deposits of the Siberian Platform.] Sibirskii Nauchno-Issledovatelskii Institut Geologii, Geofiziki a Mineralogii Syria (SNIIGGIMS), Trudy, 34(1):23-33, 1 plate. [In Russian.]

1976. Brakhiopody i Stratigrafiia Siluriiskikh Otlozhenii Severa Sibirskoi Platformy. Sibirskii Nauchno-Issledovatelskii Institul Geologii, Geofiziki i Mineralogi Syria (SNIIGGIMS), Trudy, 199:1-96, 16 plates.

Ludvigsen, $\mathrm{R}$.

1974. A New Devonian Acrotretid (Brachiopoda, Inarticulata) with Unique Protegular Ultrastructure. Neues Jahrbuch für Geologie und Paläontologie Monatshefte, 3:133-148, 2 plates.

Mabuti, S.

1937. On a Permian Brachiopod, Gemmellaroia (Gemmellaroiella) ozawai, subgen. et sp. nov. from Japan. Tokyo Imperial Academy, Proceedings, 13(1):16-19.

Maillieux, E.

1912. Apparition de deux formes siegeniennes dans les schistes de Mondrepuits. Société Belge de Géologie, de Paléontologie el d'Hydrologie, Bulletin, 25:176-180.

1935. Contribution a la connaissance de quelques brachiopodes et pélécypodes dévoniens. Musée 
Royal d'Histoire Naturelle de Belgique, Mémoire, 70:342.

1939. La faune des schistes de Barvaux-sur-Ourthe (Frasnien supérieur). Musée Royal d'Histoire Naturelle de Belgique, Bulletin, 15(53):1-8.

Makridin, V. P.

1955. [Some Jurassic Rhynchonellids from the European Part of the USSR.] Zapiski Geologicheskogo Fakulteta Kharkouskogo Gosudarstvennogo Universiteta, 12:81-91, 3 plates. [In Russian.]

1964. Brakhiopody Iurskikh Otlozhenii Russkoi Platformy i Nekotorukh Prilezhashchkh k nei Oblasti. 394 pages, 25 plates. Moscow: Ministerstvo Vysshego i Srednego Spetsialnogo Obrazovaniia USSR, Kharkovskii Gosudarstvennyi Universitet imeni A. M. Gorkogo Nauchno-Issledovatelskii Sektor.

Malygina, A. A., L. I. Kaplun, A. N. Khodalevich, and M. G. Breivel

1972. [New Representatives of Devonian Delthyriacea of the Soviet Union.] In Novyye Vidy Dreunikh Rastenii i Bespozvonochnykh SSSR, pages 198-203. Moscow: Akademiia Nauk SSSR, Nauchnyi Sovet po Probleme. [In Russian.]

Mamedov, A. B.

1976. [Rzonsnickiana, a New Devonian Rhynchonellid Genus (Brachiopoda).] Paleontologicheskii Zhurnal, 1976(1):121-123. [In Russian.]

1978. [New Representatives of Devonian Ambocoeliidae (Brachiopoda) from the Little Caucasus.] In E. A. Modzalevskaja and L. M. Donakova, Ezhegodnik Vsesoyuznogo Paleontologicheskogo Obshchest va. Akademiia Nauk SSSR, Vsesoiuznoe Paleontologicheskoe Obshchestvo, 21:197-204, 1 plate. [In Russian.]

Manankov, I. N.

1979. Strofomenidy Pozdnego Paleozoia Sibiri i Arktiki. Akademiia Nauk SSSR, Paleontologicheskii Institut, Trudy, 171:102 pages, 13 plates.

Manankov, I. N., and E. E. Pavlova

1976. [Mongolosia-a New Genus of Permian Brachiopod.] Akademiia Nauk SSSR, Akademiia Nauk MNR Paleontologiia i Biostratigrafiia Mongolii, Trudy, 3: 354-357. [In Russian.]

Mansuy, $\mathrm{H}$.

1913. Faunes des calcaires à Productus de l'Indochine, première série. Mémoire du Service Géologique de l'Indochine, 2(4): 1-133, 13 plates.

Marek, L., and V. Havlícek

1967. The Articulate Brachiopods of the Kosov Formation (Upper Ashgillian). Ústrédniho Ústavu Geologichkého Vèstnik, 42(4):275-284, 4 plates.

Marinelarena, M.M.P. de

1964. Gemelinmagas plicata, nuevo braquiópodo terciario de Patagonia y su atribución a Terebratella dorsata Gm. Ameghiniana, 3(9):266-271.
Markowskii, B. P., editor

1960. Novye Vidy Dreunikh Rastenii i Bespozvonochnykh SSSR. Part 1, 612 pages, 93 plates. Moscow: Vsesoiuznyi Nauchno-Issledovatelskii Geologicheskii Institut (VSEGEI).

1968. Nouye Vidy Dreunikh Rastenii i Bespozvonochnykh SSSR. Part 2, 440 pages, 69 plates. Moscow: Vsesoiuznyi Nauchno-Issledovatelskii Geologicheskii Institut (VSEGEI).

Martínez Chacón, M. L.

1975. Avisyrinx n. gen. (Syringothyrididae, Brachiopoda) del carbonifero de Asturias (España). Breviora Geologica Astúrica, 19(3):33-40, 1 plate.

1979. Braquiopodos carboniferos de la Cordillera Cantabrica (Orthida, Strophomenida y Rhynchonellida). Memoria del Instituto Geologico y Minero de España, 96:1-291, 32 plates.

Martínez Chacón, M. L., and C. F. Winkler Prins

1977. A Namurian Brachiopod Fauna from Meré (Province of Oviedo, Spain). Scripta Geologica, 39:1-67, 14 plates.

Martynova, M. V.

1961. Stratigrafiia i Brakhiopody Famenskogo Iarusa Zapadnoi Chasti Tsentral'nogo Kazakhstana. $\mathrm{Ma}$ terialy po Geologii Tsentral'nogo Kazakhstana, 2:1-210, 29 plates.

1970. [New Late Famennian Brachiopods of Central Kazakhstan.] Paleontologicheskii Zhurnal, 1970(1): 58-72, 2 figures, 2 plates. [In Russian.]

Martynova, M. V., and T. V. Sverbilova

1968. [First Members of the Spiriferidae from the Devonian of Kazakhstan.] Paleontologicheskii Zhurnal, 1968(2):26-31. [In Russian.]

1969. O Novom Posdnedevonskom Rode Spiriferid (Brachiopoda) iz Tsentral'nogo Kazakhstana. Vestnik Moskouskogo Universiteta, 4:96-99.

Marwick, J.

1953. Divisions and Faunas of the Hokonui System (Triassic and Jurassic). New Zealand Geological Survey, Palaeontology, Bulletin, 21:1-141, 17 plates.

Mascle, G. H., and G. Termier

1970. Nouvelles Données paléontologiques sur le permocarbonifere de Lercara Friddi (Sicile). Rivista Italiana di Paleontologia e Stratigrafia, 76(2):187-196, plates $17-18$.

Massa, D., V. Havlícek, and J. Bonnefous

1977. Stratigraphic and Faunal Data on the Ordovician of the Rhadames Basin (Libya and Tunisia). Bulletin des Centres de Recherches Exploration-Production Elf-Aquitaine, 1(1):3-27, 2 plates.

Massa, D., G. Termier, and H. Termier

1974. Le Carbonifere de Libye occidentale, stratigraphie et paléontologie. Compagnie Francaise des Pétroles, Notes et Mémoires, 11:139-206, 11 figures, 19 plates. 
Matthew, G. F.

1891. Illustrations of the Fauna of the St. John Group No.5. Royal Society of Canada, Proceedings and Transactions, series 1, 8(4):123-166, plates 11-16.

1893. Tremalobolus: An Articulate Brachiopod of the Inarticulate Order. Canadian Record of Science, 5:276279, 1 figure.

1895. Traces of the Ordovician System on the Atlantic Coast. Roval Society of Canada, Proceedings and Transactions, series 2, 1(4):253-279, 2 plates.

1897. The Oldest Siphonotrela. Geological Magazine, 4:6871.

1899. Preliminary Notice of the Etcheminian Fauna of Cape Breton. New' Brunswick Natural History Society, Bulletin, 4:198-208, 4 plates.

1901. Acrothyra, a New Genus of Etcheminian Brachiopods. Nere Brunswick Natural History Society, Bulletin, 4:303-304, 6 figures.

1902. Notes on Cambrian Fauna. Royal Society of Canada, Transactions, 8(4):93-112, 1 plate.

Maxwell, W.G.H.

1951. Upper Devonian and Middle Carboniferous Brachiopods of Queensland. Queensland Unversity Papers (Geology), 3(14):1-27, 4 plates.

1954. Upper Palaeozoic Formations in the Mt. Morgan District-Faunas. Queensland University Papers (Geology), 4(5): 1-69, 6 plates.

McClung, G., and J. Armstrong

1978. Species of Glendonia Gen. Nov. (Ingelarellinae: Spiriferida) in the Permian of Eastern Australia. Queensland Dept. of Mines, Geological Survey of Queensland, Publication, 365 (Palaeontological Paper 39): $1-9$.

McCoy, F.

1844. A Synopsis of the Characters of the Carboniferous Limestone Fossils of Ireland. 207 pages, 34 figures, 29 plates.

1851. On Some New Cambro-Silurian Fossils. Annals and Magazine of Natural History, series 2, 8:387-409.

McIntosh, M. J.

1974. Some Scottish Carboniferous Davidsoniacean Brachiopods. Scoltish Journal of Geology, 10(3): 199222, 2 figures, 6 plates.

McKellar, R. G.

1966. Devonian Marine Fossils from the Subsurface Adavale Basin, Queensland. Geological Survey of Queensland, Publication, 332:1-9, 1 plate.

1970. The Devonian Productoid Brachiopod Faunas of Queensland. Geological Survey of Queensland, Publication, 342 (Palaeontological Paper 18):1-40, 12 plates.

McLaren, D. J.

1961. Three New Genera of Givetian and Frasnian (Devonian) Rhynchonelloid Brachiopods. Institut
Royal des Sciences Nalurelles de Belgique, Bulletin, $37(23): 1-7,2$ plates.

McLearn, F. H.

1918. The Silurian Arisaig Series of Arisaig, Nova Scotia. American Journal of Science, 45:126-140.

1924. Palaeontology of the Silurian Rocks of Arisaig, Nova Scotia. Geological Survey of Canada, Memorr, 137:1-179, 30 plates.

Meek, F. B.

1865. Observations on the Microscopic Shell Structure of Spirifer cuspidalus, Sowerby, and Some Similar American Forms. Academy of Natural Sciences of Philadelphra, Proceedings, 17:275-277.

1872. Descriptions of a Few New Species, and One New Genus of Silurian Fossils, from Ohio. American Journal of Science, series 3, 4:274-281.

1873. Preliminary Palaeontological Report. 6th Annual Report of the U.S. Geological Survey of Montana, Idaho, Wyoming and Utah, 1872:429-518.

Meek, F. B., and A. H. Worthen

1870. Descriptions of New Species and Genera of Fossils from the Paleozoic Rocks of the Western States. Academy of Natural Sciences of Philadelphia, Proceedings, 22:22-56.

Megerle von Mühlfeld, J. K.

1811. Entwurf eines neuen System's der Schalthiergehäuse. Gesellschaft Naturforschender Freunde, Magazin, 5:38-72, plate 3 .

Melou, M.

1976. Orthida (Brachiopoda) de la formation de postolonnec (Ordovicien) Finistère, France. Geobios, 9(6):700-705.

Menakova, G. N.

1964. Brakhiopody iz Nizhnesiluriskikh Otlozhenii Zeravshano-Gessarskoi Gornoi Oblasti. Upravleniia Geologu i Okhrani Nedr Pr Sovete Minislerov Tadzhinskoi SSR (Paleontologiia a Stratıgrafiia), Trudy, 1:1174, 30 plates.

Mendes, J. C.

1959. Chonetacea e Productacea carboníferos da Amazônia. Sao Paulo, Universidade, Faculdade de Filosofia, Ciências e Lctras, Boletim, 236(Geologia, 17):1-83, 7 plates.

1961a. Langella, novo genero de lingulídco da série tubarão. Boletim da Universidade do Parana, Instituto de Geologia, 5:1-8, 2 plates.

1961b. Notas suplementares sôbre os braquiópodes carboníferos da Amazônia. Boletim da Sociedade Brasileira de Geologia, 10(1):5-24.

Menner, V. V., T. G. Sarytcheva, and G. E. Tschcrnjak

1970. Stratigrafiia Kamennougol'nykh i Permskikh Otlozhenii Severnogo Verkhoian'ia. Nauchno-Issledovatelskii Institut Geologiz Arktiki, Trudy, 154:1-191, 22 plates 
Mickwitz, A.

1896. Über die Brachiopodengattung Obolus Eichwald. Academie Impériale des Sciences, St. Pélersbourg. Mémoires, series $8,4(2): 1-215,3$ plates.

1909. Vorläufige Mitteilung über das Genus Pseudolingula Mickwitz. Academie Impériale des Sciences, St. Pétersbourg, Mémoires, series 6, 3:765-772, 3 figures.

Middlemiss, F. A.

1959. English Aptian Terebratulidae. Palaeontology, 2(1): 94-142, 20 figures, plates 15-18.

1968. Brachiopodes du Cretace Inferieur des Corbieres Orientales (Aude). Annales de Paleontologre, Invertebres, 54(2): 173-197, 3 plates.

1976. Lower Cretaceous Terebratulidina of Northern England and Germany and Their Geological Background. Geologisches Jahrbuch, 30:21-104.

Miloradovich, B. V.

1945. [Some Data on the Morphology of the Shells of Productidae.] Bulletin de L'Académie des Sciences de Union des Republiques Soviétiques Socialistes (Biologique), 4:485-500, 16 figures. [In Russian; English summary.]

1947. [On Two New Brachiopod Genera from the Upper Paleozoic of the Arctic.] Société Impériale des Naturalistes de Moscou, Bulletin (Geologique), 22(3):91-99. [In Russian.]

Minato, $\mathrm{M}$.

1951. On the Lower Carboniferous Fossils of the Kitakami Massif, Northeast Honshu, Japan. Hokkaido University, Journal of the Faculty of Science, series 4 (Geology and Mineralogy), 7(4):355-382, 5 plates.

1952. A Further Note on the Lower Carboniferous Fossils of the Kitakami Mountainland, Northeast Japan. Hokkaido University, Journal of the Faculty of Science, series 4 (Geology and Mineralogy), 8(2): 136-174.

1953. On Some Reticulate Spiriferidae. Palaeontological Sociely of Japan. Transactions and Proceedings, new series, 11:65-73.

Minato, M., and M. Kato

1977. A Reticulate Spiriferid from the Devonian Nakazato Formation of the Kitakami Mountains, Japan. Hokkaido University, Journal of the Faculty of Science, series 4 (Geology and Mineralogy), 18(4): 619-627, 1 plate.

Misius, P. P., and G. T. Ushatinskaia

1977. [New Ordovician and Silurian Strophomenids from Kazakhstan and Northern Khirgiz.] In G. A. Stukalina, editor, Novye Vidy Dreunikh Rasteniz i Bespozvonochnykh SSSR, 4:113-116. Moscow: Akademiia Nauk SSSR, Paleontologicheskii Institut; Nauchnyi Sovet po Probleme. [In Russian.]

Mitchell, J.

1921. Some New Brachiopods from the Middle Palaeo- zoic Rocks of New South Wales. Linnean Society of New South Wales, Proceedings, 45(2):543-551, plate 31.

Mitchell, J., and W. S. Dun

1920. The Atrypidae of New South Wales, with References to Those Recorded from Other States of Australia. Linnean Society of New South Wales, Proceedings, 45(2):266-276.

Mitchell, W. I.

1974. An Outline of the Stratigraphy and Paleoecology of the Ordovician Rocks of Central Portugal. Geological Magazine, 111(5):385-396.

1977. The Ordovician Brachiopoda from Pomeroy, Co. Tyrone. Palaeontographical Society Monograph, 130:1138, 28 plates.

Mittmeyer, H. G.

1965. Die Bornicher Schichten im Gebiet zwischen Mittelrhein und Idsteiner Senke (Taunus, Rheinisches Schiefergebirge). Notzzblatl des Hessischen Landesamtes für Bodenforschung zu Wiesbaden, 93:73-98, 5 figures 1 plate.

1972. Delthyrididae und Spinocyrtiidae (Brachiopoda) des tiefsten Ober-Ems im Mosel-Gebiet (EmsQuarzit, Rheinisches Schiefergebirge). Mainzer Geowerssenschaftluche Mitteilungen, 1:82-121, 2 plates.

Mittmeyer, H. G., and K. W. Geib

1967. Gliederung des Unterdevons im Gebiet Warmsroth-Wald-Erbach (Stromberger Mulde). Notizblatl des Hessischen Landesamles für Bodenforschung zu Wiesbaden, 95:24-44, 1 plate.

Mizens, L. I.

1975. [Levispira-a New Genus of Eifelian Atrypids of the Urals.] In Materialy po Paleontologii Srednego Paleozoia Urala i Kazakhstana. Akademiia Nauk SSSR, Ural'skii Nauchnyz Tsentr, Institut Geologii i Geokhimii, Trudy, 117:47-50. [In Russian.]

1977a. Novye Devonskie Atripidy Srednego i Severnogo Urala. In Novye Materialy po Paleontologii Urala. Akademiia Nauk SSSR, Ural'skii Nauchnyi Tsentr, Institut Geologii i Geokhimii, Trudy, 128:77-96.

1977b. [New Silurian Atrypids from the Eastern Slope of the Northern and Central Urals.] In Paleontologiia Nizhnego Paleozoia Urala. Akademiza Nauk SSSR, Ural'skii Nauchnyi Tsentr, Institut Geologii i Geokhimii, Trudy, 129:39-54. [In Russian.]

Mizens, L. I., and M. A. Rzonsnitzkaja

1979. [The Subfamily Punctatrypinae (Brachiopoda), Its Generic Composition, Distribution, and Evolution.] Paleontologicheskii Zhurnal, 1979(3):57-71. [In Russian.]

Mizens, L. I., and V. P. Sapelnikov

1975. Novyi Rod Atripid iz Silura Vostochnogo Sklona Urala. In Materialy po Paleontologii Srednego Paleozoia Urala i Kazakhstana. Akademiia Nauk 
SSSR, Ural'skii Nauchnyi Tsentr, Institut Geologii i Geokhimi, Trudy, 117:43-46.

Moberg, J. C., and C. O. Segerberg

1906. Bidrag till Kännedomen om Ceratopygeregionen med särskild hänsyn till dess Utveckling i Fogelsangstrakten. Lund Universitet, Arsskrfft, new series, 2(2): 1-113, 10 plates.

Modzalevskaja, T. L.

1970. Siluriiskie i Rannedevonskie Atiridy (Brakhiopody) Vaigacha. In S. V. Tcherkesova, editor, Stratigrafiia i Fauna Siluriiskikh Otlozhenii Vaigacha, pages 150-164, 2 plates. Leningrad: Nauchno-Issledovatelskii Institut Geologii Arktiki, Ministerstvo Geologii SSSR.

1979. K Sistematike Paleozoyskikh Atiridid. Paleontologicheskii Zhurnal, 1979(2):48-63.

Mohanti, M.

1972. The Portilla Formation (Middle Devonian) of the Alba Syncline, Cantabrian Mountains, Prov. Leon, Northwestern Spain: Carbonate Facies and Rhynchonellid Palaeontology. Leidse Geologische Mededelingen, 48(2):135-205, 10 plates.

Moisseiev, A. S.

1934. [Brachiopods of the Jurassic Deposits of the Crimea and the Caucasus.] Vsesoiuznyi Geologo-Razvedochnyi Obedineniya SSSR, Trudy, 203:1-213, 19 plates. [In Russian.]

1936. [New Triassic and Liassic Genera of the Rhynchonellidae.] Leningradskyi Obshchestva Estestvoispitatelei Otdelenie, Geologii i Mineralogie, Trudy, 65(1):39-50, plate 1. [In Russian.]

1939. [On the Stratigraphy and Brachiopods of the Lower Cretaceous Deposits of the Gagry Region (Abkhazia).] Uchenye Zapiski Leningradskii Gosudarstvennyi Universitet, 34 (Geologo-Pochvennyk Nauk, 7): 186-208, 2 plates. [In Russian.]

Möller, V. I.

1870. In Obyknovennoe zasedanie, II Fevralya 1869 Goda. Russische-Kaiserliche Mineralogische Gesellschaft zu St. Petersburg, Verhandlungen, series 2, 5:408413.

Moore, C.

1855. On New Brachiopoda, from the Inferior Oolite of Dundry, etc. Somersetshire Archaeological and Natural History Society, Proceedings, 5:107-128, plates 1-3.

Muir-Wood, H. M.

1928. The British Carboniferous Producti, II: Productus (sensu stricto) semireticulatus and longispinus Groups. Great Britain, Geological Survey (Palaeontology), Mernoir, 3(1):1-217, 12 plates.

1930. The Classification of the British Carboniferous Brachiopod Subfamily Productinae. Annals and Magazine of Natural History, series 10, 5(25):100108.
1934. On the Internal Structure of Some Mesozoic Brachiopoda. Royal Society of London, Philosophical Transactions, series B, 223:511-567, 2 plates.

1935. Jurassic Brachiopoda. In The Mesozoic Palaeontology of British Somaliland, 2:75-147, plates 7-13.

1936a. A Monograph on the Brachiopoda of the British Great Oolite Series. Palaeontographical Society Monograph, 89:1-144, 5 plates.

1936b. Brachiopoda from the Lower Lias, Green Ammonite Beds, of Dorset. Geological Society of London, Quarterly Journal, 92:472-485.

1952. Some Jurassic Brachiopoda from the Lincolnshire Limestone and Upper Estuarine Series of Rutland and Lincolnshire. Geologists' Association, Proceedings, 63(2): 113-142, plates 5-6.

1959. Report on the Brachiopoda of the John Murray Expedition. In John Murray Expedition 1933-1934, Scientific Reports, 10(6):283-31 7, 4 figures, 5 plates.

1962. On the Morphology and Classification of the Brachiopod Suborder Chonetoidea. 132 pages, 24 figures, 16 plates. London: British Museum.

1965. [New Name for Leptothyris Muir-Wood, 1959.] In Brachiopoda. In Raymond C. Moore, editor, Treatise on Invertebrate Paleontology, $\mathrm{H}(2): 855-856$.

Muir-Wood, H. M., and G. A. Cooper

1960. Morphology, Classification and Life Habits of the Productoidea (Brachiopoda). Geological Society of America, Memoir, 81:1-447, 135 plates.

Müller, O. F

1776. Zoologiae Danicae prodromus seu animalium Daniae et Norvegiae indigenarum characteres, nomina, et synonyma imprimis popularium. 282 pages. Havniae.

Munier-Chalmas, E.

1880. Sur Quelques Genres de brachiopodes. Bulletın de la Société Géologique de France, 8(4):279-280.

Nakamura, K., M. Kato, and D. R. Choi

1970. On Permundaria, a New Genus of the Brachiopod Family Linoproductidae. Hokkaido University, Journal of the Faculty of Science, series 4, 14(3):293-299, 1 plate.

Nalivkin, D. V.

1925. [The Group of Spirifer anossofi Verneuil in the Devonian of the European Part of the USSR.] Russische-Kaiserliche Mineralogische Gesellschaft zu St. Petersburg, Zapiski, 54(2):267-358. [In Russian.]

1930. [Brachiopods from the Upper and Middle Devonian of the Turkestan.] Comité Géologique, Mémoires, new series, 180:1-221, 10 plates. [In Russian.]

1937. [Brachiopoda of the Upper and Middle Devonian and Lower Carboniferous of Northeastern $\mathrm{Ka}$ zakhstan.] Tsentralnyi Nauchno-Issledovatelskii Geologo-Razvedochnyi Instztut, Trudy, 99:1-200, 39 plates. [In Russian.]

1941. Brakhiopody Glavnogo Devonskogo Polya. In M 
A. Batalina et al., Fauna Glaunogo Devonskogo Polya, 1:139-226, 8 plates. Moscow: Akademiia Nauk SSSR, Paleontologicheskii Institut.

1947. Devonien. Atlas of the Guide Forms of the Fossil Faunas of the USSR, 3: 245 pages, 56 plates. Moscow: Vsesoiuznyi Nauchno-Issledovatelskii Geologicheskii Institut (VSEGEI), Ministcrstvo Geologii SSSR. [In Russian; English title page.]

1951. Fauna Verkhnego i Srednego Devona Vostochnogo Sklona Bashkirskogo Urala. 66 pages, 9 plates. Moscow: Vsesoiuznyi Nauchno-Issledovatel'skii Geologicheskii Institut (VSEGEI) Ministerstvo Geologii SSSR.

1979. Brakhıopody Turneiskogo Iarusa Urala. 248 pages, 65 plates. Leningrad: Akademiia Nauk SSSR, Otdelenie Geologii, Geofiziki i Geokhimi.

Nazarov, B. B., and L. E. Popov

1976. [Radiolaria, Inarticulate Brachiopods and Organisms of Uncertain Systematic Position from the Middle Ordovician of Eastern Kazakhstan.] $\mathrm{Pa}$ leontologicheskii Zhurnal, 1976(4):33-42. [In Russian.]

Nekvasilova, O.

1973. The Brachiopod Genus Bohemirhynchia gen. n. and Cyclothyris McCoy (Rhynchonellidae) from the Upper Cretaceous of Bohemia. Sbornik Geologickych Věd (Paleontologie), 15:75-118, 20 figures, 12 plates.

Nelson, S. J., and C. E. Johnson

1968. Permo-Pennsylvanian Brachythyrid and Horridonid Brachiopods from the Yukon Territory, Canada. Journal of Paleontology, 42(3):715-746, plates 91-96.

Neuman, R. B.

1964. Fossils in Ordovician Tuffs, Northeastern Maine. U.S. Geological Survey Bulletin, 1181-E:1-38, 7 plates.

1971. An Early Middle Ordovician Brachiopod Assemblage from Maine, New Brunswick, and Northern Newfoundland. In J. T. Dutro, Jr., editor, Paleozoic Perspectives: A Paleontological Tribute to G. Arthur Cooper. Smithsonian Contributions to Paleobiology, 3:113-124, 2 plates.

1977. Early Ordovician (Late Arenig) Brachiopods from Virgin Arm, New World Island, Newfoundland. Geological Survey of Canada, Bulletin, 261:11-61, 8 plates.

Neuman, R. B., and D.E.B. Bates

1978. Reassessment of Arenig and Llanvirn Age (Early Ordovician) Brachiopods from Anglesey, NorthWest Wales. Palaeontology, 21(3):571-613, plates 63-68.

Neuman, R. B., and D. L. Bruton

1974. Early Middle Ordovician Fossils from the Ho- londa Area, Trondheim Region, Norway. Norsk Geologisk Tidsskrift, 54(1):69-115, 4 figures, 12 plates.

$\mathrm{Ni}$, Shi-zhao, and De-li Yang

1977. In Zhong Nan Di Qu Gu Sheng Wu Tu Ce, Er, Wan Gu Sheng Dai Bu Feng [Paleontological Atlas of Central-South China, Part 2 (Late Paleozoic Part)]. 856 pages, 253 plates. Peking: Geologic Press. [In Chinese.]

Nikiforova, O. I.

1937. [Upper Silurian Brachiopoda of the Central Asiatic Part of the U.S.S.R.] Akademiia Nauk SSSR, Paleontologicheskii Institut, Monographii po Paleontologii SSRR, 35(1):1-94, 14 plates. [In Russian.]

1960. [Kulumbella, a New Genus of the Family Stricklandiidae.] Paleontologicheskii Zhurnal, 1960(3):61-64, 1 plate. [In Russian.]

1964. [Certain New Silurian Brachiopoda from the Chatkal Mountains.] Vsesoiuznyi Nauchno-Issledovatelskii Geologicheskii Institut (VSEGEI), Trudy, 93: 81-90, 1 plate. [In Russian.]

1970. Brakhiopody Grebenskogo Horizonta Vaigacha (Pozdnii Silur). In S. V. Tcherkesova, editor, Stratigrafiia 2 Fauna Siluriiskikh Otlozhenii Vaigacha (Sbornik Statei), pages 97-149. Leningrad: NauchnoIssledovatelskii Institut Geologii Arktiki: Ministerstvo Geologii SSSR.

1978. Pogranichnye Sloi Ordovika i Silura Altae-Saianskoi Oblasti i Tian'-Shania. Akademiia Nauk SSSR, Sibirskoe Otdelenie, Institut Geologii i Geofiziki, Trudy, 397:102-126.

Nikiforova, O. I., and O. N. Andreeva

1961. [Ordovician and Silurian Stratigraphy of the Siberian Platform and Its Paleontological Basis (Brachiopoda).] Vsesoiuznyi Nauchno-Issledovatelskii Geologicheskii Institut (VSEGEI), Trudy, new series, 56:1-412, 56 plates. [In Russian.]

Nikiforova, O. I., and T. L. Modzalevskaja

1968. [A New Brachiopod Genus Dnestrina from the Silurian, Moldavia and Podolsk.] Ezhegodnik Vsesoiuznogo Paleontologicheskogo Obshchestva, 18:206-211. [In Russian.]

Nikiforova, O. I., and V. P. Sapelnikov

1971. [New Early Silurian Virgianidae (Brachiopoda).] Paleontologicheskii Zhurnal, 1971(2):47-56, 3 figures, 2 plates. [In Russian.]

1973. Nekotorye Drevnie Pentameridy Zeravshanskogo Khrebta. Akademiia Nauk SSSR, Uralskii Nauchnyi Tsentralnyi, Institut Geologii i Geografii, Trudy, 99:6481.

Nikitin, I. F.

1974. [New Middle Ordovician Plectambonitacea (Brachiopoda) from Kazakhstan.] Paleontologicheskii Zhurnal, 1974(3):55-66, 2 plates. [In Russian.] 
Nikolayev, A. A., and V. P. Sapelnikov

1969. TTwo New Genera of Late Ordovician Virgianidae.] Sverdlouskogo Ordena Trudov'ogo Krasnogo Znameni Gornogo Instituta im 5.V. Vakhrusheva, Trudy, 63:1117. [In Russian.]

Nilova, N. V.

1965. Brakhiopody Karaespinskogo Horizonta. In Straligrafiia Nizhnepaleoizorskikh i Siluriiskikh Otlozhenii Tsentral'nogo Kazakhstana, pages 91-111, 3 plates. Leningrad: VSEGEI: Tsentralno-Kazakhstanskoe Geologicheskoe Upravlenie (TSKGU) Sbornik Statei.

North, F. J.

1920. On Syringothyris Winchell and Certain Carboniferous Brachiopoda Referred to Spiriferina d'Orbigny. Geological Society of London, Quarterly Journal, 76(2): 162-227, plates 11-13.

Northrop, S. A.

1939. Paleontology and Stratigraphy of the Silurian Rocks of the Port Daniel-Black Cape Region, Gaspé. Geological Soczety of America, Special Paper, 21 : 1-302, I figure, 28 plates.

Nutsubidze, K.

1945. Les Brachiopodes du crétacé inférieure de la Géorgie occidentale. Travaux de l'Instztul Géologıque, Academie des Sciences de la RSS Géorgienne, 2(7):1-240, 4 plates.

Oehlert, D. P.

1890. Note sur différents groupes établis dans le genre Orthis et en particulier sur Rhipidomella Oehlert (= Rhipidomys Oehlert, olim). Journal of Conchyliologie, series 3, 30:366-374.

Oliveira, E. de

1934. Oliveirella, nomen novum para designar os brachiopodos do genero Brasilia Clarke 1913, da familia Centronellidae, Hall and Clark. Academia Brasileira de Sciencias, Annaes, 6(3):167-168.

Öpik, A

1930. Brachiopoda Protremata der estländischen Ordovizischen Kukruse-Stufe. Tarlu Unwersitatis (Dorpatensis), Acta and Commentationes, series A, 17(1):1262, 22 plates.

1932. Über die Plectellinen. Tartu Universitatzs (Dorpatensis), Acta and Commentationes, series A, 23(3):1-85, 12 plates.

1933a. Über Plectamboniten. Tartu Universitatis (Dorpatensis), Acta and Commentationes, 24(7):1-79, 12 plates.

1933b. Über einige Dalmanellacea aus Estland. Tartu Universitatis (Dorpatensis), Acta and Commentationes, series A, 25(1):1-25.

1934. Über Klitamboniten. Tartu Universitatis (Dorpatensis), Acta and Commentatzones, series A, 26(3):1-239, 48 plates.

1939. Brachiopoden und Ostrakoden aus dem Expan- susschiefer Norwegens. Norsk Geologisk Tidsskrift, 19:117-142, 6 plates

1953. Lower Silurian Fossils from the Illaenus Band, Heathcote, Victoria. Victoria Geological Survey, Memoirs, 19:1-42, 13 plates.

Orbigny, A. d'

1847. Considérations zoologiques et géologiques sur les brachiopodes ou palliobranches. Académe des Sciences de Paris, Comples Rendus, 25:266-269.

1850. Memoire sur les Brachiopodes. Annales des Sciences Naturelles, 3rd series (Zoologie), 13:295-353.

Ovtsharenko, V. N.

1975. [Some New Aspects of the Morphology, Systematics and Phylogeny of the Mesozoic Rhynchonellid Brachiopods.] In Voprosy Paleontologii Tadzhikistana, pages 121-138, I plate. Dushanbe: Vsesoiuznoe Paleontologicheskoe Obshchestvo. [In Russian.]

1976. [Basis of the Taxonomic Status of the Family Gusarellidae (Brachiopoda).] Akademiia Nauk Tadzhikistan SSR, Izvestiya (Otdelente Biologicheskikh Nauk), 4(65):20-29, 7 figures. [In Russian.]

1979. [A New Generic Name Proposed for the Preoccupied One in Jurassic Brachiopods.] Paleontologicheskii Zhumal, 1979(3): 126. [In Russian.]

Owen, E. F.

1962. The Brachiopod Genus Cyclothyris. British Museum (Natural History), Bulletin (Geology), 7(2):37-63, 2 plates.

1965. Some Lower Cretaceous Terebratelloidea. British Museum (Natural History), Bulletın (Geology), 11(2): 49-72, 3 plates.

1968. A Further Study of Some Cretaceous Rhynchonelloid Brachiopods. Indian Geologists Association Bulletin, 1: 17-32.

1970. A Revision of the Brachiopod Subfanily Kingeninae Elliott. British Museum (Natural History), Bulletin (Geologv), 19(2):29-83, 14 plates.

1977. Evolutionary Trends in Some Mesozoic Terebratellacea. British Museum (Natural History), Bulletin (Geology), 28(3):207-253.

Ozaki, Kin-emon

1931. Upper Carboniferous Brachiopods from North China. Shanghat Science Institute Bulletin, 1(6):1-205, 15 plates.

Paeckelmann, W.

1930. Die Fauna des deutschen Unterkarbons, I: Die Brachiopoden, I. Königlich Preussichen Geologischen Landesanstall, Abhandlungen, new series, 122:1-326, platcs 9-24.

1931a. Die Fauna des deutschen Unterkarbons, II: Die Brachiopoden, II. Königlich Preussichen Geologischen Landesanstalt, Abhandlungen, new series, 136:1-440, 41 plates. 
1931b. Versuch einer zusammenfassenden Systematik der Spiriferidae King. Neues Jahrbuch für Mineralogie, Geologie, und Palaeontologie, series B, 67:1-64.

Pajaud, D.

1963. Note sur les Thecideidae jurassiques. Société Géologique de France, Bulletin, series 7, 5(6):995-999.

1964. Ramarques sur les Thecideidae (Brachiopodes) tertiaires, sur une nouvelle forme du Miocène rhodanien: Glazewskıa demarcqui nov. gen., nov. sp. Société Géologique de France, Bulletin, series 7, 6(2):258-261.

1966a. Deux genres nouveaux de Thécidées (Brachiopodes): Parabifolium (P. priscum n. sp.) et Danella (D. fragilis n. sp.). Compte Rendu Sommaire des Séances de la Société Géologique de France, 2:70-71.

1966b. Sur deux nouvelles formes de Thécidées (Brachiopodes), dont le genre nouveau Backhausina (B. rugosa D'Orb). Compte Rendu Sommaire des Séances de la Société Géologique de France, 3:124-125.

1966c. Problèmes relatifs a la détermination des espèces chez les Moorellininae (Thecideidae, Brachiopodes). Société Géologique de France, Bulletin, series 7 , 8:630-637.

1970. Monographie des Thécidées (Brachiopodes). Memoires de la Société Géologique de France, new series, 49: 1-349, 16 plates.

1976. Les Brachiopodes du pliocène 1 de la Sierra de Santa Pola (sud d'Alicante, Espagne): Terebratula terebratula (Linné, 1758) et Phapsirhynchia sanctapaulensis nov. gen., nov. sp. Annales de la Société Géologique du Nord, 96(2):99-106, 1 plate.

Pajaud, D., and D. Patrulius

1964 [1965]. Sur la presence de trois espèces de Thecideidae (Brachiopodes) en Roumanie. Société Géologique de France, Bulletin, series 7, 6(4):579-585.

Palmer, A. R.

1954. The Faunas of the Riley Formation in Central Texas. Journal of Paleontology, 28(6): 709-786, plates $86-92$.

Pander, C. H.

1830. Beiträge zur Geognosie des Russischen Reiches. 165 pages, 31 plates.

1861. In G. von Helmersen, Die geologische Beschaffenheit des untern Narovathals und die Versandung der Narovamündung. Académie Impériale des Sci-

Paulus, B. ences, St. Pétersbourg, Bulletin, 3:46-49, plate 2.

1957. Rhynchospirifer $\mathrm{n}$. gen. im Rheinischen Devon. Rhynchospiriferinae n. subfam., Brachiopoda). Senckenbergiana Lethaea, 38(1/2):49-72, 3 plates.

Paulus, B., W. Struve, and R. Wolfart

1963. Chimaerothyris n. g. (Spiriferacea) aus dem Eifelium der Eifel. Senckenbergiana Lethaea, 44(6):459-497, plates 63-66.
Pavlova, E. E.

1965. Revision of the Genus Neophricodothyris (Order Spiriferida).] Paleontalogicheskii Zhurnal, 1965(2): 133-137. [In Russian.]

1969. [The Development of Brachiopods of the Family Reticulariidae.] Akademiia Nauk SSSR, Paleontologicheskii Institut, Trudy, 120:1-129, 13 plates. [In Russian.]

Pearson, A. B.

1977. Rhaetian Brachiopods of Europe. Neue Denkschriften des Naturhistorischen Museums in Wien, 1:1-85, 22 figures, 7 plates.

Peetz, H. von

1898. Hemiplethorhynchus Novyi Podrod Roda Camarotoechia Hall. Travaux Société Histoire Naturelle St. Pétersbourg, 29(1):178-182, 4 figures.

1901. Beiträge zur Kenntniss der Fauna aus den devonischen Schichten am Rande des Steinkohlenbassins von Kuznetz. Travaux Section Geologique du Cabinet de la Majesté St. Pétersbourg, 4:1-394, 6 plates.

Pelman, Yu. L.

1977. Early and Middle Cambrian Inarticulate Brachiopods from the Siberian Platform.] Akademiia Nauk SSSR, Sibirskoe Otdelenie, Instıtut Geologii i Geofizikz, Trudy, 316:1-168, 24 plates. [In Russian.]

Peou, S.

1979. A New Athyridacean Brachiopod from the Carboniferous of Rawdon Vale, New South Wales. Alcheringa, 3(3):189-195, 7 figures.

Percival, I. G.

1978. Inarticulate Brachiopods from the Late Ordovician of New South Wales, and Their Palaeoecological Significance. Alcheringa, 2:117-141.

1979a. Ordovician Plectambonitacean Brachiopods from New South Wales. Alcheringa, 3(2):91-116.

1979b. Late Ordovician Articulate Brachiopods from Gunningbland, Central Western New South Wales. Proceedings of the Linnean Society of New South Wales, 103(3):175-187, 2 plates.

Perner, $J$.

1903. Gasteropodes. In J. Barrande, Systême silurien du centre de la Bohême, 4:1-164, 21 plates. Prague.

Perry, D. G., and B.D.E. Chatterton

1979. Late Early Triassic Brachiopod and Conodont Fauna, Thaynes Formation, Southeastern Idaho. Journal of Paleontology, 53(2):307-319, 6 figures, 2 plates.

Pettitt, N. E.

1950. A Monograph on the Rhynchonellidae of the Brtish Chalk. Part 1, pages 1-26, 2 plates. London: Palaeontographical Society.

1954. A Monograph on the Rhynchonellidae of the Britzsh 
Chalk. Part 2, pages 27-52, 1 plate. London: Palaeontographical Society.

Philip, G. M.

1962. The Palaeontology and Stratigraphy of Siluro-Devonian Sediments of the Tyers Area, Gippsland, Victoria. Royal Society of Victoria, Proceedings, 75(2): 123-246, plates 11-35.

Phillips, J.

1841. Figures and Descriptions of the Palaeozoic Fossils of Cornwall, Devon, and West Somerset. Geological Survey of Great Britain, Memoir, 1:1-231, 60 plates.

Pitrat, C. W.

1975. Orthospirifer New Genus of Devonian Spinocyrtiid Brachiopods. Journal of Paleontology, 49(2):387-394, 2 plates.

Plodowski, G.

1968. Neue Spiriferen aus Afghanistan. Senckenbergzana Lethaea, 49(2/3):251-258, 1 plate.

1973. Rhynchonellacea aus den Zentralkarnischen Alpen. Senckenbergiana Lethaea, 54(1):65-103, 5 plates.

Pojariskaja, G. F.

1966. [On Some Dallinidae (Brachiopoda) from the Jurassic of the Gissar Range.] Paleontologicheskii Zhurnal, 1966(3):22-30, 8 figures. [In Russian.]

Poletaev, V. I

1971. In D. E. Aisenverg, editor, Atlas fauny turneiskikh ollozhenii Donetškogo basseina [Atlas of the Tournaisian Fauna in the Donets Basin (with Description of the New Species)], pages 1-327. Kiev: Akademiia Nauk Ukrainskoi SSR, Institut Geologicheskikh Nauk.

1975. Rannekamennougolnye i Bashkirskie Gladkie Spinferidy I Atripidy Donetskogo Basseina. 140 pages, 27 plates. Kiev: Akademiia Nauk Ukrainskoi SSR, Institut Geologicheskikh Nauk.

Poli, G. S.

1791. Testacea Utriusque Siciliae Eorumque Historia Naturalis et Anatome Tabulis Aeneis Illustrata. Volume 1.

Popiel-Barczyk, E.

1968. Upper Cretaceous Terebratulide (Brachiopoda) from the Middle Vistula Gorge. Prace Muzeum Ziemi, 12:3-86.

1972. Albian-Cenomanian Brachiopods from the Environs of Annopol on the Vistula with Some Remarks on Related Species from Cracow Region. Prace Muzeum Ziemi, 20:119-149.

Popiel-Barczyk, E., and T. N. Smirnova

1978. [A New Megathyrid Genus (Brachiopoda) from the Upper Cretaceous of Poland.] Paleontologicheskii Zhurnal, 1978(3):134-137. [In Russian.]

Poulsen, C.

1943. The Fauna of the Offley Island Formation, Part II: Brachiopoda. In The Silurian Faunas of North
Greenland, II. Meddelelser om Gronland, 72(3):1-60, 6 plates.

Prendergast, K. L.

1935. Some Western Australian Upper Palaeozoic Fossils. Royal Society of Western Australia, Journal, 21:9 35,3 plates.

Prentice, J. E.

1950. The Genus Gigantella Sarycheva. Geological Magazine of London, 87(6):436-438.

Prosorovskaja, E. L.

1962. [Some New Brachiopods from the Upper Jurassic of Western Turkmen.] Vesinik Leningradskogo Universiteta (Geologii i Geografiia), 12(2):108-114, 5 figures. [In Russian.]

1968. [On a New Species of Late Jurassic Brachiopod from the Gissorskiy Range.] Ezhegodnik Russkogo Paleontologicheskogo Obshchestva, 18:244-249, 1 plate. [In Russian.]

Quenstedt, F. A.

1868-1871. Brachiopoden. In Petrefactenkunde Deutschlands, 2: 748 pages, atlas, plates 37-61. Tübingen and Leipzig.

Racheboeuf, P. R.

1976. Chonetacea (Brachiopods) from the Lower Devonian of the Laval Basin (Armorican Massif). Palaeontographica, series A, 152(1-3):14-89, 10 plates.

1979. Chonetacea (Brachiopodes) du Devonien du Boulonnais (Nord de la France). Annales Société Géologique du Nord, 98:247-261, 2 plates.

Ramovs, A.

1969. Karavankininae, Nova Poddruzina Produktid (Brachiopoda) iz Alpskih Zgornjekarbonskih in Permijskih Skladov. Jeseniski Zbornik Jeklo in Ljudje, 2:251-260, 3 plates.

Ramsbottom, W.H.C.

1952. The Fauna of the Cefn Coed Marine Band in the Coal Measures at Aberbaiden, near Tondu, Glamorgan. Great Britain, Geological Survey, Palaeontology, Bulletin, 4:8-32.

Raymond, P. E.

1911. The Brachiopoda and Ostracoda of the Chazy. Carnegie Museum, Annals, 7(2):215-259, plates 3336.

1923. New Fossils from the Chapman Sandstone. Boston Society of Natural History, Proceedings, 36(7):467-472, 1 plate.

Redlich, K. A.

1899. The Cambrian Fauna of the Eastern Salt-Range. Geological Survey of India, Memoirs, Palaeontologia Indica, new series, 1(1):1-13, 1 plate.

Reed, F.R.C.

1917. The Ordovician and Silurian Brachiopoda of the Girvan District. Royal Society of Edinburgh, Transactions, 51(4):795-998, 24 plates. 
1933. Notes on Some Lower Palaeozoic Fossils from the Southern Shan States. Geological Survey of India, Records, 66(2): 181-211, plate 3.

1935. Palaeontological Evidence of the Age of the Craighead Limestone. Geological Society of Glasgow, Transactions, 19:340-372.

1936. The Lower Palaeozoic Faunas of the Southern Shan States. Palaeontologia Indica, 21(3):1-130, 7 plates.

1943. Notes on Certain Upper Devonian Brachiopods Figured by Whidborne, Part 1-2. Geological Magazine of London, 80(2-3):69-78, 95-106.

1944. Brachiopoda and Mollusca from the Productus Limestone of the Salt Range. Palaeontologia Indica, new series, 23(2):1-678, 65 plates.

1949. Notes on Some Carboniferous Spiriferidae from Fife. Annals and Magazine of Natural History, series 12, 1(7):449-487, plates 7-12.

1954. Lower Carboniferous Brachiopods from Scotland. Leeds Philosophical Society, Proceedings, 6(3):180-189, 3 plates.

Renouf, J. T.

1972. Brachiopods from the Grès à Orthis monnieri Formation of Northwestern France and Their Significance in Giedinnian/Siegenian Stratigraphy of Europe. Palaeontographica, series A, 139(4-6):89170, 11 figures 6 plates.

Retzius, A. J.

1781. Crania oder Todtenkopfs-Muschel. Schriften der Berlinschen Gesellschaft Naturforschender Freunde, 2:66-76, plate 1 .

Richardson, J. R.

1973. Studies on Australian Cainozoic Brachiopods, 2: The Family Laqueidae (Terebratellidae). Royal Society of Victoria, Proceedings, 86(1):117-126.

Rioult, $M$.

1966. Présence de Sclérites de Brachiopodes dans 1 'Oxfordien de Normandie. Société Linnéenne de Normandie, Bulletin, series 10, 6:71-76, 1 plate.

Roberts, J.

1963. A Lower Carboniferous Fauna from Lewinsbrook, New South Wales. Royal Society of New South Wales, Journal and Proceedings, 97:1-29, 6 plates.

1964. Lower Carboniferous Brachiopods from Greenhills, New South Wales. Journal of the Geological Society of Australia, 11(2):173-194. .

1971. Devonian and Carboniferous Brachiopods from the Bonaparte Gulf Basin, Northwestern Australia. Australia, Bureau of Mineral Resources, Geology and Geophysics, Bulletin, 122:1-319, 104 figures, 59 plates.

1976. Carboniferous Chonetacean and Productacean Brachiopods from Eastern Australia. Palaeontology, 19(1):17-77, plates 3-13.
Roberts, J., J. W. Hunt, and D. M. Thompson

1976. Late Carboniferous Marine Invertebrate Zones of Eastern Australia. Alcheringa, 1:197-225, 17 figures.

Rollet, A. (= A. Boullier)

1964. Etude des térébratules des Marnes dites à Rhynchonella varians du jura septentrional. Annales Scientific de l'Université de Besançon, series 2, 19:27-44, 2 plates.

1966. Définition d'un genre de térébratulidés: Gallientthyris. Comple Rendu des Séances de la Sociélé Géologique de France, 8:304-306.

1972. Argovithyris nov. gen. et $A$. birmensdorfensis (Brachiopoda) des couches a spongiaires de l'oxfordien moyen du jura. Geobios, 5(1):91-119, 14 figures, 1 plate.

Rollier, L.

1919. Synopsis des spirobranches (brachiopodes) jurassiques celto-Souabes. Société Paléontologique de la Suisse, Mémoires, 44(4):279-422.

Rong, Jia-yu

1974. In Xi Nan Di Qu Di Ceng Gu Sheng Wu Shou Ce [A Handbook of the Stratigraphy and Paleontology in Southwest China]. 454 pages, 202 plates. Peking. [In Chinese.]

Rong, Jia-yu, Han-kui Xu, and Xue-Chang Yang

1974. In Xi Nan Di Qu Di Ceng Gu Sheng Wu Shou Ce [A Handbook of the Stratigraphy and Paleontology in Southwest China]. 454 pages, 202 plates. Peking. [In Chinese.]

Rong, Jia-yu, and Xue-chang Yang

1974. In Xi Nan Di Qu Di Ceng Gu Sheng Wu Shou Ce [A Handbook of Stratigraphy and Paleontology in Southwest China]. 454 pages, 202 plates. Peking. [In Chinese.]

Rōōmusoks, A. K.

1956. [Luhaia a New Genus of Strophomenids from the Upper Ordovician of Estonia.] Akademiia Nauk SSSR, Doklady, 106(6): 1091-1092, 1 plate. [In Russian.]

1959. [Strophomenoidea of the Ordovician and Silurian of Estonia, 1: Genus Sowerbyella Jones.] Tartu Universitatis (Dorpatensis) Acta and Commentationes, 75 11-41, 8 plates. [In Russian.]

1963. [Strophomenoidea of the Ordovician and Silurian of Estonia, 2: New Genera and Species from the Kharovsky Series.] Eesti NSV Teaduste Akadeemia, Toimetised, Bioloogiline Seeria, 12(3):231-241, 2 plates. [In Russian.]

Rosenkrantz, A.

1964. Notes on Some Cranias from Central Poland. Acta Palaeontologica Polonica, 9(4):513-538, 5 plates.

Ross, R. J., Jr.

1959. Brachiopod Fauna of Saturday Mountain For- 
mation, Southern Lemhi Range, Idaho. U.S. Geological Surcey, Professional Paper, 294-L:437-461, 3 plates.

1970. Ordovician Brachiopods, Trilobites and Stratigraphy in Eastern and Central Nevada. U.S. Geological Survey, Professional Paper, 639:1-103, 19 plates.

1971. A New Middle Ordovician Syntrophopsid Genus. In J. T. Dutro, Jr., editor, Paleozoic Perspectives: A Paleontological Tribute to G. Arthur Cooper. Smithsonian Contributions to Paleobiology, 3:125-128.

Rotai, A. P.

1931. NNew Representatives of Brachiopoda from the Lower Carboniferous of the Donetz Basin.] Glavnogo Geologo-Razzedochnogo Upravleniya, Trudy, 73:134, 2 plates. [In Russian.]

Rousselle, L.

1977. Spiriférines du Lias moyen et supérieur au Maroc (Rides prérifaines: Moyen Atlas) et en Espagne (Chaine celtibérique orientale). Notes du Senuce Geologique du Maroc, Noles el Memoires, 268, 38:153175,15 figures, 1 plate.

Rowell, A. J.

1962. The Genera of the Brachiopod Superfamilies Obolellacea and Siphonotretacea. Journal of Paleontology, 36(1):136-152, 2 plates.

1963. Some Nomenclatural Problems in the Inarticulate Brachiopods. Genlogical Magazine, 100(1):33-43.

1966. Revision of Some Cambrian and Ordovician Inarticulate Brachiopods. University of Kansas Paleonlological Contributions, 7:1-36, 33 figures, 4 plates.

1977. Early Cambrian Brachiopods from the Southwestern Great Basin of California and Nevada. Journal of Paleontology, 51 (1):68-85.

Rowell, A. J., and R. A. Henderson

1978. New Genera of Acrotretids from the Cambrian of Australia and the United States. Unversity of Kansas Paleontological Contributions, 93:1-12, 2 plates.

Rozman, Kh. S.

1962. IStratigraphy and Brachiopods of the Famennian Stage of the Mugodzhar and Adjacent Areas.] Akademiia Nauk SSSR, Institul Geologicheskogo, Trudy, 50:1-196, 49 figures, 31 plates. [In Russian.]

1967. New Early Paleozoic Genus Eoconchidium (Pentameracea) from the Selennyakh Ridge (North-castern USSR). Paleontologicheskn Zhurnal, 1967(2):6268. [In Russian.]

1969. [Late Ordovician Brachiopods of the Siberian Platform.] Paleontologicheskii Zhurnal, 1969(3):86108. [In Russian.]

1977. [A Pseudopunctate Davidsonacean (Brachiopoda) from the Lower Ashgillian of Mongolia.] Paleontologicheskil Zhurnal, 1977(4):124-127. [In Russian.]

1978. Pogranichnyi Sloi Ordovika i Silura Altae-Saian- skoi Oblatsi i Tian-Shania. Akademiia Nauk SSSR, Sibirskoe Otdelenie, Institut Geologii , Geofiziki, Trudy, 397:75-102.

Rübel, M. P.

1961. [Lower Ordovician Brachiopods of the Superfamilies Orthacea, Dalmanellacea, and Syntrophiacea of Eastern Baltic.] Eesti NSV Teaduste Akadeemia Geoloogia Instiluudi Uurimused, Trudy, 6:141-226, 24 figures, 27 plates. [In Russian.]

Rübel, M. P., and T. Modzalevskaja

1967. Novye Siluriiskii Brakhiopody Semeistva Athyrididae. Eest NSV Teaduste Akadeemia Toimetised, (Keemıa-Geoloogıa), 16(3):238-249, 3 figures, 4 plates.

Rukavischnikova, T. B.

1956. [Ordovician Brachiopods from Kazakhstan.] Akademiia Nauk SSSR, Geologicheskii Instilul, Trudy, 1: 105-168, 5 plates. [In Russian.]

1977. [New Early Silurian Nalivkiniids from Eastern Kazakhstan.] In G. A. Stukalina, editor, Novye Vidy Dreunikh Rastenn ? Bespozvonochnyk SSSR, 4:134-137. Moscow: Akademiia Nauk SSSR, Paleontologicheskii Institut; Nauchnyi Sovet po Probleme. [In Russian.]

Rukavischnikova, T. B., and V. P. Sapelnikov

1973. Novye Pozdneashgillskie Pentameracea Kazakhstan. Akademiia Nauk SSSR, Uralskï Nauchnyi Tsentr, Institut Geologit i Geokhimi, Trudy, 99:89-109.

Ruzhentsev, B. E., and T. G. Sarytcheva

1965. Razvitie i Smena Morskikh Organizmov na Rubezhe Paleozoiia i Mezozoiia. Akademiia Nauk SSSR, Paleontologicheskii Instıtul, Trudy, 108:1-431, 58 plates.

Rzonsnitzkaja, M. A.

1952. Spriferidy Devonskzkh Ollozheniz Okrain Kuznetskogo Basseina |Spiriferids from Devonian Deposits at the Edge of the Kuznetsk Basin]. 232 pages, 25 plates. Moscow: Vsesoiuznyi Nauchno-Issledovatelskii Geologicheskii Institut (V'SEGEI).

1959. [Systematics of the Rhynchonellids.] Paleontologicheskii Zhurnal, 1959(1):25-36. [In Russian.]

1960. Rod Gruenwaldtia Tschernyschew v Devone SSSR. Nauchno-Issledoratelskii Instrtut Geologii Arktiki Mnnisterstva Geologii z Okhrany Nedr SSSR; Sbornik Statei po Paleontologii i Biostratigrafii, 20:45-50, 2 plates.

1964. [On Devonian Atrypidae from the Kuznetz Basin.] I'sesomznyi Nauchno-Issledovatelskii Geologicheskii Institut (ISEGEI), Trudy, 93:91-112, 5 figures, 2 plates. [In Russian.]

1973. Brakhiopody. In Stratıgrafiya SSSR, Devonskaya Sistema, 2:376 pages. Moscow: Ministerstvo Geologii SSSR, Akademiia Nauk SSSR, Ministerstvo Vysshego i Srednego Spetsial'nogo Obrazovaniya SSSR.

1975. Biostratigrafiia Devona Okrain Kuznetskogo Bas- 
seina. Vsesoiuznyı Ordena Lenina Nauchno-Issledovatelskii Geologicheskii Institut, Trudy, 244:1-232, 34 plates.

1977. [New Devonian Rhynchonellids of Central Asia and the Urals.] In G. A. Stukalina, editor, Noyve Vidy Dreunikh Rastenii i Bespozvonochnykh SSSR, 4: 127-130. Moscow: Akademiia Nauk SSSR, Paleontologicheskii Institut: Nauchnyi Sovet po Probleme. [In Russian.]

1978. Obzor Devonskikh Rinkhonellid Okrain Kuznetskogo Basseina. Ezhegodnik Vsesoiuznogo Paleontologicheskogo Obshchesiva, 21:174-196.

Rzonsnitzkaja, M. A., B. K. Licharew, and V. P. Makridin 1960. Mshanki, Brakhiopody. In T. G. Sarytcheva, editor, Otryad Rhynchonellid. In Yu. A. Orlov, editor, Osnovy Paleontologii, pages 239-257.

Rzonsnitzkaja, M. A., and L. I. Mizens

1977. Novyi Diagnoz Roda Vagrania i Novyi Rod Totia (Brachiopoda). Ezhegodnik Vsesoiuznogo Paleontologicheskogo Obshchestua, 19:92-99, 1 plate.

Sahni, M. R.

1925. Morphology and Zonal Distribution of Some Chalk Terebratulids. Annals and Magazine of Natural History, series 9, 16:353-385, 4 plates.

1929. A Monograph of the Terebratulidae of the British Chalk. Palaeontographical Society [Monographs], 1927:1-62, 10 plates.

1955. Recent Researches in the Palaeontologic Division, Geological Survey of India. Current Science Bangalore, 24(6): 187-188.

1960. Revision of the Cretaceous Terebratulidae of Southern India with Descriptions of two Species from the East Coast Gondwanas. Geological Survey of India, Memoirs, Palaeontologia Indica, 35(1):1-45, 5 plates.

Sahni, M. R., and J. P. Srivastava

1956. Discovery of Eurydesma and Conularia in the Eastern Himalaya and Description of Associated Faunas. Palaeontological Society of India, Journal, 1(1):202214, 4 plates.

St. Joseph, J.K.S.

1942. A New Pentamerid Brachiopod Genus from Yass, New South Wales. Annals and Magazine of Natural History, series 11, 9(51):245-252.

Saito, K.

1936. Older Cambrian Brachiopoda, Gastropoda, etc., from North-western Korea. Tokyo Imperial University, Faculty of Science, Journal, 4(3):345-367, 3 plates.

Salmon, E. S.

1942. Mohawkian Rafinesquininae. Journal of Paleontology, 16(5):564-603, 8 figures, plates 85-87.

Salter, J. W.

1866. Appendix: On the Fossils of North Wales. Great
Britain Geological Survey, Memoirs, 3:240-381, 26 plates.

Sandberger, C.L.F.

1855. Über Anoplotheca, eine neue Brachiopoden-Gattung. Sitzungsberichte der K. Akademie der Wissenschafien zu Wien, 16:5-8, 1 plate.

Sando, W. J.

1957. Beekmantown Group (Lower Ordovician) of Maryland. Geological Society of America, Memoir, 68: $1-161,15$ plates.

Sapelnikov, V. P.

1960a. Jolvia, a New Lower Wenlock Genus from the Middle Urals.] Paleontologischeskii Zhumal, 1960(4): 54-62, 2 plates. [In Russian.]

1960b. Novye Pentameraceae iz Siluriiskikh Otlozhenii Severouralskogo Raiona Sverdlovskogo Oblasti. Sverdlouskogo Gornogo Instituta imeni V. V. Vakhrusheva, Trudy, 37:106-143, 10 plates.

1963. [New Representatives of Articulate Brachiopods from the Silurian of the Urals.] Suerdlouskogo Gornogo Instituta imeni $V$. V. Vakhrusheva, Trudy, 42:1519. [In Russian.]

1976. Reveroides-Novye Siluriyskiy Rod Pentamerid s Vostochnogo Sklona Urala. In Ezhegodnik, 1975 page 3. Sverdlovsk: Institut Geologii i Geokhimi, Akademiia Nauk SSSR, Uralskii Nauchnyi Tsentr.

Sapelnikov, V. P., and A. A. Malygina

1977. A New Genus of Devonian Rhynchonellid from Southern Tian-Shan.] Akademiia Nauk SSSR, Uralskii Nauchnyi Tsentr, Instituta Geologii $i$ Geokhimii, Trudy, 129:60-69. [In Russian.]

Sapelnikov, V. P., and T. B. Rukavishnikova

1973. [Two New Genera of Early Pentameracea (Brachiopoda) from Southern Kazakhstan.] Paleontologicheskii Zhurnal, 1973(1):32-38. [In Russian.]

1975a. Novye Llandoveriiskie Pentameridy Kazakhstana. Akademiia Nauk SSSR, Uralskii Nauchnyi Tsentr, Institut Geologii $i$ Geokhimii, Trudy, 117:1-17.

1975b. Verkhneordovikskie, Silurskie i Nizhnedevonskie Pentameridy Kazakhstana. 226 pages. Moscow: Akademiia Nauk SSSR, Uralskii Nauchnyi Tsentr, Institut Geologii i Geokhimi.

1976. [Galeatellina, a New Name for a Pentamerid Genus (Brachiopoda).] Paleontologicheskii Zhurnal, 1976(2): 122. [In Russian.]

Sartenaer, P.

1961a. Late Upper Devonian (Famennian) Rhynchonelloid Brachiopods. Institut Royal des Sciences Naturelles de Belgique, Bulletin, 37(24):1-10, 2 plates.

1961b. Note nomenclatoriale: Yunnanella Grabau, Yunnanellina Grabau, Nayunnella nom. nov. (Rhynchonelles). Institut Royal des Sciences Naturelles de Belgique, Bulletin, 37(2):1-3. 
1961c. Etude nouvelle, en deux parties, du genre Camarotoechia Hall and Clarke, 1893, Deuxieme partie: Cupularostrum recticostatum n. gen., n. sp. Institut Royal des Sciences Naturelles de Belgique, Bulletin, 37(25): 1-15, 2 plates.

1965a. Trois nouveaux genres de brachiopodes Rhynchonellides du Famennien. Institul Royal des Sciences Naturelles de Belgique, Bulletin, 41(3):1-12.

1965b. Rhynchonelloidea de Shogran et Kuragh (Chitral). Italian Expeditions to the Karakorum $\left(K^{2}\right)$ and Hindu Kush, Scientific Reports, series 4 (Paleontology-Zooology-Botany), 1:55-66, 2 plates.

1966a. Ripidiorhynchus, nouveau genre de brachiopode rhynchonellide du Frasnien. Institut Royal des Sciences Naturelles de Belgique, Bulletin, 42(30):1-15, 2 plates.

1966b. Frasnian Rhynchonellida from the Ozbak-Kuh and Tabas Regions (East Iran). Iran Geological Survey Report, 6:25-54, 2 plates.

1967. Del'Importance stratigraphique des rhynchonelles famenniennes situées sous la zone à Ptychomaletoechia omaliusi (Gosselet, J., 1877), quatrième note: Tenuisinurostrum n. gen. [( T. crenulatum (Gosselet, J., $1877)=$ espèce-type)]. Institut Royal des Sciences Naturelles de Belgique, Bulletin, 43(32):1-24, 2 plates.

1968a. Stenaulacorhynchus, nouveau genre de brachiopode rhynchonellide du famennien inferieur de l'Iran oriental. Institut Royal des Sciences Naturelles de Belgique, Bulletin, 44(30):1-14.

1968b. De l'Importance stratigraphique des rhynchonelles famennienes situées sous la zone à Ptychomaletoechia omaliusz (Gosselet, J., 1877), cinquième note: $P a$ romoeopygma $\mathrm{n}$. gen. Institut Royal des Sciences $\mathrm{Na}$ turelles de Belgrque, Bulletin, 44(42):1-26.

1968c. De l'Importance stratigraphique des rhynchonelles famenniennes situées sous la zone à Ptychomaletoechia omaliusi (Gosselet, J., 1877), sixième note: Pampoecilorhynchus n. gen. Institut Royal des Sciences Naturelles de Belgique, Bulletın, 44(43):1-36, 5 plates.

1968d. Eurycolporhynchus, nouveau genre de brachiopode rhynchonellide du givetien superieur. Senckenbergiana Lethaea, 49:565-574.

1970. Nouveaux Genres rhynchonellides (brachiopodes) du paleozoique. Institut Royal des Sciences Naturelles de Belgique, Bulletin, 46(32):1-32.

1971. Genres rhynchonellides (brachiopodes) nouveaux. Institut Royal des Sciences Naturelles de Belgique, Bulletin, 47(4): 1-7, I plate.

1972. De l'Importance stratigraphique des rhynchonellides famenniens situes au-dessus de la zone à Ptychomaletoechia dumonti (Gosselet, J., 1877), première note: Cavatisinurostrum n. gen. Institul Royal des Sciences Naturelles de Belgique, Bulletin, 48(2):122.
1975. Eumetabolotoechia, nouveau genre rhynchonellide (brachiopode) du givetien d'Amerique du Nord. Institut Royal des Sciences Naturelles de Belgique, Bulletin, 51(6): 1-8.

1977. Un Nouveau Genre de rhynchonellide de la fin du frasnien. Annales de la Sociélé Géologique du Nord, 97(1):67-76.

1979a. Deux Nouveaux Genres de rhynchonellides frasniens precedemment inclus dans le genre Calvinaria (Brachiopoda). Geobios, 12(4):535-547, 2 plates.

1979b. Parvulaltarostrum, genre rhynchonellide (brachiopode) nouveau du frasnien de L'Australie de L'Ouest. Institut Royal des Sciences Naturelles de Belgique, Bulletin, 51(16):1-4.

Sartenaer, P., and G. Plodowski

1975. Importance stratigraphique et repartition geographique du genre rhynchonellide Araratella n. gen. du famennien superieur. Institut Royal des Sciences Naturelles de Belgique, Bulletin, 51 (8): 1-34, 3 plates.

Sartenaer, P., and Kh. S. Rozman

1968. [Physetorhyncha, New Genus of Late Famennian Rhynchonellids.] Paleontologicheskii Zhurnal, 1968 (2):137-139. [In Russian.]

Sarytcheva, T. G.

1949. [Morphology, Ecology and Evolution of Carboniferous Producti of the Moscow Basin: Genera Drctyoclostus, Pugilis and Antiquatonia.] Akademiia Nauk SSSR, Paleontologicheskii Institut, Trudy, 18:1304, 118 figures, 36 plates. [In Russian.]

1964. [Oldhaminoid Brachiopods from the Permian of Trans-Caucasia.] Paleontologicheskii Zhurnal, 1964 (3):58-72, 2 figures, 2 plates. [In Russian.]

1966. [Chonopectella Sarytcheva nom. nov., a New Generic Name.] Paleontologicheskii Zhurnal, 1966(1): 135. [In Russian.]

1968. [Early Palaeozoic Brachiopods of Eastern Kazakhstan.] Akademiza Nauk SSSR, Paleontologicheskii Institut, Trudy, 121:1-212, 23 plates. [In Russian.]

Sarytcheva, T. G., and M. Legrand-Blain

1977. [The Family Semiplanidae (Brachiopoda), Its Composition and Development.] Paleontologicheskii Zhurnal, 1977(2):70-82. [In Russian.]

Sarytcheva, T. G., B. K. Licharew, and A. N. Sokolskaja

1960. Mshanki, Brakhiopody. In T. G. Sarytcheva, editor, Otryad Productida. In Yu. A. Orlov, editor, Osnovy Paleontologii, pages 221-238.

Sarytcheva, T. G., and A. N. Sokolskaja

1952. [A Description of the Palaeozoic Brachiopoda of the Moscow Basin.] Akademiia Nauk SSSR, Paleontologicheskii Institut, Trudy, 38:1-307, 231 figures, 71 plates. [In Russian.]

Sarytcheva, T. G., A. N. Sokolskaja, G. A. Besnossova, and S. V. Maksimova

1963. [Carboniferous Brachiopods and Paleogeography 
of the Kuznetsk Basin.] Akademiia Nauk SSSR, Paleontologicheskii Institut, Trudy, 95:1-547, 151 figures, 64 plates. [In Russian.]

Sarytcheva, T. G., and J. B. Waterhouse

1972. [Some Brachiopods of the Family Retariidae from the Permian of Northern Canada.] Paleontologicheskii Zhurnal, 1972(4):62-74, 2 plates. [In Russian.]

Savage, N. M.

1968a. Planicardinia, a New Septate Dalmanellid Brachiopod from the Devonian of New South Wales. Palaeontology, 11(4):627-632, 1 figure, 1 plate.

1968b. Australirhynchia, a New Lower Devonian Rhynchonelloid Brachiopod from New South Wales. $\mathrm{Pa}$ laeontology, 11(5):731-735, 1 plate.

1970. New Atrypid Brachiopods from the Lower Devonian of New South Wales. Journal of Paleontology, 44(4):655-668, 3 plates.

1971. Brachiopods from the Lower Devonian Mandagery Park Formation, New South Wales. Palaeonlology, 14(3):387-422, 6 plates.

1974. The Brachiopods of the Lower Devonian, Maradana Shale, New South Wales. Palaeontographica, series A, 146(1-3):1-51, 11 plates.

Savage, N. M., G. D. Eberlein, and M. Churkin, Jr.

1978. Upper Devonian Brachiopods from the Port Refugio Formation, Suemez Island, Southeastern Alaska. Journal of Paleontology, 52(2):370-393.

Savage, T. E.

1913. Stratigraphy and Paleontology of the Alexandrian Series in Illinois and Missouri. Illinois Geological Survey Bulletin, 23:1-124, 7 plates.

Schauroth, K. F.

1854. Ein Beitrag zur Palaeontologie des deutschen Zechsteingebirges. Zeitschrift der Deutschen Geologischen Gesellschaft, 6:539-577.

Schellwien, E.

1900. Beiträge zur Systematik der Strophomeniden des oberen Palaeozoicum. Neues Jahrbuch für Mineralogie, Geologie, und Palaeontologie, 1:1-15, 1 plate.

Schlotheim, E. von

1820. Die Petrefactenkunde auf ihrem jetzigen Standpunkte durch die Beschreibung einer Sammlung versteinerter und fossiler $\ddot{U}$ Uerreste der Tier- und Pflanzenreichs der Vorwelt Erläutert. Volume 1, 378 pages. Gotha.

Schmidt, F.

1888. Über eine neuentdeckte untercambrische Fauna in Estland. Académie Impériale des Sciences, St. Pétersbourg, Mémoires, series 7, 36(2):1-27, 2 plates.

Schmidt, $\mathrm{H}$.

1941. Die mitteldevonischen Rhynchonelliden der Eifel. Senckenbergische Naturforschende Gesellschaft, Abhandlungen, 459:1-79, 7 plates.

1942. Die Rhynchonelliden des wetteldorfer Richt. schnittes. Senckenbergiana, 25(4-6):389-404, 18 figures.

1943. Die Terebratulidae des wetteldorfer Richtschnit tes. Senckenbergiana, 27(2):67-75.

1954. Sphaerirhynchia (Estonirhynchia) estonica n. subgen. n. sp. Senckenbergiana, 35(3-4):235-245, 12 figures.

1955. Devonische Gattungen der Rhynchonellacea (Brach.). Senckenbergiana Lethaea, 36(1-2):115-122.

1964. Neue Gattungen palaeozischer Rhynchonellacea (Brạchiopoda). Senckenbergiana Lethaea, 45(6):505506.

1967. Aratanea monodi n.g., n.sp. (Brachiopoda; W-Sahara). Senckenbergiana Lethaea, 48(2):91-97.

1975. Septalariinae (Brachiopoda, Rhynchonellida) im Devon westlich und ostlich des Rheins. Senckenbergiana Lethaea, 56(2-3):85-121, 19 figures, 7 plates.

Schuchert, C.

1910. On the Brachiopod Genus Syringolhyris in the Middle Devonian of Missouri. American Journal of Science, 30:223-224.

1913. Class Brachiopoda. In The Lower Devonian Deposits of Maryland, pages 290-449, plates 53-74. Baltimore: Maryland Geological Survey.

Schuchert, C., and G. A. Cooper

1930. Upper Ordovician and Lower Devonian Stratigraphy and Paleontology of Percé, Quebec, Part 2: New Species from the Upper Ordovician of Percé. American Journal of Science, series 5, 20:265-288, 3 plates.

1931. Synopsis of the Brachiopod Genera of the Suborders Orthoidea and Pentameroidea, with Notes on the Telotremata. American Journal of Science, series 5, 22:241-251.

1932. Brachiopod Genera of the Suborders Orthoidea and Pentameroidea. Peabody Museum of Natural History, Memoir, 4(1): 1-270.

Schuchert, C., and C. M. LeVene

1929. New Names for Brachiopod Homonyms. American Journal of Science, 17:119-122.

Schulz, E.

1914. Über einige Leitfossilien der Stringocephalenschichten der Eifel. Verhandlungen des Naturhistorischen Vereins der Preussischen Rheinlande, 70:335383, 38 figures, 11 plates.

Scupin, $\mathrm{H}$.

1896. Versuch einer Classification der Gattung Spirifier. Neues Jahrbuch für Mineralogie, Geologie, und Palaeonlologie, 2:239-248.

Sdzuy, K.

1955. Die Fauna der Leinitz-Schiefer (Tremadoc). Senckenbergische Naturforschende Gesellschaft, Abhandlungen, 492: 1-73, 8 plates.

Seguenza, G.

1885. Al Sistema giurassico nel territorio Taormina. Il 
Naturalista Siciliano Giomale di Scienze Naturali, 1884 1885:251-253.

Seidlitz, W. von

1913. Misólia, eine neue Brachiopoden-Gattung aus den Athyridenkalken von Buru und Misól. Palaeontographica, supplement 4, part 2, pages 163-193, plates 12-14.

Seifert, I.

1963. Die Brachiopoden des oberen Dogger der schwäbischen Alb. Palaeontographica, series A, 121(4-6): 156-203, plates 10-13.

Semichatova, S. V.

1936. [Contributions to the Stratigraphy of the Middle and Lower Carboniferous in the European Part of the USSR.] Sociélé Impériale des Naturalistes de Moscou, Bulletin, new series (Géologique), 14(3): 189224, 3 plates. [In Russian.]

Severgina, L. G.

1978. Brakhiopody i Stratigraphiia Verkhnego Ordovika Gornogo Altaia, Al Salaira i Gornoishorii. Akademiza Nauk SSSR, Sibirskoe Otdelenie, Institul Geologii i Geofizzki, Trudy, 405:1-152, 19 plates.

Shaler, N. S.

1865. List of the Brachiopoda from the Island of Anticosti Sent by the Museum of Comparative Zoology to Different Institutions in Exchange for Other Specimens, with Annotations. Harvard University, Museum of Comparative Zoology, Bulletm, 1:6170 .

Sharpe, D.

1848. On Trematis, a New Genus Belonging to the Family of Brachiopodous Mollusca. Geological Society of London, Quarterly Journal, 4:66-69, 3 figures.

Sheng, Hui-bin

1975. [Silurian Brachiopods from Atrypella Bed of Kuangyuan, Sichuan.] Professional Papers of Siratigraphy and Palaeontology, 2:78-88, 2 plates. [In Chinese.]

Shiells, K.P.G.

1966. A New Productid Brachiopod from the Upper Visean of Scotland. Palaeontology, 9(3):426-447.

Siblik, M.

1965. Some New Liassic Brachiopods. Geologický Sbornik, 16(1):73-82.

1967. New Species of Domerian Rhynchonelloids from Slovakia. Geologický Sbornik, 18(1):151-168.

1977. Rod Gemerilhyris gen. n. (Brachiopoda) ze Slovenského triasu. Západné Karpaty (Paleontológia), 2-3: 203-220, plates $108-110$

Sidiachenko, A. I.

1961. The Upper Devonian Cyrtospiriferid Subgenus Dmitria.] Paleontologicheskii Zhurnal, 1961(2):80-85, 1 figure, I plate. [In Russian.]

Siehl, A.

1962. Der Greifensteiner Kalk (Eiflium, Rheinisches
Schiefergebirge) und seine Brachiopodenfauna, 1: Geologie, Atrypacea und Rostrospiracea. Palaeonlographica, series A, 119(5-6):173-221, 18 plates.

Siemiradzki, J

1909. Zibory L. Zejsznera z kieleckiego dowonu. Sprawozdanie Komisyi Fizyograficznej Academia Umiejetnosci, 43:62-94.

1922. Katalog Systematyczny Zbivrów Paleontologicznych Muzeum im Dzieduszyckich we Lwowie. Rozprawy \& Wiadomośc z Muzeum im Dzieduszyckich, 5-6: 151-178.

Simakov, K. V.

1970. [The Carboniferous of Omolonian and Southwestern Part of Kolymian Massifs.] Akademiia Nauk SSSR, Sibirskoe Otdelenie, Institut Geologii i Geofiziki, Trudy, 60:81-95. [In Russian.]

Simorin, A. M.

1956. Stratigrafia Brakhiopody Karagandinskogo Basseina. 296 pages, 28 plates. Alma-Ata: Akademiia Nauk Kazakhskoi SSR.

Sinclair, G. W.

1945. Some Ordovician Lingulid Brachiopods. Royal Society of Canada, Transactions, series 3, 39(4):55-82, 4 plates.

1946. Bancrofina, a New Brachiopod Name. Journal of Paleontology, 20(3):295.

Smirnova, T. N.

1960. [A New Subfamily of the Lower Cretaceous Dallinids.] Paleontologicheskii Zhurnal, 1960(2):114-120, plate 11. [In Russian.]

1962. [New Data on Lower Cretaceous Dallinids (Brachiopoda).] Paleontologicheskii Zhurnal, 1962(2): 97-105. [In Russian.]

1966. [On the Systematics of the Early Cretaceous Terebratulids of the Subfamily Cancellothyrinae.] $\mathrm{Pa}$ leontologicheskii Zhurnal, 1966(3):31-41, 8 figures. [In Russian.]

1969a. [Ontogeny and Phylogeny of Early Cretaceous Brachiopods of the Suborder Thecideidina.] $\mathrm{Pa}$ leontologicheskii Zhurnal, 1969(1):74-89. [In Russian.]

1969b. [A New Tithonian-Valanginian Terebratulid Genus.] Paleontologicheskii Zhumal, 1969(3):144-146. [In Russian.]

1972. Rannemelovye Brakhiopody Kryma i Severnogo Kavkaza. 143 pages, 13 plates. Moscow: Akademiia Nauk SSSR, Nauchnyi Sovet po Probleme "Puti i Zakonomernosti Istoricheskogo Razvitiia Zhivotnykh i Rastitelnykh Organizmov."

1973. A Contribution to the Study of Ontogenetic Changes in Rhynchonellids (Brachiopods).] Paleontologicheskii Zhurnal, 1973(4):40-46, 4 figures. [In Russian.]

1974. [A New Terebratulid Family (Brachiopoda) from 
the Lower Cretaceous of Northwestern Kamchatka.] Paleontologicheskii Zhurnal, 1974(3):47-54, 9 figures. [In Russian.]

1975a. Revision of the Uppcr Tithonian Brachiopods from Inwald (Polish Carpathians). Acta Geologica Polonica, 25(1):115-141, 17 figures, 4 plates.

1975b. [A New Early Cretaceous Dallinid Genus (Brachiopoda).] Paleontologicheskii Zhurnal, 1975(1): 120-123. [In Russian.]

1975c. [New Berriasian and Lower Hauterivian Terebratulids of the Russian Platform.] Paleontologicheskii Zhurnal, 1975(3):70-82. [In Russian.]

Smirnova, T. N., and M. A. Pergament

1969. [New Early Cretaceous Brachiopods from Kamchatka.] Paleontologicheskii Zhurnal, 1969(4):34-40, 3 figures. [In Russian.]

Smirnova, T. N., and G. P. Terekhova

1972. [First Finds of Early Cretaceous Brachiopods in the Anadyr' River Basin.] Paleontologicheskii Zhurnal, 1972(2):71-81, 5 figures. [In Russian.]

Sobolev, L. P.

1976. A New Ecardine Brachiopod Genus from the Upper Cambrian of the Dzhagdy Range (Kha. barovsk Territory).] Paleontologicheskii Zhurnal, 1976(2):131-133. [In Russian.]

Sokolskaja, A. N.

1941. [Lower Carboniferous and Devonian-Carboniferous Brachiopods of the Moscow Basin (Tschernyschino, Upa and Malevka-Muraevnya Beds).] Akademiia Nauk, Paleontologicheskii Institut, Trudy, 12(2): 1-138, 39 figures, 12 plates. [In Russian.]

1948. [Evolution of the Genus Productella Hall and Related Forms in the Paleozoic of the Moscow Region.] Akademïa Nauk, Paleontologicheskii Institul, Trudy, 14(3): 1-168, 10 plates. [In Russian.]

1950. [Chonetidae of the Russian Platform.] Akademiia Nauk, Paleontologicheskii Institut, Trudy, 27:1-108, 25 figures, 13 plates. [In Russian.]

1960. Mshanki, Brakhiopody. In T. G. Sarytcheva, editor, Otryad Strophomenida. In Yu. A. Orlov, editor, Osnovy Paleontologii, pages 206-220.

Solomina, R. V., and G. E. Tschernjak

1961. [Orulgania a New Spiriferid Genus from the Upper Palaeozoic of the Arctic.] Paleontologicheskii Zhurnal, 1961(3):61-66. [In Russian.]

Sowerby, J.

1812-1815. The Mineral Conchology of Great Britain. Volume 1, 234 pages, 102 plates.

1815-1818. The Mineral Conchology of Great Britain. Volume 2, 235 pages, plates $103-203$.

Spjeldnaes, $\mathrm{N}$.

1957. The Middle Ordovician of the Oslo Region, Norway, 8: Brachiopoda of the Suborder Stropho- menoidea. Norsk Geologisk Tidsskrift, 37(1):1-214 14 plates.

Spriestcrsbach, J.

1925. Die Oberkoblenzschichten des bergischen Landes und Sauerlandes. Königlich Preussichen Geologischen Landesanstalt, Jahrbuch, 45:367-450, plates 15-17.

1942. Lenneschicfer (Stratigraphie, Fazies, und Fauna). Abhandlungen des Reichsamts für Bodenforschung, 203: 1-219, 10 plates.

Stainbrook, M. A.

1943a. Strophomenacea of the Cedar Valley Limestone of Iowa. Journal of Paleontology, 17(1):39-59, plates 6-7.

1943b. Spiriferacea of the Cedar Valley Limestone of Iowa. Journal of Paleontology, 17(5):417-450, 14 figures, plates 65-70.

1945. Brachiopoda of the Independence Shale of Iowa. Geological Society of America, Memoir, 14:1-74, 2 figures, 6 plates.

1947. Brachiopoda of the Percha Shale of New Mexico and Arizona. Journal of Paleontology, 21 (4):297-328, plates $44-47$.

1950. Brachiopoda and Stratigraphy of the Aplington Formation of Northern Iowa. Journal of Paleontology, 24(3):365-385, 2 plates.

1951. Substitution for the Preoccupied Brachiopod Name, Hystricina. Washington Academy of Sciences, Journal, 41(6): 196 .

Stehli, F. G.

1954. Lower Leonardian Brachiopoda of the Sierra Diablo. American Museum of Natural History, Bulletin, 105:257-358, plates 17-27.

1955a. A New Devonian Terebratuloid Brachiopod with Preserved Color Pattern. Journal of Paleonlology, 29(5):868-870, 1 plate.

1955b. Elliottella, New Name for Psilonotus Stehli. Joumal of Paleontology, 29(4):711.

1955c. Notes on Permian Rhynchonellids. Washington Academy of Sciences, Journal, 45(3):70-74, 1 plate.

1956. Notes on Oldhaminid Brachiopods. Journal of Paleontology, 30(2):305-313, 2 plates.

1961a. New Terebratuloid Genera from Australia. Journal of Paleontology, 35(3):451-456, 1 plate.

1961b. New Genera of Upper Paleozoic Terebratuloids. Journal of Paleontology, 35(3):457-466, 1 plate.

1964. New Names for Two Homonyms. Journal of Paleontology, 38(3):610.

Steinich, G.

1963. Drei neue Brachiopodengattungen der Subfamilie Cancellothyrinae Thomson. Geologie, 12(6):732 740, 8 figures.

1967. Neue Brachiopoden aus der ruegener Schreibkreide (Unter-Maastricht), 1: Draciinae-eine 
neue Unterfamilie der Cancellothyrididae Thomson. Geologie, 16(10):1145-1155, 1 plate.

1968a. Neue Brachiopoden aus der ruegener Schreibkreide (Unter-Maastricht), 2: Die Platidiidae Thomson. Geologie, 17(2):192-209, 1 plate.

1968b. Neue Brachiopoden aus der ruegener Schreibkreide (Unter-Maastricht), 3: Dalligas nobilis gen. et spec. nov. und Kingena sp. Geologie, 17(3):336347,1 plate.

Steinmann, G., and H. Hoek

1912. Das Silur und Cambrium des Hochlandes von Bolivia und ihre Fauna. Neues Jahrbuch für Mineralogie, Geologie und Paläontologie, 34:176-252.

Stenzel, H. B.

1964. Stratigraphic and Paleoecologic Significance of a New Danian Brachiopod Species from Texas. Sonderdruck aus der Geologischen Rundschau, 54:619-631, 5 figures.

Stepanov, D. L.

1934. [The Brachiopoda of the Bryozoa Limestones of the Kolwa River Region (Northern Ural), Part 1: Strophomenacea.] Neftianoi Geologo-Razvedochnyi Institut (NGRI), Trudy, series A, 37:1-63, 4 plates. [In Russian.]

1937. [On Some Upper Carboniferous Brachiopoda of the Urals.] Uchenie Zapisk Leningradskii Universitet (Geologo-Pochvenno-Geographicheskaya), 16(4) 3:144-150. [In Russian.]

1975. [Paleontological Atlas of the Carboniferous in the Urals.] Vsesoiuznyz Nauchno-Issledovatelskii GeologoRazvedochnyi Neftianoi Institut (VNIGNI), Trudy, 383: 1-358, 100 plates. [In Russian.]

Stepanov, D. L., M. V. Kulikov, and A. A. Sultanaev

1975. Stratigrafiia i Brakhiopody Verkhnepermskikh Otlozhenii Poluostrova Kanin. Vestnik Leningradskogo Universiteta (Geologia i Geografiia), 6(1):51--65, 3 plates.

Stoyanow, A.

1910. O novom rode Brachiopoda. Académie Impériale des Sciences, St. Pétersbourg, Bulletin, series 6, 11:853855, 2 figures.

Strand, E.

1928. Miscellanea nomenclatorica zoologica et palaeontologica, I-II. Archiv für Naturgeschichte (Berlin), 92(A8):37-38.

Struve, W.

1961. Zur Stratigraphie der südlichen Eifler Kalkmulden (Devon: Emsium, Eifelium, Givetium). Senckenbergiana Lethaea, 42(3/4):291-345, 3 plates.

1963. Alatiformia n.g. (Spiriferacea). Senckenbergiana Lethaea, 44(6):499-500.

1964a. Mimatrypa n.g. (Atrypidae Palaferellinae). Senckenbergiana Lethaea, 45(5):433-440, 5 figures.

1964b. Erörterung des Alters der Refrath-Schichten und
Darstellung einiger devonischer Atrypinae. Senckenbergiana Lethaea, 45(6):523-532, 2 figures.

1964c. Strömungs-Orientierung bei Bodenverwachsenen, Schlosstragenden Brachiopoden. Natur und $\mathrm{Mu}$ seum, 94(12):515-529, 13 figures.

1965a. Atrythyris n.g. und ihre Arten (Athyridae). Senckenbergiana Lethaea, 46(2/3):217-228, 9 figures.

1965b. Beiträge zur Kenntnis devonischer Brachiopoden, 14: Über Geranocephalus (Stringomimus) n. sg. und Verwandte. Senckenbergiana Lethaea, 46(4/6):459472.

1966. Einige Atrypinae aus dem Silurium und Devon. Senckenbergiana Lethaea, 47(2):123-163.

1970. "Curvate Spiriferen" der Gattung Rhenothyris und einige andere Reticulariidae aus dem rheinischen Devon. Senckenbergiana Lethaea, 51(5/6):449-577, 12 figures, 15 plates.

1978. Fixo-Sessile Brachiopoden aus dem Rheinischen Devon: Schuchertellopsis (Krejcigrafella) und Auchmerella (Strophomenida). Senckenbergiana Lethaea, 59(1/3):93-115.

Stubblefield, C. J.

1939. Some Devonian and Supposed Ordovician Fossils from South-West Cornwall. Geological Survey of Great Britain, Bulletin, 2:63-71, plate 4.

Stuckenberg, A.

1905. Die Fauna der obercarbonischen Suite des Wolgadurchbruches bei Samara. Comıté Géologique, Mémoires, new series, 23:1-144, 13 plates.

Su, Yang-zheng

1976. In Hua Bei Di Qu Gu Sheng Wu Tu Ce, Nei Meng Gu Feng Ce, Yi Gu Sheng Dai Bu Feng [Palaeontological Atlas of Northern China (Inner Mongolia Part)]. Volume 1, 502 pages, 232 plates. Peking. [In Chinese.]

Sučić-Protić, Z.

1969. Mesozoic Brachiopoda of Yugoslavia, Middle Liassic Brachiopoda of the Yugoslav CarpathoBalkanids [part 1]. Universtty of Belgrade Monograph (Belgrade), 1:1-214, 6 figures, 57 plates.

1971. Mesozoic Brachiopoda of Yugoslavia, Middle Liassic Brachiopoda of the Yugoslav CarpathoBalkanids [part 2]. University of Belgrade Monograph (Belgrade), 5:1-63, 41 plates.

Suess, E.

1851. Ueber ein neues Brachiopoden-Geschlecht Merista. Jahrbuch der Kaiserlich-Koniglichen Geologischen Reichsanstalt, 2(4):150.

1855. Ueber Meganteris, eine neue Gattung von Terebratuliden. Akademie der Wissenschaften Wien, Mathematische-Naturwissenschaftliche Klasse, Denkschriften, 18:51-64, 3 plates.

1859a. Die Brachiopoden der stramberger Schichten. Bei- 
träge zur Palaeontographie von Oesterreich, 1(2):15-58, 6 plates.

1859b. Ueber die Wohnsitze der Brachiopoden. Sitzungsberichte der Mathematisch-Naturwissenschaftlichen Klasse, Akademie der Wissenschafien, Wien, 37:185248.

Sutherland, P. K., and F. H. Harlow

1973. Pennsylvanian Brachiopods and Biostratigraphy in Southern Sangre de Cristo Mountains, New Mexico. New Mexico Bureau of Mines and Mineral Resources, Memoir, 27:1-173, 18 plates.

Sutton, A. H.

1938. Taxonomy of Mississippian Productidae. Journal of Paleontology, 12(6):537-569, plates 62-66.

Tachibana, $\mathrm{K}$.

1964. [Upper Devonian and Lowest Carboniferous Formations in the Vicinity of Minamiiwairi, Higashiyamamachi, Iwate Prefecture, Pt. 1 (Study of the Devonian-Carboniferous Boundary in the Southwestern Part of the Kitakami Mountainland. 1).] Faculty of Liberal Arts, Nagasaki University, Natural Science, Bulletin, 4:31-43, 1 plate. [In Japanese; English abstract.]

Talent, J. A.

1956. Devonian Brachiopods and Pelecypods of the Buchan Caves Limestone, Victoria. Royal Society of Victoria, Proceedings, new series, 68:1-56, 15 figures, 5 plates.

1963. The Devonian of the Mitchell and Wentworth Rivers. Geological Survey of Victoria, Memoirs, 24:1118,78 plates.

Tcherkesova, S. V.

1965. [Nordotoechia-A New Genus of Early Devonian Rhynchonellid.] Uchenye Zapiski, Paleontologiia $i$ Biostratigrafiia, Nauchno-Issledovatelskii Institut Geologii Arktiki Ministerstva Geologii SSSR, 10:79-92. [In Russian.]

1967. New Genera of the Family Uncinulidae Rzhonsnitzkaia (Brakhiopody) from the Lower Devonian of Nova Zemlya.] Uchenye Zapiski, Paleontologiia i Biostratigrafiia, Nauchno-Issledovatelskii Institut Geologii Arktiki Ministerstva Geologii SSSR, 18:5-22. [In Russian.]

1973. [The Vascular System in Pentamerids.] Paleontologicheskii Zhurnal, 1973(4):25-34, 4 figures, 2 plates. [In Russian.]

1976. [Spiriferid Morphology.] Paleontologicheskii Zhurnal, 1976(3):87-101. [In Russian.]

Tchorszhewsky, E. S.

1971. [A New Genus of Jurassic Terebratulid from the Penine Slope (Zakarpat) Zone.] Vestnik Kharkovskogo Universiteta (Geologia), 72:62-65, 2 figures. [In Russian.]

1972. [Tchegemithyrididae-a New Family of Jurassic
Terebratuloidea.] Vestnik Kharkouskogo Universiteta (Geologia), 86:35-41. [In Russian.]

1974. [New Data Regarding the Inner Structures of the Shells and Systematics of Jurassic Terebratuliid Brachiopods from the Superfamily Terebratuloidea Gray, 1840 and Loboidothyrididoidea Makridin, 1964.] Vestnik Kharkouskogo Universiteta, 108(5):42-58. [In Russian.]

Teichert, C.

1937. Ordovician and Silurian Faunas from Arctic Canada. In Report of the 5th Thule Expedition, 19211924, 1(5):1-169, 24 plates.

Temple, J. T.

1965. Upper Ordovician Brachiopods from Poland and Britain. Acta Palaeontologia Polonica, 10(3):379-427, 3 figures, 21 plates.

1968. The Lower Llandovery (Silurian) Brachiopods from Keisley, Westmoreland. Palaeontological Society Monograph, 521: 58 pages, 10 plates. [Issued as volume 122(1968):1-58, plates 1-10.]

Termier, G., and O. Monod

1978. Inarticulate Brachiopods from Cambro-Ordovician Formations in the Western Taurus (Turkey). Bulletin of the Geological Society of Turkey, 21:145152, 4 plates.

Termier, G., and H. Termier

1974. Les Brachiopodes cambriens de la Montagne Noire (Sud de la France). Géologie Méditerranéenne, $1(2): 35-52,15$ figures.

Termier, G., H. Termier, A. F. de Lapparent, and P. Marin

1974. Monographie du permo-carbonifère de Wardak (Afghanistan central). Documents des Laboratoires de Geologie de la Faculte des Sciences de Lyon, hors series, 2:1-167, 25 figures, 38 plates.

Termier, G., H. Termier, and D. Pajaud

1966. Découverte d'une Thécidée dans le permien du Texas. Comple Rendu des Séances de l'Academie des Sciences, series D, 263:332-335, 2 figures.

Termier, $\mathrm{H}$.

1936. Études géologiques sur le Maroc Central et le Moyen Atlas Septentrional, 3: Paléontologie. Maroc Service Geologique du Division des Mines et de la Géologie, Notes et Mémoires, 33:1087-1421, 23 plates.

Termier, H., and G. Termier

1960. Contribution à la classification des brachiopodes: Le Lophophore des Collophides nov. ord. Société Géologique de France, Bulletin, series 7, 1(3):233-244.

1970. Les Productoïdés du Djoulfien (Permien supérieur) dans la Téthys orientale: Essai sur l'agonie d'un phylum. Annales de la Société Géologique du Nord, 90(4):443-461.

Thomas, G. A.

1958. The Permian Orthotetacea of Western Australia. 
Australia Bureau of Mineral Resources, Geology and Geophysics, Bulletin, 39:1-115, 10 figures, 22 plates.

Thomas, H. D.

1937. Plicatoderbya, a New Permian Brachiopod Subgenus. Journal of Paleontology, 11 (1):13-18, plate 13.

Thomas, I.

1910. The British Carboniferous Orthotetinae. Great Britain Geological Survey Mernoir, 1(2):83-134, plate 13.

1914. The British Carboniferous Producti, I: Genera Pustula and Overtonia. Great Britain Geological Survey Memoir, 1(4):197-366, plates 17-20.

Thomson, J. A.

1915a. Brachiopod Morphology; Types of Folding in the Terebratulacea. Geological Magazine (London), 2: $71-76$.

1915b. The Genera of Recent and Tertiary Rhynchonellids. Geological Magazine (London), 2:387-392.

1915c. A New Genus and Species of the Thecidiinae (Brachiopoda). Geological Magazine (London), 2: $461-464$.

1915d. Brachiopod Genera: The Position of Shells with Magaselliform Loops, and of Shells with Bouchardiform Beak Characters. New Zealand Institute, Transactions and Proceedings, 47:392-403.

1916a. Additions to the Knowledge of the Precent and Tertiary Brachiopoda of New Zealand and Australia. New Zealand Institute, Transactions and Proceedings, 48:41-47, plate 1.

1916b. On the Classification of the Terebratellidae. Geological Magazine (London), 3:496-505.

1918. Australasian Antarctic Expedition, 1911-14, under the Leadership of Sir Douglas Mawson, D.Sc., B.E., Brachiopoda. Scientific Reports, series C, 4(3): 1-76, 4 plates.

1920. The Notocene Geology of the Middle Waipara and Weka Pass District, North Canterbury, New Zealand. New Zealand Institute, Transactions and Proceedings, 52:322-415, 9 figures, plates 16-27.

1926. A Revision of the Subfamilies of the Terebratulidae (Brachiopoda). Annals and Magazine of Natural Hislory, series 9, 18:523-530.

1927. Brachiopod Morphology and Genera (Recent and Tertiary). New Zealand Board of Science and Art, Manual, 7:1-338, 103 figures, 2 plates.

Thomson, M.R.A., and E. F. Owen

1979. Lower Cretaceous Brachiopoda from South-eastern Alexander Island. British Antartic Survey Bulletın, 48: 15-36.

Tien, C. C.

1938. Devonian Brachiopoda of Hunan. Palaeontologia Sinica, new series B, 4:1-192, 22 plates.
Tillman, C. G

1967. Silicified Rhynchonellid Brachiopods from Beds of New Scotland Age (Early Devonian) Virginia and West Virginia. Journal of Paleonlology, 41(5): 1247-1255.

Titova, M. V.

1977. [Late Cretaceous Cancellothyrididae (Brachiopoda) of Turkmenia.] Paleontologicheskii Zhurnal, 1977(4):73-85. [In Russian.]

Tjazheva, A. P.

1972. [New Devonian Athyracids from the Urals.] In I. E. Zanina, editor, Novye Vidy Dreunikh Rastenii $i$ Bespozvonochnykh SSSR, [3]:205-207. Moscow: Akademiia Nauk SSSR, Nauchnyi Sovet po Probleme. [In Russian.]

Tjazheva, A. P., and R. A. Zhavoronkova

1972. Korally i Brakhiopody Pogranichnykh Otlozhenii Silura i Nizhnego Devona Zapadnogo Sklona Iuzhnogo Urala. 183 pages. Moscow: Akademiia Nauk SSSR, Bashkirskii Filial Institut Geologii.

Tjazheva, A. P., R. A. Zhavoronkova, and A. A. Garifullina 1976. Korally z Brakhiopody Nizhnego Devona Iuzhnogo Urala. 226 pages, 51 plates. Moscow: Akademiia Nauk SSSR, Bashkirskii Filial, Institut Geologii.

Tokuyama, A.

1957a. On Some Upper Triassic Spiriferinoids from the Sakawa Basin in Prov. Tosa, Japan. Palaeontological Society of Japan, Transactions and Proceedings, new series, 27:99-106, 1 plate.

1957b. On the Late Triassic Rhynchonellids of Japan. Japanese Journal of Geology and Geography, 28:121137, 2 plates.

1958a. On Some Terebratuloids from the Late Jurassic Torinosu Series in Shikoku, Japan. Japanese Journal of Geology and Geography, 29(1-3):119-131, 1 plate.

1958b. On Some Terebratuloids from the Middle Jurassic Naradani' Formation in Shikoku, Japan. Japanese Journal of Geology and Geography, 29(1-3):1-10, 1 plate.

Tomlin, J. R. Le B.

1930. Some Preoccupied Generic Names. Proceedings of the Malacological Society of London, 19:22-24.

Tong, Zheng-xiang

1978. In Xi Nan Di Qu Gu Sheng Wu Tu Ce, Si-Chuan Feng $\mathrm{Ce}$ (Paleontological Atlas of Southwestern China, Sichuan Province], 2: 684 pages, 191 plates. Peking: Southwestern Geological Research Institute. [In Chinese.]

Torley, K

1934. Die Brachiopoden des Massenkalkes der oberen Givel-Stufe von Bilveringsen bei Iserlohn. Senckenbergische Naturforschende Gesellschaft, Abhandlungen, 43(3):69-148, 82 figures, 9 plates. 
Trechmann, C. T.

1918. The Trias of New Zealand. Geological Society of London, Quarterly Journal, 73(3): 165-246, plates 1725.

Tschernyschew, B. B.

1937. Siluriiskie Brakhiopody Mongolii i Tuvy. Akademiia Nauk SSSR, Mongol'skoi Komissii, Trudy, 29(5): 1-94, 5 plates.

Tschernyschew, T. N.

1885. Die Fauna des unteren Devon am West-Abhange des Urals. Comilé Géologique, Mémoires, 3(1):1-107, 9 plates.

1902. Die Obercarbonischen Brachiopoden des Ural und des Timan. Comité Géologique, Mémorres, 16(2):1749,63 plates.

Tuluweit, $\mathbf{K}$.

1965. Terebratulidae und Zeilleriidae (Brachiopoda) des mittleren Lias Nordwestdeutschlands. Neues Jahrbuch für Gcologie und Palaeontologie, Abhandlungen, 122: 50-126, 36 figures, 5 plates.

Twenhofel, W. H.

1914. The Anticosti Island Faunas. Canada Geological Survey, Department of Mines and Resources, Bulletin, 3:139, 1 plate.

Ulrich, E. O.

1886. Descriptions of New Silurian and Devonian Fossils. American Paleontology Contributions, 1:3-35, 2 figures, 3 plates.

1889. On Lingulasma, a New Genus, and Eight New Species of Lingula and Trematis. American Geologist, 3:377-391, 9 figures.

Ulrich, E. O., and G. A. Cooper

1936a. New Silurian Brachiopods of the Family Triplesiidae. Journal of Paleontology, 10(5):331-347, plates 48-50.

1936b. New Genera and Species of Ozarkian and Canadian Brachiopods. Journal of Paleonlology, 10(7): 616-631.

1937. Cambrotrophia, New Name for Eostrophia Ulrich and Cooper, not Dall, J. Journal of Paleontology, 11(1): 78.

1938. Ozarkian and Canadian Brachiopoda. Geological Society of America, Special Paper, 13:1-323, 14 figures, 58 plates.

1942. New Genera of Ordovician Brachiopods. Journal of Paleontology, 16(5):620-626, plate 90.

Ushatinskaia, G. T.

1977a. [New Silurian Cyrtinids from Central Kazakhstan.] In G. A. Stukalina, editor, Novye Vidy Drevnikh Rastenii \& Bespozvonochnykh SSSR, 4:137-138. Moscow: Akademiia Nauk SSSR, Paleontologicheskii Institut; Nauchnye Sovet po Probleme. [In Russian.]

1977b. [A New Chonetid (Brachiopoda) from the Lower
Devonian of Central Kazakhstan.] Paleontologicheskii Zhurnal, 1977(1):42-47. [In Russian.]

Ustritsky, V.I.

1962. On the Origin of the Family Horridoniidae. $P a$ leontologicheskii Zhurnal, 1962 (3):57-60, I plate. [In Russian.]

1967. K Sistematike i Filogenii Khoristitov. Uchenye Zapiski, Paleontologiia i Biostratigrafiia, Nauchno-Issledovatelskii Institut Geologii Arkiki Ministerstva Geologii SSSR, 19:35-40.

1971. Biostratigrafia Verkhnego Paleozoia Arktiki. Nauchno-Issledovatel'skii Institut Geologii Arktiki Ministerstva Geologii SSSR, Trudy, 164:1-279.

Ustritsky, V. I., and G. E. Tschernjak

1963. [Biostratigraphy and Brachiopods of the Upper Paleozoic of Taimyr.] Nauchno-Isseldovatelskii Institut Geologii Arktiki, Trudy, 134:1-139, 47 plates. [In Russian.]

Vandercammen, A.

1955. Septosyringothyris demaneti, nov. gen., nov. sp., un syringothyride nouveau du dinantien de la Belgique. Instztut Royal des Sciences Naturelles de Belgique, Bulletin, 31(30): 1-6, 1 plate.

1957. Revision de Spirifer euryglossus Schnur 1851, = Minatothyris nov. gen. euryglossa (Schnur). Senckenbergiana Lethaea, 38(3/4):177-193, 3 plates.

Vanuxem, L.

1842. Geology of New York, Part 3, Comprising the Survey of the Third Geological District. In Natural History of New York, 4(3): 1-306, 80 figures. Albany.

Veevers, J. J

1959. Devonian Brachiopods from the Fitzroy Basin, Western Australia. Australia Bureau of Mineral Resources, Geology and Geophysics, Bulletin, 45:1-220, 18 plates.

Verneuil, E. de

1845. Paléontologie, Mollusques, Brachiopodes. In R. I. Murchison, E. de Verneuil, and A. de Keyserling, Géologie de la Russie d'Europe et des Montagnes de l'Oural, 2(3): 17-395, 43 plates.

Vincent, E. G.

1893. Contribution à la paléontologie des terrains tertaires de la Belgique: Brachiopodes. Sociélé Royale Malacologique de Belgique, Annales, 28:38-64, plates $3-4$.

Vörös, A.

1978. Viallihyris gen. n. (Terebratulida, Brachiopoda) from the Mediterranean Lower Jurassic. Annales Historico-Naturales Musez Nationalis Hungarici, 70:6168,1 plate.

Waagen, W. H.

1882-1885. Brachiopoda. In Salt Range Fossils [part 4(2)]. Palaeontologia Indica, Memoir, series 13, 1:329 770, plates 25-86. [Pages 329-390, plates 25-28, 
December 1882; pages 391-546, plates 29-49, August 1883; pages 547-610, plates 50-57, May 1884; pages 611-728, plates 58-81, December 1884; pages $729-770$, plates $82-86$, July 1885.]

Waern, B., P. Thorslund, and G. Henningsmoen

1948. Deep Boring through Ordovician and Silurian Strata at Kinnekulle, Vestergötland. Upsala University, Geological Instztution, Bulletin, 32:374-432, plates 23-25.

W'alcott, C. D.

1884. Paleontology of the Eureka District, Nevada. U.S. Geological Survey, Monograph, 8:1-298, 24 plates.

1885. Palaeontologic Notes. American Journal of Science, series 3, 29:114-117, 8 figures.

1889. Description of a New Genus and Species of Inarticulate Brachiopod from the Trenton Limestone. U.S. National Museum, Proceedings, 12:365-366, 4 figures. [Advance copy distributed 10 Dec 1889.]

1897. Cambrian Brachiopoda: Genera Iphidea and Yorkia with Descriptions of New Species of Each and of the Genus Acrothele. U.S. National Museum, Proceedings, 19:707-718.

1901. Cambrian Brachiopoda: Obolella, Subgenus Glyptıas; Bicia; Obolus, Subgenus Westonia; with Descriptions of New Species. U.S. National Museum, Proceedings, 23:669-695.

1902. Cambrian Brachiopoda: Acrotreta, Linnarssonella, Obolus, with Descriptions of New Species. U.S. National Museum, Proceedings, 25:577-612.

1905. Cambrian Brachiopoda with Descriptions of New Genera and Species. U.S. National Museum, Proceedings, 28:227-337.

1908. Cambrian Geology and Palaeontology, No. 3: Cambrian Brachiopoda, Descriptions of New Genera and Species. Smithsonian Miscellaneous Collections, 53:53-137.

1912. Cambrian Brachiopoda. U.S. Geologıcal Survey, Monograph, 51(1):1-872, 76 figures; 51(2):1-363, 104 plates.

Walmsley, V. G., and A. J. Boucot

1975. The Phylogeny, Taxonomy and Biogeography of Silurian and Early to Mid-Devonian Isorthinae (Brachiopoda). Palaeontographica, series A, 148(13):34-108, 8 figures, 10 plates.

Walmsley, V. G., A. J. Boucot, C. W. Harper, and N. M. Savage

1968. Visbyella-a New Genus of Resserellid Brachiopod. Palaeontology, 11(2):306-316.

Wang, Su-ming

1978. In Xia Dong Di Qu Zhen Dan Ji Zhi Er Die Ji Di Chen Gu Sheng $W u$ [Stratigraphy and Paleontology of Sinian to Permian of the Eastern Part of Yangtze Gorge]. 381 pages, 113 plates. Peking: Geological Press. [In Chinese.]
Wang, Yü

1949. Maquoketa Brachiopoda of Iowa. Geological Society of America, Memoir, 42:1-55, 12 plates.

1955. New Genera of Brachiopods. Scientia Sinica, 4(2): 327-357, 6 plates.

Wang, Yü, Yu-kan Ching, and Da-wei Fang

1964. Zhong Guo Di Wan Zu Dong Wu Hua Shi, Shang Ce [Brachiopod Fossils of China, Volume 1]. 354 pages, 58 plates. Peking: Scientific Press. [In Chinese.]

1966. Wan Zu Dong Wu Hua Shi [Fossil Brachiopoda]. 702 pages, 567 figures. Peking: Scientific Press. [In Chinese.]

Wang, Yü, Chang-ming Yu, and Qi Wu

1974. [Advances in the Devonian Biostratigraphy of South China.] Memoirs of Nanking Institute of Geology and Palaeontology, 6:1-71, 19 plates. [In Chinese.]

Wang, Yü, and Ruifang Zhu

1979. [Beiliuan (Middle Middle Devonian) Brachiopods from South Guizhou and Central Guangxi.] $P_{a}$ laeontologia Sinica, new series B, 158(15):1-95, 34 plates. [In Chinese.]

Wanner, J., and H. Sieverts

1935. Zur Kenntnis der permischen Brachiopoden von Timor, 1: Lyttoniidae und ihre biologische und stammes-geschichtliche Bedeutung. Neues Jahrbuch für Mineralogie, Geologie, und Palaeontologie, series B, 74:201-281, 25 figures, 4 plates.

Warren, P. S.

1937. A Rhynchonellid Brachiopod from the Bearpaw Formation of Saskatchewan. Royal Society of Canada, Transactions, series 3, 31:1-4, 1 plate.

Waterhouse, J. B.

1964. Permian Brachiopods of New Zealand. New Zealand Geological Survey, Palaeontological Bulletin, 35: 1287, 81 figures, 37 plates.

1966. Lower Carboniferous and Upper Permian Brachiopods from Nepal. Jahrbuch Geologische Bundesanstalt, 12:5-99, 16 plates.

1967a. Upper Permian (Tatarian) Brachiopods from New Zealand. New Zealand Journal of Geology and Geophysics, 10(1):74-118, 5 plates.

1967b. Proposal of Series and Stages for the Permian in New Zealand. Royal Society of New Zealand, Transactions (Geology), 5(6):161-180.

1968a. The Classification and Descriptions of Permian Spiriferida (Brachiopoda) from New Zealand. $\mathrm{Pa}-$ laeontographica, series A, 129(1-3):1-94, 19 figures, 18 plates.

1968b. New Species of Megousia Muir-Wood and Cooper and Allied New Genus from the Permian of Australia and North America. Journal of Paleontology, 42(5): 1171-1185, 3 plates.

1970. Permian Brachiopod Retimarginifera n. gen. n. sp. 
from the Byro Group of Carnarvon Basin, Western Australia. Royal Society of Western Australia, Journal, 53(4):120-128.

1973. New Brachiopod Genera from the New Zealand Permian. Royal Society of New Zealand, Journal, 3(1): 35-42, 6 plates.

1975. New Permian and Triassic Brachiopod Taxa. University of Queensland, Department of Geology, Papers, $7(1): 1-23,2$ plates.

1976. New Occurrences of Brachiopod and Bivalve Species from the Arthurton Group of South Otago and Southland, New Zealand with Comments on Structure and Stratigraphy. Royal Society of New Zealand, Journal, 6(2):227-252, 7 figures.

1978. Permian Brachiopoda and Mollusca from NorthWest Nepal. Palaeontographica, series A, 160(1-6): 1-175, 9 figures, 26 plates.

Waterhouse, J. B., and V. J. Gupta

1977. Brachiopods from the Carboniferous Fenestella Shale of Kashmir. Recent Researches in Geology, 3:1503.

1978a. Early Permian Fossils from the Bijni Tectonic Unit, Garhwal Himalaya. Recent Researches in $\mathrm{Ge}$ ology, 4:410-437.

1978b. Early Carboniferous Brachiopods from the Syringothyris Limestone and Fenestella Shales of Kashmir. Contributions to Himalayan Geology, 1:106-146, 16 plates.

Waterhouse, J. B., and S. Piyasin

1970. Mid-Permian Brachiopods from Khao Phrik, Thailand. Palaeontographica, series A, 135:83-197, 22 figures, 19 plates.

Watson, D.M.S.

1917. Poikilosakos, a Remarkable New Genus of Brachiopods from the Upper Coal Measures of Texas. Geological Magazine, new series, 4:212-219, plate 14.

Wedekind, R.

1926. Die devonische Formation. In W. Salomon, Grundzüge der Geologie, 2:194-226, 6 plates.

Weir, J.

1925. Brachiopoda, Lamellibranchiata, Gastropoda and Belemnites. In B.K.N. Wyllie, and W. R. Smellie, The Collection of Fossils and Rocks from Somaliland. Monographs of the Geological Department of the Hunterian Museum, Glasgow University, 1(6):80-85, plates 11-12.

Weisbord, N. E.

1926. Venezuelan Devonian Fossils. Bulletin of American Paleontology, 11(46):9-24, 5 plates.

Weller, S.

1905. Paraphorhynchus, a New Genus of Kinderhook Brachiopoda. Academy of Science of St. Louis, Transactions, 15:259-264, plate 1 .
1910. Internal Characters of Some Mississippian Rhynchonelliform Shells. Geological Society of America, Bulletin, 21:497-516, 18 figures.

1911. Genera of Mississippian Loop-bearing Brachiopoda. Journal of Geology, 19:439-448.

1914. The Mississippian Brachiopoda of the Mississippi Valley Basin. Illinois State Geological Survey, Monograph, 1:1-508, 83 plates.

Weller, S., and S. St. Clair

1928. Geology of Ste. Genevieve County, Missouri. Missouri Bureau of Geology and Mines, 2nd series, 22:1352,5 figures, 25 plates.

Westbroek, P.

1967. Morphological Observations with Systematic Implications on Some Palaeozoic Rhynchonellida from Europe, with Special Emphasis on the Uncinulidae. Leidse Geologische Mededelingen, 41:1-82, 14 plates.

Westphal, $\mathrm{K}$.

1970. Die Terebratulidae (Brachiopoda) des tieferen Weissjura der schwäbischen Alb. Jahresbericht und Mitteilungen Oberrheinische Geologische Verein, new series, $52: 33-70$.

Weyer, D.

1967. Kitakamithyris Minato 1951 (Brachiopoda, Spiriferida) aus dem Etroeungt (Oberdevon) und Tournai (Unterkarbon) des rheinischen Schiefergebirges. Geologie, 16(4):433-451, 3 figures, 2 plates.

1972. Rozmanaria, ein neues Rhynchonellida-Genus aus dem europaischen Oberfamenne (Brachiopoda, Oberdevon). Geologie, 21(1):84-99, 4 plates.

Whidborne, G. F.

1893. A Monograph of the Devonian Fauna of the South of England. Volume 2, part 3, pages 89-160, plates 7-17. London: Palaeontographical Society.

White, C. A

1865. Descriptions of New Species of Fossils from the Devonian and Carboniferous Rocks of the Mississippi Valley. Boston Society of Natural History, Proceedings, 1862, 9:8-33, 5 figures.

White, C. A., and O. St. John

1867. Descriptions of New Subcarboniferous Coal-Measure Fossils, Collected upon the Geological Survey of Iowa, Together with a Notice of New Generic Characters Involved in Two Species of Brachiopoda. Chicago Academy of Sciences, Transactions, 1 115-127.

Whitehouse, F. W

1928. Notes on Upper Palaeozoic Marine Horizons in Eastern and Western Australia. Australian and New Zealand Association for the Advancement of Science, Report for 1926, Transactions, section C, 18:281-283.

Whitfield, R. P.

1890. Description of a New Genus of Inarticulate 
Brachiopodous Shell. Amencan Museum of Natural History, Bulletin, 3:121-122, 8 figures.

1891. Notes on Some Fossils from Bolivia Collected by Mr. A. F. Wendt and Description of a Remarkable New Genus and Species of Brachiopod. In A. F. Wendt, The Potsi, Bolivia, Silver-District. Transactions of the American Institute of Mining Engineering, 19: $104-107$.

1896. Description of a New Genus of Fossil Brachiopod from the Lower Helderberg Limestones. American Museum of Natural History, Bulletin, 8:231-232, 5 figures.

1908. Notes and Observations of Carboniferous Fossils and Semifossil Shells Brought Home by Members of the Peary Expedition of 1905-1906. American Museum of Natural History, Bulletin, 24:51-58, 4 plates.

Whittington, H. B., and A. Williams

1955. The Fauna of the Derfel Limestone of the Arenig District, North Wales. Roval Society of London, Philosophical Transactions, series B, 238(658):397-430, 6 figures, 3 plates.

Willard, B.

1928. The Brachiopods of the Ottosee and Holston Formations of Tennessee and Virginia. Hariard University, Museum of Comparative Zoologv, Bulletin, 68(6): 255-292, 3 plates.

Williams, A.

1950. New Stropheodontid Brachiopods. Washington Academy of Scuences Journal, 40(9):277-282, 17 figures, I plate.

1951. Llandovery Brachiopods from Wales with Special Reference to the Llandovery District. Geological Society of London, Quarterly Journal, 107(1):85-136, plates 3-8.

1962. The Barr and Lower Ardmillan Series (Cardoc) of the Girvan District, South-West Ayrshire, with Descriptions of the Brachiopoda. Geological Society of London, Memoir, 3:1-267, 13 figures, 25 plates.

1963. The Caradocian Brachiopod Faunas of the Bala District, Merionethshire. Britzsh Museum (Nalural Hislory) Bulletin (Geology), 8(7):327-471, 13 figures, 16 plates.

1974. Ordovician Brachiopoda from the Shelve District, Shropshire. British Museum (Natural Hisiory), Bulletin (Geology), Supplement 2:1-163, 11 figures, 28 plates.

Williams, A.J.

1977. Insight into Lingulid Evolution from the Late Devonian. Alcheringa, 1(3):401-406, 5 figures.

Williams, H. S.

1908. Dalmanellas of the Chemung Formation and a Closely Related New Brachiopod Genus Thiemella.
U.S. National Museum, Proceedings, 34:35-64, plates 2-4.

Williams, H. S., and C. L. Breger

1916. The Fauna of the Chapman Sandstone of Maine Including Descriptions of Some Related Species from the Moose River Sandstone. U.S. Geological Survey, Professional Paper, 89:1-347, 27 plates.

Williams, J. S

1943. Stratigraphy and Fauna of the Louisiana Limestone of Missouri. U.S. Geological Survey, Professional Paper, 203:1-133, 9 plates.

Wilson, A. E

1913. A New Brachiopod from the Base of the Utica. Canada Geological Survey, Department of Mines and Resources, Bulletin, 1:81-86, plate 8.

1926. An Upper Ordovician Fauna from the Rocky Mountains, British Columbia. Canada Geological Survey, Department of Mines and Resources, Bulletin, 44: $1-34,5$ plates.

1932. Ordovician Fossils from the Region of Cornwall, Ontario. Royal Society of Canada, Transactions, series 3, 26(4):373-404, 6 plates.

1944. Rafinesquina and Its Homomorphs [sic] Öpikina and Öpikinella, from the Ottawa Limestone of the Ottawa-St. Lawrence Lowlands. Royal Society of Canada, Tranactions, series 3, 38(4):145-203, 10 figures, 2 plates.

1945. Strophomena and Its Homomorphs [sic] Trigrammaria and Microtrypa from the Ottawa Limestone of the Ottawa-St. Lawrence Lowlands. Royal Society of Canada, Transactions, 39(4):121-150, 2 plates.

Wiman, C.

1905. Studien über des nordbalt ische Silurgebiet. Upsala Unwersity, Geological Institution, Bulletin, 6:12-76, 4 plates.

Winchell, A

1863. Description of the Fossils of the Yellow Sandstones Lying beneath the Burlington Limestone at Burlington, Iowa. Academy of Natural Sciences of Philadelphia, Proceedings, series 2, 7:2-25.

Winchell, N. H., and C. Schuchert

1892. Preliminary Descriptions of New Brachiopoda from the Trenton and Hudson River Groups of Minnesota. American Geologist, 9:284-294.

Winters, S. S

1963. Supai Formation (Permian) of Eastern Arizona. Geological Society of America, Memoir, 89:1-99, 9 plates.

Wirth, E.

1936. Über "Clitambonites" giraldi Martelli and Yangizeella poloi Martelli aus den Ordoviz Chinas. Palaeontologische Zeitschrift (Berlin), 18:292-302, plate 20. 
Wiśniewska, M.

1932. Les Rhynchonellidés du jurassique sup. de Pologne. Palaeontologia Polonica, 2(1):1-71, 6 plates.

Wiśniewska-Żelichowska, M.

1978. Middle Jurassic Brachiopods of the Order Rhynchonellida in the Czestochowa-Wielun and $\mathrm{Za}$ wiercie-Olkusz Areas.] Biuletyn Instytutu Geologicznego, 10(304):65-151, 18 figures, 21 plates. [In Polish; English summary.]

Wolfart, R., F. Bender, and V. Stein

1968. Stratigraphie und Fauna des Ober-Ordoviziums (Caradoc-Ashgill) und Unter-Silurs (Unter-Llandovery) von Sudjordanien. Geologisches Jahrbuch, 85:517-563.

Wright, A. D.

1963. The Fauna of the Portrane Limestone, I: The Inarticulate Brachiopods. British Museum (Natural History) Bulletin (Geology), 8(5):221-254, 4 plates.

1964. The Fauna of the Portrane Limestone, II. Brtish Museum (Natural History) Bulletin (Geology), 9(6): 157-256, 11 plates.

1968. A New Genus of Dicoelosiid Brachiopod from Dalarna. Arkiv for Zoologi, series 2, 22(2):127-138, 1 plate.

Wright, J. D.

1975. Two Species of a New Brachiopod Genus from Middle Devonian Beds of Ontario and Michigan. Contributions from the Museum of Paleontology, University of Michigan, 24(16):171-180, 4 plates.

Xian, Shi-yuan

1978a. In Xi Nan Di Qu Gu Sheng Wu Tu Ce, Gui Zhou Feng Ce, Yi Han Wu Ji-Ni Pen Ji [Paleontological Atlas of Southwestern China, Guizhou (Kweichow) Province, Volume 1 (Cambrian-Devonian)]. 843 pages, 214 plates. Peking. [In Chinese.]

1978b. In Xi Nan Di Qu Gu Sheng Wu Tu Ce, Si-Chuan Feng Ce, Yi Zhen Dan Ji Zhi Ni Peng Ji [Paleontological Atlas of Southwestern China, Sichuan Province, Volume 1 (Sinian-Devonian)]. 617 pages, 185 plates. Peking. [In Chinese.]

19.79. In Xi Bei Di Qu Gu Sheng Wu Tu Ce, Qing-hai Feng Ce, Yi [Paleontological Atlas of Northwestern China, Qinghai Province, Volume 1 (Lower Paleozoic-Cenozoic)]. 393 pages, 96 plates. Peking. [In Chinese.]

$\mathrm{Xu}, \mathrm{Han}-\mathrm{kui}$

1977. [Early Middle Devonian Plicanoplids from Nandan of Guangxi.] Acta Palaeontologica Sinica, 16(1): 59-70, 3 plates. [In Chinese.]

1978. In Xia Dong Di Qu Zhen Dan Ji Zhi Er Die Ji Dl Chen $G u$ Sheng $W u$ [Stratigraphy and Paleontology of Sinian to Permian of the Eastern Part of Yangtze Gorge]. 381 pages, 113 plates. Peking: Geological Press. [In Chinese.] 1979a. Brachiopods from the Tangxiang Formation (Devonian) in Nandan of Guangxi.] Acta Palaeontologica Sinica, 18(4):362-382, 4 plates. [In Chinese.]

1979b. In Xi Ber Di Qu Gu Sheng Wu Tu Ce, Qing-hal Feng $\mathrm{Ce}, \quad Y_{i}$ [Paleontological Atlas of Northwestern China, Qinghai Province, Volume 1 (Lower Paleozoic-Cenozoic)]. 393 pages, 96 plates. Peking. [In Chinese.]

Xu, Han-kui, Jia-yu Rong, and Di-yong Liu

1974. In Xi Nan Di Qu Di Ceng Gu Sheng Wu Shou Ce [A Handbook of the Stratigraphy and Paleontology in Southwest China]. 454 pages, 202 plates. Peking. [In Chinese.]

$\mathrm{Xu}$, Jin Jian

1978a. In Xi Nan Di Qu Gu Sheng Wu Tu Ce, Si-Chuan Feng Ce, Yi Zhen Dan Ji Zhi Ni Peng Ji [Paleontological Atlas of Southwestern China, Sichuan Province, Volume 1 (Sinian-Devonian)]. 617 pages, 185 plates. Peking. [In Chinese.]

1978b. In Xi Nan Di Qu Gu Sheng Wu Tu Ce, Si-Chuan Feng $C e, \operatorname{Er}$ [Paleontological Atlas of Southwestern China, Sichuan Province, Volume 2]. 684 pages, 191 plates. Peking. [In Chinese.]

Yabe, H., and K. M. Hatai

1934. The Recent Brachiopod Fauna of Japan, 1: New Genera and Subgenera. Proceedings of the Imperial Academy of Japan, 10(9):586-589, 4 figures.

1941. On Some Brachiopoda from Kagosima-ken, Kyûsyu. Palaeontological Society of Japan, Transactions, 134:491-495, 1 plate.

Yadrenkina, A. G.

1972. [New Early Ordovician Pentamerids from the Russian Platform.] In I. E. Zanina, editor, Novye Vidy Dreunikh Rasteniz a Bespozvonochnykh SSSR, [3]: 177-180. Moscow: Akademiia Nauk SSSR, Nauchnyi Sovet po Probleme. [In Russian.]

1977. Problemy Stratigrafii Ordovika i Silura Sibiri. Akademiza Nauk SSSR, Sibirskoe Otdelenie, Institute Geologii i Geofiziki, Trudy, 372:1-140, 15 plates.

Yan, Shi-pu

1959. [A New Genus of Visean Spiriferid_Grandispirifer, gen. nov.] Acta Palaeontologica Sinica, 7(2):111-120, 4 figures, 2 plates. [In Chinese.]

Yanagida, J.

1970. Permian Brachiopods from Khao Phrik, near Rat Buri, Thailand. Geology and Palaeontology of Southeast Asia, 8:69-96, plates 14-16.

Yancey, T. E.

1978. Brachiopods and Molluscs of the Lower Permian Arcturus Group, Nevada and Utah, Part 1: Brachiopods, Scaphopods, Rostroconchs, and Bivalves. Bulletins of American Paleontology, 74(303):1367,10 plates. 
Yang, De-li

1977. In Zhong Nan Di Qu Gu Sheng Wu Tu Ce, Er, Wan Gu Sheng Dai Bu Feng [Paleontological Atlas of Central-South China, Volume 2 (Late Paleozoic Part)]. 856 pages, 253 plates. Peking. [In Chinese.]

Yang, Shi-pu

1978a. [Lower Carboniferous Brachiopods of Guizhou Province and Their Stratigraphic Significance.] Professional Papers of Siraligraphy and Palaeonlology, 5: 1-200, 46 plates. [In Chinese.]

1978b. In Xi Nan Di Qu Gu Sheng Wu Tu Ce, Gui Zhou Fen Ce, Er Shi Tan Ji-Di Si Ji [Paleontological Atlas of Southwestern China, Guizhou (Kweichow) Province, Volume 2 (Carboniferous-Quaternary)]. 638 pages, 165 plates. Peking. [In Chinese.]

Yang, Tsun-yi, P. Ting, H. Yin, S. Chang, and C. Fang

1962. [The Brachiopod Fauna of Carboniferous, Permian, and Triassic in the Chilianshan Region.] Monograph on the Geology of the Chilianshan Mountains, 4(4): 1-134, 48 plates. [In Chinese.]

Yang, Tsun-yi, and Guei-yong Xu

1966. Gui Zhou Bu Shang Die Wan Lei Zhong Zhong Shan Tong $Z u$ [Triassic Brachiopods of Central Guizhou (Kweichow) Province, China]. 151 pages, 14 plates. Peking. [In Chinese; English summary.]

Ye, Song-ling, and Sheng-qui Yang

1979. [Brachiopods from the Bagon Lake Series, Northern Tibet.] Acta Palaeontologica Sinica, 18(1):64-71, 1 plate. [In Chinese.]

Yoh, Sen-shing, and Shun-liang Bai

1978. [Devonian Stratigraphy of Dale, Xiangzhou Area, Kwangsi.] I Symposium on the Devonian System of South China 1974, pages 43-63. Peking: Geological Press. [In Chinese.]

Zavodowsky, V. M.

1960. [New Species of Permian Brachiopods from Northeastern USSR.] In Nouye Vidy Dreunikh Rastenii i Bespozvonochnykh SSSR, 1:334-340. Moscow: Vsesoiuznyi Nauchno-Issledovatelskii Geologicheskii Institut, (VSEGEI), Ministerst vo Geologii SSSR. [In Russian.]

Zeng, Qin-luan (= Zheng Qing-luan)

1977. In Zhong Nan Di Qu Gu Sheng Wu Tu Ce, Yi, Zao Gu Sheng Dar Bu Feng |Paleontological Atlas of Central-South China, Volume 1 (Early Paleozoic Part)]. 470 pages, 116 plates. Peking. [In Chinese.]
Zezina, O. N.

1976. [A New Genus of Recent Terebratelloid Brachiopods from the Sublittoral of the Kurilo-Kamchatka Region.] In Donnaia Fauna Kraevykh Morei SSSR, pages 101-105. Moscow: Akademiia Nauk SSSR, Institut Okeanologii. [In Russian.]

1979. Novye Dannye o Brakhiopodakh Dal'nevostochnyka SSSR. In Biologiya Shel'fa Kuril'skikh Ostrovov, pages 222-278. Moscow: Akademiia Nauk SSSR, Dal'nevostochnyi Nauchnyi Tsentr, Institut Biologii Morya.

Zhang, Shou-xin, and Yu-kan Ching

1976. [Late Paleozoic Brachiopods from the Mount Jolmo Lungma Region.] In A Report of Scientific Expedition in the Mount Jolmo Lungma Region (19661968), Paleontology, 2:159-271, 19 plates. Peking: Science Press. [In Chinese.]

Zhao, Ruxuan

1977. In Zhong Nan Di Qu Gu Sheng Wu Tu Ce, Er, Wan Gu Sheng Dai Bu Feng [Paleontological Atlas of Central-South China, Volume 2 (Late Paleozoic Part)]. 856 pages, 253 plates. Peking. [In Chinese.]

Ziegler, A. M.

1966. Unusual Stricklandiid Brachiopods from the Upper Llandovery Beds near Presteigne, Radnorshire. Palaeonlology, 9(2):346-350, 1 plate.

Zittel, K. A. von

1870. Ueber den Brachial-Apparat bei einigen jurassischen Terebratuliden und über eine neue Brachiopodengattung Dimerella. Palaeonlographica, 17:211-222

1880. Class 2, Brachiopoda. In Handbuch der Palaeontologie, 1:641-722. München and Leipzig.

1913. Text-Book of Paleonlology. Translated by C. R. Eastman, 2nd edition, volume 1, 839 pages. London.

1934. Bespozvonochyne. In Osnovy Paleonlologii (Paleozoologiia), 1: xii + 1056 pages. Leningrad-Moscow. [Russian edition of Grundzüge der Paläonlologie, I. Abteilung: Invertebrata.]

Zugmayer, $\mathrm{H}$

1880. Ueber rhätische Brachiopoden. Silzungsberichle der Kaiserlich-Königlichen Geologischen Reichsanstall, Jahrbuch, 30:149-156.

Zyong, Suan, Khao, Rzhonsnitskaya, et al.

1968. Typical Forms of Fossil Faunas in the Devonian Deposits of North Vietnam. 123 pages. Hanoi: Chief Geologic Board of the Vietnamese Democratic Republic. [As cited in Paleontological Journal, 1977(3):333.] 




\section{REQUIREMENTS FOR SMITHSONIAN SERIES PUBLICATION}

Manuscripts intended for series publication receive substantive review within their originating Smithsonian museums or offices and are submitted to the Smithsonian Institutıon Press with approval of the appropriate museum authority on Form SI-36. Requests for special treatment-use of color, foldouts, casebound covers, etc.-require, on the same form, the added approval of designated committees or museum directors.

Review of manuscripts and art by the Press for requirements of series format and style, completeness and clarity of copy, and arrangement of all material, as outlined below, will govern, within the judgment of the Press, acceptance or rejection of the manuscripts and art.

Copy must be typewritten, double-spaced, on one side of standard white bond paper, with $11 / 4$ " margins, submitted as ribbon copy (not carbon or xerox), in loose sheets (not stapled or bound), and accompanied by original art. Minimum acceptable length is 30 pages.

Front matter (preceding the text) should include: title page with only title and author and no other information, abstract page with author/title/series/etc., following the established format, table of contents with indents reflecting the heads and structure of the paper.

First page of text should carry the title and author at the top of the page and an unnum. bered footnote at the bottom consisting of author's name and professional mailing address.

Center heads of whatever level should be typed with initial caps of major words, with extra space above and below the head, but with no other preparation (such as all caps or underline). Run-in paragraph heads should use period/dashes or colons as necessary.

Tabulations within text (lists of data, often in parallel columns) can be typed on the text page where they occur, but they should not contain rules or formal, numbered table heads.

Formal tables (numbered, with table heads, boxheads, stubs, rules) should be sub. mitted as camera copy, but the author must contact the series section of the Press for editorial attention and preparation assistance before final typing of this matter.

Taxonomic keys in natural history papers should use the alined-couplet form in the zoology and paleobiology series and the multi-level indent form in the botany series. If cross-referencing is required between key and text, do not include page references within the key, but number the keyed-out taxa with their corresponding heads in the text.

Synonymy in the zoology and paleobiology series must use the short form (taxon, author, year:page), with a full reference at the end of the paper under "Literature Cited." For the botany series, the long form (taxon, author, abbreviated journal or book title, volume, page, year, with no reference in the "Literature Cited") is optional.

Footnotes, when few in number, whether annotative or bibliographic, should be typed at the bottom of the text page on which the reference occurs. Extensive notes must appear at the end of the text in a notes section. If bibliographic footnotes are required, use the short form (author/brief title/page) with the full reference in the bibliography.

Text-reference system (author/year/page within the text, with the full reference in a "Literature Cited" at the end of the text) must be used in place of bibliographic footnotes in all scientific series and is strongly recommended in the history and technology series: "(Jones, 1910:122)" or ". . . Jones (1910:122)."

Bibliography, depending upon use, is termed "References," "Selected References," or "Literature Cited." Spell out book, journal, and article titles, using initial caps in all major words. For capitalization of titles in foreign languages, follow the national practice of each language. Underline (for italics) book and journal titles. Use the colon-parentheses system for volume/number/page citations: "10(2):5-9." For alinement and arrangement of elements, follow the format of the series for which the manuscript is intended.

Legends for illustrations must not be attached to the art nor included within the text but must be submitted at the end of the manuscript-with as many legends typed, doublespaced, to a page as convenient.

Illustrations must not be included within the manuscript but must be submitted separately as original art (not copies). All illustrations (photographs, line drawings, maps, etc.) can be intermixed throughout the printed text. They should be termed Figures and should be numbered consecutively. If several "figures" are treated as components of a single larger figure, they should be designated by lowercase italic letters (underlined in copy) on the illustration, in the legend, and in text references: "Figure 9b.." If illustrations are intended to be printed separately on coated stock following the text, they should be termed Plates and any components should be lettered as in figures: "Plate 9b.." Keys to any symbols within an illustration should appear on the art and not in the legend.

A few points of style: (1) Do not use periods after such abbreviations as "mm, ft, yds, USNM, NNE, AM, BC." (2) Use hyphens in spelled-out fractions: "two-thirds." (3) Spell out numbers "one" through "nine" in expository text, but use numerals in all other cases if possible. (4) Use the metric system of measurement, where possible, instead of the English system. (5) Use the decimal system, where possible, in place of fractions. (6) Use day/month/year sequence for dates: "9 April 1976." (7) For months in tabular listings or data sections, use three-letter abbreviations with no periods: "Jan, Mar, Jun," etc.

Arrange and paginate sequentially EVERY sheet of manuscript-including ALL front matter and ALL legends, etc., at the back of the text-in the following order: (1) title page, (2) abstract, (3) table of contents, (4) foreword and/or preface, (5) text, (6) appendixes, (7) notes, (8) glossary, (9) bibliography, (10) index, (11) legends. 
